\title{
Development and Evaluation of Stochastic \\ Rainfall Models for Urban Drought Security
}

\section{Assessment}

\author{
AFM Kamal Chowdhury \\ Graduate Certificate (Engineering Geology) \\ BSc Engineering (Civil and Environmental)
}

A thesis submitted for the degree of Doctor of Philosophy at the University of Newcastle, Australia

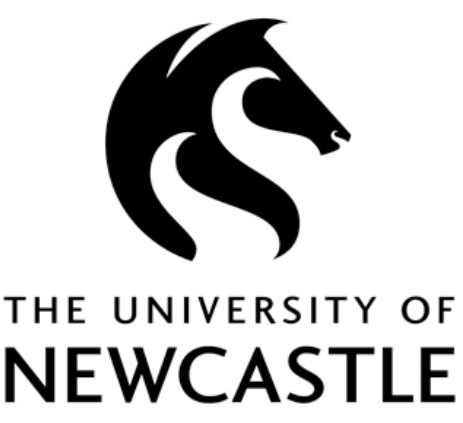

AUSTRALIA

December 2016 

The thesis contains no material which has been accepted for the award of any other degree or diploma in any university or other tertiary institution and, to the best of my knowledge and belief, contains no material previously published or written by another person, except where due reference has been made in the text. I give consent to the final version of my thesis being made available worldwide when deposited in the University's Digital Repository, subject to the provisions of the Copyright Act 1968.

AFM Kamal Chowdhury 
Page ii 
I hereby certify that the work embodied in this thesis contains published paper/s/scholarly work of which I am a joint author. I have included as part of the thesis a written statement, endorsed by my supervisor, attesting to my contribution to the joint publication/s/scholarly work.

AFM Kamal Chowdhury

Professor Garry Willgoose Principal Supervisor 


\section{Acknowledgements}

I am grateful to the almighty for giving me the strength and ability to accomplish this $\mathrm{PhD}$.

I would like to thank my supervisors, Professor Garry Willgoose, Professor George Kuczera and Dr. Natalie Lockart for their time, support and advises. I am also grateful to Dr. Anthony Kiem and all other colleagues in ESCCI-ECL project. I have learned research as well as culture and life by working with this outstanding team of ESCCI-ECL project. I would like to thank Ms. Cherie Pilgrim and Ms. Anne Ramsey for their time and supports.

I am grateful to my parents, who have dreamt and done their best to ensure my study, despite the adverse socio-economic conditions. My heartfelt thanks to my parents in law, my brothers and sisters, and all other family and friends for their prayers and encouragements. I cannot thank enough my wife, Shanta Islam. Thank you, Shanta for the support and sacrifices over the last three and half years. I am also grateful to all of my ex-teachers, including Professor Aktarul Islam Chowdhury, the supervisor of my undergraduate thesis.

Funding for this project was provided by an Australian Research Council Linkage Grant LP120200494, the NSW Office of Environment and Heritage, NSW Department of Financial Services, NSW office of Water, and Hunter Water Corporation. 


\section{Table of Contents}

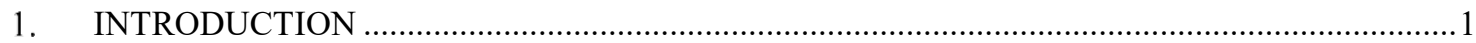

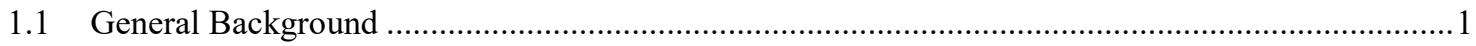

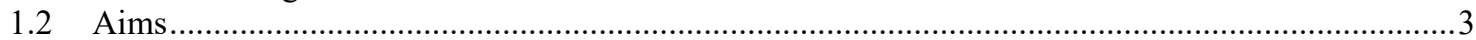

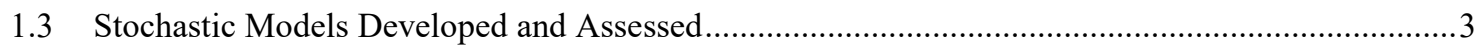

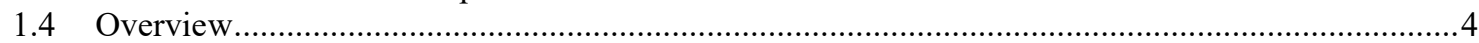

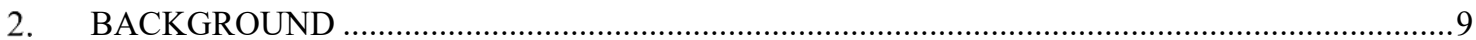

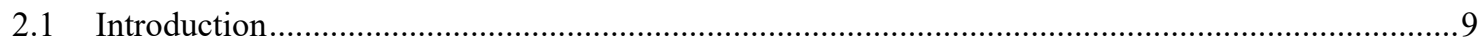

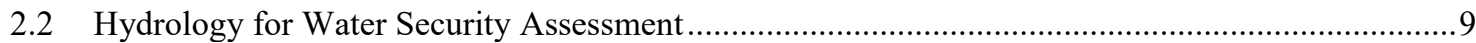

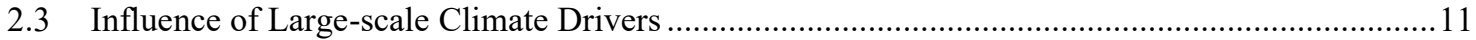

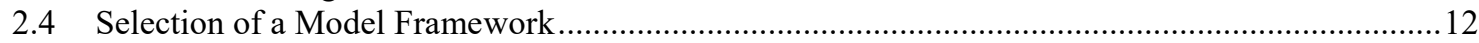

2.5 Resolving the Underestimation of Long-term Variability .............................................................

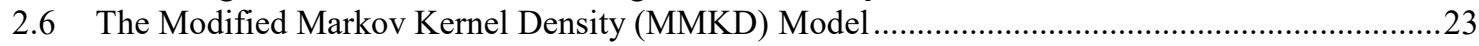

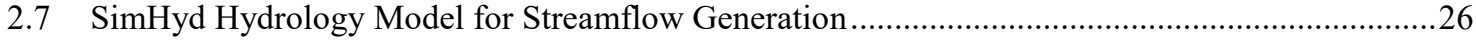

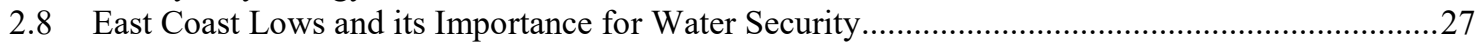

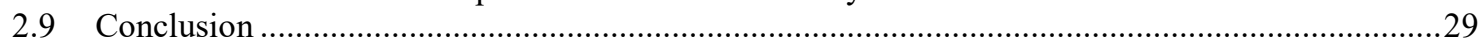

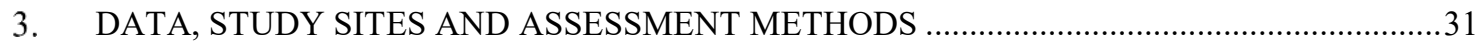

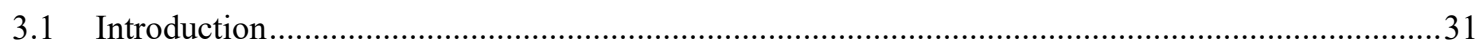

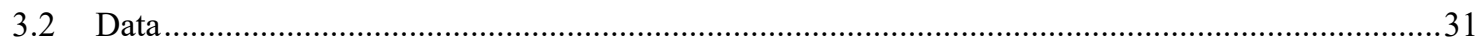

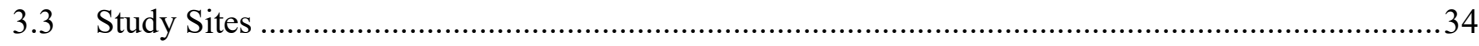

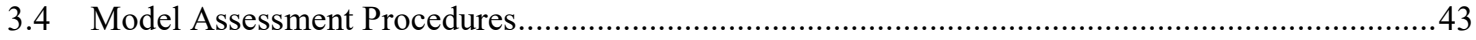

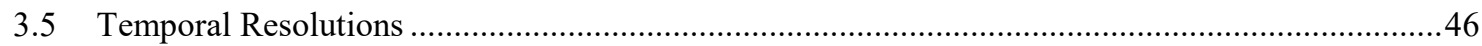

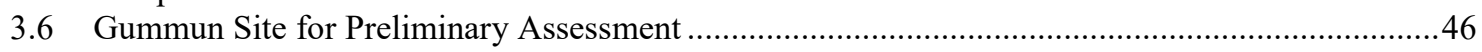

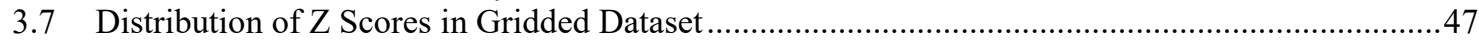

3.8 Calculation of Temporally and Spatially Averaged Statistics.........................................................48

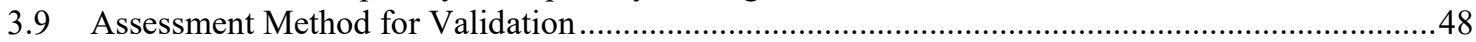

3.10 Assessment Method for Streamflow Generation .......................................................................49

4. DEVELOPMENT OF THE MARKOV CHAIN DAILY RAINFALL MODEL …..........................50

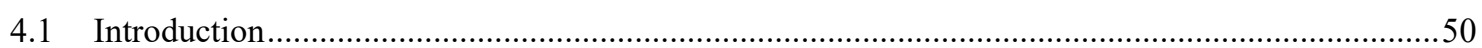

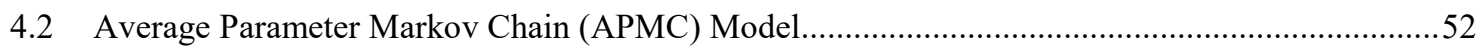

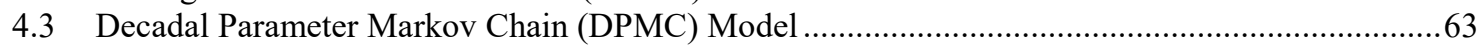

4.4 Compound Distribution Markov Chain (CDMC) Model ..................................................................71

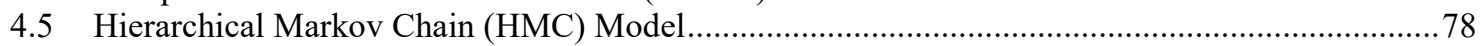

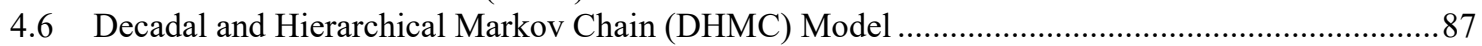

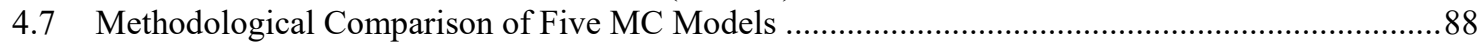

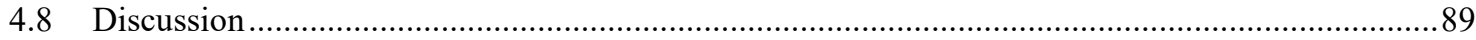

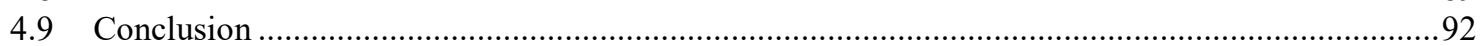

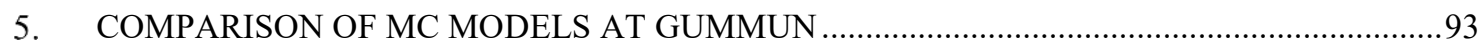

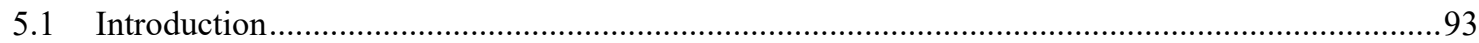

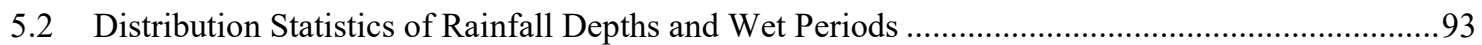

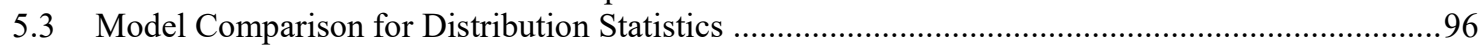

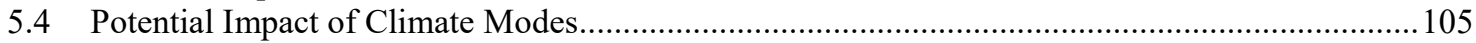

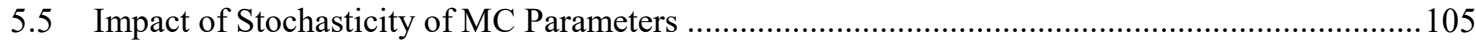

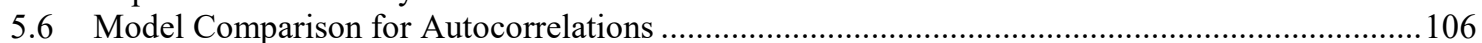

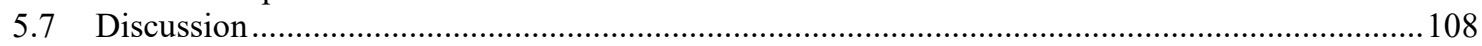

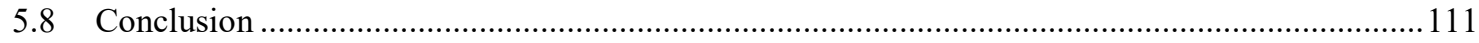




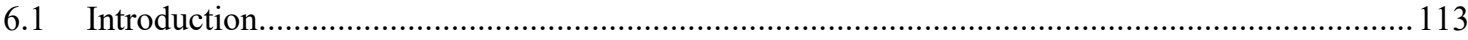

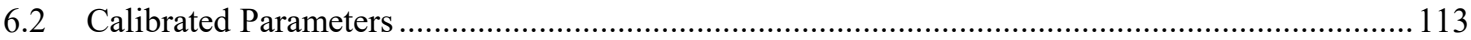

6.3 Distribution Statistics of Rainfall Depths and Wet Periods .................................................... 118

6.4 Model Comparison for Distribution of Rainfall Depths ........................................................ 122

6.5 Model Comparison for Distribution of Wet Periods .............................................................. 129

6.6 Summary of Model Comparison for Distribution Statistics........................................................ 139

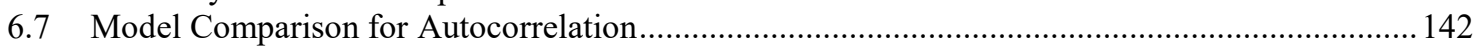

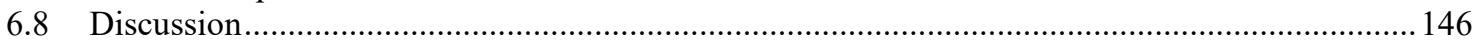

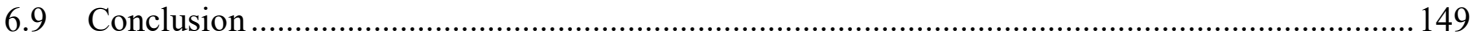

7. COMPARISON OF MC MODELS IN WILLIAMS RIVER SITE .......................................... 150

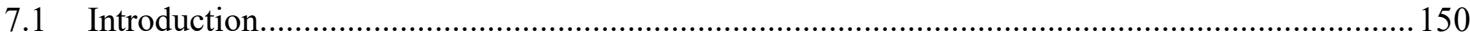

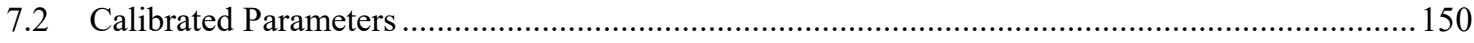

7.3 Distribution Statistics of Rainfall Depths and Wet-Dry Periods.............................................. 154

7.4 Model Comparison for Distribution of Rainfall Depths ......................................................... 157

7.5 Model Comparison for Distribution of Wet-Dry Periods ......................................................... 160

7.6 Summary of Model Comparison for Distribution Statistics................................................... 166

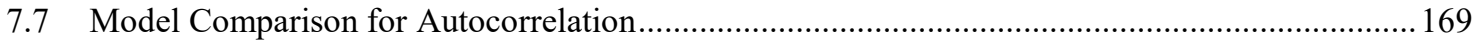

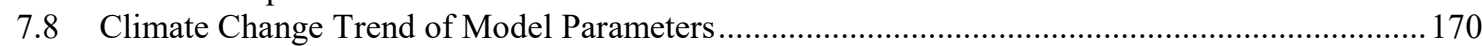

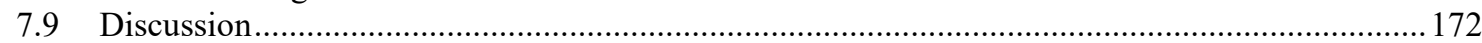

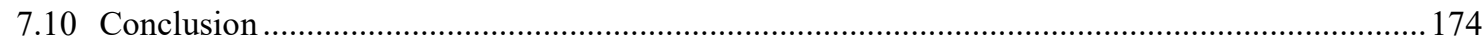

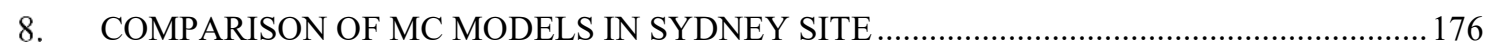

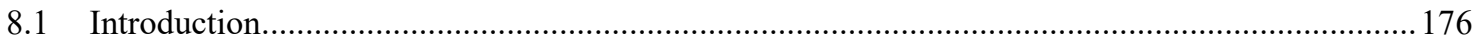

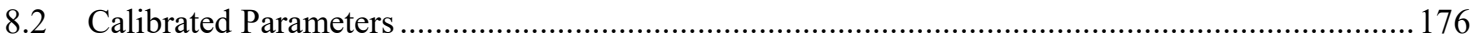

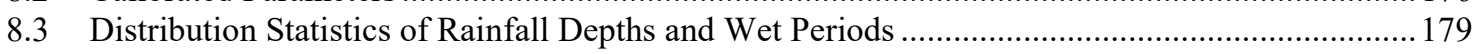

8.4 Model Comparison for Distribution of Rainfall Depths ........................................................ 181

8.5 Model Comparison for Distribution of Wet Periods .......................................................... 183

8.6 Summary of Model Comparison for Distribution Statistics................................................... 187

8.7 Model Comparison for Autocorrelation................................................................................. 190

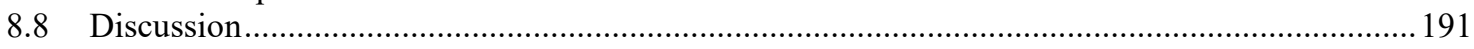

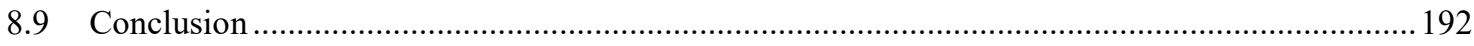

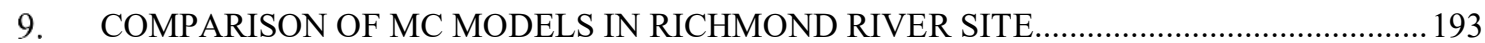

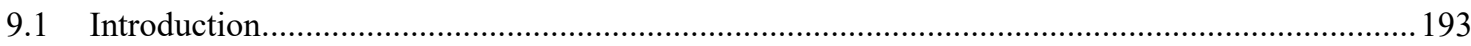

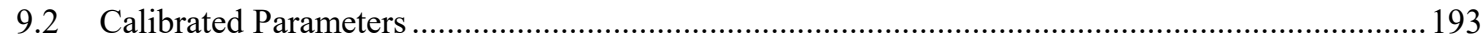

9.3 Distribution Statistics of Rainfall Depths and Wet Periods ..................................................... 196

9.4 Model Comparison for Distribution of Rainfall Depths ….................................................. 198

9.5 Model Comparison for Distribution of Wet Periods ............................................................... 199

9.6 Summary of Model Comparison for Distribution Statistics.................................................202

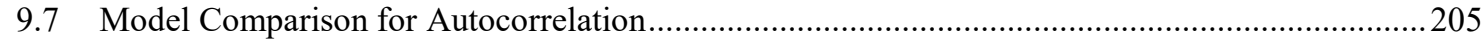

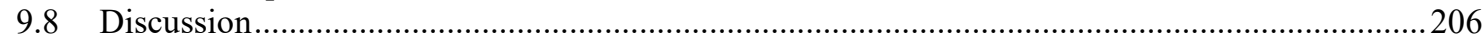

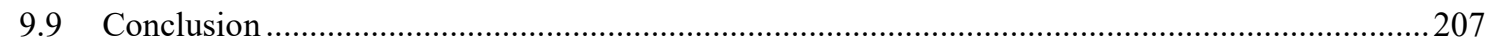

10. COMPARISON OF MC MODELS IN BEGA RIVER SITE ..............................................2208

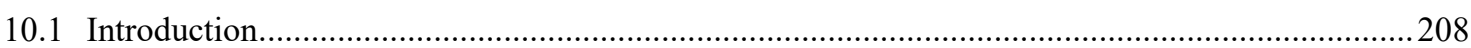

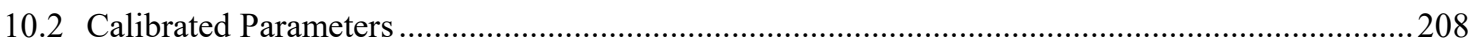

10.3 Distribution Statistics of Rainfall Depths and Wet Periods ...................................................211

10.4 Model Comparison for Distribution of Rainfall Depths ...........................................................2212

10.5 Model Comparison for Distribution of Wet Periods.................................................................2214

10.6 Summary of Model Comparison for Distribution Statistics.......................................................2218

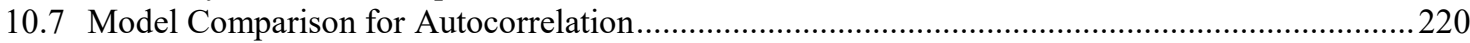

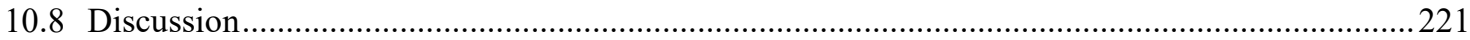

10.9 Conclusion 


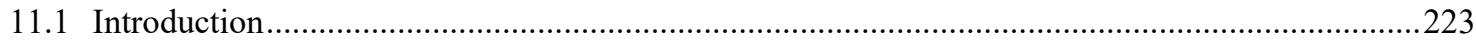

11.2 Five Case Study Sites along East Coast of Australia ................................................................223

11.3 Rainfall Variability along the East Coast of Australia ..............................................................224

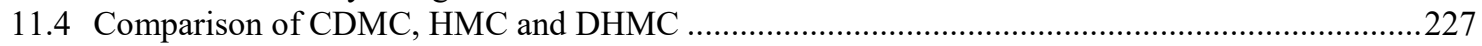

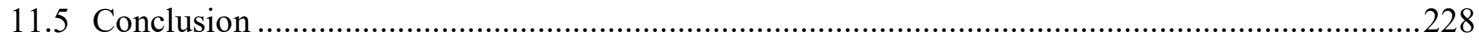

12. COMPARISON OF MC AND MMKD FOR RAINGAUGE DATA AROUND AUSTRALIA..229

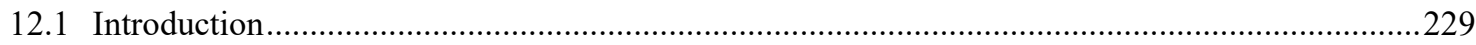

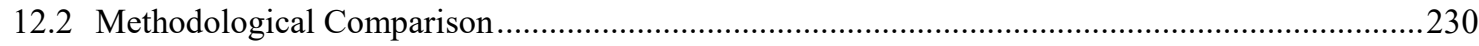

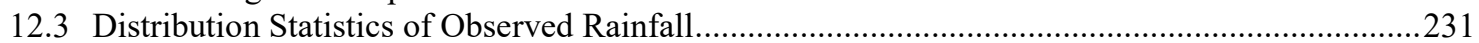

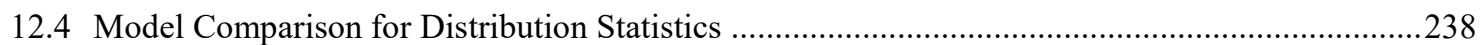

12.5 Autocorrelations of Monthly Wet Days and Rainfall Depth...................................................249

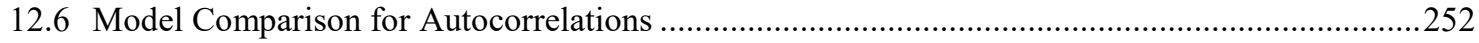

12.7 Insights for Simulation of Mean of Multiyear Rainfall Depths ..............................................253

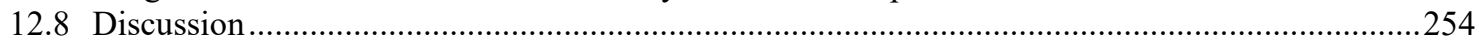

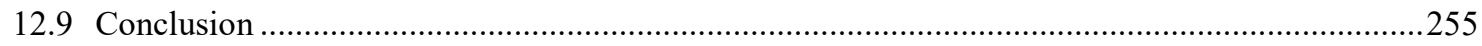

13. COMPARISON OF MC AND MMKD FOR RAINGAUGE DATA AROUND SYDNEY ........257

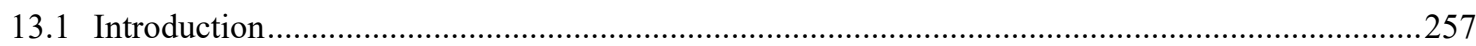

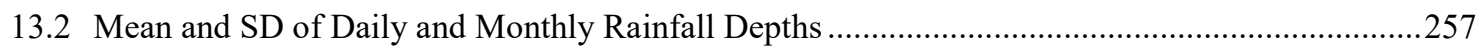

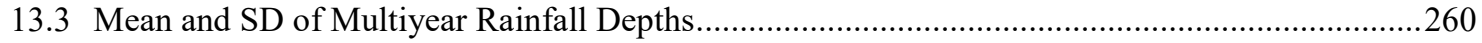

13.4 Mean and SD of Monthly and Multiyear Number of Wet Days ..............................................261

13.5 Mean and SD of Monthly and Annual Mean Length of Wet Spells .........................................263

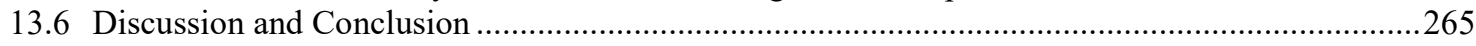

14. COMPARISON OF MC AND MMKD FOR VALIDATION PERIODS .................................266

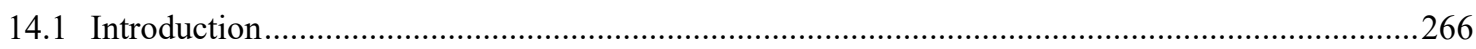

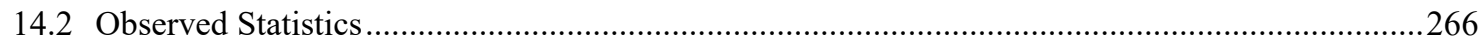

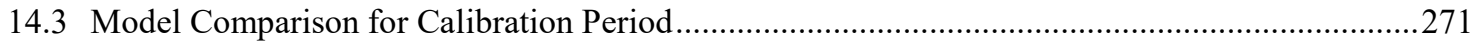

14.4 Model Comparison for Validation Period .............................................................................2 271

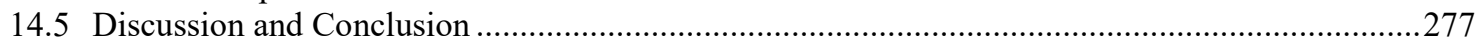

15. COMPARISON OF MC AND MMKD FOR STREAMFLOW GENERATION .......................279

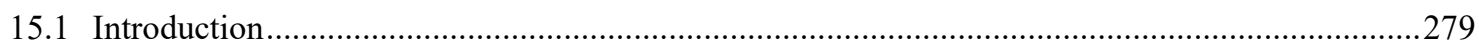

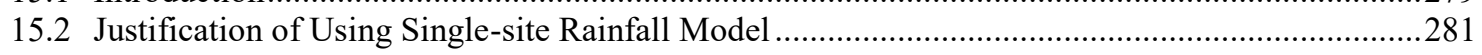

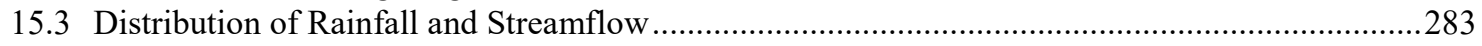

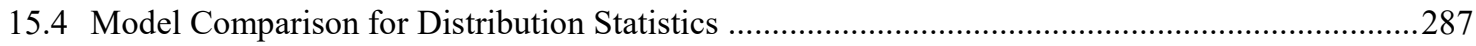

15.5 Model Comparison for Autocorrelation.............................................................................298

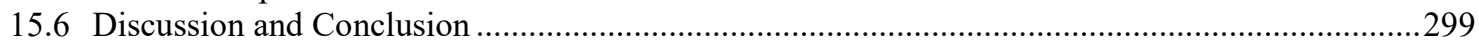

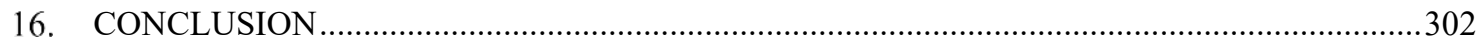

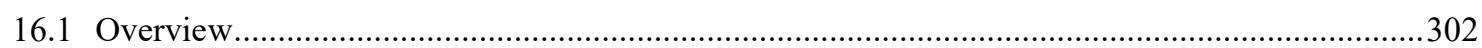

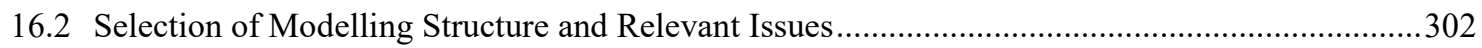

16.3 Development of Markov Chain Daily Rainfall Models ........................................................303

16.4 Comparison of Five MC Models for Rainfall Generation …................................................303

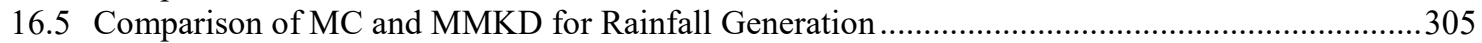

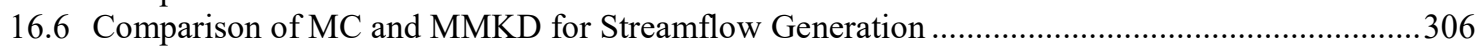

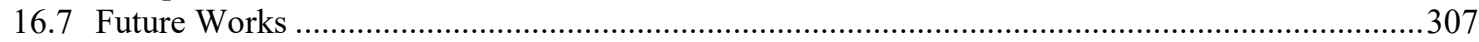

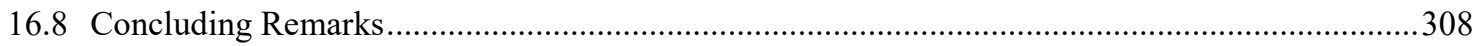

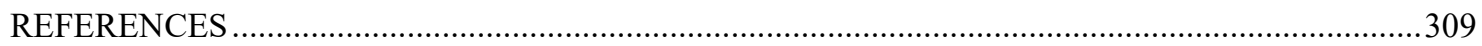

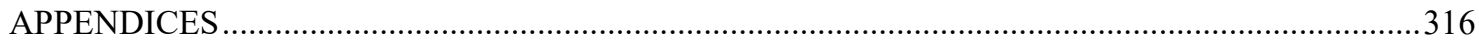




\section{List of Figures}

Figure 1.1: Flowchart of the chapter arrangement of this study. ..................................................... 5

Figure 3.1: Elevation map for NARCliM data boundary in the eastern seaboard of Australia. ................32

Figure 3.2: Location of the five case study sites (in rectangular boxes) of this study. The green circles

indicate the major cities/towns along the east coast of NSW.

Figure 3.3: NARCliM elevation map (at $10 \mathrm{~km}$ resolution) of the case study area in Goulburn River site.

Figure 3.4: NARCliM elevation map (at $10 \mathrm{~km}$ resolution) of the case study area in Williams River site.

White region is in ocean.

Figure 3.5: NARCliM elevation map (at $10 \mathrm{~km}$ resolution) of the case study area in the Sydney site. The white region is in the ocean.

Figure 3.6: NARCliM elevation map (at $10 \mathrm{~km}$ resolution) of the case study area in Richmond River site.

The white region is in the ocean.

Figure 3.7: NARCliM elevation map (at $10 \mathrm{~km}$ resolution) of the case study area in Bega River site. The white region is in the ocean. 38

Figure 3.8 Location map of 12 raingauge stations around Australia. 39

Figure 3.9: Location map of 30 raingauge stations around Sydney (from Mehrotra et al. [2015]). Station

numbers correspond to the station names listed in Table 3.2.

Figure 3.10: The Grahamstown, Tillegra and Chichester sub-catchments of Williams River site are shown in left. The location and elevation of the sub-catchments area within Williams River site are shown in right. The boxes represent the $10 \mathrm{~km} \times 10 \mathrm{~km}$ NARCliM grid pixels. The (x, y) coordinates shown in the left figure corresponds to the $(\mathrm{x}, \mathrm{y})$ coordinates of right figure. The distance scale refers to the left hand figure with the catchment boundaries.

Figure 3.11: Location of Gummun and Blackville, and 17 other raingauge stations within the NARCliM data boundary at Goulburn River site in the east coast of Australia.

Figure 4.1: Schematic of the APMC. Compare the red boxes with the same of Figure 4.10, Figure 4.21,

Figure 4.30 and Figure 4.31 to see the changes in DPMC, CDMC, HMC and DHMC respectively.

Figure 4.2: MC parameters P00 and P11 (transition probabilities of dry-to-dry and wet-to-wet day respectively), and Gamma distribution parameters $\mu$ and $\sigma$ (mean and SD of wet day rainfall depths respectively) of APMC in RCM2 and AWAP. The parameters are shown for each month, where the first month is January.

Figure 4.3: Month-to-month autocorrelations of monthly rainfall depths and number of wet days in

RCM2 and AWAP

Figure 4.4: Distribution of wet day rainfall depths in RCM2 and APMC simulation for January, April, July, and September (typical months)......

Figure 4.5: Distribution of wet day rainfall depths between $0.3 \mathrm{~mm}$ and $1 \mathrm{~mm}$ in RCM2 and APMC simulation for January, April, July and September (typical months) 
Figure 4.6: Gamma distribution with different $\alpha$ and $\beta$.

Figure 4.7: Changes of $\mu$ and $\sigma$ with changes of $\alpha$ and $\beta$......

Figure 4.8: Box-cox transformation of wet day rainfall depths in RCM2 using $\lambda=0.1402$ (left).

Correlation of transformed rainfall depths in successive wet days (right).

Figure 4.9: Simulation of wet and dry spells in APMC for January (a typical month) for RCM2

Figure 4.10: Schematic of the DPMC. Compare the red boxes with the same of Figure 4.1 Figure 4.21,

Figure 4.30 and Figure 4.31 to see the relative differences in APMC, CDMC, HMC and DHMC respectively.

Figure 4.11: Comparison of the decadal variability of the DPMC parameters (wet-to-wet probabilities P11 and mean wet day rainfalls $\mu$ ) with the APMC parameters. The coloured lines are the values of DPMC parameters and the thick black lines are the values of APMC parameters.

Figure 4.12: Sampling distributions of DPMC parameters (P11 and $\mu$ ) for January and July as two representative months. The blue horizontal bars are the $5^{\text {th }}$ to $95^{\text {th }}$ percentile bounds, the boxes are the $25^{\text {th }}$ to $75^{\text {th }}$ percentile bounds, and the red horizontal bars are the $50^{\text {th }}$ percentiles of the $6 \times$ 1000 realisations of simulated parameter. The red dots show the DPMC parameter values, calculated from each of the 10-year samples of RCM2 data at Gummun. The green dots show the respective APMC value of a parameter.

Figure 4.13: Parameters (P11 and $\mu$ ) of sub-samples of RCM2 according to the two IPO (positive and negative) and three ENSO phases (La Niña, Neutral and El Niño).

Figure 4.14: Correlation between the 12x 60 values of each parameter (P00, P11, $\mu$, and $\sigma$ ) for RCM2 and ONI, nino3.4, MEI, PDOI climate indices.

Figure 4.15: Correlation between the $12 \mathrm{x} 60$ values of each parameter (P00, P11, $\mu$, and $\sigma$ ) for RCM2 and SOI, SAMI, DMI, and IPOI climate indices.

Figure 4.16: Boxplot distribution of $\mu$ and $\sigma$ for every month. Each boxplot is fitted to the 60 values a parameter for respective month. The blue horizontal bars are the $5^{\text {th }}$ to $95^{\text {th }}$ percentile bounds, the boxes are the $25^{\text {th }}$ to $75^{\text {th }}$ percentile bounds, and the red horizontal bars are the $50^{\text {th }}$ percentiles. The green dots show the APMC parameters (equivalent to the average of 60 distributed values)........73

Figure 4.17: Lognormal probability plots of $\mu$ for each month for RCM2 ..........................................74

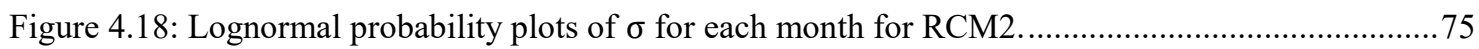

Figure 4.19: Correlation between $\log \mu$ and $\log \sigma$ for each month for RCM2 ..................................75

Figure 4.20: Month-to-month autocorrelations of $\log \mu$ and $\log \sigma$ for RCM2 and AWAP.....................76

Figure 4.21: Schematic of the CDMC (compare with the Figure 4.1, Figure 4.10, Figure 4.30 and Figure

Figure 4.22: Normal probability plots of P00 for each month for RCM2 .........................................79

Figure 4.23: Normal probability plots of P11 for each month for RCM2 ........................................79

Figure 4.24: Correlation between P00 and P11 for each month for RCM2 .......................................80

Figure 4.25: Month-to-month autocorrelations of P00 and P11 for RCM2 and AWAP. ......................80

Figure 4.26: Correlation between $\log \mu$ and P11 for each month for RCM2 .....................................81

Figure 4.27: Correlation between $\log \sigma$ and P11 for each month for RCM2 …...............................82 
Figure 4.28: Correlation between $\log \mu$ and P00 for each month for RCM2.

Figure 4.29: Correlation between $\log \sigma$ and P00 for each month for RCM2.

Figure 4.30: Schematic of the HMC (compare with the Figure 4.1, Figure 4.10, Figure 4.21 and Figure 4.31).

Figure 4.31: Schematic of the DHMC (compare with the Figure 4.1, Figure 4.10, Figure 4.21 and Figure

Figure 5.1: Mean and SD of rainfall depths for RCM2 and AWAP at daily and monthly resolutions. ....94

Figure 5.2: Mean and SD of rainfall depths for RCM2 and AWAP at multiyear (aggregated rainfall depths for multiple overlapping years) resolutions.

Figure 5.3: Mean and SD of monthly number of wet days for RCM2 and AWAP.............................95

Figure 5.4: Mean and SD of multiyear number of wet days in RCM2 and AWAP. ............................95

Figure 5.5: Mean and SD of monthly mean length of wet spells for RCM2 and AWAP......................96

Figure 5.6: Comparison of APMC, DPMC, CDMC, HMC, and DHMC to reproduce the mean and SD of daily (wet day) rainfall depths for RCM2 and AWAP.

Figure 5.7: Comparison of APMC, DPMC, CDMC, HMC, and DHMC to reproduce the mean and SD of monthly rainfall depths for RCM2 and AWAP.

Figure 5.8: Comparison of APMC, DPMC, CDMC, HMC and DHMC to reproduce the mean and SD of multiyear rainfall depths for RCM2 and AWAP.

Figure 5.9: Comparison of APMC, DPMC, CDMC, HMC and DHMC to reproduce the mean and SD of monthly number of wet days for RCM2 and AWAP. 100

Figure 5.10: Comparison of APMC, DPMC, CDMC, HMC and DHMC to reproduce the mean and SD of multiyear number of wet days for RCM2 and AWAP. The Z scores of APMC and CDMC for AWAP are out of the +6 range.

Figure 5.11: Comparison of APMC, DPMC, CDMC, HMC, and DHMC to reproduce the mean and SD of monthly mean length of wet spells for RCM2 and AWAP. ..... 102

Figure 5.12: Comparison of APMC, DPMC, CDMC, HMC, and DHMC to reproduce the mean and SD of annual mean length of wet spells for RCM2 and AWAP.

Figure 5.13: Comparison of DPMC and other DPMC-like models (e.g. model with sub-samples of 5-year length, model with sub-samples according to IPO and ENSO phases) to reproduce the mean and SD of monthly rainfall depths for RCM2.

Figure 5.14: Comparison of HMC and other HMC-like models (e.g. model with lag-1 autocorrelation equation and model with multivariate sampling for stochastic MC parameters) to reproduce the mean and SD of monthly rainfall depths for RCM2.

Figure 5.15: Comparison of APMC, DPMC, CDMC, HMC and DHMC to reproduce the month-to-month autocorrelations of monthly rainfall depths and monthly number of wet days for RCM2.

Figure 5.16: Comparison of APMC, DPMC, CDMC, HMC and DHMC to reproduce the month-to-month autocorrelations of monthly rainfall depths and monthly number of wet days for AWAP. 108

Figure 6.1: Intra-annual and spatial variability of dry-to-dry transition probabilities (P00). The colourbars indicate the parameter values. 
Figure 6.2: Intra-annual and spatial variability of wet-to-wet transition probabilities (P11). The

colourbars indicate the parameter values.

Figure 6.3: Intra-annual and spatial variability of the mean of wet day rainfall depths ( $\mu$ ) for all RCMs and AWAP at Goulburn. The colourbars indicate the parameter values.

Figure 6.4: Intra-annual and spatial variability of the SD of wet day rainfall depths $(\sigma)$ for all RCMs and

AWAP at Goulburn. The colourbars indicate the parameter values. 116

Figure 6.5: Correlation between elevation and model parameters for each month. 117

Figure 6.6: Mean and SD of rainfall depths at daily, monthly and multiyear resolutions.

Figure 6.7: Intra-annual and spatial variability of the mean monthly number of wet days. 120

Figure 6.8: Intra-annual and spatial variability of the SD of monthly number of wet days 120

Figure 6.9: Mean and SD of number of wet days at monthly and multiyear resolutions.

Figure 6.10: Mean and SD of mean length of wet spells at monthly and annual resolutions. 121

Figure 6.11: Z Scores of CDMC, HMC and DHMC for the mean of wet day rainfall depths. 123

Figure 6.12: Z Scores of CDMC, HMC and DHMC for the SD of wet day rainfall depths. 124

Figure 6.13: Z Scores of CDMC, HMC, and DHMC for the SD of monthly rainfall depths.... 126

Figure 6.14: Z Scores of CDMC, HMC and DHMC for the mean annual rainfall depths.

Figure 6.15: Z Scores of CDMC, HMC and DHMC for the SD of multiyear rainfall depths. 128

Figure 6.16: Z Scores of CDMC, HMC and DHMC for the mean of monthly number of wet days.......130

Figure 6.17: Z Scores of CDMC, HMC and DHMC for the SD of monthly number of wet days. 131

Figure 6.18: Z Scores of CDMC, HMC and DHMC for the mean of multiyear number of wet days.....132

Figure 6.19: Z Scores of CDMC, HMC and DHMC for the SD of multiyear number of wet days. .......133

Figure 6.20: Z Scores of CDMC, HMC and DHMC for the mean of monthly mean length of wet spells.

Figure 6.21: Z Scores of CDMC, HMC and DHMC for the SD of monthly mean length of wet spells. 136 Figure 6.22: Z Scores of CDMC, HMC and DHMC for the mean of annual mean length of wet spells. 137 Figure 6.23: Z Scores of CDMC, HMC and DHMC for the SD of annual mean length of wet spells....138 Figure 6.24: Month-to-month autocorrelations of monthly rainfall depths and number of wet days in all

RCMs and AWAP at Goulburn.

Figure 6.25: Comparison of CDMC, HMC and DHMC to reproduce the month-to-month autocorrelations of monthly rainfall depths for all RCMs and AWAP.

Figure 6.26: Comparison of CDMC, HMC and DHMC to reproduce the month-to-month autocorrelations of monthly number of wet days for all RCMs and AWAP.

Figure 7.1: Intra-annual and spatial variability of dry-to-dry and wet-to-wet transition probabilities. The colourbars indicate the parameter values.

Figure 7.2: Intra-annual and spatial variability of mean and SD of wet day rainfall depths. The colourbars indicate the parameter values. .152

Figure 7.3: Correlation between elevation and model parameters (APMC values)..... 153

Figure 7.4: Mean and SD of rainfall depths at daily, monthly and multiyear resolutions. .154

Figure 7.5: Mean and SD of number of wet and dry days at monthly and annual resolutions. 155 
Figure 7.6: Mean and SD of mean length of wet and dry spells at monthly and annual resolutions. 156

Figure 7.7: Z Scores of CDMC, HMC and DHMC for the SD of multiyear rainfall depths.... .158

Figure 7.8: 100 CDMC realisations of SD of multiyear rainfall depths (dashed lines) for RCM2 data at two NARCliM pixels. The green solid lines indicate the expected values with $95 \%$ confidence limit. The red lines indicate the RCM2 values. 159

Figure 7.9: Z Scores of CDMC, HMC and DHMC for the mean of monthly mean length of wet spells.

Figure 7.10: Z Scores of CDMC, HMC and DHMC for the mean of monthly mean length of dry spells. 162

Figure 7.11: Z Scores of CDMC, HMC and DHMC for the SD of monthly number of wet days. 163

Figure 7.12: Z Scores of CDMC, HMC and DHMC for the SD of monthly mean length of dry spells. 164 Figure 7.13: Z Scores of CDMC, HMC and DHMC for the SD of annual mean length of wet spells... 165 Figure 7.14: Z Scores of CDMC, HMC and DHMC for the SD of multiyear number of wet days. ....... 166 Figure 7.15: Month-to-month autocorrelations of monthly rainfall depths in all RCMs and AWAP. .... 169 Figure 7.16: Month-to-month autocorrelations of monthly number of wet days. 169

Figure 7.17: Intra-annual and spatial variability of model parameters (APMC values) for reanalysis and CCCMA 3.1 of RCM2. 171

Figure 8.1: Intra-annual and spatial variability of dry-to-dry and wet-to-wet transition probabilities. The colourbars indicate the parameter values.

Figure 8.2: Intra-annual and spatial variability of mean and SD of wet day rainfall depths. The colourbars indicate the parameter values. 178

Figure 8.3: Correlation between elevation and model parameters for each month. 179

Figure 8.4: Spatial variability of the SD of multiyear rainfall depths. 180

Figure 8.5: Spatial variability of the SD of multiyear number of wet days. 180

Figure 8.6: Z Scores of CDMC, HMC and DHMC for the SD of multiyear rainfall depths...... 182

Figure 8.7: Z Scores of CDMC, HMC and DHMC for the SD of monthly number of wet days. ........... 184

Figure 8.8: Z Scores of CDMC, HMC and DHMC for the SD of multiyear number of wet days. 186

Figure 8.9: Z Scores of CDMC, HMC and DHMC for the SD of annual mean length of wet spells...... 187 Figure 8.10: Month-to-month autocorrelations of monthly rainfall depths in all RCMs and AWAP.... 190 Figure 8.11: Month-to-month autocorrelations of monthly number of wet days in all RCMs and AWAP.

Figure 9.1: Intra-annual and spatial variability of dry-to-dry and wet-to-wet transition probabilities. The colourbars indicate the parameter values.

Figure 9.2: Intra-annual and spatial variability of mean and SD of wet day rainfall depths. The colourbars indicate the parameter values.

Figure 9.3: Correlation between elevation and model parameters for each month. .............................196

Figure 9.4: Spatial variability of the SD of multiyear rainfall depths. ............................................... 197

Figure 9.5: Spatial variability of the SD of multiyear number of wet days. ........................................ 197

Figure 9.6: Z Scores of CDMC, HMC and DHMC for the SD of multiyear rainfall depths................. 199 
Figure 9.7: Z Scores of CDMC, HMC and DHMC for the SD of monthly number of wet days. ..........200

Figure 9.8: Z Scores of CDMC, HMC and DHMC for the SD of multiyear number of wet days. .........201

Figure 9.9: Z Scores of CDMC, HMC and DHMC for the SD of annual mean length of wet spells. .....202

Figure 9.10: Month-to-month autocorrelations of monthly rainfall depths in all RCMs and AWAP....205

Figure 9.11: Month-to-month autocorrelations of monthly number of wet days in all RCMs and AWAP.

Figure 10.1: Intra-annual and spatial variability of dry-to-dry transition probabilities. ......................209

Figure 10.2: Intra-annual and spatial variability of wet-to-wet transition probabilities. .......................209

Figure 10.3: Intra-annual and spatial variability of mean wet day rainfall depths.............................209

Figure 10.4: Intra-annual and spatial variability of SD of wet day rainfall depths............................210

Figure 10.5: Correlation between elevation and model parameters for each month.............................210

Figure 10.6: Spatial variability of the SD of multiyear rainfall depths............................................211

Figure 10.7: Spatial variability of the SD of multiyear number of wet days. .....................................211

Figure 10.8: Z Scores of CDMC, HMC and DHMC for the SD of multiyear rainfall depths. ..............213

Figure 10.9: Z Scores of CDMC, HMC and DHMC for the SD of monthly number of wet days. ........215

Figure 10.10: Z Scores of CDMC, HMC and DHMC for the SD of multiyear number of wet days. .....217

Figure 10.11: Z Scores of CDMC, HMC and DHMC for the SD of annual mean length of wet spells. .217

Figure 10.12: Month-to-month autocorrelations of monthly rainfall depths. ...................................220

Figure 10.13: Month-to-month autocorrelations of monthly number of wet days.

Figure 12.1: Mean and SD of monthly number of wet days and rainfall depth for the coastal (top 6 panels), inland (middle 4 panels), and monsoonal (bottom 2 panels) stations around Australia. Each left $\mathrm{y}$-axis denotes mean and SD of number of wet days per month, and each right $\mathrm{y}$-axis denotes mean and SD of monthly rainfall depths. Note the change in vertical scales.

Figure 12.2: SD of multiyear number of wet days (each left y-axis) and multiyear rainfall depth (each right y-axis) for 1 to 10 overlapping years. Note the change in vertical scale. ...........................236

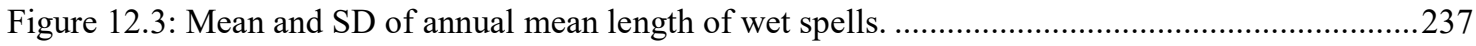

Figure 12.4: Comparison of model performances for mean of daily rainfall depths............................239

Figure 12.5: Comparison of model performances for SD of daily rainfall depths. ............................240

Figure 12.6: Comparison of model performances for mean of multiyear rainfall depths......................242

Figure 12.7: Comparison of model performances for SD of multiyear rainfall depths. .......................243

Figure 12.8: Comparison of model performances for mean of monthly number of wet days. ..............245

Figure 12.9: Comparison of model performances for SD of monthly number of wet days....................246

Figure 12.10: Comparison of model performances for SD of multiyear number of wet days...............247

Figure 12.11: Comparison of models for mean of annual mean length of wet spells.........................248

Figure 12.12: Comparison of models for SD of annual mean length of wet spells.

Figure 12.13: Month-to-month autocorrelations of monthly number of wet days and monthly rainfall depths for coastal stations.

Figure 12.14: Month-to-month autocorrelations of monthly number of wet days and monthly rainfall depths for the inland and monsoonal stations. 251 
Figure 12.15: Comparison of model performances for autocorrelations of monthly number of wet days and monthly rainfall depths for Perth as a representative station.

Figure 12.16: Comparison of DHMC, MMKD and Combined Model (occurrence from MMKD and depth from DHMC) to reproduce the mean of multiyear rainfall depths. .253

Figure 13.1: Comparison of model performances for mean of wet day rainfall depths. .......................258

Figure 13.2: Comparison of model performances for SD of wet day rainfall depths...........................258

Figure 13.3: Comparison of model performances for mean of monthly rainfall depths.......................259

Figure 13.4: Comparison of model performances for SD of monthly rainfall depths. .........................259

Figure 13.5: Comparison of model performances for mean of annual rainfall depths. ........................260

Figure 13.6: Comparison of model performances for SD of multiyear rainfall depths.......................2260

Figure 13.7: Comparison of model performances for mean monthly number of wet days. ....................261

Figure 13.8: Comparison of model performances for SD of monthly number of wet days. .................262

Figure 13.9: Comparison of model performances for mean of annual number of wet days..................262

Figure 13.10: Comparison of model performances for SD of multiyear number of wet days................263

Figure 13.11: Comparison of model performances for mean of monthly mean length of wet spells. .....264

Figure 13.12: Comparison of model performances for SD of monthly mean length of wet spells. ........264

Figure 13.13: Comparison of model performances for mean (left) and SD (right) of the annual mean

length of wet spells

Figure 14.1: Mean and SD of the daily and monthly rainfall depths. The daily statistics are the mean and

SD for the days on which rain occurs and does not include dry days. 268

Figure 14.2: Mean and SD of the multiyear rainfall depths. ..........................................................268

Figure 14.3: Mean and SD of the monthly (left) and multiyear (right) number of wet days.................269

Figure 14.4: Mean and SD of the monthly mean length of wet spells............................................2270

Figure 14.5: Comparison of performances to reproduce the mean and SD of daily and monthly rainfall depths for four validation periods.

Figure 14.6: Comparison of performances to reproduce the mean and SD of multiyear rainfall depths for four validation periods.

Figure 14.7: Comparison of performances to reproduce the mean and SD of monthly number of wet days in four validation periods.

Figure 14.8: Comparison of performances to reproduce the mean and SD of multiyear number of wet days in four validation periods. 275

Figure 14.9: Comparison of performances to reproduce the mean and SD of monthly mean length of wet spells in four validation periods. 276

Figure 14.10: Comparison of performances to reproduce the mean and SD of annual mean length of wet spells for four validation periods. 276

Figure 15.1: The boxes in the right figure show three pixel windows (around Gummun) of three different sizes. The figures in left panel show changes of the wet-to-wet probabilities (P11) and mean of wet day rainfall depths $(\mu)$ with the changes in size of pixel windows. 282 
Figure 15.2: Distribution of correlation of daily rainfall timeseries (rts) between pairs of pixels at different distances for RCM2 at Williams River site

Figure 15.3: Distribution statistics of daily rainfall depths and streamflow volumes for the calibration (1980-2009) and validation (1950-1979) periods at Grahamstown catchment

Figure 15.4: Distribution statistics of daily rainfall depths and streamflow volumes for the calibration (1980-2009) and validation (1950-1979) periods at Tillegra catchment.

Figure 15.5: Distribution statistics of multiyear rainfall depths and streamflow volumes for the calibration (1980-2009) and validation (1950-1979) periods at Grahamstown.

Figure 15.6: Distribution statistics of multiyear rainfall depths and streamflow volumes for the calibration (1980-2009) and validation (1950-1979) periods at Tillegra

Figure 15.7: Model comparison to reproduce the distribution statistics of daily rainfall and streamflow for calibration (1980-2009) period of RCM1 at Grahamstown.

Figure 15.8: Model comparison to reproduce the distribution statistics of daily rainfall and streamflow for validation (1950-1979) period of RCM1 at Grahamstown.

Figure 15.9: Model comparison to reproduce the distribution statistics of daily rainfall and streamflow for calibration (1980-2009) period of RCM1 at Tillegra

Figure 15.10: Model comparison to reproduce the distribution statistics of daily rainfall and streamflow for validation (1950-1979) period of RCM1 at Tillegra

Figure 15.11: Model comparison to reproduce the distribution statistics of multiyear rainfall and streamflow for calibration (1980-2009) period of RCM1 at Grahamstown.

Figure 15.12: Model comparison to reproduce the distribution statistics of multiyear rainfall and streamflow for validation (1950-2079) period of RCM1 at Grahamstown.

Figure 15.13: Model comparison to reproduce the distribution statistics of multiyear rainfall and streamflow for calibration (1980-2009) period of RCM1 at Tillegra.

Figure 15.14: Model comparison to reproduce the distribution statistics of multiyear rainfall and streamflow for validation (1950-2079) period of RCM1 at Tillegra.

Figure 15.15: Model Comparison for the autocorrelations of monthly rainfall (left panel) and streamflow (right panel) for RCM1 at Grahamstown. .298

Figure 15.16: Model Comparison for the autocorrelations of monthly rainfall (left panel) and streamflow (right panel) for RCM1 at Tillegra. 299 


\section{List of Tables}

Table 2.1: Pre-calibrated SimHyd parameters for three sub-catchments of the Williams River site.

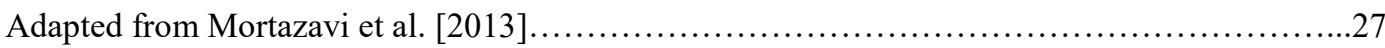

Table 3.1: Geographical features (location and elevation) of the 12 raingauge stations.

Table 3.2: List of 30 raingauge stations around Sydney. Daily rainfall data of 1979-2008 period for each

of these raingauge stations are obtained from Mehrotra et al. [2015]............................................. 40

Table 3.3: Area of the Grahamstown, Tillegra and Chichester sub-catchments........................................ 41

Table 4.1: Methodological comparison among five MC models of this study.........................................8

Table 5.1: Mean and SD of annual mean length of wet spells ( $\mu$ wet) for RCM2 and AWAP. ................96

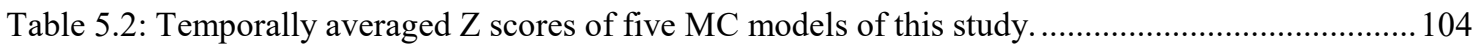

Table 6.1: Spatially and temporally averaged APMC values of model parameters. ............................... 115

Table 6.2: Average of the absolute values of correlation coefficients between elevation and parameters.

Table 6.3: Spatially and temporally averaged values of the mean and SD of rainfall depth and wet period statistics at annual and multiyear resolutions.

Table 6.4: Spatial (all pixels) and temporal (all months and years) average of the absolute values of $Z$ scores for mean and SD of rainfall depths.

Table 6.5: Spatial (all pixels) and temporal (all lags 1-12) average of the absolute values of $Z$ scores for mean and SD of wet period statistics.

Table 6.6: Spatial (all pixels) and temporal (all lags 1-12) average of autocorrelations of monthly rainfall depths and number of wet days. 142

Table 7.1: Spatially and temporally averaged APMC values of model parameters. 151

Table 7.2: Average of the absolute values of correlation coefficients between elevation and parameters.

Table 7.3: Spatially and temporally averaged values of the mean and SD of rainfall depth and wet-dry period statistics at annual and multiyear resolutions.

Table 7.4: Spatially and temporally average of the absolute value of Z scores for mean and SD of rainfall depths.

Table 7.5: Spatially and temporally average of the absolute value of $Z$ scores for mean and SD of wet period statistics.

Table 7.6: Spatial (all pixels) and temporal (all lags 1-12) average of autocorrelations of monthly rainfall depths and number of wet days.

Table 7.7: Spatially and temporally averaged APMC values of model parameters for reanalysis and four GCMs of RCM2. 172

Table 8.1: Spatially and temporally averaged APMC values of model parameters. 177

Table 8.2: Average of the absolute values of correlation coefficients between elevation and parameters. 
Table 8.3: Spatially and temporally averaged values of the mean and SD of rainfall depth and wet period statistics at annual and multiyear resolutions

Table 8.4: Spatially and temporally average of the absolute value of $\mathrm{Z}$ scores for mean and SD of rainfall depths.

Table 8.5: Spatially and temporally average of the absolute value of $Z$ scores for mean and SD of wet period statistics.

Table 8.6: Spatial (all pixels) and temporal (all lags 1-12) average of autocorrelations of monthly rainfall depths and number of wet days.

Table 9.1: Spatially and temporally averaged APMC values of model parameters. .194

Table 9.2: Average of the absolute values of correlation coefficients between elevation and parameters.

Table 9.3: Spatially and temporally averaged values of the mean and SD of rainfall depth and wet period statistics at annual and multiyear resolutions

Table 9.4: Spatially and temporally average of the absolute value of Z scores for mean and SD of rainfall depths

Table 9.5: Spatially and temporally average of the absolute value of Z scores for mean and SD of wet period statistics.

Table 9.6: Spatial (all pixels) and temporal (all lags 1-12) average of autocorrelations of monthly rainfall depths and number of wet days.

Table 10.1: Spatially and temporally averaged APMC values of model parameters. 208

Table 10.2: Average of the absolute values of correlation coefficients between elevation and parameters.

Table 10.3: Spatially and temporally averaged values of the mean and SD of rainfall depth and wet period statistics at annual and multiyear resolutions.

Table 10.4: Spatially and temporally average of the absolute value of Z scores for mean and SD of rainfall depths.

Table 10.5: Spatially and temporally average of the absolute value of Z scores for mean and SD of wet period statistics.

Table 10.6: Spatial (all pixels) and temporal (all lags 1-12) average of autocorrelations of monthly rainfall depths and number of wet days.

Table 11.1: Average of the elevation-parameters correlations (absolute values).

Table 11.2: Spatially and temporally averaged values of the mean and SD of rainfall depth at annual and multiyear resolutions.

Table 11.3: Spatially and temporally averaged values of the mean and SD of wet period statistics at annual and multiyear resolutions.

Table 11.4: Comparison of MC models for mean and SD of rainfall depths.

Table 11.5: Comparison of MC models for mean and SD of wet period statistics...... 228

Table 12.1: Methodological differences among CDMC, HMC, DHMC and MMKD.

Table 12.2: Mean of annual number of wet days and rainfall depth. 
Table 14.1: Mean and SD of the annual mean length of wet spells.

Table 15.1: Model comparison by the average of the absolute values of $Z$ scores for rainfall depths for calibration period (1980-2009).

Table 15.2: Model comparison by the average of the absolute values of $\mathrm{Z}$ scores for streamflow volume for calibration period (1980-2009). .295

Table 15.3: Model comparison by the average of the absolute values of $\mathrm{Z}$ scores for rainfall depth for validation period (1950-1979).

Table 15.4: Model comparison by the average of the absolute values of $\mathrm{Z}$ scores for streamflow volume for validation period (1950-1979) 


\section{List of Symbols and Notations}

The following is a description of the symbols and notation commonly used in this thesis.

$\begin{array}{llc}\text { Symbol } & \text { Description } & \text { Unit } \\ P_{00, i} & \text { Probability of dry-to-dry day for month } i \text {, for January, } i=1 & - \\ P_{11, i} & \text { Probability of wet-to-wet day for month } i & - \\ \mu_{i} & \text { Mean of wet day rainfall depths for month } i & \mathrm{~mm} \\ \sigma_{i} & \text { Standard deviation of wet day rainfall depths for month } i & \mathrm{~mm} \\ r_{c, i} & \text { Coefficient of correlation between } \log \mu_{i} \text { and } \log \sigma_{i} \text { values for month } i & - \\ \lambda_{\mu, i} & \text { Mean of } \log \mu_{i} \text { values for month } i & \mathrm{~mm} \\ \zeta_{\mu, i} & \text { Standard deviation of } \log \mu_{i} \text { values for month } i & \mathrm{~mm} \\ \lambda_{\sigma, i} & \text { Mean of } \log \sigma_{i} \text { values for month } i & \mathrm{~mm} \\ \zeta_{\sigma, i} & \text { Standard deviation of } \log \sigma_{i} \text { values for month } i & \mathrm{~mm} \\ \mathrm{E} & \text { Elevation } & \mathrm{m} \\ r_{0} & \text { Coefficient of correlation between Elevation and Parameters of MC } & - \\ & \text { model } & \\ r_{t s} & \text { Coefficient of correlation between rainfall timeseries in pairs of pixels } & - \\ Z & \text { Z score } & -\end{array}$




\section{Abbreviations}

The following abbreviations are commonly used in this thesis:

$\begin{array}{ll}\text { BoM } & \text { Bureau of Meteorology } \\ \text { NARCliM } & \text { NSW/ACT Regional Climate Modelling } \\ \text { RCM } & \text { Regional Climate Model } \\ \text { AWAP } & \text { Australian Water Availability Project } \\ \text { ECL } & \text { East Coast Low } \\ \text { MC } & \text { Markov Chain } \\ \text { APMC } & \text { Average Parameter Markov Chain } \\ \text { DPMC } & \text { Decadal Parameter Markov Chain } \\ \text { CDMC } & \text { Compound Distribution Markov Chain } \\ \text { HMC } & \text { Hierarchical Markov Chain } \\ \text { DHMC } & \text { Decadal and Hierarchical Markov Chain } \\ \text { MMKD } & \text { Modified Markov Kernel Density }\end{array}$




\begin{abstract}
The key objective of this study is to develop a stochastic daily rainfall model, which can be used in streamflow and reservoir water simulation for urban drought security assessment. After critically reviewing the existing rainfall simulation techniques, this study has developed a Markov Chain (MC) model for stochastic generation of daily rainfall. The MC model uses a two-state MC process with two parameters (wet-to-wet and dry-to-dry transition probabilities) to simulate rainfall occurrence and a Gamma distribution with two parameters (mean and standard deviation of wet day rainfall) to simulate wet day rainfall depths. One of the major focuses of the study is to evaluate the ability of the stochastic model to preserve the rainfall variability and autocorrelation at daily, monthly and multiyear resolutions. Preserving monthly to multiyear variabilities in a daily rainfall model is always challenging, while those longerterm variabilities are critically important for the drought security analysis of reservoirs as the reservoir water levels usually vary in monthly to multiyear resolutions. The traditional models usually underestimate the monthly to multiyear variability, which results in the overestimation of reservoir reliability. On the other hand, the daily variability is also important in many parts of the world to take the influence of short-term extreme rainfall events into account (e.g. East Coast Lows in eastern Australia, which may occur for a few days or weeks, but substantially contribute to the reservoir water level).
\end{abstract}

Five variants of the MC model with different parameterisation techniques have been tested in this study. The first model, referred to as the Average Parameter Markov Chain (APMC) model, uses deterministic parameters of MC and Gamma distribution, that is, the same parameter set is used to simulate the rainfall in all years. The second model, referred to as the Decadal Parameter Markov Chain (DPMC) model, also uses deterministic parameters of MC and Gamma distribution, but the parameters vary for each decade. The third model, referred to as the Compound Distribution Markov Chain (CDMC) model, uses deterministic parameters of MC (same as APMC) and stochastic parameters of the Gamma distribution by sampling the mean and standard deviation of wet day rainfall depths from fitted distributions for each month. The fourth model, referred to as the Hierarchical Markov Chain (HMC) model, uses stochastic parameters of both MC, by sampling wet-to-wet and dry-to-dry transition probabilities from fitted distributions, and Gamma distribution (same as CDMC). The fifth and final model, referred to as the Decadal and Hierarchical Markov Chain (DHMC) model, uses decade-varied parameters of MC (same as DPMC) and stochastic parameters of Gamma distribution (same as CDMC). 
To calibrate the model parameters and compare their performance, this study has used dynamically downscaled rainfall data produced by the NSW/ACT Regional Climate Modelling (NARCliM) project (reanalysis data for three Regional Climate Models (RCMs)), gridded data by the Australian Water Availability Project (AWAP), and ground-based data of raingauge stations. The MC models have been assessed in five catchments of coastal NSW - (i) Goulburn River site (ii) Williams River site (iii) Sydney site (iv) Richmond River site and (v) Bega River site using the NARCliM and AWAP datasets. In addition, raingauge data for 12 raingauge stations around Australia and 30 stations around Sydney have been used to compare the MC models with an existing model. To compare the model performance for streamflow generation, this study has used area-averaged rainfall data of NARCliM and AWAP in a SimHyd hydrology model for three sub-catchments of the Williams River site (i.e. Hunter Water System).

The APMC satisfactorily reproduces the variability of rainfall depths and wet periods at daily resolution only, and significantly underestimates the variability at monthly to multiyear resolutions. The DPMC also significantly underestimates the variability of rainfall depths at monthly to multiyear resolutions, but mostly preserves the variability of wet periods at monthly to multiyear resolutions. The CDMC satisfactorily reproduces the variability of rainfall depth at daily to multiyear resolutions, but significantly underestimates the variability of wet periods at multiyear resolution. The performance of CDMC for wet period variability is consistent with the respective performance of APMC, as both models use the same deterministic parameters of the MC process. The HMC also satisfactorily reproduces the variability of rainfall depths at daily to multiyear resolutions, which is consistent with CDMC as both models use the same stochastic parameters of Gamma distribution. However, the HMC can preserve the variability of wet periods at multiyear resolutions, but significantly overestimates the variability of wet periods at monthly resolution. The DHMC performs better than the other four models, and satisfactorily reproduces the variability of rainfall depths and wet periods at all resolutions, although it significantly underestimates the variability of wet days at shorter multiyear resolutions. For mean of rainfall depths and wet periods, all five MC models perform satisfactorily, although the CDMC, HMC and DHMC show a slight tendency to underestimate the mean of rainfall depths, particularly at multiyear resolutions. For month-to-month autocorrelations of monthly rainfall depths and monthly wet days, all five models perform satisfactorily, except the HMC shows a tendency to underestimate the autocorrelations. The above results suggest the following conclusions: 
- The models with deterministic parameters of Gamma distribution (e.g. APMC and DPMC) cannot reproduce the monthly to multiyear variability of rainfall depths. Stochastic parameters of Gamma distribution (e.g. CDMC, HMC and DHMC) are useful for satisfactorily reproducing the short and long-term variability of rainfall depths.

- Deterministic parameters of MC (e.g. APMC and CDMC) underestimate the multiyear variability of wet periods, while stochastic parameters of MC (e.g. HMC) overestimate the monthly variability of wet periods. Decadally varied parameters of MC (e.g. DPMC and DHMC) are better to satisfactorily reproduce the variability of wet periods at monthly to multiyear resolutions.

- The stochastic parameters of Gamma distribution (e.g. CDMC, HMC and DHMC) yield a slight underestimation of mean rainfall depths.

- The MC models are adequate to reproduce the autocorrelations of monthly rainfall depths and monthly wet days. The underestimation of the autocorrelations in HMC might be linked with the overestimation of wet period variability.

This study has compared the performance of CDMC, HMC and DHMC with an existing Modified Markov Kernel Density (MMKD) model by Mehrotra and Sharma [2007]. The MMKD uses a modified MC process with memory of past periods to simulate rainfall occurrence and resamples rainfall depths for wet days from observed records using a kerneldensity estimation. The MC models are methodologically simple and straightforward in comparison with the relative complexity of the MMKD. Despite the methodological simplicity, the DHMC shows comparable satisfactory performance as MMKD to reproduce the distribution and autocorrelations of rainfall depths and wet periods at daily to multiyear resolutions. The other two MC models, CDMC and $\mathrm{HMC}$, also show comparable performance to reproduce the distribution of rainfall depths at all resolutions, but fail to preserve the distribution of wet periods at all resolutions. However, MMKD tends to overestimate the mean of rainfall depths at all resolutions, which might be caused by the resampling of wet day rainfall depths using kernel-density estimation.

The performance of CDMC, HMC, DHMC and MMKD have also been compared for streamflow generation. The performance of each model for streamflow generation is consistent with their respective performance for the rainfall depths. The MC models perform similarly and 
slightly better than the MMKD to reproduce the distribution and autocorrelation of streamflow volume. 


\section{Introduction}

\subsection{General Background}

This study is a part of the Eastern Seaboard Climate Change Initiative - East Coast Lows (ESCCI-ECL) Project 5 of the University of Newcastle, Australia. The ESCCI-ECL Project 5 has been evaluating the influence of East Coast Lows (ECLs) on the water security of coastal New South Wales (NSW). The key objective of this study is to develop stochastic rainfall models and evaluate their ability to be used in urban drought security analysis of reservoir systems.

Urban water security concerns the entire Eastern Seaboard of Australia (ESA), particularly in coastal NSW because this region has been subject to several major droughts [Kiem and Franks, 2004; Vance et al., 2015]. This region is also in the region of influence of ECLs, which generally occur several times a year, produce substantial amounts of rainfall and streamflow, and contribute to the reservoir water supply [Verdon-Kidd et al., 2010]. Therefore, evaluating the influence of ECL on reservoir water levels is very important for the urban water security analysis of coastal NSW.

In recent years, Regional Climate Models (RCMs) have increasingly been used in urban water security analysis of reservoirs. RCMs can be used to downscale data from General Circulation Models (GCMs) to provide high-resolution gridded data. For the ESA, the NSW/ACT Regional Climate Modelling (NARCliM) project dynamically downscaled coarse resolution ( $\sim 250 \mathrm{~km} \times$ $250 \mathrm{~km}) \mathrm{GCM}$ data to produce gridded data at a $10 \mathrm{~km}$ resolution for one current and two future 20-year periods (climate change data) as well as one 60 -year historical period (reanalysis data) [Evans et al., 2014]. The ESSCCI-ECL Project 5 has been using those dynamically downscaled high-resolution NARCliM data to evaluate the ECL influence on the urban water security of reservoirs in coastal NSW. The primary reason for using the dynamically downscaled NARCliM data is its fine $(\sim 10 \mathrm{~km} \times 10 \mathrm{~km})$ spatial resolution, which is important to incorporate the ECL rainfall. Since the ECLs can occur in a small area of a catchment (e.g. ECLs of approximately 100 to $1000 \mathrm{~km}^{2}$ size), it is not possible to explicitly represent those events using point-resolution raingauge station data or coarse resolution GCM data. The fine resolution data can also capture the local catchment-scale geographical features [Fowler et al., 2007]. For example, coastal NSW catchments are typically less than $100 \mathrm{~km}$ in length, and 


\section{Introduction}

rainfall characteristics on either side near the escarpment (the Great Dividing Range) can be variable. These spatial variabilities of rainfall should be better represented in the NARCliM data in comparison with other coarser resolution data [Evans and McCabe, 2010]. Since NARCliM provides data for two future periods, in addition to the historical reanalysis data, it is possible to examine the climate change effects on the ECL characteristics and associated urban water security implications.

Although the NARCliM RCMs provide regional scale spatial data, there are two important limitations of these datasets: (1) the short-time periods (e.g. 20 or 60 years) and (2) only one realisation is provided. Therefore, the RCMs are not directly applicable to reservoir water security analysis, as long hydrologic records are required to sample extreme droughts that drive the security of the urban system [Mortazavi et al., 2013]. However, the RCM data may be suitable to calibrate stochastic rainfall models that can, in turn, be used to generate long stochastic streamflow sequences for use in reservoir reliability modelling. In this context, this study has developed and assessed stochastic rainfall models, focusing on their ability to preserve rainfall distribution and dependence at daily to multiyear resolutions.

As a requirement of the ESCCI-ECL project 5, this study used NARCliM RCMs for calibration and validation of the stochastic rainfall models. However, during the project, it became clear that validation of the models could not be comprehensively performed only using NARCliM data. Therefore, in addition to the NARCliM RCMs, this study has also used the daily rainfall data produced by Australian Water Availability Project (AWAP), which is available at a finer spatial resolution $(\sim 5 \mathrm{~km} \times 5 \mathrm{~km})$, but only for historical period. Moreover, ground-based data of 12 raingauge stations around Australia and 30 raingauge stations around Sydney have also been used in the development and assessment of the stochastic rainfall models. The gridded datasets of NARCliM and AWAP allowed this study to incorporate the spatial variability of rainfall in coastal NSW, while the raingauge data were used to cover a range of climate zones in Australia. 


\subsection{Aims}

The four main aims of this study are:

- The primary aim is to develop a stochastic daily rainfall model that can preserve the rainfall variability for both short (daily) and longer (monthly to multiyear) resolutions. Preserving short-term variability is important in terms of incorporating ECL rainfall because ECL events often occur for a short period (e.g. a few days or weeks). On the other hand, the long-term variability needs to be preserved for reservoir simulation because the reservoir water levels usually vary from monthly to multiyear resolutions.

- The second aim is to assess the performance of the developed stochastic model in rainfall generation for selected catchments along coastal NSW and other climate zones around Australia.

- The third aim is to compare the performance of the developed stochastic model in rainfall generation with an existing cutting-edge model, a semi-parametric model by Mehrotra and Sharma [2007].

- The final aim is to compare the performance of the developed model of this study and the existing semi-parametric model for streamflow generation.

\subsection{Stochastic Models Developed and Assessed}

After a comprehensive review of the existing stochastic rainfall models, this study has developed and assessed the following five variants of a Markov Chain (MC) model:

- Model 1: Average Parameter Markov Chain (APMC) model

- Model 2: Decadal Parameter Markov Chain (DPMC) model

- Model 3: Compound Distribution Markov Chain (CDMC) model

- Model 4: Hierarchical Markov Chain (HMC) model

- Model 5: Decadal and Hierarchical Markov Chain (DHMC) model.

In addition, the performance of a semi-parametric model developed by Mehrotra and Sharma [2007] has been compared with these MC models. This study refers to the semi-parametric model as Modified Markov Kernel Density (MMKD) model in further discussions. 


\section{Introduction}

\subsection{Overview}

A flowchart of the key components of this thesis is shown in Figure 1.1. This study has completed a comprehensive literature review on the stochastic rainfall modelling and relevant issues, which is presented in Chapter 2. Chapter 3 introduces the datasets, case study sites, and the assessment procedure of the performances of each stochastic model. The development of the five MC models is presented in Chapter 4. The preliminary assessment in Chapter 5 shows that the APMC and DPMC have been rejected by this study because of their significant limitations. Therefore, only the CDMC, HMC and DHMC have been further assessed in the five case study sites along the east coast of NSW. The comparative performance of CDMC, HMC and DHMC in rainfall generation for calibration period at the five case study sites is presented in Chapters 6 to 10. Chapter 11 shows the geographical comparisons of the rainfall and performance of MC models in the five case study sites. Chapters 12 and 13 show the comparison of CDMC, HMC and DHMC, with the MMKD of Mehrotra and Sharma [2007] in rainfall generation for the calibration period, while Chapter 14 shows the same comparison for independent validation periods. Chapter 15 shows the comparison of CDMC, HMC, DHMC and MMKD in streamflow generation for calibration and validation periods, followed by concluding remarks in Chapter 16. 
Background and aims of the study (Chapters 1 and 2)

Data, study site, model assessment procedures (Chapter 3)

Development of stochastic rainfall models (APMC, DPMC, CDMC,

HMC and DHMC), preliminary assessments (Chapters 4 and 5)

Comparison of CDMC, HMC and DHMC in rainfall generation for

calibration period at five case study sites along coastal NSW

(Chapters 6, 7, 8, 9 and 10)

Geographical comparison of the rainfall and model performances

among the five case study sites (Chapter 11)

\section{1}

Comparison of CDMC, HMC, DHMC and MMKD in rainfall

generation for calibration period (Chapters 12 and 13)

1

Comparison of CDMC, HMC, DHMC and MMKD in rainfall generation for validation periods (Chapter 14)

Comparison of CDMC, HMC, DHMC and MMKD in streamflow

generation for calibration and validation periods (Chapter 15)

Conclusion (Chapter 16)

Figure 1.1: Flowchart of the chapter arrangement of this study. 


\section{Introduction}

As a result of the research undertaken during the course of this thesis, the following journal and conference papers have been submitted and published:

Chowdhury, A.F.M.K., N. Lockart, G. Willgoose, G. Kuczera, A.S. Kiem, and N. Parana Manage (in revision), Development of a decadal and hierarchical Markov Chain daily rainfall model with long-term variability.

Chowdhury, A.F.M.K., N. Lockart, G. Willgoose, G. Kuczera, A.S. Kiem, and N. Parana Manage (2015), Modelling daily rainfall along the east coast of Australia using a compound distribution Markov Chain model, 36th Hydrology and Water Resources Symposium, December 2015, Hobart, Australia.

Kiem, A.S., C. Twomey, N. Lockart, G. Willgoose, G. Kuczera, A.F.M.K. Chowdhury, N. Parana Manage, and L. Zhang (2016), Links between East Coast Lows and the spatial and temporal variability of rainfall along the eastern seaboard of Australia, Journal of Southern Hemisphere Earth System Science, 66, 162-176.

Lockart, N., G. Willgoose, G. Kuczera, A.S. Kiem, A.F.M.K. Chowdhury, N. Parana Manage, L. Zhang, and C. Twomey (2016), Case study on the use of dynamically downscaled GCM data for assessing water security on coastal NSW, Journal of Southern Hemisphere Earth System Science, 66, 177-202.

Lockart, N., G. Willgoose, G. Kuczera, A.S. Kiem, A.F.M.K. Chowdhury, and N. Parana Manage (2015), Use of NARCliM rainfall data for simulating streamflow in the Williams River Catchment, 36th Hydrology and Water Resources Symposium, December 2015, Hobart, Australia.

Parana Manage, N., N. Lockart, G. Willgoose, G. Kuczera, A.S. Kiem, A.F.M.K. Chowdhury, L. Zhang, and C. Twomey (2016), Statistical testing of dynamically downscaled data for the Upper Hunter region, New South Wales, Australia, Journal of Southern Hemisphere Earth System Science, 66, 203-227. 
Parana Manage, N., N. Lockart, G. Willgoose, G. Kuczera, A.S. Kiem, and A.F.M.K. Chowdhury (2015), Testing the statistics of dynamically downscaled rainfall data for the east coast of NSW, 36th Hydrology and Water Resources Symposium, December 2015, Hobart, Australia.

Findings of this thesis have also been presented in the following conferences:

Chowdhury, A.F.M.K., N. Lockart, G. Willgoose, G. Kuczera, A.S. Kiem, and N. Parana Manage, (2016), Comparison of two stochastic daily rainfall models and their ability to preserve multiyear rainfall variability, European Geosciences Union General Assembly, April 2016, Vienna, Austria.

Chowdhury, A.F.M.K., N. Lockart, G. Willgoose, G. Kuczera, N. Parana Manage, and A.S. Kiem (2015), A stochastic model for rainfall generation with long-term variability Calibration to NARCliM data at catchments with characteristic influence of east coast lows, Australian Meteorological and Oceanographic Society Conference, July 2015, Brisbane, Australia.

Chowdhury, A.F.M.K., N. Lockart, G. Willgoose, G. Kuczera, and N. Parana Manage (2015), Development of a compound distribution Markov Chain model for stochastic generation of rainfall with long term variability, European Geosciences Union General Assembly, April 2015, Vienna, Austria.

Chowdhury, A.F.M.K., N. Lockart, G. Willgoose, G. Kuczera, and N. Parana Manage (2014), Stochastic simulation of rainfall using a Markov Chain model calibrated to dynamically downscaled data, American Geophysical Union Fall Meeting, December 2014, San Francisco, USA.

Lockart, N., G. Willgoose, G. Kuczera, A.S. Kiem, A.F.M.K. Chowdhury and N. Parana Manage (2015): Use of a stochastic rainfall generation model calibrated to NARCliM data to simulate runoff in the Lower Hunter for water security assessment, Australian Meteorological and Oceanographic Society Conference, July 2015, Brisbane, Australia. 
Parana Manage, N., N. Lockart, G. Willgoose, G. Kuczera, A.S. Kiem, and A.F.M.K. Chowdhury (2016), Evaluation of spatial correlations of dynamically downscaled rainfall data for eastern Australia, European Geosciences Union General Assembly, April 2015, Vienna, Austria.

Parana Manage, N., N. Lockart, G. Willgoose, G. Kuczera, A.S. Kiem, and A.F.M.K. Chowdhury (2015), Statistical validation of dynamically downscaled climate data for the Hunter Region of New South Wales, Australia, Australian Meteorological and Oceanographic Society Conference, July 2015, Brisbane, Australia.

Parana Manage, N., N. Lockart, G. Willgoose, G. Kuczera, A.S. Kiem, and A.F.M.K. Chowdhury (2014), Statistical testing of dynamically downscaled rainfall data for the east coast of Australia, American Geophysical Union Fall Meeting, December 2014, San Francisco, USA. 


\section{Background}

\subsection{Introduction}

This chapter presents a critical review of previous stochastic rainfall models. A number of models were reviewed, focusing on their suitability for reservoir simulation and incorporation of ECL influence. This review will show that the existing models have limitations for applying in the examination of ECL influence on the urban water security of reservoirs. It will also form the motivation of developing a new stochastic rainfall model, which is the primary contribution of this study. In addition, a brief review on the ECL and its influence on water security will be presented at the end of this chapter.

\subsection{Hydrology for Water Security Assessment}

The capacity and prerequisite of water infrastructure in terms of ECL influence (see section 2.8) may be optimally designed and managed by water network simulation software like REALM [Diment, 1991] and WATHNET [Kuczera, 1997], which are currently being used by most of the large Australian cities in their water headworks management. These water resource assessment techniques are based on network linear programming and they require long synthetic hydrological time series.

\subsubsection{Why Use a Stochastic Model?}

Certain water infrastructure simulation models, like REALM [Diment, 1991] and WATHNET [Kuczera, 1997], are operational in major Australian cities for their headworks water management. In coastal NSW, WATHNET5 [Kuczera et al., 2009] has been used in two major urban water systems - the Sydney and Hunter Water - particularly in optimising water supply headworks operating rules and drought security optimisation with respect to climate change projections [Cui and Kuczera, 2005; Mortazavi et al., 2012; Mortazavi et al., 2013]. This software is based on network linear programming, and the optimal design and management of water networks is done with stochastic simulation models to ensure that solutions are robust to uncertainties in the system [Kuczera et al., 2009]. In order to determine the importance of ECLs on the water system by incorporating them into water infrastructure simulation models, a stochastic simulation model is needed to provide the long timeseries of climate variables (e.g. rainfall, streamflow) that are required by the water infrastructure models. 
The observed rainfall data generally provides a single realisation of a short record, often not more than a few decades. The direct application of these data in hydrological and agricultural systems may not provide the necessary robustness in identification and implication of extreme conditions. Particularly in urban water security analysis of reservoirs, long-term hydrologic records are required to sample extreme droughts that drive the security of the urban system [Mortazavi et al., 2013]. However, the observed data may still be suitable to calibrate stochastic rainfall models that can, in turn, be used to generate stochastic streamflow sequences for use in reservoir reliability modelling. In addition to historical and current scenarios, the stochastic models are useful to evaluate the climate and hydrological characteristics of future climate change scenarios [Semenov, 2008; Alfieri et al., 2014; Glenis et al., 2015].

\subsubsection{Why Simulate Rainfall Instead of Streamflow?}

The reason behind the simulation of rainfall instead of streamflow is the availability of highresolution data by NARCliM and AWAP. NARCliM and AWAP have provided gridded rainfall data at $10-\mathrm{km}$ and $5-\mathrm{km}$ fine resolutions respectively. A comprehensive description of the NARCliM and AWAP data used in this study is presented in Chapter 3 at section 3.2.

The major advantages of simulating NARCliM and AWAP data include their spatial distribution and finer resolution to obtain improved understanding of ECL effects on the water system concerning their small size and the NSW orography. There are high spatial variations in Australian climate, particularly in the NSW climate. In many cases, the climate variabilities are influenced by local orographic conditions. The studies of natural climate variability show that the hydroclimate east of the Great Dividing Range responds differently to large-scale ocean drivers than regions west of the Divide [Verdon-Kidd and Kiem, 2009]. Chiew and Leahy [2003] also found similar differences between the east (coastal) and west (inland) of the Great Dividing Range. North-east of NSW is dominated by summer rainfall, with relatively dry winters. On the other hand, southern NSW experiences regular winter rainfall from cold fronts and cut-off lows navigating south-eastern Australia. A large region of western NSW is evenly governed by summer and winter rainfall. The range supports the high rainfall near the coast and forms a strong east-to-west drop at annual average rainfall, from more than $1800 \mathrm{~mm}$ along the north-east coast to less than $200 \mathrm{~mm}$ in the north-west of NSW [DECCW, 2010]. These spatial variabilities of NSW rainfall are expected to be better understood by the analysis of finer resolution NARCliM [Evans et al., 2013; Ji et al., 2016] and AWAP data. Therefore, the rainfall simulations are expected to be superior in comparison to simulating point-based streamflow records. 


\subsection{Influence of Large-scale Climate Drivers}

Australia's climate is characterised by dry and wet periods comprising of a number of sharp transitions or sudden shifts between the two extremes. Particularly, the east-Australian rainfall variability has been described as among the highest in the world [Pittock et al., 2006]. The temporal variability of east-Australian rainfall is broadly influenced by different large-scale climate drivers, such as El Niño/Southern Oscillation (ENSO), Inter-decadal Pacific Oscillation (IPO) and Southern Annular Mode (SAM), which regulate the rainfall variations in intra-annual seasonal to inter-annual and multi-decadal resolutions [Verdon-Kidd et al., 2004; Duc et al., 2015]. Srikanthan and Stewart [1992] found that these complex circulations of climatic systems produce long periods of alternating wet and dry years in Australian rainfall time series.

\subsubsection{Influence of El Niño/Southern Oscillation (ENSO)}

The influence of ENSO on Australia is particularly severe in a global context and it predominantly regulates the dry and wet periods [Allan et al., 1996; Risbey et al., 2009]. Assessing documentary, historical instrumental and modern rainfall observations, Gergis and Ashcroft [2013] showed statistically significant relationships between ENSO and droughts and wet years in eastern NSW back to 1788 using a Chi-square test. It suggested a fluctuating relationship between ENSO and east-Australian rainfall variations over the past 220 years, and noticed that the coastal NSW rainfall-ENSO relationship is not as pronounced as in inland areas of eastern Australia. Power et al. [1999] also observed the weaker relationship between ENSO and coastal NSW rainfall variability. Despite this weak ENSO-rainfall relationship, Harper and Granger [2000] found some evidence of a relationship between ENSO and ECL variability. They observed higher intensity of ECL during periods of highly positive values of the Southern Oscillation Index (SOI), which is a measure of the ENSO (the two conditions of ENSO, La Niña and El Niño are represented by positive and negative values of SOI respectively). Hopkins and Holland [1997] observed increased frequency of ECLs during the periods of shift between El Niño and La Niña conditions.

\subsubsection{Influence of Inter-Decadal Pacific Oscillation (IPO)}

There are influences of IPO on the rainfall variability of coastal NSW. The IPO indicates the inter-decadal sea surface temperature variability in the Pacific Ocean. Regardless of the weak relationship of ENSO-rainfall variability, Power et al. [1999] found that the year-to-year eastAustralian rainfall variability is closely related to the year-to-year ENSO variability in the 
negative (cool) phase of IPO with correlation coefficient around 0.7. However, they did not find a robust relationship of year-to-year ENSO-rainfall variability in the positive (warm) phase of IPO (correlation coefficient around 0.1). Speer [2008] also found that more NSW extreme annual rainfall totals occurred in the negative phase of the IPO than the positive phase of IPO. Kiem and Franks [2004] found that the IPO modulation has a multi-decadal effect on the drought risk in eastern Australia.

\subsubsection{Influence of Southern Annular Mode (SAM)}

Wiles et al. [2009] identified a strong correlation between SAM and the east-Australian rainfall events, including ECL. Speer [2008] described the prolonged drought in east Australia since 2002 as an effect of reduction in mid-latitude low-pressure systems due to the predominance of positive values of the SAM index since the late 1990s, while SAM might have been affected by natural or anthropogenic influence. Hendon et al. [2007] found a strong correlation of cool season ECL-rainfall and SAM, but a weaker SAM influence on non-ECL rainfall in the eastern seaboard.

The above review shows that the temporal variabilities (particularly the inter-annual, multiyear variability) of rainfall in east Australia are linked to large-scale climate drivers. Therefore, this study has tested variants of the MC model (e.g. DPMC) with parameters varying decade-todecade.

\subsection{Selection of a Model Framework}

In this study, a stochastic model has been developed based on the widely used two-part Markov Chain (MC) model framework. However, other models were also reviewed before selecting the MC model. This section will present a justification for using a MC model framework instead of another model.

\subsubsection{Why the Rainfall Model at Daily Resolution?}

In reviewing the previous models, a primary focus was on the ability of a model to preserve both the short and long-term rainfall variability. The short-term variability is important because the ECLs often occur for a short period (e.g. only a few days), but produce a substantial amount of rainfall [Verdon-Kidd et al., 2010]. Capturing these short-term extreme events in the model 
is one of the main objectives of this study. Consequently, the selection of a model framework required a method that can operate at daily resolution and thus incorporate the short-term variability. A model with a finer temporal resolution (e.g. hourly) might be better but not essential for the purposes of this study, particularly as the spatial resolution is $100 \mathrm{~km}^{2}$.

\subsubsection{Key Requirements of the Ability of Daily Rainfall Model}

There is a major issue in the use of stochastic daily rainfall models. The daily models generally efficiently preserve the short-term daily rainfall variability (since they are calibrated to the daily resolution data) but tend to underestimate the longer-term rainfall variability of monthly and multiyear resolutions. Such underestimation is critically important for the application of these models in hydrological planning and design. Preserving the long-term variability is important for drought security analysis of reservoirs because the reservoir water levels usually vary at monthly to multiyear resolutions. The underestimation of longer-term variability of rainfall may cause an overestimation of reservoir reliability in urban water planning [Frost et al., 2007]. Therefore, preserving key statistics of wet and dry spells, and rainfall depths in all (daily to multiyear) resolutions is important in stochastic rainfall simulation. Accordingly, this review of the stochastic rainfall models focused on the models' ability to preserve both the short and longer-term variability of rainfall, and in turn their suitability to be used for the analysis of ECL influence on the urban water security of reservoirs.

\subsubsection{Types of Models Reviewed}

There are a number of stochastic rainfall models available to simulate rainfall timeseries of different temporal resolutions, such as hourly, daily, monthly and annual timeseries. Previously, comprehensive reviews were done by Srikanthan and McMahon [2001], Sharma and Mehrotra [2010], and Ailliot et al. [2015]. Based on the framework of simulation process, the stochastic models which were reviewed in this study can be classified into the following categories:

- Autoregressive Moving Average (ARMA) models,

- Point Process models,

- Markov Chain (MC) models. 


\subsubsection{Autoregressive Moving Average (ARMA) Models}

The Box and Jenkins [1970] type of ARMA models are one of the commonly used parametric approaches for stochastic rainfall simulation. Salas [1993] described the general form of the $\operatorname{ARMA}(\mathrm{p}, \mathrm{q})$ process as:

$$
z_{t}=\mu+\sum_{i=1}^{p} \emptyset_{i}\left(z_{t-i}-\mu\right)-\sum_{i=1}^{q} \theta_{i} \varepsilon_{t-i}+\varepsilon_{t}
$$

Equation 2.1

where $z_{t}$ represents the rainfall timeseries at $t$ temporal resolution, $\mu$ is the mean of the timeseries, $\varnothing$ and $\theta$ are the autoregressive and moving average parameters respectively, and $\varepsilon_{t}$ is a noise term. The noise term $\varepsilon_{t}$ is assumed to be an uncorrelated Gaussian random variable, with zero mean and variance $\sigma_{\varepsilon}^{2}$. Since $\varepsilon_{t}$ is a Gaussian random variable, the rainfall timeseries is also required to be Gaussian distributed or able to be transformed to Gaussian distribution.

Daily rainfall timeseries are rarely Gaussian distributed, and instead are often highly skewed. Therefore, it is a common practice to transform the distribution of rainfall timeseries into an approximate Gaussian distribution using a standard transformation process, frequently a BoxCox transformation. The parameters calibrated to the transformed series are then used in the model to generate the synthetic rainfall timeseries. This method of rainfall modelling has been found to be successful for stochastic simulation of monthly and annual rainfall timeseries [GilAlana, 2009]. However, the daily rainfall timeseries is far from being Gaussian distributed with a large number of zero values (dry days with no rainfall). It is generally difficult to transform a daily rainfall series into an approximately Gaussian distribution. In case of drought security analysis, the model should properly represent the run of contiguous dry and wet spells, which the traditional ARMA model is unable to provide [Chang et al., 1984]. Moreover, the transformation can affect the dependence structure of the original rainfall series, which is one of the essential properties to be preserved in the model [Montanari et al., 1997].

As an alternative, Montanari et al. [1997] developed a Fractionally Differenced Autoregressive Integrated Moving Average (FARIMA) model which can work with a non-Gaussian noise and does not require a transformation. But, they only applied the FARIMA model to a monthly rainfall timeseries in Genoa, Italy, and did not consider the model suitable for daily rainfall timeseries. Dastorani et al. [2016] also found that the ARIMA-type models are useful for monthly rainfall forecasting. For Australian data, McMahon et al. [2008] found that the 
traditional autoregressive lag-1, AR (1) models cannot satisfactorily reproduce the multiyear variability of wet and dry periods that characterise Australia's climate. They used an ARMA $(1,1)$ model to simulate six-monthly (not daily) rainfall data, which reproduced the multiyear behaviours better than an AR (1) model.

Despite the ARMA modelling framework not being considered for this study, one important conclusion of this review is that the rainfall in many parts of the world shows significant month-to-month and/or year-to-year autocorrelations, particularly at monthly and annual aggregated timeseries. Such autocorrelation characteristics of rainfall are critical for the drought security analysis considering the long runs of wet and dry periods at intra- and interannual resolutions. Therefore, the autocorrelation should be carefully assessed in the observed data used in this study. If there is any significant autocorrelation in the observed data, it should be properly reproduced by the intended model of this study.

\subsubsection{Point Process Models}

A number of previous studies focused on the application of a stochastic point process to simulate rainfall timeseries, mainly in hourly resolution [Chun et al., 2013; Kaczmarska et al., 2014]. In these models, a point process is used to generate the arrival of rainfall cells and independent distributions are used to model the rainfall depth and duration. Two of the most popular point process models are the Neyman-Scott Rectangular Pulses (NSPR) model [Cowpertwait et al., 1996; Burton et al., 2008; Tarpanelli et al., 2012] and the Bartlett-Lewis Rectangular Pulses (BLRP) model [Rodríguez-Iturbe et al., 1987; Hanaish et al., 2013; Kim et al., 2016]. Generally, the Neyman-Scott process assumes the cell arrival times are exponentially distributed and the number of cells in a rainstorm is Poisson distributed, while the Bartlett-Lewis process assumes the cell arrival time as Poisson distributed and the storm duration as exponentially distributed [Onof and Wheater, 1993].

Cowpertwait et al. [1996] tested two approaches of the NSPR by fitting the model to hourly rainfall data in the United Kingdom (UK). As daily data are more commonly available than hourly data, they also proposed a relatively complex method of fitting the NSPR model to daily data by estimating the sample hourly variance from the sample daily variance using a derived empirical relationship. One of the two model approaches which involved a lag-1 autocorrelation in fitting procedure was found to inappropriately simulate the dry spell sequences and proportion of dry days. The other approach which involved the transition 
probabilities of wet and dry spells in fitting procedure improved the model ability to reproduce the dry spell sequences and proportion of dry days. They used an exponential distribution to simulate the rainfall cell intensity, while the model underestimated the observed extremes for return periods in excess of five years. An alternative approach of using Weibull distribution for cell intensity simulation showed better performance of reproducing the extremes. However, the model with exponential distribution was successfully used in the storm sewer rehabilitation studies in the UK to predict the frequency of system overloading in existing and upgraded conditions, because the events with return periods of more than five years were not the primary concern in that regard.

The BLRP model by Rodríguez-Iturbe et al. [1987] was found to underestimate the proportion of wet periods. Later, this deficiency was overcome by randomising the parameters of the cell duration simulation process [Rodriguez-Iturbe et al., 1988]. Generally, the parameter of exponential distribution of the cell duration was assumed to be Gamma distributed and chosen randomly varying from storm to storm. Onof and Wheater [1994] fitted a BLRP model to data from UK and they also found that the BLRP with randomised parameters reproduced the observed rainfall characteristics better than the basic BLRP. However, they found the inadequate reproduction of autocorrelations and extreme values in the randomised BLRP for return periods greater than the length of the dataset.

Although this study has not considered modelling at hourly resolution, there are some important conclusions from the above literature review:

- The use of transition probabilities has certain advantages in reproducing the wet and dry spell sequences.

- The use of stochastic parameters (randomly selected from a fitted distribution) is also useful to improve the model performance in reproducing the observed characteristics.

- Selecting suitable distributions for stochastic sampling of the parameters is important for model performance. 


\subsubsection{Markov Chain Models}

Using a Markov Chain (MC) model [Gabriel and Neumann, 1962], which does not require any transformation of probability distributions, is very common. A typical MC rainfall model is composed of two parts: a rainfall occurrence model that uses a transition probability between wet and dry days, and a rainfall magnitude model that uses a probability distribution of wet day rainfall depths (commonly a Gamma distribution) fitted to the observed data. The two-part MCGamma model is one of the most popular parametric models for daily rainfall simulation, primarily proposed by Richardson [1981] and known as Weather Generator (WGEN). In addition to rainfall, the WGEN also simulates temperature and solar radiation.

\subsubsection{Modelling rainfall occurrence using the MC process}

The first component of the MC model defines wet and dry days. This is determined by the state and order of the Markov process. Most MC models [Richardson, 1981; Wilks, 1998; Baigorria and Jones, 2010] use a simple two-state, first-order approach, that is, a day can be either 'wet' or 'dry' (two-state) and the state of the current day is only dependent on the state of the preceding day (first-order). Other models use higher states and orders - examples include, the four-state model [Jothityangkoon et al., 2000], alternating renewal process models with exponential [Green, 1964], mixed geometric [Racsko et al., 1991] and negative binomial [Wilby et al., 1998] distribution of wet and dry spell (continuous wetness and dryness) lengths, bivariate mixed distribution model [Li et al., 2013], and multi-order models [Wilks, 1999a; Lennartsson et al., 2008]. These models are more complex as the number of parameters required in the model increases with the number of states and orders of the Markov process. However, the two-state, first-order MC model can often reproduce the statistics of wet and dry periods as well as these higher state/order models [Roldan and Woolhiser, 1982; Chen and Brissette, 2014].

Similar to WGEN, Dubrovský et al. [2004] developed a four-variate (solar radiation, minimum and maximum temperatures, and rainfall) daily weather generator, known as Met\&Roll. In a basic version of their rainfall model, they used a two-state, first-order MC process for rainfall occurrence simulation. In the modified versions of the model, they used second- and third-order MC processes. To compare the MC models with different orders they tested the ability of the models to reproduce (i) the distribution functions of length of dry and wet spells, (ii) the number of spells longer than 10 days, and (iii) the maximum length of the spells. They found that the distribution of the length of dry spells was not satisfactorily modelled by the two-state, 
first-order MC, which also underestimated the frequency of occurrence of long dry spells. The reproduction of dry spell distribution improved in the second-order model, while the third-order model had negligible improvement. However, the second-order model still underestimated the number of long dry spells and the maximum length of the dry spells in all seasons, and the same statistics of wet spells in winter. These underestimations were also apparent for the thirdorder MC. They recommended that, rather than trying an increased order MC, one should consider other approaches for better reproduction of wet and dry days. Mehrotra and Sharma [2007] proposed a modified MC process using memory of past wet periods, which has been found to reproduce the wet and dry spell statistics reasonably well. They also tested a firstorder and a second-order process in their modified MC model and found that the second-order process provided marginal improvements over the first-order process. Another important finding of Dubrovský et al. [2004] and Mehrotra and Sharma [2007] was that the order of MC generally had no effect on the variability of monthly rainfall depths.

Modelling wet and dry conditions based on their transition probabilities of occurrence provides a mechanism to mimic the wet and dry periods, while many non-MC models do not explicitly simulate the wet and dry periods [Thyer and Kuczera, 2000; Ng and Panu, 2010]. Daily rainfall is continuous with discrete days of exact zeros (no rain) and, therefore, many rainfall models have difficulty with the mixture of discrete and continuous features [Dunn, 2004]. The MC model can incorporate both these features by determining wet and dry days first and then generating rainfall depths only for the wet days [Dunn, 2004]. This model is also useful for simulating intra-annual, and intra- to inter-decadal rainfall variability [Srikanthan and McMahon, 2001]. Inter-decadal variability exists in Australian rainfall due to the influence of numerous large-scale climatic mechanisms, such as the ENSO, IPO, and SAM [Verdon-Kidd et al., 2004; Vance et al., 2015].

\subsubsection{Modelling rainfall depths using suitable distribution}

The second component of the MC model is the probability distribution for the wet day rainfall. As the distribution of wet day rainfall is generally right-skewed [Hundecha et al., 2009], it is common practice to use right-skewed exponential type distributions. Common distributions include Gamma distribution [Stern and Coe, 1984; Dubrovský et al., 2004; Srikanthan, 2005; Wang and Nathan, 2007; Chen et al., 2010; Beecham et al., 2014], skewed normal distribution [Chapman, 1998], kappa distribution [Mielke, 1973], Weibull distribution [Sharda and Das, 2005], truncated normal distribution [Hundecha et al., 2009], and kernel-density estimation techniques [Harrold et al., 2003]. A number of other studies fitted a mixture of two or more 
distributions, for example mixed exponential distribution [Wilks, 1999a; Liu et al., 2011], Gamma and generalised Pareto distribution [Furrer and Katz, 2008], and transformed normal and generalised Pareto distribution [Lennartsson et al., 2008]. However, the Gamma distribution is the most commonly used distribution, because it has two simple parameters calculated from the mean and standard deviation of wet day rainfall and adequately represents the rainfall distribution functions. The parameterisation and application of the distribution in the model is straightforward. Although the Gamma distribution has been found to be appropriate for simulating most of the variability of rainfall depth [Bellone et al., 2000; Vrac and Naveau, 2007; Liu et al., 2011], the major drawback of using a Gamma distribution is that its tail is too light to capture heavy rainfall intensities [Vrac and Naveau, 2007]. Therefore, direct use of a Gamma distribution usually causes an underestimation of standard deviation and extreme rainfall depths at monthly to multiyear resolutions.

\subsection{Resolving the Underestimation of Long-term Variability}

A number of methods have been developed in an attempt to resolve the underestimation of long-term variability. The major approaches for resolving this issue include:

- Models with mixed distributions,

- Nesting-type models,

- Models with rainfall-climate index correlation,

- Models with modified Markov Chain.

\subsubsection{Models with Mixed Distributions}

The models with mixed distributions concentrate on the upper tail behaviour of the probability distribution of wet day rainfall depths. Since a single component distribution cannot incorporate the extreme rainfall depths well, a mixture of distributions is introduced. The mixture models are usually based on the extreme value theory developed by Fisher and Tippett [1928]. Rainfall above a threshold depth is defined as 'extreme' and two separate distributions are used to simulate the 'extreme' and 'small' rainfall amounts. Wilks [1999a] proposed a mixture of two exponential distributions with one shape parameter, but two scale parameters which are used to incorporate the extreme and small rainfall depths respectively. In other models, the 'extreme' rainfall depths are modelled by a generalised Pareto distribution (GPD) [Vrac and Naveau, 2007] or stretched exponential distribution [Wilson and Toumi, 2005], while small rainfall 
depths are modelled by a Gamma distribution. Nonetheless, these models have difficulty in defining the threshold corresponding to the 'extreme value'. Wilson and Toumi [2005] defined extreme rainfall as daily totals with exceedance probability less than $5 \%$. Although Vrac and Naveau [2007] used a dynamic mixture to avoid choosing a threshold for 'extreme', Furrer and Katz [2007] described the method as over-parameterised. Recently, Naveau et al. [2016] proposed a new model with smooth transition between the 'small rainfall' and 'extreme rainfall' simulation process to generate low, moderate and heavy rainfall depths without selecting a threshold.

\subsubsection{Nesting-Type Models}

Nesting models adjust the daily rainfall series at different temporal resolutions to obtain statistics that are optimal for all resolutions. These models initially generate a daily rainfall series, which is then modified to adjust the monthly and yearly statistics [Wang and Nathan, 2007; Chen et al., 2010].

For Australian data, Srikanthan and McMahon [1985] proposed a multi-state Transition Probability Matrix (TPM) model. Boughton [1999] observed that the daily TPM model underestimates the standard deviation of annual rainfall, and he proposed an empirical adjustment (known as TPMb) to scale the rainfall depths on wet days to match the observed annual standard deviation. Although the TPMb model was able to preserve the standard deviation of annual rainfall, Srikanthan et al. [2005] noted that it consistently overestimated the mean annual rainfall and failed to preserve the monthly autocorrelation. In a modified nested TPM (mTPM) model, Srikanthan [2005] proposed empirical adjustments of rainfall distributions at monthly and annual resolution, that is, the daily model is used to drive a monthly model and the resulting monthly model is used to drive an annual model. Several other models [Dubrovský et al., 2004; Wang and Nathan, 2007; Srikanthan and Pegram, 2009; Chen et al., 2010] use similar methods. They generally generate a daily rainfall series, then the generated daily rainfall data are aggregated to monthly rainfall values, and these monthly values are modified by a lag-1 autoregressive monthly rainfall model. The modified monthly rainfall values are aggregated to annual rainfall values and these values are then modified by a lag-1 autoregressive annual model [Srikanthan and Pegram, 2009].

The nesting-type models generally perform well to reproduce the rainfall variability at all resolutions. Dubrovský et al. [2004] also showed satisfactory performance of their nesting-type 
model (a modified version of the Met\&Roll model discussed in 2.4.6.1) to reproduce the variability of monthly streamflow characteristics and the frequency of extreme streamflow. Although the nesting-type models preserve the daily, monthly and yearly statistics, they are generally based on statistical adjustments and thus have a weak physical basis. Moreover, these models often only focus on preserving daily, monthly and yearly statistics for water supply issues and do not consider persistence for multiyear resolutions [Piantadosi et al., 2009]. Hong et al. [2016] proposed a copula-based weather generator (cWGEN) that generates daily and monthly rainfall in two separate pools by using parameters calibrated to daily data. The cWGEN performed better than the traditional WGEN of Richardson [1981], particularly to reproduce the extreme dry events. However, the performance of cWGEN was not satisfactory for short record length of data [Hong et al., 2016]. In another nesting-type model, Fatichi et al. [2011] initially used an AR (1) model to generate an annual rainfall series and NSRP model to generate an hourly rainfall series, then the hourly rainfalls are adjusted based on the statistics of annual rainfall. However, while their model preserved the mean rainfall but underestimated the monthly standard deviation.

\subsubsection{Model Using Correlation Between Rainfall and Climate Indices}

Some parametric models introduced the influence of the large-scale climate mechanisms in parameterisation [Hansen and Mavromatis, 2001; Katz et al., 2003; Furrer and Katz, 2007]. Bardossy and Plate [1992] used the correlation between atmospheric circulation patterns and rainfall in a transformed conditional multivariate autoregressive AR (1) model for daily rainfall simulation. Katz and Parlange [1993] developed a model with parameters conditional on the ENSO index. Yunus et al. [2016] developed a generalised linear model for daily rainfall by using ENSO indices (e.g. SOI and nino3.4) as predictors. Although the climate indices were often not strongly correlated to the rainfall, Katz and Zheng [1999] described it as a diagnostic element to detect a 'hidden' (i.e. unobserved) index which could be used to obtain long-term variability. They used a specialised statistical technique, known as the expectationmaximisation algorithm [McLachlan and Krishnan, 1997], to estimate the 'hidden' index and rainfall parameters. Thyer and Kuczera [2000] developed a hidden state MC model for annual data, while Ramesh and Onof [2014] developed a hidden state MC model for daily data. The major drawback of this model approach is that the 'hidden' index is unobserved and its origin is unknown. 


\subsubsection{Model with Modified Markov Chain}

Modified MC models concentrate not only on the order of MC, but also introduce modifications to the parameterisation of the MC process to better reproduce the rainfall variability.

The transition probabilities are modified by considering their long-term variability. The dry and wet spell lengths are conditioned on previous wet and dry spells respectively, and the wet day rainfall is modelled using a nonparametric kernel-density simulator conditional on previous time step rainfall [Lall et al., 1996; Harrold et al., 2003]. While this approach provides satisfactory rainfall variability up to monthly resolutions, at a cost of greater complexity, it has been found to overestimate the annual standard deviation in some locations [Srikanthan et al., 2005]. Moreover, the nonparametric kernel-density techniques usually used resampling of observed data [Rajagopalan and Lall, 1999]. While these models perform reasonably well, they usually cannot generate extreme values higher than the observed extremes, because only the original observations are resampled in the model [Sharif and Burn, 2006].

Mehrotra and Sharma [2007] proposed a semi-parametric Markov model, which was further evaluated by Mehrotra et al. [2015]. To incorporate the long-term variability, they modified the transition probabilities of the MC process by taking the memory of past wet periods into account, while the wet day rainfall depths were simulated by a nonparametric kernel-density process. Since the performance of the MC models developed in this study have been compared with the performance of this semi-parametric model, this semi-parametric model is discussed in greater detail in the next section.

\subsubsection{Summary}

The MC model approaches for resolving the underestimation of long-term variability involve subjective assumptions and limitations. The first approach focused only on the high rainfall values and ignored the small rainfalls, which may be important for drought occurrence in reservoirs. Moreover, defining a certain rainfall depth as an extreme value has a weak physical basis. The second approach used empirical adjustment factors, generally without physical foundation. The third approach modified the transition probabilities of wet and dry periods to obtain long term variability. These models were found to overestimate annual rainfall variability. 


\subsection{The Modified Markov Kernel Density (MMKD) Model}

A number of parametric and nonparametric models have been proposed to resolve the underestimation of longer resolution variability. The semi-parametric Modified Markov Kernel Density (MMKD) model proposed by Mehrotra and Sharma [2007] is possibly one of the best daily rainfall models for this issue in existing literature. Mehrotra et al. [2015] has provided an $\mathrm{R}$ software package for daily rainfall simulation, based on the methodology of MMKD.

\subsubsection{Model Structure}

The MMKD is a two-part daily rainfall model. In the first part, rainfall occurrences are generated using a two-state, first-order Markov process. To incorporate monthly to multiyear variability, the transition probabilities (Markov parameters) are modified at each time step based on the aggregated wetness over pre-specified period/s of time in the recent past. In the second part, wet day rainfall depths are simulated using a nonparametric kernel-density estimation technique. The $\mathrm{R}$ package of Mehrotra et al. [2015] provides a multisite version of the MMKD using a spatial dependence matrix to incorporate the spatial correlations. However, in this study (Chapters 12 to 15), the MMKD has been run as a single-site model for each daily rainfall timeseries, without using any spatial dependence matrix.

For the single-site MMKD, at each time step $t$, the first-order traditional transitional probabilities of wet and dry days $P\left(O_{t} \mid O_{t-1}\right)$ are modified using a continuous predictor $X_{t}$ as conditioning variables in the form of $P\left(O_{t} \mid O_{t-1}, X_{t}\right)$ given by $\left(O_{t}\right.$ refers to rainfall occurrence at time $t$ ):

$$
P\left(O_{t} \mid O_{t-1}, X_{t}\right)=\frac{P\left(O_{t}=1, O_{t-1}=i\right)}{P\left(O_{t-1}=i\right)} \times \frac{f\left(X_{t} \mid O_{t}=1, O_{t-1}=i\right)}{f\left(X_{t} \mid O_{t-1}=i\right)}
$$

The function of continuous predictor $X_{t}$ characterises the wetness over some period prior to time $t$. The $X_{t}$ is often approximated as a multivariate normal and the typical first-order transitional probability $P\left(O_{t}=1, O_{t-1}=i\right)$ is expressed as $p_{1 i}$ (with $p_{0 i}$ equal to $1-p_{1 i}$ ), when Equation 2.2 [Mehrotra and Sharma, 2007; Mehrotra et al., 2015] becomes: 


$$
\begin{aligned}
P\left(O_{t} \mid O_{t-1}, X_{t}\right)= & p_{1 i} \\
& \times \frac{f\left(X_{t} \mid O_{t}=1, O_{t-1}=i\right)}{\left[f\left(X_{t} \mid O_{t}=1, O_{t-1}=i\right) p_{1 i}\right]+\left[f\left(X_{t} \mid O_{t}=0, O_{t-1}=i\right) p_{0 i}\right]}
\end{aligned}
$$

Equation 2.3

where $X_{t}$ is the aggregated wetness predictor variable over the recent past, given by:

$$
X_{t} \in\left\{X_{j_{1} t}, X_{j_{2} t}, \ldots \ldots, X_{j_{m} t}\right\} ; \quad X_{j_{i} t}=\frac{1}{j_{i}} \sum_{l=1}^{j_{i}} O_{t-l}
$$

where $m$ is the number of predictors, $O_{t-l}$ is the rainfall occurrence on the previous $l$ th day, and $X_{j_{i} t}$ is the wetness over the preceding $j_{i}$ days. Mehrotra et al. [2015] recommended that users should conduct a sensitivity analysis to select the optimal set of predictors (by checking with different number of preceding wet days) for better reproduction of the long-term rainfall variability.

In the second part of the model, the wet day rainfall depth is generated by the kernel-density estimation technique and by conditioning rainfall depth on the previous day's rainfall. Both the occurrence and depth model use a moving window of pre-specified number of days (e.g. 30 days) to reproduce the seasonal variations.

\subsubsection{Variants of MMKD}

Mehrotra et al. [2015] assessed the following variants of the MC process with different lengths of days for state of MC (denoted by $\mathrm{L}_{\mathrm{s}}$ ) and the predictor for wetness of past period (denoted by $\left.\mathrm{L}_{\mathrm{w}}\right)$ :

- $\mathrm{L}_{\mathrm{s}}(1)=$ wet or dry state of previous one day as predictor (basic first-order MC),

- $\mathrm{L}_{\mathrm{s}}(2)=$ wet or dry state of previous two days as predictor (basic second-order MC),

- $\mathrm{L}_{\mathrm{s}}(1) \mathrm{L}_{\mathrm{w}}(30)=$ state of previous one day and wetness of previous 30 days as predictor,

- $\mathrm{L}_{\mathrm{s}}(2) \mathrm{L}_{\mathrm{w}}(30)=$ state of previous two days and wetness of previous 30 days as predictor,

- $\mathrm{L}_{\mathrm{s}}(1) \mathrm{L}_{\mathrm{w}}(90)=$ state of previous one day and wetness of previous 90 days as predictor,

- $\mathrm{L}_{\mathrm{s}}(2) \mathrm{L}_{\mathrm{w}}(90)=$ state of previous two days and wetness of previous 90 days as predictor,

- $\mathrm{L}_{\mathrm{s}}(1) \mathrm{L}_{\mathrm{w}}(180)=$ state of previous one day and wetness of previous 180 days as predictor, 
- $\mathrm{L}_{\mathrm{s}}(2) \mathrm{L}_{\mathrm{w}}(180)=$ state of previous two days and wetness of previous 180 days as predictor,

- $\quad \mathrm{L}_{\mathrm{s}}(1) \mathrm{L}_{\mathrm{w}}(365)=$ state of previous one day and wetness of previous 365 days as predictor,

- $\mathrm{L}_{\mathrm{s}}(2) \mathrm{L}_{\mathrm{w}}(365)=$ state of previous two days and wetness of previous 365 days as predictor,

- $\quad \mathrm{L}_{\mathrm{s}}(1) \mathrm{L}_{\mathrm{w}}(30,365)=$ state of previous one day and wetness of previous 30 and 365 days as predictor,

- $\mathrm{L}_{\mathrm{s}}(2) \mathrm{L}_{\mathrm{w}}(30,365)=$ state of previous two days and wetness of previous 30 and 365 days as predictor.

\subsubsection{Performance of MMKD}

Mehrotra et al. [2015] calibrated the different variants of MMKD to 30-year (1979-2008) raingauge data for 30 stations around Sydney (see Section 3.3.7), and found:

- $\mathrm{L}_{\mathrm{s}}(2)$ provides marginal improvements over $\mathrm{L}_{\mathrm{s}}(1)$ in rainfall generation, although $\mathrm{L}_{\mathrm{s}}(2)$ provides the best representation of the distribution of yearly maximum wet spell lengths.

- $\quad \mathrm{L}_{\mathrm{s}}(1) \mathrm{L}_{\mathrm{w}}(30)$ and $\mathrm{L}_{\mathrm{s}}(2) \mathrm{L}_{\mathrm{w}}(30)$ best reproduced the Standard Deviation (SD) of monthly wet days.

- $\mathrm{L}_{\mathrm{s}}(1) \mathrm{L}_{\mathrm{w}}(90$ or 180$)$ and $\mathrm{L}_{\mathrm{s}}(2) \mathrm{L}_{\mathrm{w}}(90$ or 180$)$ best reproduced the SD of wet days at annual resolution.

- $\mathrm{L}_{\mathrm{s}}(1) \mathrm{L}_{\mathrm{w}}(365)$ and $\mathrm{L}_{\mathrm{s}}(2) \mathrm{L}_{\mathrm{w}}(365)$ best reproduced the SD of wet days at annual and multiyear resolutions.

- $\quad \mathrm{L}_{\mathrm{s}}(1) \mathrm{L}_{\mathrm{w}}(30,365)$ and $\mathrm{L}_{\mathrm{s}}(2) \mathrm{L}_{\mathrm{w}}(30,365)$ best reproduced the monthly and multiyear variability of rainfall depths and wet periods.

\subsubsection{Summary}

In a traditional two-part MC model structure, MMKD uses the MC parameters modified by using memory of pre-specified past period/s to simulate rainfall occurrence, while wet day rainfall depths are resampled from the observed records using a nonparametric kernel-density estimation technique and by conditioning rainfall depth on the previous day's rainfall depth. For seasonality, MMKD uses a moving window of a pre-specified number of days (e.g. 30 days) for both rainfall occurrence and depth simulation. By calibrating to Sydney data, 
Mehrotra et al. [2015] found that the MMKD with second-order MC process provided marginal improvement over the MMKD with first-order MC process. They also found that the MMKD with the memory of past 30 and 365 days satisfactorily reproduced the monthly and multiyear variability of rainfall depths and wet periods. The similarities and differences of the MMKD and the MC models developed in this study are discussed in section 12.2.

\subsection{SimHyd Hydrology Model for Streamflow Generation}

This study has used a SimHyd hydrology model [Chiew et al., 2002; Chiew and Siriwardena, 2005; Vaze et al., 2011] for synthetic streamflow generation to assess the performance of stochastic models in streamflow generation. For three small-sized sub-catchments (less than $200 \mathrm{~km}^{2}$ area) of the Williams River site, this study has used the single-site stochastic rainfall models calibrated to area-averaged rainfall data, as Buchtele et al. [1999] found that the singlesite rainfall generators can be used for small river catchment streamflow modelling.

SimHyd is a lumped conceptual model for daily streamflow generation. The SimHyd model was previously calibrated to the Williams River site of Lower Hunter by Hunter Water Corporation (HWC) and used by several other studies [HWC, 2012; WBM, 2012; Mortazavi et al., 2013; Lockart et al., 2016]. The calibrated parameters of SimHyd (e.g. store capacity, infiltration loss, soil moisture) for Lower Hunter and a comprehensive description of the model is available in Mortazavi et al. [2013]. However, to generate streamflow using the precalibrated SimHyd for Lower Hunter, this study has only used area-averaged daily rainfall data and monthly mean daily catchment average potential evapotranspiration data, while other parameters (see Table 2.1) remain unchanged as Mortazavi et al. [2013]. The potential evapotranspiration data was provided by HWC and previously used by Lockart et al. [2016]. Andréassian et al. [2004] justified the use of the monthly mean daily catchment average potential evapotranspiration as errors in potential evapotranspiration generally induce much smaller output errors compared to the errors in rainfall. 
Table 2.1: Pre-calibrated SimHyd parameters for three sub-catchments of the Williams River site. Adapted from Mortazavi et al. [2013].

\begin{tabular}{|l|l|c|c|c|}
\hline \multirow{2}{*}{ Parameters } & \multicolumn{2}{|c|}{ Description } & \multicolumn{2}{c|}{ Sub-catchments of Williams River site } \\
\cline { 3 - 5 } & & Grahamstown & Tillegra & Chichester \\
\hline Intcap & Interception store capacity $(\mathrm{mm})$ & 2.28 & 4.92 & 0.5 \\
\hline Coeff & Maximum infiltration loss $(\mathrm{mm})$ & 200 & 293 & 85 \\
\hline sq & Infiltration loss exponent & 1.5 & 9.6 & 3.6 \\
\hline smsCap & Soil moisture store capacity (mm) & 332 & 239 & 435 \\
\hline sub & $\begin{array}{l}\text { Constant of proportionality in } \\
\text { interflow equation }\end{array}$ & 0.311 & 0.003 & 0.167 \\
\hline crak & $\begin{array}{l}\text { Constant of proportionality in } \\
\text { groundwater recharge equation }\end{array}$ & 0.828 & 0.534 & 0.728 \\
\hline k & $\begin{array}{l}\text { Baseflow linear recession } \\
\text { parameter }\end{array}$ & 0.292 & 0.151 & 0.061 \\
\hline initSFrac & Initial soil moisture store fraction & 0.001 & 0.001 & 0.001 \\
\hline initGW & Initial groundwater store fraction & 0.173 & 0.22 & 0.22 \\
\hline
\end{tabular}

\subsection{East Coast Lows and its Importance for Water Security}

As a part of the overarching ESCCI-ECL project, the stochastic rainfall model developed in this study will be used for the evaluation of ECL influence on the urban water security of coastal NSW. Therefore, this study has briefly reviewed the existing literature of ECL to understand the key features of the ECL which are needed to be considered in the development of a stochastic rainfall model. This section will present an overview of the ECL and its importance for water security, followed by a discussion about the selection of five case study sites of this thesis considering the varying ECL impacts along the east coast of NSW.

\subsubsection{What is East Coast Low?}

East Coast Low (ECL) is an intense maritime low-pressure system which occurs several times a year in the east-Australian coasts along the Great Dividing Range, including southern Queensland, New South Wales and eastern Victoria, roughly between $20^{\circ} \mathrm{S}$ and $40^{\circ} \mathrm{S}$. It is a system with a closed cyclonic circulation at the surface, forming and/or intensifying in a maritime environment within the vicinity of the east coast, and usually moves parallel to the coast [Wiles et al., 2009]. It may occur at any time of a year but is most common in autumn and winter (i.e. March to August) while the frequency of ECL occurrence is usually the highest in the month of June [Holland et al., 1987]. ECL is one of the most dangerous weather systems to affect the densely populated east-Australian coast. ECLs can generate heavy rainfall, often resulting in major flooding, and also gale force winds, resulting in large swells, rough seas, and 
wind damage to infrastructure. Hopkins and Holland [1997] estimated that ECLs are responsible for approximately $7 \%$ of major Australian disasters. The Pasha Bulker storm of 2007 was marked as the eighth-most-expensive disaster in Australia's history, causing a loss of around A\$1.35bn in coastal NSW [McAneney et al., 2007].

\subsubsection{Intra-annual Variability}

Generally, ECL develops in a baroclinic environment which exhibits an area of strong surface temperature and pressure gradients. The strong temperature gradient forms due to the inclusion of cold continental air flowing offshore with the warm ocean current, called East Australian Current, that flows southward along the Australian east coast. The location of the warm current and the strength of the cold-air outbreak occurring inland determine the strength of the temperature gradient which usually peaks on approaching the winter [Harper and Granger, 2000]. Hence, the majority of ECLs occur at the transition of autumn to winter as residual heat in the oceans from the summer months creates strong temperature gradients with cold continental air masses from winter cold fronts. This review suggests that the intra-annual variability of the rainfall in coastal NSW is important, which needs to be well-preserved by the developed stochastic model for incorporating the seasonal event such as ECL.

\subsubsection{Short Duration ECL Events}

The ECL can often occur for short period, such as for a few days or weeks, but can generate a substantial amount of rainfall to contribute to the reservoir level [Verdon-Kidd et al., 2010]. This suggests that the short-term daily variability of rainfall is also important for incorporating ECL influence. Therefore, this study has developed the rainfall model to generate rainfall at daily resolution by using parameters calibrated to the daily timeseries of observed rainfall.

\subsubsection{Spatial Variability}

ECLs may vary in size from meso-scale (approximately $10 \mathrm{~km}$ to $100 \mathrm{~km}$ ) to synoptic scale (approximately $100 \mathrm{~km}$ to $1,000 \mathrm{~km}$ ) and are greatly influenced by the local orographic conditions. The mountainous Great Dividing Range is an important factor in ECL formation as it may enhance the cyclogenesis by funnelling southerly winds (in the Southern Hemisphere) back into the system, tightening the clockwise rotation of the low [Carpenter, 2007]. Therefore, this study has used the high-resolution NARCliM data, which are adequate to incorporate the small-sized ECL events and their link with local orography [Evans et al., 2013]. Moreover, 
Kiem et al. [2016] found that the influence of different sub-categories of ECLs are different along the eastern seaboard of Australia. It justifies the selection of five case study sites of this study (see section 3.3), which are selected to capture the different types of ECLs with different degree of influence in the different part of the eastern seaboard.

\subsubsection{Influence of ECL on Water Security of Coastal NSW}

ECL is not just a devastating meteorological event. ECLs and their associated rainfall are important for generating significant inflows into major water storages along coastal NSW [Speer et al., 2009; Wiles et al., 2009]. For example, ECLs, including the Pasha Bulker storm, had a prominent influence on Hunter Water reservoirs, enabling the region to escape the worst of the drought in the period 2000-2007. Without the ECL events, the capacity of the Hunter Water system would have been about $35 \%$ capacity instead of the $97 \%$ that actually occurred after the Pasha Bulker storm [Verdon-Kidd et al., 2010]. Hopkins and Holland [1997] estimated that ECLs are responsible for $16 \%$ of all heavy rainfall events. Pepler and Rakich [2010] found that ECLs are the most important contributor to Sydney's high inflow events, particularly in recent years. Pepler et al. [2014] found that ECLs are responsible for around 23\% of annual rainfall and nearly $40 \%$ of heavy rain events along the east coast. These experiences motivated the establishment of the ESCCI-ECL project to evaluate the water security implications of ECLs in the eastern seaboard.

\subsection{Conclusion}

The literature review presented in this chapter shows that the stochastic models with parameters calibrated to the available rainfall data of short record lengths are useful for the assessment of urban drought security. To incorporate the ECL influence in the urban drought security assessment of reservoirs, the stochastic model should capture the rainfall distribution and autocorrelations at both short (daily) and longer (monthly to multiyear) resolutions, because the ECLs often occur for a few days or weeks, but substantially contribute to the reservoir water level, while the reservoir water level generally varies at monthly to multiyear resolutions. Therefore, this study has developed and evaluated the stochastic models for simulation of daily rainfall data, and assessed the ability of the models to reproduce the rainfall distribution at daily, monthly and multiyear resolutions. This study has assessed the ability of the models to reproduce the monthly autocorrelations of rainfall. 
Among the different model structures, the two-part MC models are most widely used and suitable for adequate reproduction of the variability of rainfall and wet-dry periods. However, the daily rainfall models often tend to underestimate the rainfall variability at monthly to multiyear resolutions. The available models that attempted to resolve the underestimation of long-term variability have certain advantages, but also limitations. Therefore, this study has developed MC models for daily rainfall simulation focusing on their ability to preserve the rainfall characteristics at daily to multiyear resolutions (Chapters 4 and 5). The performance of the developed MC models is assessed (using gridded data of NARCliM and AWAP) in five case study sites along the east coast of NSW, which were selected based on their ECL influence and importance for urban water security (Chapters 6 to 10). Moreover, the stochastic models developed in this study have been compared with one of the existing models that attempted to resolve the underestimation of long-term variability (Chapters 12 and 13). In addition to calibration, the comparative performances of the stochastic models have been assessed for the validation period (Chapter 14) and streamflow generation (Chapter 15). 


\section{Data, Study Sites and Assessment Methods}

\subsection{Introduction}

This chapter presents the datasets used to calibrate the parameters of the rainfall models developed in this thesis (APMC, DPMC, CDMC, HMC, DHMC and MMKD) and assess their ability to reproduce observed rainfall. This chapter also introduces the case study sites for which the performance of the rainfall models has been assessed. Finally, the assessment procedures of model performance will be presented.

\subsection{Data}

In the development and assessment of the stochastic rainfall models, this study has used the following three types of daily rainfall data:

- Dynamically downscaled, high-resolution climate model data produced by the NSW/ACT Regional Climate Modelling (NARCliM) project.

- High-resolution gridded data produced by the Australian Water Availability Project (AWAP).

- Ground-based observations of raingauge data from the observatory stations of the Bureau of Meteorology (BoM), Australia.

\subsubsection{NARCliM Data}

This study has used the Regional Climate Model (RCM) data produced by the NARCliM project. The NARCliM project dynamically downscaled coarse resolution $(\sim 250 \mathrm{~km} \times 250$ $\mathrm{km})$ Global Circulation Model $(\mathrm{GCM})$ data to a $10 \mathrm{~km} \times 10 \mathrm{~km}$ spatial resolution for the eastern seaboard of Australia [Evans et al., 2014]. It used a Weather Research and Forecasting (WRF) model to dynamically downscale climate data from a number of GCMs for high emission scenario A2 of Intergovernmental Panel on Climate Change [IPCC, 2007]. Each GCM dataset was downscaled using three different configurations of WRF, referred to as RCM1, RCM2 and RCM3. For the reanalysis simulations the three RCMs were run to produce three reanalysis datasets for each year from 1950 to 2009 [Evans et al., 2013]. In addition to these reanalyses, four climate change GCM projections (MIROC-medres 3.2, ECHAM5, 
CCCMA 3.1 and CSIRO mk3.0) from Coupled Model Inter-comparison Project 3 (CMIP3) that best reproduce the east-Australian climate [Evans and McCabe, 2010; Evans et al., 2013] were downscaled. Each of these latter datasets has daily data for three time periods: 1990-2010, 2020-2040 and 2060-2080. This study has used the NARCliM reanalysis data (for 1950-2009 period) of three RCMs available at $10 \mathrm{~km}$ and hourly resolution for five case study sites (see section 3.3). The future period GCM data of RCM2 has been used for Williams River site only (see section 3.3.2 and 7.8). The NARCliM data used in this study to test the models are not bias corrected. The bias corrected data was not used in this thesis because it only became available towards the end of the $\mathrm{PhD}$ candidature and a separate study [Parana Manage, 2016] has shown that bias correction does not substantially change the relationship between statistics averaged over a day versus averaged over a year or longer. Since the stochastic models assessed in this study are calibrated to daily rainfall timeseries, the hourly resolution NARCliM data have been aggregated to daily resolution. NARCliM has also provided elevation data at 10 $\mathrm{km}$ resolution. Figure 3.1 shows the area of NARCliM data boundary in the eastern seaboard of Australia with elevation.

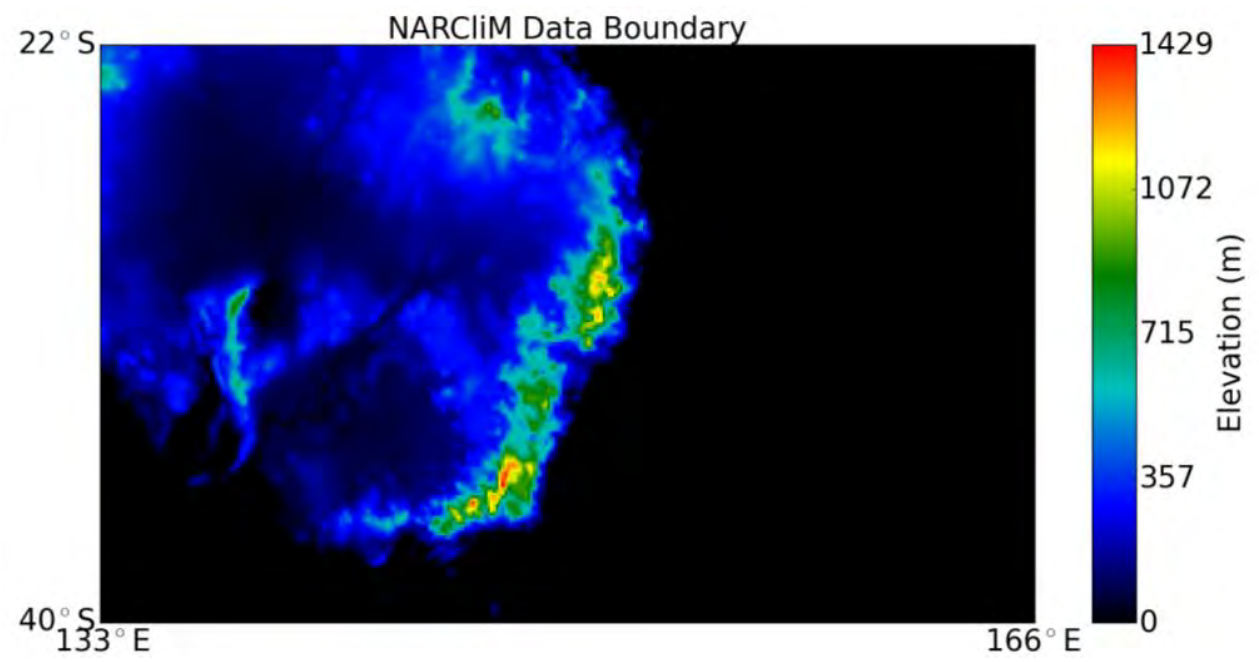

Figure 3.1: Elevation map for NARCliM data boundary in the eastern seaboard of Australia.

\subsubsection{AWAP Data}

The AWAP has produced spatially distributed gridded data at $5 \mathrm{~km} \times 5 \mathrm{~km}$ fine resolutions for all of Australia, which was analytically derived from the ground-based observations of BoM raingauge stations [Raupach et al., 2009]. Similar to NARCliM reanalysis data, this study has used the daily resolution AWAP data for the period 1950-2009. Also for consistency with NARCliM spatial resolution, the $5 \mathrm{~km} \times 5 \mathrm{~km}$ resolution AWAP data are aggregated to $10 \mathrm{~km}$ 
$\times 10 \mathrm{~km}$ resolution. Weighted average of the daily rainfall depth of four AWAP pixels (2 pixel $\times 2$ pixel) that approximately align with a NARCliM pixel are adapted as a rainfall depth over $10 \mathrm{~km} \times 10 \mathrm{~km}$ area.

\subsubsection{Raingauge Data}

The daily resolution ground-based observations of raingauge data are available from the BoM, Australia website $[B o M, 2013]$. These point data are available for a number of stations around Australia, with record lengths generally varying between 30 to 100 years. However, for many of the stations, the data contains a considerable number of missing observations. This study has used raingauge data for 12 stations around Australia and 30 stations around Sydney for a 30year period of 1979-2008. This study has also used a 150-year daily rainfall data for the period 1860-2009 for the Sydney Observatory Hill station (Figure 3.8).

\subsubsection{Data for Streamflow Generation}

This study has compared the performances of rainfall models for streamflow generation for three sub-catchments of the Williams River site (Grahamstown, Tillegra and Chichester) (Figure 3.10). For each sub-catchment, area-averaged daily rainfall (for 1950-2009) from overlaying pixels of RCM1 and AWAP are used for streamflow generation using a SimHyd hydrology model. In addition to the two datasets of area-averaged daily rainfall, the monthly mean daily catchment average potential evapotranspiration data (provided by Hunter Water Corporation, sourced from Scientific Information for Land Owners (SILO) database [Jeffrey et al., 2001], and previously used by Lockart et al. [2016]) have been used in the SimHyd model for streamflow generation for each sub-catchment. Other parameters of the SimHyd hydrology model remain the same as the Hunter Water Corporation's calibrated model (previously used by Mortazavi et al. [2013]).

\subsubsection{Climate Indices}

The relationships between parameters of the models of this study and large-scale climate drivers (e.g. IPO, ENSO) have been evaluated using the monthly timeseries of the following climate indices for period 1950-2009, collected from different sources [Folland, 2008; NOAA, 2014]: (i) Oceanic Nino Index (ONI), (ii) Nino3.4, (iii) Multivariate ENSO Index (MEI), (iv) Pacific Decadal Oscillation Index (PDOI) index, (v) Southern Oscillation Index (SOI), (vi) 
Southern Annular Mode Index (SAMI), (vii) Dipole Mode Index (DMI), and (viii) Interdecadal Pacific Oscillation Index (IPOI).

\subsection{Study Sites}

The performance of the stochastic rainfall models developed in this study have been assessed by using NARCliM and AWAP data for the following five case study sites along the east coast of NSW (Figure 3.2): Goulburn River site, Williams River site, Sydney site, Richmond River site, and Bega River site.

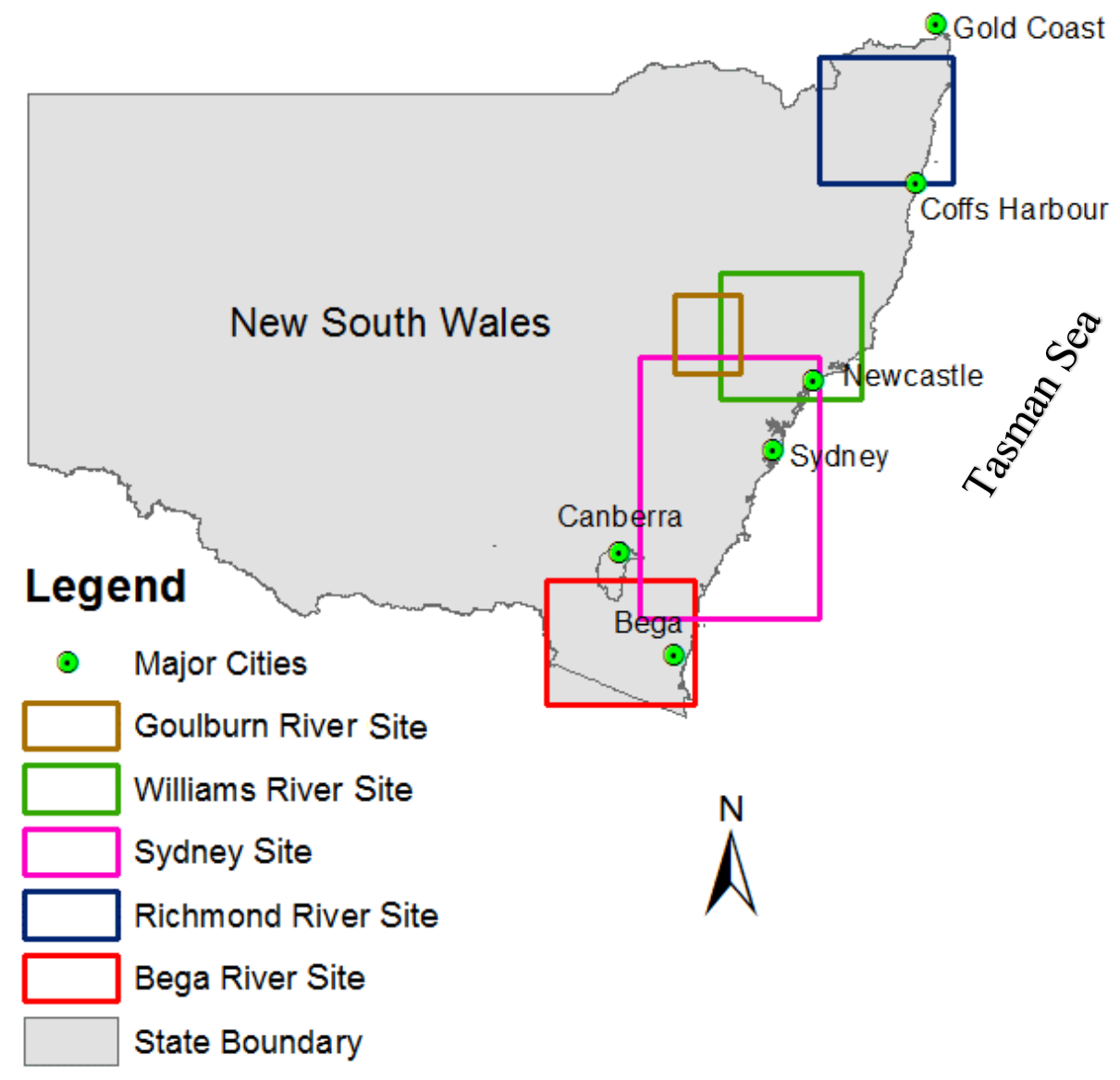

Figure 3.2: Location of the five case study sites (in rectangular boxes) of this study. The green circles indicate the major cities/towns along the east coast of NSW.

This study has also compared the performance of the CDMC, HMC and DHMC against MMKD using raingauge data from 12 stations around Australia and 30 stations around Sydney. In addition, the performances of the three stochastic rainfall models to generate streamflow have been assessed by using NARCliM and AWAP data for three sub-catchments of the Williams River site: Grahamstown, Tillegra and Chichester. 


\subsubsection{Goulburn River Site}

Figure 3.3 shows the elevation map of the case study area in the Goulburn River site located in the Upper Hunter Region of NSW, Australia (see Figure 3.2). The study area is $100 \mathrm{~km}$ (eastwest) $\times 130 \mathrm{~km}$ (north-south), roughly bounded by $149.83^{\circ} \mathrm{E}$ to $150.73^{\circ} \mathrm{E}$ and $31.75^{\circ} \mathrm{S}$ to $32.82^{\circ} \mathrm{S}$ and based around the town of Merriwa. NARCliM divided the area into $10 \times 13$ grid pixels (a total of 130 pixels) of $10 \mathrm{~km}$ resolution. The site has mountain ranges along the northern and southern boundaries. The western boundary of the site is also the western catchment divide but comprises low rolling hills that are significantly lower in elevation than the northern and southern boundaries. The Goulburn River flows across the middle of the site from the west to the east. The highest elevation in the NARCliM elevation map is about $830 \mathrm{~m}$ dropping down to $150 \mathrm{~m}$ in the river. This study has compared the CDMC, HMC and DHMC in rainfall generation for calibration period using NARCliM RCMs and AWAP datasets for each pixel of the Goulburn River site (see Chapter 6).

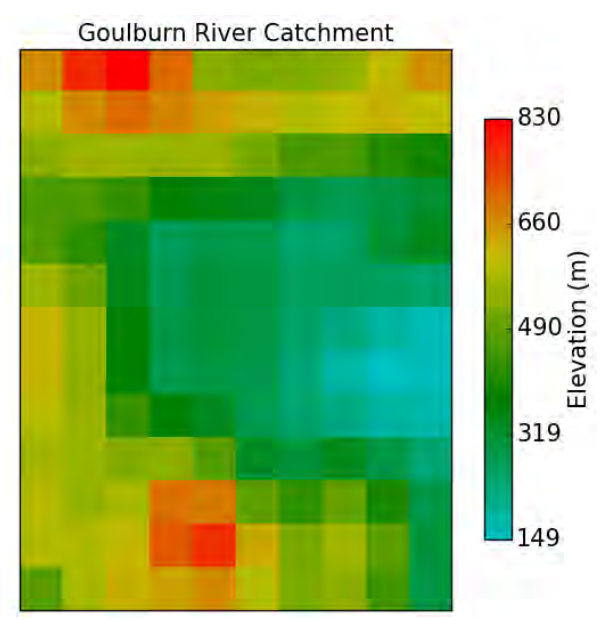

Figure 3.3: NARCliM elevation map (at $10 \mathrm{~km}$ resolution) of the case study area in Goulburn River site.

\subsubsection{Williams River Site}

Figure 3.4 shows the elevation map of the case study area in the Williams River site, located in central east coast of NSW, Australia (Site 2 in Figure 3.2). It is $200 \mathrm{~km} \times 200 \mathrm{~km}$ in area, roughly bounded by $150.45^{\circ} \mathrm{E}$ to $152.40^{\circ} \mathrm{E}$ and $31.35^{\circ} \mathrm{S}$ to $33.30^{\circ} \mathrm{S}$ and based around the city of Newcastle and the Hunter Water urban water supply catchments. NARCliM divided the area into $20 \times 20$ grid pixels (a total of 400 pixels) of $10 \mathrm{~km}$ resolution. However, this study has not assessed 43 pixels located in the ocean (south-east corner of Figure 3.4); therefore 357 pixels are assessed. The highest elevation of the region in the NARCliM elevation map is $1070 \mathrm{~m}$ at the north edge, with the elevation gradually decreasing southwards to the ocean. 
This study has compared the CDMC, HMC and DHMC in rainfall generation using NARCliM reanalysis of three RCMs and AWAP datasets for each of the 357 pixels of the Williams River site (see Chapter 7). In addition, the climate change trend of rainfall characteristics is examined using future period GCM data (MIROC-medres 3.2, ECHAM5, CCCMA 3.1 and CSIRO mk3.0) of RCM2. The RCM2 is chosen because the calibration results for reanalysis period show that the RCM2 has more similarities with the AWAP (gridded data analytically derived from the ground-based observations), while all three RCMs are wetter than AWAP.

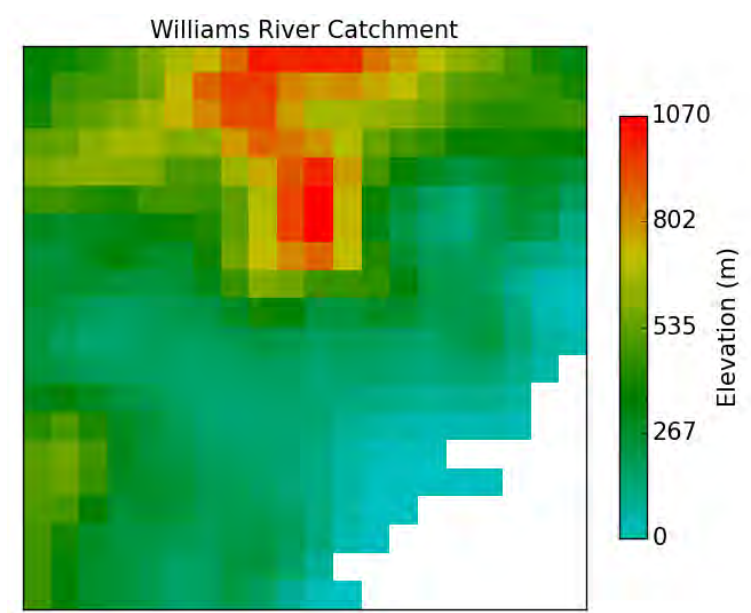

Figure 3.4: NARCliM elevation map (at $10 \mathrm{~km}$ resolution) of the case study area in Williams River site. White region is in ocean.

\subsubsection{Sydney Site}

Figure 3.5 shows the elevation map of the case study area in the Sydney site. It is $250 \mathrm{~km}$ (eastwest $) \times 410 \mathrm{~km}$ (north-south) in area (i.e. $25 \times 41$ NARCliM grid pixels of $10 \mathrm{~km}$ resolution), roughly bounded by $149.36^{\circ} \mathrm{E}$ to $151.80^{\circ} \mathrm{E}$ and $32.60^{\circ} \mathrm{S}$ to $36.17^{\circ} \mathrm{S}$ and based around the city of Sydney and the Sydney Water urban water supply catchments. The highest elevation in the NARCliM elevation map is about $1150 \mathrm{~m}$ in the west of the study area, which gradually decreases to the ocean in the east, but with a steep escarpment adjacent to the coastline. This study has compared the CDMC, HMC and DHMC in rainfall generation using NARCliM RCMs and AWAP datasets for 652 pixels on the land (see Chapter 8). 


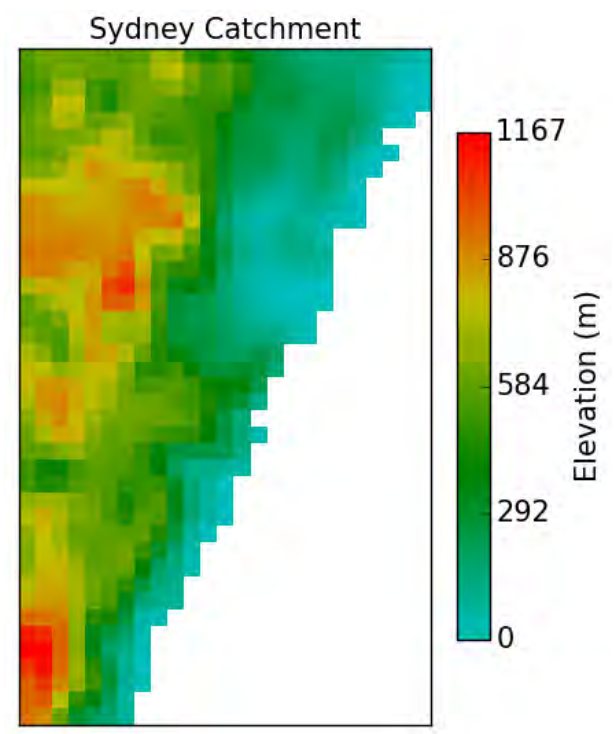

Figure 3.5: NARCliM elevation map (at $10 \mathrm{~km}$ resolution) of the case study area in the Sydney site. The white region is in the ocean.

\subsubsection{Richmond River Site}

Figure 3.6 shows the elevation map of the case study area in the Richmond River site, located in the north-eastern border of the NSW coast. It is $200 \mathrm{~km} \times 200 \mathrm{~km}$ in area consisting $20 \times 20$ NARCliM grid pixels and based around the town of Lismore and the Richmond River catchment. It is bounded by $151.81^{\circ} \mathrm{E}$ to $153.64^{\circ} \mathrm{E}$ and $28.48^{\circ} \mathrm{S}$ to $30.22^{\circ} \mathrm{S}$. Mountainous areas of about $1200 \mathrm{~m}$ elevation are in the south-west corner, which descends to around $800 \mathrm{~m}$ to the north, and meets the ocean in the east. The comparison of CDMC, HMC and DHMC in rainfall generation for 331 pixels on the land is presented in Chapter 9.

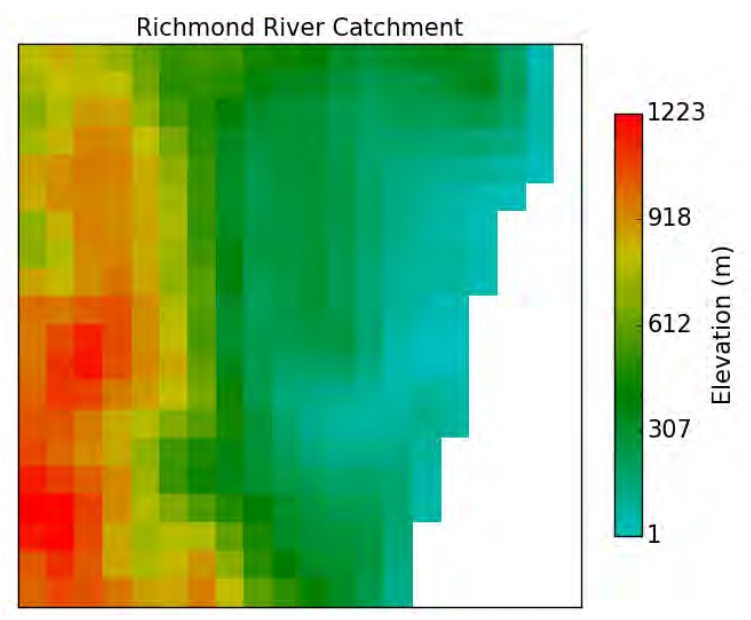

Figure 3.6: NARCliM elevation map (at $10 \mathrm{~km}$ resolution) of the case study area in Richmond River site. The white region is in the ocean. 


\subsubsection{Bega River Site}

Figure 3.7 shows the elevation map of the case study area in the Bega River site, located in the south-eastern border of the NSW coast. It is $200 \mathrm{~km} \times 200 \mathrm{~km}$ in area, bounded by $148.07^{\circ} \mathrm{E}$ to $150.11^{\circ} \mathrm{E}$ and $35.63^{\circ} \mathrm{S}$ to $37.33^{\circ} \mathrm{S}$ and is based around the town of Bega and the Bega River catchment. Pixels on the east edge are in the ocean. Mountainous areas gradually rise from the south-west corner to the middle of north edge to a maximum elevation of about $1400 \mathrm{~m}$, which descends on both sides and meets the ocean in the east. The comparison of CDMC, HMC and DHMC for 374 pixels on the land is presented in Chapter 10.

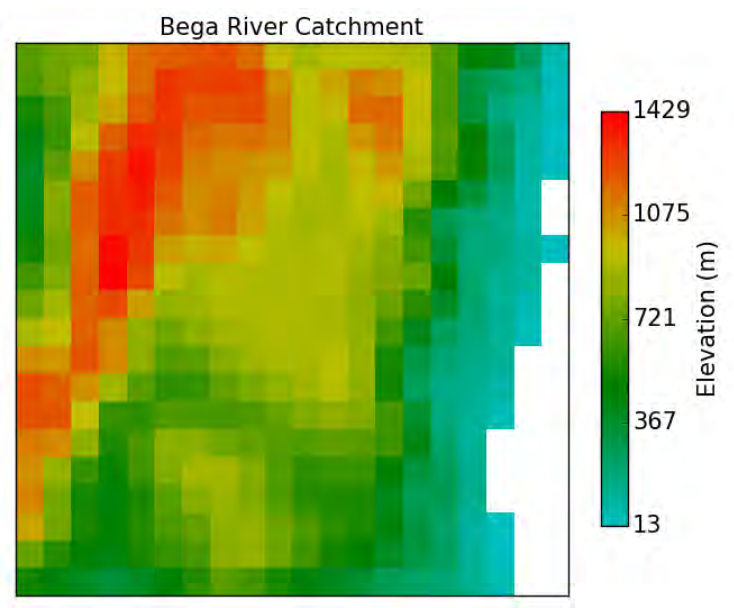

Figure 3.7: NARCliM elevation map (at $10 \mathrm{~km}$ resolution) of the case study area in Bega River site. The white region is in the ocean.

\subsubsection{Raingauge Stations around Australia}

In Chapter 12, this study compares the performance of CDMC, HMC, DHMC and MMKD in rainfall generation for the calibration period using raingauge data of 12 stations around Australia (Figure 3.8). Based on climate zone, these 12 stations are divided into three categories: coastal stations, inland stations and monsoonal stations. For each station, 30-year daily rainfall data for 1979-2008 has been used.

Table 3.1 shows the geographical features (location and elevation) of each station. Geographically, the coastal and monsoonal stations are at lower elevations of $2 \mathrm{~m}$ to $51 \mathrm{~m}$ above mean sea level except the Melbourne station at $113 \mathrm{~m}$ above mean sea level. The inland stations are generally at a higher elevation of $212 \mathrm{~m}$ to $376 \mathrm{~m}$ above mean sea level, except Mildura at $50 \mathrm{~m}$ elevation. 


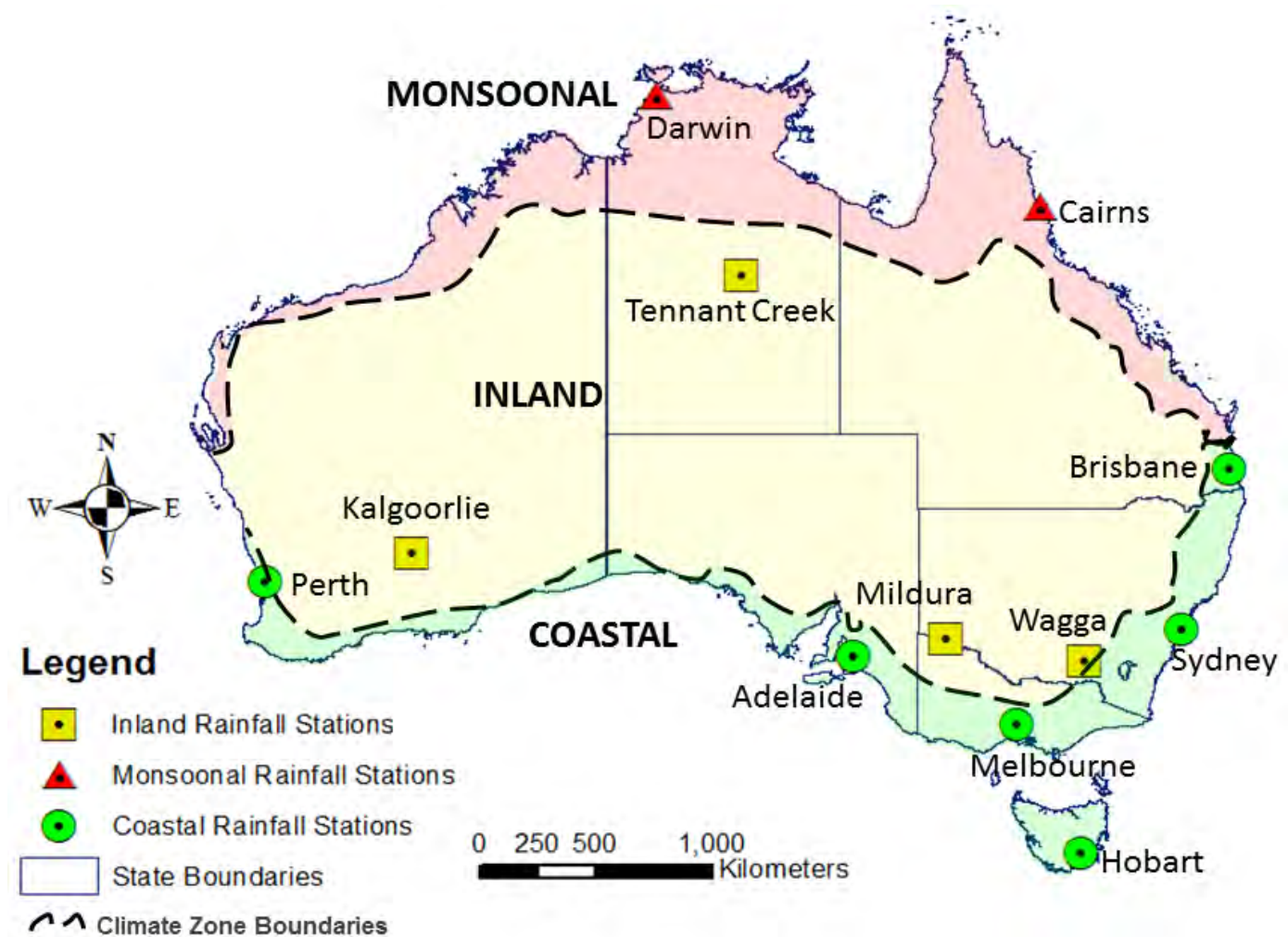

Figure 3.8 Location map of 12 raingauge stations around Australia.

Table 3.1: Geographical features (location and elevation) of the 12 raingauge stations.

\begin{tabular}{llcccc}
\hline Climate Zone & Location & $\begin{array}{c}\text { BoM's Station } \\
\text { Number }\end{array}$ & $\begin{array}{c}\text { Latitude } \\
\left({ }^{\circ} \mathbf{S}\right)\end{array}$ & $\begin{array}{c}\text { Longitude } \\
\left({ }^{\circ} \mathbf{E}\right)\end{array}$ & $\begin{array}{c}\text { Elevation (m) } \\
\text { Coastal }\end{array}$ \\
& Adelaide & 023034 & 34.95 & 138.52 & 2 \\
& Brisbane & 040212 & 27.43 & 153.07 & 9 \\
& Hobart & 094029 & 42.89 & 147.33 & 51 \\
& Melbourne & 086282 & 37.67 & 144.83 & 113 \\
& Perth & 009151 & 31.96 & 115.79 & 20 \\
& Sydney & 066062 & 33.86 & 151.21 & 39 \\
\hline Inland & Wagga Wagga & 072150 & 35.16 & 147.46 & 212 \\
& Mildura & 076031 & 34.24 & 142.09 & 50 \\
& Tennant Creek & 015135 & 19.64 & 134.18 & 376 \\
& Kalgoorlie & 012038 & 30.78 & 121.45 & 365 \\
\hline Monsoonal & Cairns & 031011 & 16.87 & 145.75 & 2 \\
& Darwin & 003003 & 12.42 & 130.89 & 30 \\
\hline
\end{tabular}


3. Data, Study Sites and Assessment Methods

\subsubsection{Raingauge Stations around Sydney}

In Chapter 13, this study compares the CDMC, HMC, DHMC and MMKD in rainfall generation for the calibration period using raingauge data of 30 stations around Sydney (see Figure 3.9 and Table 3.2). This study has obtained the dataset from Mehrotra et al. [2015].

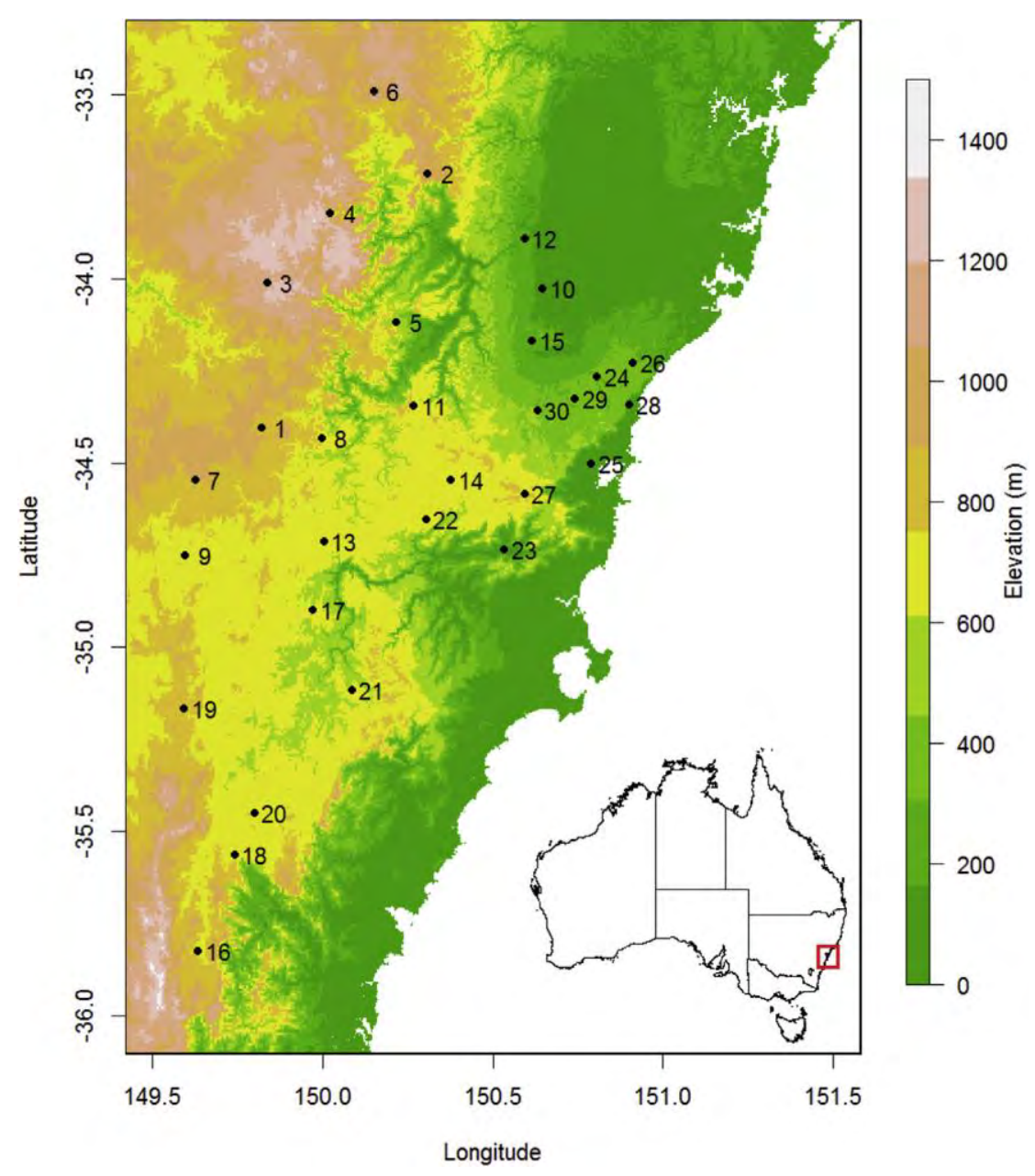

Figure 3.9: Location map of 30 raingauge stations around Sydney (from Mehrotra et al. [2015]). Station numbers correspond to the station names listed in Table 3.2.

Table 3.2: List of 30 raingauge stations around Sydney. Daily rainfall data of 1979-2008 period for each of these raingauge stations are obtained from Mehrotra et al. [2015].

\begin{tabular}{lllll}
\hline 1. Taralga & 7. Woodhouselee & 13. Marulan & 19. Mount Fairy & 25. Dapto \\
2. Katoomba & 8. Bannaby & 14. Moss Vale & 20. Braidwood-Wall. & 26. Dark Forest \\
3. Gurnang & 9. Goulburn & 15. Picton & 21. Nerriga & 27. Robertson \\
4. Oberon & 10. Camden & 16. Braidwood Kraw. & 22. Bundanoon & 28. Woonona \\
5. Yerranderie & 11. High Range & 17. Bungonia & 23. Kangaroo & 29. Cordux \\
6. Lithgow & 12. Warragamba & 18. Majors Creek & 24. Cataract & 30. Avon \\
\hline
\end{tabular}




\subsubsection{Grahamstown, Tillegra and Chichester Sub-Catchments}

Figure 3.10 shows the area, location, and elevation of sub-catchments of the Williams River site used in a SimHyd hydrology model by Hunter Water Corporation. This study has compared the performance of CDMC, HMC, DHMC and MMKD in streamflow generation for the calibration and validation periods at the Grahamstown, Tillegra and Chichester sub-catchments. Geographically, the sub-catchments are in the south-eastern part of the case study area. Tillegra and Chichester sub-catchments are at higher elevations, which includes the mountainous region in the north. The Grahamstown sub-catchment is at lower elevation of the southern part.

Table 3.3 shows the area of the three sub-catchments. The total area of both Tillegra and Chichester are about $200 \mathrm{~km}^{2}$, while Grahamstown is about $120 \mathrm{~km}^{2}$.

Table 3.3: Area of the Grahamstown, Tillegra and Chichester sub-catchments.

\begin{tabular}{cc|cc|cc}
\hline \multicolumn{2}{c|}{ Grahamstown } & \multicolumn{2}{c|}{ Tillegra } & \multicolumn{2}{c}{ Chichester } \\
\hline Grid (x,y) & Area $\mathbf{( k m}^{\mathbf{2}}$ ) & Grid (x,y) & Area $\left.\mathbf{( k m}^{\mathbf{2}}\right)$ & Grid (x,y) & Area $\mathbf{( k m}^{\mathbf{2}}$ ) \\
\hline 14,7 & 15.152 & 11,13 & 26.463 & 11,13 & 9.471 \\
13,6 & 1.149 & 12,13 & 0.501 & 12,13 & 36.830 \\
14,6 & 67.681 & 11,12 & 11.546 & 13,13 & 6.775 \\
14,5 & 31.545 & 12,12 & 32.401 & 12,12 & 62.018 \\
15,6 & 0.587 & 12,11 & 70.303 & 13,12 & 55.526 \\
15,5 & 3.494 & 13,11 & 23.324 & 12,11 & 4.118 \\
& & 12,10 & 10.488 & 13,11 & 24.013 \\
& & 13,10 & 20.665 & & \\
\hline Total Area & $\mathbf{1 1 9 . 6 1}$ & \multicolumn{4}{|c|}{} \\
\hline
\end{tabular}


3. Data, Study Sites and Assessment Methods

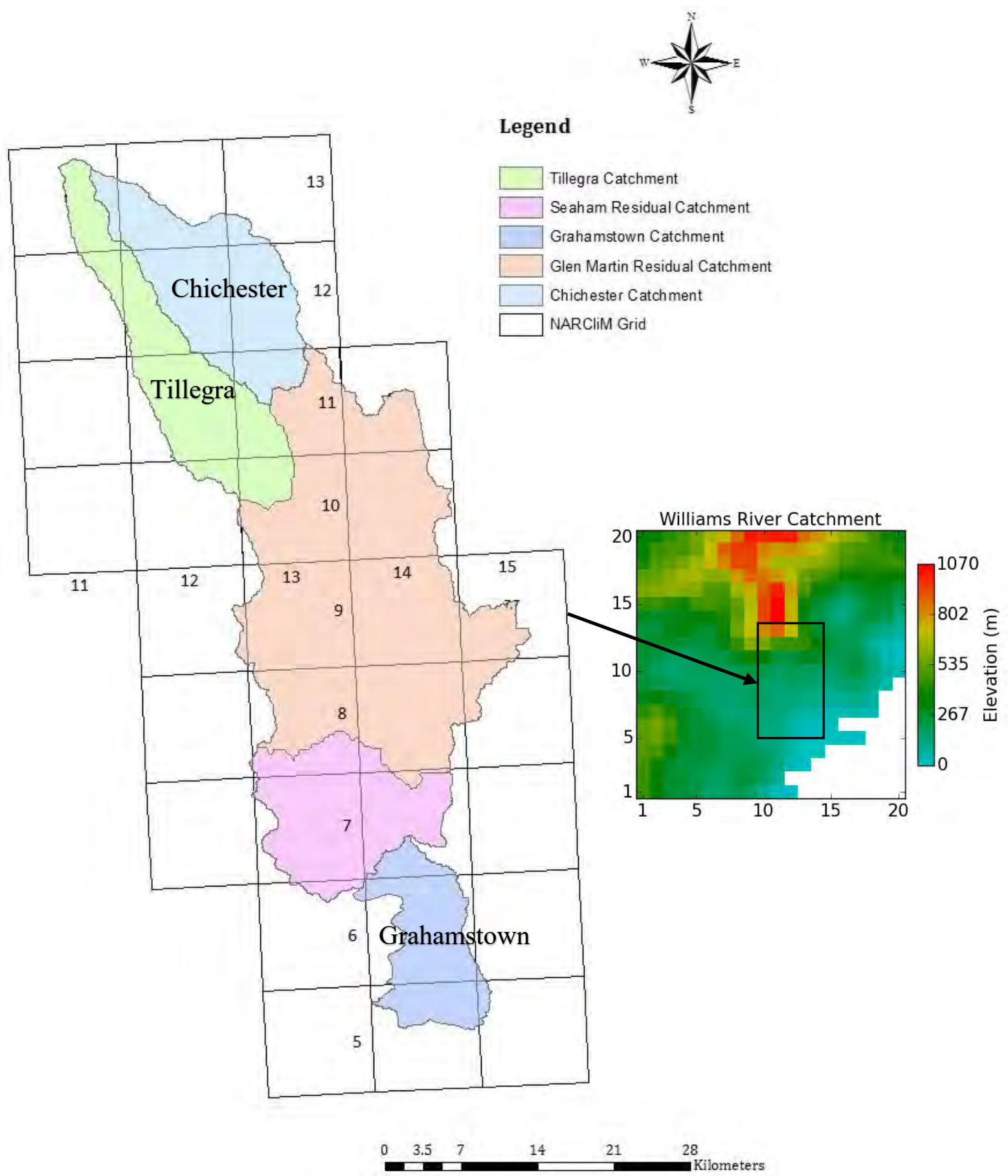

Figure 3.10: The Grahamstown, Tillegra and Chichester sub-catchments of Williams River site are shown in left. The location and elevation of the sub-catchments area within Williams River site are shown in right. The boxes represent the $10 \mathrm{~km} \times 10 \mathrm{~km}$ NARCliM grid pixels. The (x, y) coordinates shown in the left figure corresponds to the $(x, y)$ coordinates of right figure. The distance scale refers to the left hand figure with the catchment boundaries. 


\subsection{Model Assessment Procedures}

This section presents the assessment procedures for the stochastic models used in this study (APMC, DPMC, CDMC, HMC, DHMC and MMKD). Each of the models has been assessed to understand its ability to reproduce the distribution and autocorrelation statistics of the observed rainfall (i.e. the data to which the parameters of respective model are calibrated). The assessment criteria of each model considers its ability to reproduce:

- Distribution of rainfall depths,

- Distribution of wet periods,

- Autocorrelation of rainfall depths,

- Autocorrelation of wet periods.

The performance of the models to reproduce the distribution and autocorrelation statistics have been assessed for daily to multiyear resolutions to evaluate the suitability of the model to be used in water security analysis.

\subsubsection{Distribution of Rainfall Depth}

This study has assessed the performance of each model in reproducing the following distribution statistics of rainfall depths of observed data:

- Mean and standard deviation (SD) of daily (wet day) rainfall depths,

- Mean and SD of monthly rainfall depths (aggregated rainfall depths at monthly resolution),

- Mean and SD of multiyear rainfall depths (aggregated rainfall depths in multiple overlapped years, from 1 to 10 years).

The daily and monthly resolution statistics are assessed for each month to understand the intraannual seasonal variability. The multiyear statistics are assessed from 1 to 10 overlapped years to understand the inter-annual and intra-decadal variability. The mean, SD, skewness, the $5^{\text {th }}$ and $95^{\text {th }}$ percentiles of rainfall depths are assessed at daily to multiyear resolutions. However, the skewness is not usually important for monthly to multiyear resolution aggregation [Wang 
and Nathan, 2007], while the results of the $5^{\text {th }}$ and $95^{\text {th }}$ percentiles do not largely influence the overall discussions of the model performance. Therefore, this study focuses on the ability of each model to reproduce the mean and SD of rainfall depths at daily to multiyear resolutions.

\subsubsection{Distribution of Wet Periods}

A key requirement of a daily rainfall model is that it should simulate the distribution of wet and dry periods well. This study has examined the performance of the stochastic rainfall models in reproducing the wet period statistics, as the dry period statistic are complementary to the wet period statistics (see section 7.5.2 and 7.5.3). The following statistics are used to assess the distribution of wet periods:

- Mean and SD of number of wet days (total number of wet days) at monthly and multiyear resolutions

- Mean and SD of mean length of wet spells $\mu_{w e t}$ at monthly and annual resolutions (see sections 3.4.2.1 and 3.4.2.2 below).

\subsubsection{Monthly mean length of wet spells $\mu_{\text {wet }}$}

To estimate the observed values of the monthly mean length of wet spells, $\mu_{w e t}$, the wet spells (i.e. 1, 2, 3, 4 ... consecutive wet days) are extracted for each month of each year from the observed data. Then, for a month (e.g. January, 1950), the $\mu_{w e t}$ is calculated as:

$$
\mu_{w e t}=\frac{\sum(\text { length of wet spells })}{\left.\sum \text { (occurrences of wet spells }\right)}
$$

Equation 3.1

For a 60-year (e.g. 1950-2009) daily rainfall timeseries, the above equation gives $60 \mu_{w e t}$ values for each of the twelve months. Then, the mean and SD of monthly $\mu_{\text {wet }}$ are calculated from these values for each month.

\subsubsection{Annual mean length of wet spells $\mu_{w e t}$}

For annual mean length of wet spell statistics, the wet spells are extracted for each year from the observed data and the annual $\mu_{w e t}$ values are estimated using Equation 3.1. Then, the mean and SD of annual $\mu_{w e t}$ are calculated from the set of $\mu_{\text {wet }}$ values (e.g. 60 values for a 60 -year data, one for each year). 


\subsubsection{Calculation of $Z$ Scores}

For the distribution statistics of rainfall depths and wet period, this study has calculated the expected value and $95 \%$ confidence limit (2SD) of a model simulation. The $\mathrm{Z}$ scores are calculated to quantify the performance of a model in preserving rainfall distribution statistics (e.g. mean and $\mathrm{SD}$ of rainfall depth). The calculation of $\mathrm{Z}$ score of a statistic is as follows:

- Run the model (e.g. APMC) using parameters calibrated to the observed data 1000 times, with each run being the same length as the observed data.

- Calculate the desired statistics (e.g. mean and SD of the daily, monthly and multiyear rainfall depths) in each run, which gives 1000 realisations of each statistic.

- For each statistic, calculate the mean (expected value) and SD (error limit) of the 1000 realisations.

- Calculate the Z Score of a statistic by comparing the expected value with the respective observed value (calculated from the observed data), as follows:

$$
\mathrm{Z} \text { Score }=\frac{\text { Observed value }- \text { Expected value }}{\mathrm{SD}} \quad \text { Equation } 3.2
$$

- A Z Score between -2 and +2 for a statistic indicates that the observed value falls within the $95 \%$ confidence limits of the simulated rainfall assuming a normal distribution approximates the sampling distribution of $\mathrm{Z}$.

A Z Score less than -2 or greater than +2 suggests that the statistic is over- or under-estimated respectively in the model simulation. An example of this $\mathrm{Z}$ score calculation has been presented in Appendix A (see Figure A.1).

\subsubsection{Autocorrelation of Rainfall Depths and Wet Days}

This study has assessed the performance of each model in reproducing the following autocorrelation of observed data:

- Month-to-month autocorrelation of monthly rainfall depths,

- Month-to-month autocorrelation of monthly number of wet days. 


\subsection{Temporal Resolutions}

For temporal resolutions:

- The 'daily' resolution for rainfall depths in Chapters 4 to 14 indicate the rainfall depths in wet days only. However, in Chapter 15, the 'daily' resolution indicates the rainfall depths (similarly, streamflow volumes) of all days.

- The 'monthly' resolution indicates the aggregation of rainfall depths (similarly, wet days, or streamflow volumes) in months.

- The 'multiyear' resolution indicates aggregation of rainfall depths (similarly, wet days or streamflow volumes) in 1, 2, 3, 4, 5, 6, 7, 8, 9 and 10 overlapping years.

In this thesis, the first month of calibration and simulation is January, accordingly the first month is January for the daily and monthly resolution statistics presented.

\subsection{Gummun Site for Preliminary Assessment}

Chapter 4 describes the development process of the MC models of this study. In that model development process, a timeseries of daily rainfall data is required to calibrate the parameters of each model and test the associated model assumptions. Therefore, this study has used NARCliM reanalysis of RCM2 data (aggregated to daily resolution) for 1950-2009 period in a $10 \mathrm{~km} \times 10 \mathrm{~km}$ NARCliM pixel that overlaid the Gummun raingauge station of the Goulburn River Site (Figure 3.11). In addition to the RCM2 data, AWAP data at daily and $10 \mathrm{~km}$ resolution has been used for the same period and same location at Gummun. Chapter 4 describes the development and calibration process of each MC model using these two datasets at Gummun, while Chapter 5 provides the assessment of the performances of each MC model to reproduce the key statistics of the two datasets (see section 3.4 for the assessment procedure).

Figure 3.11 shows the location of total 19 raingauge stations including Gummun and Blackville (two primary stations) within the NARCliM boundary of the Goulburn River site. The data from RCM2 and AWAP pixels that overlay the 19 stations were used in the preliminary model development and assessment of this study. In Chapter 6, results for all pixels (including these 19 pixels) within the NARCliM data boundary of Goulburn River site are discussed. 


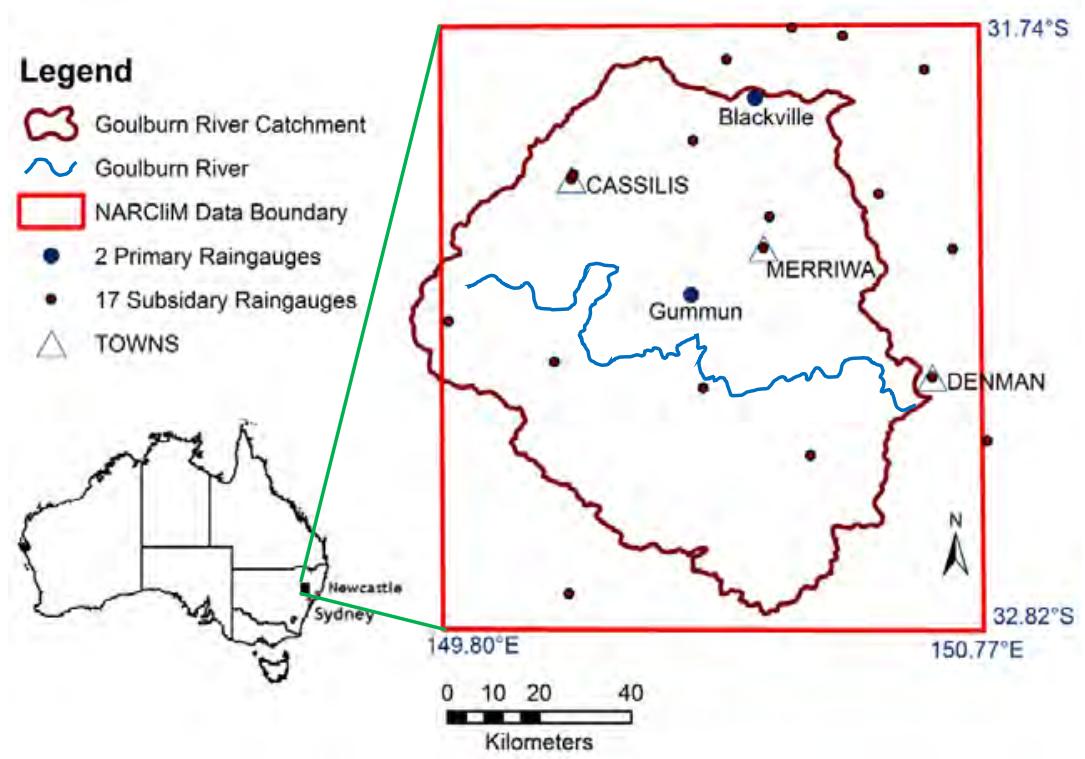

Figure 3.11: Location of Gummun and Blackville, and 17 other raingauge stations within the NARCliM data boundary at Goulburn River site in the east coast of Australia.

\subsection{Distribution of Z Scores in Gridded Dataset}

In each of five case study sites along the NSW coast, the CDMC, HMC and DHMC models are calibrated to NARCliM RCMs and AWAP data for each pixel of a site. Running each calibrated model 1000 times, the $\mathrm{Z}$ scores of the distribution statistics of rainfall depths and wet period in each pixel have been calculated by using Equation 3.2.

For example, for each of the above-mentioned distribution statistics in the Goulburn River site (Chapter 6), the Z scores from all 130 pixels are plotted in histograms. For the daily and monthly resolution statistics, the histograms are plotted for $\mathrm{Z}$ scores of all pixels and all months (e.g. $130 \times 12 \mathrm{Z}$ scores for daily mean rainfall depths). Similarly, for multiyear resolution statistics, histograms are plotted for $Z$ scores of all pixels and each of the ten overlapping years (e.g. $130 \times 10 \mathrm{Z}$ scores for multiyear mean of rainfall depths). In addition to histogram plots, for each month and each multiyear aggregate, $\mathrm{Z}$ scores of 130 pixels are plotted in gridded maps and compared to the corresponding maps of topography and observed statistics. 


\subsection{Calculation of Temporally and Spatially Averaged Statistics}

The temporally and spatially averaged statistics are calculated to present simple summaries.

\subsubsection{Temporally Averaged Statistics}

For temporally averaged value of a statistic, this study has calculated:

- Average of the absolute values of the statistic for all 12 months at daily and monthly resolution.

- Average of the absolute values of the statistic for one to 10 overlapping years at multiyear resolution.

\subsubsection{Spatially and Temporally Averaged Statistics}

For spatially and temporally averaged value of a statistic, this study has calculated:

- Average of the absolute values of the statistic for all 12 months and all pixels at daily and monthly resolution.

- Average of the absolute values of the statistic for one to 10 overlapping years and all pixels at multiyear resolution.

\subsection{Assessment Method for Validation}

Chapter 14 provides the comparison of CDMC, HMC, DHMC and MMKD for the validation period, that is, for a rainfall sample with record length and/or period different than the calibration period. A 150-year daily rainfall data for the Sydney Observatory Hill station shown in Figure 3.8 is used for this validation-comparison. From the 150-year rainfall data of 1860 2009, a 30-year sample of 1979-2008 has been used to calibrate the parameters of each model. Then, the performances of each model are validated using the following samples of different record lengths, while some of these samples overlap the periods:

- A 30-year validation period of 1940-1969,

- A 60-year validation period of 1910-1969,

- A 100-year validation period of 1910-2009 (note that this period partially includes the calibration period), 
- A 150-year validation period of 1860-2009 (note that this period also partially includes the calibration period).

Each model has been run 100 times using the parameters calibrated to the 1979-2008 sample. For the calibration period, the $\mathrm{Z}$ scores of the distribution statistics of rainfall depths and wet periods are calculated by comparing the mean (expected value) and SD (error limit) of the 100 realisations of a statistic with the respective observed value calculated from the calibration data (see Equation 3.2 at section 3.4.3). However, to calculate the $\mathrm{Z}$ score of a statistic for a validation period, the observed value of the statistic calculated from the respective validation period is used in Equation 3.2 instead of the observed value calculated from the calibration period.

\subsection{Assessment Method for Streamflow Generation}

For each sub-catchment of the Williams River site (see sections 3.2.4 and 3.3.8), the 60-year sample of each dataset (RCM1 and AWAP) has been divided into two 30-year samples of 1950-1979 and 1980-2009. The parameters of each model are calibrated to the 1980-2009 sample (i.e. the calibration period), while the 1950-2009 sample is used for validation. Using the calibrated parameters, 100 realisations of rainfall are generated using each model (CDMC, HMC, DHMC and MMKD). Then, the SimHyd model is used to generate streamflow of the calibration period, validation period, and the 100 simulated rainfall series of each model. Using the 100 realisations of rainfall series, and the 100 realisations of streamflow series (generated by SimHyd), the $\mathrm{Z}$ scores of distribution statistics (mean, SD, $5^{\text {th }}$ and $95^{\text {th }}$ percentiles) of rainfall depths and streamflow volumes are calculated for both calibration and validation periods using the same methods described in sections 3.4.3 and 3.9 respectively. In addition, the month-to-month autocorrelations of monthly rainfall depths and streamflow volumes are assessed for the respective calibration and validation periods, and the simulation of each model. 


\section{Development of the Markov Chain Daily Rainfall Model}

\subsection{Introduction}

The literature review (Chapter 2) has showed the necessity for the development of a daily rainfall model for the analysis of ECL influence on the water security of coastal NSW. Chapter 2 also highlighted a key issue of daily rainfall simulation, which is the underestimation of observed rainfall variability in longer resolutions (e.g. monthly, annual and multiyear), which usually results in overestimation of reservoir reliability in urban water planning. In this context, this chapter details the development of a Markov Chain (MC) model for stochastic generation of daily rainfall. This MC model uses a two-state MC process with two parameters (wet-to-wet and dry-to-dry transition probabilities) for simulating rainfall occurrence and a two parameter Gamma distribution (mean and SD of wet day rainfall) for simulating wet day rainfall depths.

Five variants of the MC model, with gradually increasing complexity of parameterisation techniques, are developed and assessed. Starting with a very simple model against which the performances of the other models will be compared, each of the successive models provides better performance in reproducing the variability and dependence of observed rainfall in various resolutions. The five models are listed and discussed below:

- Average Parameter Markov Chain (APMC) model: This model uses deterministic parameters of MC and Gamma, that is, the same parameter set is used to simulate the rainfall in all years. These parameters are different for each month of year (see section 4.2). The APMC can only satisfactorily reproduce the daily variability of rainfall depths and systematically underestimates the monthly to multiyear variability. Moreover, the APMC preserves the variability of wet periods only at monthly resolution and underestimates it at multiyear resolutions (see section 5.3). Therefore, this study has rejected the APMC.

- Decadal Parameter Markov Chain (DPMC) model: This model uses deterministic parameters of MC and Gamma, but sets of parameters are used to simulate rainfall in each decade with the parameters changing each decade (see section 4.3). The DPMC, with higher numbers of parameters, performs better than APMC to reproduce the wet period variabilities, but performs in a similar way to APMC to simulate the rainfall 
depths and systematically underestimates the rainfall depth variabilities at monthly to multiyear resolutions (see section 5.3). Like the APMC, the DPMC has been rejected by this study. However, these two models provided important information, underpinning the other three MC models with their improved performance in reproducing observed variability.

- Compound Distribution Markov Chain (CDMC) model: The long-term variability (e.g. monthly to multiyear resolution variability) of rainfall depths in each model is predominantly governed by the year-to-year variability of the Gamma parameters, rather than the variability of the MC parameters. Therefore, this third model uses stochastic parameters in the Gamma distribution (by sampling the mean and SD of wet day rainfall depths from fitted distributions) along with the deterministic MC parameters from the APMC (see section 4.4). The CDMC satisfactorily reproduces the variability of rainfall depth in all resolutions. It also satisfactorily reproduces the autocorrelations of monthly rainfall depths and monthly number of wet days (see section 5.6). However, just like APMC the CDMC underestimates the variability of the number of wet days for multiyear resolutions (see section 5.3). This indicates the limitations of averaged APMC values of MC parameters in reproducing the variability of wet and dry periods in the longer resolutions. Therefore, further modifications have been required to introduce the year-to-year variability of MC parameters in the model.

- Hierarchical Markov Chain (HMC) model: The deterministic APMC values of MC parameters in CDMC were found to be inadequate for simulating the variability of the wet and dry period lengths. This fourth model uses stochastic parameters of the MC process (by sampling wet-to-wet and dry-to-dry transition probabilities from fitted distributions) in addition to the stochastic parameters of Gamma distribution in CDMC (see section 4.5). The HMC preserves the rainfall depth variabilities for all resolutions and wet period variabilities for multiyear resolutions. However, it tends to overestimate the variability and underestimate the autocorrelation of the monthly number of wet days (see section 5.3 and 5.6).

- Decadal and Hierarchical Markov Chain (DHMC) model: The fifth model uses decadally varied MC parameters (same as DPMC) in addition to the stochastic parameters of Gamma distribution (same as CDMC). The DHMC preserves the rainfall depth and wet period variabilities for all resolutions (see section 4.6, 5.3 and 5.6). 
This chapter will discuss the methodologies (e.g. calibration and simulation procedures) and the associated assumptions of the five variants of MC model using the RCM2 and AWAP data at Gummun (see section 3.6). The preliminary assessment of the performances of each model to reproduce the distribution and autocorrelations of the RCM2 and AWAP at Gummun is presented in Chapter 5 .

\subsection{Average Parameter Markov Chain (APMC) Model}

The first MC model - the APMC - is a traditional two-part MC-Gamma distribution model. This is similar to the rainfall generator proposed by Richardson [1981], widely known as the Weather Generator (WGEN) model. The exception is that the parameters of WGEN were smoothed with Fourier harmonics, which has not been done in the case of APMC parameters. Models similar to APMC were also used by Wang and Nathan [2007] and Chen et al. [2010], which they referred to as 'Basic' and 'WeaGETS' models respectively, and used to compare the robustness of their more sophisticated models in preserving rainfall distribution and dependence in various temporal resolutions. Although APMC is not the final model of this study, it forms the baseline of the modelling approaches against which the more sophisticated models were compared, which have been gradually improved in the further development of relatively complex but robust models.

\subsubsection{Model Structure}

The APMC simulates the daily rainfall in two steps: daily rainfall occurrence (i.e. wet and dry day) simulation by first-order Markov Chain, and wet day rainfall depth simulation by Gamma distribution. To incorporate the seasonal variability in the model, the APMC uses a separate set of parameters for each month, where the first month of the simulation is January. The reason for using monthly varying parameters is discussed in section 4.2.3.1.

\subsubsection{Rainfall occurrence simulation by first-order Markov Chain}

A first-order two-state MC model assumes that the daily rainfall occurrence fluctuates between 'wet' (rain) and 'dry' (no rain) states and that the occurrence of a wet or a dry day is dependent only on the rainfall state of the previous day. 
The transition probability matrix $P_{i}$, for month $i$, describes the conditional daily rain state probabilities:

$$
P_{i}=\left[\begin{array}{ll}
P_{00, i} & P_{01, i} \\
P_{10, i} & P_{11, i}
\end{array}\right]
$$

with $P_{00, i}+P_{01, i}=1$ and $P_{10, i}+P_{11, i}=1$

For a month $i$, these transition probabilities are:

- $\quad P_{00, i}=$ probability of dry day after a dry day,

- $P_{01, i}=$ probability of wet day after a dry day,

- $P_{10, i}=$ probability of dry day after a wet day,

- $\quad P_{11, i}=$ probability of wet day after a wet day.

In the case of APMC, for a month $i$, two transition probabilities of interest, $P_{00, i}$ (dry-to-dry probability) and $P_{11, i}$ (wet-to-wet probability) are calculated from the entire period of data record. This gives 12 values (for each month of the year) of $P_{00}$ and 12 values of $P_{11}$, which are the deterministic MC parameters to be used in the simulation of wet and dry days in the model. However, these two parameters of transition probabilities only determine a day to be wet or dry after another day. Therefore, at the beginning of the simulation, it is also required to determine the wet or dry condition of the first day of January for the first simulation year. This is achieved by using an unconditional probability of dry days $\left(\pi_{0}\right)$ only for January (it is simply the percentage of dry days calculated from all January days in the data record). Cox and Miller [1977] showed that the unconditional probabilities of dry $\left(\pi_{0}\right)$ and wet $\left(\pi_{1}\right)$ days are also linked to the conditional probabilities of dry-to-dry $\left(P_{00}\right)$ and wet-to-wet $\left(P_{11}\right)$ days. This relation is as follows:

$$
\pi_{0}=\frac{1-P_{11}}{2-P_{00}-P_{11}}
$$

where $\pi_{0}+\pi_{1}=1$ 
In total, the APMC requires $24(2 \times 12)$ parameters of $P_{00}$ and $P_{11}$ for wet and dry day occurrence simulation. In the model calibration, these deterministic MC parameters are calculated from the daily rainfall data that are to be modelled (e.g. RCM2 data at Gummun). To calculate these parameters, a day with rainfall depth of $0.3 \mathrm{~mm}$ and above has been considered as wet day, otherwise it was considered as a dry day. The reason for choosing $0.3 \mathrm{~mm}$ as wet day rainfall depth threshold is discussed in section 4.2.3.2.

The occurrences of wet and dry days are simulated in the following steps of Monte-Carlo simulation process:

1. For each day of the simulated timeseries, a random number is generated from a uniform distribution between 0 and 1 .

2. For the first day of the series, the generated random number is compared against the calibrated $\pi_{0}$ value of January. If the random number is less than or equal to $\pi_{0}$, the first day of the series will be a dry day, else it will be a wet day.

3. If the previous day was dry, for the next day, a random number is generated and is compared against the calibrated $P_{00, i}$ value of month $i$ (e.g. $P_{00,1}$ for January). If the random number is less than or equal to $P_{00, i}$ (i.e. transition of dry-to-dry day has occurred), the day will be a dry day, else it will be a wet day.

4. If the previous day was wet, for the next day, a random number is generated and is compared against the calibrated $P_{11, i}$ value of month $i$ (e.g. $P_{11,1}$ for January). If the random number is less than or equal to $P_{11, i}$ (i.e. transition of wet-to-wet day has occurred), the day will be a wet day, else it will be a dry day.

5. After each dry day, repeat step 3 to determine the dry or wet condition of the next day.

6. After each wet day, repeat step 4 to determine the dry or wet condition of the next day.

7. For the first day of next month (e.g. first day of February), the generated random number is compared against the calibrated $P_{00}$ or $P_{11}$ value of that month depending on the dry or wet (respectively) condition of the last day of previous month (e.g. January).

\subsubsection{Rainfall depth simulation by Gamma distribution}

After simulation of the rainfall occurrence using MC parameters, the next step is to generate rainfall depths for the wet days. The rainfall depth for dry days is zero. The APMC rainfall 
depth simulation process assumes that (i) daily rainfall depth for wet days follows a Gamma distribution, and (ii) the depth of rainfall for a wet day is independent of the depth of rainfall of the preceding day. Justifications of these two assumptions are presented in sections 4.2.3.3 and 4.2.3.5 respectively.

A random variable $X$ that is Gamma-distributed with a shape parameter $\alpha$ and a scale parameter $\beta$ is denoted by $X \sim G(\alpha, \beta)$ with pdf:

$$
f(x)= \begin{cases}\frac{1}{\Gamma(\alpha) \beta^{\alpha}} x^{\alpha-1} e^{-x / \beta} & x>0 \\ 0 & x \leq 0\end{cases}
$$

where the Gamma function $\Gamma(\alpha)$ is defined as

$$
\Gamma(\alpha)=\int_{0}^{\infty} x^{\alpha-1} e^{-x} d x
$$

where mean $\mu=\alpha \beta$ and variance $\sigma^{2}=\alpha \beta^{2}$.

The distribution is completely defined by the mean $(\mu)$ and standard deviation $(\sigma)$. Therefore, for a month $i$, the $\alpha_{i}$ and $\beta_{i}$ parameters of the Gamma distribution can be calculated from $\mu_{i}$ and $\sigma_{i}$ of the wet day rainfall as:

$$
\beta_{i}=\frac{\sigma_{i}^{2}}{\mu_{i}} \quad \text { and } \quad \alpha_{i}=\frac{\mu_{i}}{\beta_{i}}
$$

Since both $\alpha_{i}$ and $\beta_{i}$ are directly proportional to and can be derived from $\mu_{i}$ and $\sigma_{i}$ of wet day rainfall of the month $i$ (see section 4.2.3.4), during calibration of the model it is convenient to calculate the $\mu_{i}$ and $\sigma_{i}$ values from the daily rainfall data to be modelled. The appropriate ratios of the $\mu_{i}$ and $\sigma_{i}$ (Equation 4.5) can then be used in the rainfall depth generation process using the Gamma distribution. Therefore, $\mu_{i}$ and $\sigma_{i}$ will be referred to as the Gamma distribution parameters in further discussions of this study.

In calibration of APMC, deterministic values of $\mu_{i}$ and $\sigma_{i}$ are calculated from the entire period of data record for each month (e.g. for the first month January, from a 60-year daily rainfall 
data, the $\mu_{1}$ and $\sigma_{1}$ are calculated from the wet day rainfall depths in 60 Januaries). This gives 12 values of $\mu$ and 12 values of $\sigma$.

In simulations with APMC, the rainfall depth for each wet day of a month $i$ is generated using the $\mu_{i}$ and $\sigma_{i}$ values of the respective month using the Gamma distribution. In generating the rainfall depth for a wet day, if a random sample from the Gamma distribution gives a rainfall depth less than $0.3 \mathrm{~mm}$ then the rainfall for that day is set to $0.3 \mathrm{~mm}$ (i.e. the threshold rainfall depth), while the rainfall depths for dry days are set to $0.0 \mathrm{~mm}$ (see section 4.2.3.3).

\subsubsection{Schematic of APMC}

Figure 4.1 shows a schematic of the APMC. The red dashed boxes show the key parts of the model, that is, the use of MC parameters to simulate the dry and wet days, and use of Gamma parameters to simulate rainfall depths for wet days. The red dashed boxes in Figure 4.1 can be compared with the red boxes in Figure 4.10, Figure 4.21, Figure 4.30 and Figure 4.31 to see the sequential changes in the successive developments of the MC model.

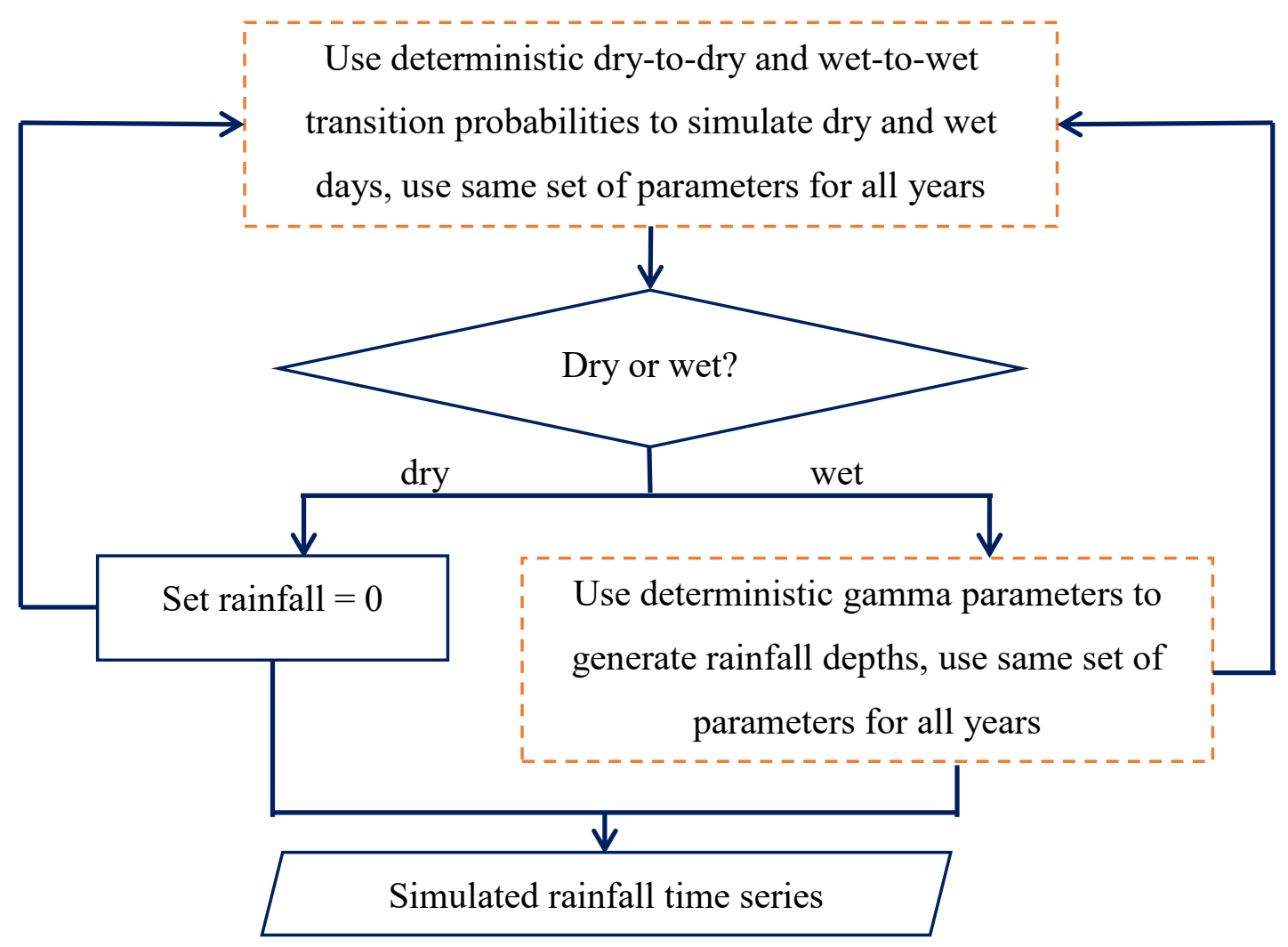

Figure 4.1: Schematic of the APMC. 


\subsubsection{Justifications of Model Assumptions}

This section will present the assessments done in this study to justify the key assumptions of the APMC. These assumptions are also used for other MC models (DPMC, CDMC, HMC and DHMC).

\subsubsection{Use of monthly varying parameters}

Figure 4.2 shows the APMC parameters $\left(P_{00}, P_{11}, \mu\right.$, and $\left.\sigma\right)$ calibrated to RCM2 and AWAP at Gummun. For RCM2, the parameters show a systematic monthly variation with a higher probability of wet days in summer (December-February) and higher probability of dry days in winter (June-August). Similarly, there is greater rainfall on wet days (higher mean and SD) in summer and less rainfall in winter, which is consistent with DECCW [2010] estimates of NSW rainfall. Autumn (March-May) has less frequent heavy rainfall days while spring (SeptemberNovember) has more frequent low rainfall days. The seasonal variabilities are similar in AWAP for $P_{11}, \mu$, and $\sigma$, while only $P_{00}$ is mostly stable across the months. The clear variation in parameter values between the months justifies the use of monthly varying parameters.

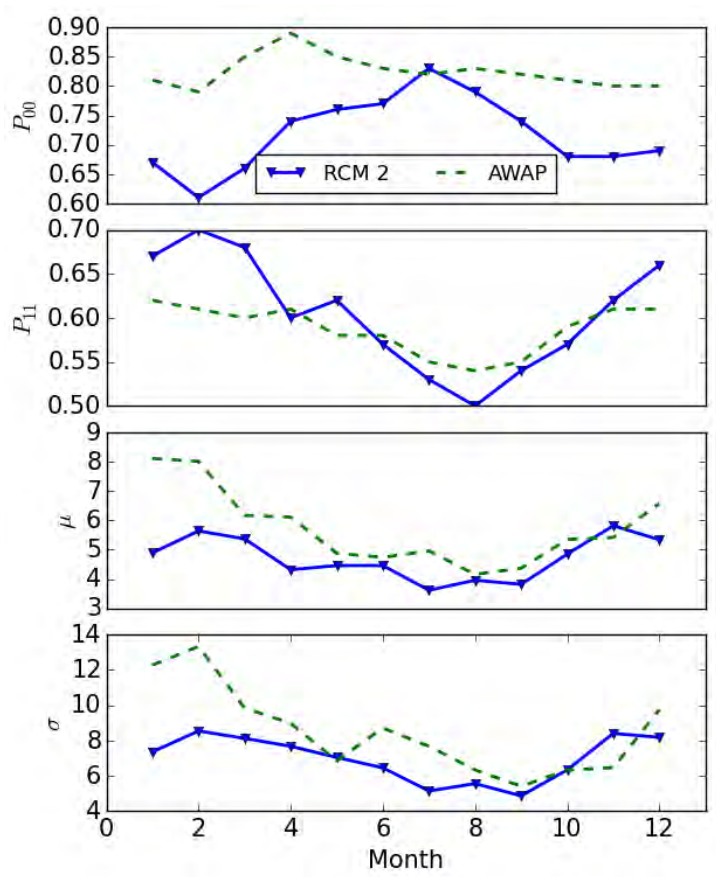

Figure 4.2: MC parameters $P_{00}$ and $P_{11}$ (transition probabilities of dry-to-dry and wet-to-wet day respectively), and Gamma distribution parameters $\mu$ and $\sigma$ (mean and SD of wet day rainfall depths respectively) of APMC in RCM2 and AWAP. The parameters are shown for each month, where the first month is January. 
Figure 4.3 shows the month-to-month autocorrelations of monthly rainfall depths and number of wet days for RCM2 and AWAP. For both datasets, the autocorrelations show clear seasonal signals between lag-1 (i.e. January) and lag-12 (i.e. December), although the autocorrelations are weaker in AWAP than RCM2. This also supports the use of monthly varying parameters in APMC.
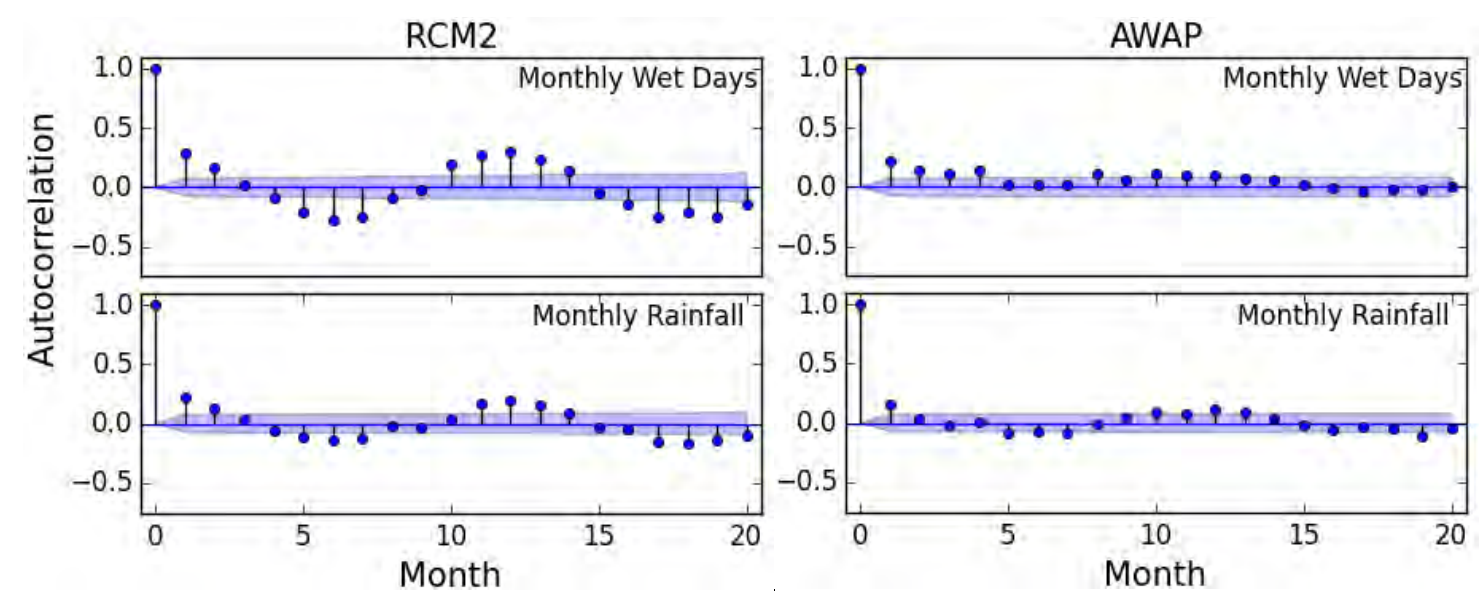

Figure 4.3: Month-to-month autocorrelations of monthly rainfall depths and number of wet days in RCM2 and AWAP.

\subsubsection{Threshold wet day rainfall depths}

In calibration of the APMC parameters, this study has considered a day as a 'wet day' if rainfall depth of that day is above $0.3 \mathrm{~mm}$, otherwise it is considered as a dry day (zero rainfall). Some other previous studies, including Mehrotra et al. (2015), also used the $0.3 \mathrm{~mm}$ threshold to define wet days. Moreover, examination of the NARCliM reanalysis data (for all RCMs) highlighted a considerable number of days with rainfall depths between 0.0 and $0.3 \mathrm{~mm}$. When these rain days with less than $0.3 \mathrm{~mm} /$ day were considered to be wet days, they yielded more wet-to-wet probabilities $P_{11}$, but less mean wet day rainfall depths $\mu$ (see Figure B.1 at Appendix B.1). In addition, the minimum daily rainfall in the AWAP data is $0.1 \mathrm{~mm}$, and about $10 \%$ of the rainfall days have a value of $0.1 \mathrm{~mm}$. Therefore, this study has used the $0.3 \mathrm{~mm}$ threshold for all datasets (e.g. NARCliM RCMs, AWAP and raingauge).

\subsubsection{Gamma distribution for wet day rainfall depths simulation}

Figure 4.4 shows the comparison between the distribution of wet day rainfall depths in RCM2 (for four typical months) and the distribution of wet day rainfall depths in APMC, generated by using Gamma distribution with parameters calibrated to the RCM2. This comparison shows that the distribution of wet day rainfall depths of the RCM2 has been well reproduced in 
APMC, which also justifies the use of Gamma distribution to simulate wet day rainfall depths. This finding is also true for the AWAP data (see Figure B.2 at Appendix B.1).
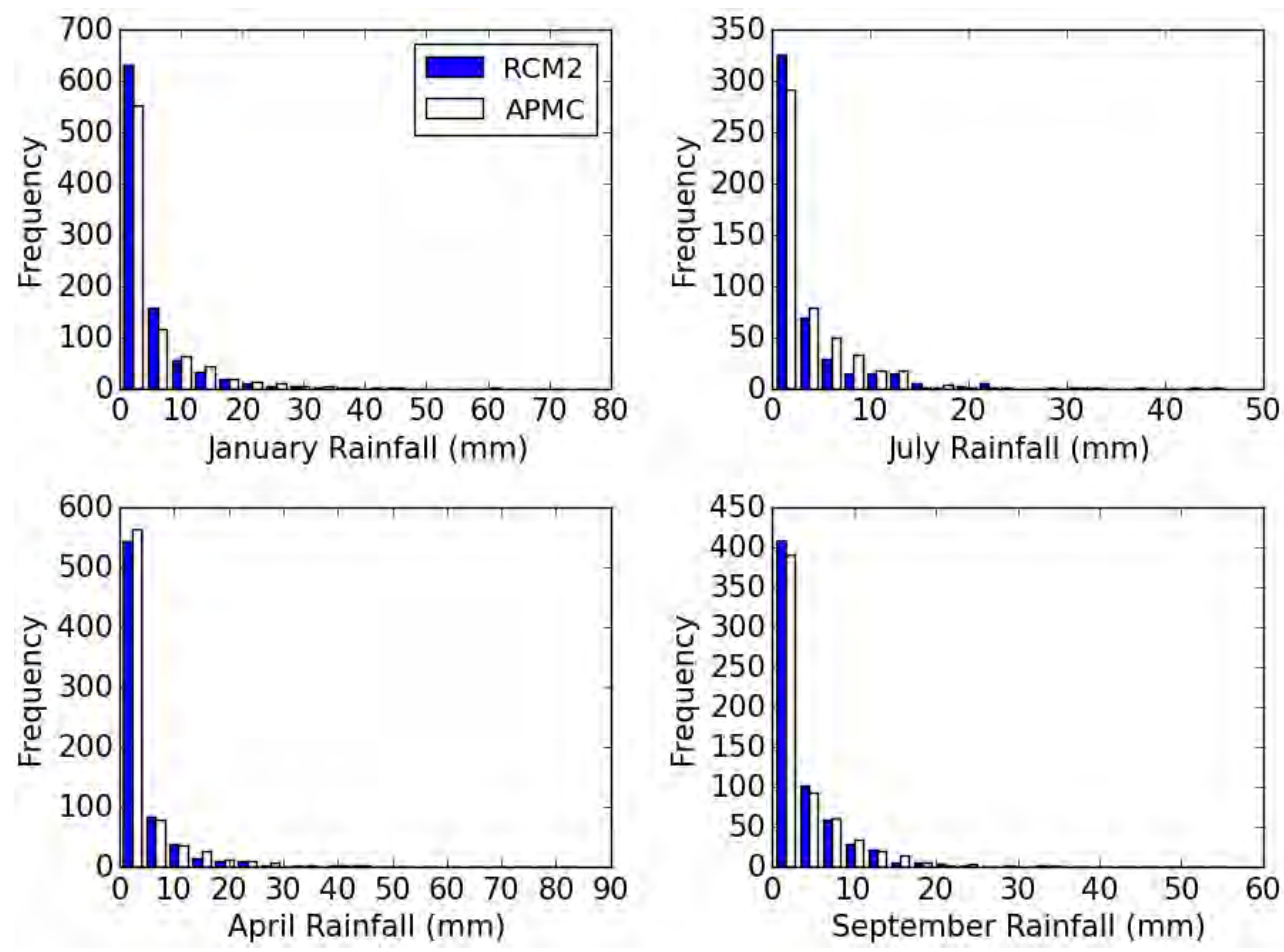

Figure 4.4: Distribution of wet day rainfall depths in RCM2 and APMC simulation for January, April, July, and September (typical months).

Figure 4.5 shows the comparison of the distribution of wet day rainfall depths for rainfall depths between $0.3 \mathrm{~mm}$ and $1.0 \mathrm{~mm}$. It shows that the APMC generates a considerably higher number of wet days with $0.3 \mathrm{~mm}$ rainfall depth than the RCM2. This is because in generation of rainfall depths for wet days in APMC, if a random sample from the Gamma distribution gives a rainfall depth less than $0.3 \mathrm{~mm}$ then the rainfall for that day is set to $0.3 \mathrm{~mm}$. However, Figure 4.4 suggests that it does not affect the overall distribution of wet day rainfall depths in APMC as the frequency of minimum rainfall depths in APMC and RCM2 are very much similar. Similar investigation for the AWAP data also suggests that setting the $0.3 \mathrm{~mm}$ as minimum rainfall depth does not affect the overall distribution of wet day rainfall depths in APMC (compare between Figure B.2 and B.3 at Appendix B.1). 

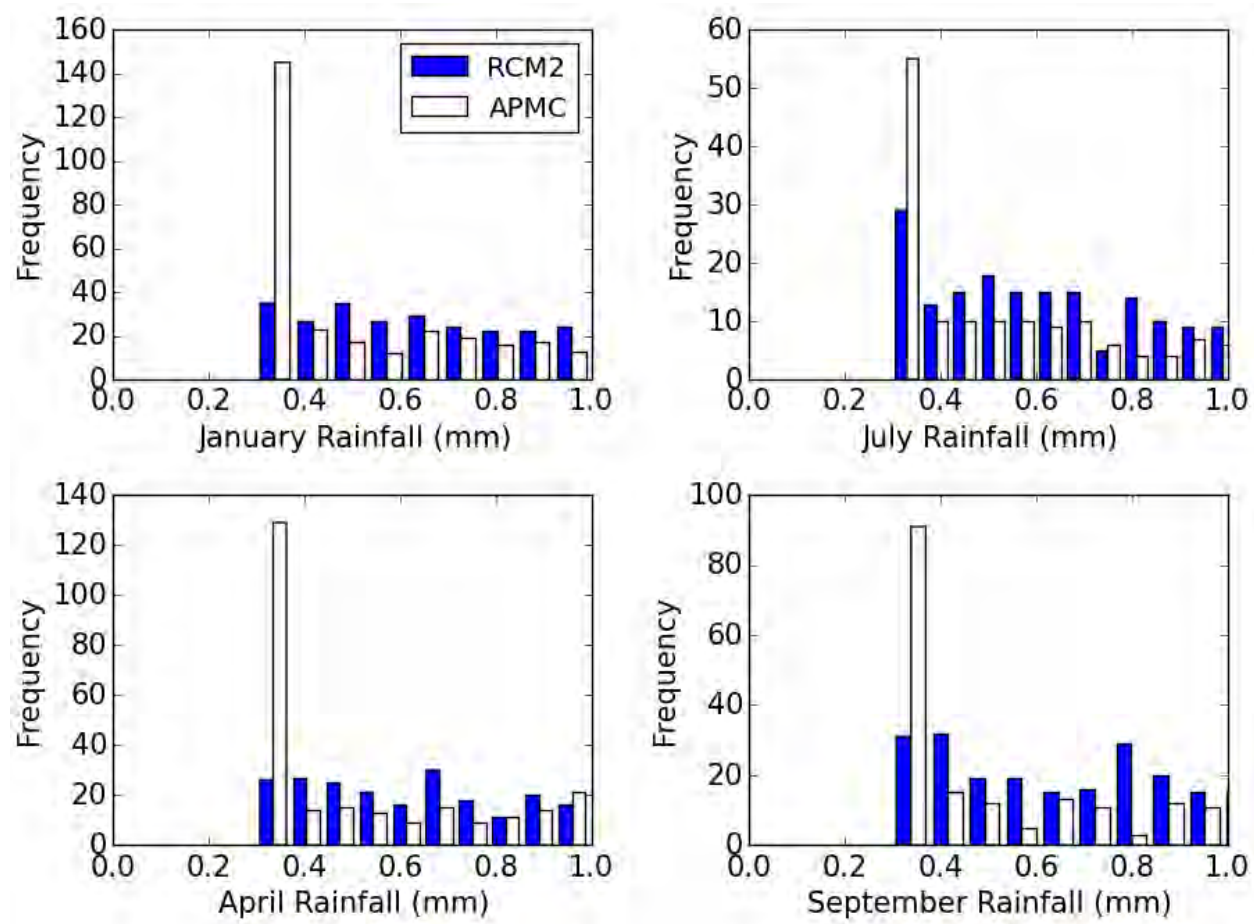

Figure 4.5: Distribution of wet day rainfall depths between $0.3 \mathrm{~mm}$ and $1 \mathrm{~mm}$ in RCM2 and APMC simulation for January, April, July and September (typical months).

\subsubsection{Characteristics of Gamma distribution parameters}

Figure 4.6 and Figure 4.7 show the characteristics of Gamma distribution parameters, which indicate how the distribution changes with different combinations of parameter values. These results also justify the use of $\mu$ and $\sigma$ as Gamma distribution parameters instead of $\alpha$ and $\beta$ in this thesis. The $\alpha$ and $\beta$ parameters of rightly skewed Gamma distribution are positive. The shape parameter $\alpha$ determines the skewness of the distribution. For $\alpha<1$, the distribution tends to infinity at minimum rainfall and decreases monotonically for increasing rainfall. With an increase in $\alpha$ value, the distribution becomes less skewed, the peak of the distribution moving away from the minimum value with a broader distribution, indicating a decline of low rainfall events. The Gamma distribution turns out to be an exponential distribution with $\alpha=1$, and it approaches a normal distribution for larger values of $\alpha$ (Figure 4.6). The scale parameter $\beta$ determines the dispersion of the gamma distribution. The higher the $\beta$ is, the higher the gamma curve dispersion will be, which indicates existence of heavy rainfall days. The modes shift to the right as the ratio of $\alpha / \beta$ increases (Figure 4.6). However, both $\alpha$ is directly proportional to the $\mu$ of wet day rainfall if $\beta$ is kept constant and vice versa. The $\beta$ is directly proportional to $\sigma$ if $\alpha$ is kept constant, while $\alpha$ and $\sigma$ have a non-linear relation (Figure 4.7). Since $\alpha$ and $\beta$ are derived from $\mu$ and $\sigma$, it is convenient to calculate the $\mu$ and $\sigma$ values from the observed daily rainfall in calibration and then use their appropriate ratios (Equation 4.5) in the rainfall depth 
generation process by Gamma distribution. Therefore, $\mu$ and $\sigma$ have been referred to the Gamma distribution parameters in this study.
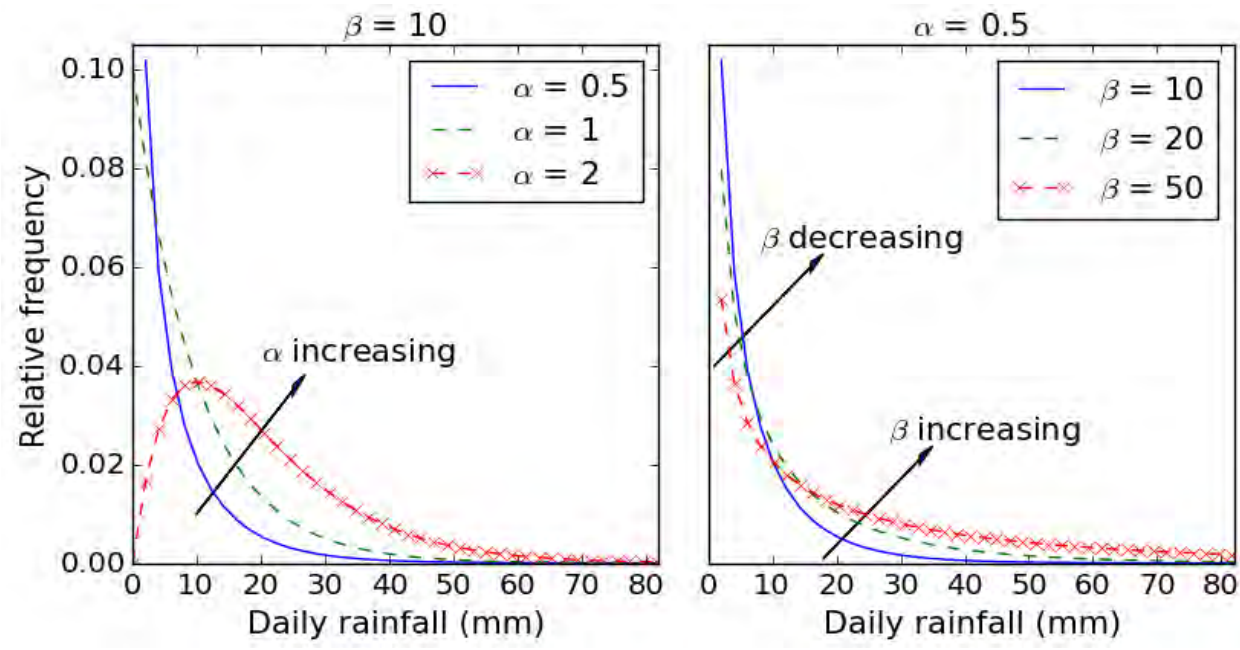

Figure 4.6: Gamma distribution with different $\alpha$ and $\beta$.

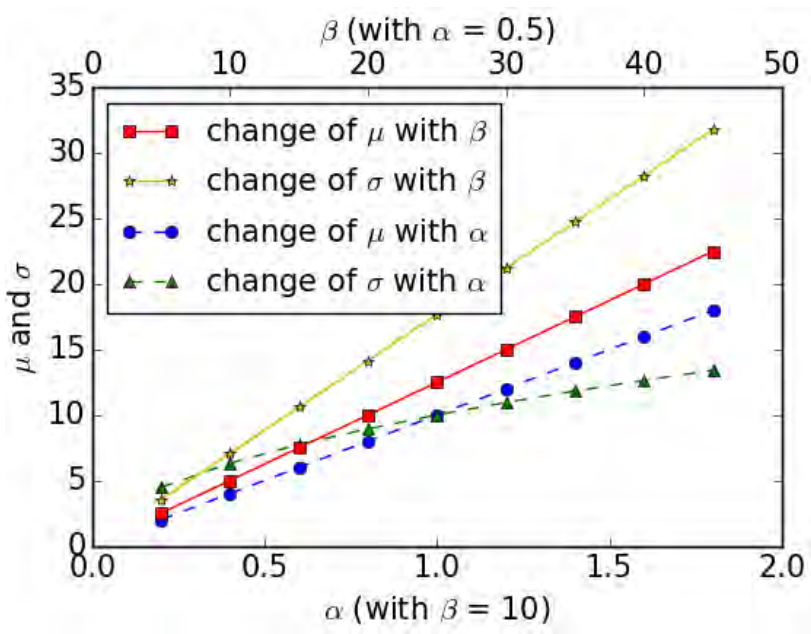

Figure 4.7: Changes of $\mu$ and $\sigma$ with changes of $\alpha$ and $\beta$.

\subsubsection{Independence of rainfall depths in successive wet days}

The APMC assumes that the rainfall depth for a particular day is independent of the rainfall depth of the preceding day (although the occurrence of rainfall in a day is dependent on the wet or dry state of the previous day). To validate this assumption, this study has examined the correlation of rainfall depths for two successive wet days. First, only a Box-Cox transformation (no standardization) with lambda $\lambda$ value of 0.1402 was used to transform the skewed wet day rainfall depths into an approximately normal distribution to avoid sampling bias. The transformed rainfall of a day is then plotted against the same of the previous day, which shows a very weak correlation $\left(r^{2}=0.065\right)$ among the rainfall depths of successive wet days (Figure 
4.8). Investigating the same relationship for each individual month, this study has found similar independence of the wet day rainfall depths irrespective of seasonal variations. The conclusion is that the underlying assumption of daily independence of the APMC is consistent with the respective characteristic of the calibration data. The relevant analysis of AWAP data also shows a very weak correlation $\left(r^{2}=0.062\right)$ among the rainfall depths of successive wet days (see Figure B.4 at Appendix B.1).
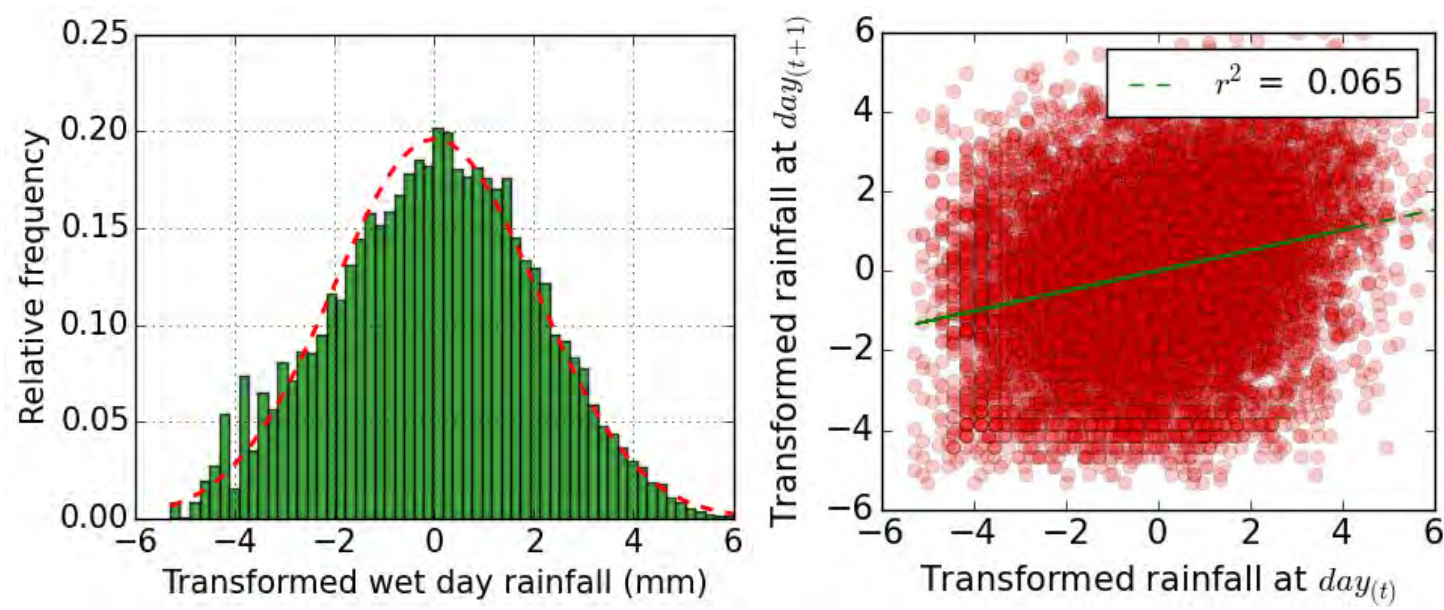

Figure 4.8: Box-cox transformation of wet day rainfall depths in RCM2 using $\lambda=0.1402$ (left). Correlation of transformed rainfall depths in successive wet days (right).

\subsubsection{Simulation of wet and dry spells}

Figure 4.9 shows the relative frequencies (in log scale) of the occurrence of wet and dry spells in RCM2 and APMC (for January as a typical month). This indicates that the first-order MC process simulates wet and dry spells with an exponentially distributed frequency of occurrence, which is consistent with the findings of Green [1964]. It also indicates that the APMC simulation has mostly mirrored the spell properties of RCM2 data with a mild divergence for the longer spells. The performance of the MC models to simulate the wet and dry spells is further assessed in this study by calculating the distribution of mean length of wet spells at monthly and annual resolutions using the method described in section 3.4.2. 


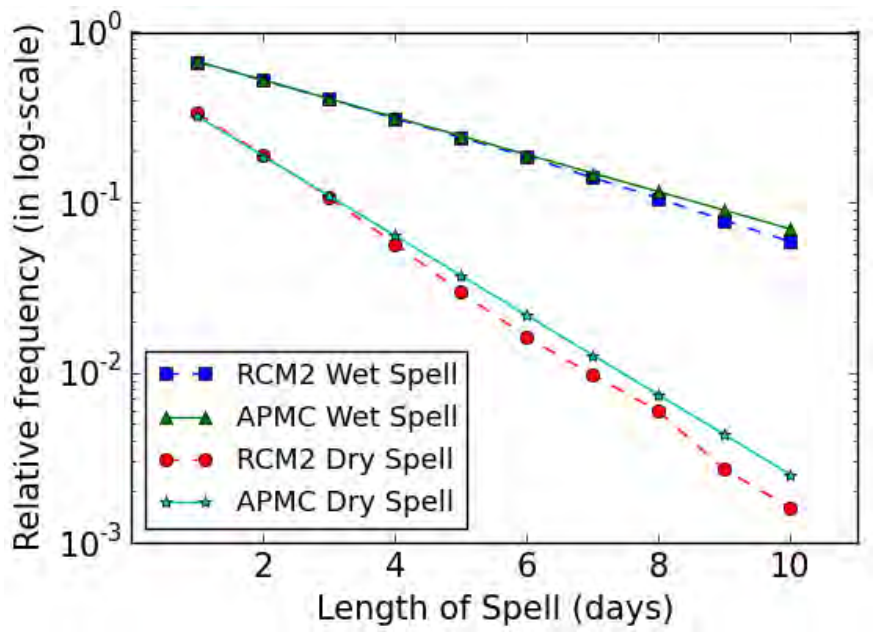

Figure 4.9: Simulation of wet and dry spells in APMC for January (a typical month) for RCM2.

\subsubsection{Summary of APMC}

The APMC uses deterministic parameters of MC (i.e. the transition probabilities of wet-to-wet day and dry-to-dry day) to simulate rainfall occurrence and deterministic parameters of Gamma distribution (i.e. mean and SD of wet day rainfall depths) to simulate rainfall depths in wet days. This section has discussed the structure of the APMC with calibration and simulation methods, and justified the associated assumptions of the APMC. However, the discussion in Chapter 5 will show that the APMC cannot satisfactorily reproduce the SD of rainfall depth at monthly to multiyear resolutions, although it reproduces the mean in all resolutions and SD at the daily resolutions very well. Therefore, this study has rejected the APMC because it underestimates longer resolution rainfall depth variability (see Chapter 5 for details). In the subsequent modifications of the MC model, this study will overcome this limitation by introducing different approximations.

\subsection{Decadal Parameter Markov Chain (DPMC) Model}

\subsubsection{Model Structure}

The DPMC is the second model developed in this thesis. It assumes that the inter-annual rainfall variability can be captured by the decade-to-decade variability of the parameters. The idea is to divide the observed rainfall sample into sub-samples of 10-year lengths (e.g. dividing a 60 -year rainfall sample into six sub-samples of 10 -years in length). Then, $4 \times 12$ parameters of $P_{00}, P_{11}, \mu$, and $\sigma$ (one set of four parameters for each of the 12 months) are calculated from each of the sub-samples. The simulation proceeds in a way similar to the APMC, except that 
the parameters are varied from decade to decade. The idea of the model was that decadal variability of parameters would increase the multiyear variability that APMC failed to capture.

\subsubsection{Schematic of DPMC}

Figure 4.10 shows a schematic of the DPMC. The DPMC uses deterministic parameters similar to those used in the APMC. However, in each DPMC simulation, separate sets of MC-Gamma parameters are used to simulate rainfall for each 10-year period (e.g. six sets of parameters for a 60-year simulation). The red dashed boxes of Figure 4.10 can be compared with those of Figure 4.1, Figure 4.21, Figure 4.30 and Figure 4.31 to see the differences with APMC, CDMC, HMC and DHMC respectively.

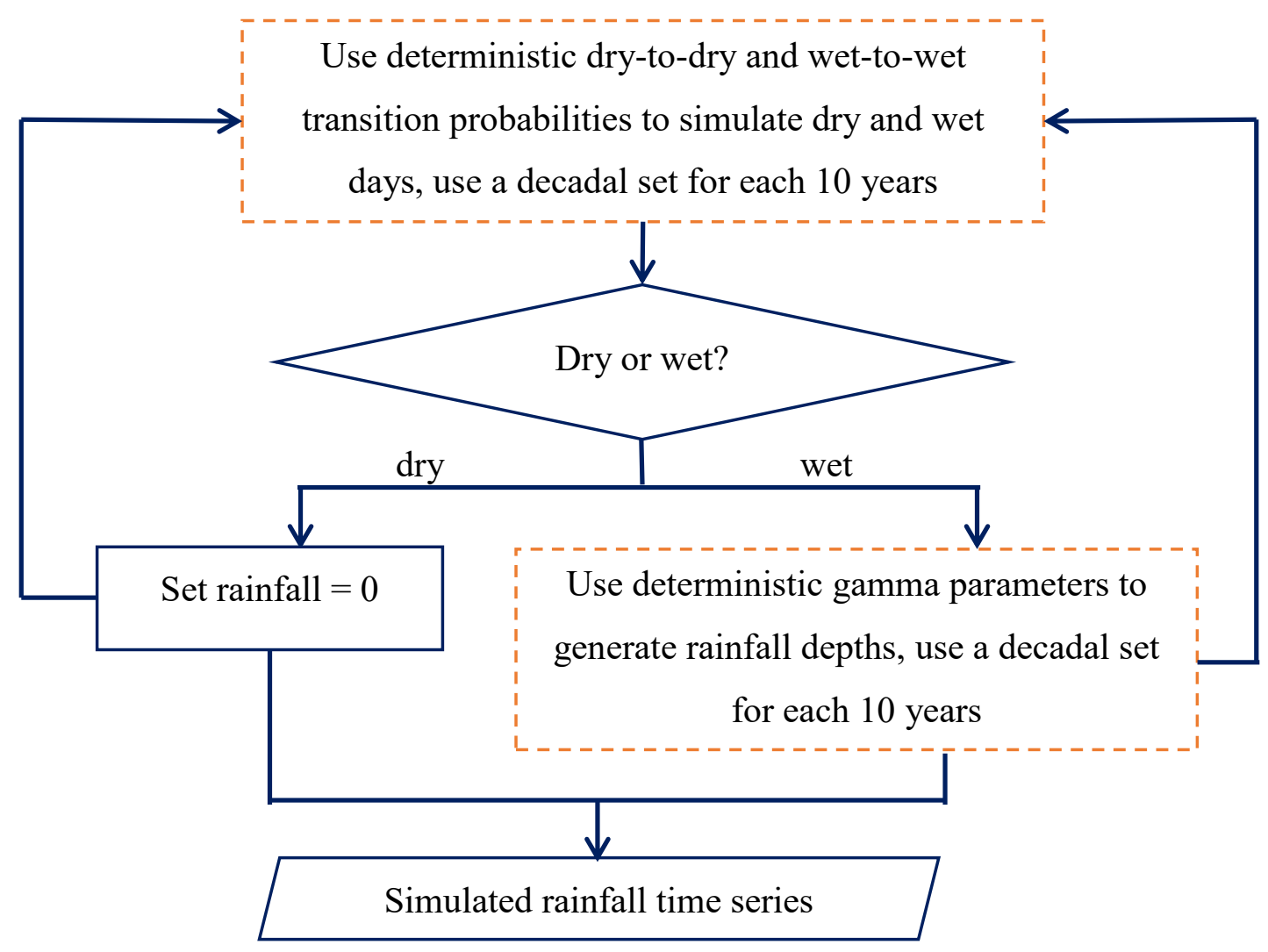

Figure 4.10: Schematic of the DPMC. Compare the red boxes with the same of Figure 4.1 Figure 4.21, Figure 4.30 and Figure 4.31 to see the relative differences in APMC, CDMC, HMC and DHMC respectively. 


\subsubsection{Calibration of DPMC Parameters to RCM2}

In calibration of DPMC, the MC-Gamma parameters of $P_{00}, P_{11}, \mu$, and $\sigma$ are calculated from each of the 10-year sub-samples (e.g. 1950-59, 1960-69) of the RCM2 data. Figure 4.11 shows the $P_{11}$ and $\mu$ values for each decade for the DPMC along with the 60 -year averages used by the APMC (from above). The DPMC values of wet-to-wet transition probability $\left(P_{11}\right)$ and rainfall depths $(\mu)$ show clear variability among the decades and deviate from the APMC values. DPMC parameters of AWAP also show similar decadal variability (see Figure B.5 at Appendix B.2).
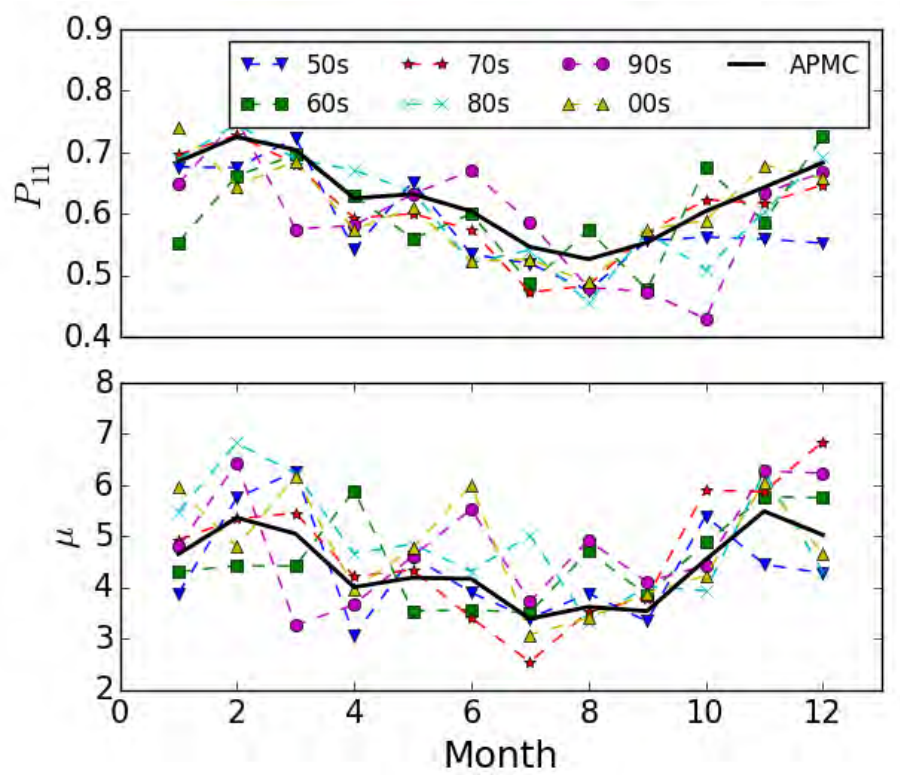

Figure 4.11: Comparison of the decadal variability of the DPMC parameters (wet-to-wet probabilities $\boldsymbol{P}_{11}$ and mean wet day rainfalls $\boldsymbol{\mu}$ ) with the APMC parameters. The coloured lines are the values of DPMC parameters and the thick black lines are the values of APMC parameters. 


\subsubsection{Statistical Significance of the Decadal Variability of DPMC Parameters}

The use of decadally varied parameters in the model is subject to the question of how significant the decadal variability of these parameters is - is the decadal variability statistically significant or just sampling variability? Therefore, this section examines the statistical significance of the decadal variability of DPMC parameters as follows:

- Similar to section 3.4.3, six APMC are run using each of the six sets of DPMC parameters (calibrated to the each 10-year sample as mentioned above).

- Models for each decade are run 1000 times with each run being 10-years long (i.e. six sets of 1000 runs, each with a different decadal parameters).

- The $4 \times 12$ values of $P_{00}, P_{11}, \mu$, and $\sigma$ are calculated for each decadal simulated rainfall series. This gives 1000 realisations of simulated parameters for every decadal sample.

- Then, for each month, the $6 \times 1000$ realisations of the simulated parameters in addition to the calibrated DPMC parameter values are plotted in boxplot distributions.

- The boxplots indicate the range of sampling variability of the calibrated parameters.

- For each month, the range of simulated parameters and the DPMC parameter values for the six decades are compared to each other and with the corresponding APMC value (60-year averaged value) to understand the statistical significance of the decadal variability of DPMC parameters.

Figure 4.12 shows the sampling distributions of the DPMC parameters $P_{11}$ and $\mu$ for January (summer) and July (winter) as representative months (the results for the other two parameters and other months are similar). As shown by the green dots and blue horizontal bars, the APMC value of most of the parameters are within the $95 \%$ confidence interval of the simulated DPMC parameters. Also, the sampling distribution of the decadal parameters in one decade overlaps the distribution for other decades. The above two observations indicate that the decadal variations of the parameters are not statistically significant from each other. This finding is also consistent for AWAP (see Figure B.6 at Appendix B.2). 

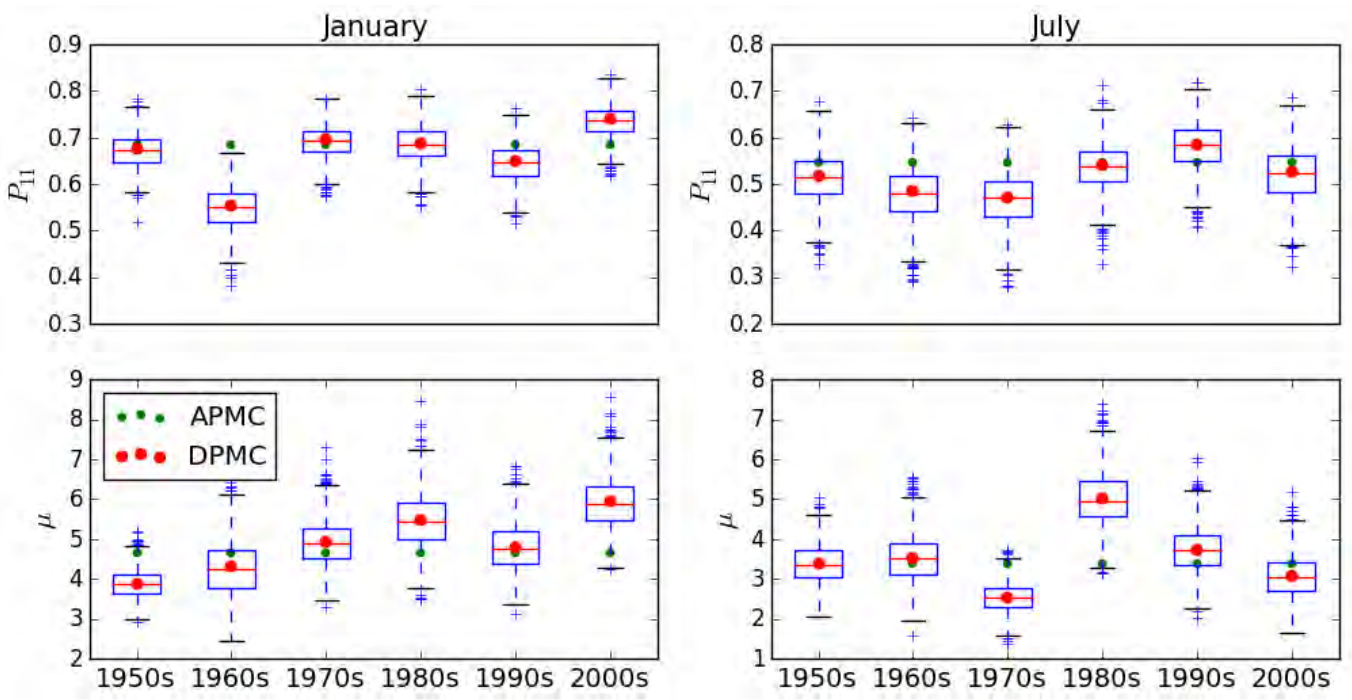

Figure 4.12: Sampling distributions of DPMC parameters $\left(P_{11}\right.$ and $\left.\mu\right)$ for January and July as two representative months. The blue horizontal bars are the $5^{\text {th }}$ to $95^{\text {th }}$ percentile bounds, the boxes are the $25^{\text {th }}$ to $7^{\text {th }}$ percentile bounds, and the red horizontal bars are the $50^{\text {th }}$ percentiles of the $6 \times$

1000 realisations of simulated parameter. The red dots show the DPMC parameter values, calculated from each of the 10-year samples of RCM2 data at Gummun. The green dots show the respective APMC value of a parameter.

\subsubsection{Potential Impact of Climate Modes}

This study has also investigated other sub-sampling approaches of the MC-Gamma parameters similar to the DPMC, which include:

- models with separate parameters for sub-samples of 5-year lengths, assuming more interannual variability can be introduced

- models with separate parameters for sub-samples divided according to the phases of large resolution climate drivers (e.g. IPO and ENSO) as the inter-annual rainfall variabilities of east Australia are linked with these climate drivers (see section 2.3).

\subsubsection{Using 5-year sub-samples}

The parameter calibration and simulation processes of this model are similar to those of the DPMC, except the 60-year rainfall sample of RCM2 was divided into 12 sub-samples of 5-year lengths, instead of six sub-samples of 10-year lengths (see Figure B.7 at Appendix B.3). The idea was that the use of varying parameters for simulation of each 5-year rainfall might introduce more inter-annual variability. However, this model also underestimated the SD of rainfall depths at monthly and multiyear resolutions, and hence has been rejected by this study. 


\subsubsection{Using sub-samples divided according to IPO and ENSO phases}

In these models, this study has calibrated the MC-Gamma parameters to sub-samples of rainfall timeseries divided according to the phases of IPO (e.g. positive and negative) and ENSO (La Niña, Neutral and El Niño). Since previous studies [Verdon-Kidd et al., 2004] found that the inter-annual variabilities of east-Australian rainfall are influenced by these large-scale climate drivers (also see section 2.3), the idea behind these models was to introduce more inter-annual variability to the model by simulating rainfall for different phases of climate drivers with parameters calibrated to respective phases. This model is very similar to DPMC, except that the sub-samples are different. The following two types of models have been tested:

- For IPO phases, the 60-year RCM2 data was divided into two sub-samples according to the positive and negative IPO index. Then the MC-Gamma parameters $\left(P_{00}, P_{11}, \mu\right.$, and $\sigma$ ) are calibrated to each sub-sample. In simulation, the rainfall for each phase of IPO was modelled by using parameters of respective phase.

- For ENSO, the RCM2 data was divided into three sub-samples according to ONI index: La Niña $(\mathrm{ONI} \leq-0.5)$, Neutral $(-0.5<\mathrm{ONI}<0.5)$, and El Niño $(\mathrm{ONI} \geq 0.5)$. Similar to DPMC, the MC-Gamma parameters are calibrated to each sub-sample and the rainfall data for each phase of ENSO was modelled by using parameters of the respective phase.

The parameters calibrated to the rainfall sub-samples of IPO phases show that the negative IPO phases were wetter than the positive IPO phases. For ENSO phases, the calibrated parameters show that the La Niña states and El Niño were the wettest and driest respectively, while the parameters of Neutral phase mostly coincided with the 60-year mean (Figure 4.13).

Similar to DPMC, these models also systematically underestimated the SD of rainfall depths at monthly and multiyear resolutions. This supports the findings of the DPMC that the deterministic parameters of divided sub-samples are not adequate to capture the long-term variability of rainfall depths. 

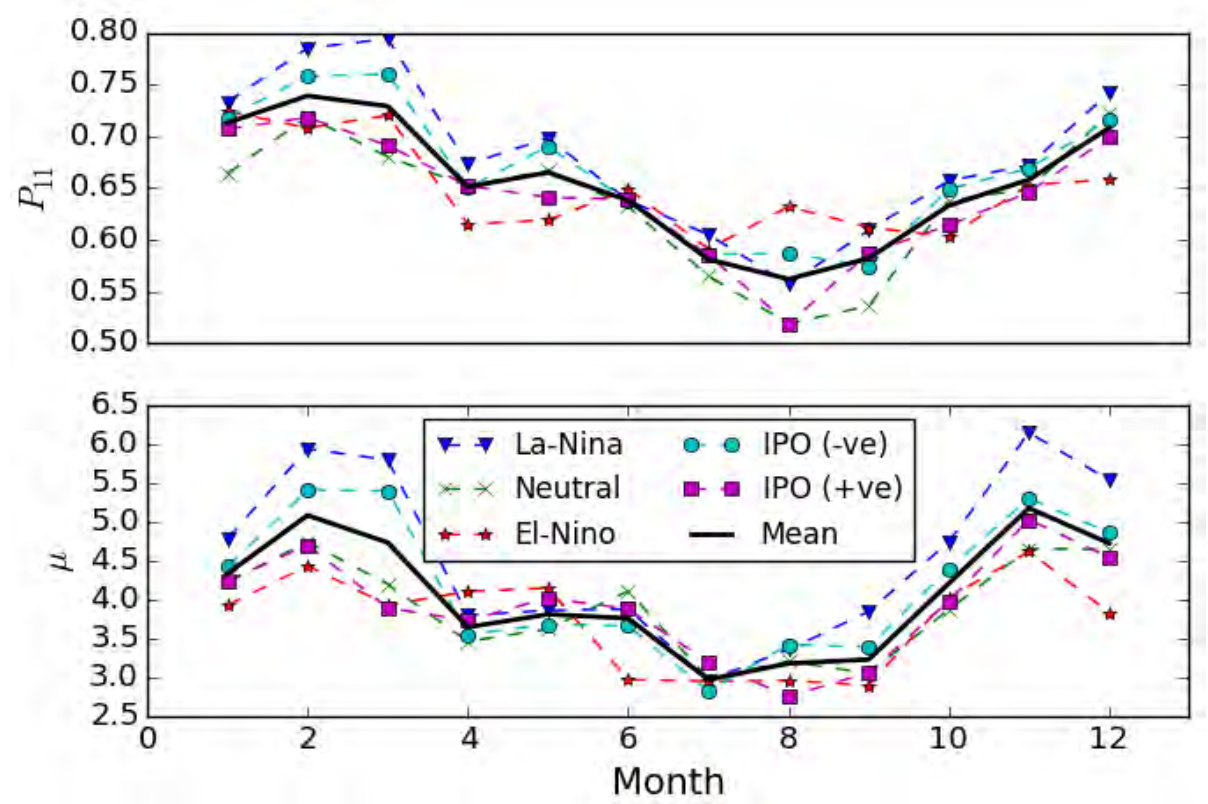

Figure 4.13: Parameters $\left(P_{11}\right.$ and $\left.\mu\right)$ of sub-samples of RCM2 according to the two IPO (positive and negative) and three ENSO phases (La Niña, Neutral and El Niño).

For further investigation, this study has calculated the MC-Gamma parameters for each month of each year, that is, $12 \times 60$ values of each parameter from the RCM2 data. Then, the correlations of each parameter with the monthly values of eight climate indices, ONI, nino3.4, MEI, PDOI, SOI, SAMI, DMI and IPOI (see 3.2.5) were tested (Figure 4.14 and Figure 4.15). The results show that the parameter-index correlations are very weak for all indices, suggesting the use of these indices for DPMC-like models might not be suitable to capture the multiyear variability of rainfall depths. This finding was also true for AWAP data (see Figure B.8-B.9 at Appendix B.3).

The above evaluations of the climate-driver based models only focused on the ability of the models to reproduce the multiyear variability of rainfall depths and did not consider their ability to reproduce the multiyear variability of wet periods. As it was realized at the end of this study that the decade-varied MC parameters used in the final model DHMC (see section 4.6) can preserve the multiyear variability of wet periods, it might be worthwhile for a future study to further investigate the climate-driver based models focusing their ability to reproduce the multiyear variability of wet periods. While this study has only investigated the relationship between climate indices and model parameters (Figure 4.14 and Figure 4.15), this future study might also investigate the relationship between climate indices and parameter anomalies from the longer-term parameter value. 

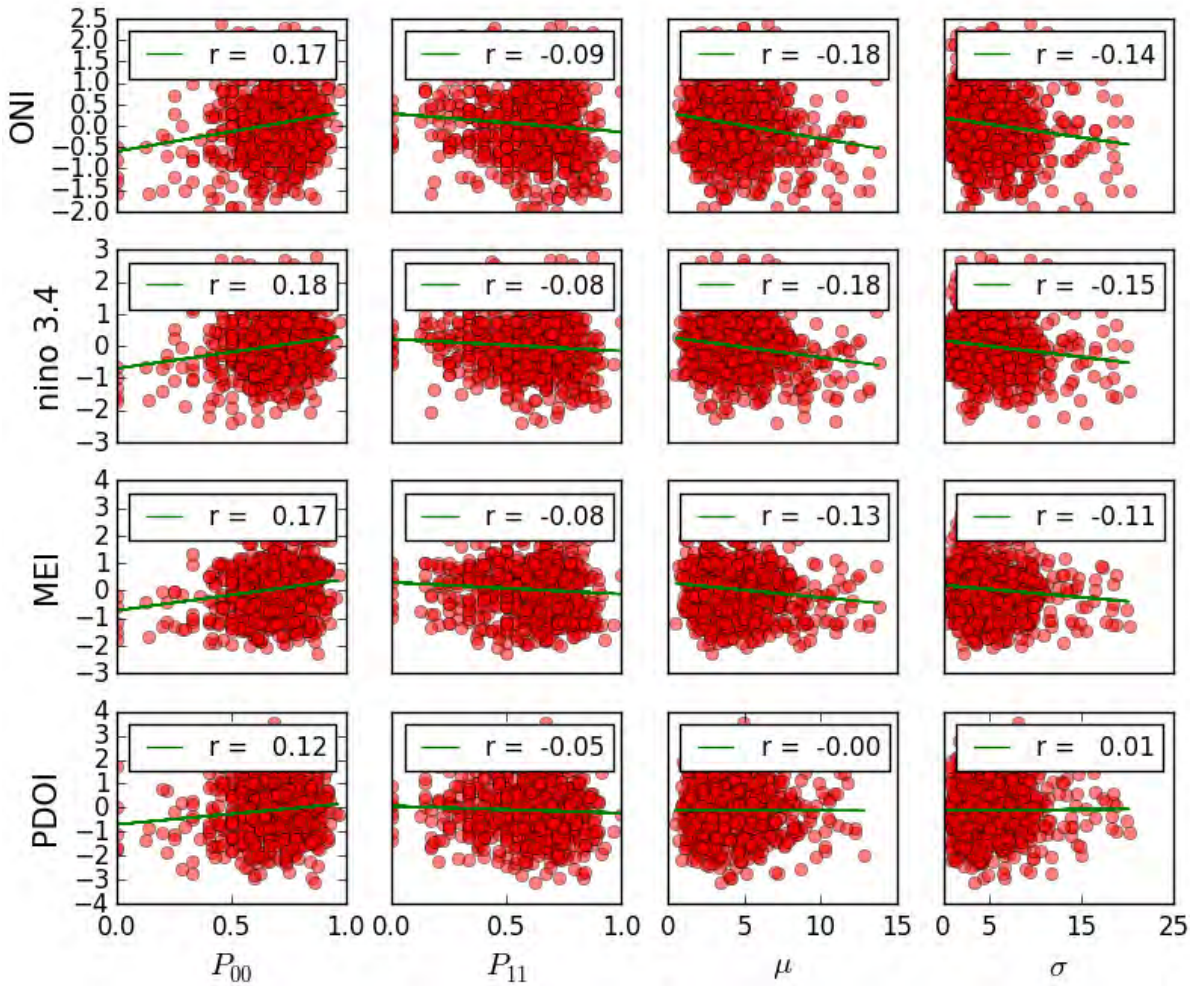

Figure 4.14: Correlation between the 12x 60 values of each parameter $\left(P_{00}, P_{11}, \mu\right.$, and $\left.\sigma\right)$ for RCM2 and ONI, nino3.4, MEI, PDOI climate indices.
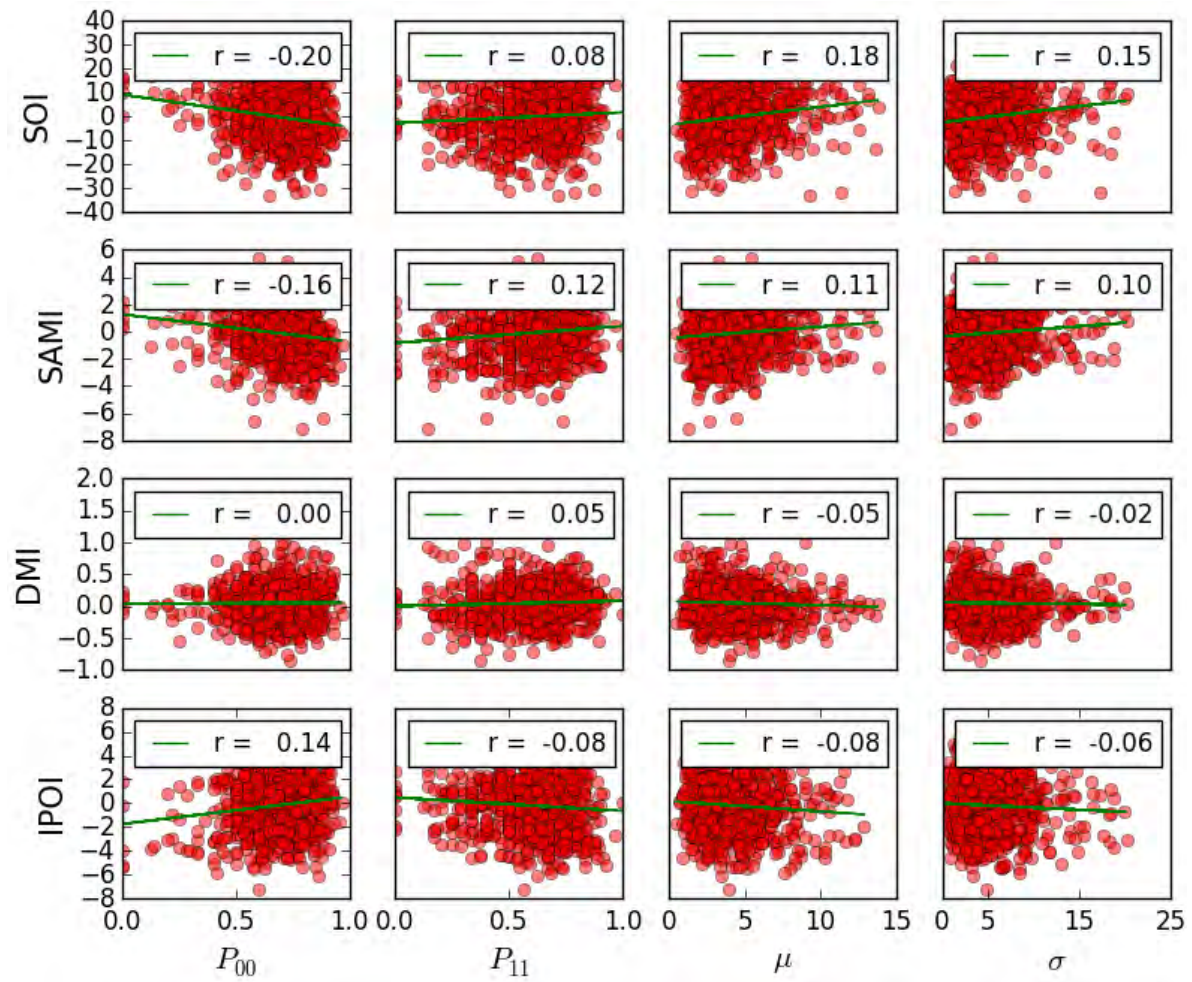

Figure 4.15: Correlation between the 12x 60 values of each parameter $\left(P_{00}, P_{11}, \mu\right.$, and $\left.\sigma\right)$ for RCM2 and SOI, SAMI, DMI, and IPOI climate indices. 


\subsubsection{Summary of DPMC}

The DPMC uses deterministic parameters similar to the APMC, except the simulation of daily rainfall in each decade (10 years) uses individual sets of parameters. A single run of 60 -year simulation uses six sets of parameters, while each parameter set is calibrated to each of the six 10-year sub-sample of the observed data. However, a relevant test of DPMC parameters has shown that the APMC parameters are mostly within the sampling variability of DPMC parameters. Chapter 5 will show that the DPMC has also failed to preserve the monthly to multiyear variability of rainfall depths, although it reproduced the wet periods better than the APMC. In addition, this study has investigated other models similar to DPMC, such as models with parameters varying for each 5-year interval (instead of 10-years as for DPMC), and models with parameters varying for phases of IPO or ENSO. However, these models also systematically underestimated the SD of rainfall depths at monthly to multiyear resolutions (see section 5.4). It suggests that the use of deterministic parameters in DPMC-like models might not be adequate to satisfactorily reproduce the SD of rainfall depths at monthly and multiyear resolutions. Therefore, like the APMC, the DPMC has been rejected by this study.

\subsection{Compound Distribution Markov Chain (CDMC) Model}

\subsubsection{Model Structure}

The previous two sections have discussed the APMC and DPMC, but they cannot satisfactorily reproduce the SD of rainfall depths for monthly to multiyear resolutions. Since both APMC and DPMC use deterministic parameters for the Gamma distribution, it suggests that the use of deterministic parameters might not be sufficient to incorporate the long-term variability of rainfall depths in the model. Accordingly, this study has examined which of the four parameters $\left(P_{00}, P_{11}, \mu\right.$ and $\sigma$ ) regulate the long-term variability of rainfall depth. While the details of this examination are available in Appendix B.4, the examination has found that variability of rainfall depths is predominantly regulated by the variability of $\mu$ and $\sigma$, rather than the variability of $P_{00}$ and $P_{11}$. This finding is also consistent with Dubrovský et al. [2004], who found that the MC parameters has marginal effect on the long-term variability of rainfall depths. Therefore, in the third MC model - the CDMC - this study has incorporated the longterm variability of rainfall depths by using random variability in $\mu$ and $\sigma$. However, for wet and dry period simulation, the CDMC still uses the deterministic parameters of $P_{00}$ and $P_{11}$, as in the APMC. 


\subsubsection{Deterministic parameters of MC process}

For month $i$ (e.g. $i=1$ for January), the CDMC uses the same deterministic parameters of $P_{00, i}$ and $P_{11, i}$ as used in APMC for simulating the occurrence of wet and dry days (see section 4.2.1.1).

\subsubsection{Stochastic parameters of Gamma distribution}

In the CDMC, $\mu_{i}$ and $\sigma_{i}$ are randomly sampled for each month of each year. The random sampling was done independently of the sampling for the preceding month/s. To estimate the distribution of $\mu_{i}$ and $\sigma_{i}$, this study has calculated $\mu_{i}$ and $\sigma_{i}$ for every month of every year from the data to be modelled. For example, from the 60-year RCM2 data, for January $(i=1)$, this study has calculated 60 samples of $\mu_{1}$ and $\sigma_{1}$ values each.

Figure 4.16 shows the boxplot distribution of $\mu_{i}$ and $\sigma_{i}$ values of RCM2 for each month, fitted to the 60 values of a parameter for month $i$. In addition, the respective APMC values are shown in green dots. The boxplots show that the value of $\mu_{i}$ and $\sigma_{i}$ for a month can vary considerably over a 60 -year period. For example, for January, the $\mu_{1}$ parameter can vary between $2 \mathrm{~mm}$ to 8 $\mathrm{mm}$, and the $\sigma_{1}$ parameter can vary between $2 \mathrm{~mm}$ to $11 \mathrm{~mm}$, while the respective APMC values of $\mu_{1}$ and $\sigma_{1}$ are around $5 \mathrm{~mm}$ and $7 \mathrm{~mm}$. This indicates that the use of an averaged value of a parameter (as for APMC) for generating rainfall depths of all years might not be sufficient to represent the range of variability of the parameter over the sampling period of the data to be modelled. Therefore, to incorporate the variability of the parameters, suitable statistical distributions are fitted to the calculated values of the parameters of each month. Then, in the simulation process, the parameters are stochastically sampled from the fitted distribution for each month of each year. 

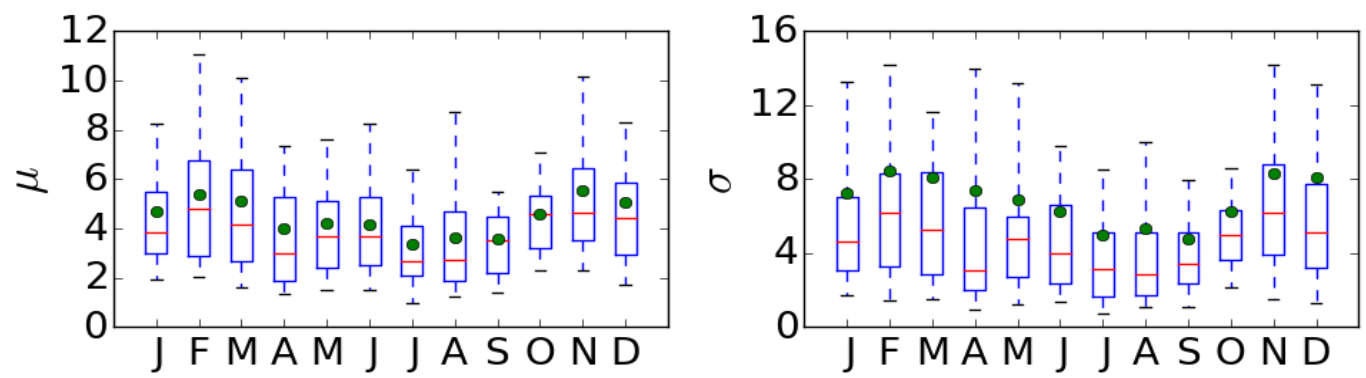

Figure 4.16: Boxplot distribution of $\mu$ and $\sigma$ for every month. Each boxplot is fitted to the 60 values a parameter for respective month. The blue horizontal bars are the $5^{\text {th }}$ to $95^{\text {th }}$ percentile bounds, the boxes are the $25^{\text {th }}$ to $75^{\text {th }}$ percentile bounds, and the red horizontal bars are the $50^{\text {th }}$ percentiles. The green dots show the APMC parameters (equivalent to the average of 60 distributed values).

By testing the probability distributions of $\mu_{i}$ and $\sigma_{i}$ values of RCM2 for each month, this study has found that both $\mu_{i}$ and $\sigma_{i}$ values for each month are lognormally distributed (i.e. best suitable distribution). Figure 4.17 and Figure 4.18 show the lognormal probability plot of $\mu_{i}$ and $\sigma_{i}$ values respectively for each month. The $r^{2}$ for $\log \mu_{i}$ and $\log \sigma_{i}$ are between 0.95 and 0.99 , indicating a very good fit of the lognormal distributions. However, in addition to the lognormally distributed $\mu_{i}$ and $\sigma_{i}$ values, this study has also found that the $\log \mu_{i}$ and $\log \sigma_{i}$ values for each month are strongly correlated. Figure 4.19 shows a very strong correlation between $\log \mu_{i}$ and $\log \sigma_{i}$ values for each month with correlation coefficient $r_{c, i}$ between 0.89 and 0.95 . The $\mu_{i}$ and $\sigma_{i}$ values of AWAP for each month also show similar lognormal distributions and strong correlations between $\log \mu_{i}$ and $\log \sigma_{i}$ (see Figure B.10-B.12 at Appendix B.5). Therefore, for each month $i$, this study has fitted a bivariate-normal distribution to the $\log \mu_{i}$ and $\log \sigma_{i}$ values with parameters $\left(\lambda_{\mu, i}, \zeta_{\mu, i}\right),\left(\lambda_{\sigma, i}, \zeta_{\sigma, i}\right)$ and $r_{c, i}$. The $\lambda$ and $\zeta$ parameters denote the mean and SD of the $\log$ variate, while $r_{c}$ is the correlation coefficient between $\log \mu$ and $\log \sigma$. Additionally, the hypothesis that $\log \mu_{i}$ and $\log \sigma_{i}$ are normally distributed is supported by the Kolmogorov-Smirnov test, with maximum deviance of around 0.09 and 0.06 for $\log \mu_{i}$ and $\log \sigma_{i}$ respectively, which are well below the critical deviance of 0.18 at $5 \%$ significance level.

At the start of each month of each year of the simulation, the $\log \mu_{i}$ is sampled from its fitted normal distribution $\log \mu_{i} \sim N\left(\lambda_{\mu_{i}}, \zeta_{\mu_{i}}^{2}\right)$ for month $i$. Then, the $\log \sigma_{i}$ is sampled from the fitted conditional normal distribution: 


$$
\log \sigma_{i} \mid \log \mu_{i} \sim N\left(\lambda_{\sigma_{i}}+\frac{\zeta_{\sigma_{i}}}{\zeta_{\mu_{i}}} r_{c, i}\left(\log \mu_{i}-\lambda_{\mu_{i}}\right), \quad\left(1-r_{c, i}^{2}\right)\left(\zeta_{\sigma_{i}}\right)^{2}\right) \quad \text { Equation } 4.6
$$

These stochastically sampled $\mu_{i}$ and $\sigma_{i}$ values are then used to generate rainfall in the wet days for the month in question, while the sequence of wet and dry days is determined using the deterministic APMC values of $P_{00, i}$ and $P_{11, i}$. However, the sampled $\mu_{i}$ and $\sigma_{i}$ values of a month $(i)$ are not correlated to the $\mu_{i-1}$ and $\sigma_{i-1}$ of the preceding month $(i-1)$ as this study has found that the month-to-month autocorrelations of $\log \mu$ and $\log \sigma$ values are not statistically significantly strong (Figure 4.20).

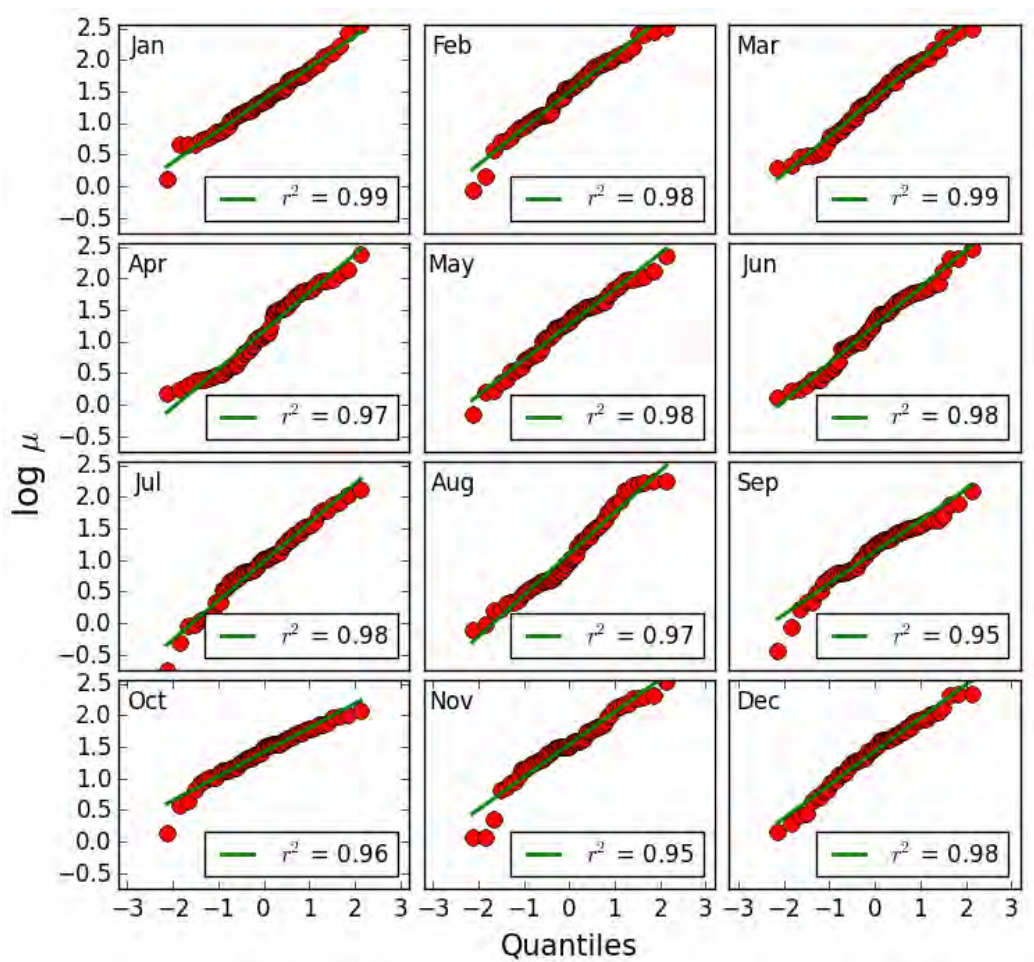

Figure 4.17: Lognormal probability plots of $\mu$ for each month for RCM2. 


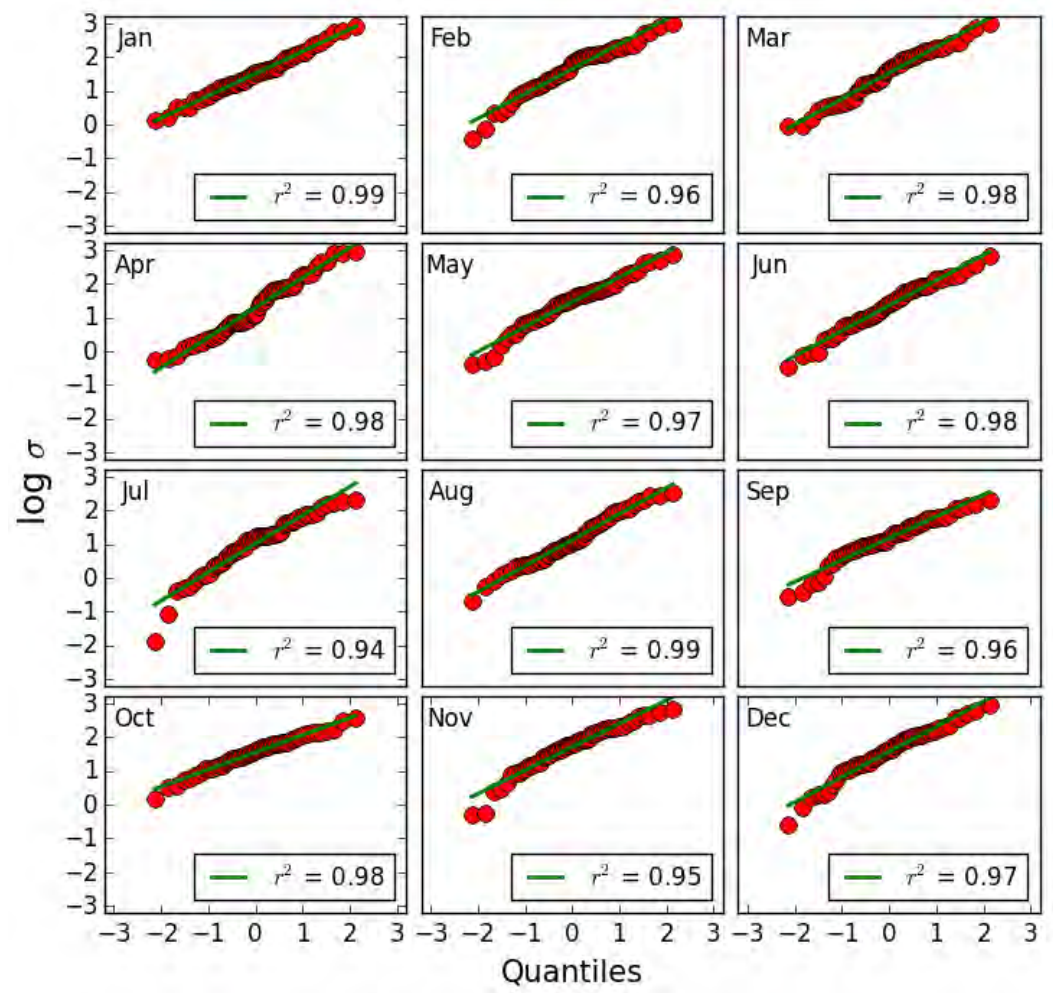

Figure 4.18: Lognormal probability plots of $\sigma$ for each month for RCM2.

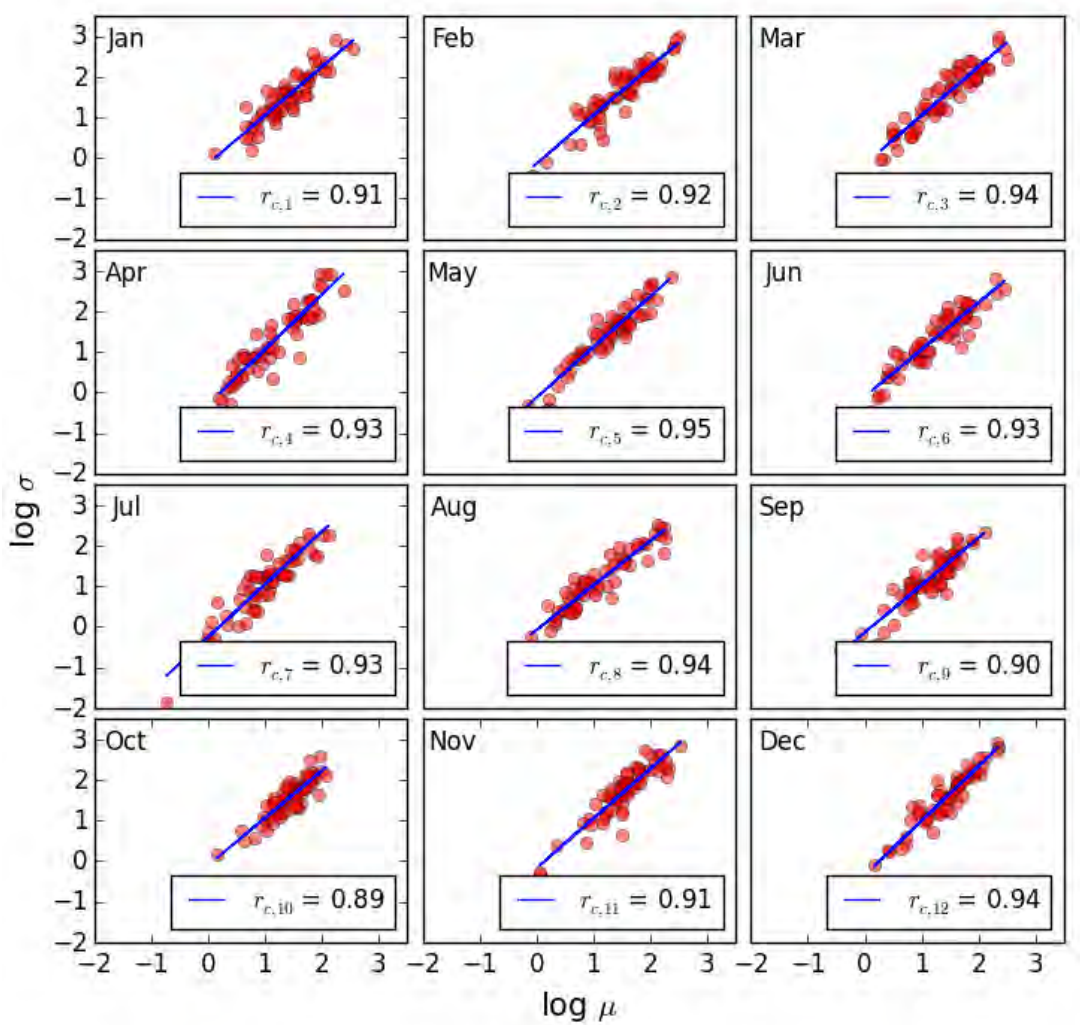

Figure 4.19: Correlation between $\log \mu$ and $\log \sigma$ for each month for RCM2. 

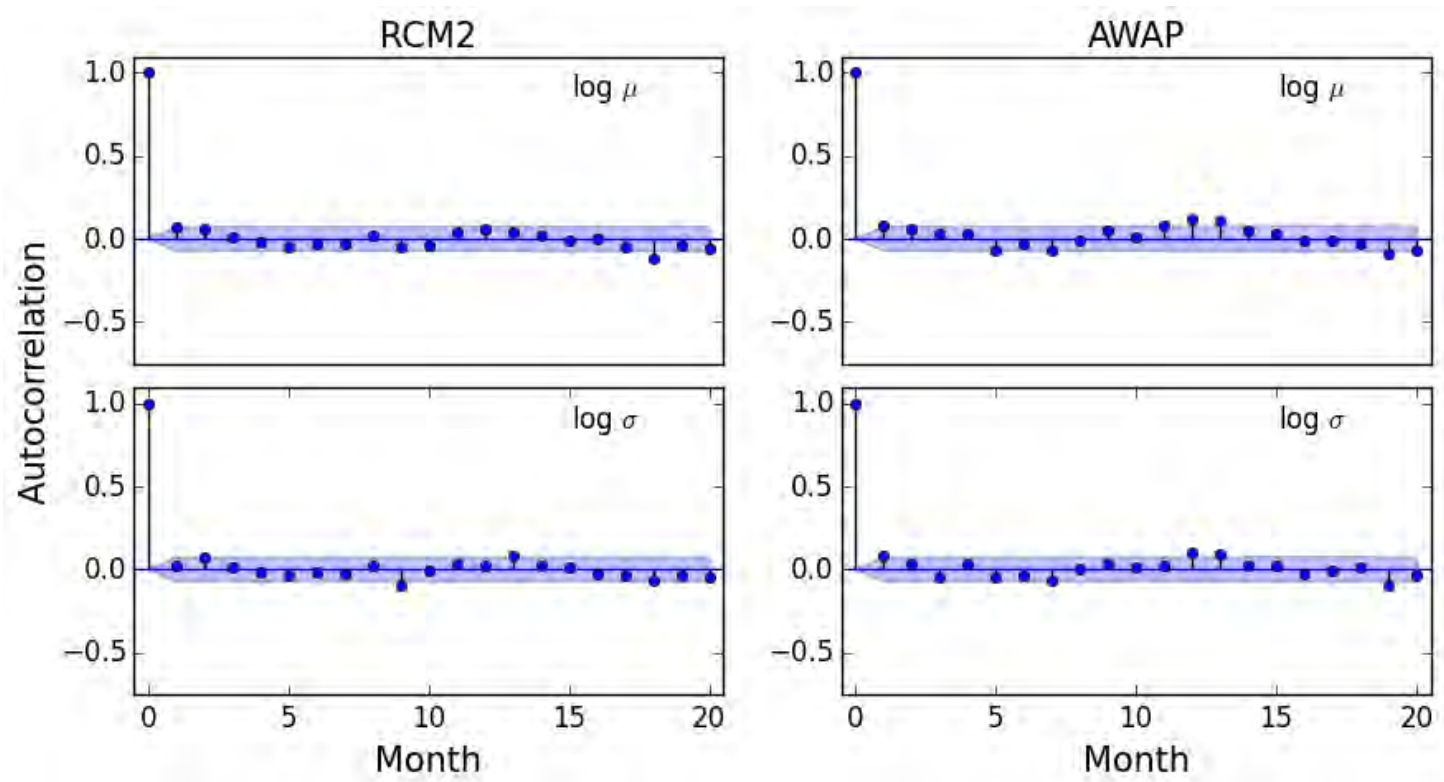

Figure 4.20: Month-to-month autocorrelations of $\log \mu$ and $\log \sigma$ for RCM2 and AWAP.

\subsubsection{Schematic of CDMC}

Figure 4.21 shows a schematic of the CDMC. The key difference between the CDMC and the APMC and DPMC (shown in Figure 4.1 and Figure 4.10 respectively) is in the parameterisation of Gamma distribution for rainfall depth generation. While APMC uses the same deterministic value of Gamma parameters for rainfall generation of all years, the CDMC uses stochastically sampled Gamma parameters in each simulation year. At the beginning of each simulation year, a set of $\mu_{i}$ and $\sigma_{i}$ values for each month $i$ (12 values of each parameter) are sampled from the fitted bivariate-lognormal distributions. The sampled $\mu_{i}$ and $\sigma_{i}$ are used in Gamma distribution to generate rainfall depths, while $\mu_{i}$ and $\sigma_{i}$ are not correlated from month to month. However, for the wet and dry day simulation, the CDMC still uses the same APMC values of $P_{00, i}$ and $P_{11, i}$ for all years' simulation. 


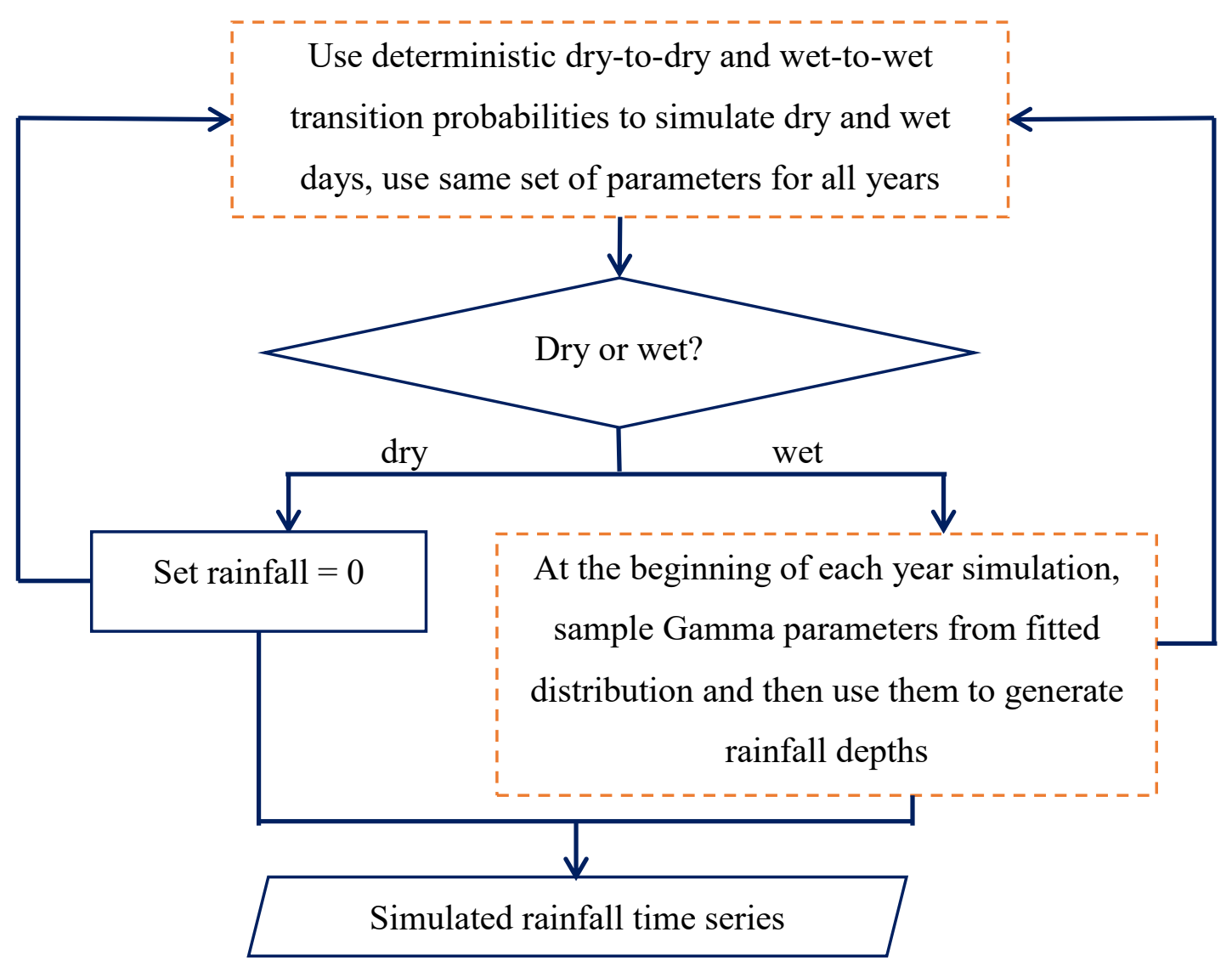

Figure 4.21: Schematic of the CDMC (compare with the Figure 4.1, Figure 4.10, Figure 4.30 and Figure 4.31).

\subsubsection{Summary of CDMC}

The key methodological difference of the CDMC and the APMC and DPMC is that the CDMC uses stochastic parameters for the Gamma distribution for rainfall depth simulation, which vary for each year of simulation, while the previous two models use the same deterministic parameters to simulate rainfall depths for all years (APMC) or each decade (DPMC). The assessments of the CDMC in subsequent chapters will show that the model can satisfactorily reproduce the mean and SD of rainfall depths in all resolutions. Reproducing the SD of rainfall depths at monthly and multiyear resolutions, in particular, is the key contribution of CDMC as these statistics were underestimated by the APMC and DPMC. It suggests that the stochastic parameterisation of Gamma distribution is useful to incorporate the long-term variability of rainfall depths. However, the further assessments show that the CDMC can satisfactorily reproduce the wet period statistics (mean and SD of wet spell lengths and number of wet days) at monthly resolution, but tends to underestimate the variability of wet periods at multiyear resolutions. It suggests that the use of APMC values of MC parameters cannot satisfactorily reproduce the SD of wet period statistics at multiyear resolution. 


\subsection{Hierarchical Markov Chain (HMC) Model}

\subsubsection{Model Structure}

The CDMC with stochastic parameters of Gamma distribution can satisfactorily preserve the rainfall depth variability in all resolutions but tends to underestimate the SD of number of wet days at multiyear resolution. Therefore, in the Hierarchical Markov Chain (HMC) model, this study has introduced stochastic parameterisation of both MC and Gamma distribution to incorporate the long-term variability of rainfall depths as well as wet periods.

\subsubsection{Stochastic parameters of MC process}

HMC introduces variability in the wet and dry period simulation by stochastically sampling the MC parameters from a truncated normal distribution. In calibration, for month $i$, the $P_{00, i}$ and $P_{11, i}$ are calculated for each month of each year from the data to be modelled. For month $i$, these $P_{00, i}$ and $P_{11, i}$ values (e.g. $60 P_{00,1}$ values for January from the 60-year RCM2 data) are found to be normally distributed with values between 0 and 1 (Figure 4.22 and Figure 4.23). Therefore, this study has fitted a truncated normal distribution, bounded by 0 and 1 to the calculated $P_{00}$ and $P_{11}$ values. In simulation, for each year, the $P_{00, i}$ and $P_{11, i}$ are sampled from their truncated normal distributions. This procedure is similar to what was done for $\mu_{i}$ and $\sigma_{i}$ to sample from bivariate-lognormal distribution. However, it does not include a bivariate distribution because the correlation between $P_{00, i}$ and $P_{11, i}$ (Figure 4.24) was weak. These findings are also supported by the same examinations of the distributions and correlations of $P_{00, i}$ and $P_{11, i}$ for AWAP (see Figure B.13-B.15 at Appendix B.6).

In the HMC, the sampled MC parameters of each month are independent of the parameters of preceding month. However, investigation of these parameters has found that there are strong month-to-month autocorrelations of the $P_{00}$ and $P_{11}$ for both RCM2 and AWAP (Figure 4.25), although the seasonal signal of the autocorrelations of AWAP are not consistent with the same of RCM2. Therefore, this study has tested an alternative to the HMC by using a lag-1 autocorrelation equation in the stochastic sampling of $P_{00, i}$ and $P_{11, i}$ from the truncated normal distribution (see section 4.5.3.1). However, as discussed in section 5.5, this alternative model has not performed significantly better than the HMC, with the cost of increased complexity, and therefore, is not considered by this study. 


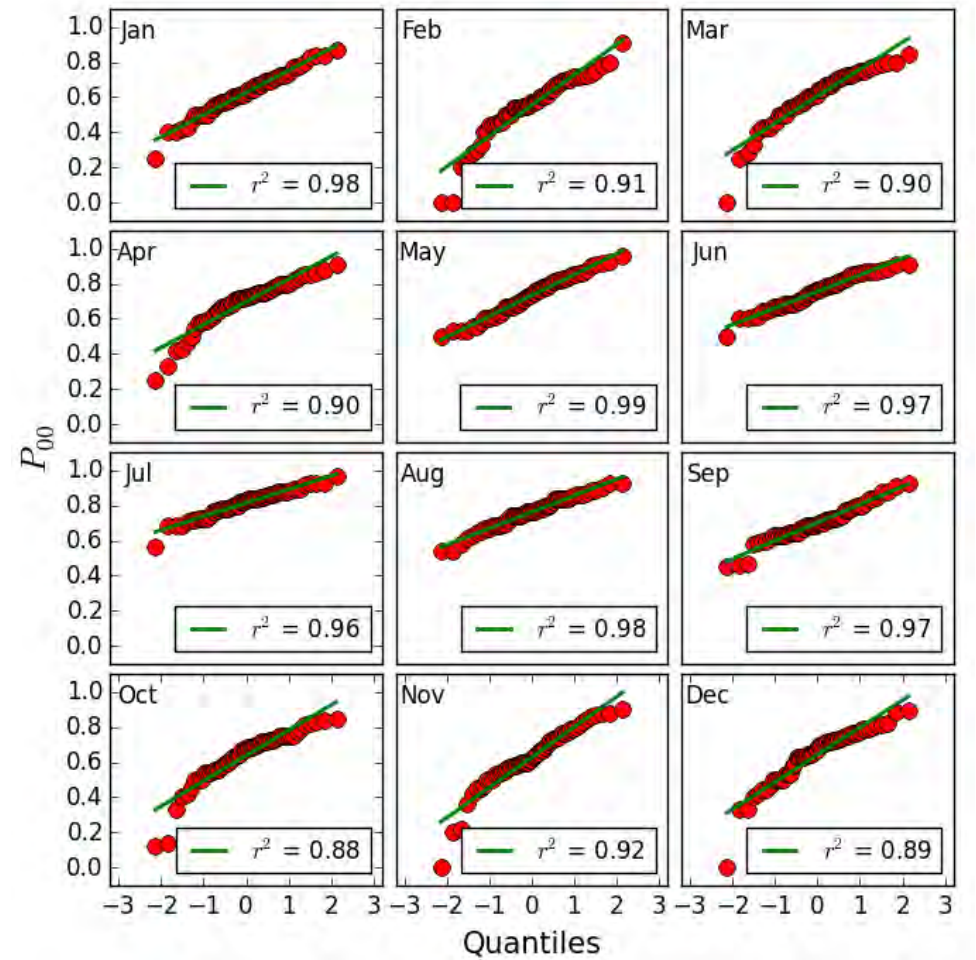

Figure 4.22: Normal probability plots of $\boldsymbol{P}_{00}$ for each month for RCM2.

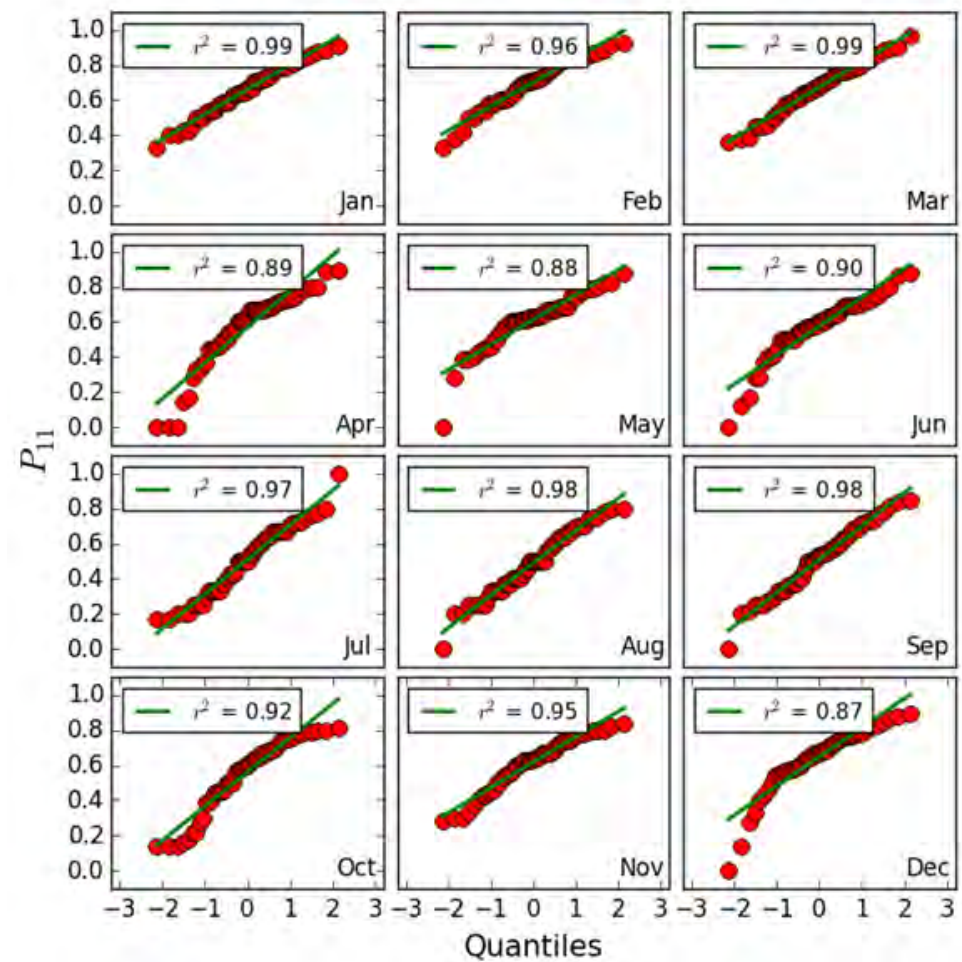

Figure 4.23: Normal probability plots of $\boldsymbol{P}_{11}$ for each month for RCM2. 


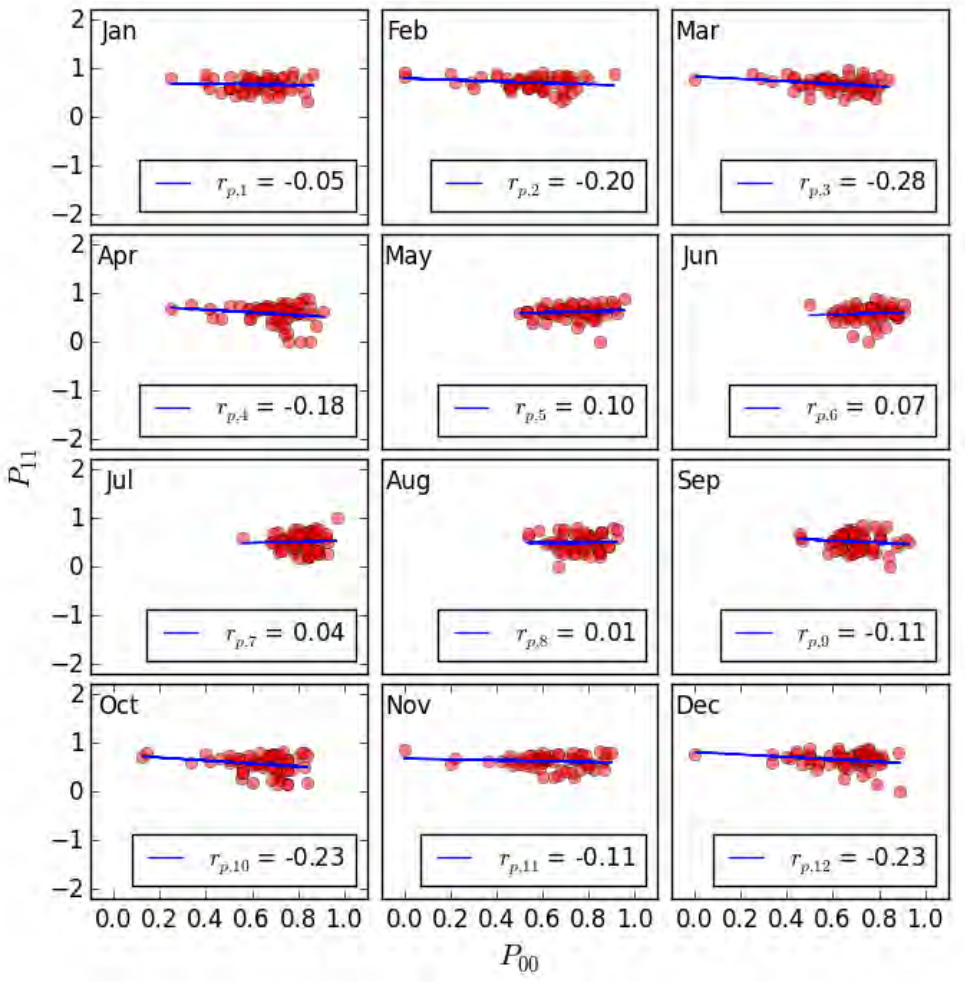

Figure 4.24: Correlation between $P_{00}$ and $P_{11}$ for each month for RCM2.
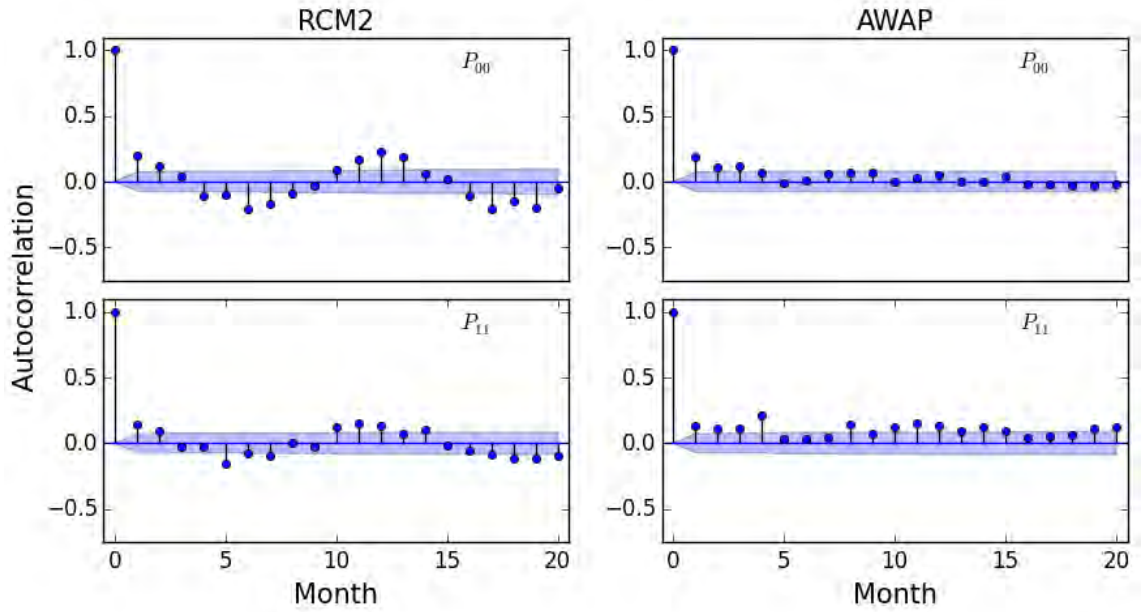

Figure 4.25: Month-to-month autocorrelations of $\boldsymbol{P}_{00}$ and $\boldsymbol{P}_{11}$ for RCM2 and AWAP. 
This study has also observed strong positive correlations of $P_{11, i}$ with the $\log \mu_{i}$ and $\log \sigma_{i}$ for most of the months (Figure 4.26 and Figure 4.27 respectively), although the correlations of $P_{00, i}$ with the $\log \mu_{i}$ and $\log \sigma_{i}$ are weak for most of the months (Figure 4.28 and Figure 4.29 respectively). While these findings are also consistent for AWAP (see Figure B.16-B.19 at Appendix B.6), this study has tested another alternative to HMC by using a multivariate sampling system for the MC and Gamma parameters of each month (see section 4.5.3.2). However, this alternative model has increased complexity and has not performed significantly better than the HMC, and therefore, is not considered by this study (see section 5.5).

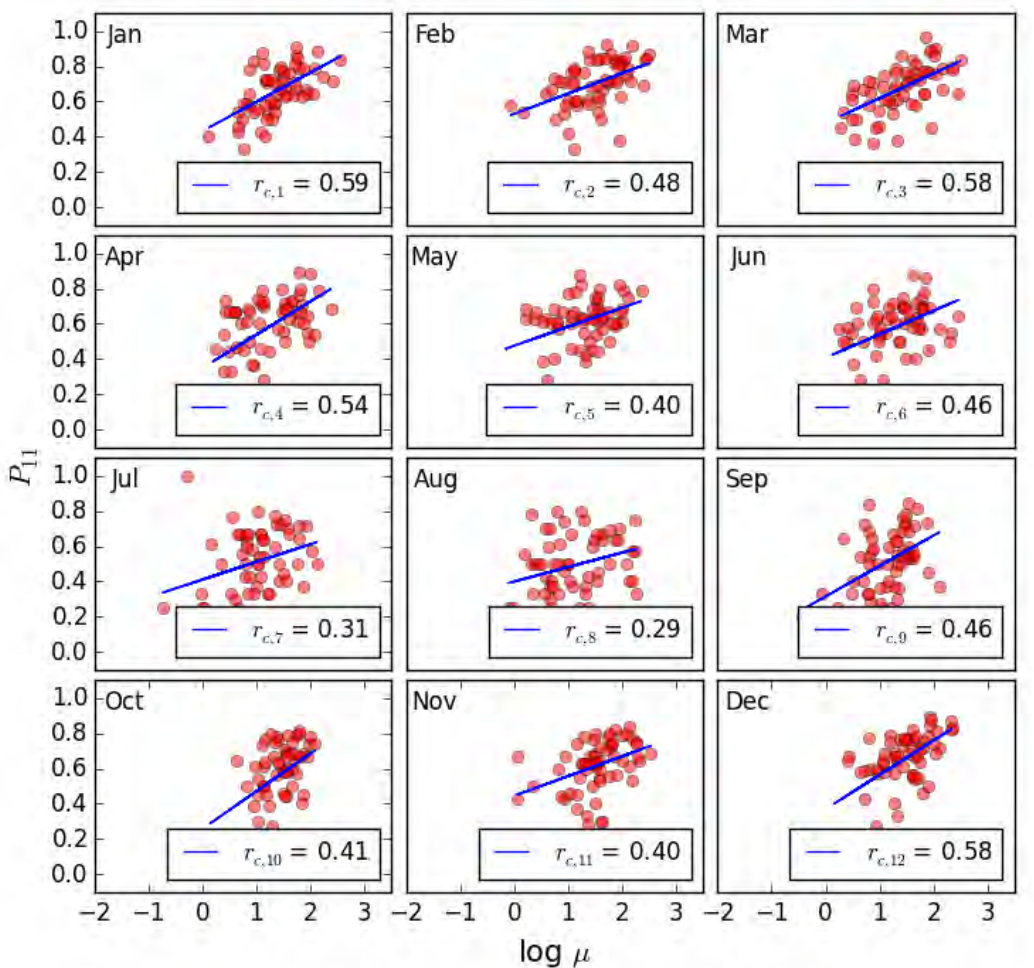

Figure 4.26: Correlation between $\log \mu$ and $P_{11}$ for each month for RCM2. 

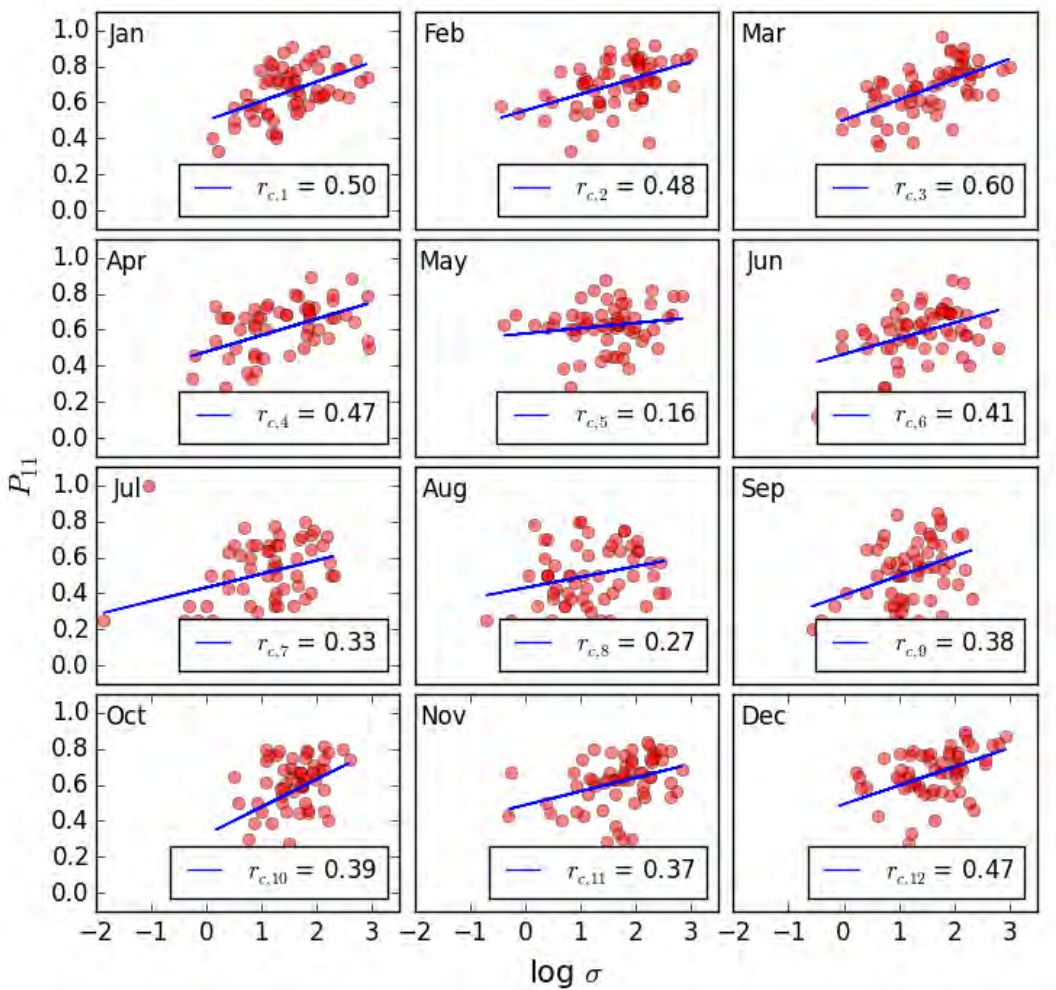

Figure 4.27: Correlation between $\log \sigma$ and $P_{11}$ for each month for RCM2.

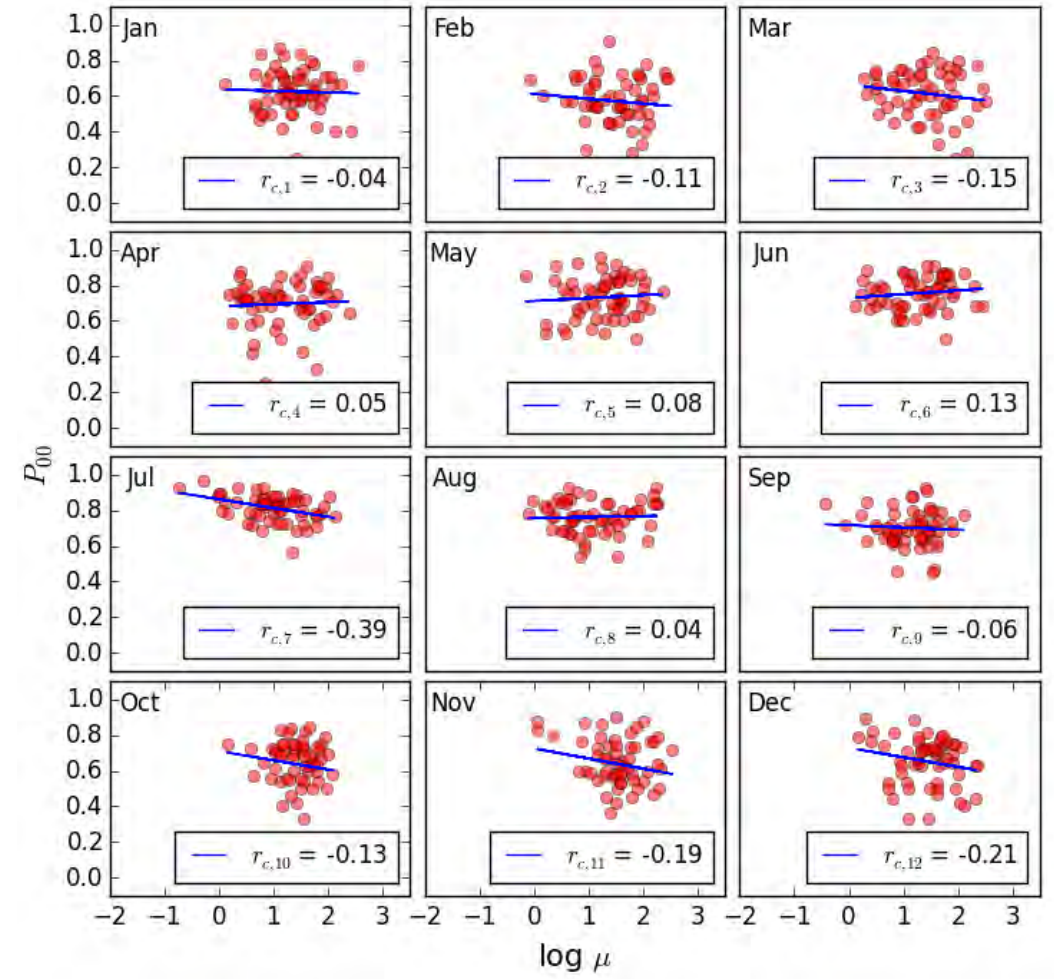

Figure 4.28: Correlation between $\log \mu$ and $P_{00}$ for each month for RCM2. 


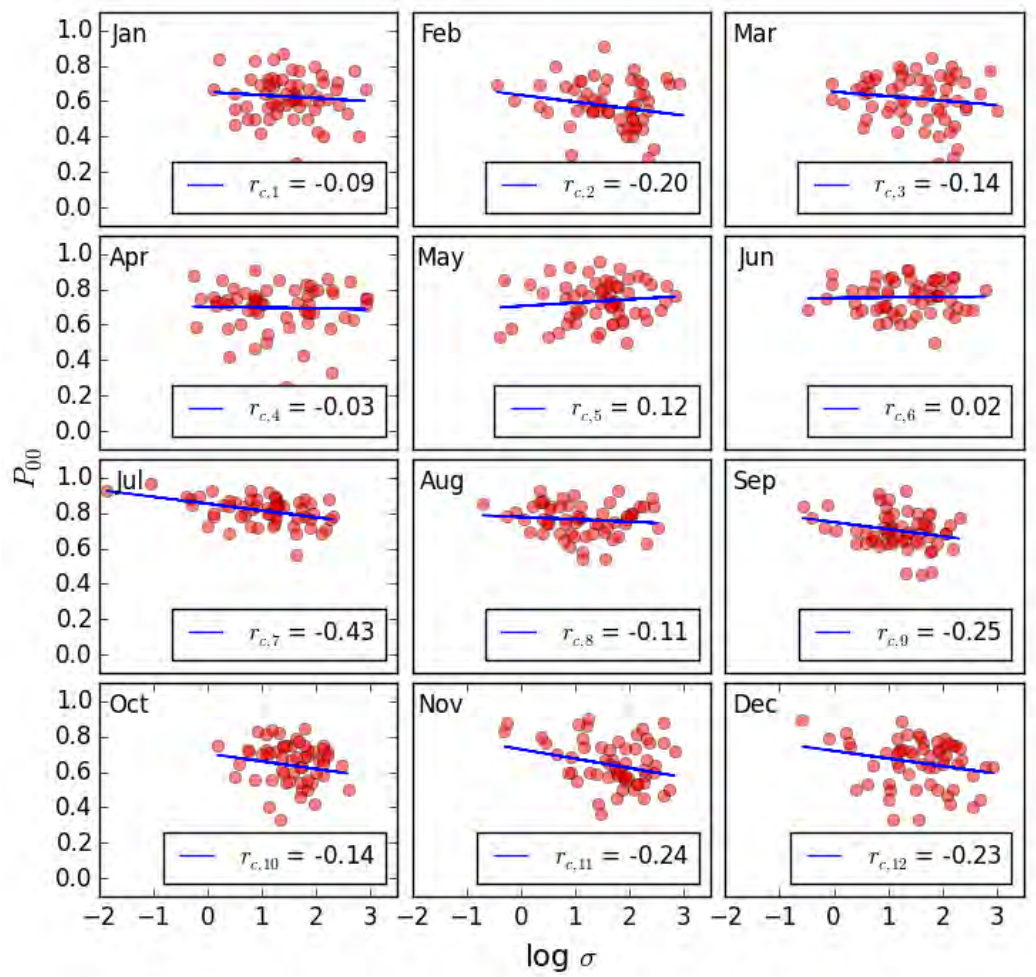

Figure 4.29: Correlation between $\log \sigma$ and $P_{00}$ for each month for RCM2.

\subsubsection{Stochastic parameters of Gamma distribution - similar to CDMC}

Since the stochastic parameters of Gamma distribution in the CDMC have been found to satisfactorily reproduce the key statistics of rainfall depths in all resolutions, the HMC uses the same parameterisation and simulation technique as CDMC for rainfall depth.

\subsubsection{Schematic of HMC}

Figure 4.30 shows a schematic of the HMC. The key differences between the HMC and CDMC (shown in Figure 4.21), is in the parameterisation of MC process for wet and dry day generation. In $\mathrm{HMC}$, the sets of MC parameters $P_{00, i}$ and $P_{11, i}$ for each month $i$ are sampled from fitted truncated normal distributions of respective month to generate the wet and dry days of each year simulation. The set of Gamma parameters $\mu_{i}$ and $\sigma_{i}$ are still sampled from the fitted bivariate-lognormal distributions similar to CDMC. 


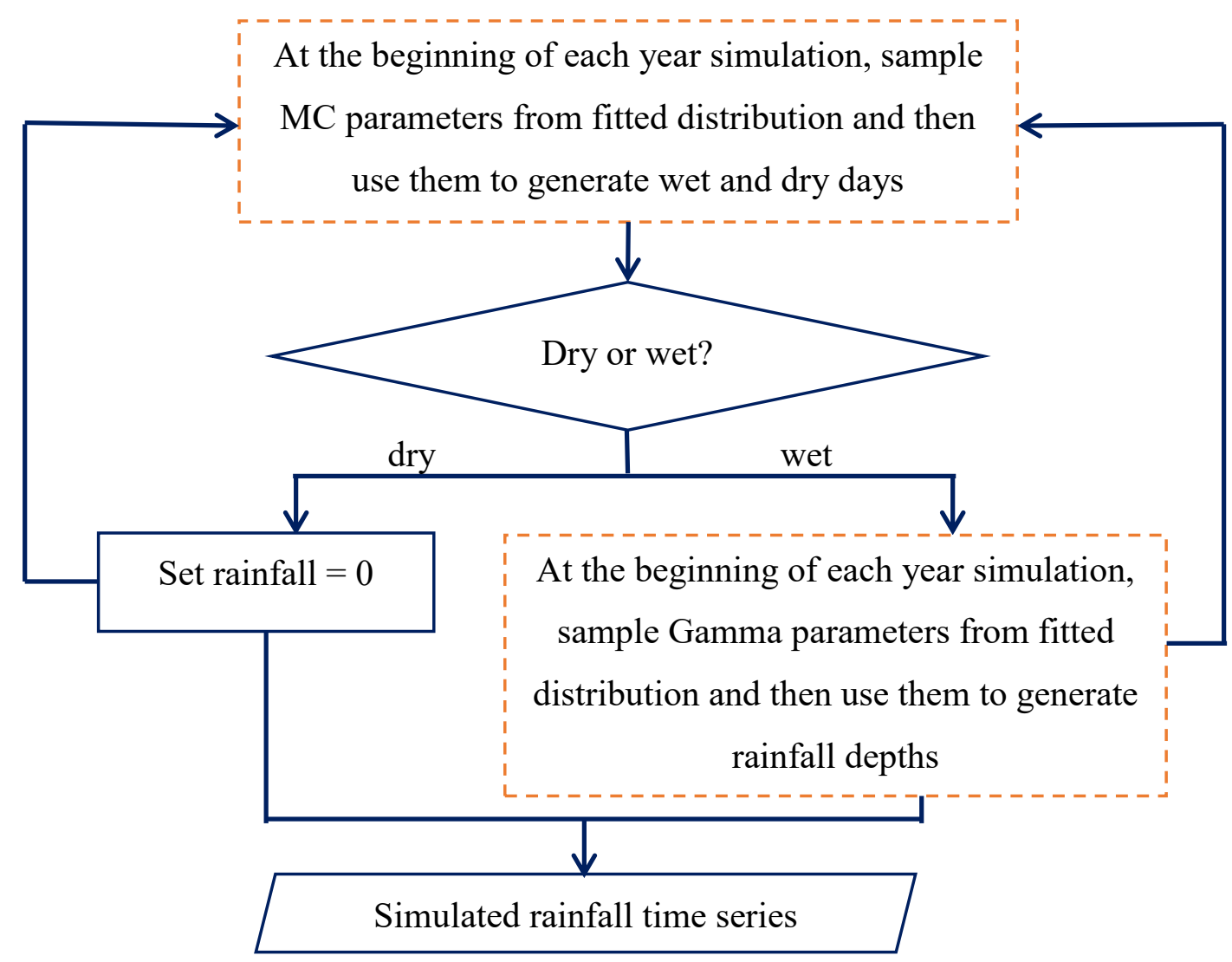

Figure 4.30: Schematic of the HMC (compare with the Figure 4.1, Figure 4.10, Figure 4.21 and Figure 4.31).

\subsubsection{Impact of Stochasticity of MC Parameters}

The overall performance of the HMC shows that it can satisfactorily reproduce the rainfall depth statistics and improve the wet period simulation in comparison with the CDMC. However, the HMC tends to overestimate the SD of wet period statistics (wet days and mean length of wet spell) and accordingly compromises the month-to-month autocorrelation of rainfall depths and wet days. This indicates that the stochastic parameterisation of MC process has introduced more variability than necessary in the wet period simulation. Therefore, this study has examined more details of the stochastic parameterisation process of both MC and Gamma distribution with an objective of improving the performance of the HMC. These alternative models have not performed better than HMC and involve increased complexity, and hence are not considered in this study, although they can still provide important insights and information for future analysis. The two types of alternative models that were examined by this study used: (i) a lag-1 autocorrelation equation for MC parameterisation and (ii) a multivariate sampling system for the MC and Gamma parameters. 


\subsubsection{Model using lag-1 autocorrelation equation in MC parameterisation}

This alternative model is based on the assumption that the inclusion of month-to-month autocorrelations of the $P_{00}$ and $P_{11}$ (Figure 4.25) can improve the simulation of the variability of wet period statistics, while the HMC does not consider the autocorrelations (parameters are sampled independently for each month) and overestimates the variability of wet period statistics. The following lag-1 autocorrelation equation has been used to modify the randomly sampled $P_{00, i}$ for month $i$ by correlating with the $P_{00, i-1}$ of month $i-1$ (preceding month):

$\frac{\overline{\overline{P_{00, i}}}-\operatorname{mean}\left(P_{00, i}\right)}{s d\left(P_{00, i}\right)}=r \times \frac{\overline{\overline{P_{00, l-1}}}-\operatorname{mean}\left(P_{00, i-1}\right)}{s d\left(P_{00, i-1}\right)}+\left(1-r^{2}\right)^{1 / 2} \frac{\overline{P_{00, l}}-\operatorname{mean}\left(P_{00, i}\right)}{s d\left(P_{00, i}\right)} \quad$ Equation 4.7

where, for a month $i$ (e.g. January),

- $\quad \overline{\overline{P_{00, \imath}}}$ is auto-correlated parameter (which is used in simulation) for month $i$

- $\overline{P_{00, l}}$ is parameter value sampled from the truncated normal distribution for month $i$

- $\quad r$ is lag-1 autocorrelation coefficient (constant for all month)

- $\quad$ mean $\left(P_{00, i}\right)$ is mean of the parameter values calculated from observed data for month $i$

- $\quad s d\left(P_{00, i}\right)$ is $\mathrm{SD}$ of the parameter values calculated from observed data for month $i$

- $\overline{\overline{P_{00, l-1}}}$ is auto-correlated parameter for month $i-1$ (preceding month)

- mean $\left(P_{00, i-1}\right)$ is mean of the parameter values calculated from observed data for month $i-1$

- $\quad s d\left(P_{00, i-1}\right)$ is $\mathrm{SD}$ of the parameter values calculated from observed data for month $i-1$.

An equivalent equation is used to correlate the randomly sampled $P_{11, i}$ of month $i$ with $P_{11, i-1}$ of month $i-1$. However, Chapter 5 will show that this alternative model has increased complexity and has not performed significantly better than the HMC, and therefore, is not considered by this study.

\subsubsection{Model using multivariate distribution}

This alternative model is based on the assumption that the inclusion of the correlations of $P_{11, i}$ with the $\mu_{i}$ and $\sigma_{i}$ (Figure 4.26 and Figure 4.27 respectively) can improve the simulation of the 
variability of wet period statistics, while HMC samples the $P_{11, i}$ independent of the $\mu_{i}$ and $\sigma_{i}$ (although the $\mu_{i}$ and $\sigma_{i}$ are correlated to each other) and overestimate the variability of wet period statistics. Therefore, this alternative model uses a multivariate sampling of these three parameters, while $P_{00, i}$ remains independent as it is not strongly correlated to any of the parameters. However, this alternative model is not considered by this study as it has not performed significantly better than the HMC with a cost of increased complexity.

\subsubsection{Summary of HMC}

The main objective of the HMC is to introduce more variability in the parameterisation of MC process so that the SD of wet period statistics at multiyear resolutions can be preserved, which has been significantly underestimated by CDMC. Therefore, in the HMC, the MC parameters are also stochastically sampled from fitted truncated normal distributions in addition to the stochastic parameters of Gamma distribution, when CDMC only uses the stochastic parameters of Gamma distribution with deterministic parameters (i.e. APMC) of MC. The stochastically sampled MC and Gamma parameters of each month are not correlated to the respective parameters of preceding months.

The subsequent chapters will show that the HMC performs in a similar way to the CDMC for rainfall depth simulation as both models use the same stochastic parameterisation of Gamma distribution for wet day rainfall depth generation. The use of stochastic MC parameters that vary for each year of HMC simulation has also been found useful to improve the simulation of the variability of wet periods at multiyear resolutions. However, the model tends to significantly overestimate the SD of wet period statistics at monthly resolution. This indicates that the stochastic parameterisation of the MC process has introduced too much variability in the wet period simulation of HMC. This study found strong month-to-month autocorrelations in the MC parameters as well as strong correlations between the wet-to-wet probabilities and Gamma parameters (mean and SD of wet day rainfall depths). Based on these investigations, this study tested two other alternatives to the HMC by using a lag-1 autocorrelation equation and using a multivariate sampling of the parameters respectively. However, these alternative models have not resulted in any significant improvement in the performances of the HMC and, hence, are not considered by this study. 


\subsection{Decadal and Hierarchical Markov Chain (DHMC) Model}

The CDMC, with APMC values of MC parameters, significantly underestimates the wet period variability at multiyear resolutions, while the HMC, with stochastic MC parameters, significantly overestimates the wet period variability at monthly resolution. However, this study has found that the DPMC can satisfactorily preserve the wet period variability at both monthly and multiyear resolutions, although it underestimates the rainfall depths variability. Therefore, in the Decadal and Hierarchical Markov Chain (DHMC) model, this study has used the DPMC values of MC parameters (those vary for each decade of simulation) for simulation of wet and dry days, while the stochastic parameters of Gamma distribution (same as CDMC) are used for simulation of wet day rainfall depths. Figure 4.31 shows a schematic of the DHMC.

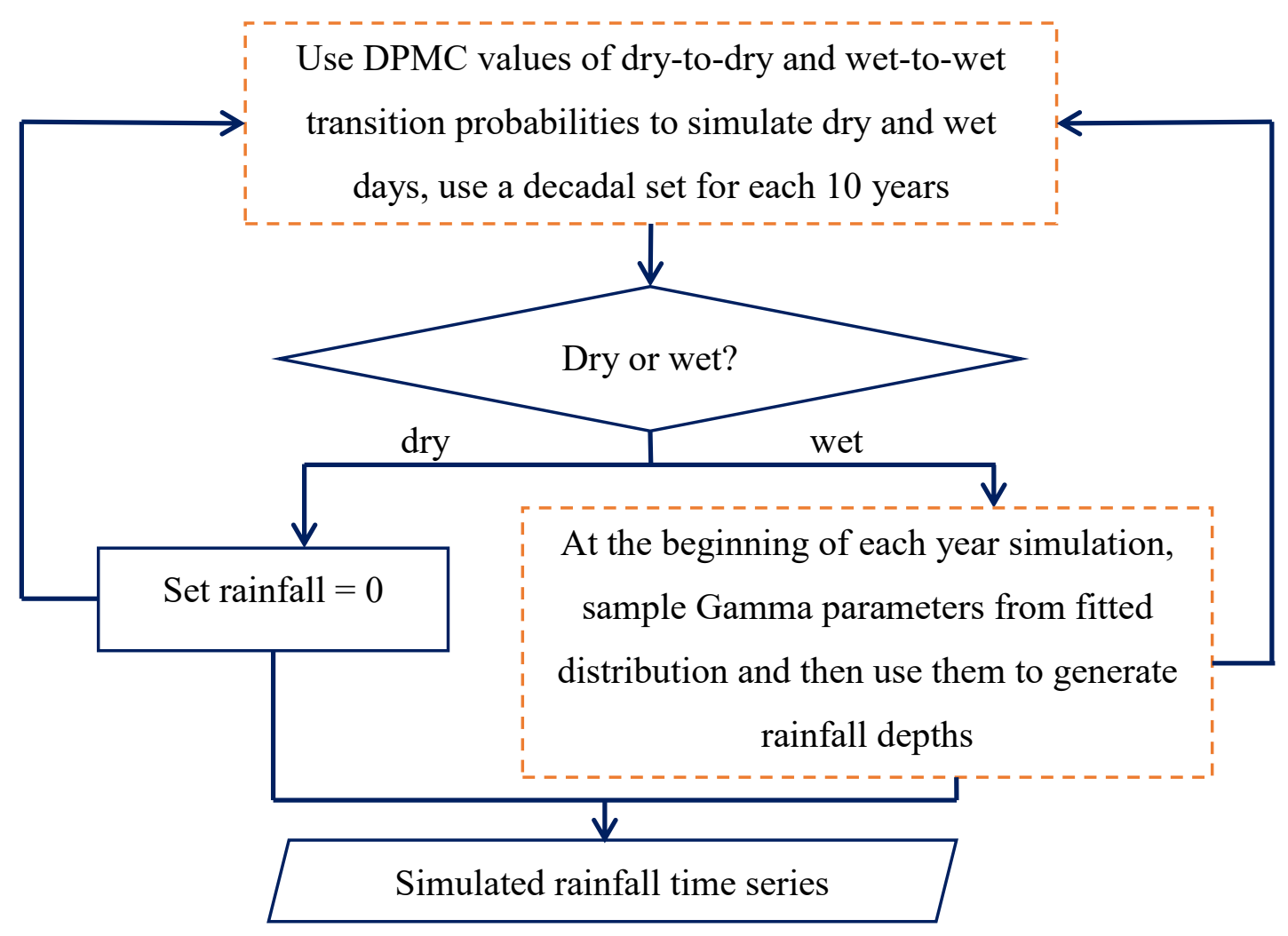

Figure 4.31: Schematic of the DHMC (compare with the Figure 4.1, Figure 4.10, Figure 4.21 and Figure 4.30). 


\subsection{Methodological Comparison of Five MC Models}

The key common features in the five MC models of this study are:

- They use first-order MC parameters (i.e. transition probabilities of dry-to-dry and wet-towet days) to simulate the rainfall occurrences and Gamma distribution with two parameters (mean and SD of wet day rainfall depths) to simulate rainfall depths in wet days.

- Simulation of rainfall depth for each wet day is independent of the rainfall depth of the preceding day.

- They use separate sets of parameters for each month (e.g. 12 sets of MC and Gamma parameters) to reproduce seasonal variability.

The other key methodological comparisons among the five MC models are shown in Table 4.1.

Table 4.1: Methodological comparison among five MC models of this study.

\begin{tabular}{|c|c|c|}
\hline & Wet and dry day simulation & Wet day rainfall depth simulation \\
\hline APMC & $\begin{array}{l}\text { Uses deterministic MC parameters, } \\
\text { same set of parameters for each } \\
\text { simulation year. }\end{array}$ & $\begin{array}{l}\text { Uses deterministic Gamma parameters, } \\
\text { same set of parameters for each } \\
\text { simulation year. }\end{array}$ \\
\hline DPMC & $\begin{array}{l}\text { Uses decadally varied deterministic } \\
\text { MC parameters. }\end{array}$ & $\begin{array}{l}\text { Uses decadally varied deterministic } \\
\text { Gamma parameters. }\end{array}$ \\
\hline CDMC & Same as APMC. & $\begin{array}{l}\text { Uses stochastic parameters (sampled } \\
\text { from fitted bivariate-lognormal } \\
\text { distribution) of Gamma distribution, } \\
\text { parameters vary for each year } \\
\text { simulation. }\end{array}$ \\
\hline HМC & $\begin{array}{l}\text { Uses stochastic MC parameters } \\
\text { (sampled from fitted truncated normal } \\
\text { distribution), parameters vary for each } \\
\text { year simulation. }\end{array}$ & Same as CDMC. \\
\hline DHMC & Same as DPMC. & Same as CDMC. \\
\hline
\end{tabular}




\subsection{Discussion}

The primary motivation of this study is to develop a stochastic rainfall generation model that can match not only the short resolution (daily) variability, but also the longer resolution (monthly to multiyear) variability. Preserving long-term variability in rainfall models has been a difficult challenge for which a number of solutions have been proposed in the stochastic rainfall generation literature. The solutions developed and tested by this study are relatively simple MC models: two MC parameters of two-state, first-order processes defining the wet and dry days, and two Gamma-distribution parameters defining the rainfall depths in wet days. For seasonal variability, the models operate at daily time step with monthly varying parameters for each of 12 months. Starting with the simplest MC-Gamma modelling approach with deterministic parameters, this study has developed and assessed three other variants of the MCGamma modelling approach. The key finding is that if the parameters of Gamma distribution are randomly sampled from fitted distributions prior to simulating the rainfall for each year, the variability of rainfall depths at long resolutions can be preserved, while the variability of wet periods can be preserved by decadally varied parameters. This is a straightforward enhancement to the traditional simplest MC model, which is both objective and parsimonious. The five MC models investigated in this study are:

- The APMC, where the four parameters $\left(P_{00}, P_{11}, \mu\right.$, and $\left.\sigma\right)$ vary monthly (e.g. 12 set parameters for 12 month of simulation) and are same for the same month each year

- The DPMC, where the four parameters vary monthly and for each decade (i.e. individual set of APMC-like parameters for each decade of simulation)

- The CDMC, where the four parameters vary monthly and the parameters $P_{00}$ and $P_{11}$ are the same from year to year, but the parameters $\mu$ and $\sigma$ are stochastically sampled for each year from fitted bivariate-lognormal distributions of respective month

- The HMC, where the four parameters vary monthly, but the parameters $P_{00}$ and $P_{11}$ are stochastically sampled for each year from fitted truncated normal distributions of respective month, while the parameters $\mu$ and $\sigma$ are stochastically sampled for each year from fitted bivariate-lognormal distributions of respective month

- The DHMC, where the $P_{00}$ and $P_{11}$ vary monthly and for each decade (same as DPMC), while the parameters $\mu$ and $\sigma$ are stochastically sampled for each year from fitted bivariate-lognormal distributions of respective month (same as CDMC). 


\section{Development of the Markov Chain Daily Rainfall Model}

The investigation started with the simplest model, APMC, where the parameters are averaged over the entire period of the dataset (e.g. 60-year for the RCM2 reanalysis and AWAP data used in this chapter). The four parameters are calculated for each month separately, as a related study on the same datasets [Parana Manage, 2016] found strong seasonality at the daily and monthly level. The APMC can successfully replicate the intra-annual seasonal variability and adequately simulate the mean rainfall depths in all resolutions, but it failed to preserve the monthly to multiyear variability of rainfall depths.

A slightly more complicated model, DPMC, has used deterministic but decadally varying parameters. It is based on the idea that, while the APMC parameters fitted to the whole 60-year data failed to mimic certain extreme dry or wet years in particular decades, the incorporation of decadal variability into the fitted parameters might enhance the model's performance in preserving the long-term variability. However, a relevant test of decadal parameters showed that the parameter variabilities between decades are mostly within the sampling variability of the APMC parameters. Accordingly, the model assessment has also found that the DPMC failed to preserve long-term variability of rainfall depths, although it preserves the variability of wet periods. This study has also investigated other models similar to DPMC, such as a model with parameters varying for each 5-year period (instead of 10-years as for DPMC) and a model with parameters varying for phases of IPO or ENSO. However, these models also systematically underestimated the SD of rainfall depths at monthly to multiyear resolutions. It suggests that the use of deterministic parameters in DPMC-like models might not be adequate to satisfactorily reproduce the SD of rainfall depths at all resolutions.

The third model, CDMC, has been found to satisfactorily preserve the long-term variability of rainfall depths in addition to the intra-annual seasonal variability. In developing the CDMC, a relevant test has found that the long-term variability of rainfall depths in the model is predominantly governed by the variability of $\mu$ and $\sigma$, rather than the variability of $P_{00}$ and $P_{11}$. Consequently, the CDMC resolves the problem of underestimating long-term variability of rainfall depths by treating $\mu$ and $\sigma$ as random variables, while uses the average APMC values of $P_{00}$ and $P_{11}$ for wet and dry day simulation. Sampling $\mu$ and $\sigma$ from the bivariate-lognormal distribution at the start of each year simulation provides a simple and explicit mechanism for replicating the long-term variability. In comparison with the past approaches of using empirical adjustment factors [Boughton, 1999], this approach has a stronger physical basis because the parameter variability comes from an underlying distribution over the sampling period. Also, the CDMC does not require a rainfall threshold (to separate moderate and extreme rainfalls) for 
separate distributions of moderate and extreme rainfalls [Wilson and Toumi, 2005]. In addition to the distribution statistics (mean and SD) of rainfall depths in all resolutions, the CDMC can satisfactorily reproduce the month-to-month autocorrelation of rainfall depths and number of wet days. It can also satisfactorily reproduce the mean and SD of number of wet days and mean length of wet spells at monthly resolution, but tends to underestimate the SD of number of wet days at multiyear resolutions.

The fourth model of this study, HMC, has used stochastic MC parameters $P_{00}$ and $P_{11}$ sampled from fitted truncated normal distributions for each year simulation, in addition to the stochastic Gamma parameters used in CDMC. The use of stochastic MC parameters, which vary for each year, has been found useful in introducing more variability in the wet periods simulation, which was underestimated by CDMC. The performance of the HMC in reproducing the mean and SD of rainfall depths in all resolutions has been found satisfactory and similar to the CDMC's performance as both models use same parameterisations of Gamma distribution for rainfall depth simulation. However, the HMC shows a consistent tendency to overestimate the SD of monthly and multiyear wet spell statistics and tends to underestimate the month-to-month autocorrelations of rainfall depths and wet days, while these statistics have been satisfactorily reproduced by the CDMC. The consistent tendency of overestimating the wet periods variability and the compromised autocorrelations indicate that the range of variability introduced by the stochastic MC parameters might be excessive. This study further examined this overestimation issue by testing two other variants of the HMC by using a lag-1 autocorrelation equation in the stochastic parameterisation of the MC process and using a multivariate sampling of MC-Gamma parameters respectively. However, these alternative models are not considered by this study as they have not performed significantly better than the HMC and involved increased complexity.

The fifth and final model, DHMC, used the DPMC values of MC parameters (those vary for each decade of simulation) for simulation of wet and dry days, while the stochastic parameters of Gamma distribution (same as CDMC) are used for simulation of wet day rainfall depths. The DHMC is found to satisfactorily preserve the variabilities of wet periods and rainfall depths for all resolutions.

In this chapter, this study used only the RCM2 and AWAP data to develop the MC models. Later in this thesis, the developed models will be further assessed using all RCMs (RCM1, RCM2 and RCM3), AWAP and raingauge data. 


\section{Development of the Markov Chain Daily Rainfall Model}

\subsection{Conclusion}

The primary objective of this $\mathrm{PhD}$ study is to develop a stochastic rainfall model which can be used to incorporate the influence of ECL rainfall (in addition to the non-ECL rainfall) in the urban water security assessments of reservoirs. The ECLs are typical east-Australian extreme storm events which usually vary from mesoscale to synoptic resolution, occur for a few days, but produce substantial amount of streamflow and contribute to the reservoir water levels. Therefore, the major focus of this study is to develop a stochastic rainfall model that can satisfactorily reproduce the short-term (e.g. daily) and longer-term (e.g. monthly to multiyear) variability and dependence of observed rainfall as the ECLs are usually short-term events (e.g. for a few hours or days), while the corresponding reservoir water levels vary at monthly to multiyear resolutions. However, this study has not explicitly examined the ability of the developed model to incorporate the influence of ECL, which is the subject of a future study.

This chapter has described the methodology of five MC models which have been developed and examined by this study. Of the five models of this study, the first two models (APMC and DPMC) use deterministic parameters of Gamma distribution and underestimate the monthly and multiyear variability of rainfall depths. The third model, CDMC, with stochastic parameters for the Gamma distribution and preserves the variability of rainfall depths in all resolutions. However, the CDMC tends to underestimate the multiyear variability of number of wet days. The fourth model, HMC, with stochastic parameters of both MC and Gamma distribution, preserves the rainfall depths variability in all resolutions, introduces more variability in the wet period simulation and preserves the multiyear variability of number of wet days. However, the HMC tends to significantly overestimate the wet period variability at monthly resolution and underestimate the month-to-month autocorrelations of rainfall depths and wet days. This overestimation of wet period variability in the HMC indicates that the stochastic parameterisation of the MC process has introduced more variability than necessary in the wet period simulation of the HMC. The fifth and final model, DHMC, has used decadally varied MC parameters (same as DPMC) and stochastic parameters of Gamma distribution (same as CDMC). The DHMC preserves the rainfall depths as well as wet period variabilities at all resolutions. 


\section{Comparison of MC Models at Gummun}

\subsection{Introduction}

This chapter presents a comparison of the performances of the APMC, DPMC, CDMC, HMC and DHMC to reproduce the distribution and autocorrelation of the rainfall depths and wet period statistics (see section 3.4) for RCM2 and AWAP at Gummun. The models should satisfactorily preserve these statistics for the hydrology applications of the models. In addition, the performances of the models that are similar to DPMC (as discussed in section 4.3.5) will be compared with the performances of DPMC, and the performances of the models that are similar to HMC (as discussed in section 4.5.3) will be compared with performances of HMC.

\subsection{Distribution Statistics of Rainfall Depths and Wet Periods}

This section presents the rainfall depth and wet period statistics (e.g. mean, SD) of the RCM2 and AWAP at daily, monthly and multiyear resolutions. The comparative assessments of the ability of the APMC, DPMC, CDMC, HMC and DHMC to reproduce these statistics will be presented in the latter sections of this chapter. However, this section is not meant to be a comprehensive comparison of the NARCliM reanalysis RCM2 with AWAP since that is the subject of another study [Parana Manage, 2016], but the comparison here highlights the rainfall characteristics that need to be accommodated in the models developed in this thesis.

\subsubsection{Mean and SD of Rainfall Depths at Daily, Monthly and Multiyear Resolutions}

Figure 5.1 and Figure 5.2 show the mean and SD of rainfall depths at daily (wet day rainfall depths), monthly and multiyear resolutions for RCM2 and AWAP. The daily and monthly rainfall show typical seasonality of east Australia with wet summers and dry winters. While the statistics are similar in both datasets, the mean and SD of daily rainfall depths are slightly higher in AWAP than RCM2, particularly in summer. In addition, the SD of multiyear rainfall depths is considerably higher in AWAP. This indicates that the AWAP rainfall is more variable than RCM2. However, the mean of multiyear rainfall depths is slightly higher in RCM2. 

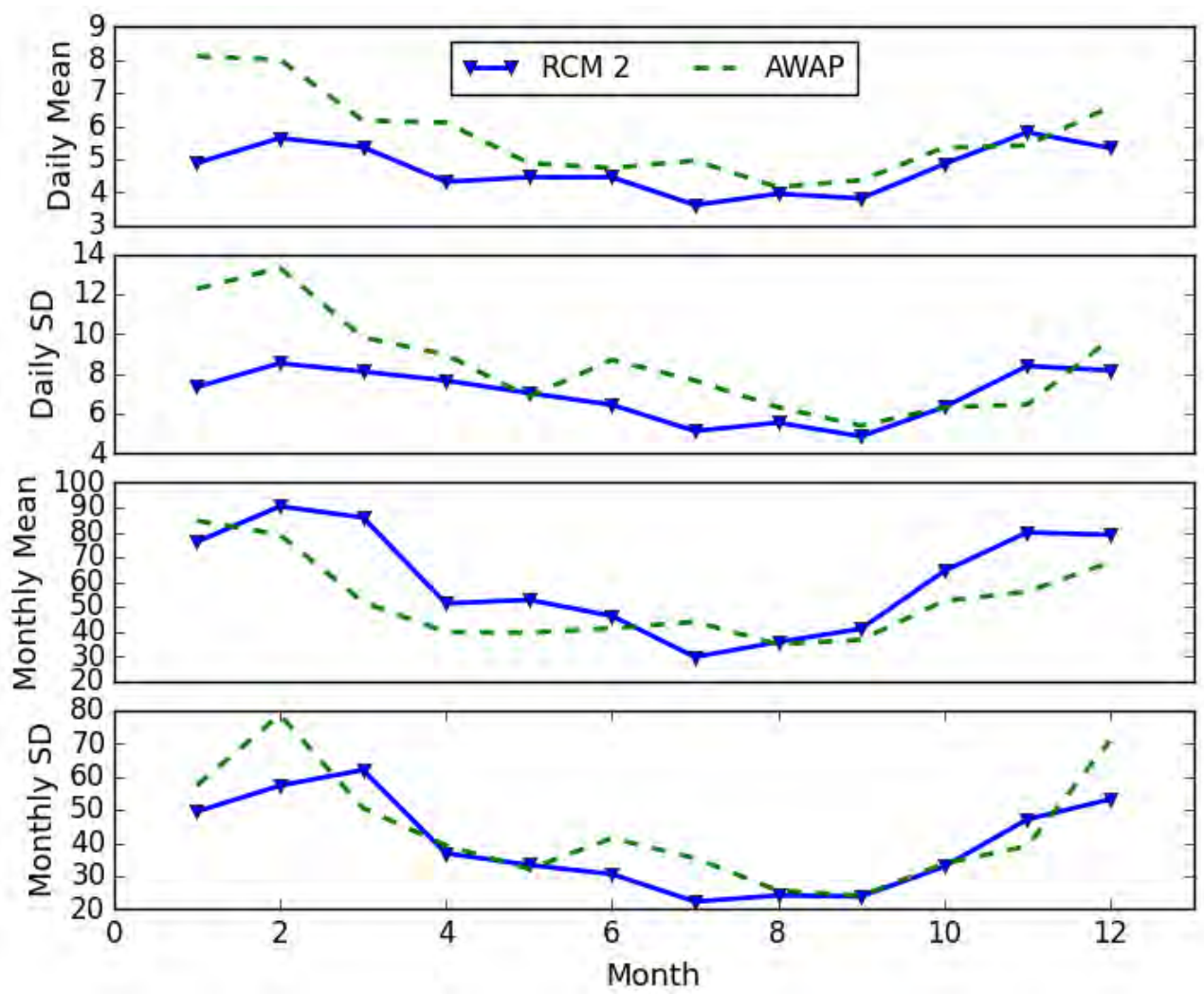

Figure 5.1: Mean and SD of rainfall depths for RCM2 and AWAP at daily and monthly resolutions.

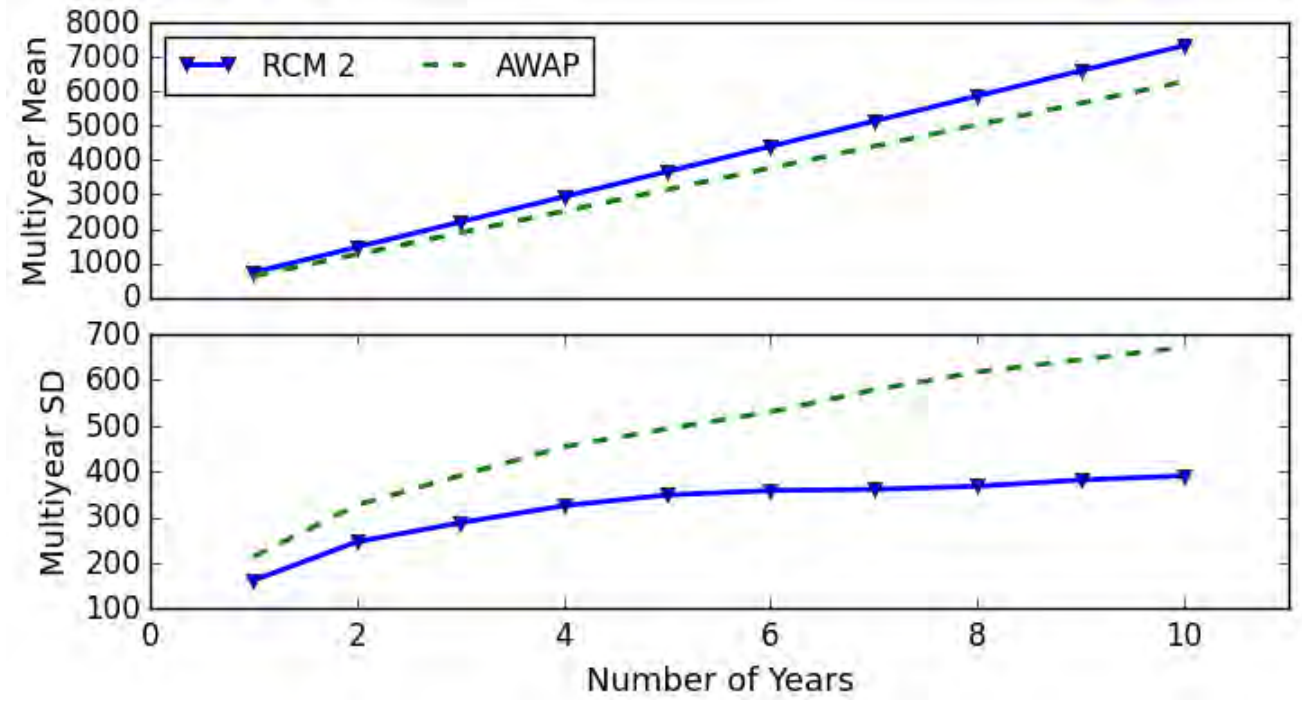

Figure 5.2: Mean and SD of rainfall depths for RCM2 and AWAP at multiyear (aggregated rainfall depths for multiple overlapping years) resolutions. 


\subsubsection{Mean and SD of Number of Wet Days at Monthly and Multiyear Resolutions}

Figure 5.3 shows the mean and SD of number of wet days for RCM2 and AWAP at monthly resolution. It shows that the mean and SD of monthly number of wet days has the typical seasonality of wet summer and dry winter, while any seasonal signal in AWAP is less clear. The mean of monthly number of wet days is higher in RCM2, but the SD of monthly number of wet days is higher in AWAP. This indicates that AWAP is more variable with lower mean than RCM2. The mean and SD of multiyear number of wet days show similar trends (Figure 5.4).

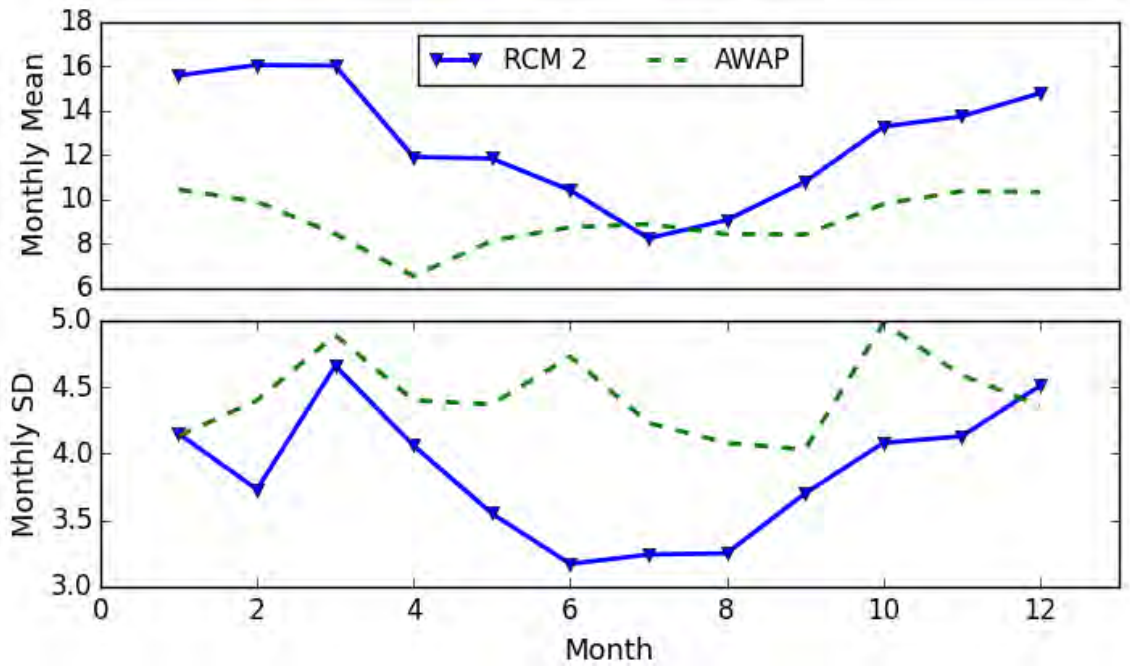

Figure 5.3: Mean and SD of monthly number of wet days for RCM2 and AWAP.

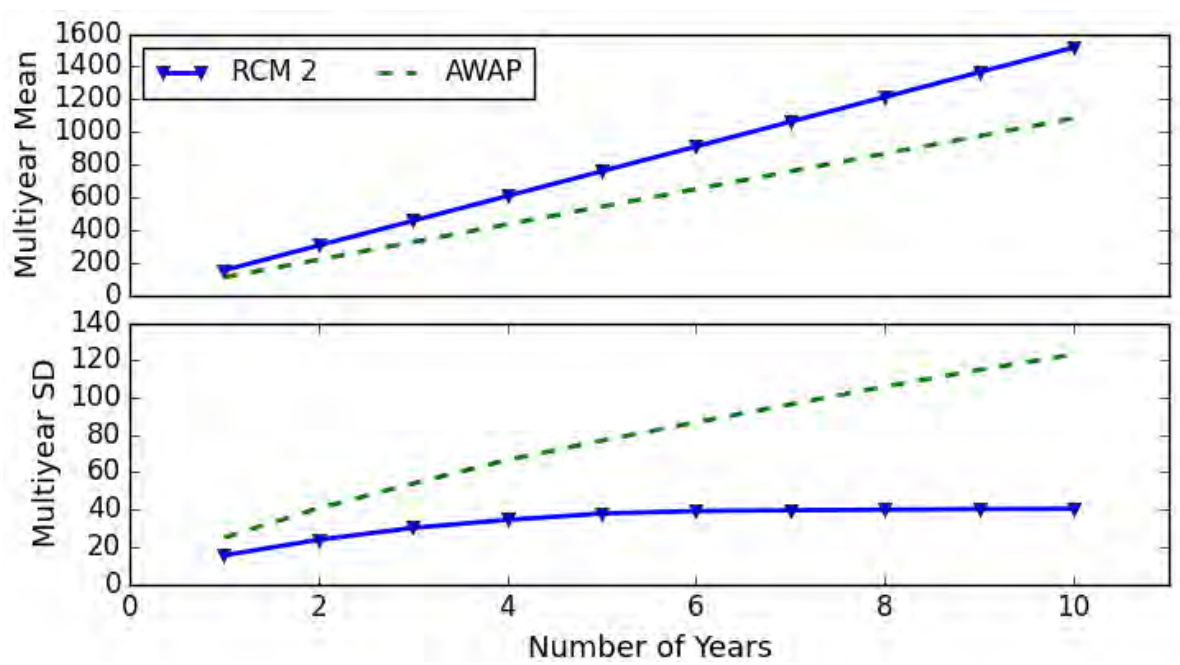

Figure 5.4: Mean and SD of multiyear number of wet days in RCM2 and AWAP. 


\subsubsection{Mean and SD of Mean Length of Wet Spells at Monthly and Annual Resolutions}

Figure 5.5 shows that the seasonal trends for mean and SD of monthly mean length of wet spells are mostly similar between RCM2 and AWAP, although the summer wet spell lengths of RCM2 are considerably longer than the same of AWAP. While the statistics in both datasets are similar, the variability in AWAP is still slightly higher with a lower mean than RCM2. A similar trend has been found for the mean and SD of annual mean length of wet spells (Table 5.1).

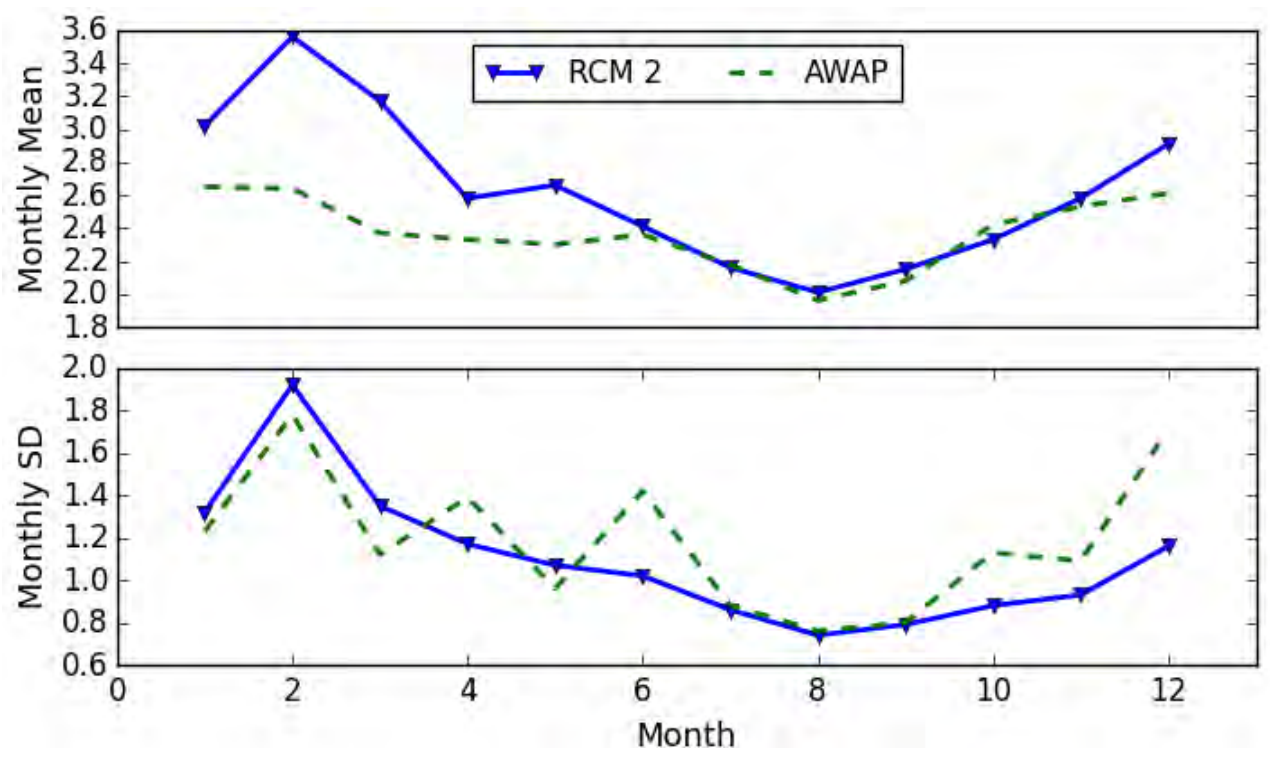

Figure 5.5: Mean and SD of monthly mean length of wet spells for RCM2 and AWAP.

Table 5.1: Mean and SD of annual mean length of wet spells $\left(\mu_{w e t}\right)$ for RCM2 and AWAP.

\begin{tabular}{ccc}
\hline Data & mean of $\mu_{\text {wet }}$ & SD of $\mu_{\text {wet }}$ \\
\hline RCM2 & 2.60 & 0.31 \\
AWAP & 2.42 & 0.49 \\
\hline
\end{tabular}

\subsection{Model Comparison for Distribution Statistics}

This section will present the comparison of the performances of the MC models (APMC, DPMC, CDMC, HMC and DHMC) to reproduce the mean and SD of rainfall depths and wet period statistics (i.e. number of wet days and mean length of wet spell) for RCM2 and AWAP at daily, monthly and multiyear resolutions. 


\subsubsection{Mean and SD of Daily Rainfall Depths}

Figure 5.6 shows the $\mathrm{Z}$ scores of the five MC models for mean and SD of daily (wet day) rainfall depths of RCM2 and AWAP. All five models preserve the mean and SD of daily rainfall depths with $\mathrm{Z}$ scores between -2 and +2 for all months. The performances of APMC and DPMC are similar, while performances of CDMC, HMC and DHMC are similar. The APMC and DPMC perform better than the CDMC and HMC with $\mathrm{Z}$ scores closer to zero for both statistics in each dataset. For mean of daily rainfall depths, the CDMC, HMC and DHMC show a consistent tendency to underestimate the statistic with positive $\mathrm{Z}$ scores (although mostly less than +1 ) for most of the months.
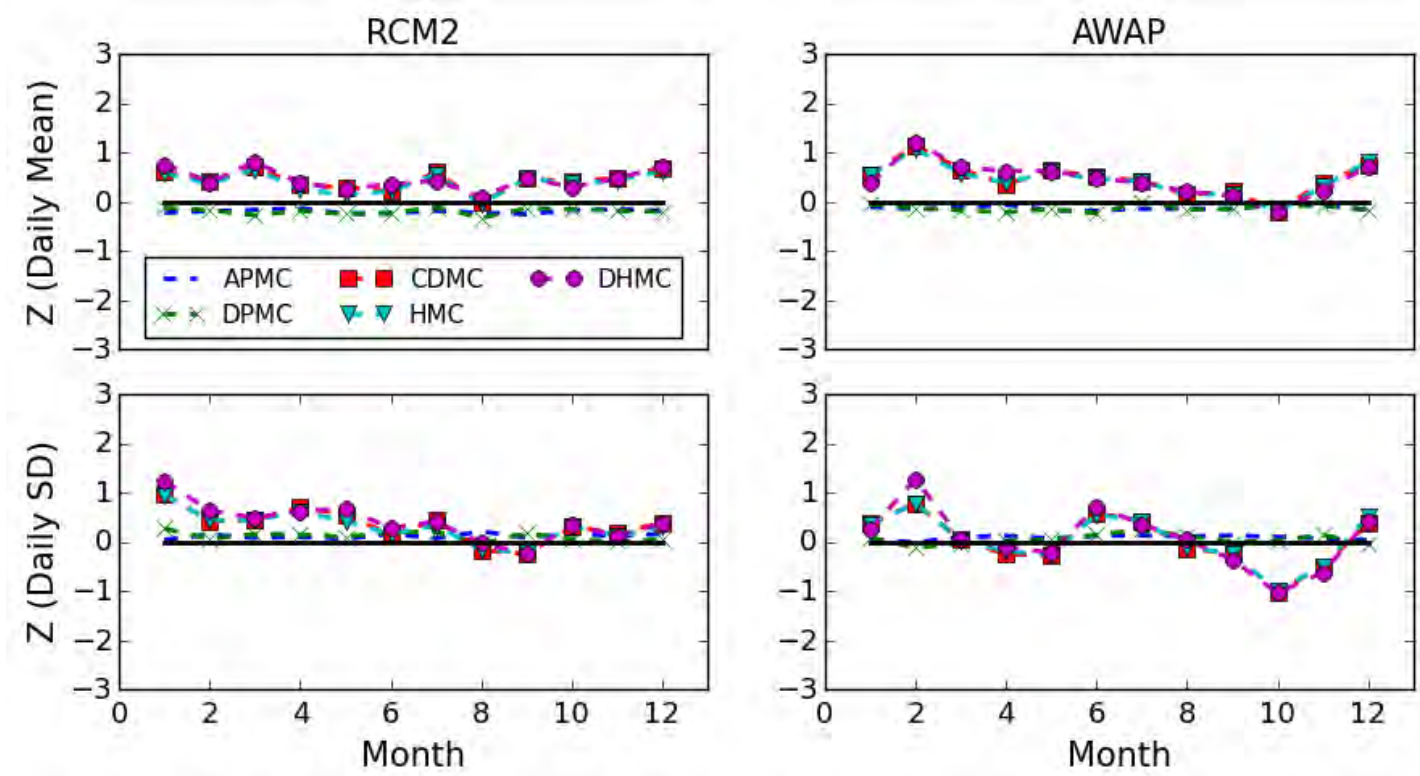

Figure 5.6: Comparison of APMC, DPMC, CDMC, HMC, and DHMC to reproduce the mean and SD of daily (wet day) rainfall depths for RCM2 and AWAP.

\subsubsection{Mean and SD of Monthly Rainfall Depths}

Figure 5.7 shows the $\mathrm{Z}$ scores of the five MC models for mean and $\mathrm{SD}$ of monthly rainfall depths of RCM2 and AWAP. The APMC and DPMC preserve only the mean of monthly rainfall depths and significantly underestimate $(Z$ score $>+2)$ the SD of monthly rainfall depths mainly for the summer months, while the DPMC performs slightly better than APMC. The CDMC, HMC and DHMC (similar performance) perform better and preserve both the mean and SD of monthly rainfall depths. 

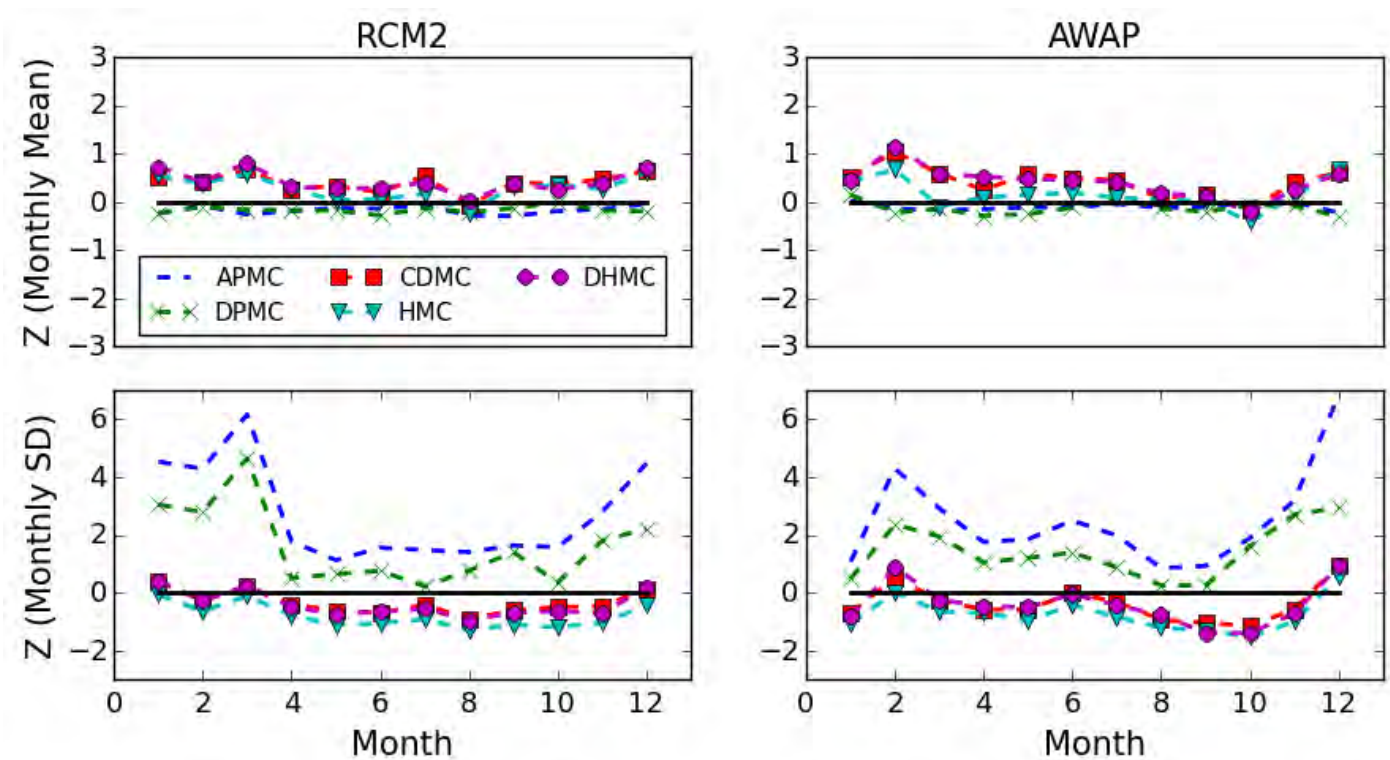

Figure 5.7: Comparison of APMC, DPMC, CDMC, HMC, and DHMC to reproduce the mean and SD of monthly rainfall depths for RCM2 and AWAP.

\subsubsection{Mean and SD of Multiyear Rainfall Depths}

Figure 5.8 shows the $\mathrm{Z}$ scores of the five models for mean and SD of multiyear rainfall depths of RCM2 and AWAP. Similar to monthly statistics, the APMC and DPMC preserve only the mean of multiyear rainfall depths and significantly underestimate the SD of multiyear rainfall depths. The CDMC, HMC and DHMC perform better than the APMC and DPMC, and preserve both the mean and SD of multiyear rainfall depths. For mean of multiyear rainfall depths, the APMC and DPMC (models with deterministic Gamma parameters) tend to overestimate the statistic, while CDMC, HMC and DHMC (models with stochastic Gamma parameters) tend to underestimate the statistic. 

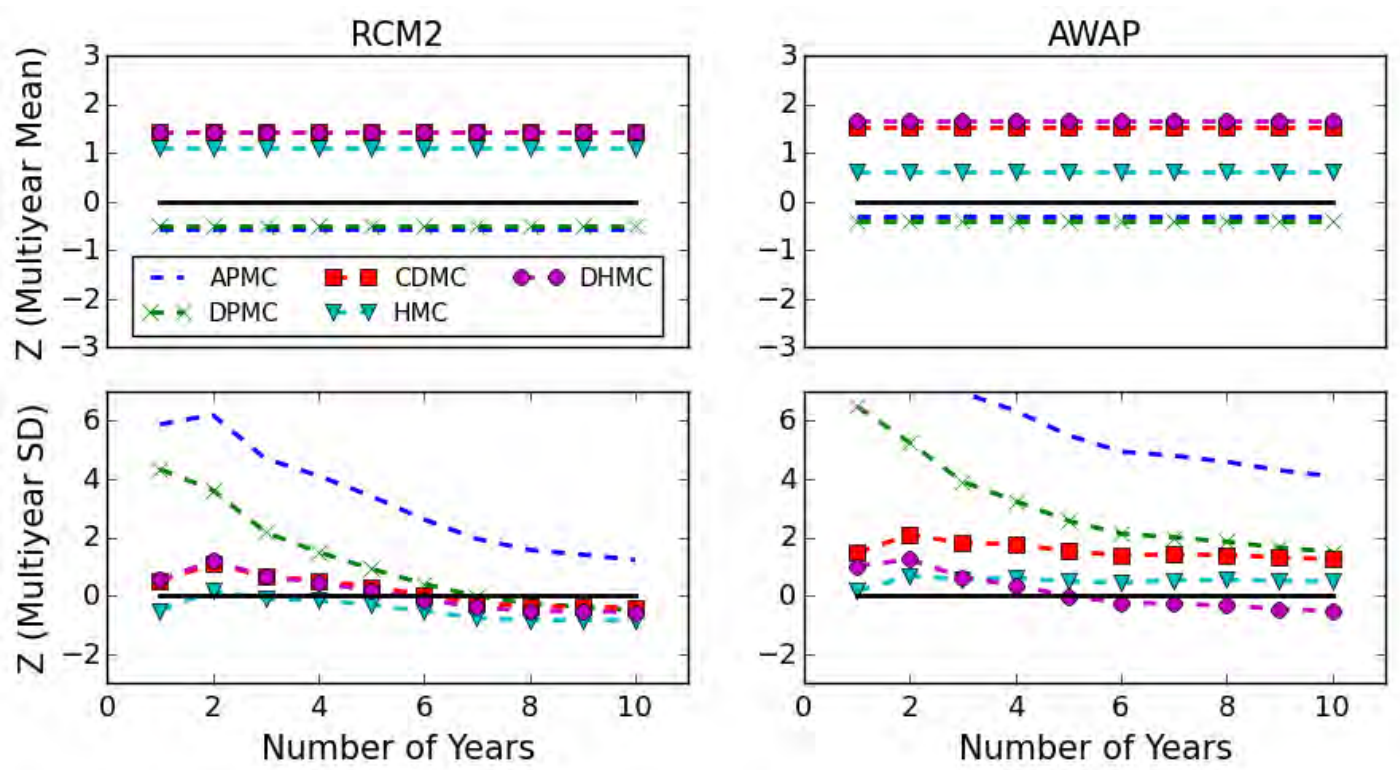

Figure 5.8: Comparison of APMC, DPMC, CDMC, HMC and DHMC to reproduce the mean and SD of multiyear rainfall depths for RCM2 and AWAP.

\subsubsection{Mean and SD of Monthly Number of Wet Days}

Figure 5.9 shows the $\mathrm{Z}$ scores of the five models for mean and SD of monthly number of wet days for RCM2 and AWAP. For mean of monthly number of wet days, all five models preserve the statistic, while the HMC shows a consistent tendency to overestimate the statistic for AWAP. For SD of monthly number of wet days, the APMC, DPMC, CDMC and DHMC show similar trends of performances, and preserve the statistic for RCM2. However, the APMC and CDMC ( $Z$ scores are very similar) tend to significantly underestimate the SD of monthly number of wet days for AWAP, while with a similar trend DPMC and DHMC ( $Z$ scores are very similar) perform better than the APMC and CDMC and preserve the statistic. For SD of monthly number of wet days, the performance of the HMC is not satisfactory as the model significantly overestimates the statistic for both datasets. 

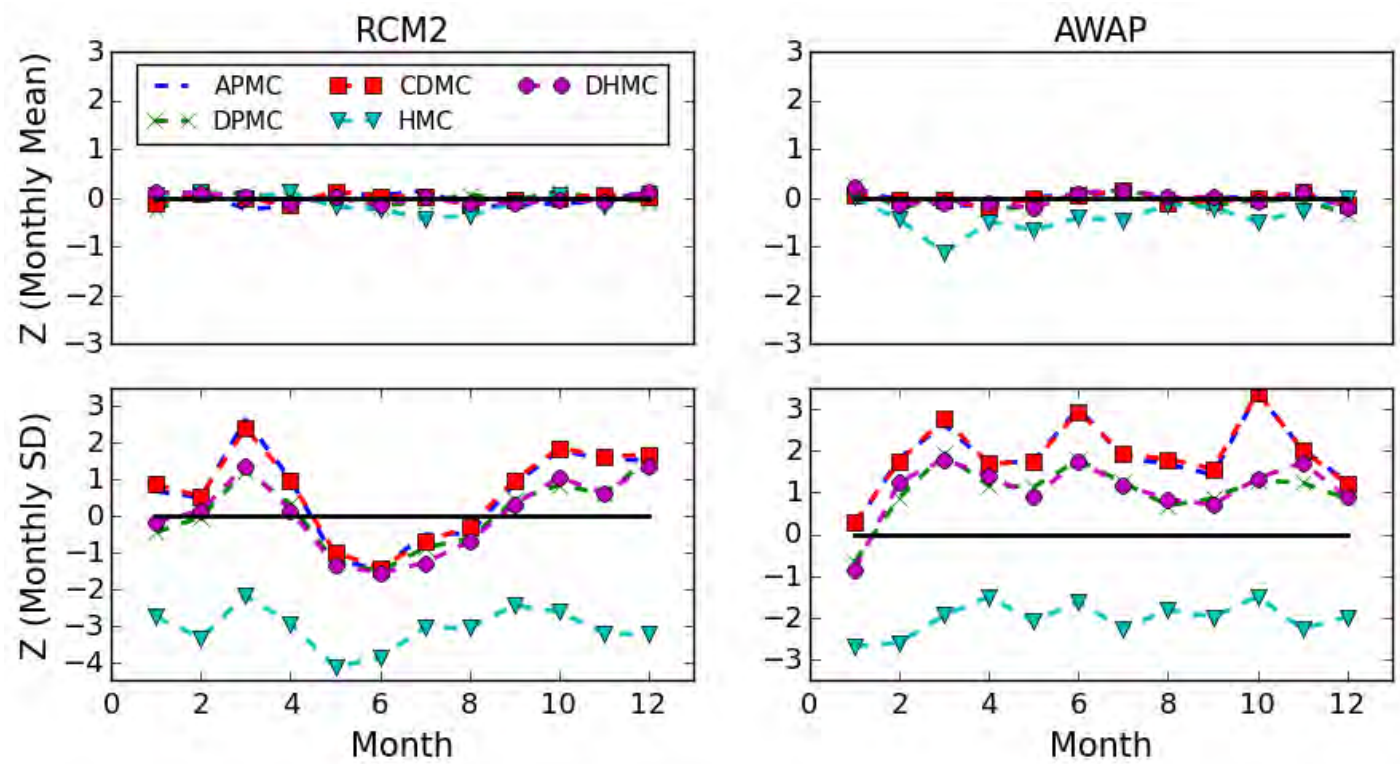

Figure 5.9: Comparison of APMC, DPMC, CDMC, HMC and DHMC to reproduce the mean and SD of monthly number of wet days for RCM2 and AWAP.

\subsubsection{Mean and SD of Multiyear Number of Wet Days}

Figure 5.10 shows the $\mathrm{Z}$ scores of the five models for mean and SD of multiyear number of wet days for RCM2 and AWAP. Similar to monthly resolution, all five models preserve the mean of multiyear number of wet days for RCM2 and AWAP, while only the HMC shows a consistent tendency to overestimate the statistic. For SD of multiyear number of wet days, the APMC and CDMC (perform similarly) preserve the statistic only at longer multiyear resolutions (e.g. five to 10 overlapping years) for RCM2, but significantly underestimate the statistic at shorter multiyear resolutions (e.g. one to four overlapping years) for RCM2 and all multiyear resolutions for AWAP ( $Z$ scores are greater than +2$)$. The DPMC and DHMC satisfactorily preserve the SD of multiyear number of wet days for both datasets with a tendency to underestimate the statistic at shorter multiyear resolutions (e.g. one to four overlapping years). The HMC also preserves the SD of multiyear number of wet days for RCM2, but shows a tendency to overestimate the statistic. However, for AWAP, the HMC significantly underestimates the SD of multiyear number of wet days. 

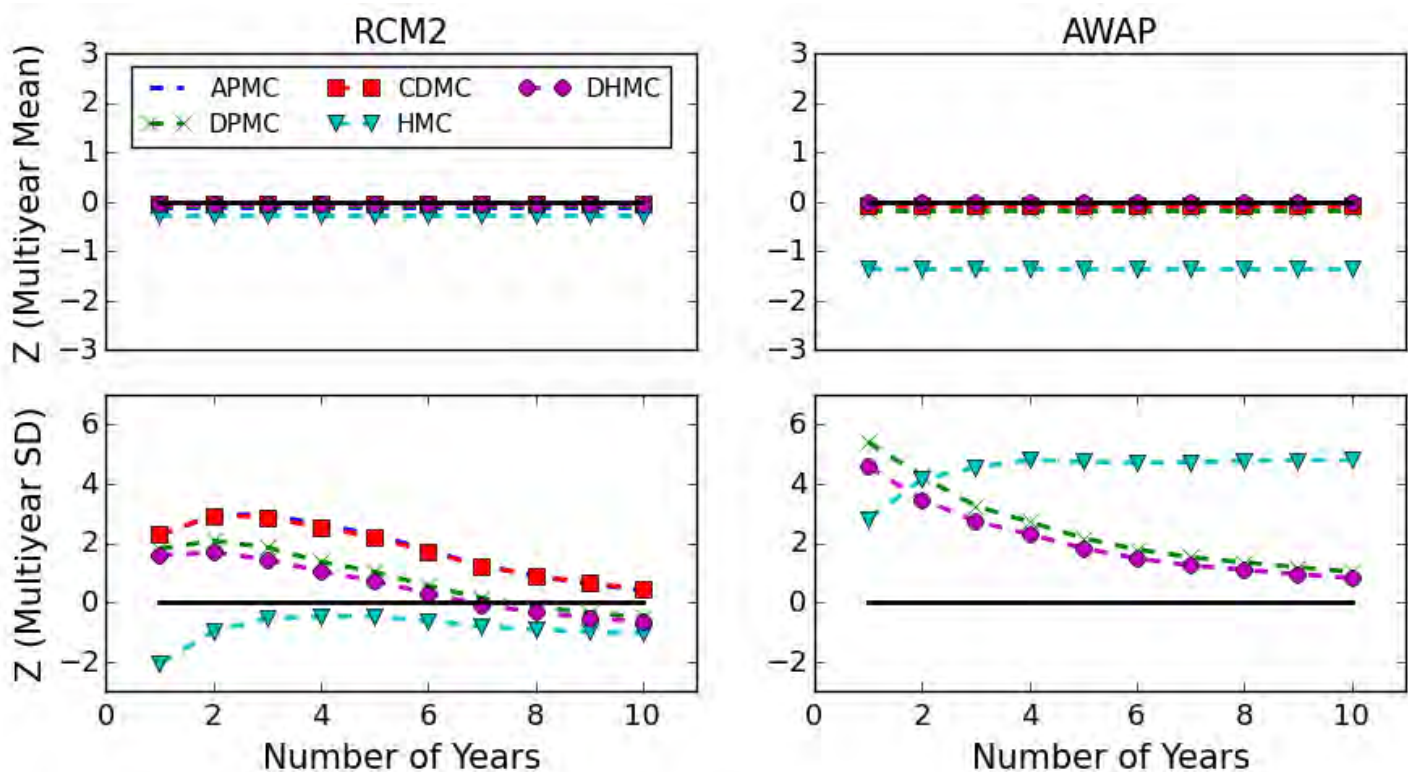

Figure 5.10: Comparison of APMC, DPMC, CDMC, HMC and DHMC to reproduce the mean and SD of multiyear number of wet days for RCM2 and AWAP. The Z scores of APMC and CDMC for AWAP are out of the +6 range.

\subsubsection{Mean and SD of Monthly Mean Length of Wet Spells}

Figure 5.11 shows the $\mathrm{Z}$ scores of the five models for mean and SD of monthly mean length of wet spells for RCM2 and AWAP. The comparative performances of each model for the mean and SD of monthly mean length of wet spells are consistent with their respective performances for monthly number of wet days. The DPMC and DHMC (similar performance) perform better than APMC and CDMC (similar performance), while performance of these four models is satisfactory. However, the HMC tends to overestimate the statistics with negative $\mathrm{Z}$ scores for most of the months. 

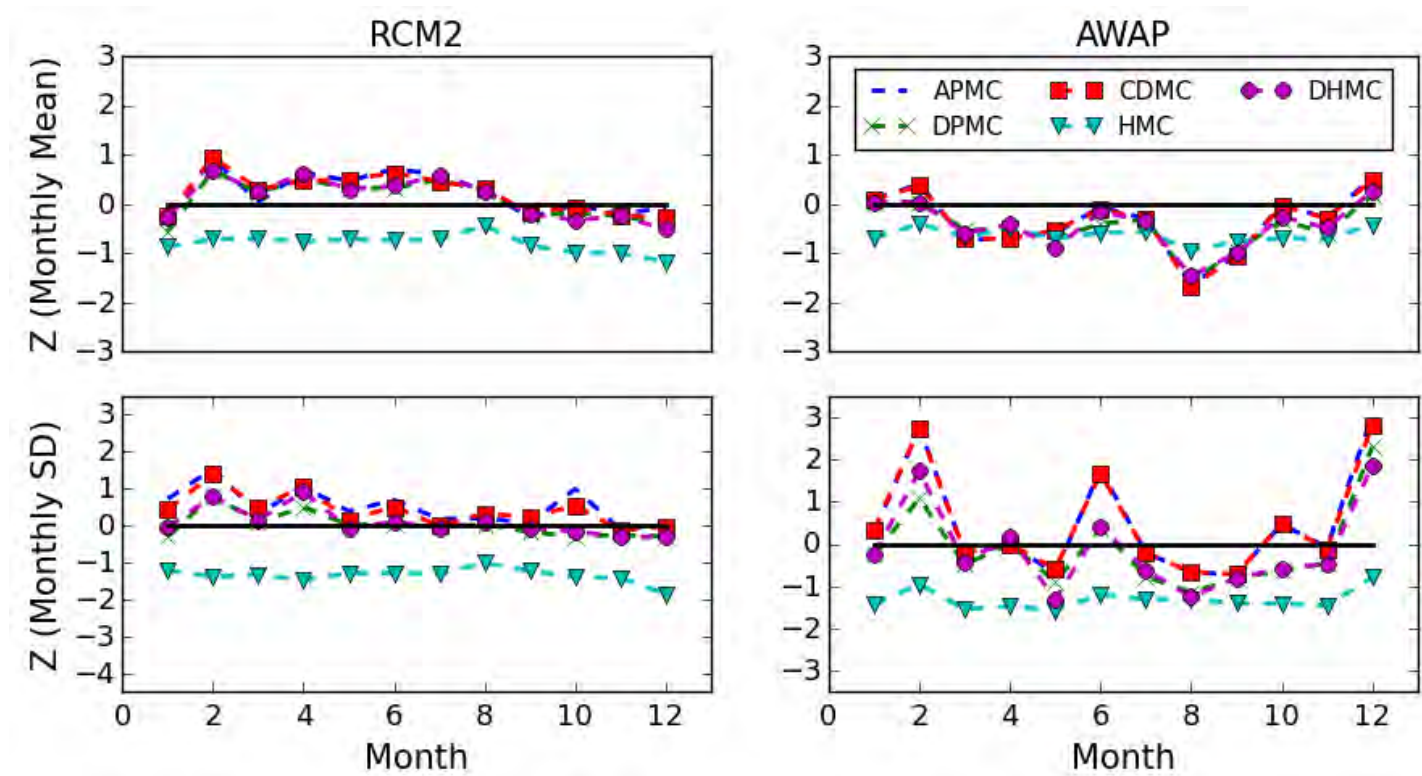

Figure 5.11: Comparison of APMC, DPMC, CDMC, HMC, and DHMC to reproduce the mean and SD of monthly mean length of wet spells for RCM2 and AWAP.

\subsubsection{Mean and SD of Annual Mean Length of Wet Spells}

Figure 5.12 shows the $\mathrm{Z}$ scores of the five models for mean and SD of annual mean length of wet spells for RCM2 and AWAP. All five models preserve the mean of annual mean length of wet spells. The APMC and CDMC perform similarly, and preserve the SD of annual mean length of wet spells for RCM2 but significantly underestimate the statistic for AWAP. However, the DPMC and DHMC preserve the variability for both datasets with a tendency of underestimation. The HMC preserves the SD of annual mean length of wet spells for AWAP but significantly overestimate the statistic for RCM2. 

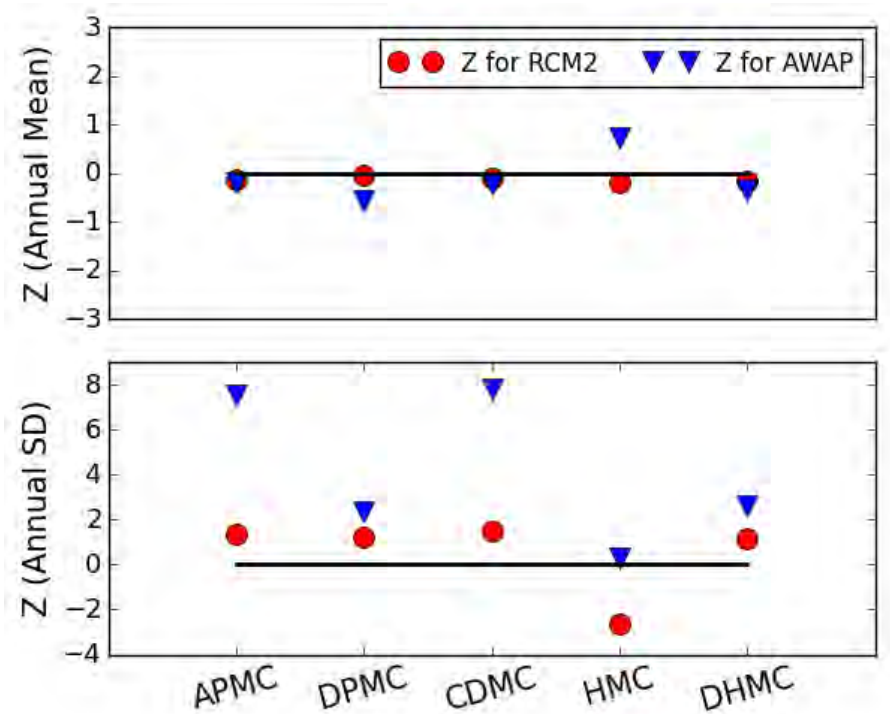

Figure 5.12: Comparison of APMC, DPMC, CDMC, HMC, and DHMC to reproduce the mean and SD of annual mean length of wet spells for RCM2 and AWAP.

\subsubsection{Summary}

Table 5.2 shows the temporally averaged $\mathrm{Z}$ scores (calculated as per section 3.8.1) of the five MC models. The performance of the DHMC is the best for both rainfall depth and wet period simulation. The CDMC and HMC perform well for the rainfall depth, but fail to preserve the long-term SD of wet periods. The DPMC performs well for wet periods, but fails to preserve the long-term SD of rainfall depths. The APMC fails to preserve the long-term SD of both rainfall depths and wet periods. The performance of each model is better for RCM2 than for AWAP, particularly for SD of wet periods, probably because AWAP has higher SD of wet periods than RCM2. 
Table 5.2: Temporally averaged $Z$ scores of five MC models of this study.

\begin{tabular}{|c|c|c|c|c|c|c|c|}
\hline & \multirow{2}{*}{ Statistics } & \multirow{2}{*}{ Data } & \multicolumn{5}{|c|}{ Temporally Averaged Z Scores } \\
\hline & & & APMC & DPMC & CDMC & $\mathrm{HMC}$ & DHMC \\
\hline \multirow{12}{*}{ 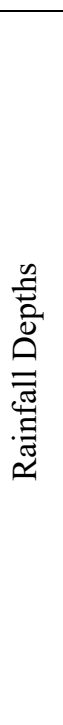 } & \multirow{2}{*}{ Daily Mean } & R2 & 0.19 & 0.19 & 0.44 & 0.40 & 0.46 \\
\hline & & AW & 0.12 & 0.13 & 0.50 & 0.47 & 0.49 \\
\hline & \multirow{2}{*}{ Daily SD } & $\mathrm{R} 2$ & 0.12 & 0.12 & 0.41 & 0.37 & 0.45 \\
\hline & & AW & 0.09 & 0.09 & 0.42 & 0.41 & 0.46 \\
\hline & Monthly & $\mathrm{R} 2$ & 0.17 & 0.16 & 0.41 & 0.32 & 0.42 \\
\hline & Mean & AW & 0.10 & 0.17 & 0.45 & 0.25 & 0.45 \\
\hline & \multirow{2}{*}{ Monthly SD } & $\mathrm{R} 2$ & 2.74 & 1.60 & 0.47 & 0.80 & 0.54 \\
\hline & & AW & 2.50 & 1.43 & 0.63 & 0.85 & 0.71 \\
\hline & Multiyear & $\mathrm{R} 2$ & 0.55 & 0.49 & 1.43 & 1.09 & 1.42 \\
\hline & Mean & AW & 0.31 & 0.41 & 1.54 & 0.62 & 1.66 \\
\hline & \multirow{2}{*}{ Multiyear SD } & $\mathrm{R} 2$ & 3.31 & 1.41 & 0.45 & 0.50 & 0.52 \\
\hline & & AW & 5.82 & 3.06 & 1.54 & 0.51 & 0.51 \\
\hline \multirow{8}{*}{ 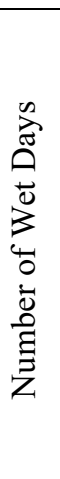 } & Monthly & $\mathrm{R} 2$ & 0.14 & 0.09 & 0.07 & 0.16 & 0.08 \\
\hline & Mean & AW & 0.08 & 0.14 & 0.09 & 0.39 & 0.12 \\
\hline & \multirow{2}{*}{ Monthly SD } & $\mathrm{R} 2$ & 1.18 & 0.81 & 1.19 & 3.07 & 0.84 \\
\hline & & AW & 1.89 & 1.14 & 1.92 & 2.01 & 1.22 \\
\hline & Multiyear & $\mathrm{R} 2$ & 0.12 & 0.06 & 0.03 & 0.27 & 0.05 \\
\hline & Mean & AW & 0.02 & 0.17 & 0.08 & 1.34 & 0.02 \\
\hline & \multirow{2}{*}{ Multiyear SD } & $\mathrm{R} 2$ & 1.80 & 0.98 & 1.78 & 0.89 & 0.84 \\
\hline & & AW & 9.74 & 2.47 & 9.98 & 4.49 & 2.06 \\
\hline \multirow{8}{*}{ 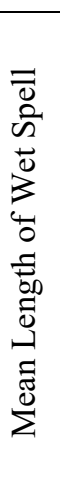 } & Monthly & $\mathrm{R} 2$ & 0.37 & 0.37 & 0.38 & 0.80 & 0.38 \\
\hline & Mean & AW & 0.53 & 0.51 & 0.53 & 0.63 & 0.49 \\
\hline & \multirow{2}{*}{ Monthly SD } & $\mathrm{R} 2$ & 0.53 & 0.24 & 0.44 & 1.34 & 0.26 \\
\hline & & AW & 0.91 & 0.77 & 0.88 & 1.32 & 0.82 \\
\hline & \multirow{2}{*}{ Annual Mean } & $\mathrm{R} 2$ & 0.12 & 0.05 & 0.11 & 0.21 & 0.15 \\
\hline & & AW & 0.21 & 0.57 & 0.24 & 0.73 & 0.34 \\
\hline & \multirow{2}{*}{ Annual SD } & $\mathrm{R} 2$ & 1.38 & 1.25 & 1.52 & 2.64 & 1.17 \\
\hline & & AW & 7.59 & 2.38 & 7.81 & 0.29 & 2.63 \\
\hline & \multirow{2}{*}{ Sum } & $\mathbf{R 2}$ & 12.72 & 7.82 & 9.13 & 12.86 & 7.58 \\
\hline & & $\mathbf{A W}$ & 29.91 & 13.44 & 26.61 & 14.31 & 11.98 \\
\hline
\end{tabular}

$\mathrm{R} 2=\mathrm{RCM} 2$ and $\mathrm{AW}=\mathrm{AWAP}$ 


\subsection{Potential Impact of Climate Modes}

Since the DPMC significantly underestimates the SD of rainfall depths at monthly and multiyear resolutions, the major target of the other models similar to DPMC (see section 4.3.5) was to preserve the SD of rainfall depths at monthly and multiyear resolutions. However, as shown in Figure 5.13, these alternative models also significantly underestimate the SD of rainfall depths at monthly resolution (accordingly at multiyear resolutions) with performances similar to the DPMC.

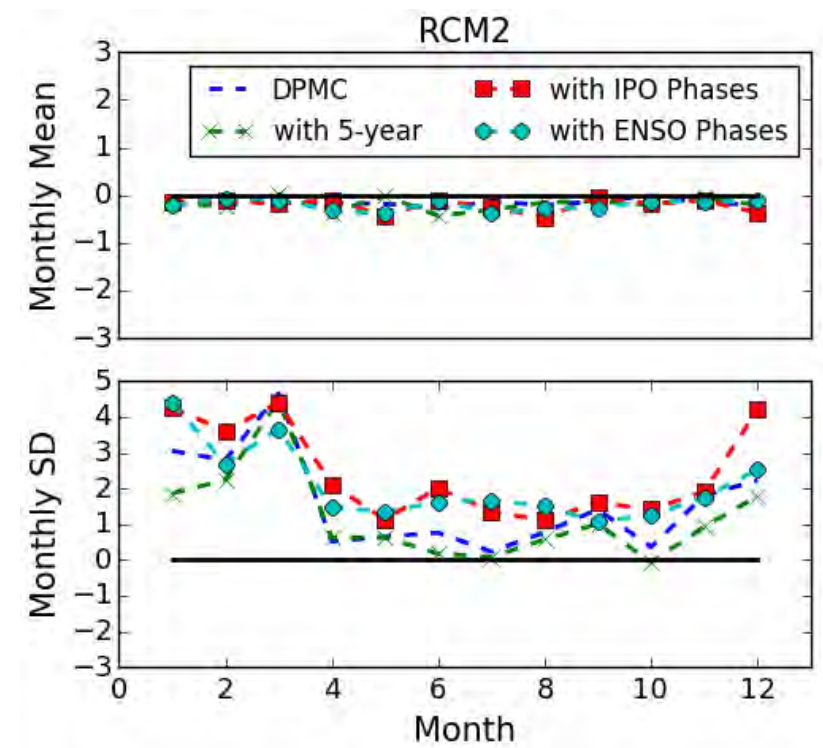

Figure 5.13: Comparison of DPMC and other DPMC-like models (e.g. model with sub-samples of 5-year length, model with sub-samples according to IPO and ENSO phases) to reproduce the mean and SD of monthly rainfall depths for RCM2.

\subsection{Impact of Stochasticity of MC Parameters}

Since the HMC significantly overestimates the SD of number of wet days and mean length of wet spells at monthly resolution, the major target of the other models similar to HMC (see section 4.5.3) was to preserve these two statistics. However, as shown in Figure 5.14, these alternative models also significantly overestimate the SD of monthly number of wet days (similarly the SD of monthly mean length of wet spells) with performances similar to the HMC. 

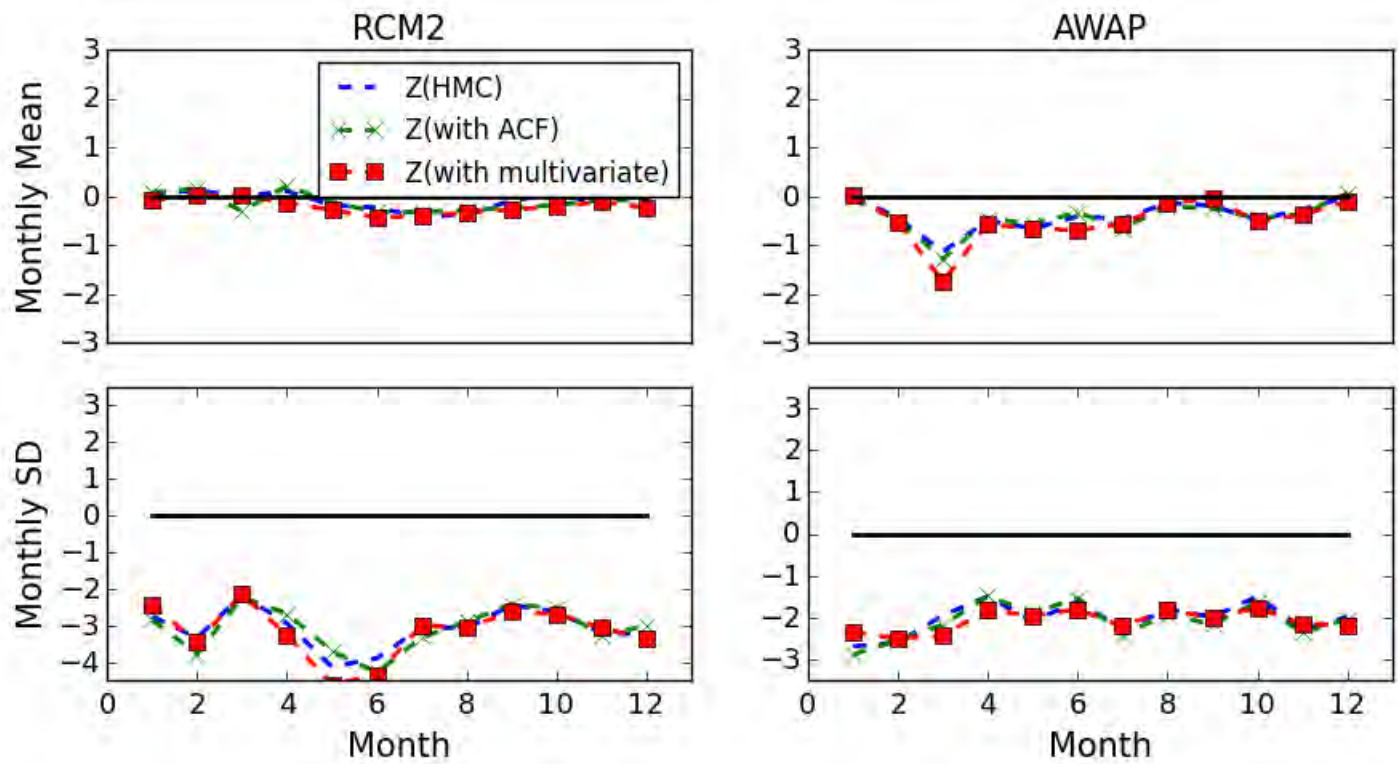

Figure 5.14: Comparison of HMC and other HMC-like models (e.g. model with lag-1 autocorrelation equation and model with multivariate sampling for stochastic MC parameters) to reproduce the mean and SD of monthly rainfall depths for RCM2.

\subsection{Model Comparison for Autocorrelations}

Figure 5.15 and Figure 5.16 show comparisons of the five MC models for the month-to-month autocorrelations of the monthly number of wet days and monthly rainfall depths for RCM2 and AWAP respectively.

Figure 5.15 shows that the lag-1 and lag-12 autocorrelations of monthly rainfall depths and number of wet days (i.e. monthly total rainfall depths and wet days) are strong in RCM2 data, which has been reproduced very well in the corresponding APMC, DPMC, CDMC and DHMC simulations. The performances of the APMC, DPMC, CDMC and DHMC are mostly similar. The seasonality and the coefficient of correlations are similar in the RCM2 and the corresponding APMC, DPMC, CDMC and DHMC simulations, although the APMC and DPMC show a tendency to overestimate the autocorrelations of monthly rainfall depths. Figure 5.15 also shows that the HMC has replicated the seasonality of RCM2 well, but the coefficients of correlations are considerably weaker in the HMC-simulated series than the same in RCM2. This performance of the HMC is inferior to the performance of the APMC, DPMC, CDMC and DHMC.

Figure 5.16 shows that the lag-1 and lag-12 autocorrelations of monthly rainfall depths are not strong in AWAP data, and the autocorrelations in corresponding CDMC and HMC simulations 
are even weaker. This indicates that the CDMC and HMC are not satisfactorily replicating the weak lag-1 autocorrelation. In contrast, the APMC and DPMC tend to slightly overestimate the autocorrelations of monthly rainfall depths, and the DHMC performs better and satisfactorily to reproduce the autocorrelations. For monthly number of wet days, only the lag-1 autocorrelation is slightly strong in AWAP. Only the DPMC and DHMC reproduce the autocorrelations well, while the other three models tend to underestimate the autocorrelations.
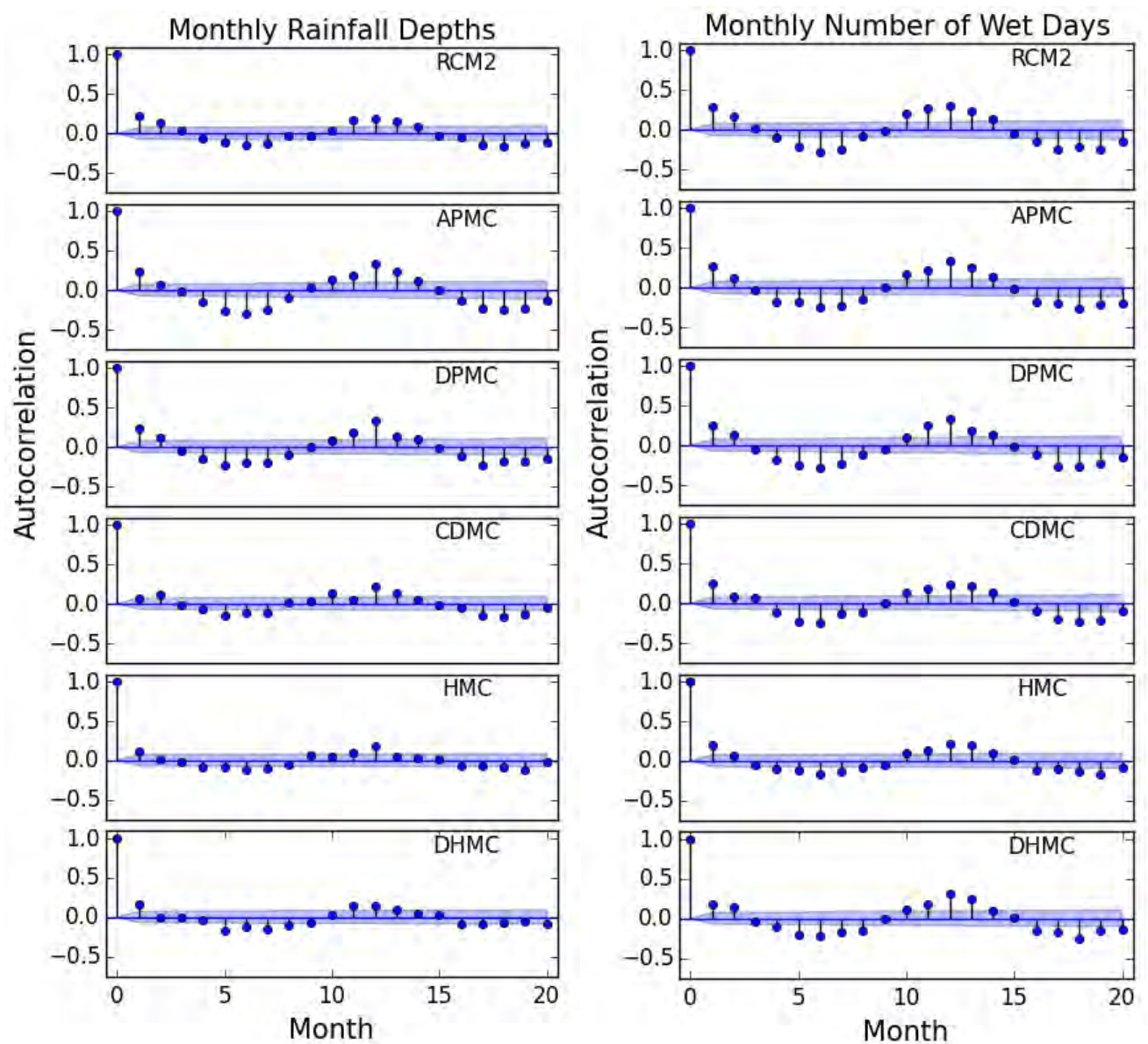

Figure 5.15: Comparison of APMC, DPMC, CDMC, HMC and DHMC to reproduce the month-tomonth autocorrelations of monthly rainfall depths and monthly number of wet days for RCM2. 

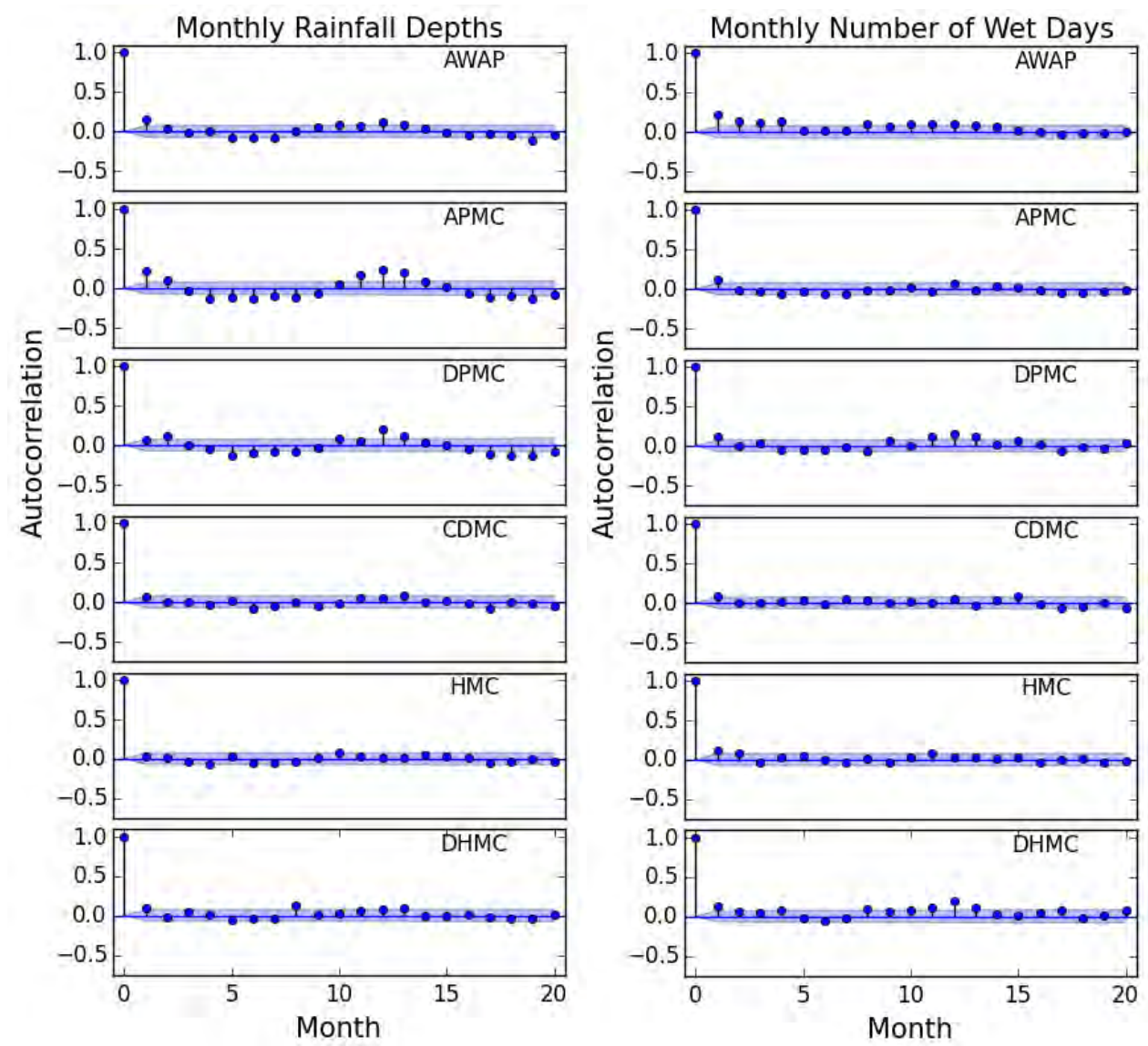

Figure 5.16: Comparison of APMC, DPMC, CDMC, HMC and DHMC to reproduce the month-tomonth autocorrelations of monthly rainfall depths and monthly number of wet days for AWAP.

\subsection{Discussion}

In this chapter, the parameters of the APMC, DPMC, CDMC, HMC and DHMC have been calibrated to NARCliM reanalysis of RCM2 and AWAP data for the 1950-2009 period at Gummun (located in the Goulburn River Site of upper Hunter region of NSW). The distribution (mean and SD) and autocorrelation of rainfall depths and wet period statistics (number of wet days and mean length of wet spells) of these two datasets are calculated at different temporal (i.e. daily, monthly and multiyear) resolutions. The daily and monthly statistics indicate typical eastern Australia seasonality of wet summers and dry winters in both datasets. Although the objective of this chapter is not to compare the characteristics of these two datasets, the analysis shows that the rainfall depths distributions in both datasets are similar, but the wet period statistics of AWAP have considerably more variability than the same of RCM2, and accordingly the month-to-month autocorrelations of rainfall depths and wet days in AWAP are significantly weaker than the same of RCM2. To compare the performances of the APMC, DPMC, CDMC, HMC and DHMC, the calculated distribution and autocorrelation statistics of the RCM2 and AWAP were compared with the respective statistics calculated from each 
model. The comparative assessments for distribution statistics were done by $\mathrm{Z}$ score calculation, as described in section 3.4.3.

The performances of each of the five models to reproduce the statistics of RCM2 and AWAP presented in this chapter have also been discussed in the model development chapter (Chapter 4). The performances of each model to reproduce the distribution statistics of rainfall depths are consistent for both RCM2 and AWAP as the distribution statistics of rainfall depths are also similar in both datasets. However, the performances of each model to reproduce the wet period statistics are clearly better for RCM2 than for AWAP, while the wet period statistics of both datasets are considerably different. However, irrespective of data sensitivity, the overall comparative performances of the models are as follows:

- For simulation of the distribution of rainfall depths, the performances of the APMC and DPMC with deterministic Gamma parameters are similar, although DPMC with more (e.g. six times more) parameters performs slightly better. The performances of CDMC, HMC and DHMC are similar as they use the same stochastic parameters of Gamma distribution.

- For mean and SD of daily rainfall depths, all five models perform satisfactorily. Good reproduction of daily statistics is expected as the model parameters are calibrated to daily timeseries. While the APMC and DPMC reproduce the statistics almost exactly, the CDMC, HMC and DHMC show a slight tendency to underestimate the statistics. This indicates that the stochastic parameters of these three models slightly affected their performances at daily resolution compared to the APMC and DPMC with deterministic parameters.

- At monthly to multiyear resolution, the APMC and DPMC can only reproduce the mean of rainfall depths, but significantly underestimate the SD of rainfall depths. The underestimation of rainfall variability at monthly to multiyear resolutions by APMClike models with deterministic parameters is well known in the stochastic daily rainfall simulation, as described in section 2.5. Although the DPMC uses more parameters than the APMC, the DPMC has not provided considerably improved performance in reproducing the $\mathrm{SD}$ of rainfall depths at monthly to multiyear resolutions. Other models similar to DPMC (e.g. models with parameters varying for each 5-year and models with parameters varying for phases of IPO or ENSO) show similar performances to the DPMC and systematically underestimate the SD of rainfall depths at monthly to 
multiyear resolutions. This suggests that the use of deterministic parameters in DPMClike models might not be adequate to satisfactorily reproduce the SD of rainfall depths at all resolutions.

- While the APMC and DPMC, with deterministic parameters for the Gamma distribution, significantly underestimate the SD of rainfall depths at monthly to multiyear resolutions, the CDMC, HMC and DHMC, with stochastic parameters for the Gamma distribution, preserves the SD of rainfall depths (in addition to mean) at monthly to multiyear resolutions. This indicates that the stochastic parameters for the Gamma distribution are useful to incorporate the longer-term variability of rainfall depths. However, both models show a tendency to underestimate the mean of rainfall depths at all resolutions.

- For simulation of the distribution of wet periods, the performances of the APMC and CDMC are similar as both models use the same deterministic MC parameters. The DPMC and DHMC perform better than the APMC and CDMC, while DPMC and DHMC use more (e.g. six times more) deterministic MC parameters. The performances of the HMC, with stochastic MC parameters, is different from the other four models with deterministic MC parameters.

- For mean of wet period statistics (e.g. number of wet days and mean length of wet spells) at monthly to multiyear resolutions, the performances of the five MC models are mostly comparable and satisfactory, although the HMC tends to slightly overestimate the statistics.

- For SD of monthly wet period statistics, the APMC and CDMC reproduce the statistics well, while the DPMC and DHMC perform better than the APMC and CDMC. However, the HMC tends to significantly overestimate the SD of monthly wet period statistics. This indicates that the stochastic MC parameters of the HMC introduce excessive variability in the wet period simulation at monthly resolution. This study has further examined this overestimation issue by testing some other variants of the HMC with different stochastic parameterisation of the MC process, but they did not perform better than the HMC.

- For SD of wet period statistics at annual and multiyear resolutions, the APMC and CDMC perform satisfactorily to reproduce the SD of annual mean length of wet spells, but show a tendency to significantly underestimate the SD of multiyear number of wet days. This suggests that the APMC values of MC parameters (same parameter for all year simulation) have limitations to reproduce the wet period variability at multiyear 
resolutions. However, the DPMC and DHMC with decadally varied MC parameters shows better and satisfactory performances to reproduce the SD of annual mean length of wet spells and SD of multiyear number of wet days. This suggests that the decadally varied MC parameters can significantly improve the simulation of wet period variability, although the decadally varied Gamma parameters cannot improve the simulation of rainfall depths variability. However, the HMC preserves the SD of annual mean length of wet spells and SD of multiyear number of wet days with a tendency of overestimation. This suggests that the stochastic MC parameters are useful to improve the simulation of wet periods variability at multiyear resolutions, although they significantly overestimate the wet periods variability at monthly resolution.

- The autocorrelation assessments have shown that the CDMC and DHMC can satisfactorily reproduce the strong lag-1 and lag-12 autocorrelations of monthly rainfall depths and monthly number of wet days. However, the HMC tends to underestimate the autocorrelations with a possible influence of excessive variability in wet period simulation at monthly resolution. The APMC and DPMC tend to overestimate the autocorrelations, particularly for monthly rainfall depths.

\subsection{Conclusion}

Each model developed in this study has advantages and disadvantages. The APMC and DPMC with deterministic parameters are found to significantly underestimate the variability of rainfall depths at monthly to multiyear resolutions. This systematic underestimation of the rainfall depths variability at monthly to multiyear resolutions is critical for using the models in urban water security assessment as the reservoir water levels usually vary at these longer resolutions. Therefore, because of their inability to reproduce the rainfall depths variability at monthly to multiyear resolutions, this study has rejected the APMC and DPMC (although DPMC performs satisfactorily for wet periods variability). The CDMC, HMC and DHMC with stochastic parameters of Gamma distribution are found to preserve the rainfall depths variability at all resolutions, but the CDMC and HMC have limitations in reproducing the variability of wet periods. The CDMC with APMC values of MC parameters tends to underestimate the multiyear variability of wet periods, while the HMC with stochastic MC parameters tends to overestimate the monthly variability of wet periods. However, the DHMC with decadally varied MC parameters (same as DPMC) performs better than the CDMC and HMC, and preserves the wet period variability at all resolutions. 
In subsequent chapters of this thesis, the CDMC, HMC and DHMC will be further assessed by calibrating them to the daily rainfall data for the case study sites. Moreover, this chapter has used only RCM2 and AWAP data. In the subsequent chapters, this study will assess the model performances using gridded data for all NARCliM RCMs and AWAP. 


\section{Comparison of MC Models in Goulburn River Site}

\subsection{Introduction}

The previous chapter calibrated the five MC models developed in this study (APMC, DPMC, CDMC, HMC and DHMC) to RCM2 and AWAP at a single location (Gummun) in the Goulburn River site, and examined their performances to reproduce the distribution and autocorrelation characteristics of RCM2 and AWAP. This chapter extends this analysis for all NARCliM pixels (see section 3.3.1) of the Goulburn River site using all RCMs (RCM1, RCM2 and RCM3) and AWAP, and focuses on the spatial characteristics in this site. However, instead of five models, this chapter will examine only the CDMC, HMC and DHMC, as the APMC and DPMC have been rejected by this study in the previous chapter.

This case study area was chosen because it has a major hydrology field site with extensive field instrumentation [Rüdiger et al., 2007], and it has been the subject of numerous hydrology research studies [Martinez et al., 2010; Chen et al., 2015].

\subsection{Calibrated Parameters}

This section will present the parameters of MC models (APMC values of $P_{00}, P_{11}, \mu$, and $\sigma$ ) calibrated to the RCM1, RCM2, RCM3 and AWAP for each of the 130 pixels of the Goulburn River site. The APMC values of the parameters are plotted because $P_{00}$ and $P_{11}$ are the same in the APMC and CDMC, and equal to the average values of the distributed $P_{00}$ and $P_{11}$ values (e.g. 60 samples for each month) of the HMC. Similarly, the $\mu$ and $\sigma$ in the APMC are equal to the average values of the distributed $\mu$ and $\sigma$ values of the CDMC and HMC. In addition to the intra-annual and spatial variability of parameters, this section will present the correlation between model parameters and elevation (E) for the pixels of this site. 


\subsubsection{Intra-annual and Spatial Variability of Parameters}

Figure 6.1-6.4 show the intra-annual as well as spatial variability of $P_{00}, P_{11}, \mu$, and $\sigma$ (averaged APMC values) respectively for all RCMs and AWAP in the Goulburn River site. Table 6.1 shows the spatially and temporally averaged values of the four parameters (calculated as per section 3.8.2).

The intra-annual variability of the parameters for all RCMs and AWAP suggests that the rainfall in the Goulburn River site follows the typical east-Australian seasonality of wet summers and dry winters, with larger probability of wet days and mean daily rainfall depths in summer than winter.

One advantage of having a gridded dataset of continuous rainfall data is that it is possible to systematically examine the spatial variation of model parameters, and infer physical processes and linkages with the rainfall model structure and site topography. The wet-to-wet probability $P_{11}$ is highest in the mountains and lowest in the lower elevations near the river for all seasons (Figure 6.2), while the spatial distribution of the dry-to-dry probability $P_{00}$ is the opposite (Figure 6.1). Similarly, the $\mu$ of wet day rainfall depths are highest in the mountains and gradually decrease with decreasing elevation (Figure 6.3), while the spatial distribution of $\sigma$ values are similar to $\mu$ (Figure 6.4). This suggests more wet days and higher intensity rainfall at higher elevations, and more dry days and lower intensity rainfall at low elevations. The orographic signal is stronger in $P_{00}$ and $P_{11}$ than in $\mu$ and $\sigma$.

The intra-annual and spatial variability of parameters are mostly consistent among four datasets. While RCM2 is more similar to AWAP than RCM1 and RCM3, the drier (higher $P_{00}$ and lower $\mu$ ) pixels are typically drier in AWAP than RCM2. The dry-to-dry probability in AWAP ranges from 0.59 to 0.86 compared with 0.47 to 0.84 for RCM2. The wet-to-wet probability in AWAP and RCM2 ranges from $0.51-0.78$ and $0.44-0.82$ respectively. The mean wet day rainfall in AWAP and RCM2 ranges from $3.5 \mathrm{~mm}-8.6 \mathrm{~mm}$ and $3.8 \mathrm{~mm}-10.5 \mathrm{~mm}$ respectively. This indicates that the RCM2 data is wetter than the AWAP. The trends of intraannual and spatial variability of the parameters in RCM1 and RCM3 are similar to that of the RCM2. However, the rank of overall wetness in the four datasets varies in the order of RCM3 > RCM1 > RCM2 > AWAP (Table 6.1). 
6. Comparison of MC Models in Goulburn River Site

Table 6.1: Spatially and temporally averaged APMC values of model parameters.

\begin{tabular}{ccccc}
\hline Parameter & RCM1 & RCM2 & RCM3 & AWAP \\
\hline$P_{00}$ & 0.68 & 0.71 & 0.72 & 0.79 \\
$P_{11}$ & 0.62 & 0.61 & 0.59 & 0.63 \\
$\mu$ & 6.41 & 5.52 & 7.08 & 5.43 \\
$\sigma$ & 9.64 & 8.12 & 10.75 & 8.46 \\
\hline
\end{tabular}

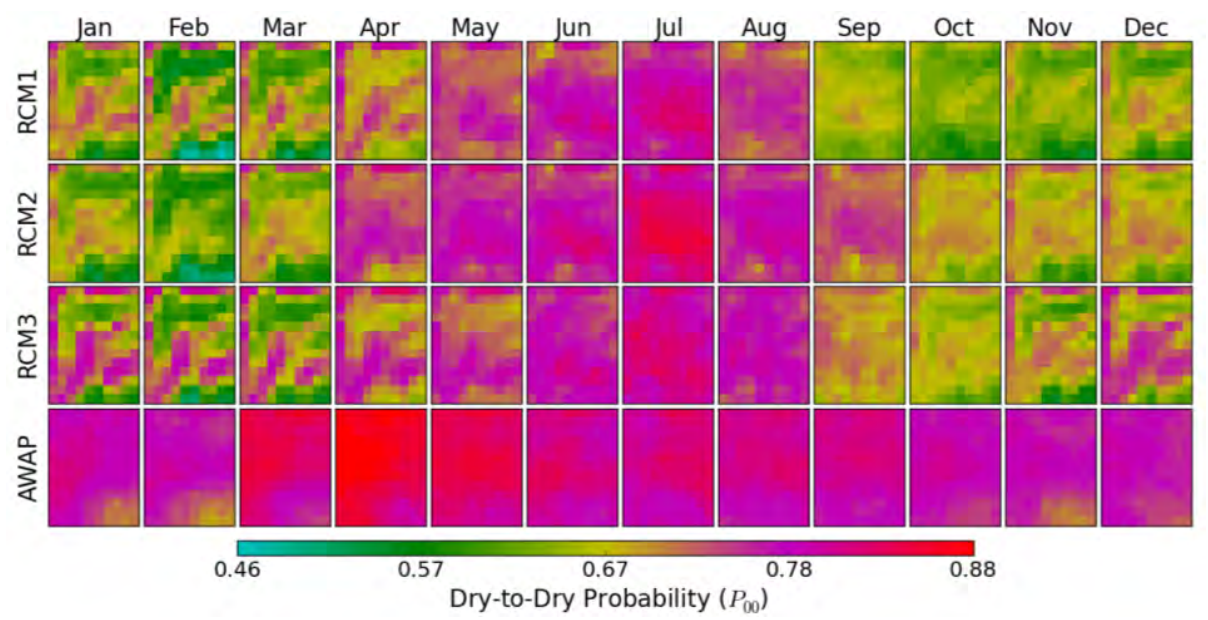

Figure 6.1: Intra-annual and spatial variability of dry-to-dry transition probabilities $\left(P_{00}\right)$. The colourbars indicate the parameter values.

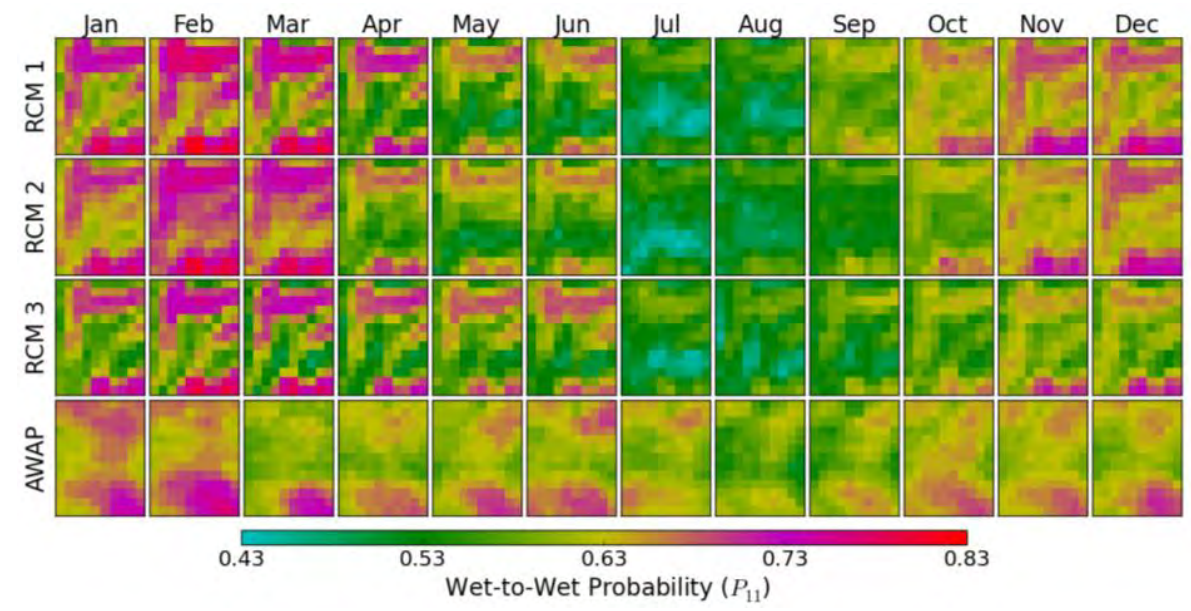

Figure 6.2: Intra-annual and spatial variability of wet-to-wet transition probabilities $\left(P_{11}\right)$. The colourbars indicate the parameter values. 


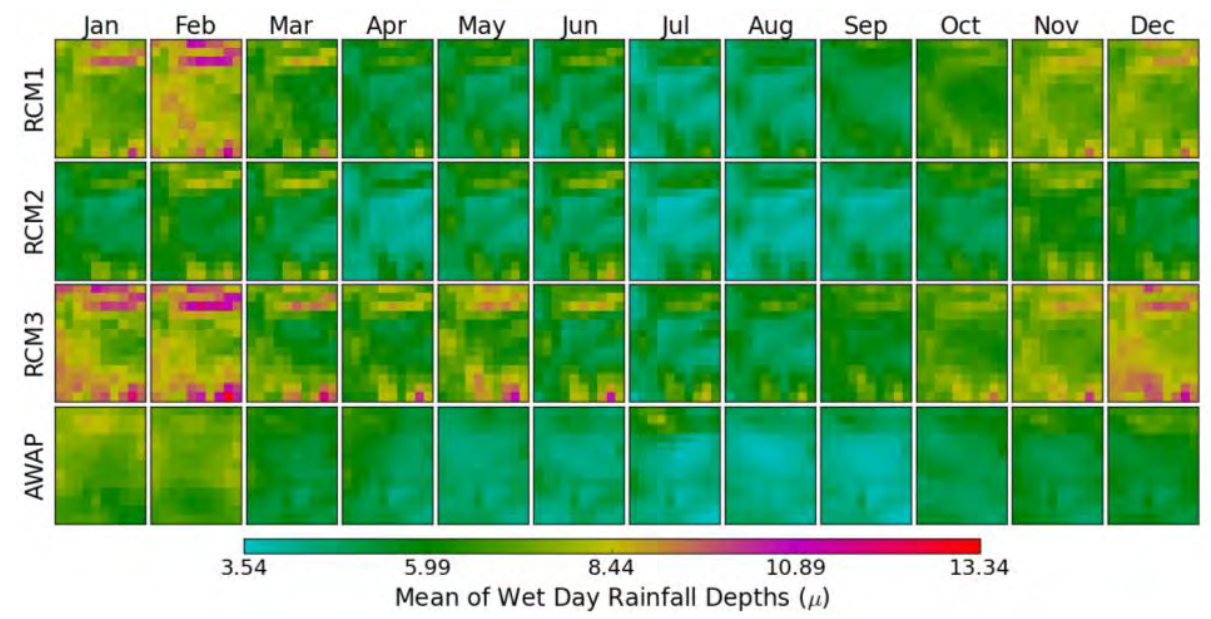

Figure 6.3: Intra-annual and spatial variability of the mean of wet day rainfall depths $(\mu)$ for all RCMs and AWAP at Goulburn. The colourbars indicate the parameter values.

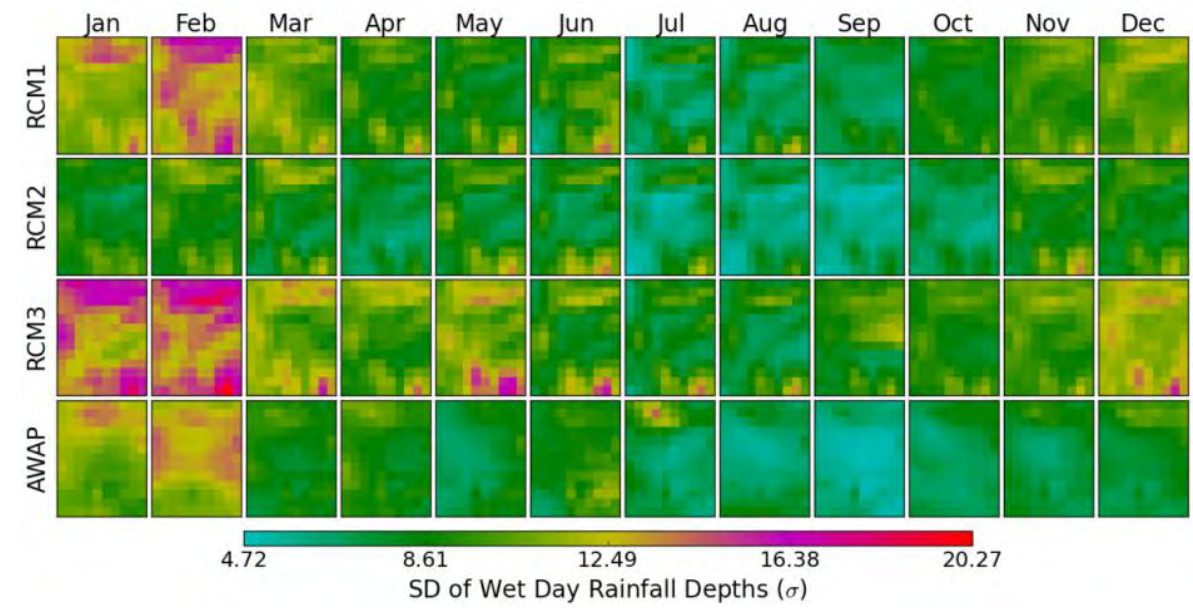

Figure 6.4: Intra-annual and spatial variability of the SD of wet day rainfall depths $(\sigma)$ for all RCMs and AWAP at Goulburn. The colourbars indicate the parameter values.

\subsubsection{Correlation between Elevation and Parameters}

This study has assessed the orographic influence on the calibrated parameters by correlating the APMC parameter values at each pixel with their corresponding elevations (E). The strength of the orographic influence is expressed by the correlation coefficient $r_{o}$ (subscript 'o' indicates orographic correlation) between the parameters and the elevations.

Figure 6.5 shows the correlation coefficients $r_{o}$ between elevation and model parameters for each month of all four datasets, while 
Table 6.2 shows averages of the absolute values of the correlation coefficients. For RCM1, RCM2 and AWAP, the elevation vs $P_{00}$ correlations are negative and strong in winter, indicating drier condition at lower elevation in winter. The elevation vs $P_{00}$ correlations for RCM3 are positive in autumn and spring, and not as strong as for other three datasets. For both $P_{11}$ and $\mu$, the elevation vs parameter correlations tend to be positive (i.e. wetter condition at higher elevation) for almost all months of all datasets, except elevation vs $\mu$ correlation for February in AWAP. In general, the elevation vs $P_{11}$ and elevation vs $\mu$ correlations are stronger in summer for the RCMs, but stronger in winter for AWAP. The correlations of elevation vs $\sigma$ mostly follow the trend of elevation vs $\mu$ correlations (Figure 6.5). Overall, the correlations with model parameters are strongest in RCM2, followed by RCM1 and AWAP, while the correlations are weaker in RCM3 ( 
Table 6.2).
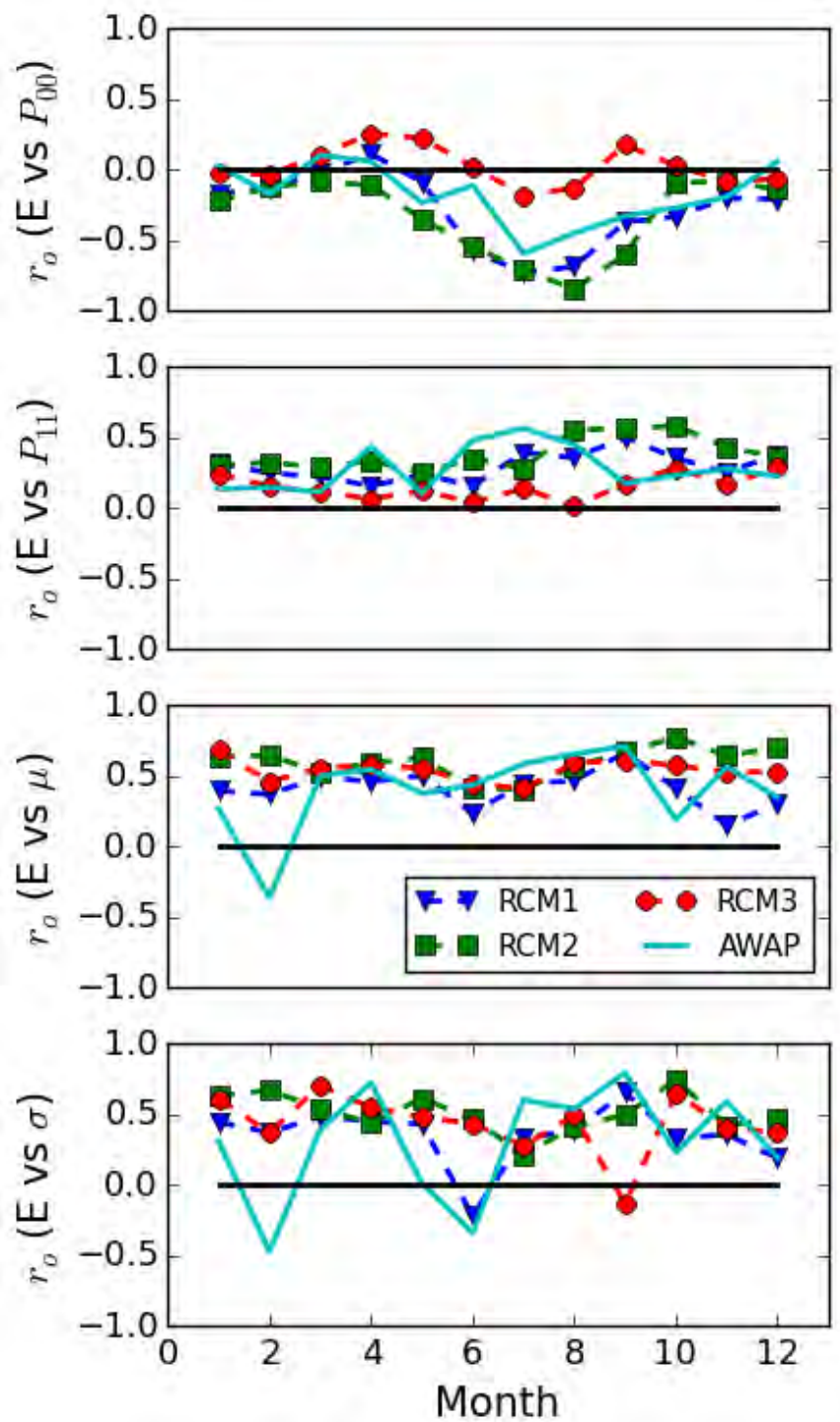

Figure 6.5: Correlation between elevation and model parameters for each month. 
Table 6.2: Average of the absolute values of correlation coefficients between elevation and parameters.

\begin{tabular}{lcccc}
\hline Correlation & RCM1 & RCM2 & RCM3 & AWAP \\
\hline E vs $P_{00}$ & 0.30 & 0.33 & 0.11 & 0.22 \\
E vs $P_{11}$ & 0.30 & 0.38 & 0.15 & 0.28 \\
E vs $\mu$ & 0.41 & 0.60 & 0.54 & 0.46 \\
E vs $\sigma$ & 0.39 & 0.51 & 0.46 & 0.43 \\
\hline
\end{tabular}

\subsection{Distribution Statistics of Rainfall Depths and Wet Periods}

This section will present the distribution statistics (i.e. mean and SD) of the rainfall depths and wet periods (i.e. number of wet days and mean length of wet spells) calculated from each RCM and AWAP for daily, monthly and multiyear resolutions. The subsequent sections will present the assessment of the performances of the CDMC, HMC and DHMC to reproduce these statistics.

\subsubsection{Mean and SD of Rainfall Depths at Daily, Monthly and Multiyear Resolutions}

The intra-annual and spatial variability of the mean and SD of wet day rainfall depths shown in Figure 6.3 and Figure 6.4 respectively indicate higher rainfall with higher variability in higher elevation and vice-versa. The spatial variability of the mean and SD of rainfall depths at monthly and multiyear resolutions also show a similar link between rainfall and local orography, and hence shown in Figure C.1-C.4 at Appendix C.1.

Figure 6.6 shows histograms of the mean and SD of rainfall depths for all 130 pixels and for all months at daily (those shown in Figure 6.3 and Figure 6.4) and monthly resolutions, and all years at multiyear resolution. It shows that the range of mean rainfall depths are similar in the RCMs, while AWAP is considerably drier than the RCMs. Moreover, the SD of rainfall depths are also lower in AWAP compare to the RCMs. 

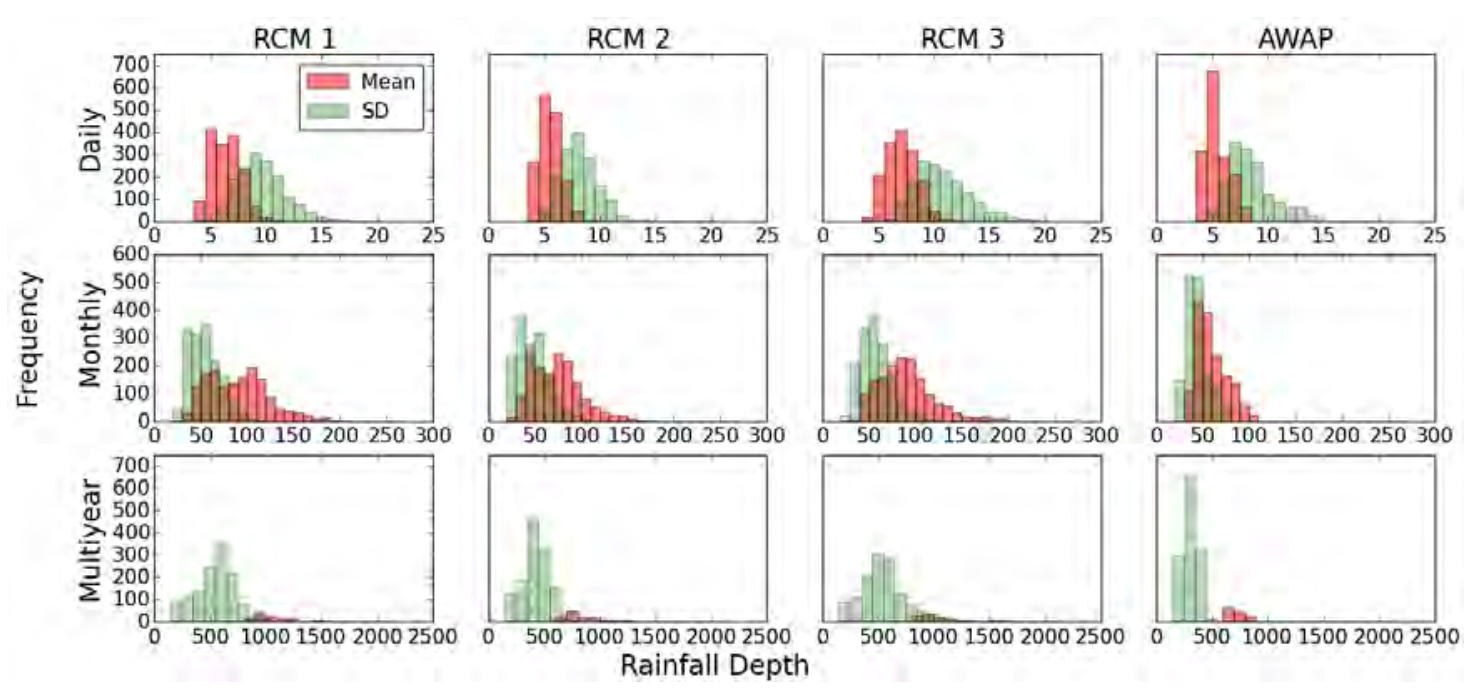

Figure 6.6: Mean and SD of rainfall depths at daily, monthly and multiyear resolutions.

\subsubsection{Mean and SD of Number of Wet Days at Monthly and Multiyear Resolutions}

Figure 6.7 and Figure 6.8 show the intra-annual and spatial variability of mean and SD (respectively) of monthly number of wet days for all RCMs and AWAP at Goulburn, which indicate wetter summers than winters and wetter conditions at higher elevation than lower elevation. However, the SD of monthly number of wet days are higher for autumn (e.g. for March) and spring (e.g. for October) for all datasets (Figure 6.8). The spatial variability of mean and SD of multiyear number of wet days shown in Figure C.5-C.6 at Appendix C.1 also indicates link between the number of wet days and local orography.

Figure 6.9 (typical of Figure 6.6) shows the overall distribution of the mean and SD of number of wet days at monthly and multiyear resolutions. It shows that the AWAP is considerably drier than the RCMs with lower mean of number of wet days (similar to rainfall depth shown in Figure 6.6). However, the SD of number of wet days are highest in the AWAP, followed by RCM2, RCM1 and RCM3 at both monthly and multiyear resolution. This suggests that the AWAP is drier than the RCMs with lower wet days (Figure 6.9) and rainfall depths (Figure 6.6), while the AWAP has higher variability of wet days but lower variability of rainfall depths. 


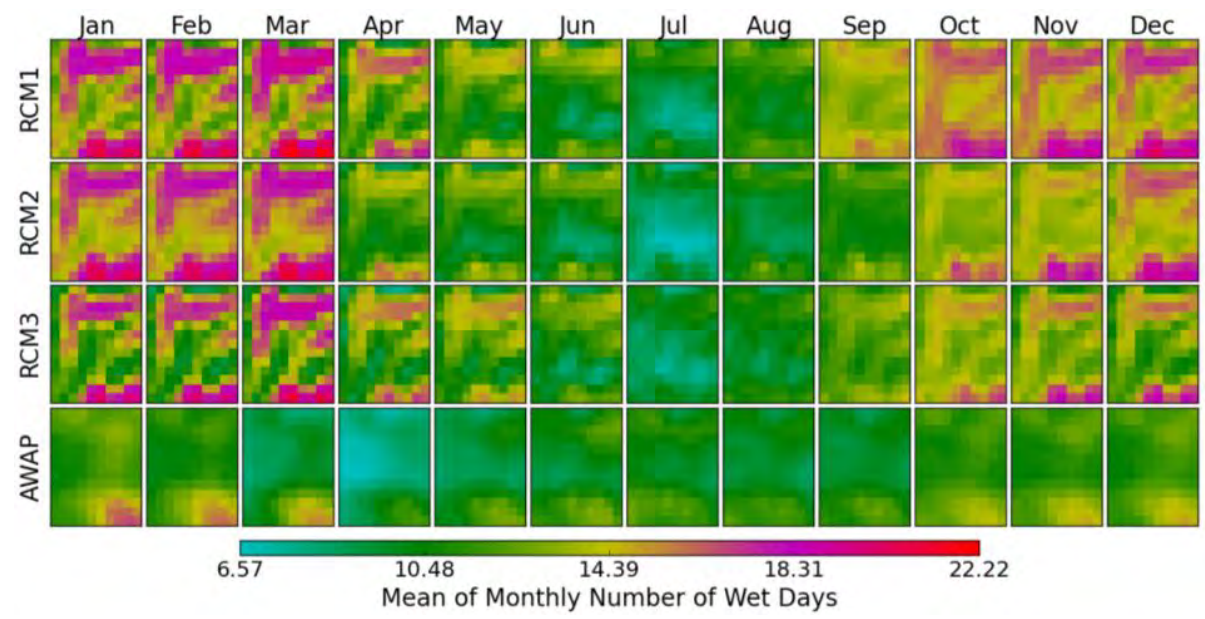

Figure 6.7: Intra-annual and spatial variability of the mean monthly number of wet days.

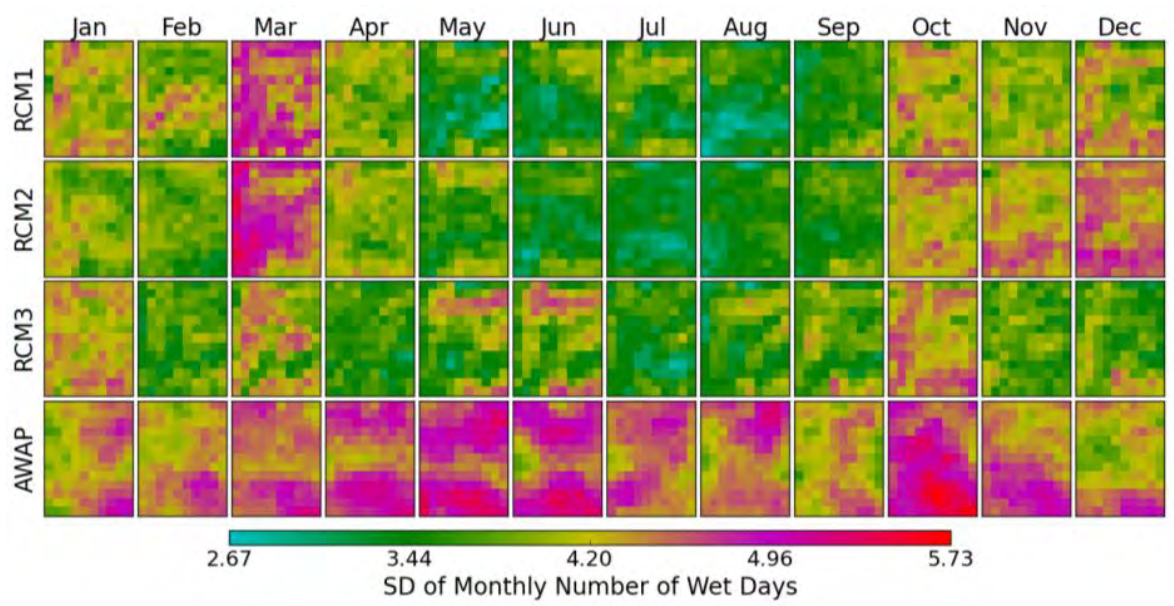

Figure 6.8: Intra-annual and spatial variability of the SD of monthly number of wet days.
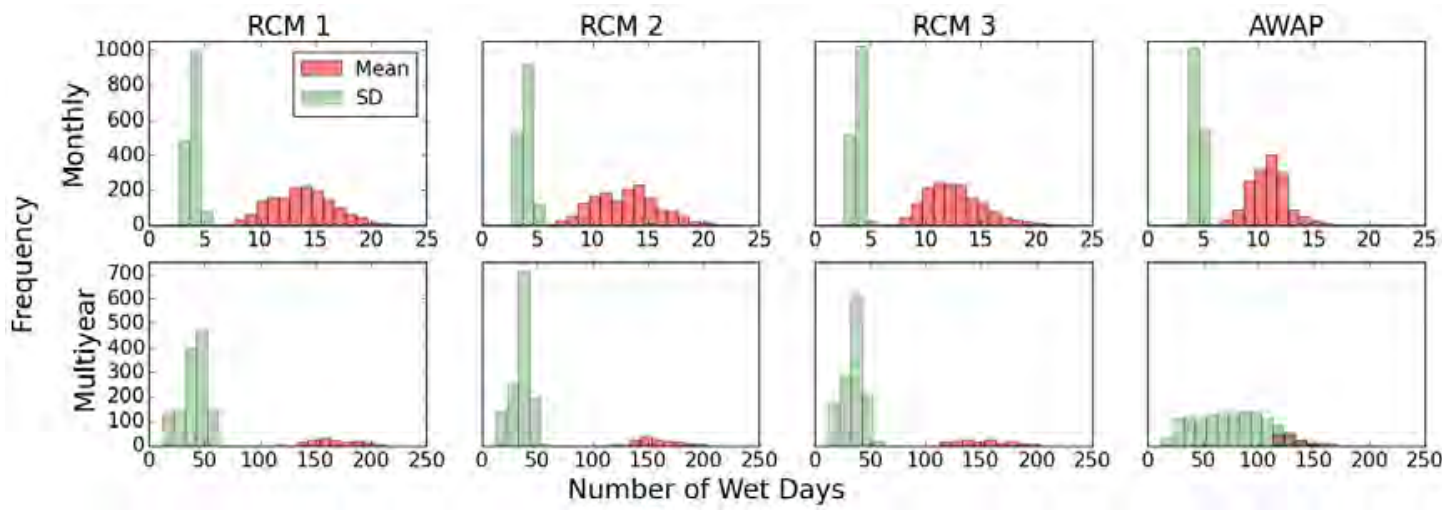

Figure 6.9: Mean and SD of number of wet days at monthly and multiyear resolutions. 


\subsubsection{Mean and SD of Mean Length of Wet Spells at Monthly and Annual Resolutions}

The intra-annual and spatial variability of the mean and SD of mean length of wet spells at monthly and annual resolutions are consistent with the respective characteristics of the number of wet days (see Figure C.7-C.10 at Appendix C.1). Figure 6.10 (typical of Figure 6.6) shows that the mean of monthly mean length of wet spells is similar in three RCMs, while the AWAP is less skewed with lower mean of monthly mean length of wet spell than the RCMs. Moreover, the AWAP shows more variability of wet spell lengths than the RCMs, which is consistent with the variability of wet days discussed in section 6.3.2.
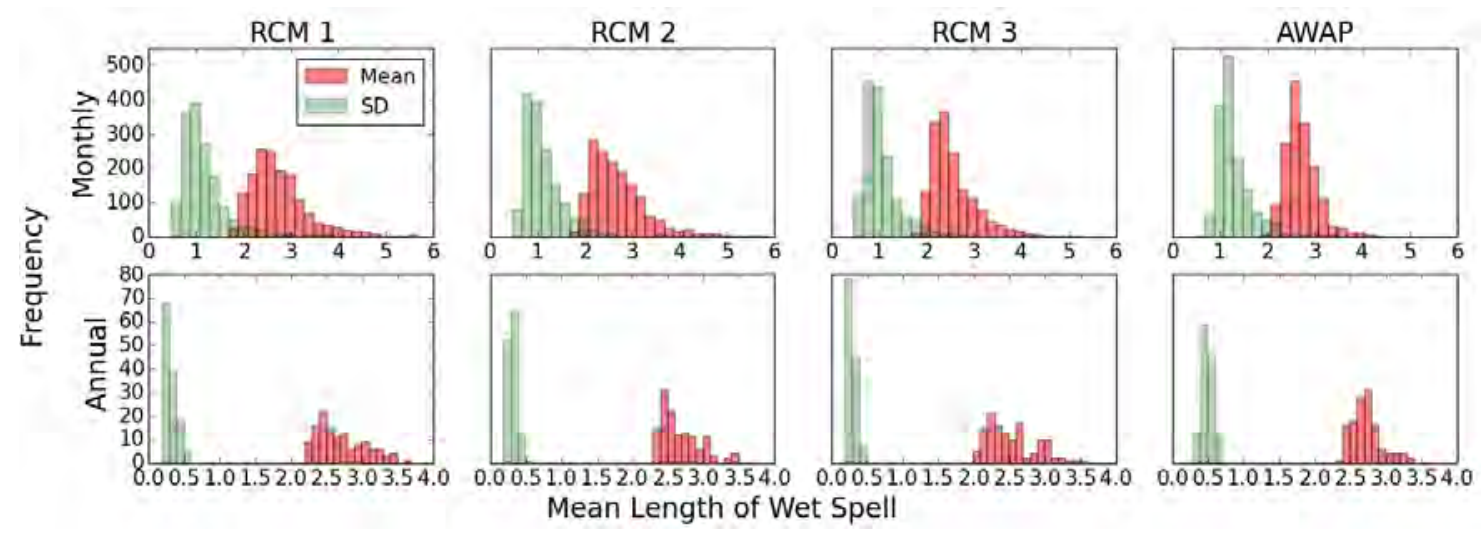

Figure 6.10: Mean and SD of mean length of wet spells at monthly and annual resolutions.

\subsubsection{Summary}

Table 6.3 shows the spatially and temporally averaged values of the mean and SD of rainfall depths and wet period statistics at annual and multiyear resolutions. It shows that the AWAP is drier than the RCMs (lower mean rainfall depths and number of wet days) with lower SD of rainfall depths but higher SD of wet days. Although the AWAP has lower mean annual number of wet days, the mean of annual mean length of wet spell in AWAP is similar to the RCMs. This indicates that the wet spells in AWAP might be relatively longer, while the RCMs might have more short wet spells. Among the three RCMs, the RCM2 is more similar to AWAP with an overall wetness in the order of RCM $3>$ RCM1 $>$ RCM2 $>$ AWAP. 
Table 6.3: Spatially and temporally averaged values of the mean and SD of rainfall depth and wet period statistics at annual and multiyear resolutions.

\begin{tabular}{cccccc}
\hline \multicolumn{2}{c}{ Statistics } & RCM1 & RCM2 & RCM3 & AWAP \\
\hline Rainfall & Annual Mean & 1087 & 884 & 1086 & 711 \\
\cline { 2 - 6 } Depth (mm) & Multiyear SD & 543 & 421 & 535 & 300 \\
\hline Number of & Annual Mean & 165 & 156 & 150 & 130 \\
\cline { 2 - 6 } Wet Days & Multiyear SD & 40 & 35 & 34 & 75 \\
\hline Mean Length & Annual Mean & 2.71 & 2.67 & 2.53 & 2.72 \\
\cline { 2 - 6 } of Wet Spell & Annual SD & 0.32 & 0.32 & 0.29 & 0.49 \\
\hline
\end{tabular}

\subsection{Model Comparison for Distribution of Rainfall Depths}

This section presents the comparative performances of CDMC, HMC and DHMC to reproduce the distribution of rainfall depths for each pixel of the Goulburn River site by using the assessment procedure described in section 3.4.

\subsubsection{Mean and SD of Daily Rainfall Depths}

Figure 6.11 and Figure 6.12 show comparisons of how well the models reproduce the mean and SD (respectively) of daily rainfall depths and show that models perform similarly and satisfactorily to reproduce the mean and SD of daily rainfall depths with $\mathrm{Z}$ scores mostly between -1 and +1 . Satisfactory reproduction of daily mean and SD of rainfall depths is anticipated, as these two statistics are also parameters of the MC models. However, the $\mathrm{Z}$ scores of the three MC models are mostly positive, indicating a tendency of the models to underestimate the mean and SD of daily rainfall depths. This consistent tendency of these models to underestimate the mean and SD of daily rainfall depths might be due to the use of stochastic parameters of Gamma distribution, as Chapter 5 shows that the APMC and DPMC with deterministic parameters of Gamma distribution do not consistently underestimate the mean and SD. These performances of the MC models for mean and SD of daily rainfall depths are consistent with their respective performances for a single pixel at Gummun. Performance of each model is mostly consistent for all months and in the entire site. This indicates that the CDMC, HMC and DHMC have performed satisfactorily, irrespective of the intra-annual and spatial variability of rainfall depths shown in section 6.3.1. Moreover, all three models have performed similarly for each of the four datasets. 


\section{Comparison of MC Models in Goulburn River Site}
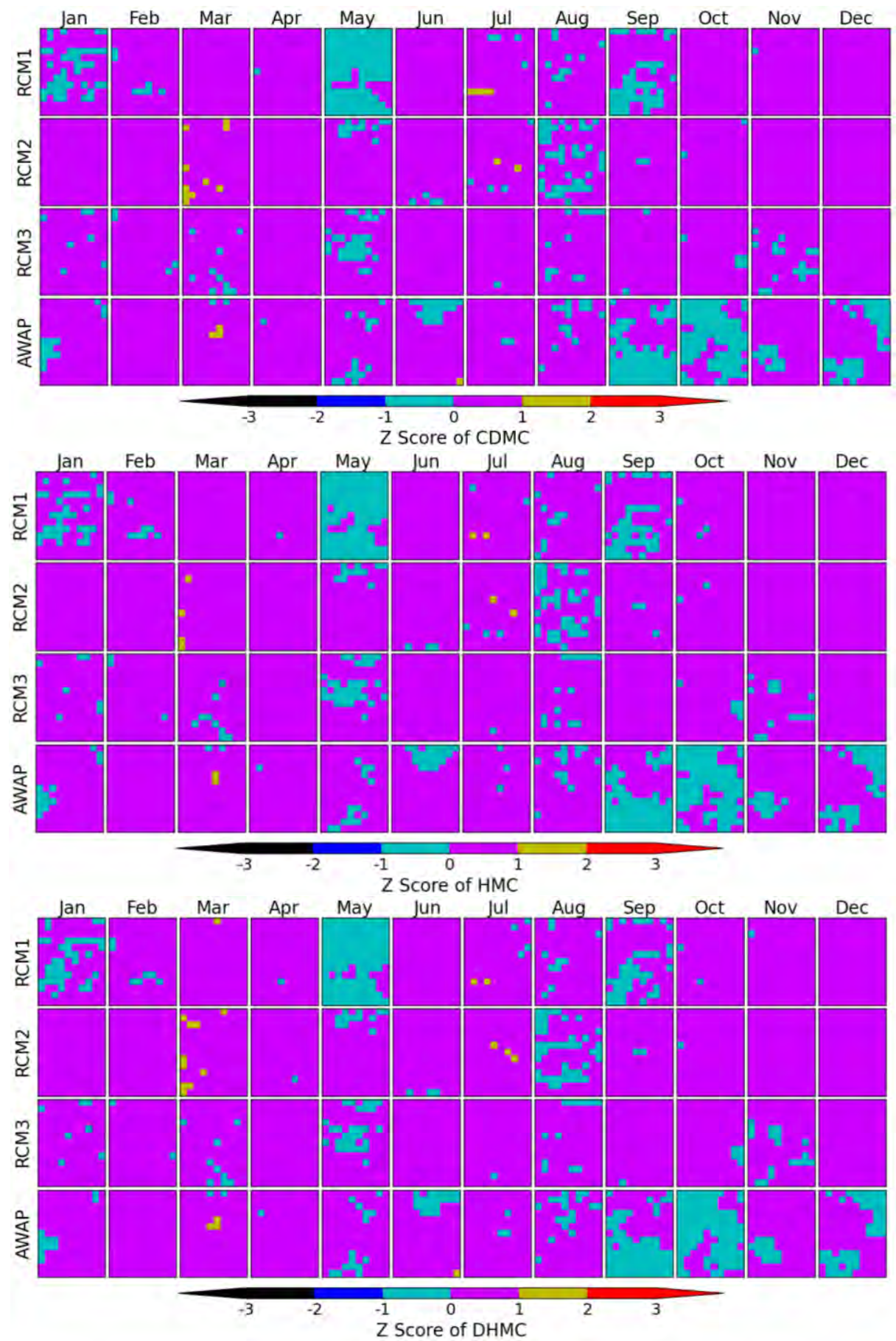

Figure 6.11: Z Scores of CDMC, HMC and DHMC for the mean of wet day rainfall depths. 
6. Comparison of MC Models in Goulburn River Site
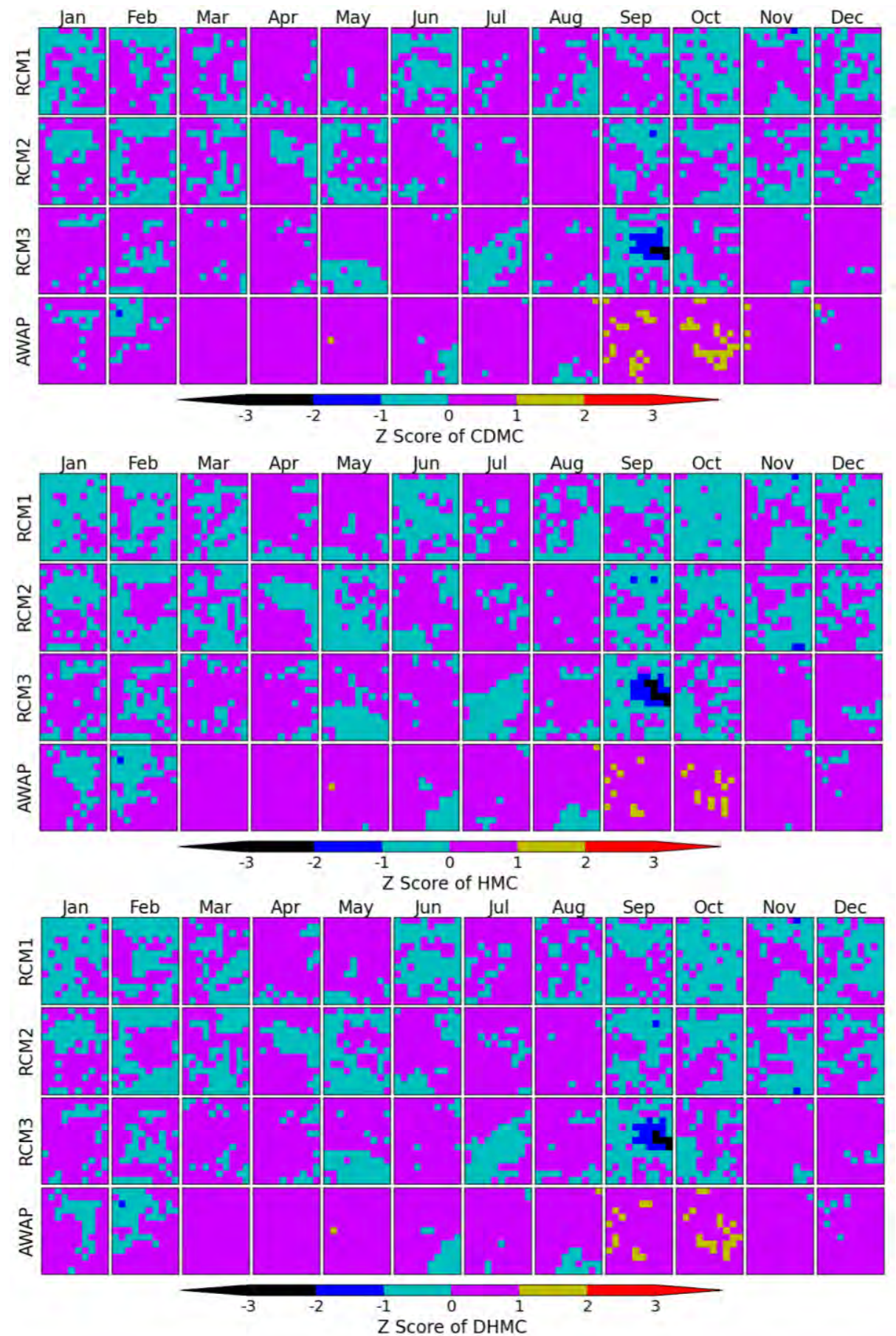

Figure 6.12: Z Scores of CDMC, HMC and DHMC for the SD of wet day rainfall depths. 


\subsubsection{Mean and SD of Monthly Rainfall Depths}

Performances of the three models to reproduce the mean of monthly rainfall depths are similar, being satisfactory and consistent with their performance for mean of daily rainfall depths (see Figure C.11 at Appendix C.2).

Figure 6.13 shows the comparison of the MC models to reproduce the SD of monthly rainfall depths. Each model shows satisfactory performance to reproduce the SD of monthly rainfall depths with a tendency to overestimate the SD. However, the HMC shows a more consistent tendency to overestimate the SD of monthly rainfall depths than the CDMC and DHMC. This excessive overestimation of the SD of monthly rainfall depths in the HMC might be due to its use of stochastic MC parameters. Moreover, the degree of overestimation of the SD by each model is higher for the AWAP compared to RCMs, which might be because the AWAP has lower SD of rainfall depths than the RCMs, as discussed in section 6.3.1.

Similar to daily statistics, these results for mean and SD of monthly rainfall depths are also consistent with the corresponding findings of previous chapter for a single pixel at Gummun. 
6. Comparison of MC Models in Goulburn River Site
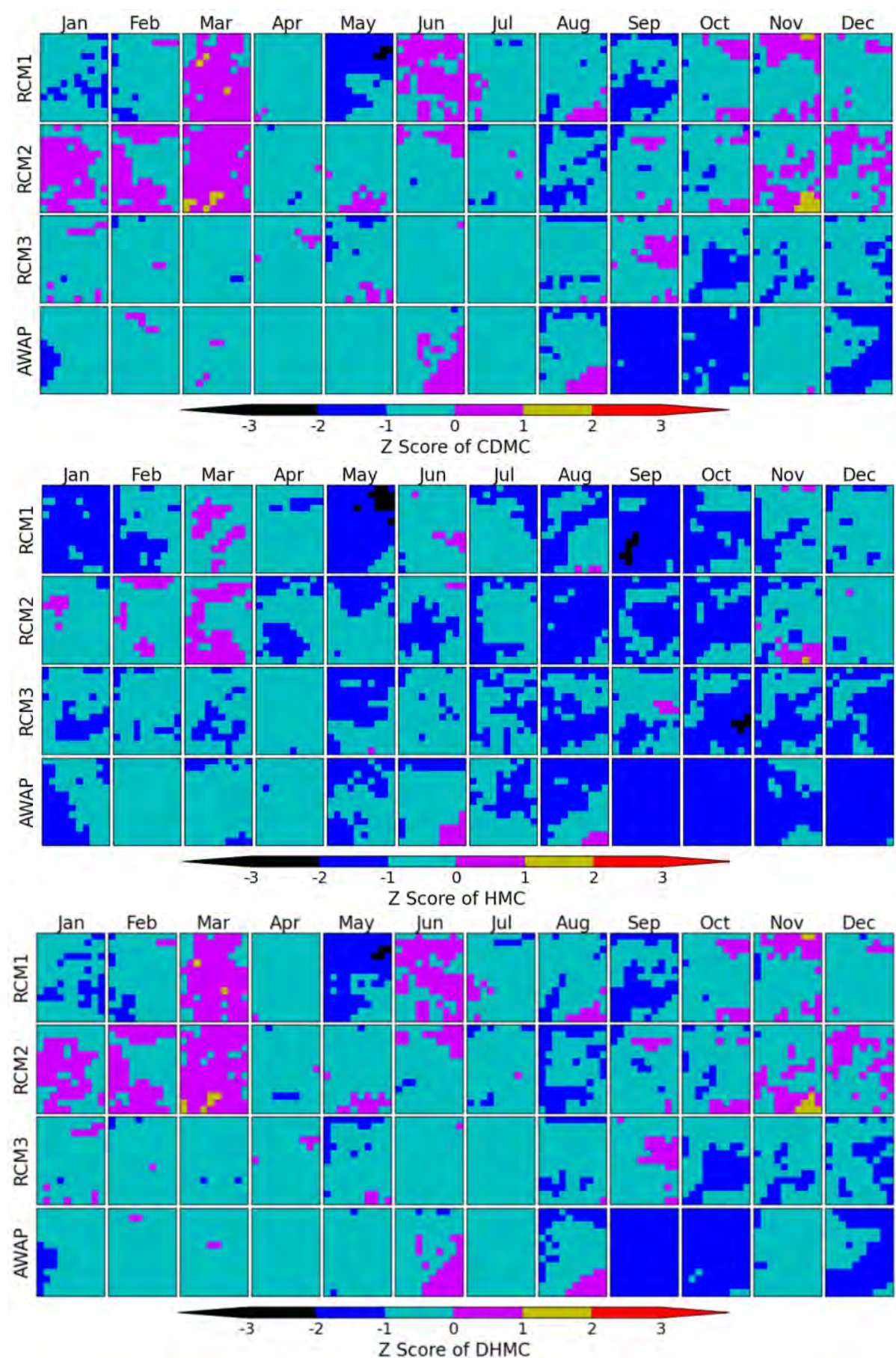

Figure 6.13: Z Scores of CDMC, HMC, and DHMC for the SD of monthly rainfall depths. 


\subsubsection{Mean and SD of Multiyear Rainfall Depths}

Similar to daily and monthly resolutions, the CDMC, HMC and DHMC show similar satisfactory performance to reproduce the mean of annual rainfall depths with a tendency to underestimate the mean (Figure 6.14). While this underestimation of mean might be caused by the use of stochastic Gamma parameters, the HMC performs slightly better than the CDMC and DHMC, with a lower degree of underestimation, which might be due to the HMC's use of stochastic MC parameters. The degree of underestimation of each model is higher for RCM2 (driest among three RCMs) compared to the RCM1, RCM3 and AWAP.

Figure 6.15 shows the model comparison to reproduce the SD of multiyear rainfall depths. Each model shows satisfactory performance to reproduce the SD of multiyear rainfall depths with a tendency of underestimation for the RCMs and overestimation for the AWAP, which might be linked with the higher SD in RCMs and lower SD in AWAP respectively (see Figure 6.6 in section 6.3.1). The degree of underestimation for RCMs is higher in the CDMC, while the degree of overestimation for AWAP is higher in the HMC and DHMC. This indicates that the use of stochastically varied and decadally varied MC parameters in HMC and DHMC respectively might introduce more variability (compared to the CDMC) of rainfall depths at multiyear resolutions, which is better for the simulation of rainfall with higher variability (e.g. RCMs) but excessive for rainfall with lower variability (e.g. AWAP). These performances of the MC models are consistent with their respective performance for Gummun.

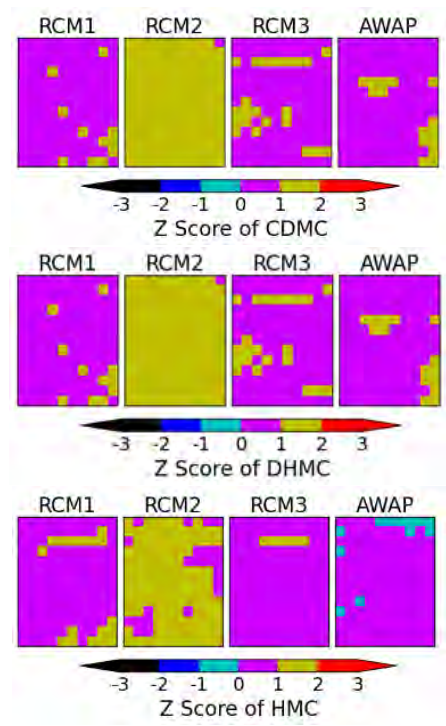

Figure 6.14: Z Scores of CDMC, HMC and DHMC for the mean annual rainfall depths. 

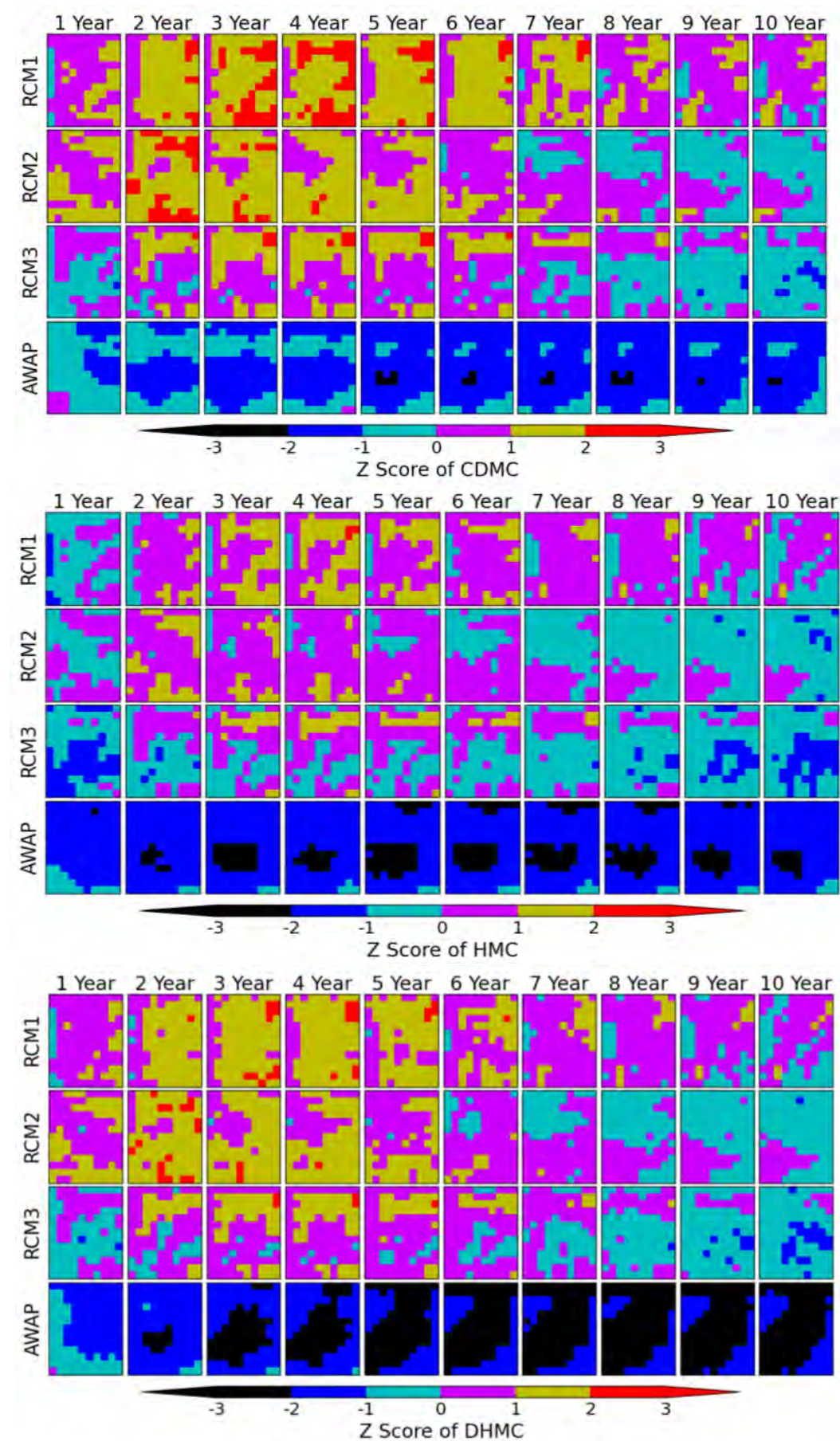

Figure 6.15: Z Scores of CDMC, HMC and DHMC for the SD of multiyear rainfall depths. 


\subsubsection{Overall Distribution of Z Scores}

The overall distribution of $\mathrm{Z}$ scores of CDMC, HMC and DHMC for mean and SD of rainfall depths at all resolutions are shown in Figure C.12 at Appendix C.2 (plotted as per section 3.7). A summary of the comparative performances of the MC models will be presented in section 6.6 .

\subsection{Model Comparison for Distribution of Wet Periods}

This section presents the comparison of how well the CDMC, HMC and DHMC reproduce the distribution of wet period statistics (number of wet days and mean length of wet spells) for each pixel of the Goulburn River site by using the assessment procedure described in section 3.4.

\subsubsection{Mean and SD of Monthly Number of Wet Days}

Figure 6.16 shows the comparison of how well the MC models reproduce the mean of monthly number of wet days. All three models satisfactorily reproduce the mean of monthly number of wet days with $\mathrm{Z}$ scores mostly between -1 and +1 . However, the HMC tends to overestimate the statistic for winter (drier months) as well as for AWAP (drier dataset).

Figure 6.17 shows the comparison of MC models to reproduce the SD of monthly number of wet days. For the RCMs, the CDMC preserves the statistic for most of the months, although it significantly underestimated the SD of the number of wet days for some months, such as March and October. However, the SD of monthly number of wet days in the RCMs are also higher for these months compared to other months (Figure 6.8). This indicates that the CDMC cannot reproduce the high variability of the monthly number of wet days. This is also supported by the similar poor performance of the CDMC for AWAP, while AWAP shows more variability of monthly number of wet days compared to the RCMs. The HMC has significantly overestimated the SD of the monthly number of wet days for all months and in most of the pixels of each dataset, which might be due to the use of stochastic MC parameters in the HMC. The DHMC with decadally varied MC parameters performs considerably better than the CDMC and HMC, and generally preserves the statistic for most of the months in each dataset. The performance of the three models in the entire site is mostly consistent with their respective performance for Gummun, discussed in the previous chapter. 
6. Comparison of MC Models in Goulburn River Site
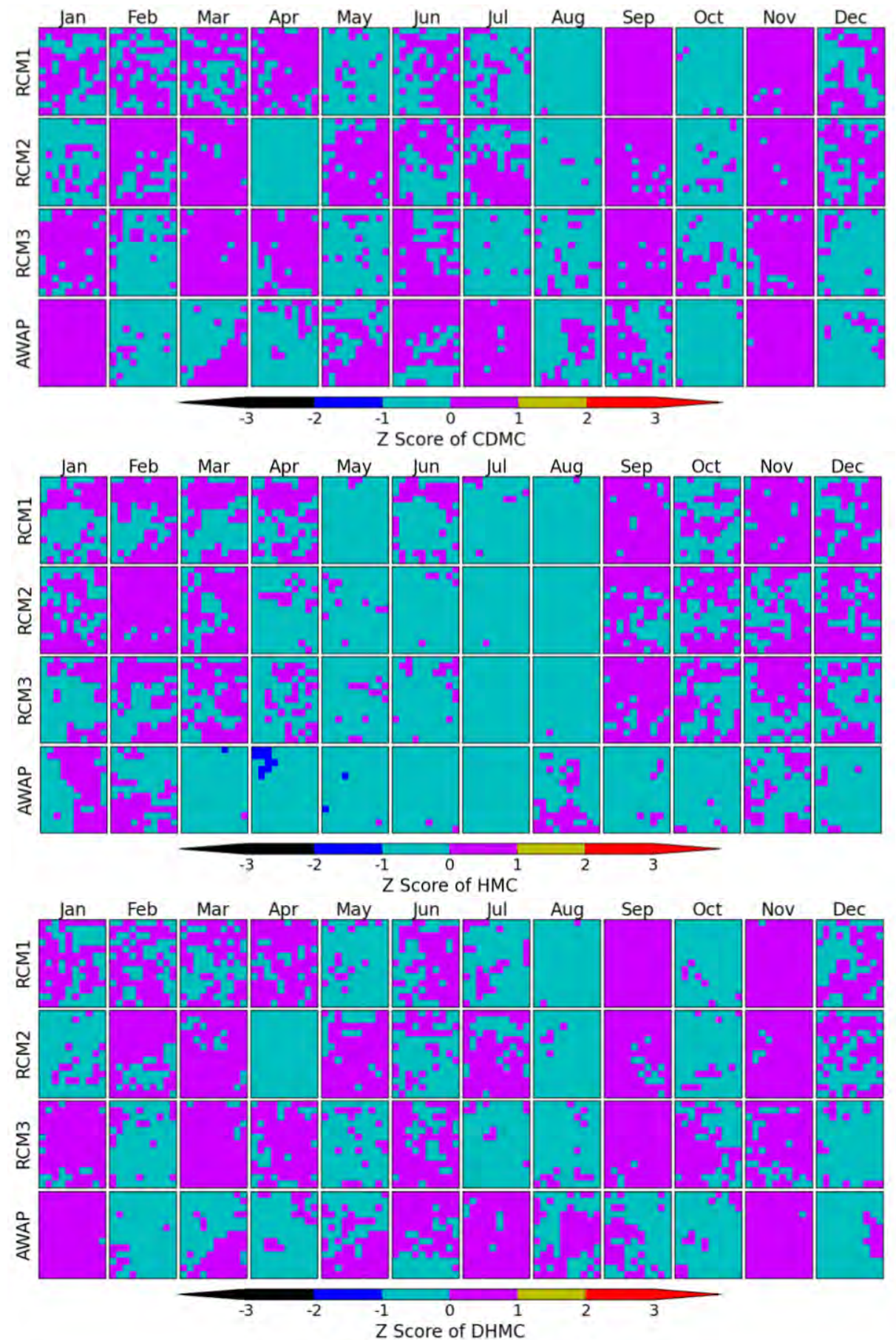

Figure 6.16: Z Scores of CDMC, HMC and DHMC for the mean of monthly number of wet days. 

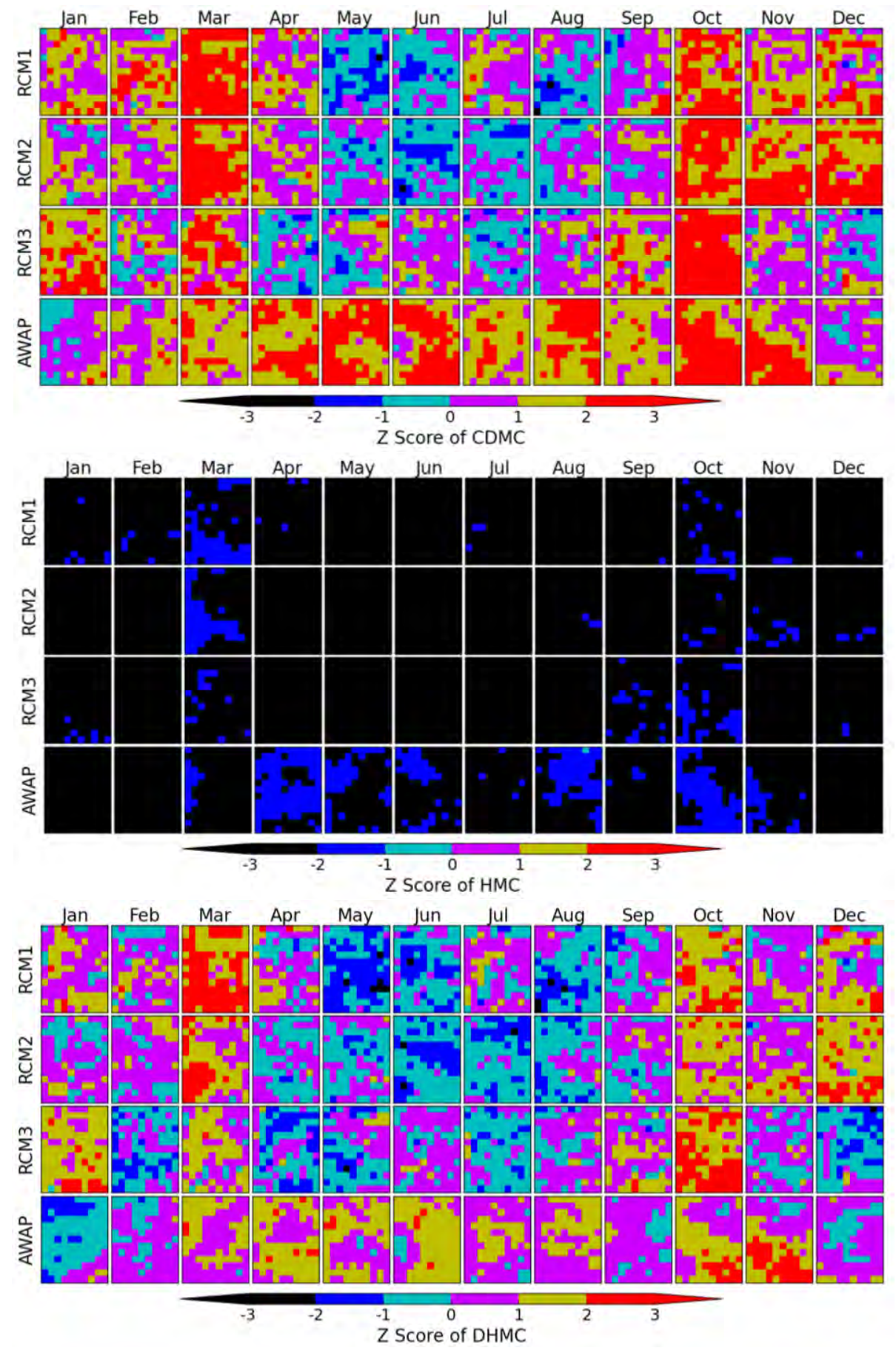

Figure 6.17: Z Scores of CDMC, HMC and DHMC for the SD of monthly number of wet days. 


\subsubsection{Mean and SD of Multiyear Number of Wet Days}

Similar to monthly resolutions, the CDMC and DHMC perform similarly and satisfactorily to reproduce the mean of multiyear number of wet days, while the HMC tends to overestimate the mean (see Figure 6.18, Z scores for only annual resolution are shown as $\mathrm{Z}$ scores for 2 to 10 multiyear resolutions are same as $\mathrm{Z}$ scores for annual resolution).

For SD of multiyear rainfall depths, the CDMC tends to significantly underestimate the statistic for most of the multiple years of each dataset (Figure 6.19). However, the HMC satisfactorily reproduces the SD of multiyear rainfall depths for most of the multiple years of each RCM, but significantly underestimate the SD for AWAP (the AWAP has higher variability of multiyear number of wet days compared to RCMs, as shown in Figure 6.9). The DHMC mostly preserves the SD of multiyear number of wet days for all datasets with a tendency to underestimate the statistic at shorter multiyear resolution (Figure 6.19). Overall, the HMC and DHMC perform satisfactorily and better than the CDMC to reproduce the SD of multiyear number of wet days.
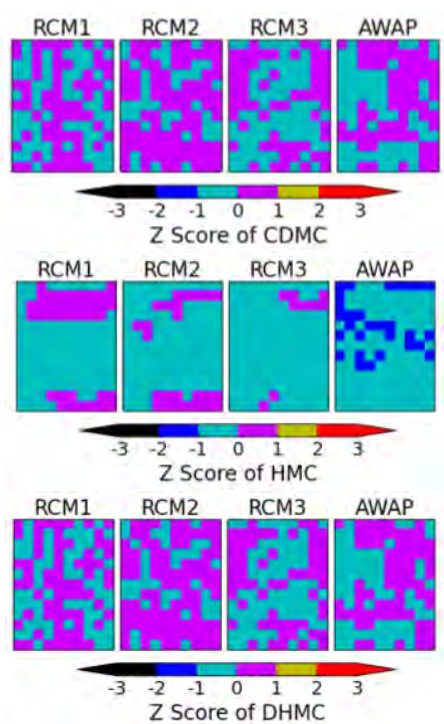

Figure 6.18: Z Scores of CDMC, HMC and DHMC for the mean of annual number of wet days. 

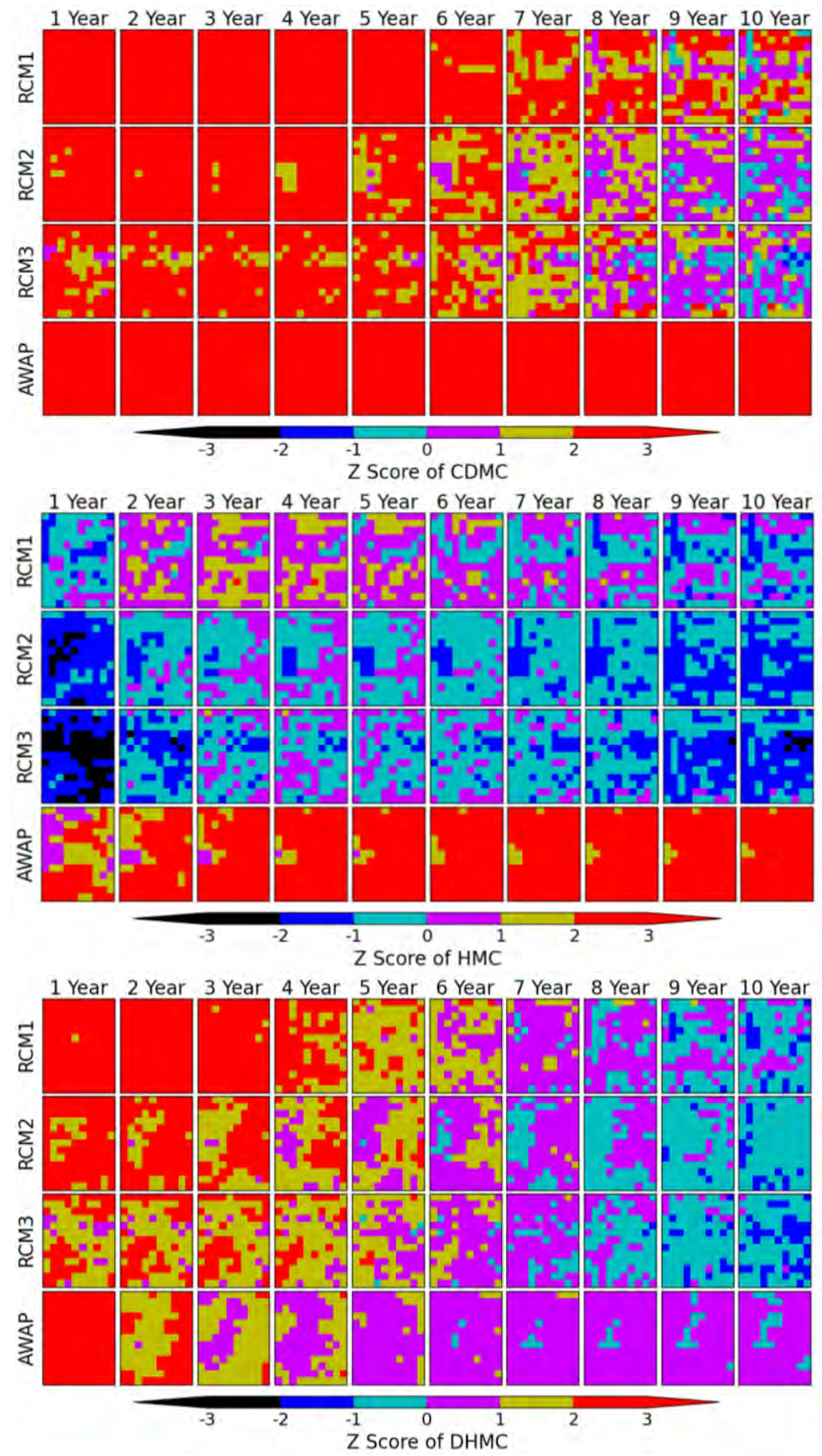

Figure 6.19: Z Scores of CDMC, HMC and DHMC for the SD of multiyear number of wet days. 


\subsubsection{Mean and SD of Monthly Mean Length of Wet Spells}

Figure 6.20 shows the comparison of $\mathrm{MC}$ models to reproduce the mean of monthly mean length of wet spells, while Figure 6.21 shows the same comparison to reproduce the SD of monthly mean length of wet spells.

The DHMC performs better (compared to CDMC and HMC) and satisfactorily to reproduce the mean and SD of monthly mean length of wet spells for each dataset. The CDMC performs mostly similar to the DHMC to reproduce the mean (Figure 6.20), but tends to underestimate the SD (Figure 6.21). However, the HMC shows a consistent tendency to overestimate both mean and SD. This suggests that the use of decadally varied MC parameters is better to reproduce the variability of monthly wet spell lengths, while the use of deterministic and stochastic MC parameters cause under- and over-estimation of the variability respectively. The performance of each model is slightly better for RCMs, as each model shows a more consistent tendency to overestimate the mean of monthly wet spell lengths for AWAP, which is drier than the RCMs. 


\section{Comparison of MC Models in Goulburn River Site}
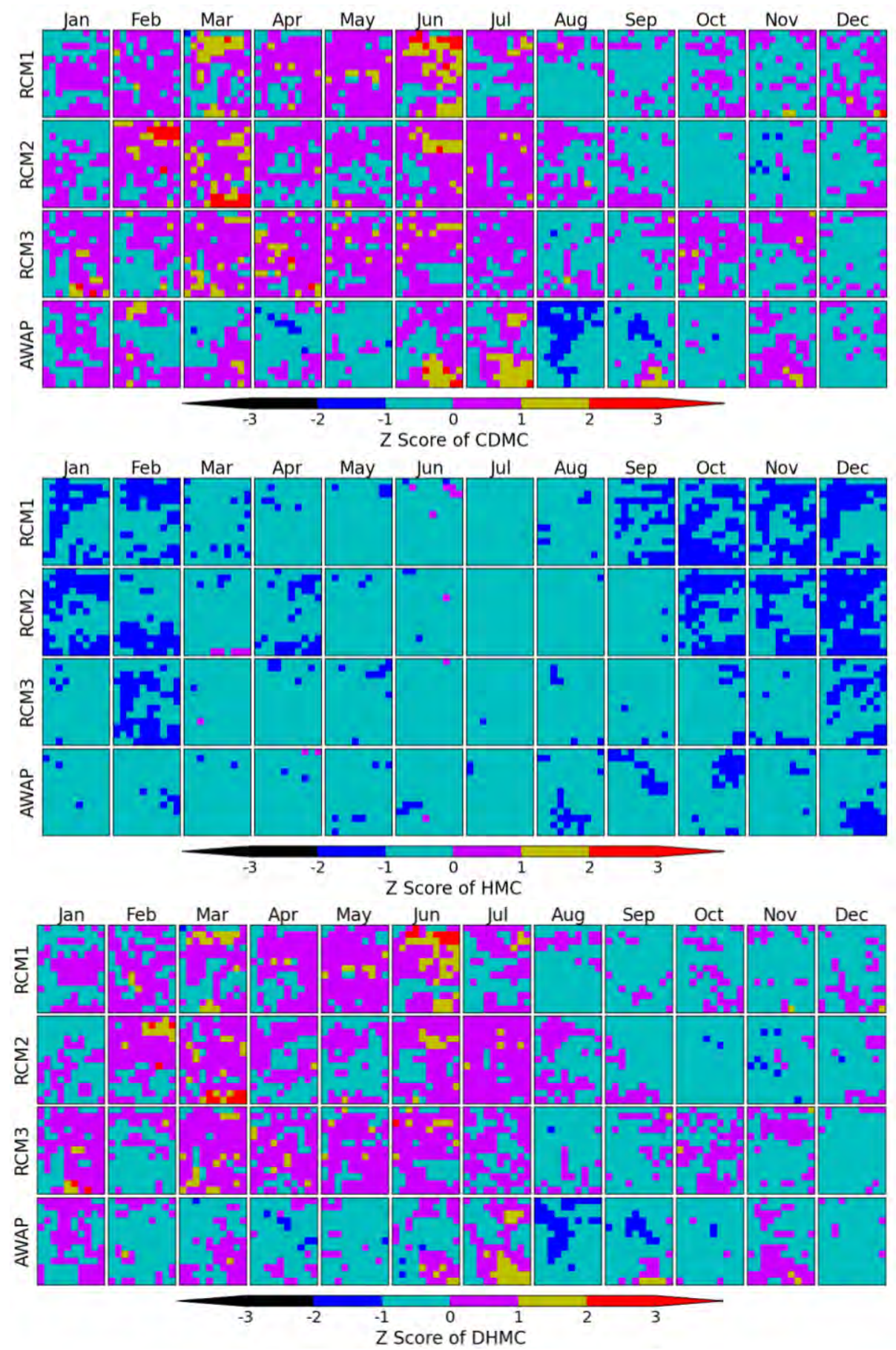

Figure 6.20: Z Scores of CDMC, HMC and DHMC for the mean of monthly mean length of wet spells. 

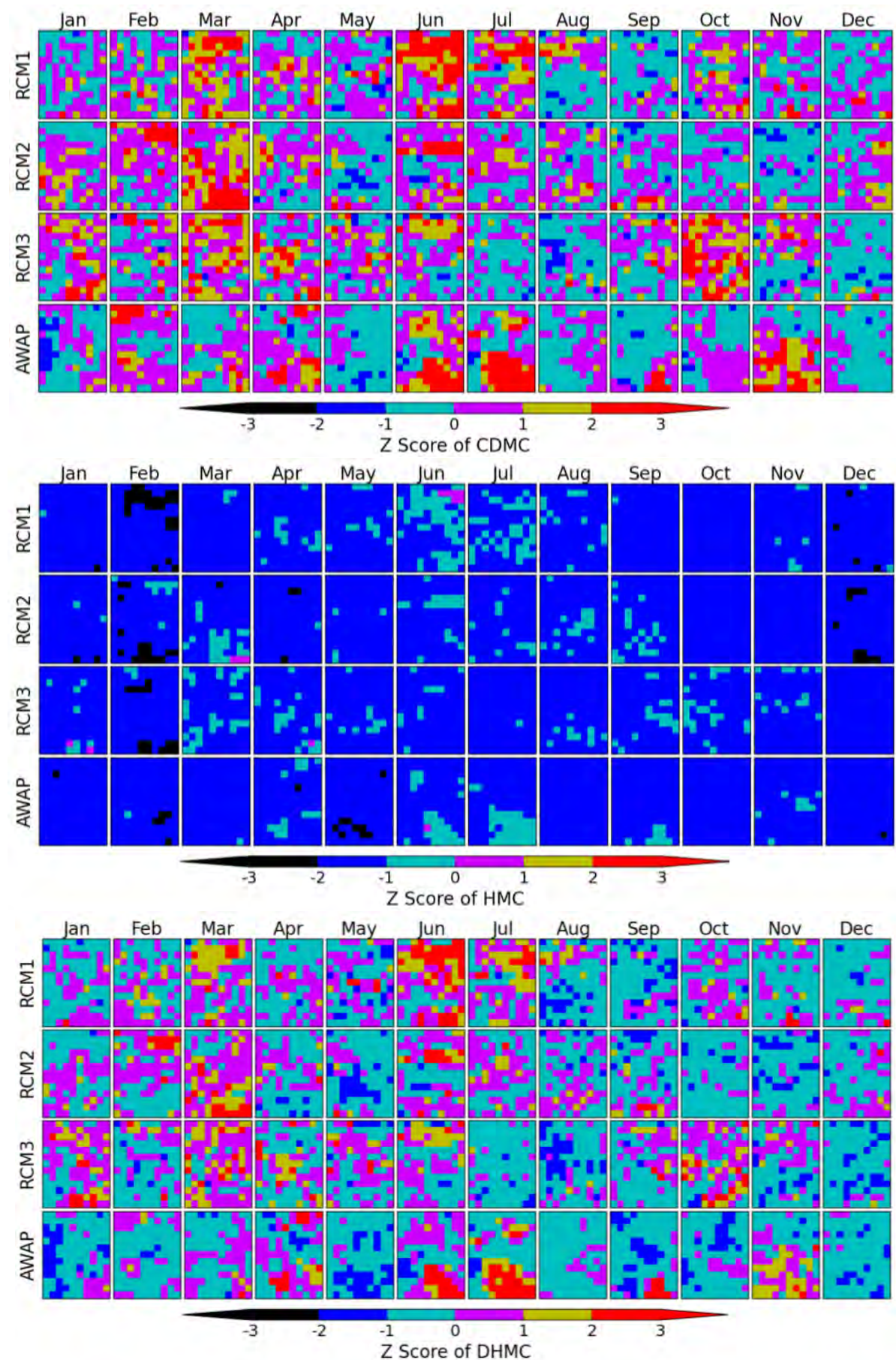

Figure 6.21: Z Scores of CDMC, HMC and DHMC for the SD of monthly mean length of wet spells. 


\subsubsection{Mean and SD of Annual Mean Length of Wet Spells}

Figure 6.22 shows the comparison of MC models to reproduce the mean of annual mean length of wet spells, while Figure 6.23 shows the same comparison for SD. The mean of annual mean length of wet spells has been preserved very well in CDMC, HMC and DHMC for all four datasets, although the HMC tends to underestimate and the DHMC tends to overestimate the mean for AWAP (drier dataset).

For SD of annual mean length of wet spells, the CDMC tends to underestimate, and the HMC tends to overestimate the SD for both RCMs and AWAP. However, the CDMC's performance is better for the RCMs compared to AWAP, while AWAP has a higher SD of wet spell lengths compared to the RCMs (see section 6.3.3). In contrast, the HMC performs better for AWAP compared to the RCMs. This suggests that the deterministic MC parameters in the CDMC might be adequate to reproduce the SD of annual mean length of wet spells for datasets with lower variability of wet spells (e.g. RCMs), while stochastic MC parameters in the HMC might be adequate to reproduce the SD for datasets with higher variability of wet spells (e.g. AWAP). However, the DHMC with decadally varied MC parameters performs considerably better than the CDMC and HMC, and mostly preserves the SD of annual mean length of wet spells for all datasets.

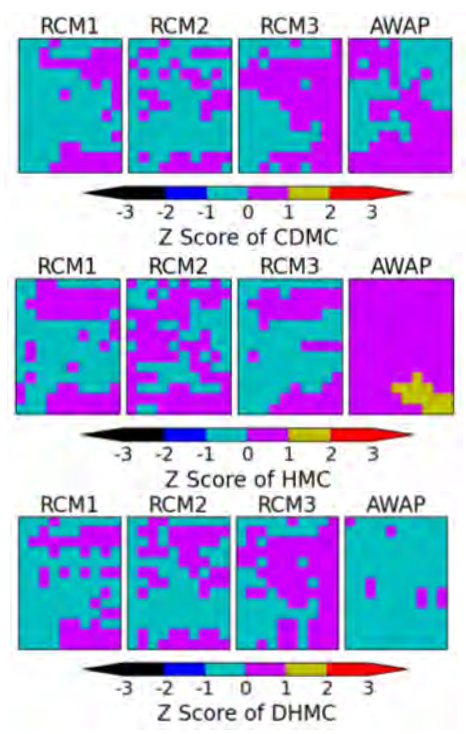

Figure 6.22: Z Scores of CDMC, HMC and DHMC for the mean of annual mean length of wet spells. 

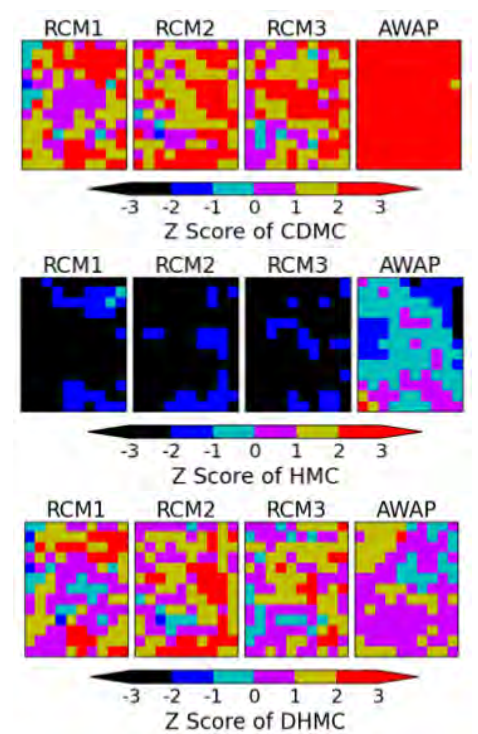

Figure 6.23: Z Scores of CDMC, HMC and DHMC for the SD of annual mean length of wet spells.

\subsubsection{Overall Distribution of Z Scores}

The overall distribution of $Z$ scores of the CDMC, HMC and DHMC for mean and SD of wet period statistics at monthly to multiyear resolutions are shown in Figure C.13 at Appendix C.3 (plotted as per the section 3.7). While the key points are already covered in the previous sections, it shows the ranges of $\mathrm{Z}$ scores of each model which indicate the overall competency of the respective model for the simulation of wet period statistics. For example, the SD of the multiyear number of wet days has been significantly underestimated in the CDMC for all datasets. While the $\mathrm{Z}$ scores for the RCMs range from 0 to 6 , the $\mathrm{Z}$ scores for AWAP range from 6 to 12. These $\mathrm{Z}$ scores that are too high indicate how poorly the CDMC performed in reproducing the variability of multiyear number of wet days, particularly for the higher variability of AWAP, as shown in Figure 6.9. It also denotes the improvement in the DHMC as the $\mathrm{Z}$ scores for the SD of multiyear number of wet days are mostly within the -2 and +2 limit and not greater than +4 . 


\subsection{Summary of Model Comparison for Distribution Statistics}

Table 6.4 and Table 6.5 show the spatially and temporally averaged $\mathrm{Z}$ scores (calculated as per section 3.8.2) for distribution statistics of rainfall depths and wet periods respectively. While the detailed results of each model have been discussed in sections 6.4 and 6.5, Table 6.4 and Table 6.5 show an overall summary of the model comparison, as discussed below.

For mean and SD of rainfall depths, performances of the three models are mostly similar as they use the same stochastic parameters of Gamma distribution for simulation of wet day rainfall depths. However, the HMC performs slightly better than the CDMC and DHMC, which might be because it uses stochastic MC parameters (see Table 6.4 and section 6.4).

For mean and SD of wet period statistics (i.e. number of wet days and mean length of wet spells), the DHMC clearly performs better than the CDMC and HMC. Particularly, the DHMC satisfactorily reproduces the $\mathrm{SD}$ of wet period statistics, while the CDMC tends to underestimate and the $\mathrm{HMC}$ tends to overestimate the SD. This indicates the advantage of using decadally varied MC parameters (in DHMC) over the deterministic and stochastic MC parameters (in CDMC and HMC respectively) to reproduce the wet period variability (see Table 6.5 and section 6.5).

For both rainfall depths and wet periods, performance of each model is inferior for AWAP compared to their respective performance for RCMs (see Table 6.4 and Table 6.5). This might be because the rainfall characteristics of AWAP are different to the RCMs. AWAP is drier than the RCMs with lower mean of rainfall depths and wet days, while AWAP has lower variability of rainfall depth but higher variability of wet periods compared to the RCMs (see section 6.3). 
6. Comparison of MC Models in Goulburn River Site

Table 6.4: Spatial (all pixels) and temporal (all months and years) average of the absolute values of $\mathrm{Z}$ scores for mean and SD of rainfall depths.

\begin{tabular}{|c|c|c|c|c|c|}
\hline \multirow{2}{*}{ Statistics } & \multirow{2}{*}{ Models } & \multicolumn{4}{|c|}{ Spatially and Temporally Averaged Z Scores } \\
\hline & & RCM1 & $\mathrm{RCM} 2$ & RCM3 & AWAP \\
\hline \multirow{3}{*}{ Daily Mean } & CDMC & 0.31 & 0.42 & 0.29 & 0.30 \\
\hline & $\mathrm{HMC}$ & 0.30 & 0.41 & 0.28 & 0.28 \\
\hline & DHMC & 0.31 & 0.42 & 0.29 & 0.29 \\
\hline \multirow{3}{*}{ Daily SD } & CDMC & 0.21 & 0.24 & 0.29 & 0.49 \\
\hline & $\mathrm{HMC}$ & 0.20 & 0.22 & 0.24 & 0.41 \\
\hline & DHMC & 0.20 & 0.22 & 0.26 & 0.44 \\
\hline \multirow{3}{*}{ Monthly Mean } & CDMC & 0.29 & 0.39 & 0.27 & 0.28 \\
\hline & $\mathrm{HMC}$ & 0.27 & 0.34 & 0.22 & 0.21 \\
\hline & DHMC & 0.29 & 0.39 & 0.27 & 0.28 \\
\hline \multirow{3}{*}{ Monthly SD } & CDMC & 0.57 & 0.49 & 0.59 & 0.7 \\
\hline & HMC & 0.91 & 0.77 & 0.94 & 1.00 \\
\hline & DHMC & 0.59 & 0.51 & 0.62 & 0.73 \\
\hline \multirow{3}{*}{ Multiyear Mean } & CDMC & 0.82 & 1.36 & 0.85 & 0.76 \\
\hline & $\mathrm{HMC}$ & 0.70 & 1.15 & 0.65 & 0.31 \\
\hline & DHMC & 0.82 & 1.35 & 0.86 & 0.76 \\
\hline \multirow{3}{*}{ Multiyear SD } & CDMC & 1.15 & 0.88 & 0.63 & 1.22 \\
\hline & HMC & 0.61 & 0.51 & 0.57 & 1.66 \\
\hline & DHMC & 0.87 & 0.78 & 0.57 & 1.97 \\
\hline \multirow{3}{*}{ Sum } & CDMC & 3.35 & 3.78 & 2.92 & 3.75 \\
\hline & HМC & 2.99 & 3.40 & 2.90 & 3.87 \\
\hline & DHMC & 3.08 & 3.67 & 2.87 & 4.47 \\
\hline
\end{tabular}


Table 6.5: Spatial (all pixels) and temporal (all lags 1-12) average of the absolute values of $Z$ scores for mean and SD of wet period statistics.

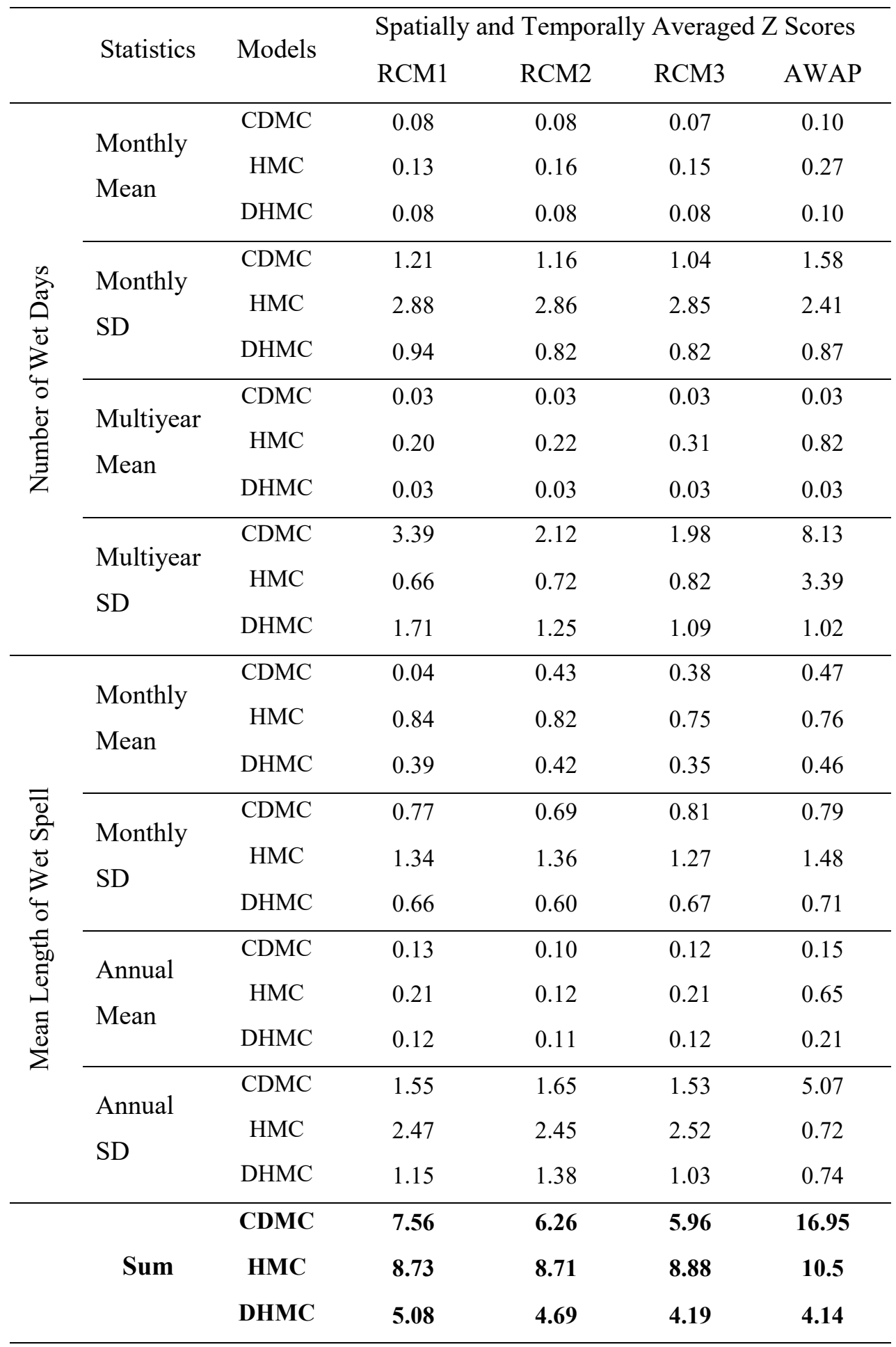




\subsection{Model Comparison for Autocorrelation}

Chapter 5 showed that the autocorrelations of monthly rainfall depths and number of wet days follow clear seasonal signals between lag-1 and lag-12. Therefore, this study has examined lag-1 to lag-12 autocorrelations for each pixel of the Goulburn River site. Figure 6.24 shows the autocorrelations of monthly rainfall depths and number of wet days in RCMs and the AWAP. Figure 6.25 and Figure 6.26 show the ability of the CDMC, HMC and DHMC to reproduce the autocorrelations of monthly rainfall depths and number of wet days respectively. Table 6.6 shows the spatially and temporally averaged autocorrelation coefficients.

Figure 6.24 shows that the month-to-month autocorrelations of monthly wet days are stronger than the monthly rainfall depths in all datasets. The autocorrelations (for both wet days and rainfall depths) are stronger with clear seasonal signals in RCM2 and RCM1 followed by RCM3, while AWAP has relatively weak autocorrelations. Table 6.6 shows that the CDMC and DHMC satisfactorily reproduce the autocorrelations of both monthly rainfall depths and number of wet days, while the HMC reproduces the autocorrelations of monthly rainfall depths mostly well but underestimates the autocorrelations of monthly wet days (consistent with results discussed in Chapter 5). The underestimation of the autocorrelations of monthly wet days in the HMC might be linked to its overestimation of the SD of monthly wet days. Figure 6.25 and Figure 6.26 show that all three MC models reproduce the seasonal and spatial pattern of the coefficient of correlations satisfactorily.

Table 6.6: Spatial (all pixels) and temporal (all lags 1-12) average of autocorrelations of monthly rainfall depths and number of wet days.

\begin{tabular}{|c|c|c|c|c|c|}
\hline & & \multicolumn{4}{|c|}{ Spatially Averaged Autocorrelation Coefficients } \\
\hline & & RCM1 & $\mathrm{RCM} 2$ & RCM3 & AWAP \\
\hline \multirow{4}{*}{$\begin{array}{l}\text { ㄱ. } \\
\text { 营 } \\
\sum\end{array}$} & In Data & 0.13 & 0.09 & 0.07 & 0.06 \\
\hline & $\mathrm{CDMC}$ & 0.10 & 0.07 & 0.06 & 0.04 \\
\hline & $\mathrm{HMC}$ & 0.08 & 0.06 & 0.05 & 0.04 \\
\hline & DHMC & 0.10 & 0.07 & 0.06 & 0.04 \\
\hline \multirow{4}{*}{ 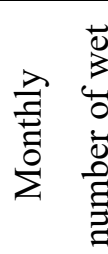 } & In Data & 0.14 & 0.17 & 0.08 & 0.09 \\
\hline & $\underset{>}{\infty} \mathrm{CDMC}$ & 0.15 & 0.17 & 0.09 & 0.04 \\
\hline & क $\mathrm{HMC}$ & 0.08 & 0.09 & 0.05 & 0.03 \\
\hline & DHMC & 0.14 & 0.17 & 0.09 & 0.07 \\
\hline
\end{tabular}




\section{Comparison of MC Models in Goulburn River Site}
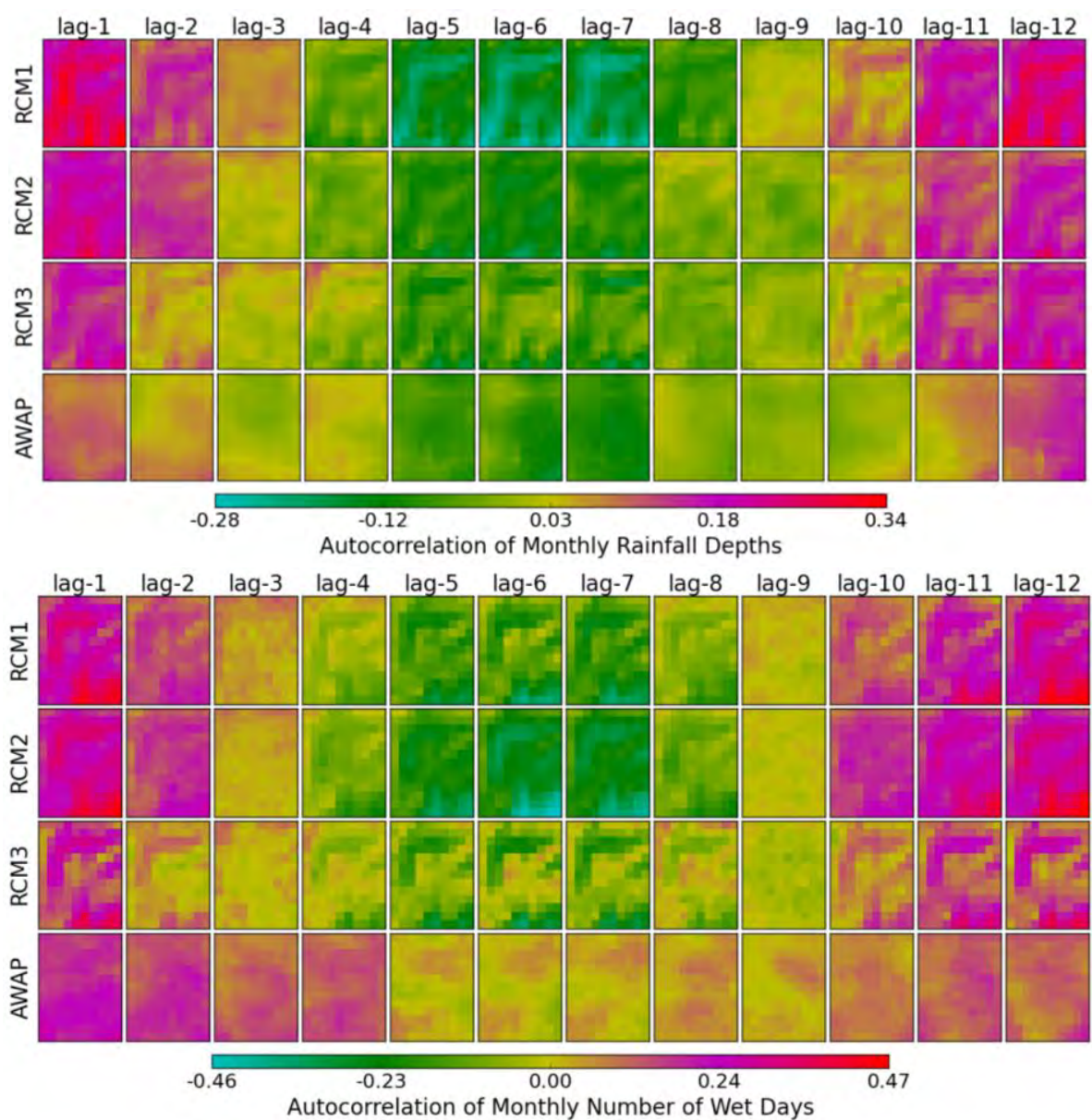

Figure 6.24: Month-to-month autocorrelations of monthly rainfall depths and number of wet days in all RCMs and AWAP at Goulburn. 
6. Comparison of MC Models in Goulburn River Site

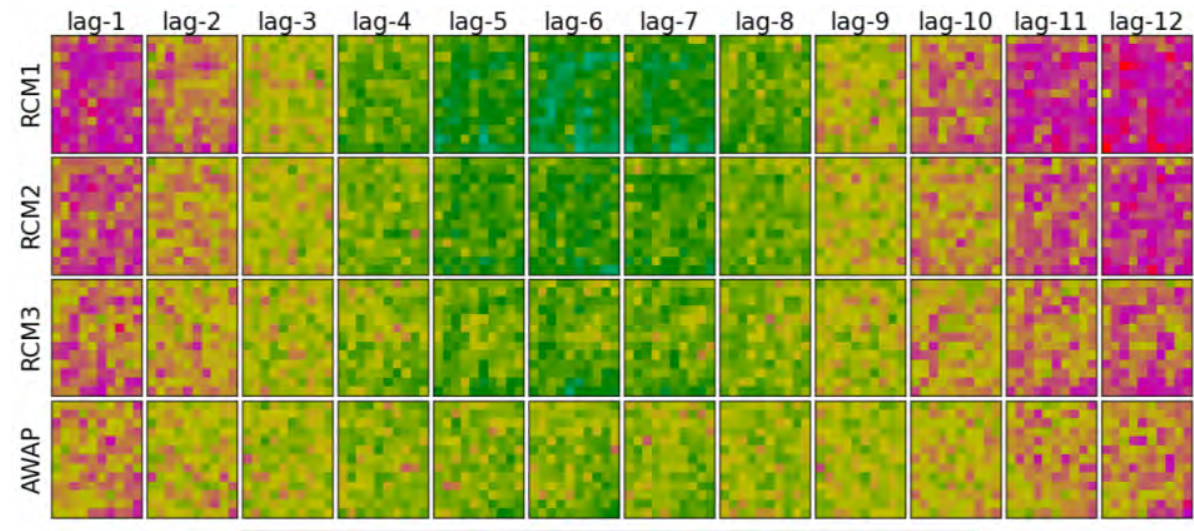

0.31

$-0.15$

0.01

0.17

0.33

CDMC Performance for Autocorrelation of Monthly Rainfall Depths

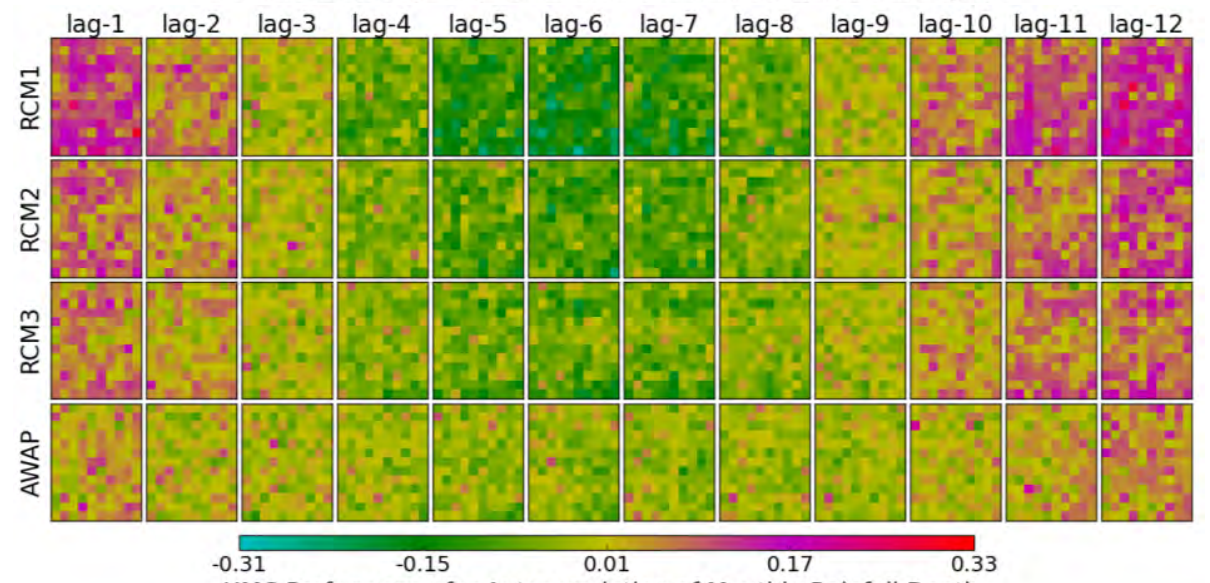

HMC Performance for Autocorrelation of Monthly Rainfall Depths

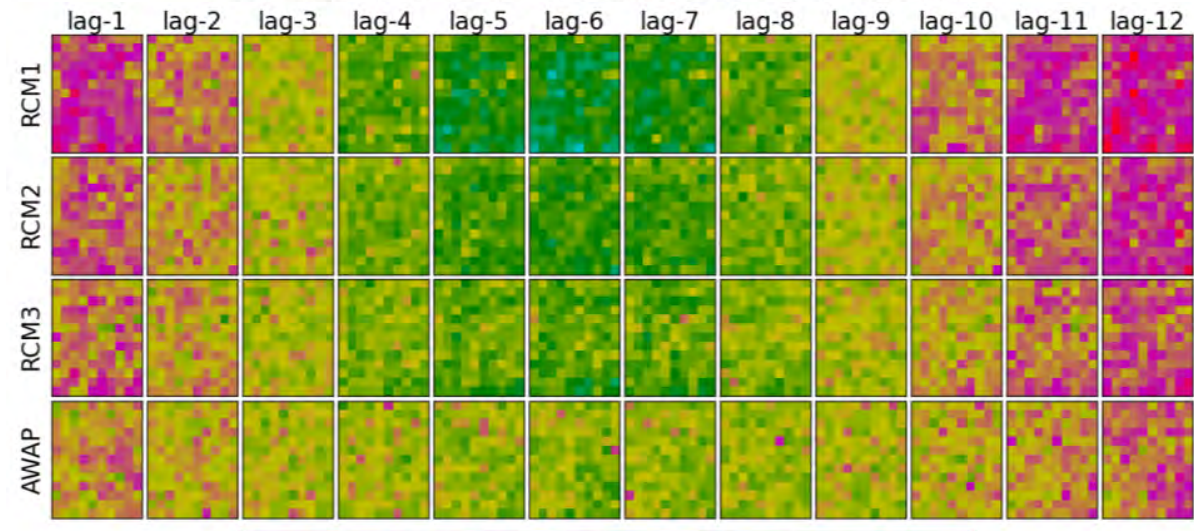

$-0.31$

$-0.15$

0.01

0.17

0.33

DHMC Performance for Autocorrelation of Monthly Rainfall Depths

Figure 6.25: Comparison of CDMC, HMC and DHMC to reproduce the month-to-month autocorrelations of monthly rainfall depths for all RCMs and AWAP. 


\section{Comparison of MC Models in Goulburn River Site}

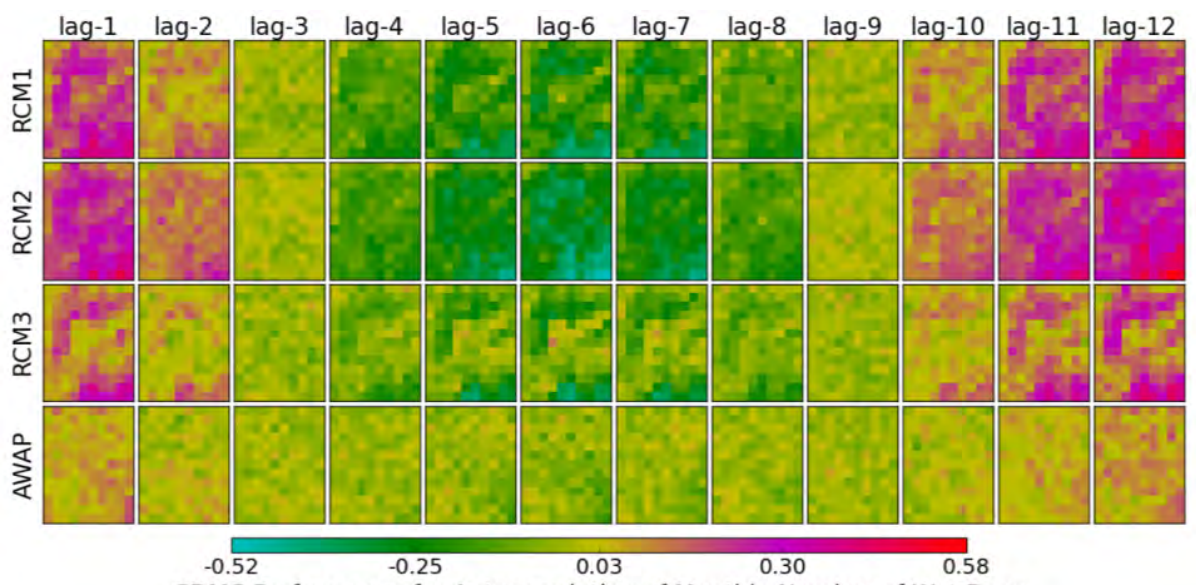

CDMC Performance for Autocorrelation of Monthly Number of Wet Days

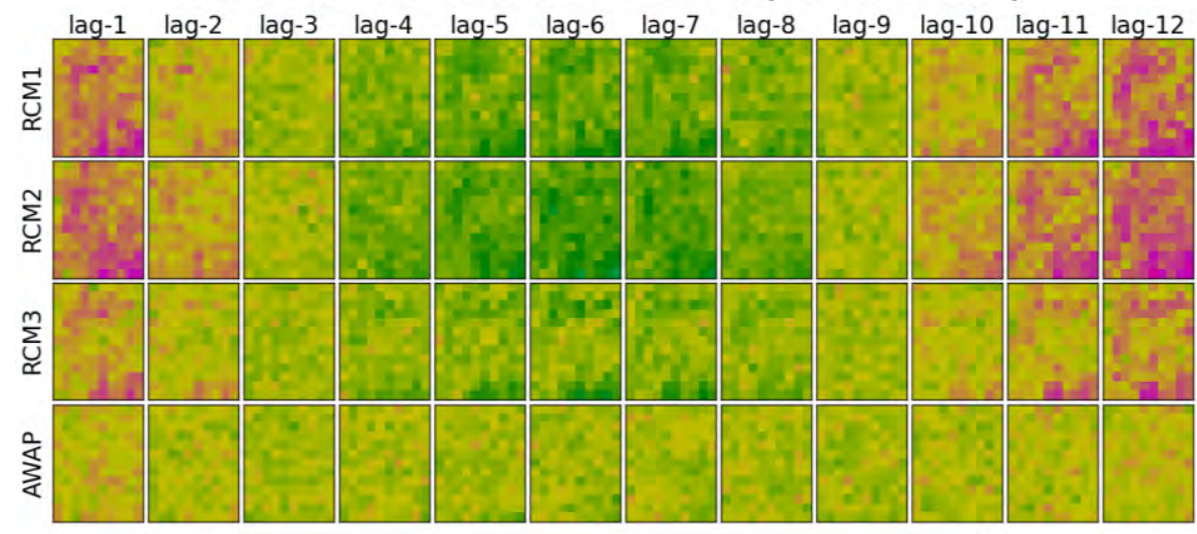

$\begin{array}{lllll}-0.52 & -0.25 & 0.03 & 0.30 & 0.58\end{array}$

HMC Performance for Autocorrelation of Monthly Number of Wet Days

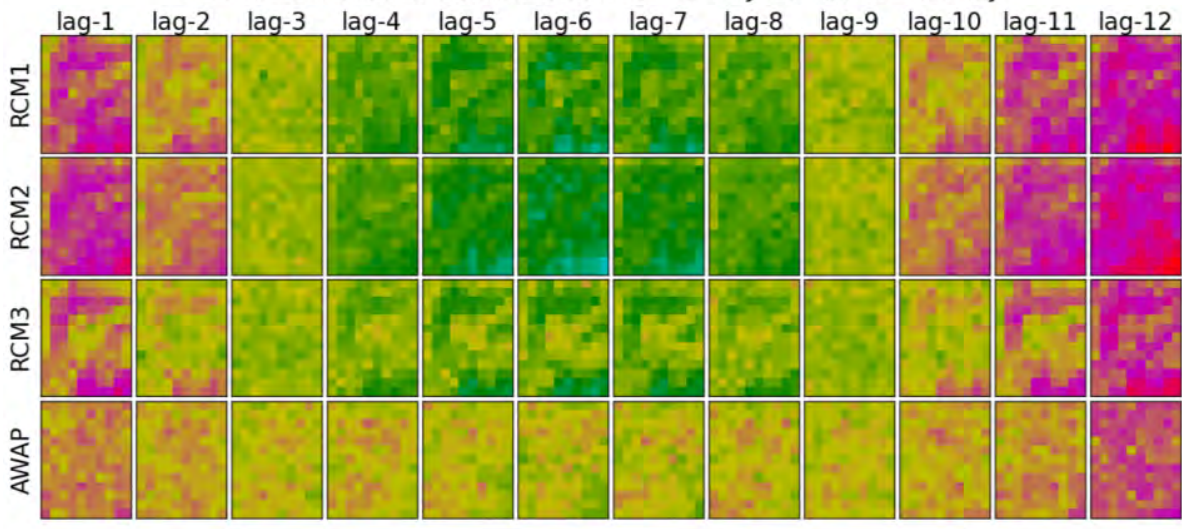

$-0.52-0.25$

0.03

0.30

0.58

DHMC Performance for Autocorrelation of Monthly Number of Wet Days

Figure 6.26: Comparison of CDMC, HMC and DHMC to reproduce the month-to-month autocorrelations of monthly number of wet days for all RCMs and AWAP. 


\subsection{Discussion}

This study has calibrated the CDMC, HMC and DHMC in the Goulburn River site of the upper Hunter region using three NARCliM RCMs (reanalysis datasets) and AWAP gridded dataset, for the 1950-2009 period. The performance of CDMC, HMC and DHMC has been assessed in reproducing the key statistics of rainfall depths and wet periods.

The calibration of the models to all 130 pixels of the study site (i.e. $100 \mathrm{~km} \times 130 \mathrm{~km}$ ) showed intra-annual seasonal variability of rainfall with wet summers and dry winters, which supports the use of the monthly varying parameters in the model. In addition, there is a strong orographic influence on model parameters and a spatial correlation between the parameters. The rainfall parameters appear to be influenced by orography, with greater wet-to-wet probability, mean and SD at higher elevations. Since the east coast of Australia is composed of a narrow coastal plain backed by a mountain range, the understanding of spatial variability and the influence of orography on the model parameters will be important to the success of a stochastic rainfall simulator. The comparison of RCMs with AWAP gridded data shows that both data have similar spatial and temporal variability, and this is consistent with them being of the same spatial resolution. However, the dry pixels are drier in AWAP than the NARCliM reanalysis of three RCMs.

The distribution of rainfall depths and wet periods are mostly similar in the RCMs, but different in the AWAP. The AWAP is drier than the RCMs with lower mean of rainfall depths and wet days. Moreover, the AWAP has lower variability of rainfall depths but higher variability of wet periods compared to the RCMs. Among the three RCMs, the RCM2 is most similar to the AWAP, particularly for rainfall depths distribution. The month-to-month autocorrelations of monthly wet days and rainfall depths are found to be stronger in RCM2 and RCM1, while the autocorrelations are very weak in RCM3 and AWAP.

The findings presented in this chapter for the performances of the CDMC, HMC and DHMC are mostly consistent with their respective performance presented in the previous chapter for a single pixel at Gummun. A summary of the comparison of each model's ability to reproduce the mean and SD of rainfall depths is provided below.

- All three MC models show similar satisfactory performances to reproduce the mean and SD of rainfall depths at all resolutions, because they use the same stochastic 
parameters of Gamma distribution to generate rainfall depths for wet days. However, the HMC performs slightly better than the CDMC and DHMC in reproducing the mean and SD of rainfall depths, which might be due to the use stochastic MC parameters in the HMC.

- Each of the models shows a tendency to underestimate the mean of rainfall depths at all resolutions and SD of rainfall depths at daily resolution, which might be caused by the stochastic Gamma parameters as the APMC and DPMC with deterministic Gamma parameters did not show such tendency.

- Each of the models shows a tendency to overestimate the monthly SD of rainfall depths, while the degree of overestimation is higher in the HMC than the CDMC and DHMC (a probable influence of stochastic MC parameters in HMC).

- At daily and monthly resolutions, performance of each model is mostly satisfactory for all months, irrespective of intra-annual variability of the rainfall depths in the data to which they are calibrated. Moreover, performances of each model are mostly satisfactory for the entire site irrespective of the spatial variability of rainfall depths in the calibration data.

- At daily and monthly resolutions, performances of each model are similar for all four datasets. However, at multiyear resolutions, each model shows satisfactory performance to reproduce the SD of multiyear rainfall depths with a tendency of underestimation for the RCMs and overestimation for the AWAP, which might be linked with the higher SD in RCMs and lower SD in AWAP respectively.

- The degree of underestimation of multiyear SD for RCMs is higher in the CDMC, while the degree of overestimation for AWAP is higher in the HMC and DHMC. This indicates that the use of stochastically varied and decadally varied MC parameters in DHMC and HMC respectively might introduce more variability (compared to CDMC) of rainfall depths at multiyear resolutions, which is better for the simulation of rainfall with higher variability (e.g. RCMs) but excessive for rainfall with lower variability (e.g. AWAP).

- There appear to systematic differences in the performance of all of the models for the RCMs versus AWAP. This suggests that there are subtle but important differences between the RCMs and AWAP in so far as it impacts on the parameterisation of rainfall models. 
A summary of comparisons of CDMC, HMC and DHMC' abilities to reproduce the mean and $\mathrm{SD}$ of wet period statistics (i.e. number of wet days and mean length of wet spell) is provided below.

- All three models can satisfactorily reproduce the mean of wet period statistics (both number of wet days and mean length of wet spells) at monthly to multiyear resolutions. However, the HMC shows a tendency to overestimate the mean with a probable influence of the stochastic MC parameters.

- The CDMC cannot satisfactorily reproduce the SD of wet period statistics (both number of wet days and mean length of wet spells), and tends to significantly underestimate at annual and multiyear resolutions (also mostly at monthly resolution). This indicates the limitation of deterministic MC parameters to reproduce the variability of wet periods.

- The HMC can mostly preserve the SD of multiyear number of wet days, but significantly overestimates the SD of wet period statistics at monthly resolution. This indicates that the stochastic MC parameters introduce excessive variability of monthly wet periods in HMC.

- The DHMC performs clearly better than the CDMC and HMC, and satisfactorily reproduces the SD of wet period statistics (both number of wet days and mean length of wet spells) at monthly to multiyear resolutions, although shows a tendency to underestimate the SD of number of wet days at shorter multiyear resolutions.

- The variability of wet period statistics is considerably higher in AWAP than the RCMs, which has been reflected in the performances of the CDMC and HMC. However, the performance of the DHMC is mostly consistent for all four datasets.

For autocorrelations, the CDMC and DHMC satisfactorily reproduce the month-to-month autocorrelations, both monthly rainfall depths and number of wet days with appropriate seasonal signal, coefficient of correlations, and spatial pattern. The HMC also mostly preserves the autocorrelations, but tends to underestimate the autocorrelations for monthly number of wet days which might be linked with the excessive variability of monthly wet periods due to the use of stochastic MC parameters. 


\subsection{Conclusion}

This study has calibrated the CDMC, HMC and DHMC and assessed their performance in reproducing the key distribution and autocorrelation statistics of NARCliM reanalysis of three RCMs as well as AWAP in the Goulburn River site. All three models have preserved the rainfall depths distributions very well in all resolutions, particularly the variability of multiyear rainfall depths. However, the CDMC has been found to underestimate the variability of multiyear wet period distributions. While the HMC improves the reproduction of multiyear variability of wet periods, it has been found to overestimate variability of monthly wet periods distributions. The HMC also underestimates the month-to-month autocorrelations of monthly wet days, while the autocorrelations are well reproduced by the CDMC. The DHMC performed better than the CDMC and HMC, and satisfactorily reproduced the distribution and autocorrelations of the rainfall depths and wet periods at daily to multiyear resolutions. 


\section{Comparison of MC Models in Williams River Site}

\subsection{Introduction}

This chapter presents the comparisons of the CDMC, HMC and DHMC after calibration to data from the Williams River site in the lower Hunter region of coastal NSW, Australia. This site has prominent ECL impacts [Verdon-Kidd et al., 2010], while it is the source of urban water supply (includes the Hunter Water Supply Network) for the Hunter and Newcastle region [Mortazavi et al., 2013]. Therefore, this analysis will provide an assessment of how well the three models perform in a region with ECL influence on rainfall. In addition, this chapter also presents the climate change trend of rainfall characteristics using future period GCM data.

\subsection{Calibrated Parameters}

This section will present the parameters of MC models (averaged APMC values of $P_{00}, P_{11}, \mu$, and $\sigma$ ) calibrated to the RCMs and AWAP for 357 land pixels of Williams River site.

\subsubsection{Intra-annual and Spatial Variability of Parameters}

Figure 7.1 and Figure 7.2 show the seasonal and spatial variability of the MC and Gamma parameters (averaged APMC values) respectively, while Table 7.1 shows the spatially and temporally averaged values of the parameters. For all four datasets, the seasonality is consistent with the typical east-Australian climate of wet summers (December-February) and dry winters (June-August), with two transitional seasons of autumn (March-May) and spring (SeptemberNovember) as shown in Figure 7.1 and Figure 7.2. Comparing the four datasets, the wet-to-wet day probability is ranked as (from wettest to driest) RCM1 $>$ AWAP $>$ RCM2 $>$ RCM3, while the wet day rainfall depths is ranked as RCM3 $>$ RCM1 $>$ RCM2 $>$ AWAP in almost all seasons. Spatially, for the three NARCliM RCMs, pixels at higher elevations are wetter (both more wet days and higher rainfall depths). In AWAP, the spatial variability of rainfall is different from the NARCliM RCMs; pixels in the low elevation coastal region are wetter than pixels at the higher elevation (the elevation map is shown Figure 3.4). Overall, the wetness of four datasets is in the order of RCM3 $>$ RCM1 $>$ RCM2 > AWAP (Table 7.1). 
Table 7.1: Spatially and temporally averaged APMC values of model parameters.

\begin{tabular}{ccccc}
\hline Parameter & RCM1 & RCM2 & RCM3 & AWAP \\
\hline$P_{00}$ & 0.64 & 0.67 & 0.66 & 0.75 \\
$P_{11}$ & 0.68 & 0.66 & 0.66 & 0.67 \\
$\mu$ & 7.34 & 6.42 & 8.77 & 5.88 \\
$\sigma$ & 11.08 & 9.67 & 13.17 & 9.86
\end{tabular}
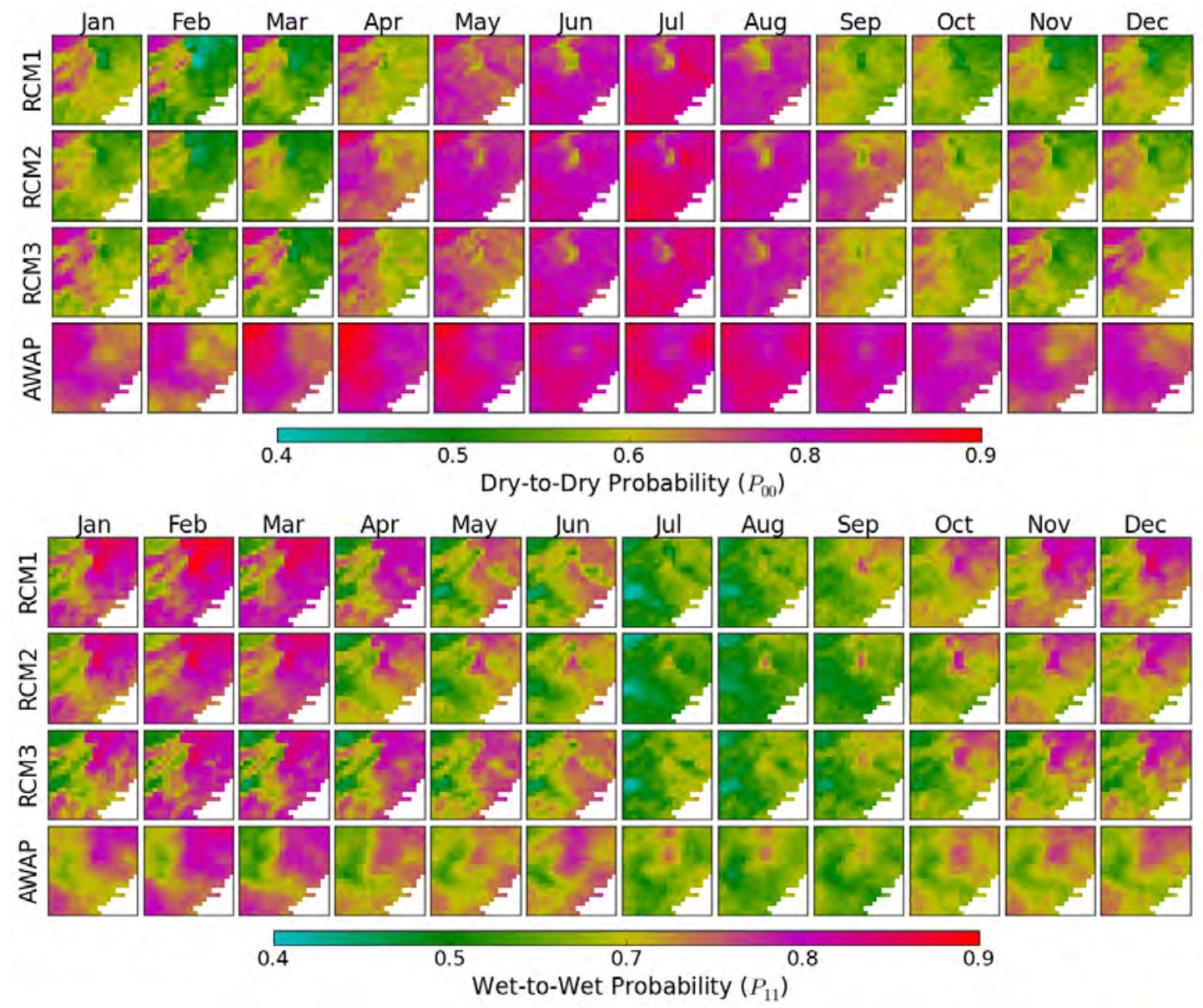

Figure 7.1: Intra-annual and spatial variability of dry-to-dry and wet-to-wet transition probabilities. The colourbars indicate the parameter values. 

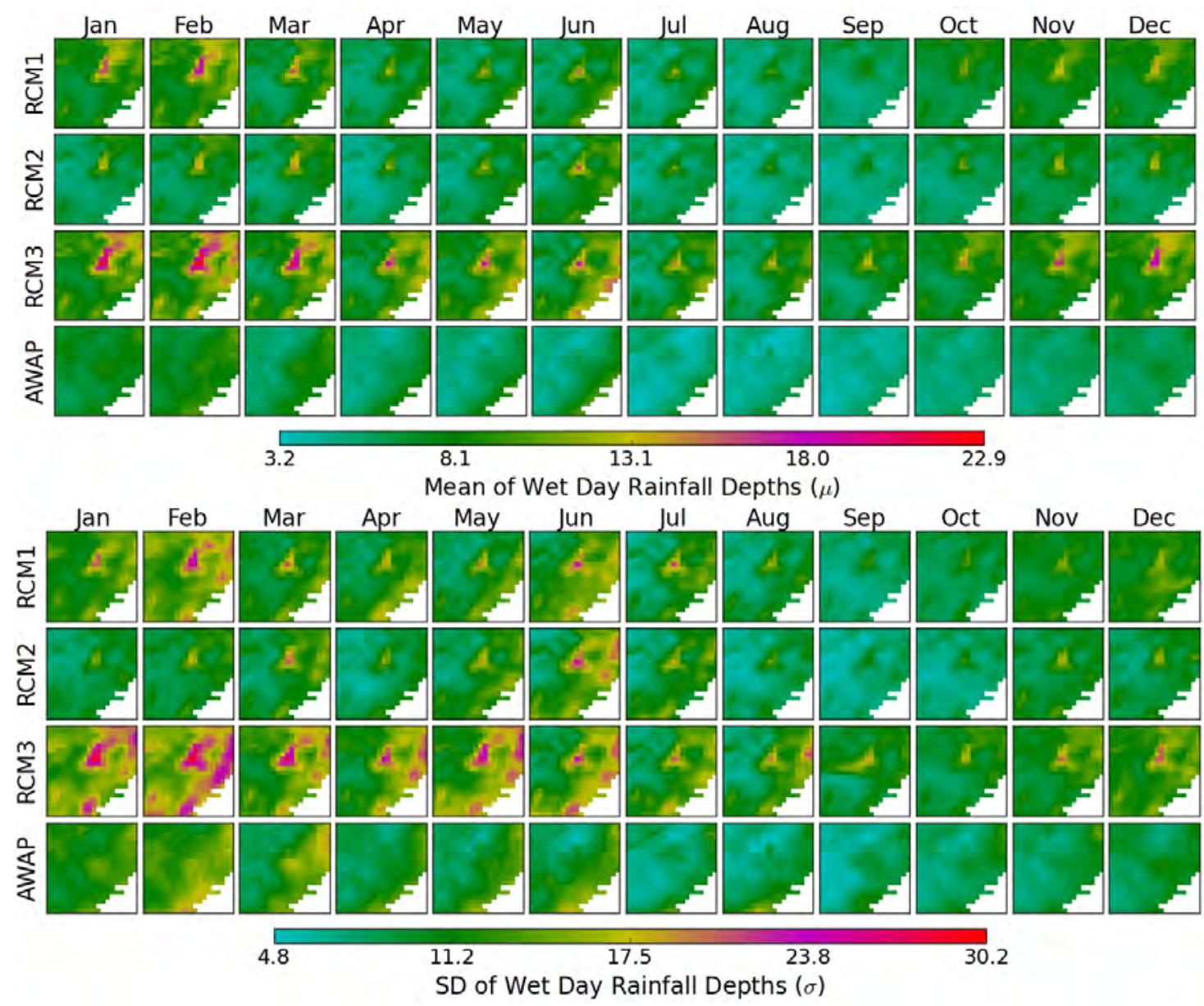

Figure 7.2: Intra-annual and spatial variability of mean and SD of wet day rainfall depths. The colourbars indicate the parameter values.

\subsubsection{Correlation between Elevation and Parameters}

The correlations between elevation and model parameters $P_{00}, P_{11}, \mu$, and $\sigma$ (averaged APMC values) are calculated to test for any orographic relationship. While the spatial distributions of parameters shown in Figure 7.1 and Figure 7.2 indicate the links of parameters with elevation, these orographic relationships for each dataset are further confirmed by the elevation-parameter correlations $r_{o}$ shown in Figure 7.3. The correlations between elevation and MC parameters (i.e. $P_{00}$ and $\left.P_{11}\right)$ for all four datasets follow a similar intra-annual trend. However, the negative correlations between elevation and $P_{00}$ are stronger in winter and weaker in summer, indicating more dry days at lower elevation, particularly in winter. The positive correlations between elevation and $P_{11}$ are stronger in summer and weaker in winter, indicating more wet days at higher elevation particularly in summer. For the three RCMs, the positive correlations between elevation and $\mu$ are stronger in summer and weaker in winter. For AWAP, stronger negative correlations between elevation and $\mu$ have been observed for February to August, while correlations for other months are weak. The correlation between elevation and $\sigma$ are relatively 
weaker but show a similar trend of correlation between elevation $\mu$. Overall, the elevationparameter correlations are stronger in RCM2 and AWAP than the RCM1 and RCM3 (Table $7.2)$.
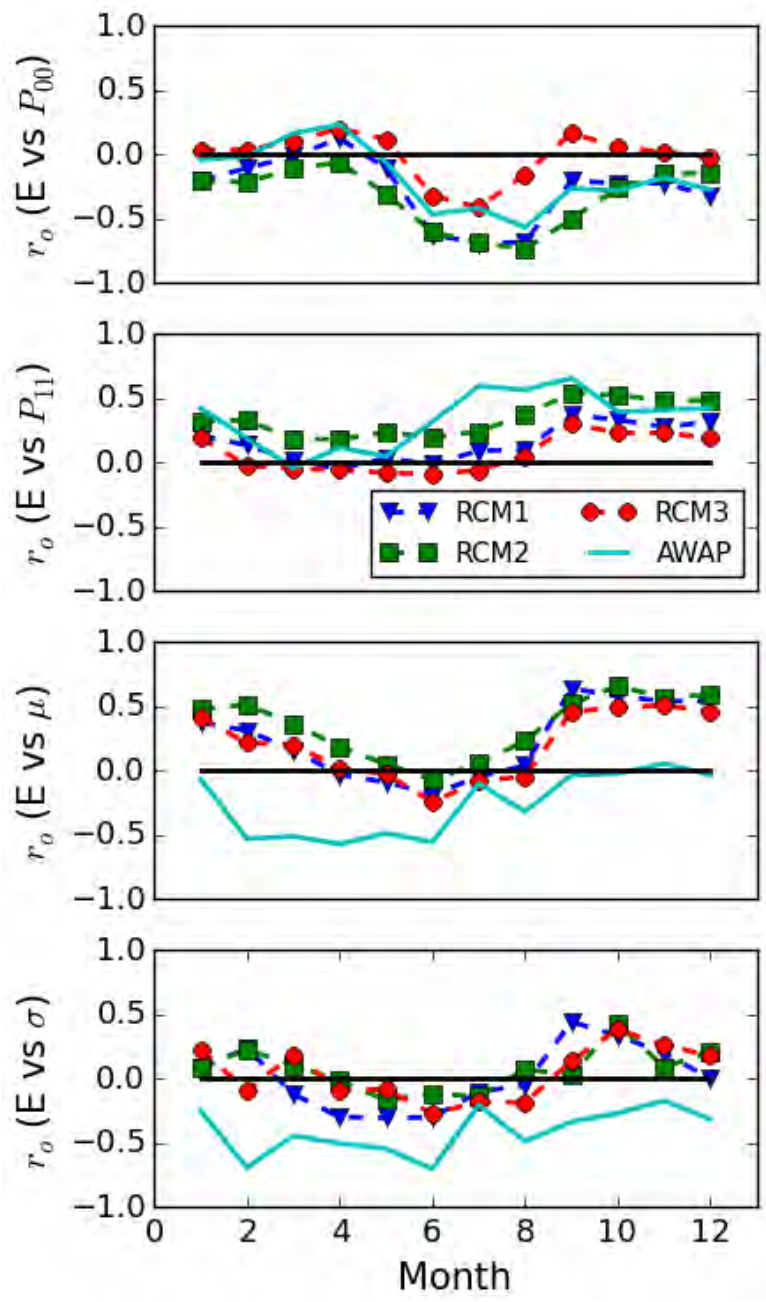

Figure 7.3: Correlation between elevation and model parameters (APMC values).

Table 7.2: Average of the absolute values of correlation coefficients between elevation and parameters.

\begin{tabular}{ccccc}
\hline Correlation & RCM1 & RCM2 & RCM3 & AWAP \\
\hline E vs $P_{00}$ & 0.3 & 0.34 & 0.14 & 0.25 \\
E vs $P_{11}$ & 0.16 & 0.34 & 0.13 & 0.35 \\
E vs $\mu$ & 0.29 & 0.36 & 0.26 & 0.28 \\
E vs $\sigma$ & 0.21 & 0.14 & 0.19 & 0.41 \\
\hline
\end{tabular}




\subsection{Distribution Statistics of Rainfall Depths and Wet-Dry Periods}

This section presents the distribution statistics (i.e. mean and SD) of the rainfall depths and wet-dry periods (i.e. number of wet and dry days, and mean length of wet and dry spells) calculated from each RCM and AWAP for daily, monthly and multiyear resolutions. The subsequent sections will assess the performances of the CDMC, HMC and DHMC to reproduce these statistics. Figures provided in this section show the distribution statistics calculated from the 357 land pixels plotted in histograms (similar to Figure 6.6). The spatial and intra-annual variability of the distribution statistics are consistent with the variability of the model parameters (section 7.2) and, hence, are shown in Appendix D.1.

\subsubsection{Mean and SD of Rainfall Depths}

Figure 7.4 shows the mean and SD of rainfall depths at daily, monthly and multiyear resolutions (see intra-annual and spatial variability in Figure D.1 to D.4 at Appendix D.1). The distribution of mean rainfall depths for all resolutions shows a similar trend as that shown in the Goulburn River site. The range of mean rainfall depths in the pixels of four datasets are similar to the rank of wetness in the order of RCM3 $>$ RCM1 $>$ RCM2 $>$ AWAP. However, the mean rainfall depths vary between $4-15 \mathrm{~mm}, 20-300 \mathrm{~mm}$, and $1000-4000 \mathrm{~mm}$ at daily, monthly and annual resolutions, respectively, for the three RCMs, while the AWAP has less respective mean rainfall at all resolutions. The SDs of rainfall depths are also in the order of RCM3 > RCM1 > RCM2 $>$ AWAP.
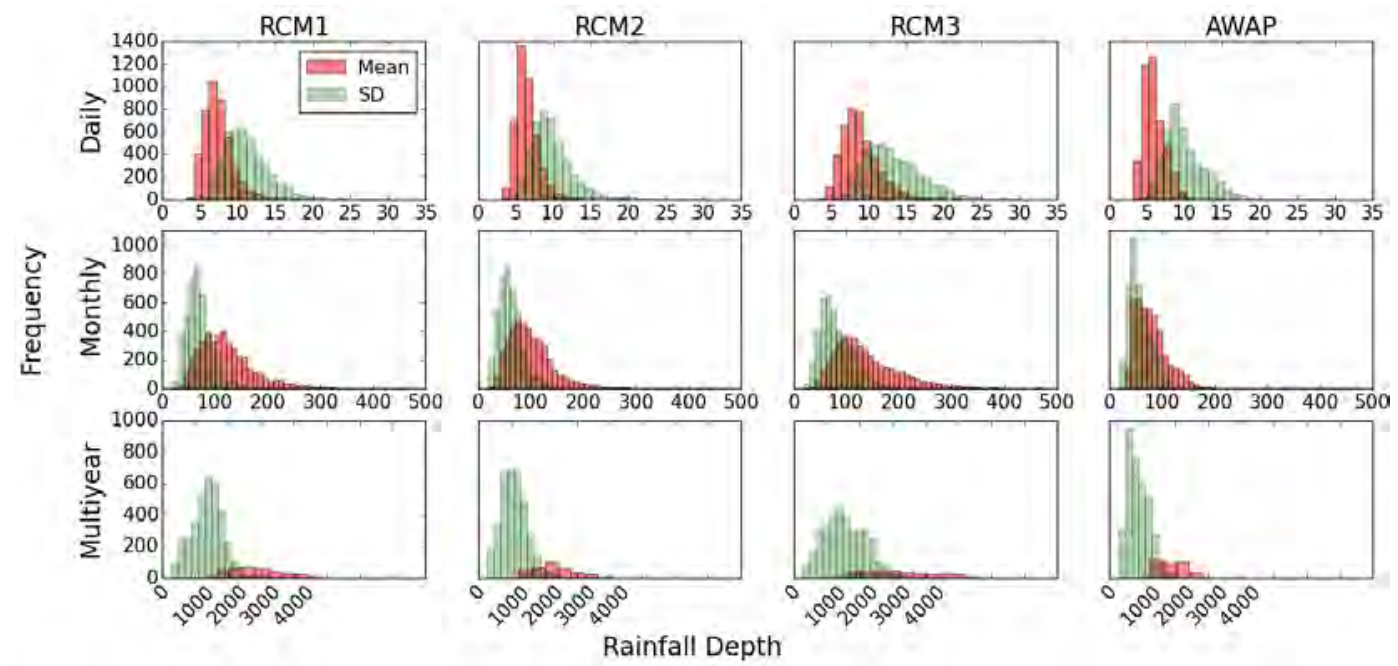

Figure 7.4: Mean and SD of rainfall depths at daily, monthly and multiyear resolutions. 


\subsubsection{Mean and SD of Wet-Dry Periods Statistics}

Figure 7.5 shows the mean and SD of number of wet and dry days, while Figure 7.6 shows mean and SD of the mean length of wet and dry spells (see intra-annual and spatial variability in Figure D.5 to D.11 at Appendix D.1). Figure 7.5 shows that the AWAP is drier than the RCMs with less wet days and higher dry days. However, the SD of wet and dry days are considerably higher in AWAP than the RCMs. Figure 7.6 shows that the mean of the mean length of wet spell is similar in all four datasets, while the mean of the mean length of dry spell is higher in AWAP (consistent with the trend of wet-to-wet and dry-to-dry probabilities shown in Figure 7.1 and Table 7.1). The SD of the mean length of wet and dry spells is also higher in AWAP than in the RCMs.

\subsubsection{Summary}

The averaged values of the statistics shown in Table 7.3 indicate that the AWAP is drier than the RCMs (rank of wetness as RCM3 $>$ RCM1 $>$ RCM2 $>$ AWAP) with lower SD of rainfall depths and higher SD of wet days (consistent with Goulburn). Although AWAP has less wet days, the mean length of wet spells of AWAP is similar to the RCMs. However, mean length of dry spells are considerably higher in AWAP than the RCMs.
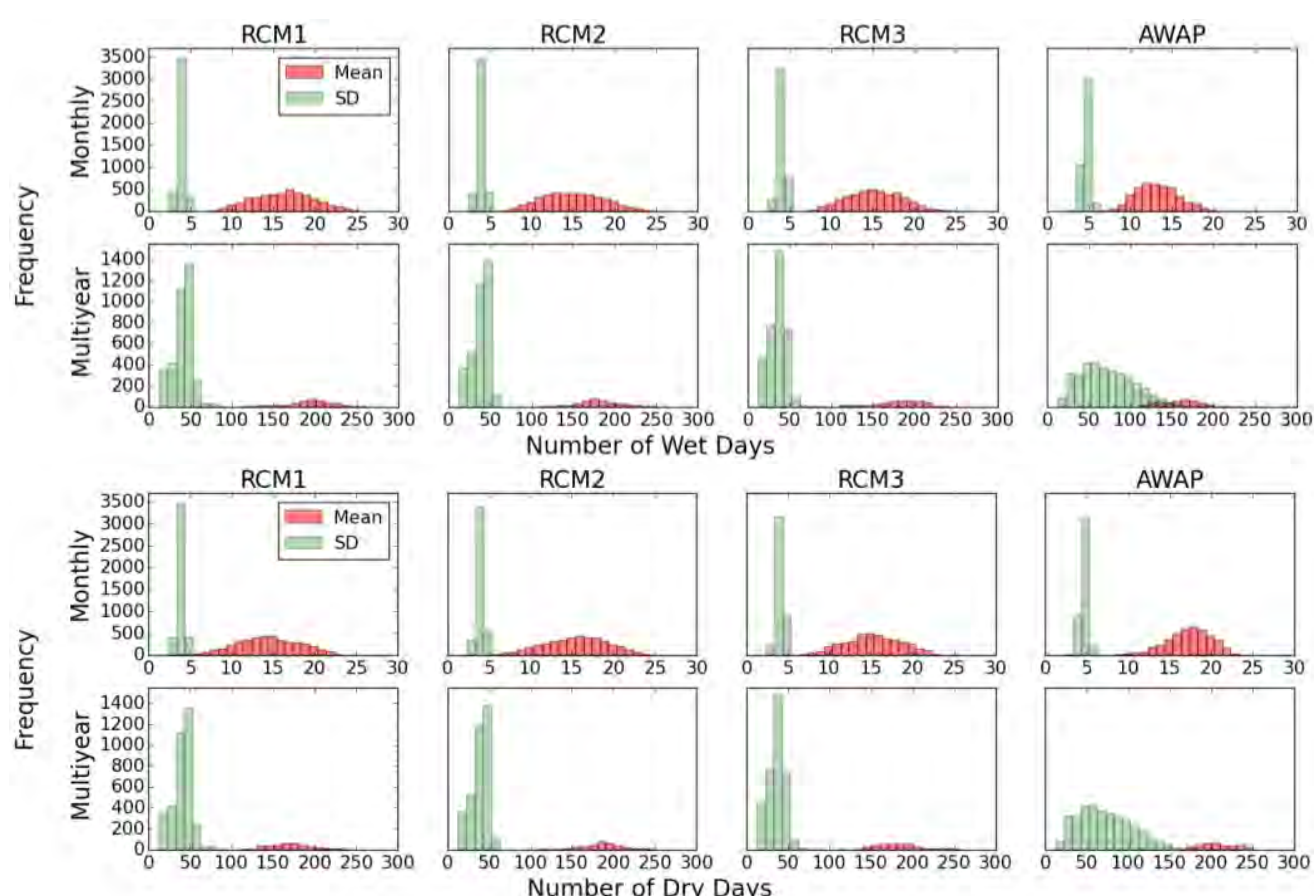

Figure 7.5: Mean and SD of number of wet and dry days at monthly and annual resolutions. 

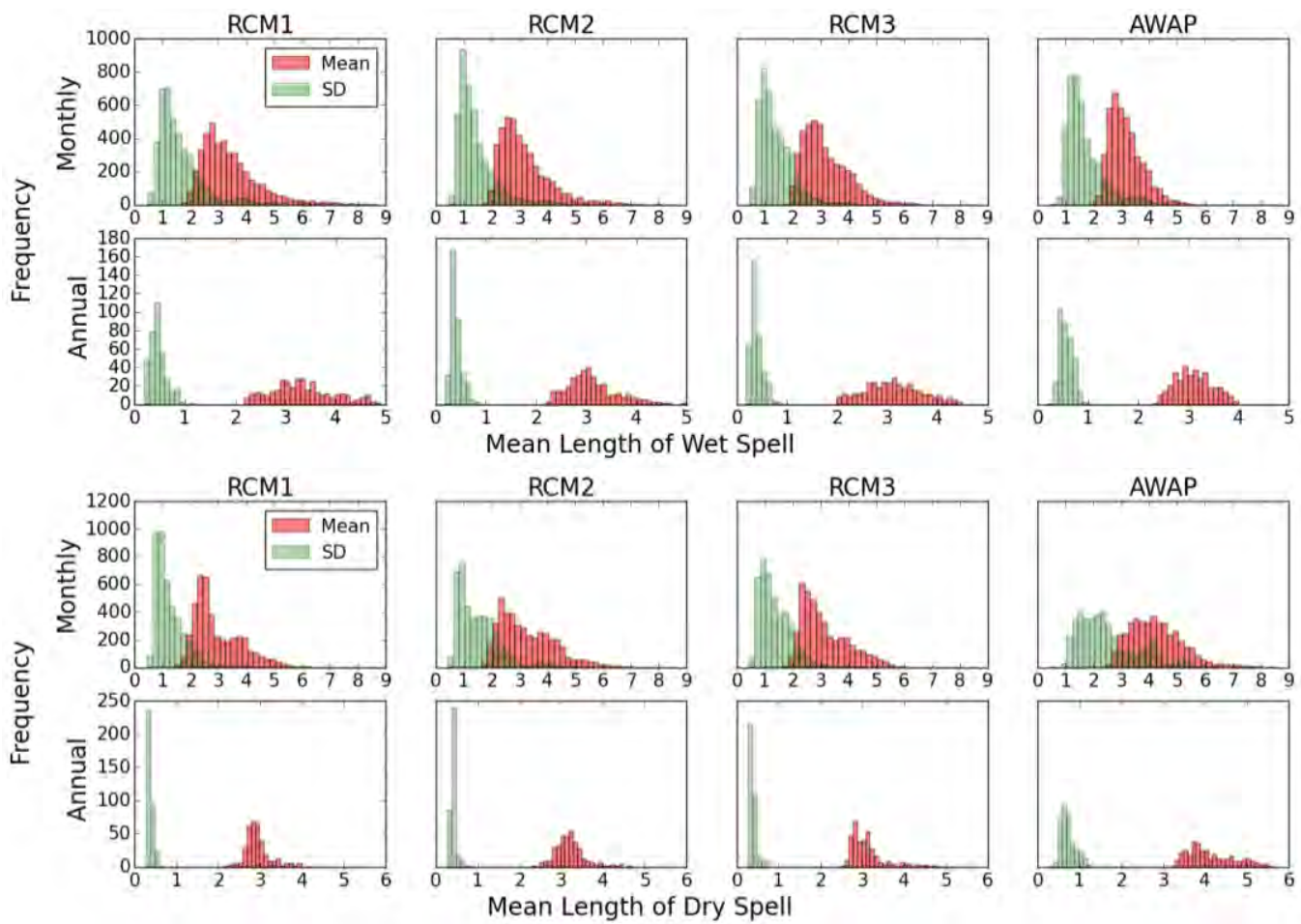

Figure 7.6: Mean and SD of mean length of wet and dry spells at monthly and annual resolutions.

Table 7.3: Spatially and temporally averaged values of the mean and SD of rainfall depth and wetdry period statistics at annual and multiyear resolutions.

\begin{tabular}{cccccc}
\hline \multicolumn{2}{c}{ Statistics } & RCM1 & RCM2 & RCM3 & AWAP \\
\hline Rainfall & Annual Mean & 1471 & 1189 & 1659 & 950 \\
\cline { 2 - 6 } Depth (mm) & Multiyear SD & 683 & 544 & 784 & 437 \\
\hline \multirow{2}{*}{$\begin{array}{c}\text { Number of } \\
\text { Wet Days }\end{array}$} & Annual Mean & 193 & 180 & 182 & 158 \\
\cline { 2 - 6 } & Multiyear SD & 40 & 38 & 35 & 73 \\
\hline Mean Length & Annual Mean & 3.35 & 3.11 & 3.11 & 3.11 \\
\cline { 2 - 6 } of Wet Spell & Annual SD & 0.47 & 0.41 & 0.4 & 0.56 \\
\hline Number of & Annual Mean & 171.9 & 185.5 & 182.9 & 207.5 \\
\cline { 2 - 6 } Dry Days & Multiyear SD & 40.3 & 38.3 & 35.4 & 72.6 \\
\hline Mean Length & Annual Mean & 2.93 & 3.20 & 3.10 & 4.13 \\
\cline { 2 - 6 } of Dry Spell & Annual SD & 0.37 & 0.41 & 0.39 & 0.71 \\
\hline
\end{tabular}




\subsection{Model Comparison for Distribution of Rainfall Depths}

This section compares the CDMC, HMC and DHMC for the mean and SD of rainfall depths (as per the assessment method discussed in section 3.4).

\subsubsection{Mean of Rainfall Depths at Daily, Monthly and Multiyear Resolutions}

For mean of rainfall depths at daily, monthly and multiyear resolutions, the three models show similar satisfactory performances with $\mathrm{Z}$ scores mostly between -1 and +1 for all four datasets. However, the $\mathrm{Z}$ scores of the models are mostly positive (see Figure D.12-D.14 at Appendix D.2), indicating a tendency for the models to underestimate the mean of rainfall depths at all resolutions. These results are consistent with the respective results for Goulburn River site.

\subsubsection{SD of Daily and Monthly Rainfall Depths}

The SD of rainfall depths at daily and monthly resolutions are also satisfactorily preserved by each of the models, with $\mathrm{Z}$ scores mostly between -1 and +1 for all four datasets (see Figure D.15-D.16 at Appendix D.2). However, each of the models tends to overestimate the SD of rainfall depths at monthly resolution (mostly negative $\mathrm{Z}$ scores). The tendency of overestimation is higher in the HMC than in the DHMC and CDMC.

\subsubsection{SD of Multiyear Rainfall Depths}

For RCMs, the CDMC, HMC and DHMC reproduce the SD of multiyear rainfall depths satisfactorily with a tendency to underestimate, while the statistics are significantly underestimated in the CDMC for a number of pixels at 2 to 5 overlapping years (Figure 7.7). For AWAP, all three models tend to overestimate the SD of multiyear rainfall depths, while HMC and DHMC significantly overestimated the statistics for a number of pixels at 6 to 10 overlapping years (Figure 7.7). It is noted that the range of multiyear SD in AWAP is considerably lower than the same in three RCMs (Figure 7.4). 

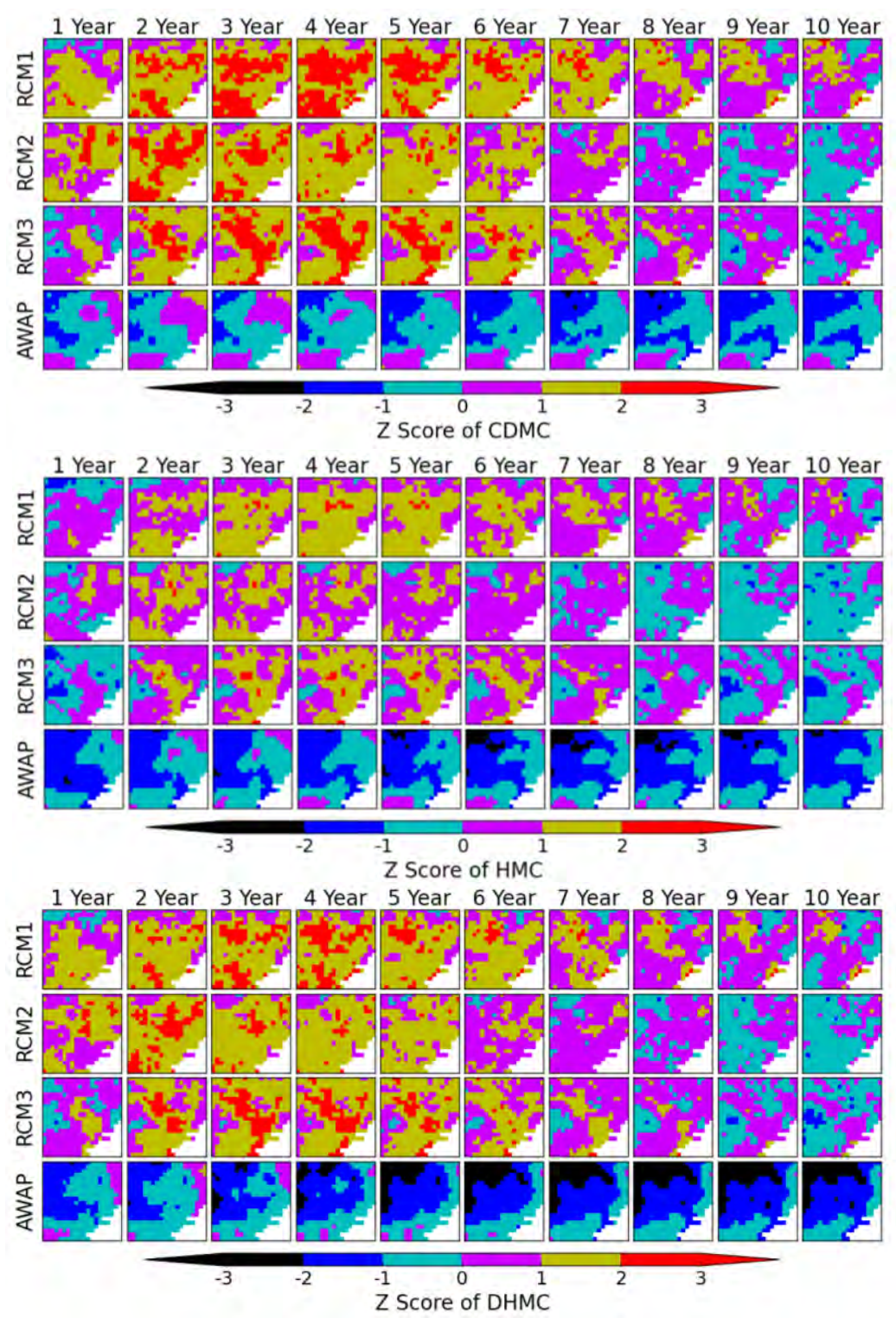

Figure 7.7: Z Scores of CDMC, HMC and DHMC for the SD of multiyear rainfall depths. 


\subsubsection{Insight on Model Performance for SD of Multiyear Rainfall Depths}

To gain further insight of the model performance in preserving multiyear SD of rainfall depths, two NARCliM pixels are selected: one (say Pixel A) where multiyear SD of rainfall depths for RCM2 is preserved in the CDMC for all overlapping years, and another (say Pixel B) where the multiyear SD of rainfall depths for RCM2 is underestimated in the CDMC for some overlapping years. For these two pixels, this study has plotted 100 samples of the 1000 realisations of the multiyear SD of rainfall depths from the CDMC simulation to examine the trend between different multiyear aggregates.

Figure 7.8 shows that the multiyear SD of rainfall depths is preserved in the CDMC for all overlapping years in Pixel A, while the same statistic is underestimated for 2, 3 and 4 overlapping years in Pixel B. For both pixels, the statistic for one overlapping period is highly correlated with successive overlapping periods. Therefore, pixels that over or underestimate the multiyear SD of rainfall depths tend to do so for several multiyear aggregates. Although the underestimations occurred in some pixels, in each pixel, the trend of multiyear SD of rainfall depths simulated by CDMC are mostly consistent with trend of multiyear SD of rainfall depths for RCM2 (statistics of the data to which the model parameters calibrated to). Moreover, the CDMC realisations represent a range of uncertainties in the rainfall variability. This suggests that the CDMC can simulate the multiyear rainfall variability, although the model is calibrated to daily data.

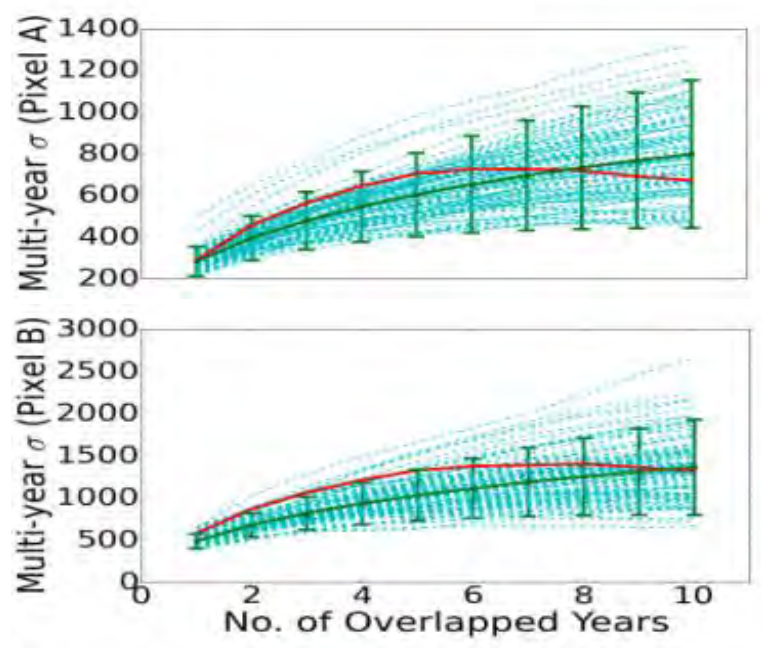

Figure 7.8: 100 CDMC realisations of SD of multiyear rainfall depths (dashed lines) for RCM2 data at two NARCliM pixels. The green solid lines indicate the expected values with $\mathbf{9 5 \%}$ confidence limit. The red lines indicate the RCM2 values. 


\subsection{Model Comparison for Distribution of Wet-Dry Periods}

This section compares the CDMC, HMC and DHMC for the mean and SD of number of wet days and mean length of wet spells (as per the assessment procedures described in section 3.4).

\subsubsection{Mean of Monthly and Annual Number of Wet Days}

For mean of number of wet days at monthly and annual resolutions, the three models show similar satisfactory performances with $\mathrm{Z}$ scores mostly between -1 and +1 for all four datasets (see Figure D.17-D.18 at Appendix D.3). However, for mean of annual number of wet days, the $\mathrm{HMC}$ tends to overestimate the statistic with negative $\mathrm{Z}$ scores for most of the pixels.

\subsubsection{Mean of Monthly and Annual Mean Length of Wet-Dry Spells}

Figure 7.9 and Figure 7.10 show the $\mathrm{Z}$ scores for mean of monthly mean length of wet and dry spells respectively. The CDMC and DHMC preserve the mean of monthly mean length of wet spells with a tendency to underestimate, while the HMC preserves the statistic with a tendency to overestimate. This performance is consistent with the performance for mean of monthly number of wet days, although performance of each model is slightly better for mean of monthly number of wet days. Performance of each model for mean of monthly mean length of dry spell is similar to the respective performance for wet spell (Figure 7.10). This justifies the assessment of wet period statistics only in this thesis.

For annual mean length of wet spells, all three models show similar satisfactory performances to reproduce the statistic with a tendency to underestimate (positive $\mathrm{Z}$ scores for most of the pixels, see Figure D.19 at Appendix D.3). 

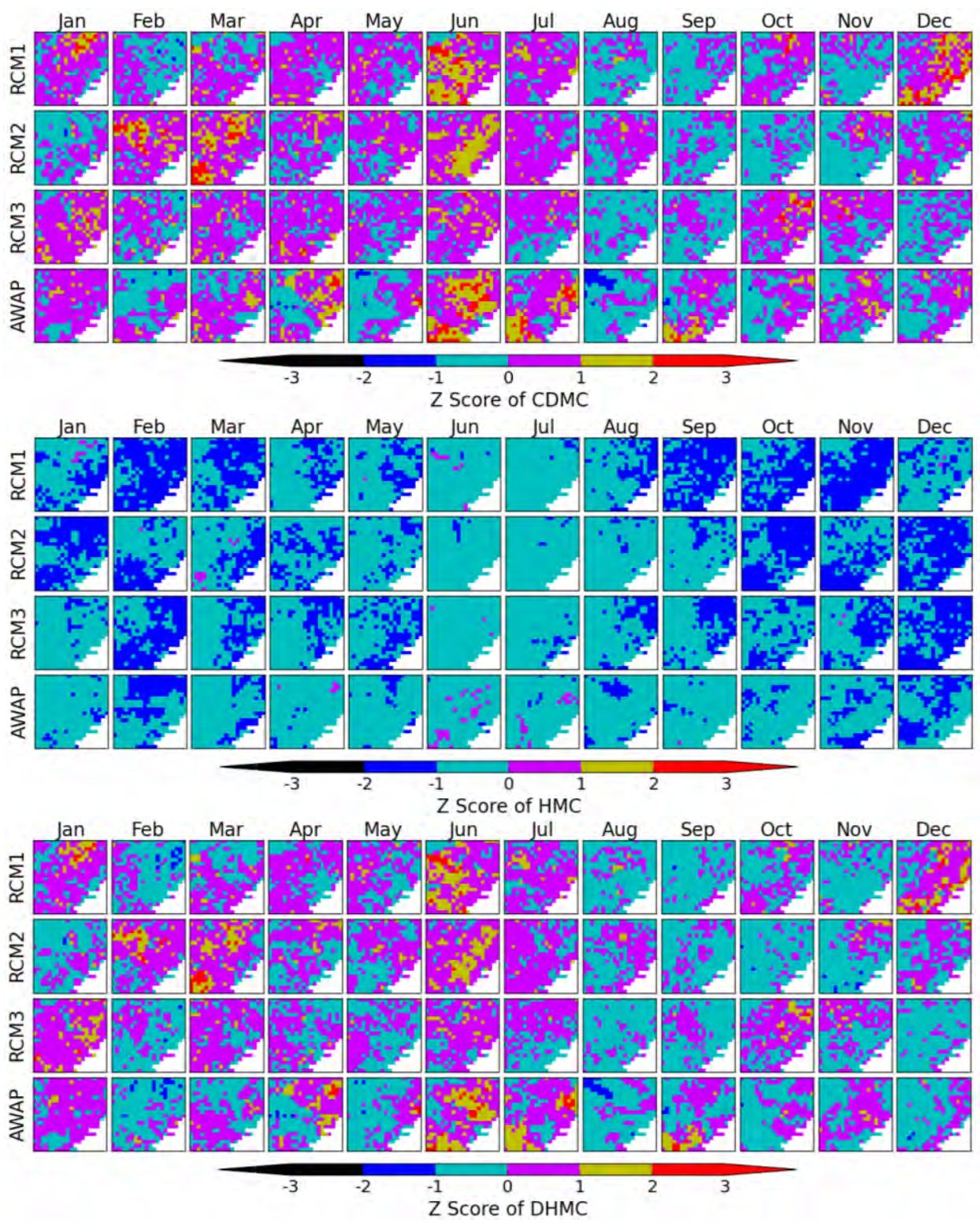

Figure 7.9: Z Scores of CDMC, HMC and DHMC for the mean of monthly mean length of wet spells. 

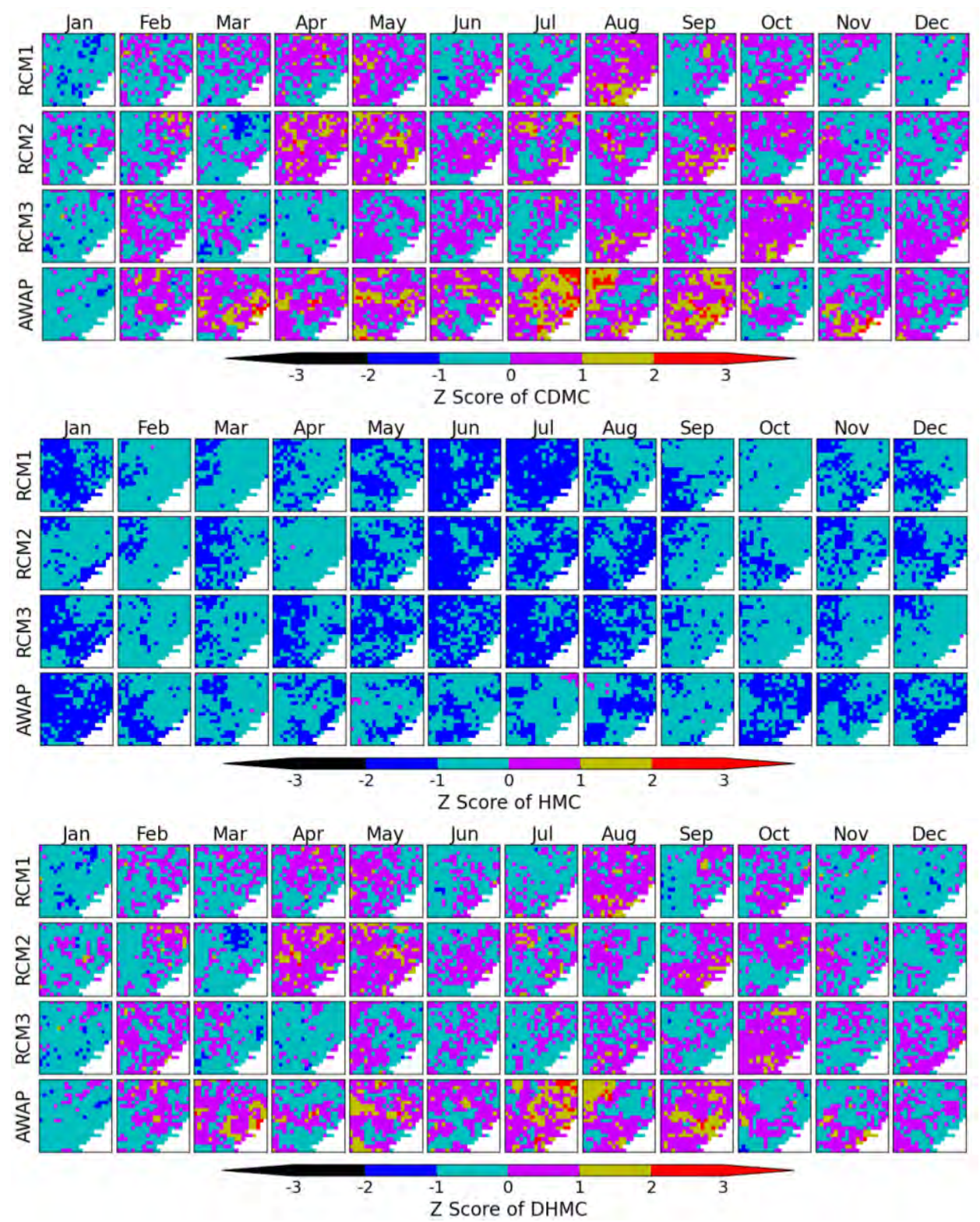

Figure 7.10: Z Scores of CDMC, HMC and DHMC for the mean of monthly mean length of dry spells.

\subsubsection{SD of Monthly Number of Wet Days and Mean Length of Wet-Dry Spells}

For SD of monthly number of wet days of all four datasets, the DHMC performs better than the CDMC and HMC, with a tendency to underestimate the statistic (Figure 7.11). Compared to DHMC, the CDMC tends to more consistently and significantly underestimate the SD of monthly number of wet days, while the HMC tends to significantly overestimate the statistic for most of the pixels of all four datasets.

For SD of monthly mean length of wet spells, the performance of each model is similar to their performance for SD of monthly number of wet days (see Figure D.20 at Appendix D.3). 
However, the models perform slightly better for SD of monthly mean length of wet spells than for SD of monthly number of wet days. Performance of each model for SD of monthly mean length of dry spell is similar to the respective performance for wet spell (Figure 7.12). This also justifies the assessment of wet period statistics only in this thesis.
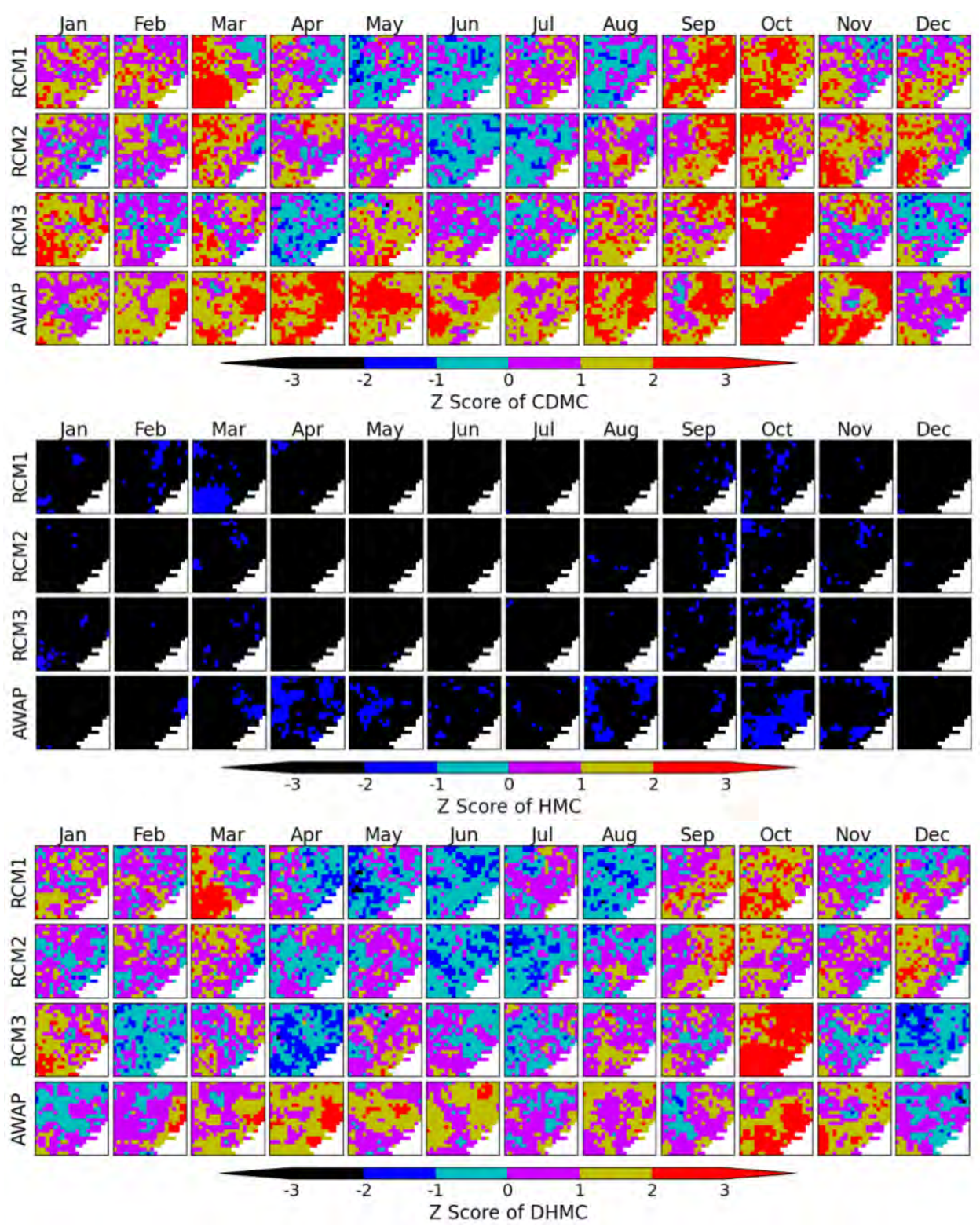

Figure 7.11: Z Scores of CDMC, HMC and DHMC for the SD of monthly number of wet days. 

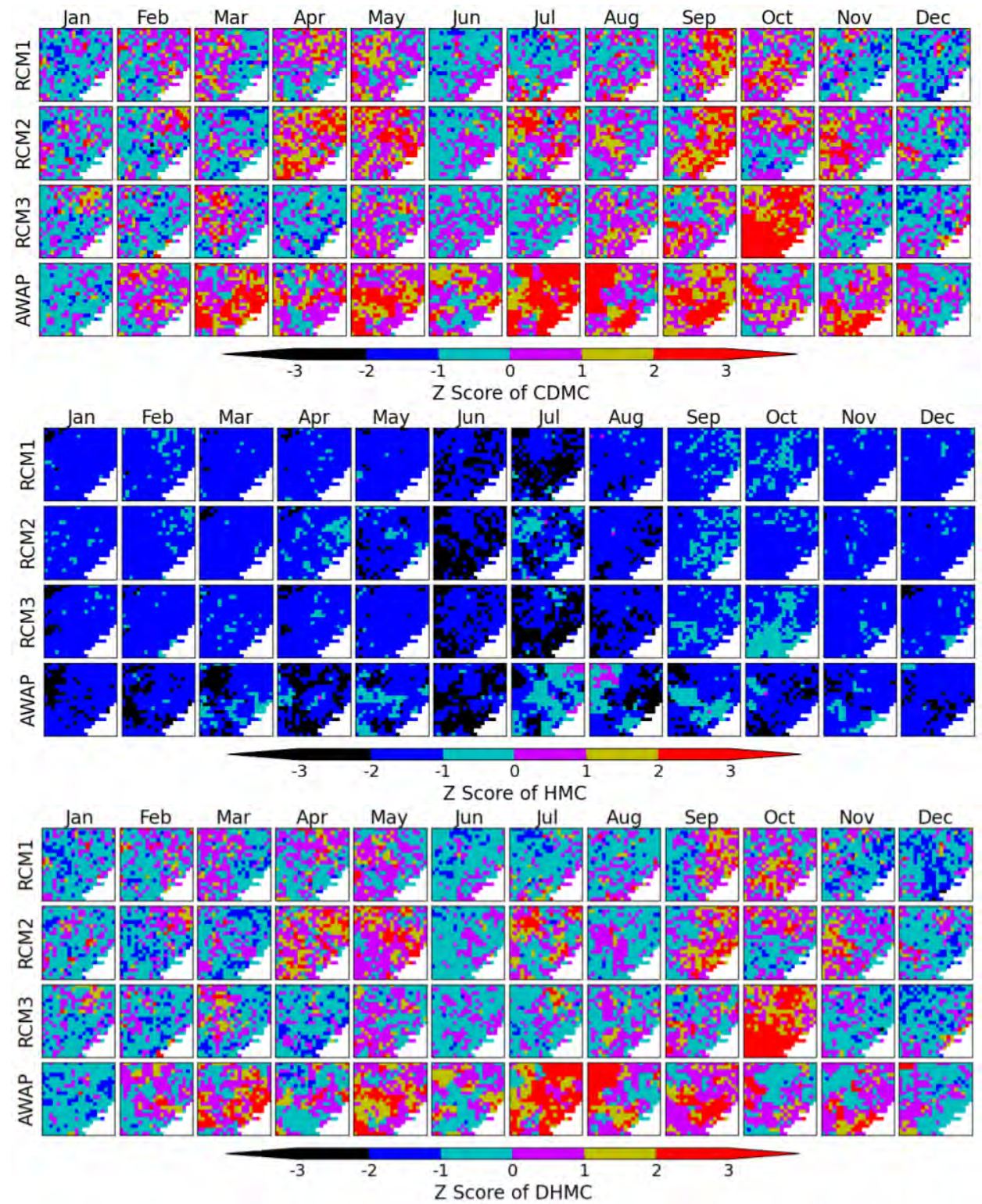

Figure 7.12: Z Scores of CDMC, HMC and DHMC for the SD of monthly mean length of dry spells. 


\subsubsection{SD of Annual Mean Wet Spell Length and Multiyear Number of Wet Days}

For SD of annual mean length of wet spells, the DHMC performs better than the CDMC and HMC to reproduce the statistic with a tendency to underestimate (Figure 7.13). The CDMC tends to significantly underestimate the statistics, while the HMC tends to significantly overestimate the statistic.

For SD of multiyear number of wet days, the HMC and DHMC perform satisfactorily and better than the CDMC to reproduce these statistics (Figure 7.14). The CDMC tends to significantly underestimate the statistic for most of the multiple years of each dataset. However, the HMC performs satisfactorily for the RCMs, but significantly underestimates the statistic for AWAP (the AWAP has higher variability of multiyear number of wet days in compare to RCMs). The DHMC mostly preserves the SD of multiyear number of wet days for all datasets with a tendency to underestimate the statistic at shorter multiyear resolution (Figure 7.14).

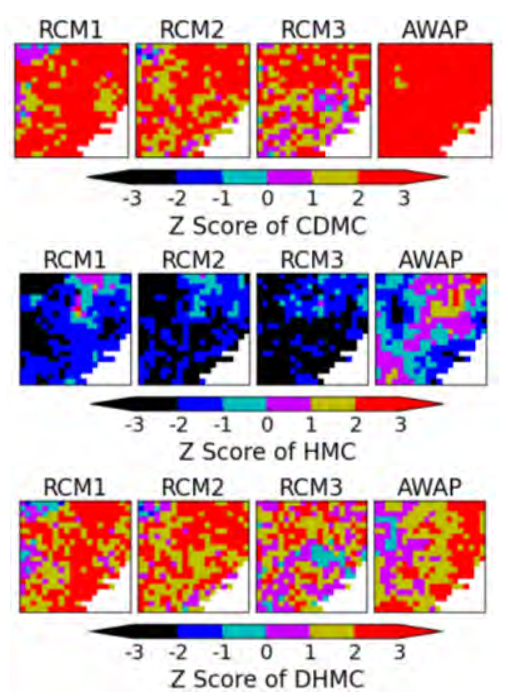

Figure 7.13: Z Scores of CDMC, HMC and DHMC for the SD of annual mean length of wet spells. 

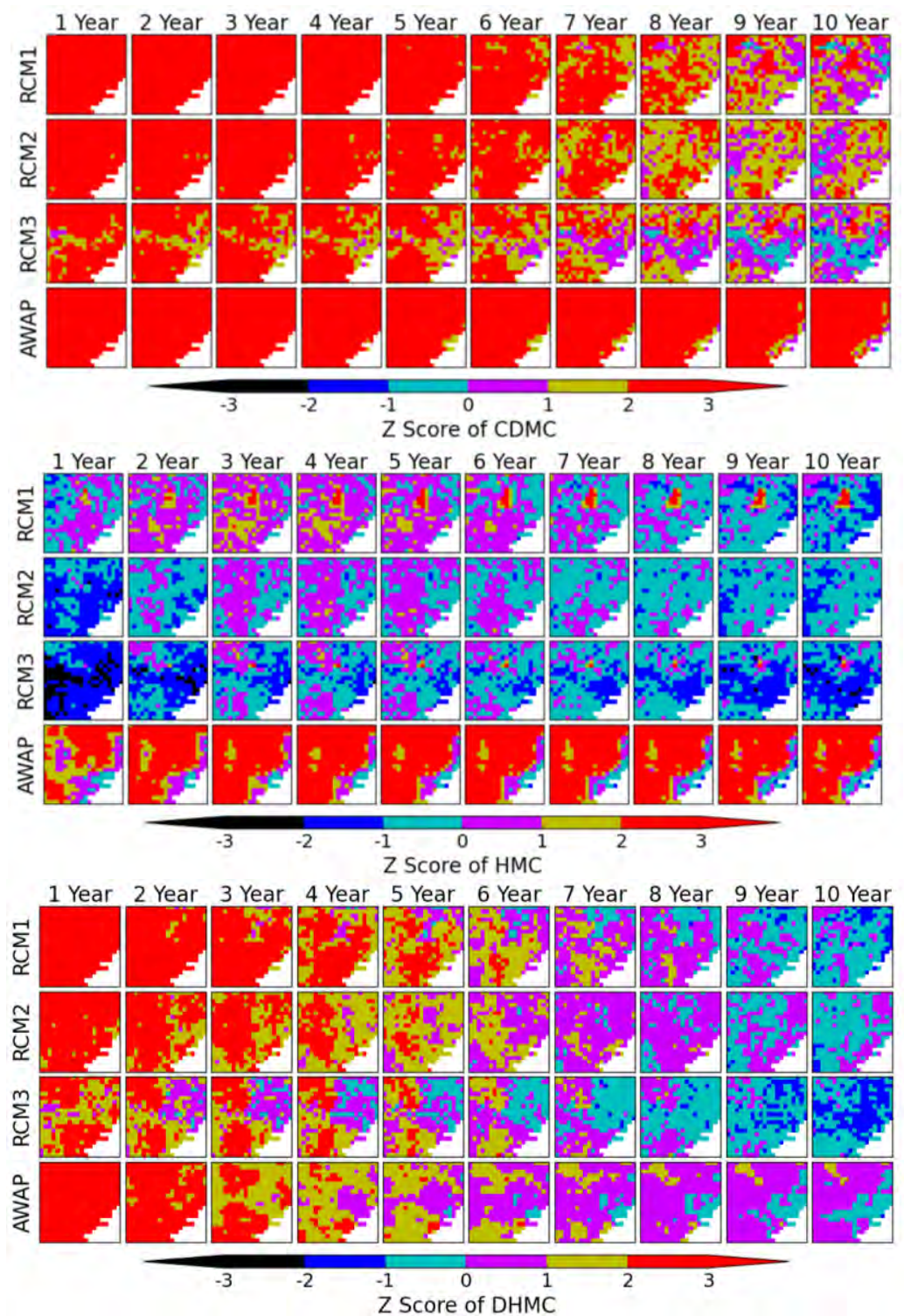

Figure 7.14: Z Scores of CDMC, HMC and DHMC for the SD of multiyear number of wet days.

\subsection{Summary of Model Comparison for Distribution Statistics}

Table 7.4 and Table 7.5 show the spatially and temporally averaged $\mathrm{Z}$ scores for mean and SD of rainfall depths and wet period statistics respectively. For mean and SD of rainfall depths, the performances of all three MC models are similar (Table 7.4). For mean and SD of wet period statistics, the DHMC performs satisfactorily and better than the CDMC and HMC (Table 7.5). These conclusions are consistent with the respective findings for Goulburn (see section 6.6). 
Table 7.4: Spatially and temporally average of the absolute value of $\mathrm{Z}$ scores for mean and SD of rainfall depths.

\begin{tabular}{|c|c|c|c|c|c|}
\hline \multirow{2}{*}{ Statistics } & \multirow{2}{*}{ Models } & \multicolumn{4}{|c|}{ Spatially and Temporally Averaged Z Scores } \\
\hline & & RCM1 & $\mathrm{RCM} 2$ & $\mathrm{RCM} 3$ & AWAP \\
\hline \multirow{3}{*}{ Daily Mean } & CDMC & 0.3 & 0.37 & 0.3 & 0.33 \\
\hline & HMC & 0.3 & 0.36 & 0.3 & 0.32 \\
\hline & DHMC & 0.3 & 0.37 & 0.31 & 0.33 \\
\hline \multirow{3}{*}{ Daily SD } & CDMC & 0.24 & 0.22 & 0.24 & 0.3 \\
\hline & $\mathrm{HMC}$ & 0.26 & 0.24 & 0.24 & 0.25 \\
\hline & DHMC & 0.25 & 0.23 & 0.23 & 0.27 \\
\hline \multirow{3}{*}{ Monthly Mean } & CDMC & 0.29 & 0.35 & 0.28 & 0.32 \\
\hline & HMC & 0.29 & 0.33 & 0.27 & 0.26 \\
\hline & DHMC & 0.29 & 0.34 & 0.28 & 0.32 \\
\hline \multirow{3}{*}{ Monthly SD } & CDMC & 0.51 & 0.44 & 0.48 & 0.55 \\
\hline & HMC & 0.79 & 0.71 & 0.78 & 0.81 \\
\hline & DHMC & 0.52 & 0.45 & 0.5 & 0.58 \\
\hline \multirow{3}{*}{$\begin{array}{c}\text { Multiyear } \\
\text { Mean }\end{array}$} & CDMC & 0.89 & 1.16 & 0.91 & 0.89 \\
\hline & HMC & 0.92 & 1.06 & 0.83 & 0.68 \\
\hline & DHMC & 0.88 & 1.15 & 0.91 & 0.89 \\
\hline \multirow{3}{*}{ Multiyear SD } & CDMC & 1.34 & 1.04 & 1.09 & 0.74 \\
\hline & $\mathrm{HMC}$ & 0.82 & 0.62 & 0.71 & 1.06 \\
\hline & DHMC & 1.11 & 0.92 & 0.92 & 1.26 \\
\hline \multirow{3}{*}{ Sum } & CDMC & 3.57 & 3.58 & 3.3 & 3.13 \\
\hline & HМC & 3.38 & 3.32 & 3.13 & 3.38 \\
\hline & DHMC & 3.35 & 3.46 & 3.15 & 3.65 \\
\hline
\end{tabular}


Table 7.5: Spatially and temporally average of the absolute value of $\mathrm{Z}$ scores for mean and SD of wet period statistics.

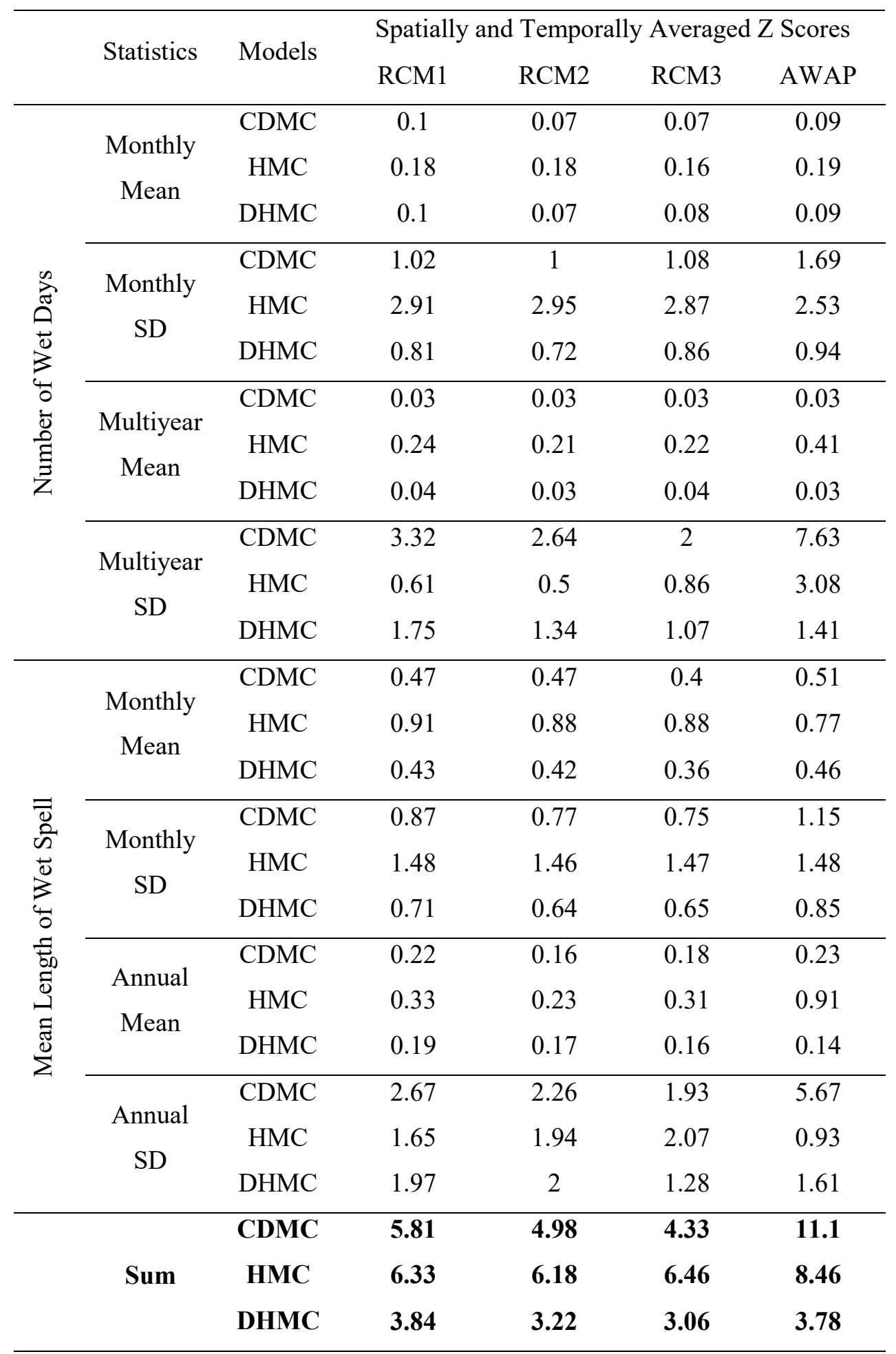




\subsection{Model Comparison for Autocorrelation}

Figure 7.15 and Figure 7.16 show that the month-to-month autocorrelations of monthly rainfall depths and monthly wet days for the Williams River site are consistent with the respective autocorrelations for the Goulburn River site (see section 6.7). The performances of the CDMC, HMC and DHMC in reproducing the autocorrelations for each pixel of the Williams River site are shown in Figure D.21-D.22 at Appendix D.4. Table 7.6 shows the spatially and temporally averaged autocorrelation coefficients. While all three models satisfactorily reproduce the seasonal and spatial patterns of the autocorrelations of monthly rainfall depths and monthly number of wet days, the HMC tends to underestimate the autocorrelations of monthly number of wet days (Figure D.21-D.22 and Table 7.6).

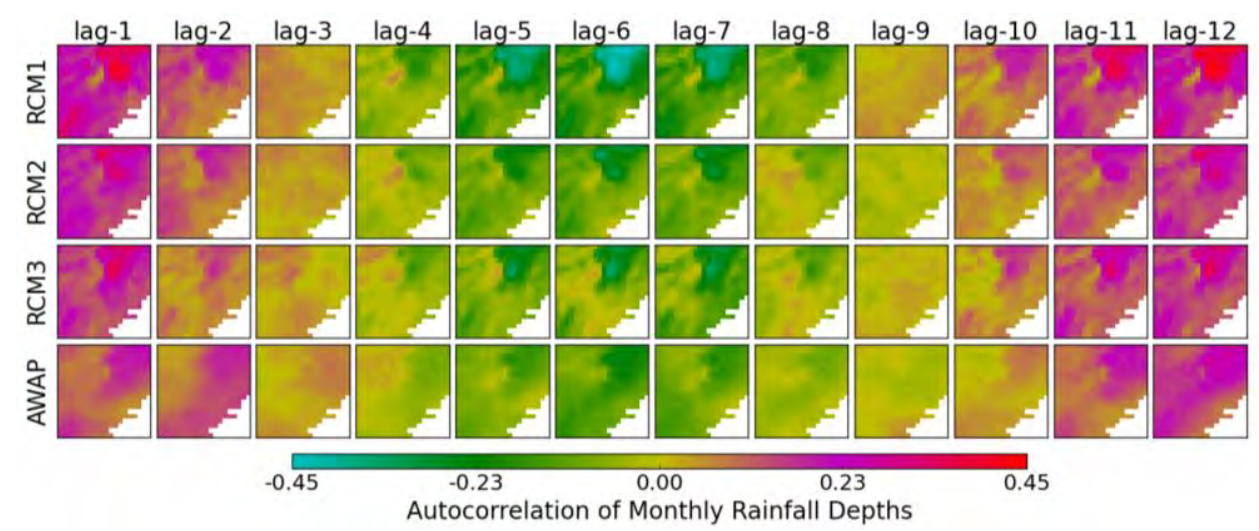

Figure 7.15: Month-to-month autocorrelations of monthly rainfall depths in all RCMs and AWAP.

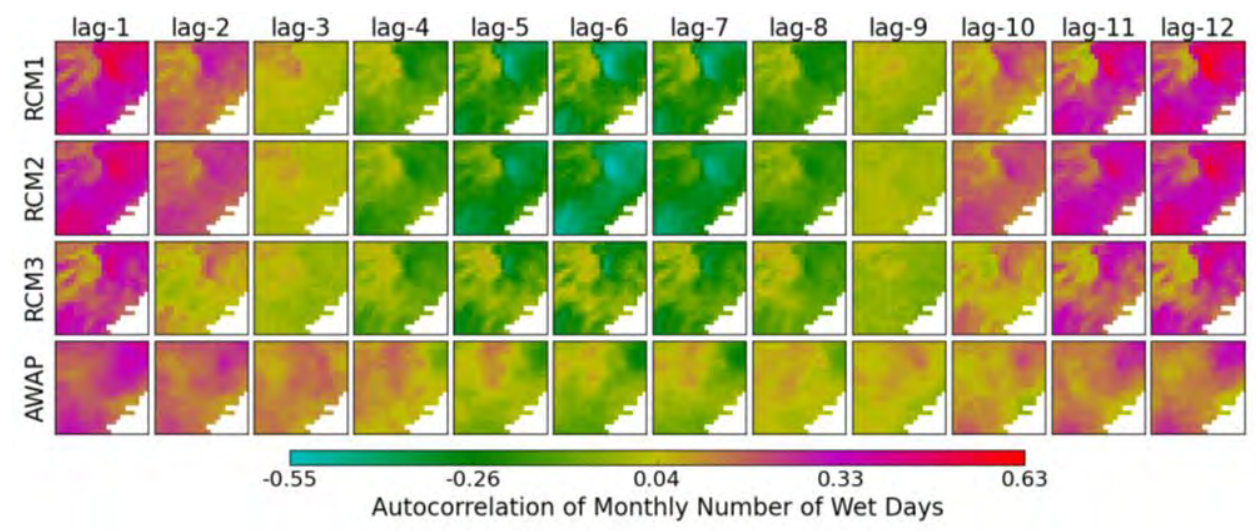

Figure 7.16: Month-to-month autocorrelations of monthly number of wet days. 
Table 7.6: Spatial (all pixels) and temporal (all lags 1-12) average of autocorrelations of monthly rainfall depths and number of wet days.

\begin{tabular}{|c|c|c|c|c|c|c|}
\hline & & & \multicolumn{4}{|c|}{ Spatially Averaged Autocorrelation Coefficients } \\
\hline & & & RCM1 & $\mathrm{RCM} 2$ & $\mathrm{RCM} 3$ & AWAP \\
\hline \multirow{4}{*}{$\begin{array}{l}\frac{\lambda}{\vec{I}} \\
\text { 范 } \\
\stackrel{\Sigma}{\Sigma}\end{array}$} & & In Data & 0.14 & 0.1 & 0.09 & 0.08 \\
\hline & & CDMC & 0.11 & 0.08 & 0.07 & 0.06 \\
\hline & & HMC & 0.1 & 0.07 & 0.07 & 0.05 \\
\hline & & DHMC & 0.11 & 0.08 & 0.08 & 0.06 \\
\hline \multirow{4}{*}{ 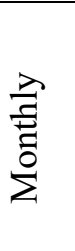 } & \multirow{4}{*}{ 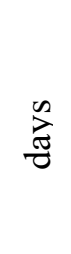 } & In Data & 0.18 & 0.21 & 0.12 & 0.11 \\
\hline & & $\mathrm{CDMC}$ & 0.19 & 0.21 & 0.13 & 0.08 \\
\hline & & $\mathrm{HMC}$ & 0.11 & 0.12 & 0.08 & 0.05 \\
\hline & & DHMC & 0.18 & 0.21 & 0.13 & 0.09 \\
\hline
\end{tabular}

\subsection{Climate Change Trend of Model Parameters}

Figure 7.17 compares the intra-annual and spatial variability of $P_{00}, P_{11}, \mu$, and $\sigma$ between NARCliM's historical (reanalysis of 1950-2009) and GCM data (CCCMA 3.1 of 1990-2009, 2020-2039, and 2060-2079) of RCM2. Table 7.7 compares the spatially and temporally averaged values of the parameters for CCCMA 3.1, CSIRO mk3.0, ECHAM5, and MIROCmedres 3.2 of RCM2, while the spatial plots for CSIRO mk3.0, ECHAM5, and MIROC-medres 3.2 are shown in Appendix D.5. This very brief analysis of possible climate change impact on the model parameters is performed to satisfy ESCCI-ECL project 5 objectives.

The intra-annual and spatial variability of model parameters in climate change periods are consistent with the historical period with summer being wetter than winter and higher elevation being wetter than lower elevation (Figure 7.17 and Appendix D.5). For CCCMA 3.1 and CSIRO mk3.0, all three climate change periods are drier than the historical period with less probability of wet days (complementary to probability of dry days) and less mean wet day rainfall depths (Table 7.7), which might be reasonable considering climate change impact. However, for ECHAM5 and MIROC-medres 3.2, the probabilities of wet days are similar to other two GCMs and less than historical (drier), but the mean wet day rainfall depths are higher than other GCMs and historical (wetter). The trend of SD of wet day rainfall depths is mostly consistent with the trend of mean, except the CSIRO mk3.0 shows lower SD with a slightly higher mean compare to CCCMA 3.1. 

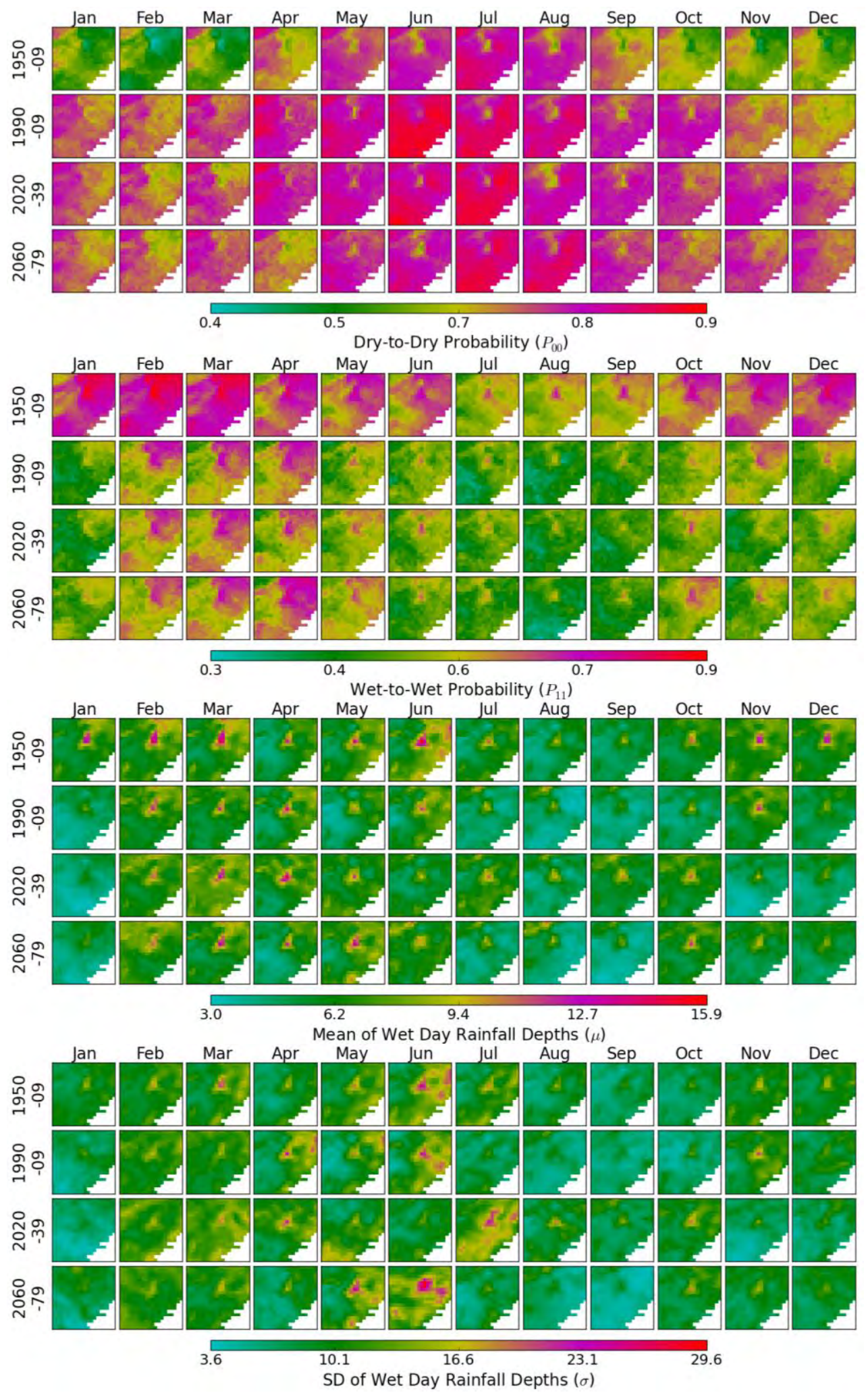

Figure 7.17: Intra-annual and spatial variability of model parameters (APMC values) for reanalysis and CCCMA 3.1 of RCM2. 
Table 7.7: Spatially and temporally averaged APMC values of model parameters for reanalysis and four GCMs of RCM2.

\begin{tabular}{c|c|cccc|cccc|cccc}
\hline & $\begin{array}{c}1950- \\
2009\end{array}$ & \multicolumn{4}{|c|}{$1990-2009$} & \multicolumn{4}{|c|}{$2020-2039$} & \multicolumn{5}{|c}{$2060-2079$} \\
& RE & CC & CS & EC & MI & CC & CS & EC & MI & CC & CS & EC & MI \\
\hline$P_{00}$ & 0.67 & 0.76 & 0.68 & 0.74 & 0.72 & 0.76 & 0.70 & 0.75 & 0.70 & 0.74 & 0.70 & 0.75 & 0.70 \\
$P_{11}$ & 0.66 & 0.53 & 0.58 & 0.54 & 0.55 & 0.53 & 0.57 & 0.54 & 0.57 & 0.54 & 0.57 & 0.55 & 0.56 \\
$\mu$ & 6.4 & 5.6 & 6.1 & 6.5 & 6.9 & 6.0 & 6.1 & 6.7 & 7.2 & 5.8 & 5.9 & 6.6 & 7.5 \\
$\sigma$ & 9.7 & 9.0 & 8.4 & 10.1 & 10.1 & 9.8 & 8.5 & 11.2 & 10.6 & 9.2 & 8.1 & 10.7 & 10.9 \\
\hline
\end{tabular}
3.2

\subsection{Discussion}

This study has calibrated the CDMC, HMC and DHMC in the Williams River site of the lower Hunter region using three NARCliM RCMs (reanalysis datasets) and the AWAP gridded dataset, for the 1950-2009 period. The performances of the three models in reproducing the key statistics of rainfall depths and wet periods have been assessed.

The intra-annual variability of the calibrated parameters to the observed datasets suggest that the rainfall in the Williams River site follows the typical east-Australian seasonality of wet summers and dry winters. Overall, the AWAP dataset is drier (lower probability of wet days and lower rainfall depths) than the RCMs in almost all seasons. While RCM2 is more similar to AWAP, the other two RCMs are considerably wetter. The spatial distributions of the parameters suggest that the rainfall depths and wet days tend to be higher in the higher elevations and lower in the lower elevations for all datasets except AWAP. In AWAP, the lower elevation coastal regions tend to be wetter than the higher elevation mountain regions. The link of model parameters to corresponding orography has been further confirmed by the correlation coefficients. Overall, the elevation-parameter correlations are strongest in RCM2, followed by RCM1, RCM3 and AWAP.

The distributions of observed mean and SD of rainfall depths at daily, monthly and multiyear resolutions indicate that the three RCMs have a similar distribution of rainfall depths, while the AWAP tends to be drier in all resolutions. In particularly, the mean and SD of multiyear rainfall in AWAP are considerably smaller than the RCMs. A similar scenario has been found for the statistics on wet periods, with the SD of multiyear number of wet days being considerably higher in AWAP than the same in RCMs. 
The CDMC, HMC and DHMC show comparable satisfactory performance to reproduce the mean and SD of rainfall depths at daily, monthly and multiyear resolutions. Although the observed statistics have been preserved within the $95 \%$ confidence interval of the model simulations, all three models tend to consistently underestimate the mean rainfall depths at all resolutions. Similarly, the models show a consistent tendency to underestimate the SD at daily resolution and overestimate the SD at monthly resolution.

As one of the key contributions of the CDMC, HMC, and DHMC, all three models have reproduced the SD of multiyear rainfall depths (at 1 to 10 year resolutions) very well, except the CDMC tends to significantly underestimate the statistic for 2 to 5 multiple years. The HMC and DHMC preserve the statistic for most of the pixels and multiple years of all datasets except overestimate in some pixels and multiple years of the AWAP, while the range of multiyear SD in AWAP is considerably lower than the same of the three RCMs.

The overall results suggest that the performances of the three models in reproducing the rainfall depths statistics are mostly similar as all three models use the same stochastic parameterisation of Gamma distribution for wet day rainfall depths simulation. However, the improved reproduction of multiyear SD in the HMC suggests that the stochastic parameterisation of the MC process, in addition to the stochastic parameters of Gamma distribution, has incorporated more variability in rainfall depths simulation of HMC than the same of CDMC (as CDMC only uses the stochastic parameters of Gamma distribution).

The assessment of wet periods statistics shows that the mean of the number of wet days and mean length of wet spells have been preserved well in CDMC, HMC and DHMC for all resolutions, although the HMC tends to overestimate the mean of wet period statistics at monthly resolution. However, for SD of wet period statistics at monthly to multiyear resolutions, the DHMC performs mostly satisfactorily (it only tends to underestimate the SD of wet days at shorter multiyear resolutions) and better than the CDMC and HMC. The CDMC mostly preserves the SD of monthly wet period statistics but tends to underestimate the SD of multiyear wet period statistics. The HMC preserves the SD of multiyear wet period statistics in all datasets except AWAP, but tends to significantly overestimate the SD of monthly wet period statistics. This suggests that the stochastic MC parameters in the HMC have introduced excessive variability in the wet period simulation process, particularly at monthly resolution. However, the SD of multiyear wet period statistics has been underestimated by both CDMC and HMC, which could be because of the very high observed multiyear SD in AWAP. 
Performance of each model for mean and SD of dry period statistics are similar to the respective performance for wet period statistics, which justifies the assessment of wet period statistics only in this thesis.

For each of the rainfall depths and wet periods statistics of all four datasets, this study has found that the $\mathrm{Z}$ scores of adjacent pixels are similar. This is likely to be the result of individual storm events affecting a large number of contiguous pixels. This interpretation is further supported by the high correlation of rainfall between adjacent pixels discussed in section 15.2 (see Figure 15.2). This also suggests that the rainfall in an individual pixel is not independent of surrounding pixels. These observations are important to understand the requirement of a singlesite or a multi-site version of the stochastic model for catchment-scale hydrology analysis.

For autocorrelation, the CDMC and DHMC performs better than the HMC to satisfactorily reproduce the autocorrelations of monthly rainfall depths and monthly number of wet days. The HMC tends to underestimate the autocorrelations.

Calibration of model parameters to NARCliM GCM data of CCCMA 3.1 and CSIRO mk3.0 (for RCM2) shows that the current (1990-2009) and future (2020-2039 and 2060-2079) periods are drier than the historical (1950-2009) reanalysis period with less probability of wet days and less mean wet day rainfall depths. This might be reasonable considering climate change impact. However, for ECHAM5 and MIROC-medres 3.2, the current and future periods show less probabilities of wet days (drier) but higher mean wet day rainfall depths (wetter) compare to the historical period. This study has assessed the NARCliM GCMs for RCM2 only as the analysis of the RCMs for reanalysis period shows that the RCM2 has more similarities to AWAP compare to RCM1 and RCM3.

The findings of this chapter for the Williams River site is consistent with the findings presented in the previous chapter for the Goulburn River site.

\subsection{Conclusion}

This study has calibrated the CDMC, HMC and DHMC in the Williams River site with the objective of assessing their ability to reproduce the variability of rainfall depths and wet periods in short as well as longer resolutions (daily, monthly and multiyear resolutions). In this regard, 
each model has advantages and limitations. The models reproduced the mean of rainfall depths and wet periods in all resolutions reasonably well. The overall performance of the DHMC is clearly better than the CDMC and HMC.

The DHMC mostly preserves the distribution and autocorrelations of rainfall depths and wet periods at daily to multiyear resolutions, although it tends to underestimate the SD of wet days at shorter multiyear resolutions. This model reproduced the SD of rainfall depths at all resolutions and SD of wet period statistics at monthly resolution, but significantly underestimated the SD of wet period statistics at multiyear resolution.

While the HMC performs in a similar way to the CDMC for other rainfall depths statistics, it improved the reproduction of SD of multiyear rainfall depths. However, the HMC only preserved the SD of multiyear wet period statistics, but significantly overestimated the SD of monthly wet period statistics. The overestimated SD of monthly wet period statistics has also affected the reproduction of month-to-month autocorrelations of rainfall depths and wet days in the HMC, while the autocorrelations are almost exactly reproduced by the CDMC and DHMC.

Despite the limitations, the models have shown an ability to reproduce most of the observed characteristics of rainfall. Therefore, the CDMC, HMC and DHMC need to be further assessed in stochastic streamflow generation to understand which of the respective advantages and limitations are important for urban drought security analysis. The further analysis of streamflow generation by using the CDMC, HMC and DHMC will be discussed in Chapter 15. The findings of this study will be an important basis for future studies to incorporate ECL influence in urban water planning and design. 


\section{Comparison of MC Models in Sydney Site}

\subsection{Introduction}

This chapter presents the comparative performances of the CDMC, HMC and DHMC in reproducing the distribution and autocorrelations of rainfall in the Sydney site of NSW, Australia. This site also has prominent ECL impacts [Pepler et al., 2014], while it is the source of urban water supply for the Sydney region [Mortazavi et al., 2013]. Several results presented for Sydney site in this chapter are similar to the results for Goulburn and Williams River sites (Chapter 6 and 7) probably because the seasonal and inter-annual rainfall variabilities of these three adjacent sites of coastal NSW (see Figure 3.2) are influenced by same type of large-scale climate drivers such as ENSO [Risbey et al., 2009]. However, the key reasons for presenting the results for Sydney site in this chapter are: (1) the site comprises both coastal and inland climate with distinctive topographical variability linked with the spatial variability of rainfall and (2) the site includes the reservoirs of Sydney water supply system, and therefore, this analysis shows the performances of the MC models at a major urban water supply site of NSW.

\subsection{Calibrated Parameters}

This section will present the parameters of the MC models (averaged APMC values of $P_{00}, P_{11}$, $\mu$ and $\sigma$ ), calibrated to the RCMs and AWAP for 652 land pixels of Sydney site (Figure 3.5).

\subsubsection{Intra-annual and Spatial Variability of Parameters}

Figure 8.1 and Figure 8.2 show the seasonal and spatial variability of the MC (i.e. $P_{00}$ and $P_{11}$ ) Gamma (i.e. $\mu$ and $\sigma$ ) distribution parameters respectively. For all four datasets, the seasonality shows wet summers (December-February) and dry winters (June-August), with two transitional seasons of autumn (March-May) and spring (September-November), which is consistent with the Goulburn and Williams River sites. Spatially (the NARCliM elevation map is shown Figure 3.5), for all four datasets, the steep escarpment adjacent to the east coastline is wetter (more wet days and higher rainfall depths) than the higher elevation regions in the west. This spatial characteristic is not consistent with the Goulburn and Williams River sites, as in both of those sites higher elevation was wetter than the lower elevation. However, the overall wetness among four datasets is consistent with Goulburn as RCM3 $>$ RCM1 $>$ RCM2 $>$ AWAP (Table 8.1). 
Table 8.1: Spatially and temporally averaged APMC values of model parameters.

\begin{tabular}{ccccc}
\hline Parameter & RCM1 & RCM2 & RCM3 & AWAP \\
\hline$P_{00}$ & 0.64 & 0.68 & 0.67 & 0.75 \\
$P_{11}$ & 0.65 & 0.63 & 0.64 & 0.66 \\
$\mu$ & 7.11 & 6.25 & 8.37 & 5.67 \\
$\sigma$ & 11.21 & 9.83 & 13.2 & 10.03 \\
\hline
\end{tabular}
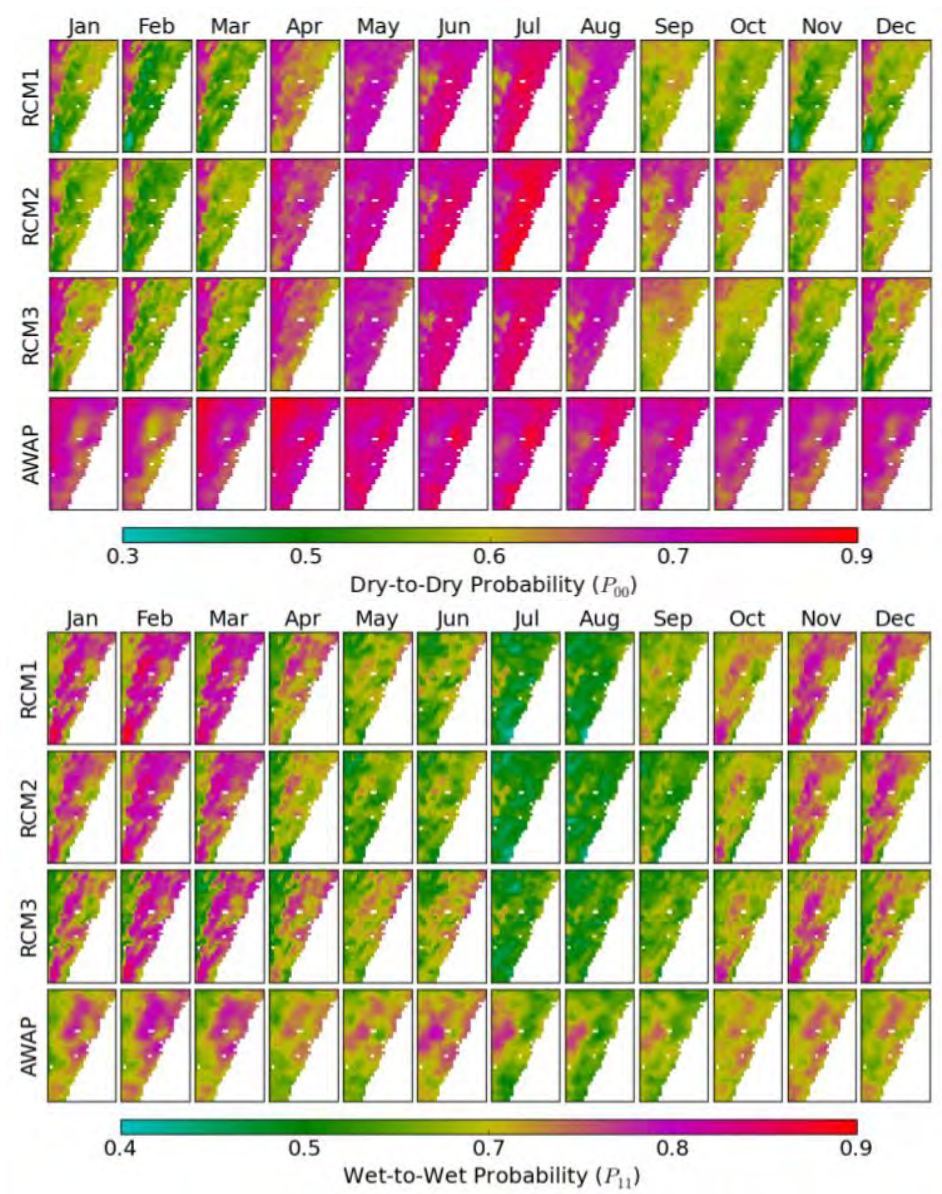

Figure 8.1: Intra-annual and spatial variability of dry-to-dry and wet-to-wet transition probabilities. The colourbars indicate the parameter values. 

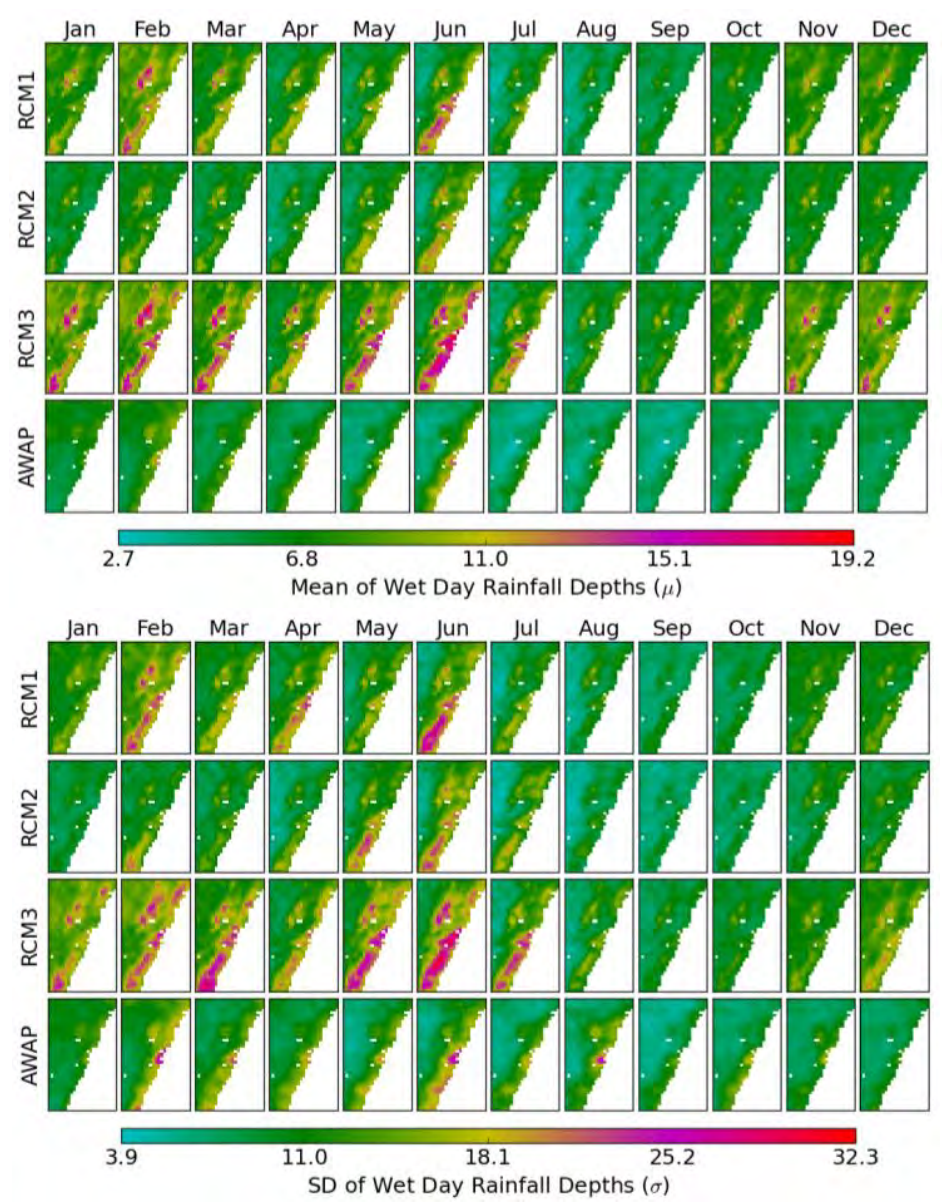

Figure 8.2: Intra-annual and spatial variability of mean and SD of wet day rainfall depths. The colourbars indicate the parameter values.

\subsubsection{Correlation between Elevation and Parameters}

The correlations between elevation and model parameters $P_{00}, P_{11}, \mu$, and $\sigma$ (averaged APMC values) are shown in Figure 8.3, which just indicate the influence of local orography on the model parameters. The elevation-parameters correlations for all four datasets follow a similar intra-annual trend. The negative correlations between elevation and $P_{00}$ are stronger in winter and weaker in summer, indicating more dry days at lower elevation, particularly in winter. The weak correlations between elevation and $P_{11}$ are mostly negative except some summer months. The correlations between elevation and $\mu$ are also mostly negative and strong in winter, indicating lower rainfall at higher elevations. The correlation between elevation and $\sigma$ are mostly strong and negative. Overall, the elevation-parameter correlations are stronger in AWAP than the RCMs (Table 8.2). 

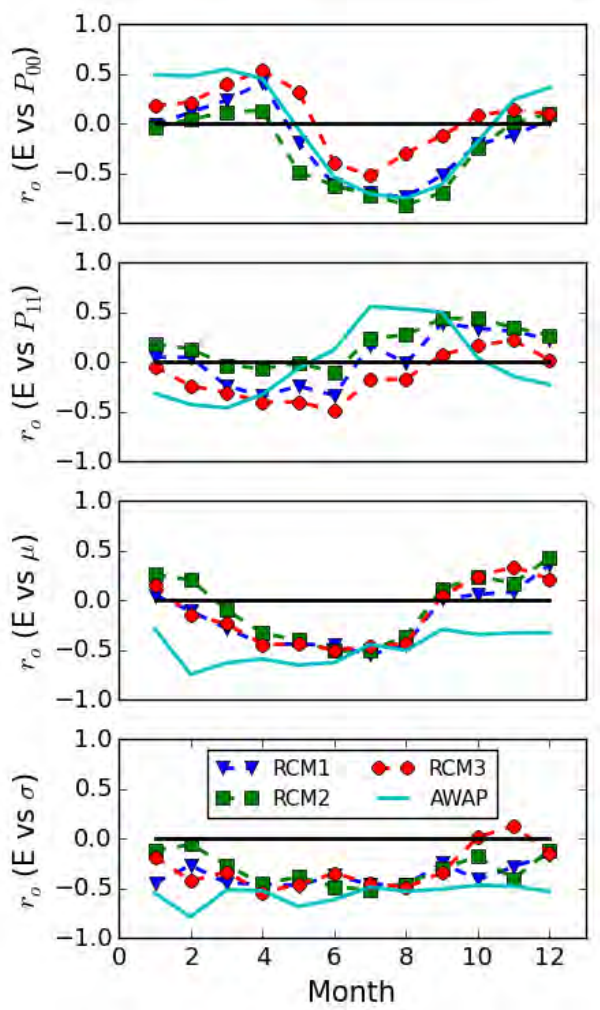

Figure 8.3: Correlation between elevation and model parameters for each month.

Table 8.2: Average of the absolute values of correlation coefficients between elevation and parameters.

\begin{tabular}{ccccc}
\hline Correlation & RCM1 & RCM2 & RCM3 & AWAP \\
\hline E vs $P_{00}$ & 0.33 & 0.34 & 0.28 & 0.45 \\
E vs $P_{11}$ & 0.23 & 0.21 & 0.23 & 0.31 \\
E vs $\mu$ & 0.27 & 0.3 & 0.31 & 0.48 \\
E vs $\sigma$ & 0.37 & 0.31 & 0.32 & 0.56 \\
\hline
\end{tabular}

\subsection{Distribution Statistics of Rainfall Depths and Wet Periods}

The spatial and intra-annual variability of the distribution statistics of rainfall depths and wet periods are mostly consistent with the variability of the model parameters (see Appendix E.1). While the RCMs show similar characteristics of rainfall depths and wet period distributions, the AWAP shows lower variability of rainfall depths (Figure 8.4) and higher variability of wet days (Figure 8.5), particularly at multiyear resolutions. Among RCMs, the RCM3 shows more variability of multiyear rainfall depths compared to the RCM1 and RCM2. Table 8.3 shows the spatially and temporally averaged values of the statistics, which indicates that the rainfall 
characteristic in each dataset for Sydney is similar to the rainfall characteristics in respective dataset for Goulburn.

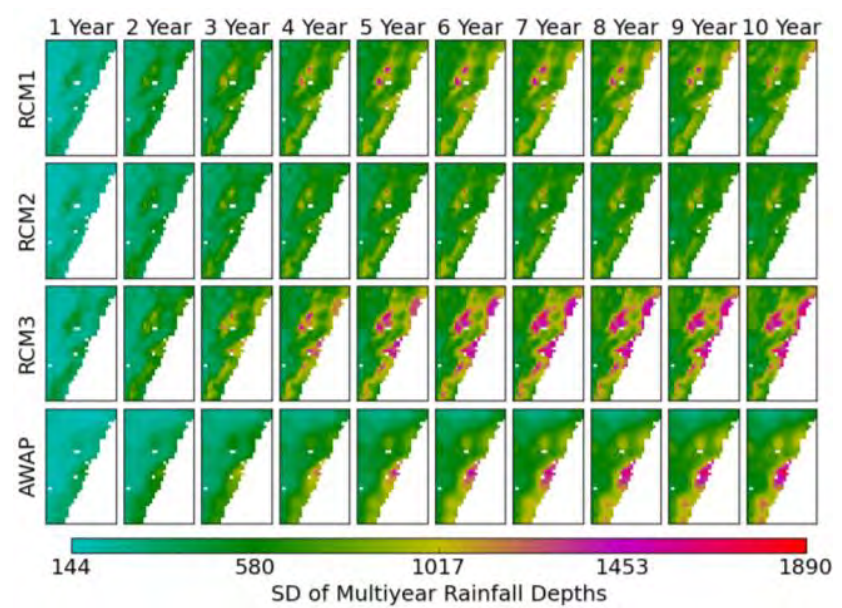

Figure 8.4: Spatial variability of the SD of multiyear rainfall depths.

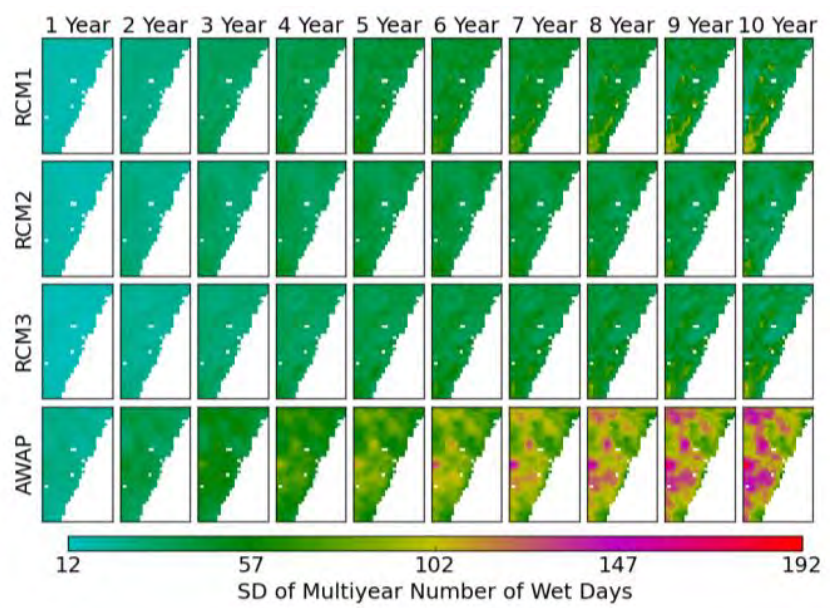

Figure 8.5: Spatial variability of the SD of multiyear number of wet days.

Table 8.3: Spatially and temporally averaged values of the mean and SD of rainfall depth and wet period statistics at annual and multiyear resolutions.

\begin{tabular}{cccccc}
\hline \multicolumn{2}{c}{ Statistics } & RCM1 & RCM2 & RCM3 & AWAP \\
\hline Rainfall & Annual Mean & 1345 & 1078 & 1495 & 876 \\
\cline { 2 - 6 } Depth (mm) & Multiyear SD & 615 & 531 & 710 & 546 \\
\hline Number of & Annual Mean & 185 & 170 & 174 & 153 \\
\cline { 2 - 6 } Wet Days & Multiyear SD & 41 & 38 & 38 & 74 \\
\hline Mean Length & Annual Mean & 3.03 & 2.87 & 2.88 & 2.96 \\
\cline { 2 - 6 } of Wet Spell & Annual SD & 0.39 & 0.38 & 0.34 & 0.55 \\
\hline
\end{tabular}




\subsection{Model Comparison for Distribution of Rainfall Depths}

This section compares the CDMC, HMC and DHMC for the mean and SD of rainfall depths (as per the assessment method discussed in section 3.4).

\subsubsection{Mean of Rainfall Depths at Daily, Monthly and Multiyear Resolutions}

For mean of rainfall depths at daily, monthly and multiyear resolutions, the three models show similar satisfactory performances with $\mathrm{Z}$ scores mostly between -1 and +1 for all four datasets. However, the $Z$ scores of the models are mostly positive (see Figure E.11-E.13 at Appendix E.2), indicating a tendency of the models to underestimate the mean of rainfall depths at all resolutions.

\subsubsection{SD of Daily and Monthly Rainfall Depths}

The three models show comparable satisfactory performances to reproduce the SD of rainfall depths at daily and monthly resolutions with $\mathrm{Z}$ scores mostly between -1 and +1 for all four datasets (see Figure E.14-E.15 at Appendix E.2). However, the models tend to overestimate the $\mathrm{SD}$ of rainfall depths at monthly resolution (mostly negative $\mathrm{Z}$ scores). The tendency to overestimate is higher in the HMC compared to the DHMC and CDMC.

\subsubsection{SD of Multiyear Rainfall Depths}

As for the Goulburn and Williams River sites, all three models satisfactorily reproduce the SD of multiyear rainfall depths with a tendency to underestimate for the RCMs and a tendency to overestimate for the AWAP (Figure 8.6). The HMC performs better than the CDMC, as the CDMC significantly underestimated the statistic for shorter multiyear resolutions (e.g. 2 to 5 overlapping years). Particularly for RCM2, Z scores of CDMC for shorter multiyear resolutions are mostly between +1 and +2 , while in a number pixels the $Z$ scores are greater than +2 . The DHMC also tends to underestimate the SD of multiyear rainfall depths at shorter multiyear resolutions, but performs better than the CDMC. 
8. Comparison of MC Models in Sydney Site
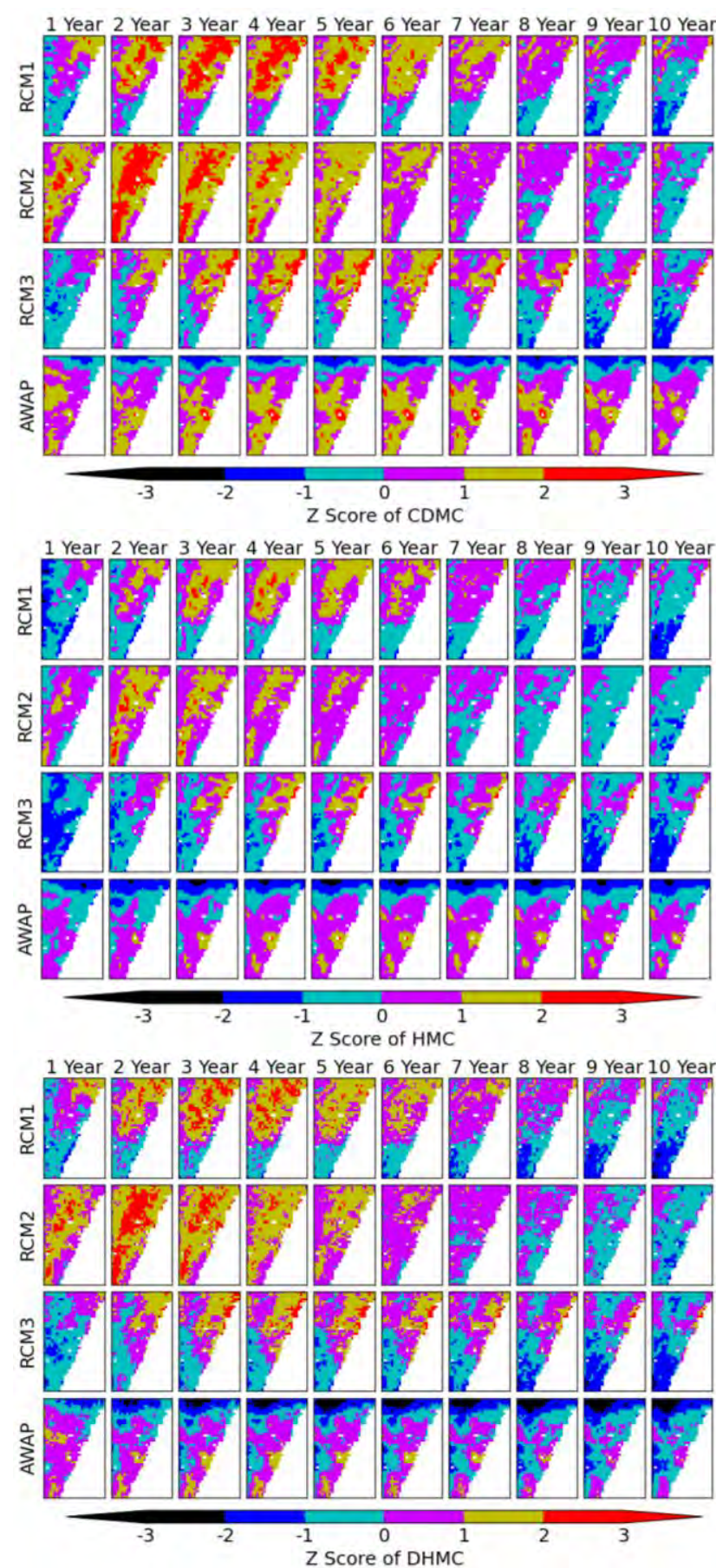

Figure 8.6: Z Scores of CDMC, HMC and DHMC for the SD of multiyear rainfall depths. 


\subsection{Model Comparison for Distribution of Wet Periods}

This section compares the CDMC, HMC and DHMC for the mean and SD of number of wet days and mean length of wet spells (as per the assessment procedures described in section 3.4).

\subsubsection{Mean of Monthly and Annual Number of Wet Days}

For the mean of number of wet days at monthly and annual resolutions, the three models show a similar satisfactory performance with $\mathrm{Z}$ scores mostly between -1 and +1 for all four datasets (see Figure E.16-E.17 at Appendix E.3). However, the DHMC and CDMC perform better than the $\mathrm{HMC}$, as the $\mathrm{HMC}$ tends to overestimate the statistics (mostly negative $\mathrm{Z}$ scores).

\subsubsection{Mean of Monthly and Annual Mean Length of Wet Spell}

For mean of monthly and annual mean length of wet spell, the performance of each model is consistent with their performance for the mean of monthly and annual number of wet days. The CDMC and DHMC perform better than the HMC, as the HMC tends to overestimate the statistics (see Figure E.18-E.19 at Appendix E.3).

\subsubsection{SD of Monthly Number of Wet Days and Monthly Mean Length of Wet Spells}

For the SD of monthly number of wet days of all four datasets, the CDMC and DHMC tend to underestimate the statistic for summer, while the HMC tends to overestimate the statistic for all months (Figure 8.7). However, the DHMC performs better than the CDMC and HMC. Compared to DHMC, the CDMC tends to more consistently and significantly underestimate the SD of monthly number of wet days, while the HMC tends to significantly overestimate the statistic for most of the pixels of all four datasets.

For SD of monthly mean length of wet spells, the performance of each model is similar to their performance for SD of monthly number of wet days (see Figure E.20 at Appendix E.3). However, each model performs slightly better for SD of monthly mean length of wet spells than for SD of monthly number of wet days. 

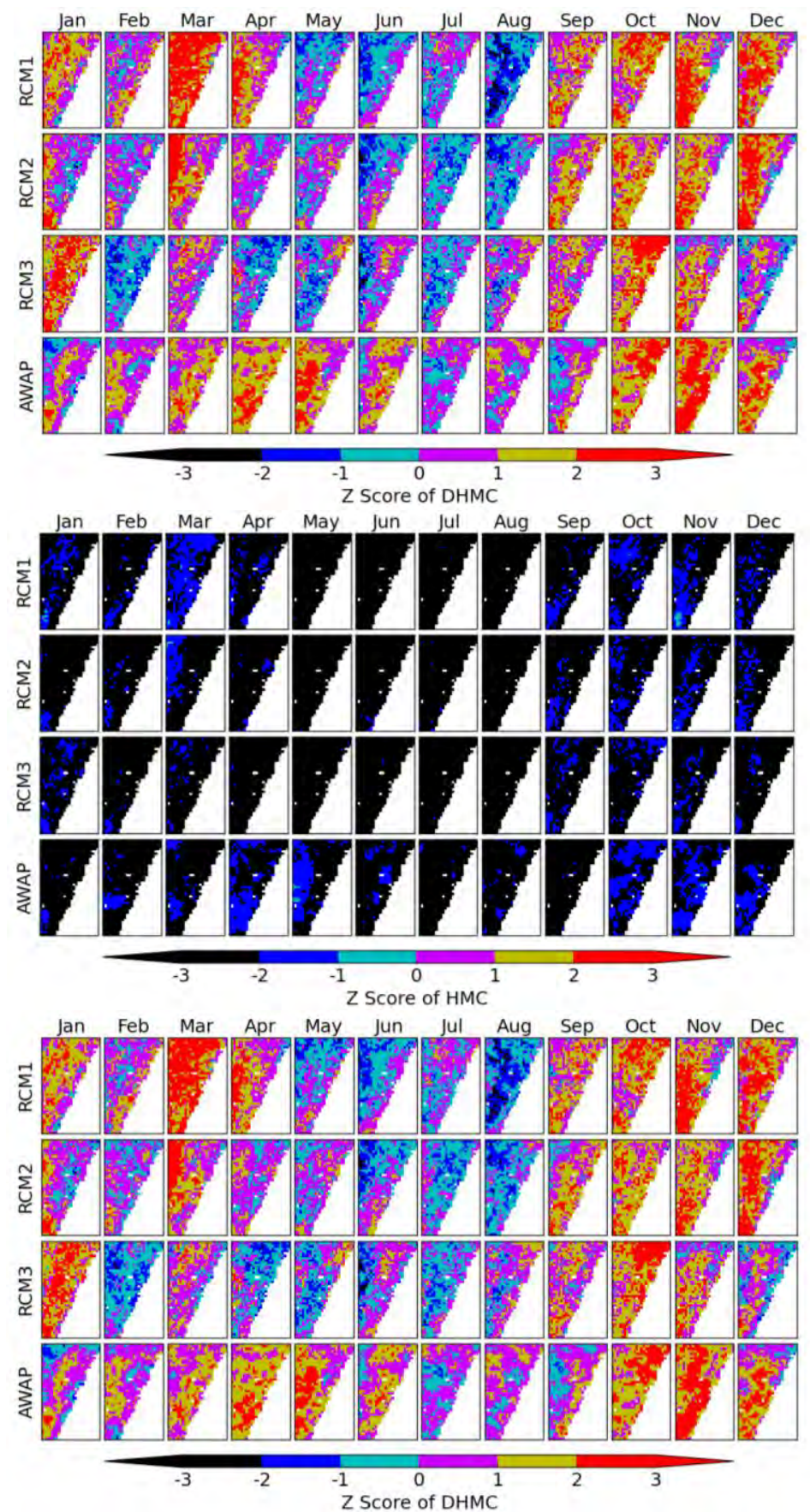

Figure 8.7: Z Scores of CDMC, HMC and DHMC for the SD of monthly number of wet days. 


\subsubsection{SD of Multiyear Number of Wet Days and Annual Mean Wet Spell Length}

For SD of multiyear number of wet days, the performance of each model in the Sydney site is consistent with their respective performances for the Goulburn and Williams River sites. The DHMC mostly preserves the SD of multiyear number of wet days for all datasets with a tendency to underestimate the statistic at shorter multiyear resolution (Figure 8.8). This performance is better than the performances of the CDMC and HMC to reproduce the statistic (Figure 8.8). The CDMC tends to significantly underestimate the statistic for most of the multiple years of each dataset. However, the HMC performs satisfactorily for the RCMs, but significantly underestimates the statistic for AWAP (the AWAP has higher variability of multiyear number of wet days than the RCMs).

For SD of annual mean length of wet spells, the DHMC performs better than the CDMC and HMC to reproduce the statistic with a tendency to underestimate (Figure 8.9). The CDMC tends to significantly underestimate the statistics, while the HMC tends to significantly overestimate the statistic. 

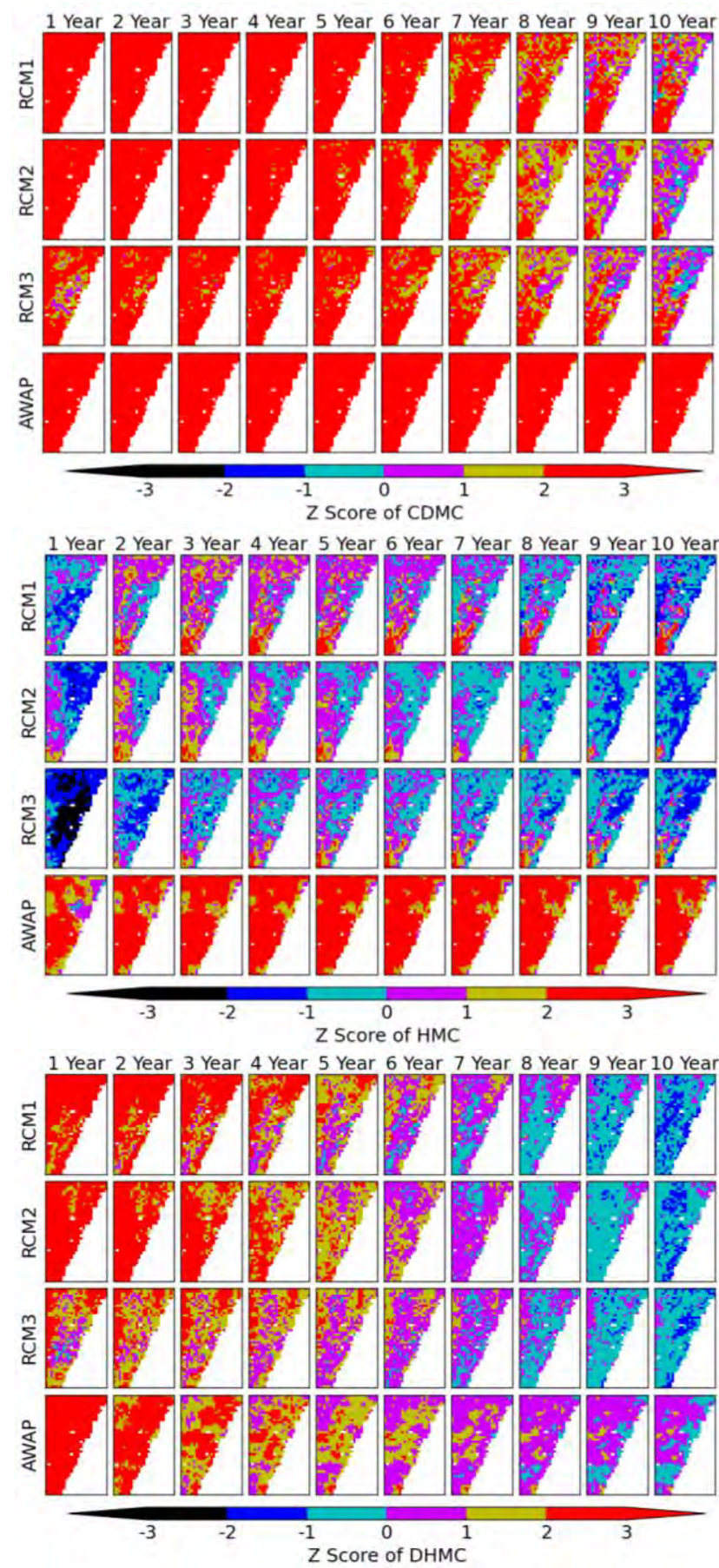

Figure 8.8: Z Scores of CDMC, HMC and DHMC for the SD of multiyear number of wet days. 

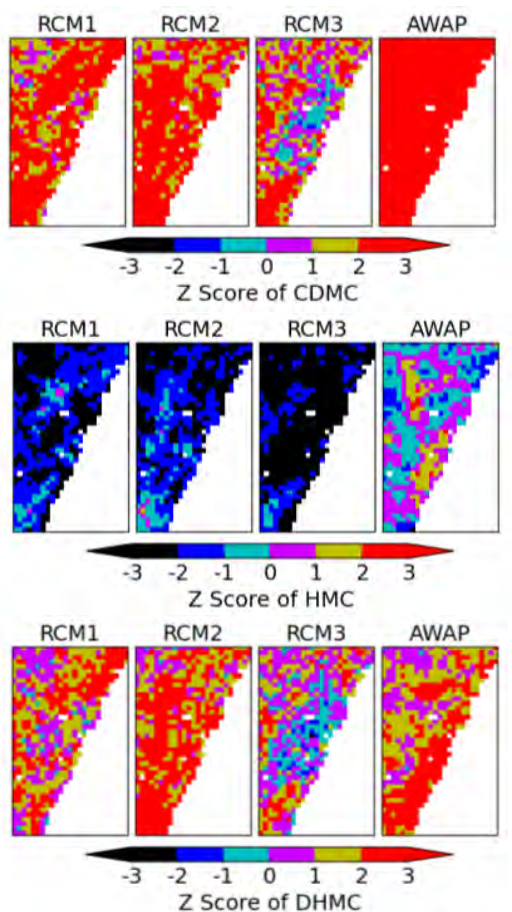

Figure 8.9: Z Scores of CDMC, HMC and DHMC for the SD of annual mean length of wet spells.

\subsection{Summary of Model Comparison for Distribution Statistics}

Table 8.4 and Table 8.5 show the spatially and temporally averaged $\mathrm{Z}$ scores for mean and SD of rainfall depths and wet period statistics respectively. The performances of all three models are similar for rainfall depths (Table 8.4), while DHMC performs better than the CDMC and HMC for wet periods (Table 8.5), which is consistent with the respective findings for Goulburn and Williams (see section 6.6 and 7.6 respectively). 
8. Comparison of MC Models in Sydney Site

Table 8.4: Spatially and temporally average of the absolute value of $\mathrm{Z}$ scores for mean and SD of rainfall depths.

\begin{tabular}{|c|c|c|c|c|c|}
\hline \multirow{2}{*}{ Statistics } & \multirow{2}{*}{ Models } & \multicolumn{4}{|c|}{ Spatially and Temporally Averaged Z Scores } \\
\hline & & RCM1 & $\mathrm{RCM} 2$ & RCM3 & AWAP \\
\hline \multirow{3}{*}{ Daily Mean } & CDMC & 0.33 & 0.45 & 0.32 & 0.36 \\
\hline & HMC & 0.32 & 0.43 & 0.31 & 0.34 \\
\hline & DHMC & 0.34 & 0.46 & 0.33 & 0.37 \\
\hline \multirow{3}{*}{ Daily SD } & CDMC & 0.25 & 0.31 & 0.25 & 0.36 \\
\hline & $\mathrm{HMC}$ & 0.25 & 0.32 & 0.25 & 0.35 \\
\hline & DHMC & 0.25 & 0.32 & 0.25 & 0.36 \\
\hline \multirow{3}{*}{ Monthly Mean } & CDMC & 0.31 & 0.42 & 0.3 & 0.34 \\
\hline & $\mathrm{HMC}$ & 0.3 & 0.38 & 0.27 & 0.28 \\
\hline & DHMC & 0.32 & 0.43 & 0.31 & 0.35 \\
\hline \multirow{3}{*}{ Monthly SD } & CDMC & 0.49 & 0.54 & 0.48 & 0.55 \\
\hline & HMC & 0.77 & 0.69 & 0.77 & 0.79 \\
\hline & DHMC & 0.52 & 0.56 & 0.51 & 0.59 \\
\hline \multirow{3}{*}{$\begin{array}{c}\text { Multiyear } \\
\text { Mean }\end{array}$} & CDMC & 0.95 & 1.41 & 0.95 & 0.98 \\
\hline & HMC & 0.89 & 1.21 & 0.85 & 0.71 \\
\hline & DHMC & 0.96 & 1.43 & 0.96 & 1 \\
\hline \multirow{3}{*}{ Multiyear SD } & CDMC & 0.93 & 1.02 & 0.77 & 0.88 \\
\hline & HMC & 0.69 & 0.58 & 0.7 & 0.68 \\
\hline & DHMC & 0.83 & 0.89 & 0.76 & 0.79 \\
\hline \multirow{3}{*}{ Sum } & CDMC & 3.26 & 4.15 & 3.07 & 3.47 \\
\hline & НMC & 3.22 & 3.61 & 3.15 & 3.15 \\
\hline & DHMC & 3.22 & 4.09 & 3.12 & 3.46 \\
\hline
\end{tabular}


Table 8.5: Spatially and temporally average of the absolute value of $\mathrm{Z}$ scores for mean and SD of wet period statistics.

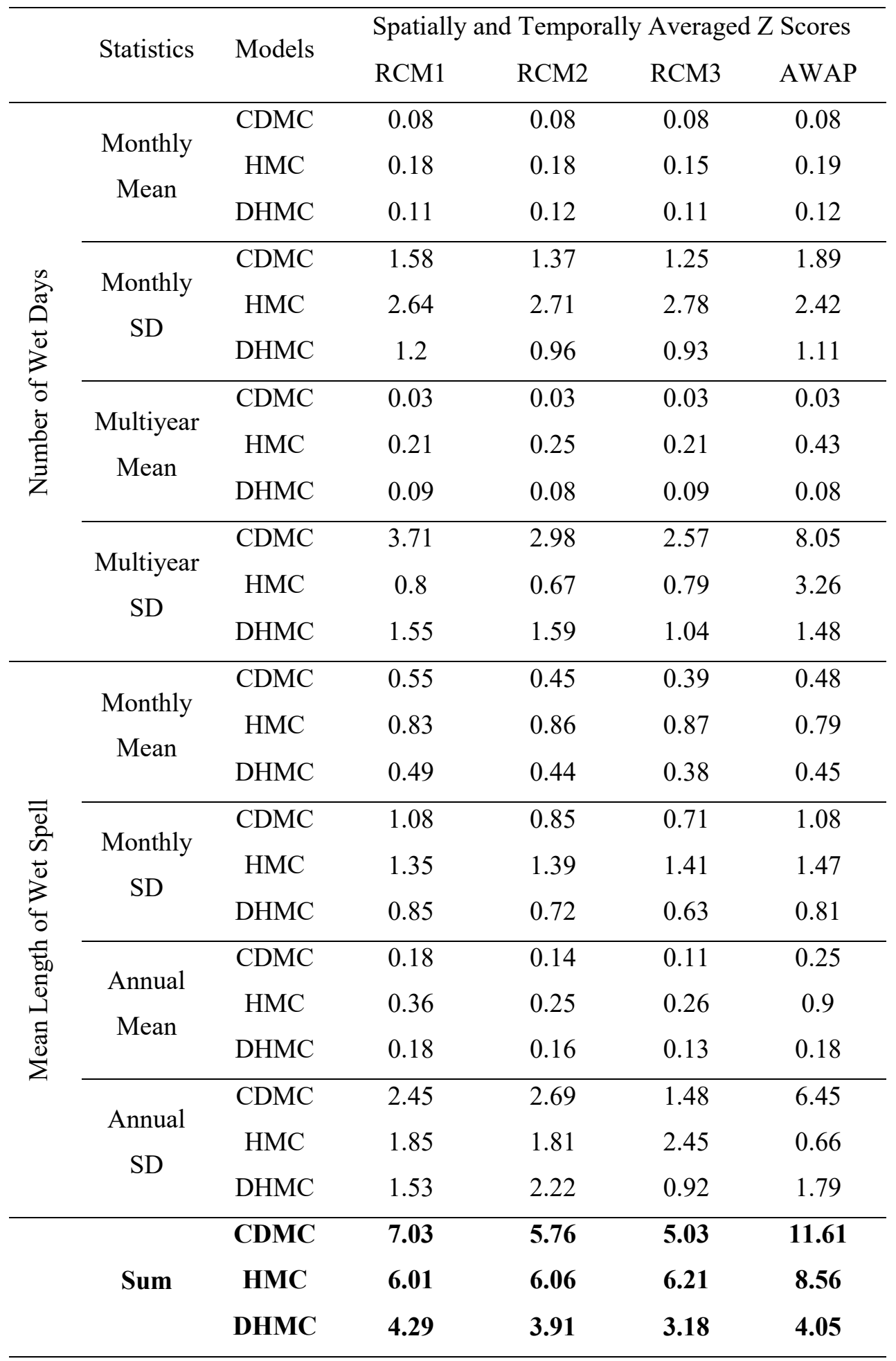




\subsection{Model Comparison for Autocorrelation}

Figure 8.10 and Figure 8.11 show that the autocorrelations of monthly rainfall depths and monthly wet days for the Sydney site are consistent (stronger in lag-1, lag-6, lag-7 and lag-12 with clear seasonal variations) with the respective autocorrelations for the Goulburn and Williams River sites, as shown in Chapters 6 and 7. The performances of the three models for the autocorrelations are also consistent with their respective performances for the previous two sites with similar performance for autocorrelation of rainfall depths while HMC tends to underestimate the autocorrelations of monthly number of wet days (see Figure E.21-E.22 at Appendix E.4 and Table 8.6).

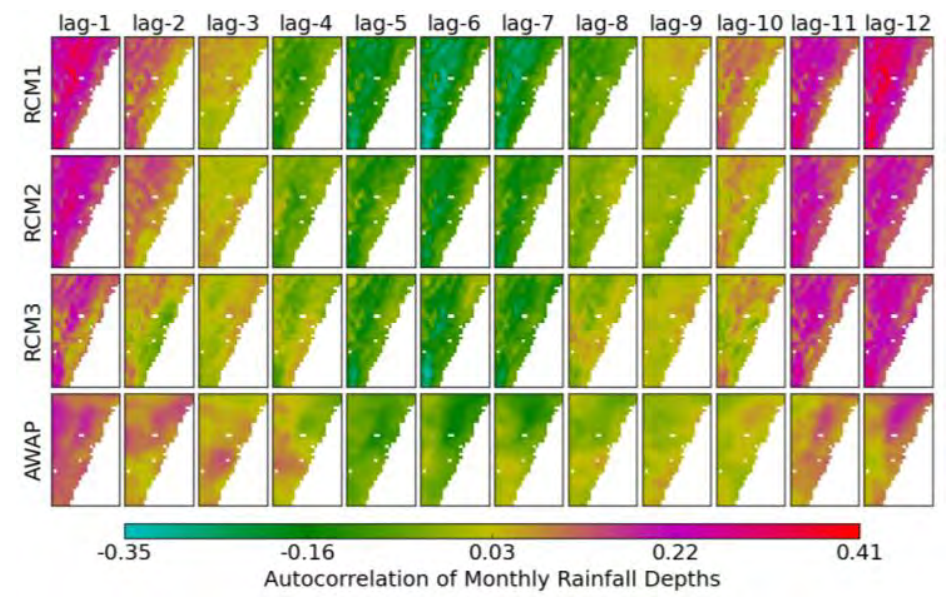

Figure 8.10: Month-to-month autocorrelations of monthly rainfall depths in all RCMs and AWAP.

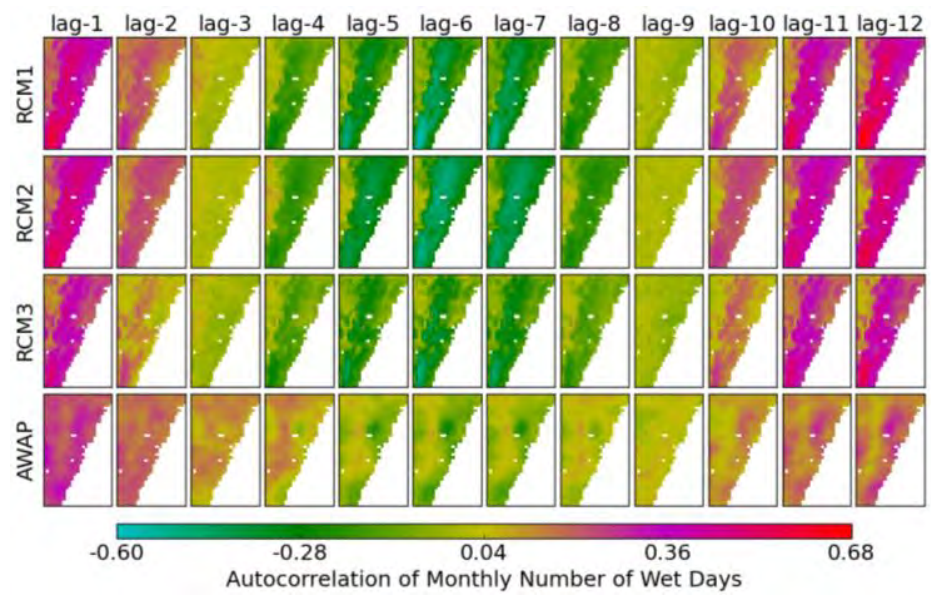

Figure 8.11: Month-to-month autocorrelations of monthly number of wet days in all RCMs and AWAP. 
Table 8.6: Spatial (all pixels) and temporal (all lags 1-12) average of autocorrelations of monthly rainfall depths and number of wet days.

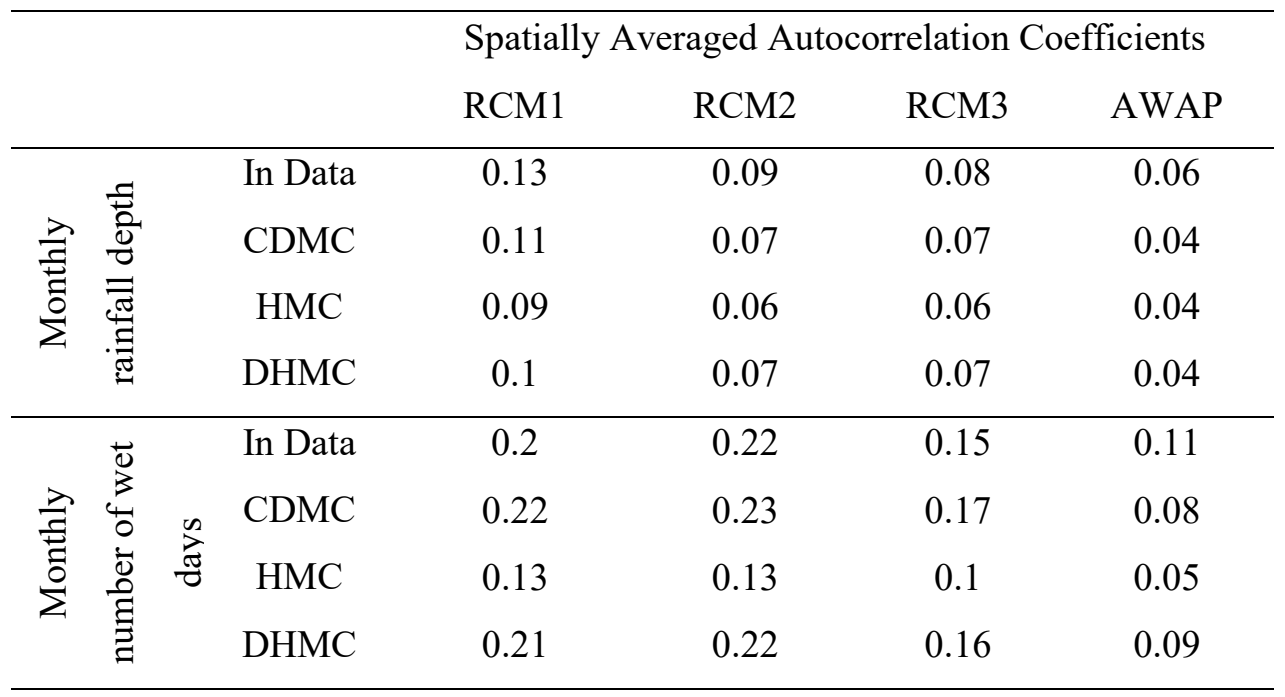

\subsection{Discussion}

This study has calibrated the CDMC, HMC and DHMC in the Sydney site using three NARCliM RCMs (reanalysis datasets) and AWAP gridded dataset, for the 1950-2009 period. The intra-annual variability of the calibrated parameters to the four datasets suggest that the rainfall in the Sydney site follows the typical east-Australian seasonality of wet summers and dry winters. Spatially, the steep escarpment adjacent to the east coastline is wetter (more wet days and higher rainfall depths) than the higher elevation regions in the west. This spatial characteristic is not consistent with the Goulburn and Williams River sites, as in both of those sites higher elevation was wetter than the lower elevation.

The performance of the three models has been assessed in reproducing the key statistics of rainfall depths and wet periods. The comparative performances of the models are very similar to their performances for the Goulburn and Williams River sites. The overall performance of the DHMC is clearly better than the CDMC and HMC as it mostly preserves the distribution and autocorrelations of rainfall depths and wet periods at daily to multiyear resolutions, except for a tendency to underestimate the SD of wet days at shorter multiyear resolutions. While other performances are mostly comparable to DHMC, the CDMC significantly underestimated the SD of wet period statistics at multiyear resolution, while the HMC significantly overestimated the SD of monthly wet period statistics. 
8. Comparison of MC Models in Sydney Site

\subsection{Conclusion}

The comparative assessments presented in this chapter suggest that the DHMC is a better model than the CDMC and HMC for stochastic simulation of rainfall in the Sydney site. The DHMC shows a better ability to preserve the distribution and autocorrelations of rainfall depths and wet periods at daily to multiyear resolutions, which are critically important for the urban water security assessment in the Sydney site considering ECL influence. This performance of DHMC is consistent irrespective of the intra-annual and spatial variability of rainfall in the site. The satisfactory performance of the DHMC is also mostly consistent for the NARCliM RCMs and AWAP datasets. Moreover, this conclusion is consistent with the conclusion for the Goulburn and Williams River sites, discussed in previous two chapters. 


\section{Comparison of MC Models in Richmond River Site}

\subsection{Introduction}

This chapter presents the comparative performances of the CDMC, HMC and DHMC to reproduce the distribution and autocorrelations of rainfall in the Richmond River site of the northern coast of NSW, Australia. This site is in the region of the greatest impact of ECLs [Kiem et al., 2016], and so this chapter will show the performances of the MC models in a site that has the greatest influence from ECLs. While several results of this chapter are similar to the results of Chapter 6,7 and 8 , still these results show the rainfall variability and performances of the MC models at a site of few hundred kilometres north of the other three sites which has distinctive storm characteristics [Kiem et al., 2016] with the influence of different large-scale climate drivers [Risbey et al., 2009].

\subsection{Calibrated Parameters}

This section will present the parameters of MC models (averaged APMC values of $P_{00}, P_{11}, \mu$ and $\sigma$ ) calibrated to the RCMs and AWAP for 331 land pixels of the Richmond River site (Figure 3.6).

\subsubsection{Intra-annual and Spatial Variability of Parameters}

Figure 9.1 and Figure 9.2 show the seasonal and spatial variability of the MC (i.e. $P_{00}$ and $P_{11}$ ) Gamma (i.e. $\mu$ and $\sigma$ ) distribution parameters respectively. For all four datasets, the seasonality shows wet summers (higher $P_{11}$ and $\mu$ ) and dry winters (higher $P_{00}$ and lower $\mu$ ), which is consistent with the Goulburn, Williams River and Sydney sites. Spatially (the NARCliM elevation map is shown Figure 3.6), for all four datasets, the summers are wetter at higher elevation, while the winters are drier at higher elevation. Similar to previous three study sites, the AWAP is drier than the RCMs with an order of wetness as RCM3 > RCM1 > RCM2 > AWAP (Table 9.1). 
Table 9.1: Spatially and temporally averaged APMC values of model parameters.

\begin{tabular}{ccccc}
\hline Parameter & RCM1 & RCM2 & RCM3 & AWAP \\
\hline$P_{00}$ & 0.61 & 0.62 & 0.65 & 0.74 \\
$P_{11}$ & 0.71 & 0.7 & 0.68 & 0.69 \\
$\mu$ & 8.25 & 7.3 & 9.79 & 6.45 \\
$\sigma$ & 12.62 & 11.49 & 15.31 & 11.51 \\
\hline
\end{tabular}
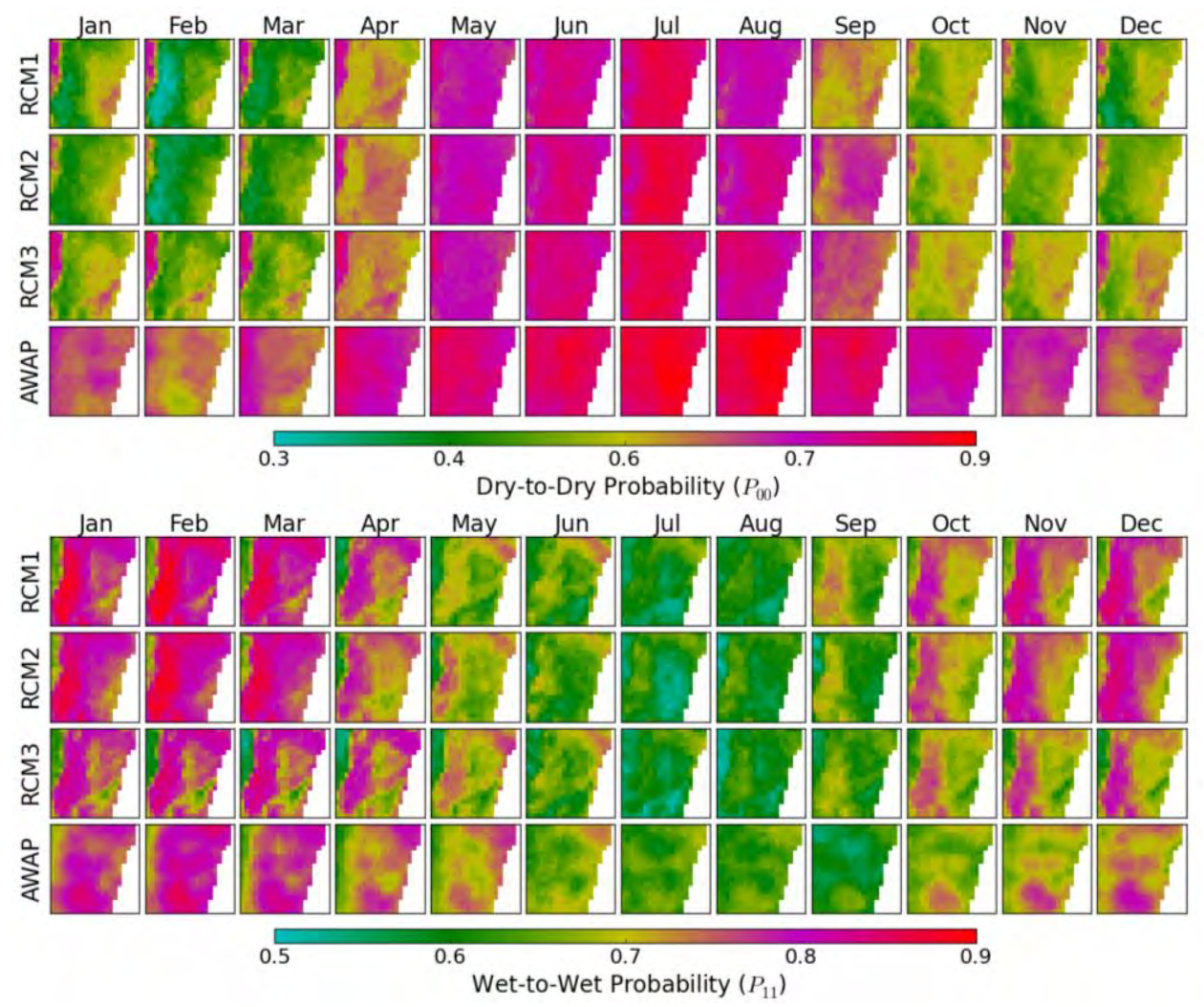

Figure 9.1: Intra-annual and spatial variability of dry-to-dry and wet-to-wet transition probabilities. The colourbars indicate the parameter values. 

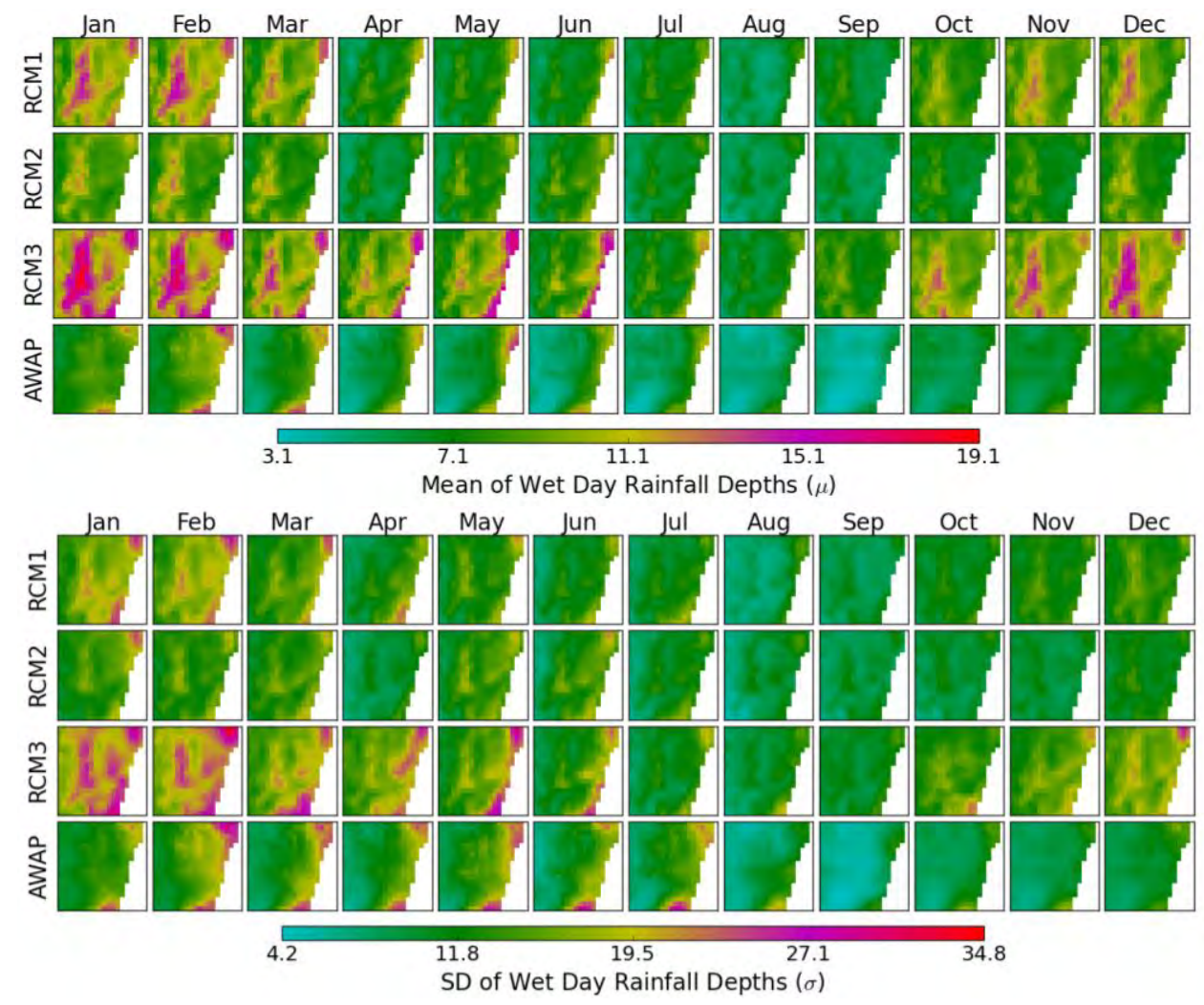

Figure 9.2: Intra-annual and spatial variability of mean and SD of wet day rainfall depths. The colourbars indicate the parameter values.

\subsubsection{Correlation between Elevation and Parameters}

The correlations between elevation and model parameters $P_{00}, P_{11}, \mu$, and $\sigma$ (averaged APMC values) are shown in Figure 9.3. These results indicate the characteristics and strength of the influence of local orography on the spatial variabilities of the model parameters. The correlations between elevation and $P_{00}$ are mostly negative for all datasets except RCM3, indicating more dry days at lower elevation. For RCMs, the correlations between elevation and $P_{11}$ are positive and strong for summer, indicating more wet days at higher elevation in summer. However, for AWAP, the correlations between elevation and $P_{11}$ are mostly negative. For RCMs, the correlations between elevation and $\mu$ are negative and strong in winter (i.e. drier at higher elevation), while positive and weak in summer (i.e. wetter at higher elevation). However, for AWAP, the correlations between elevation and $\mu$ are negative for all months. The correlation between elevation and $\sigma$ are mostly negative for all datasets, indicating more variability of rainfall depths at lower elevation. Overall, the elevation-parameter correlations are slightly stronger in AWAP than the RCMs (Table 9.2). 

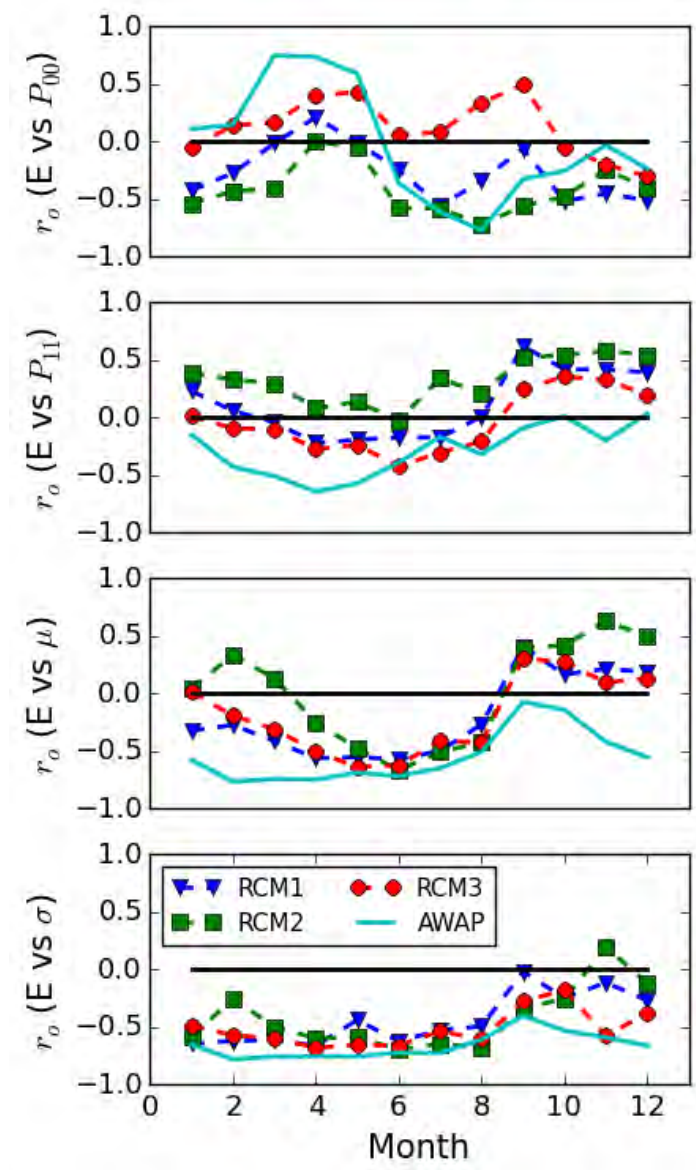

Figure 9.3: Correlation between elevation and model parameters for each month.

Table 9.2: Average of the absolute values of correlation coefficients between elevation and parameters.

\begin{tabular}{ccccc}
\hline Correlation & RCM1 & RCM2 & RCM3 & AWAP \\
\hline E vs $P_{00}$ & 0.3 & 0.42 & 0.22 & 0.41 \\
E vs $P_{11}$ & 0.25 & 0.33 & 0.23 & 0.3 \\
E vs $\mu$ & 0.37 & 0.4 & 0.33 & 0.55 \\
E vs $\sigma$ & 0.44 & 0.46 & 0.52 & 0.66 \\
\hline
\end{tabular}

\subsection{Distribution Statistics of Rainfall Depths and Wet Periods}

As for the previous three sites, the spatial and intra-annual variability of the distribution statistics are mostly consistent with the variability of the model parameters (see Appendix F.1). While the RCMs show similar characteristics of rainfall depths and wet period distributions, the AWAP shows lower variability of rainfall depths (Figure 9.4) and higher variability of wet days (Figure 9.5), particularly at multiyear resolutions (consistent with Goulburn, Williams River 
and Sydney sites). The spatially and temporally averaged statistics (Table 9.3) also show that the AWAP is drier (lower mean rainfall depths and number of wet days) than the RCMs with lower variability of rainfall depths but higher variability of wet days.

Table 9.3: Spatially and temporally averaged values of the mean and SD of rainfall depth and wet period statistics at annual and multiyear resolutions.

\begin{tabular}{cccccc}
\hline \multicolumn{2}{c}{ Statistics } & RCM1 & RCM2 & RCM3 & AWAP \\
\hline Rainfall & Annual Mean & 1774 & 1525 & 1932 & 1138 \\
\cline { 2 - 6 } Depth (mm) & Multiyear SD & 821 & 693 & 883 & 557 \\
\hline \multirow{2}{*}{$\begin{array}{c}\text { Number of } \\
\text { Wet Days }\end{array}$} & Annual Mean & 205 & 202 & 189 & 168 \\
\cline { 2 - 6 } & Multiyear SD & 44 & 36 & 46 & 70 \\
\hline Mean Length & Annual Mean & 3.76 & 3.72 & 3.37 & 3.48 \\
\cline { 2 - 6 } of Wet Spell & Annual SD & 0.54 & 0.54 & 0.47 & 0.65 \\
\hline
\end{tabular}

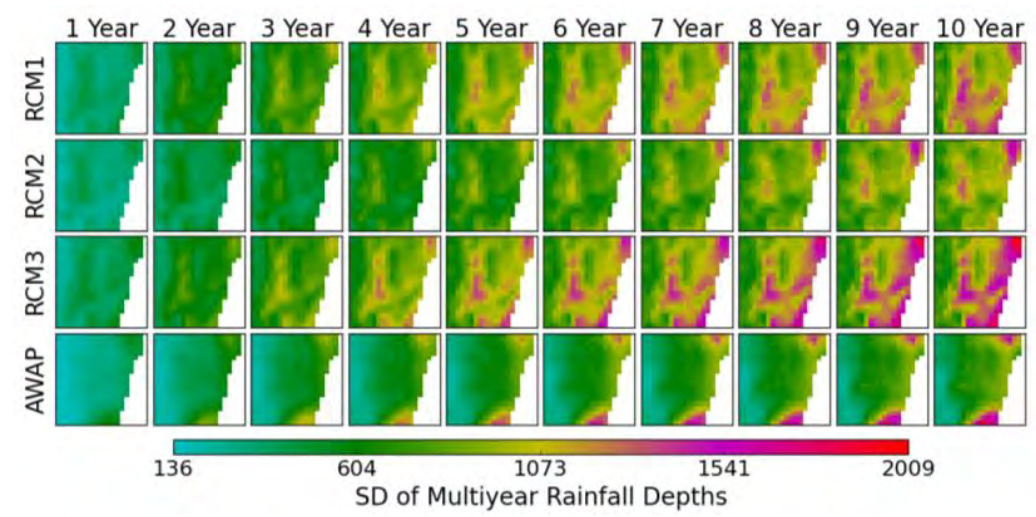

Figure 9.4: Spatial variability of the SD of multiyear rainfall depths.

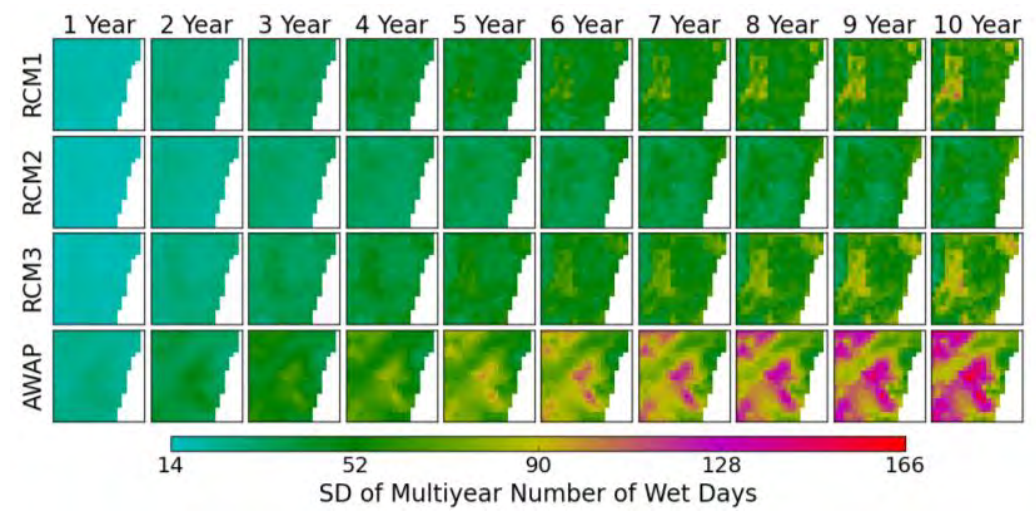

Figure 9.5: Spatial variability of the SD of multiyear number of wet days. 


\subsection{Model Comparison for Distribution of Rainfall Depths}

This section compares the CDMC, HMC and DHMC for the mean and SD of rainfall depths (as per the assessment method discussed in section 3.4).

\subsubsection{Mean of Rainfall Depths at Daily, Monthly and Multiyear Resolutions}

As for the previous three sites, the CDMC, HMC and DHMC perform similarly and satisfactorily for mean of rainfall depths at daily, monthly and multiyear resolutions, with a tendency to underestimate the mean of rainfall depths at all resolutions (see Figure F.11-F.13 at Appendix F.2).

\subsubsection{SD of Daily and Monthly Rainfall Depths}

As for previous three sites, the CDMC, HMC and DHMC show comparable satisfactory performance to reproduce the SD of rainfall depths at daily and monthly resolutions with a tendency to overestimate the SD of rainfall depths at monthly resolution (see Figure F.14-F.15 at Appendix F.2).

\subsubsection{SD of Multiyear Rainfall Depths}

As for previous three sites, all three models satisfactorily reproduce the SD of multiyear rainfall depths with a tendency to underestimate for the RCMs and a tendency to overestimate for the AWAP (Figure 9.6). The HMC performs better than the CDMC as the CDMC significantly underestimated the statistic for shorter multiyear resolutions (e.g. 2 to 5 overlapping years). The DHMC also tends to underestimate the SD of multiyear rainfall depths at shorter multiyear resolutions, but performs better than the CDMC. These results are similar to the respective results for the Goulburn, Williams River and Sydney sites. 

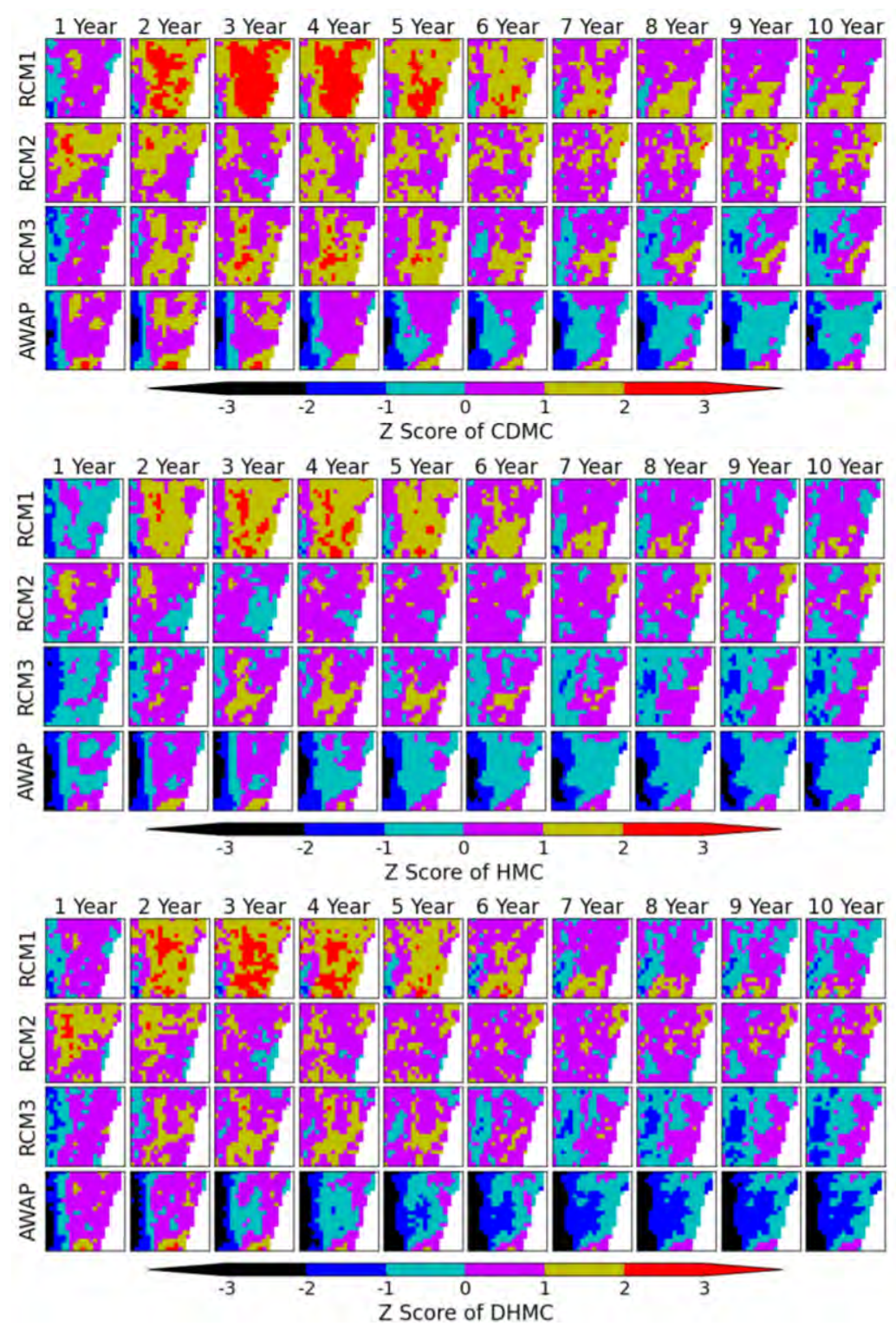

Figure 9.6: Z Scores of CDMC, HMC and DHMC for the SD of multiyear rainfall depths.

\subsection{Model Comparison for Distribution of Wet Periods}

This section compares the MC models for the mean and SD of number of wet days and mean length of wet spells (assessed as per section 3.4).

\subsubsection{Mean of Wet Period Statistics at Monthly and Annual Resolutions}

As for the previous three sites, all three MC models show similar satisfactory performance to reproduce the mean of wet period statistics (number of wet days and mean length of wet spell) at monthly and annual resolutions. However, the DHMC and CDMC perform better than the HMC, as the HMC tends to overestimate the statistics (see Figure F.16-F.19 at Appendix F.3). 


\subsubsection{SD of Monthly Number of Wet Days and Monthly Mean Length of Wet Spells}

As for the previous three sites, the DHMC performs better than the CDMC and HMC to reproduce the SD of monthly wet period statistics (number of wet days and mean length of wet spell) of all four datasets (see Figure 9.7 and Figure F.20 at Appendix F.3). Compares to the DHMC, the CDMC tends to more consistently and significantly underestimate the SD of monthly wet period statistics, while the HMC tends to significantly overestimate the statistic.
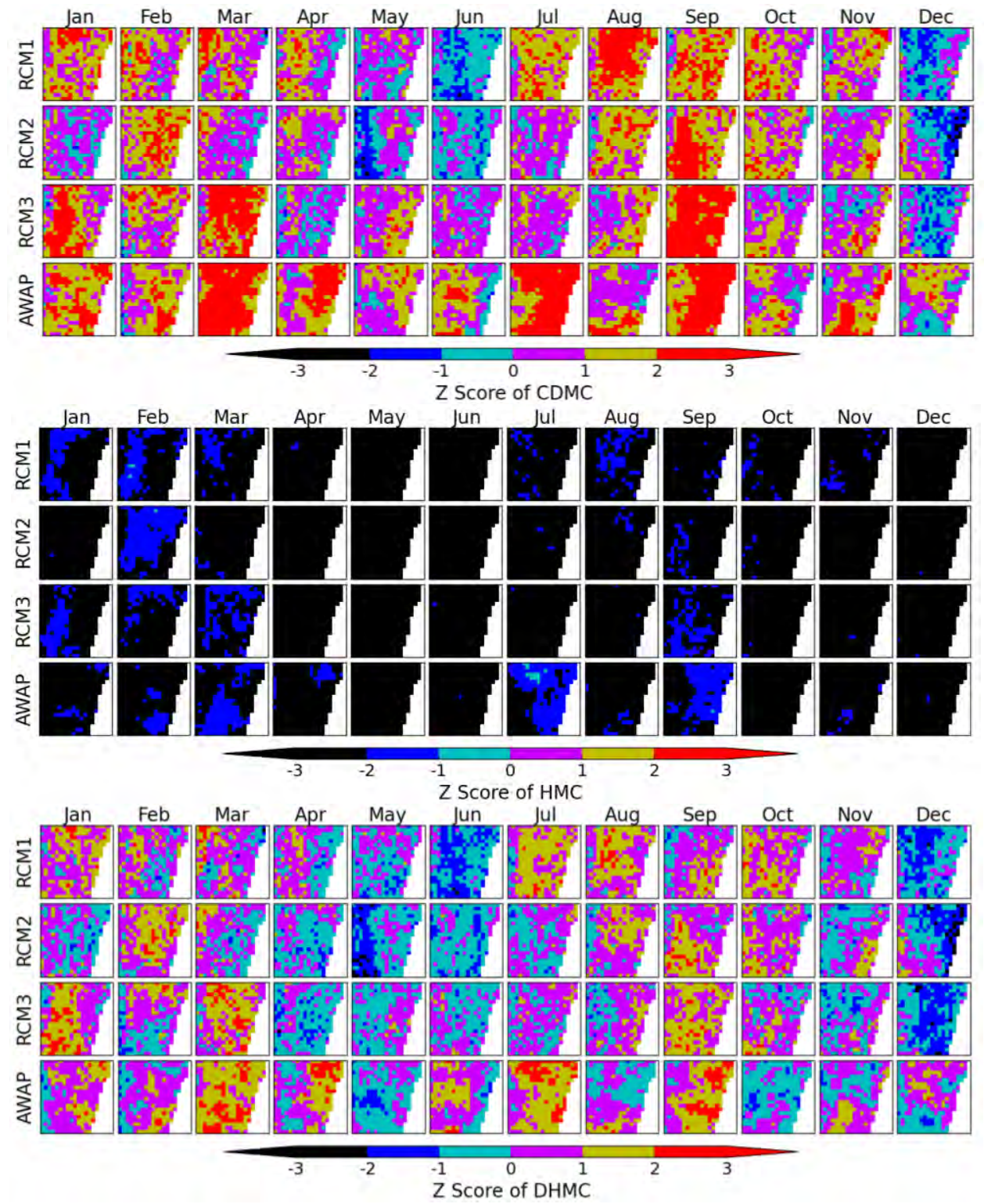

Figure 9.7: Z Scores of CDMC, HMC and DHMC for the SD of monthly number of wet days. 


\subsubsection{SD of Multiyear Number of Wet Days and Annual Mean Wet Spell Length}

For SD of multiyear number of wet days and mean length of wet spells, the DHMC performs better than the CDMC and HMC. DHMC mostly preserves the SDs with a tendency to underestimate the SD of multiyear wet days at shorter multiyear resolutions (Figure 9.8 and Figure 9.9). The CDMC tends to underestimate the SDs. However, the HMC performs satisfactorily for the SD of multiyear wet days for RCMs, but significantly underestimates for AWAP (the AWAP has higher variability of multiyear number of wet days compared to RCMs), while the HMC tends to significantly overestimate the SD of wet spell length.
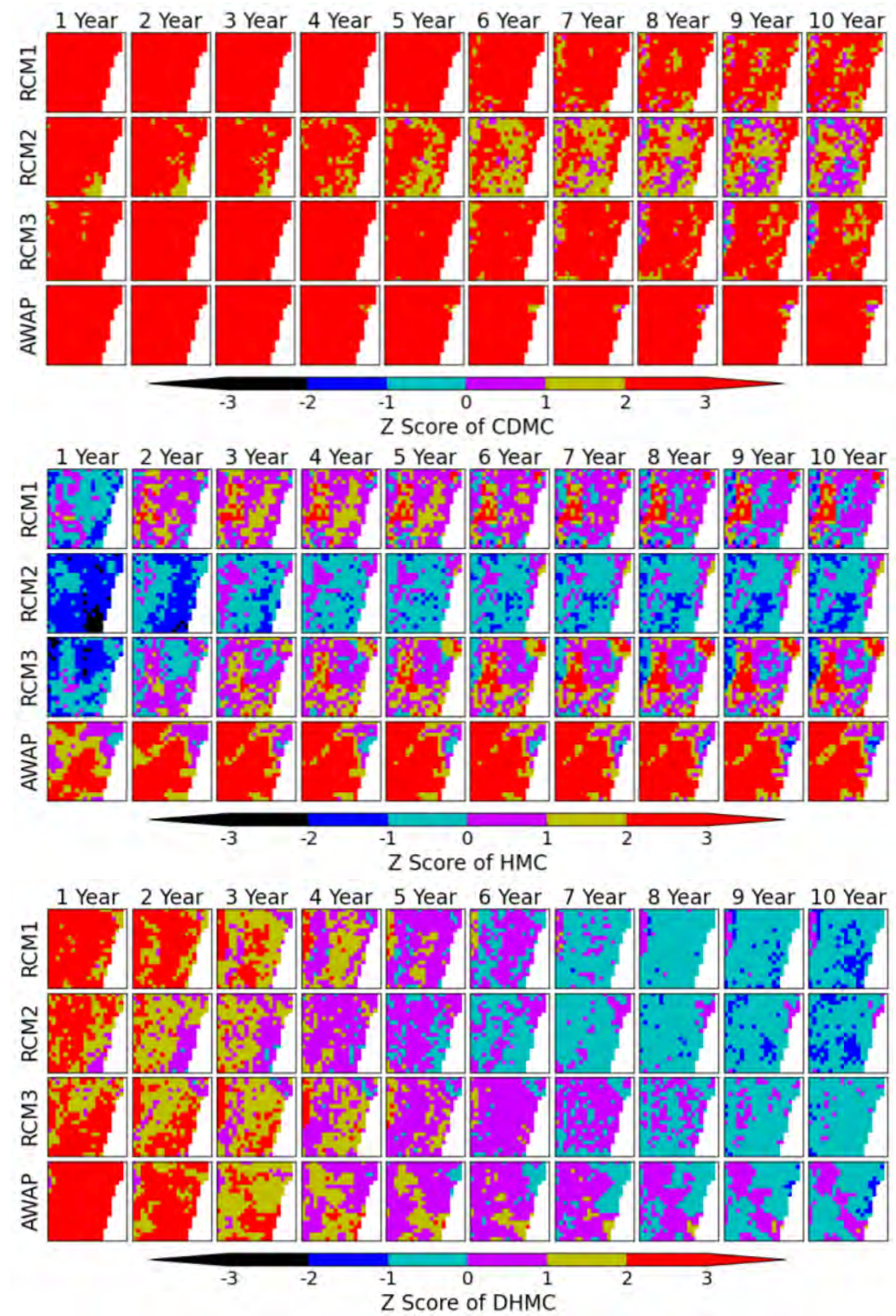

Figure 9.8: Z Scores of CDMC, HMC and DHMC for the SD of multiyear number of wet days. 


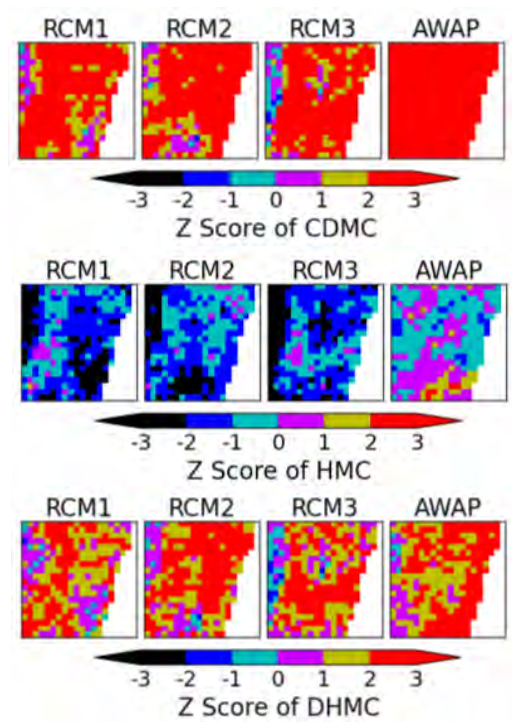

Figure 9.9: Z Scores of CDMC, HMC and DHMC for the SD of annual mean length of wet spells.

\subsection{Summary of Model Comparison for Distribution Statistics}

Table 9.4 and Table 9.5 show the spatially and temporally averaged $\mathrm{Z}$ scores for mean and SD of rainfall depths and wet period statistics respectively. The performances of all three models are similar for rainfall depths (Table 9.4), while DHMC performs better than the CDMC and HMC for wet periods (Table 9.5), which is consistent with the findings of previous three chapters. 
Table 9.4: Spatially and temporally average of the absolute value of $\mathrm{Z}$ scores for mean and SD of rainfall depths.

\begin{tabular}{|c|c|c|c|c|c|}
\hline \multirow{2}{*}{ Statistics } & \multirow{2}{*}{ Models } & \multicolumn{4}{|c|}{ Spatially and Temporally Averaged Z Scores } \\
\hline & & RCM1 & RCM2 & $\mathrm{RCM} 3$ & AWAP \\
\hline \multirow{3}{*}{ Daily Mean } & CDMC & 0.23 & 0.33 & 0.21 & 0.4 \\
\hline & HMC & 0.22 & 0.32 & 0.21 & 0.39 \\
\hline & DHMC & 0.24 & 0.34 & 0.23 & 0.41 \\
\hline \multirow{3}{*}{ Daily SD } & CDMC & 0.21 & 0.22 & 0.29 & 0.27 \\
\hline & $\mathrm{HMC}$ & 0.22 & 0.24 & 0.28 & 0.29 \\
\hline & DHMC & 0.21 & 0.23 & 0.28 & 0.28 \\
\hline \multirow{3}{*}{ Monthly Mean } & CDMC & 0.21 & 0.31 & 0.2 & 0.38 \\
\hline & HMC & 0.25 & 0.34 & 0.19 & 0.31 \\
\hline & DHMC & 0.23 & 0.32 & 0.22 & 0.38 \\
\hline \multirow{3}{*}{ Monthly SD } & CDMC & 0.57 & 0.5 & 0.7 & 0.51 \\
\hline & HMC & 0.82 & 0.69 & 0.97 & 0.68 \\
\hline & DHMC & 0.6 & 0.52 & 0.75 & 0.55 \\
\hline \multirow{3}{*}{$\begin{array}{c}\text { Multiyear } \\
\text { Mean }\end{array}$} & CDMC & 0.62 & 0.95 & 0.52 & 1.02 \\
\hline & HMC & 0.76 & 1.1 & 0.51 & 0.84 \\
\hline & DHMC & 0.63 & 0.96 & 0.53 & 1.01 \\
\hline \multirow{3}{*}{ Multiyear SD } & CDMC & 1.17 & 0.88 & 0.74 & 0.77 \\
\hline & $\mathrm{HMC}$ & 0.82 & 0.51 & 0.53 & 0.81 \\
\hline & DHMC & 0.88 & 0.67 & 0.59 & 1.14 \\
\hline \multirow{3}{*}{ Sum } & CDMC & 3.01 & 3.19 & 2.66 & 3.35 \\
\hline & HМC & 3.09 & 3.2 & 2.69 & 3.32 \\
\hline & DHMC & 2.79 & 3.04 & 2.6 & 3.77 \\
\hline
\end{tabular}


Table 9.5: Spatially and temporally average of the absolute value of $\mathrm{Z}$ scores for mean and SD of wet period statistics.

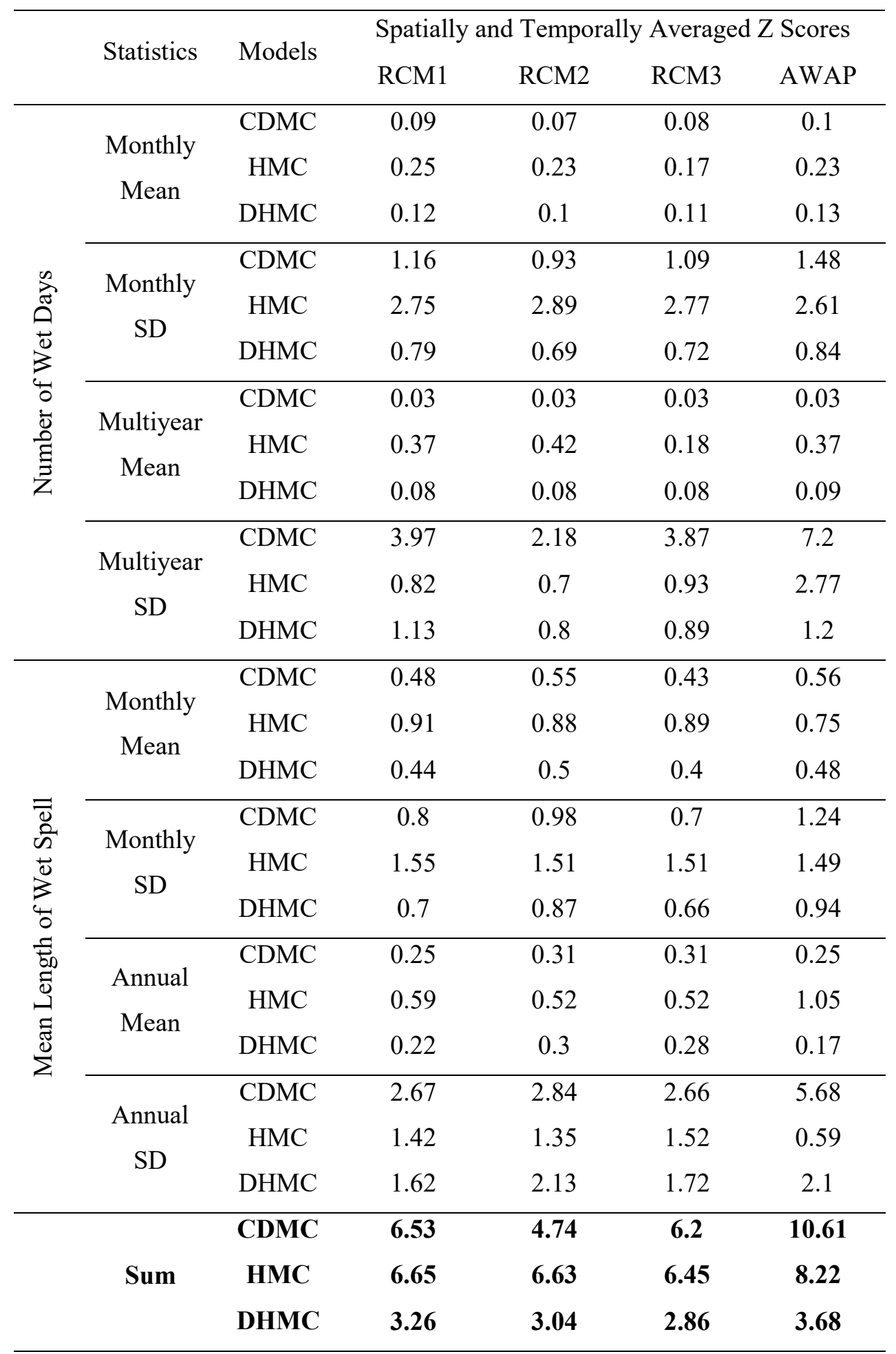




\subsection{Model Comparison for Autocorrelation}

The autocorrelations of monthly rainfall depths and monthly wet days in each RCM and AWAP for the Richmond River site show stronger positive correlations at lag-1 and lag-12, and stronger negative correlations at lag- 6 and lag-7 with gradual seasonal variations, which is consistent with the respective results for the previous three sites (Figure 9.10 and Figure 9.11). While all three models satisfactorily reproduce the seasonal and spatial patterns of the autocorrelations of monthly rainfall depths and monthly number of wet days, the HMC tends to underestimate the autocorrelations of monthly number of wet days (see Appendix F.4). The spatially and temporally averaged values of the autocorrelations shown in Table 9.6 also concludes same, which is consistent with the findings for previous three study sites.

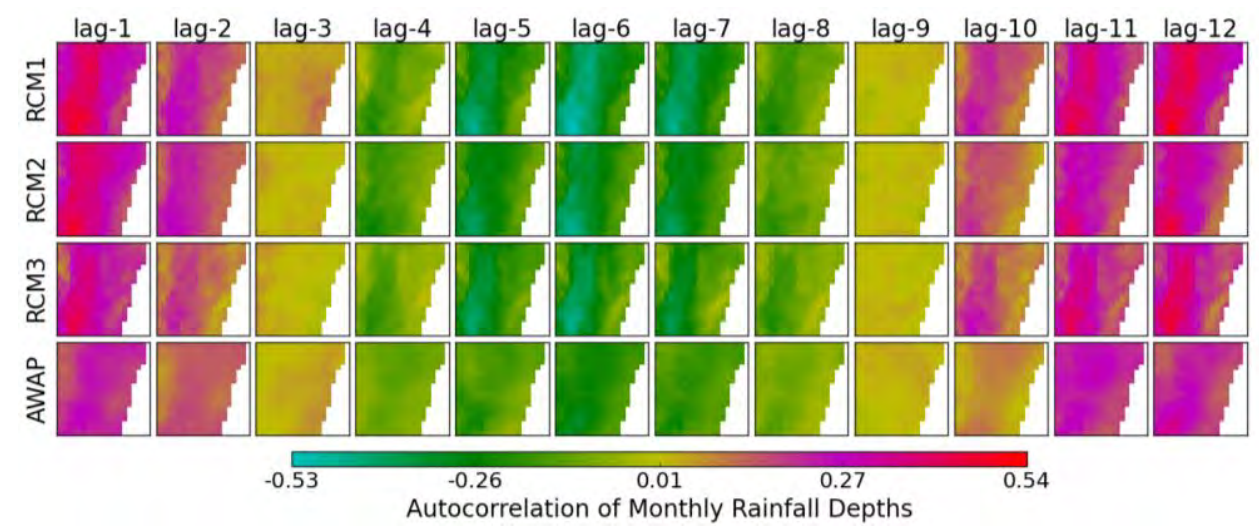

Figure 9.10: Month-to-month autocorrelations of monthly rainfall depths in all RCMs and AWAP.

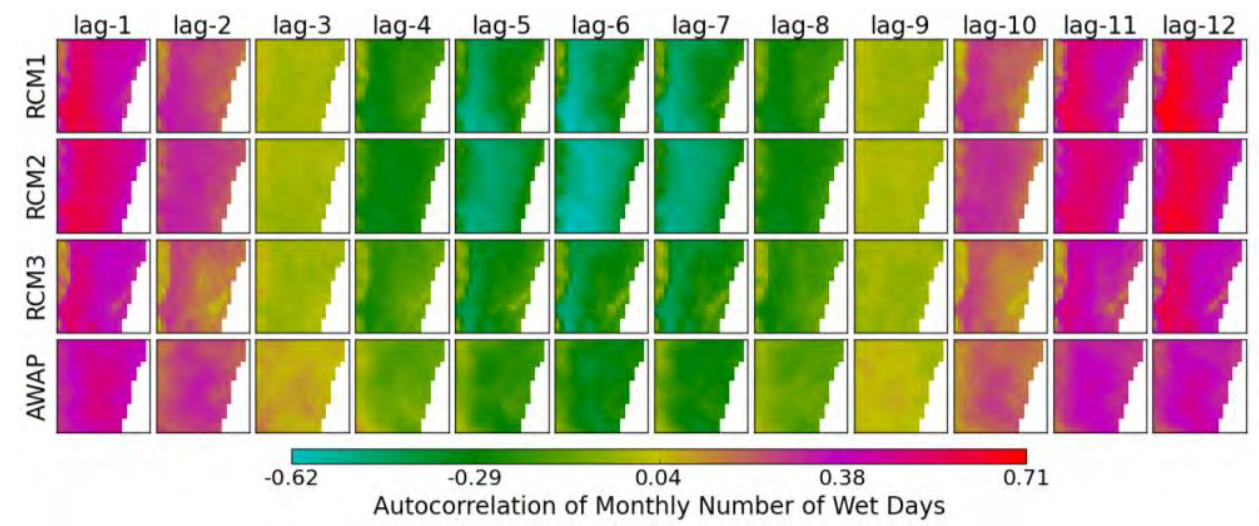

Figure 9.11: Month-to-month autocorrelations of monthly number of wet days in all RCMs and AWAP. 
Table 9.6: Spatial (all pixels) and temporal (all lags 1-12) average of autocorrelations of monthly rainfall depths and number of wet days.

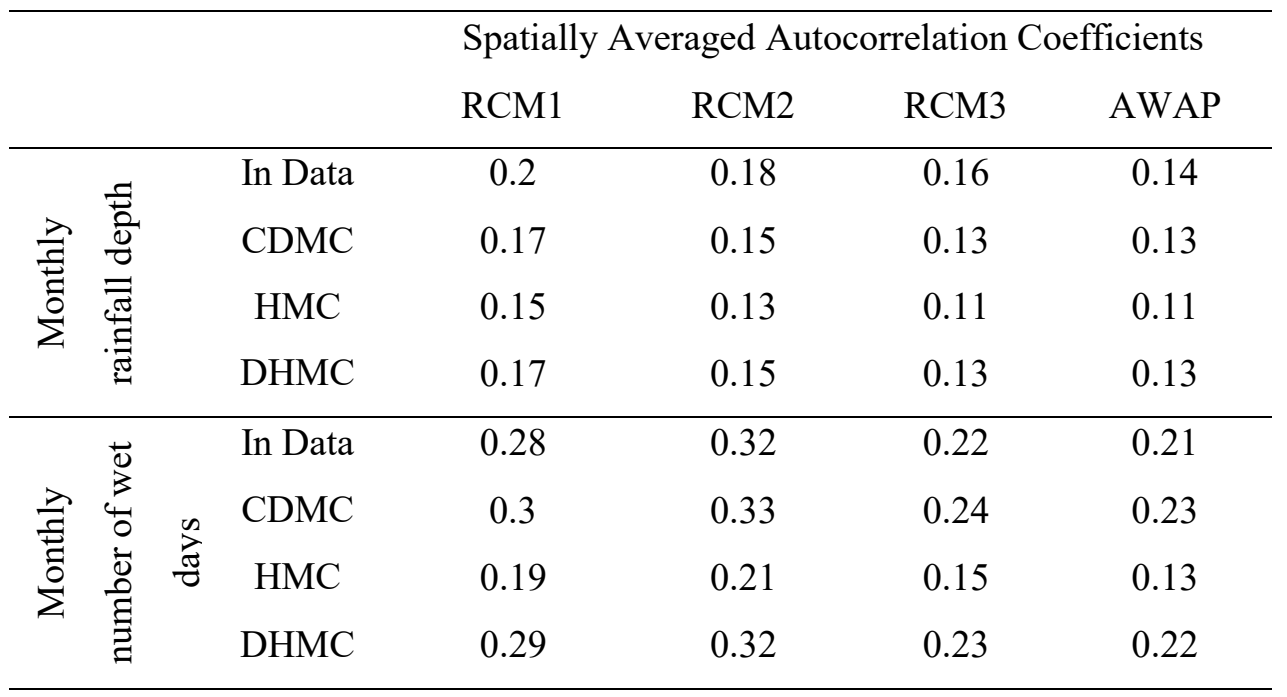

\subsection{Discussion}

This study has calibrated the CDMC, HMC and DHMC in the Richmond River site using three NARCliM RCMs and AWAP gridded dataset, for the 1950-2009 period. The intra-annual and spatial variability of rainfall in the Richmond River site is mostly consistent with intra-annual and spatial variability of Sydney site. While seasonal variability is typical of the east-Australian climate, with wet summers and dry winters, the spatial variability show that the summers are wetter at higher elevation, while the winters are drier at higher elevation.

The performance of the three models has been assessed in reproducing the key statistics of rainfall depths and wet periods. Their comparative performances are similar to their performances for the Goulburn, Williams River and Sydney sites. The overall performance of the DHMC is clearly better than the CDMC and HMC as it mostly preserves the distribution and autocorrelations of rainfall depths and wet periods at daily to multiyear resolutions, although it has a tendency to underestimate the SD of wet days at shorter multiyear resolutions. While other performances are mostly comparable to the DHMC, the CDMC significantly underestimated the SD of wet period statistics at multiyear resolution, while the HMC significantly overestimated the SD of monthly wet period statistics. 


\subsection{Conclusion}

The comparative assessments presented in this chapter suggest that the DHMC is a better model than the CDMC and HMC for stochastic simulation of rainfall in the Richmond River site. It shows the ability of the DHMC to reproduce the key characteristics of rainfall in a site with greatest ECL influence. The DHMC performs better than the other two MC models irrespective of the intra-annual and spatial variability of rainfall in the site. Each model performs better for the RCMs than the AWAP, while AWAP is drier than the RCMs with lower variability of rainfall depths but higher variability of wet days. This conclusion is consistent with the conclusion for the Goulburn, Williams River and Sydney sites, discussed in previous three chapters. 


\section{Comparison of MC Models in Bega River Site}

\subsection{Introduction}

This chapter presents the comparative performances of the CDMC, HMC and DHMC to reproduce the distribution and autocorrelations of rainfall in the Bega River site on the southern coast of NSW, Australia. This site is in the region of the least impact of ECLs [Kiem et al., 2016]. Therefore, this chapter will show the performances of the MC models in a site of the least influence of ECLs. Moreover, this chapter is the basis of the comparison (see Chapter 11) of rainfall variability and model performance in two sites at north and south border of NSW with contrasting storm characteristics.

\subsection{Calibrated Parameters}

This section presents the parameters of MC models (averaged APMC values of $P_{00}, P_{11}, \mu$ and $\sigma$ ) calibrated to the RCMs and AWAP for 374 land pixels of the Bega River site (Figure 3.7). Similar to the previous four sites, the AWAP is drier than the RCMs with an order of wetness as $\mathrm{RCM} 3>\mathrm{RCM} 1>\mathrm{RCM} 2>\mathrm{AWAP}$ (Table 10.1). Figures 10.1 to 10.4 show the seasonal and spatial variability of the $P_{00}, P_{11}, \mu$, and $\sigma$ parameters respectively. While the seasonal variability is consistent with the other four sites, the spatial variability shows that the regions east of the escarpment along the coastline (lower elevation) have a lower number of wet days but higher mean of wet day rainfall depths (similar to the Sydney site). This spatial characteristic of rainfall is further confirmed by the mostly positive correlations between elevation and $P_{11}$, and mostly negative correlations between elevation and $\mu$ (Figure 10.5), while overall elevation-parameter correlations are stronger in RCM2 (Table 10.2).

Table 10.1: Spatially and temporally averaged APMC values of model parameters.

\begin{tabular}{ccccc}
\hline Parameter & RCM1 & RCM2 & RCM3 & AWAP \\
\hline$P_{00}$ & 0.59 & 0.64 & 0.61 & 0.71 \\
$P_{11}$ & 0.67 & 0.64 & 0.66 & 0.65 \\
$\mu$ & 6.83 & 6.04 & 8.12 & 5.39 \\
$\sigma$ & 10.3 & 9.26 & 12.42 & 9.35 \\
\hline
\end{tabular}




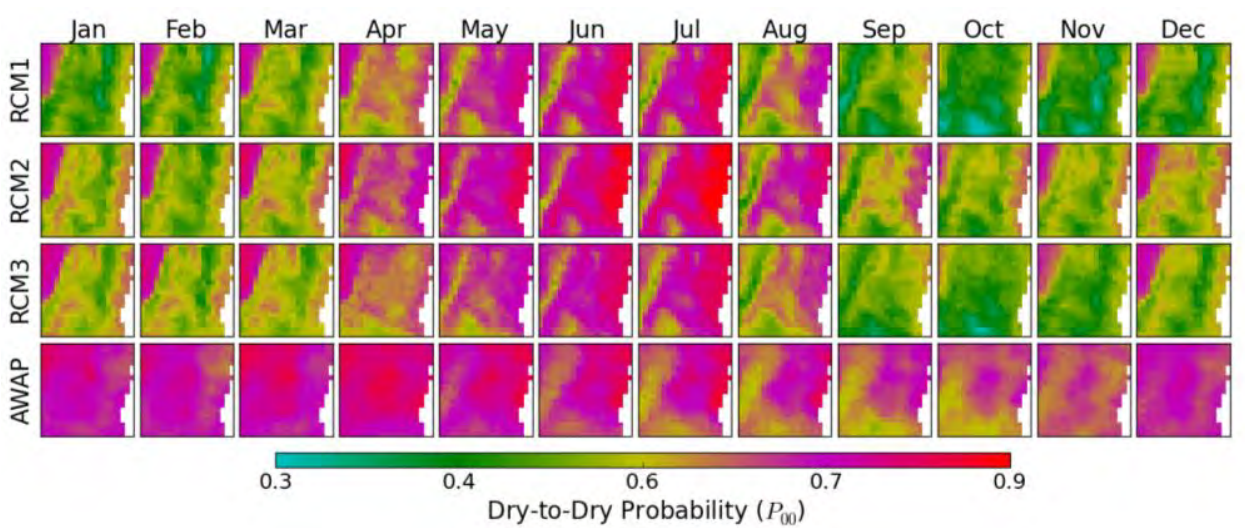

Figure 10.1: Intra-annual and spatial variability of dry-to-dry transition probabilities.

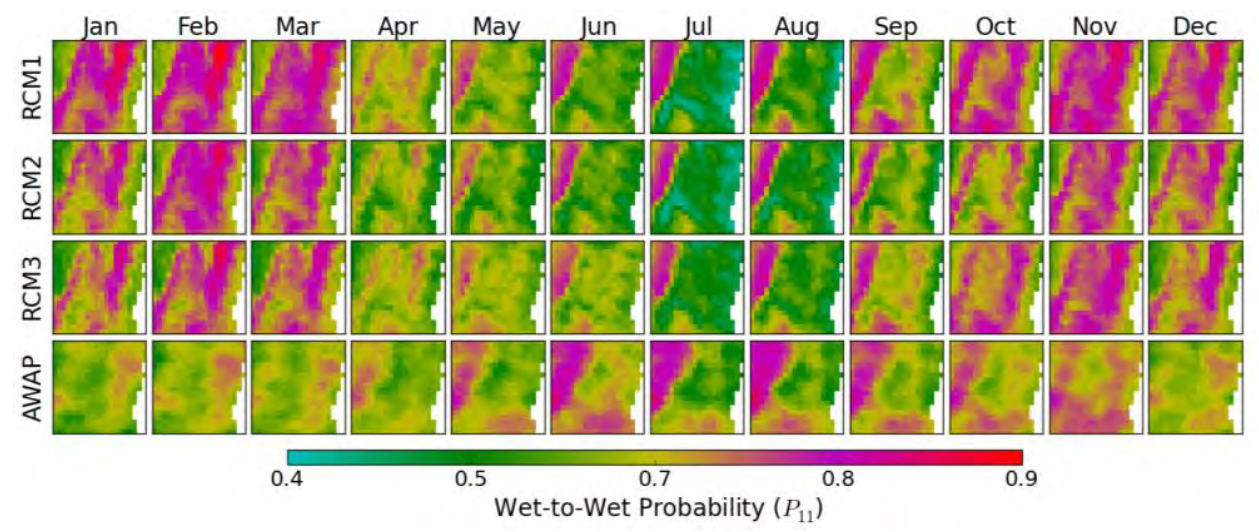

Figure 10.2: Intra-annual and spatial variability of wet-to-wet transition probabilities.

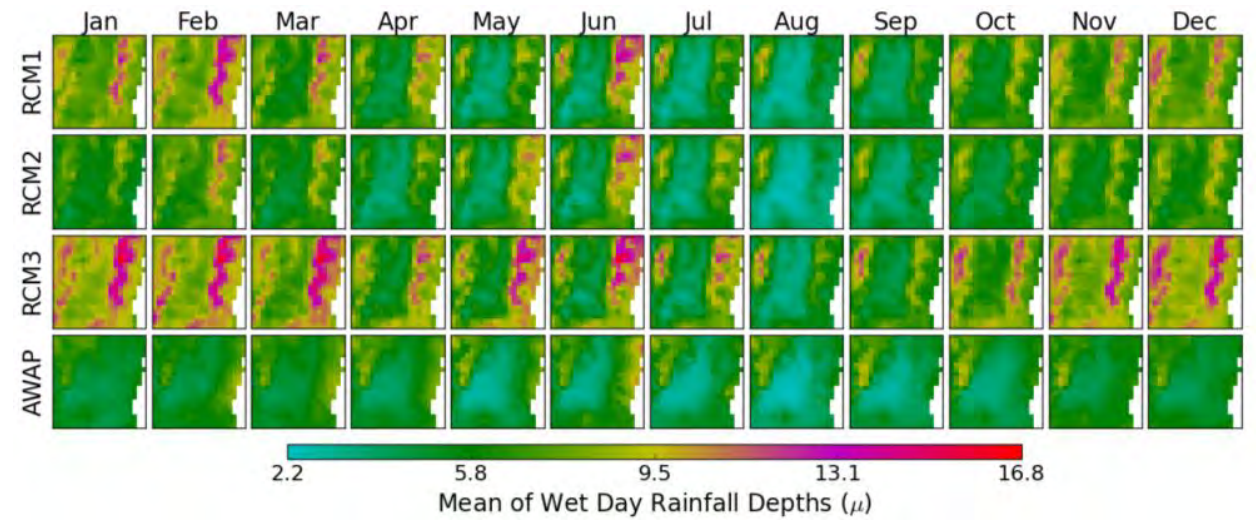

Figure 10.3: Intra-annual and spatial variability of mean wet day rainfall depths. 


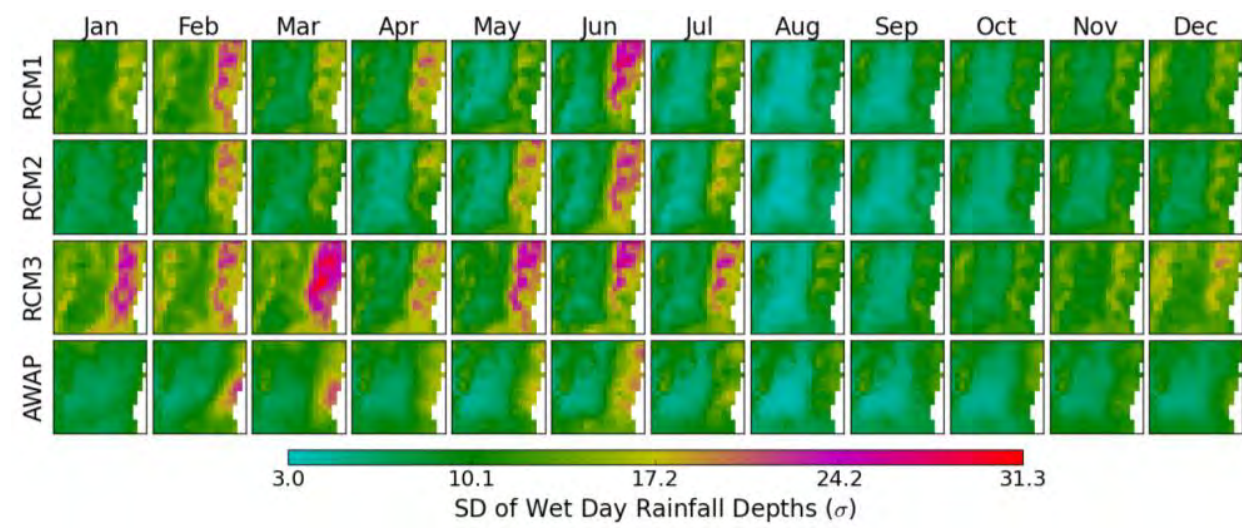

Figure 10.4: Intra-annual and spatial variability of SD of wet day rainfall depths.
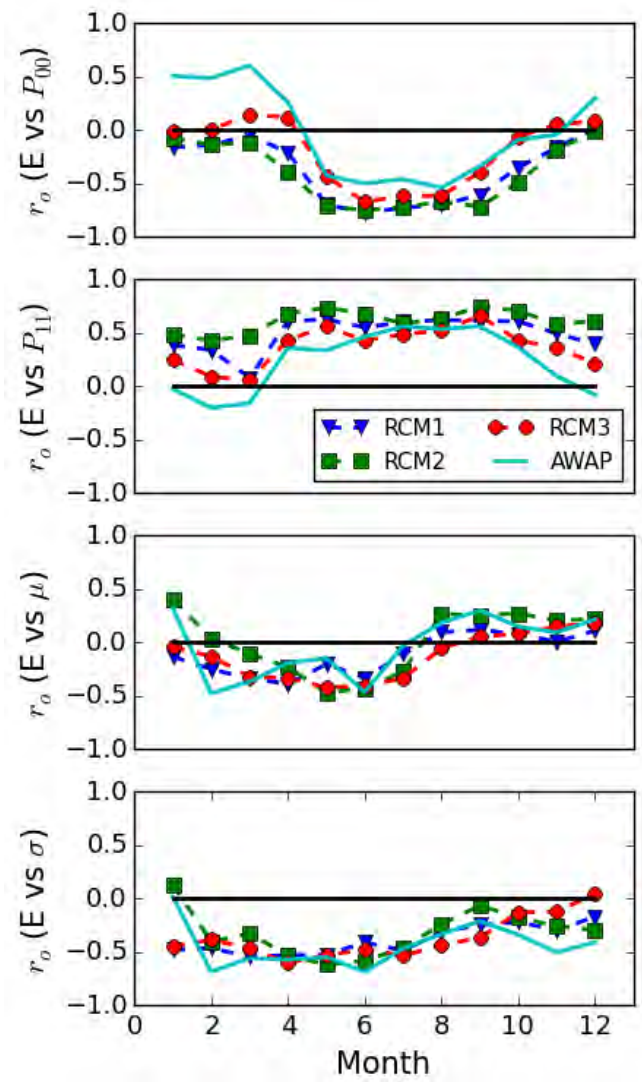

Figure 10.5: Correlation between elevation and model parameters for each month.

Table 10.2: Average of the absolute values of correlation coefficients between elevation and parameters.

\begin{tabular}{ccccc}
\hline Correlation & RCM1 & RCM2 & RCM3 & AWAP \\
\hline E vs $P_{00}$ & 0.39 & 0.42 & 0.27 & 0.38 \\
E vs $P_{11}$ & 0.49 & 0.61 & 0.37 & 0.31 \\
E vs $\mu$ & 0.18 & 0.26 & 0.21 & 0.24 \\
E vs $\sigma$ & 0.39 & 0.34 & 0.38 & 0.45 \\
\hline
\end{tabular}




\subsection{Distribution Statistics of Rainfall Depths and Wet Periods}

This study has examined the mean and SD of the rainfall depths and wet period statistics (i.e. number of wet days and mean length of wet spell) for RCMs and AWAP at daily, monthly and multiyear resolutions. The spatial and intra-annual variability of the distribution statistics are mostly consistent with the variability of the model parameters (see Appendix G.1). While the RCMs show similar characteristics of rainfall depths and wet period distributions, the AWAP shows lower variability of rainfall depths (Figure 10.6) and higher variability of wet days (Figure 10.7), particularly at multiyear resolutions. Similar to the previous four sites, the AWAP is drier than the RCMs, with lower SD of rainfall depths and higher SD of wet days (Table 10.3).

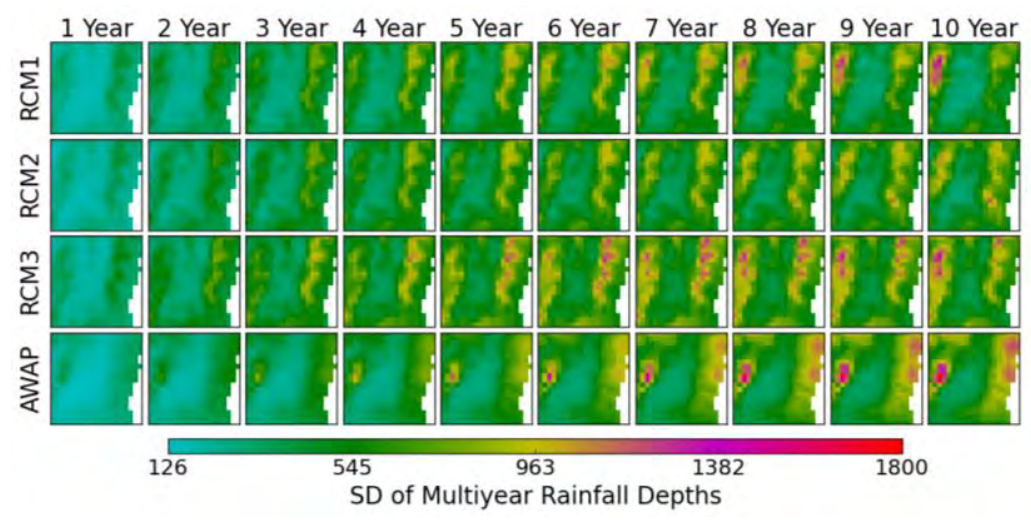

Figure 10.6: Spatial variability of the SD of multiyear rainfall depths.

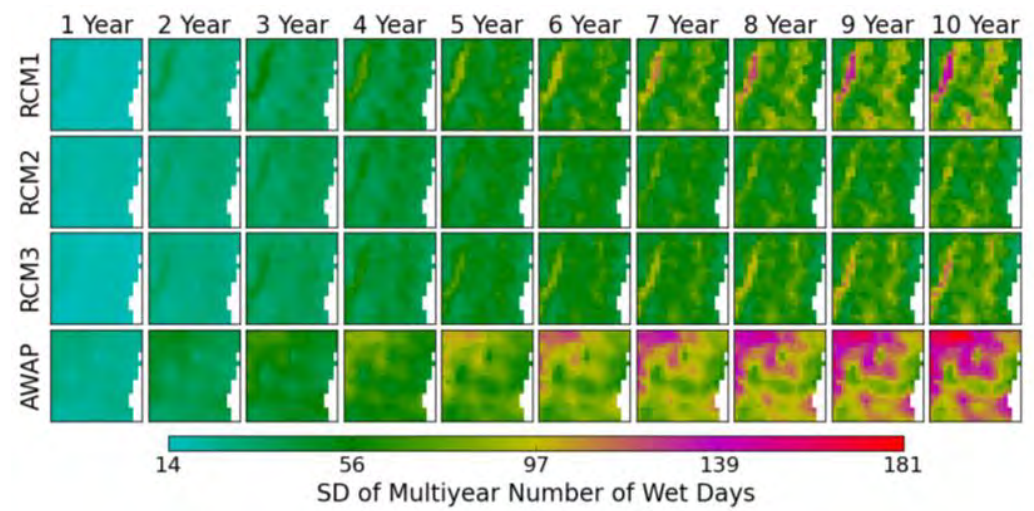

Figure 10.7: Spatial variability of the SD of multiyear number of wet days. 
Table 10.3: Spatially and temporally averaged values of the mean and SD of rainfall depth and wet period statistics at annual and multiyear resolutions.

\begin{tabular}{cccccc}
\hline \multicolumn{2}{c}{ Statistics } & RCM1 & RCM2 & RCM3 & AWAP \\
\hline Rainfall & Annual Mean & 1413 & 1108 & 1582 & 891 \\
\cline { 2 - 6 } Depth (mm) & Multiyear SD & 481 & 502 & 592 & 513 \\
\hline Number of & Annual Mean & 202 & 181 & 192 & 165 \\
\cline { 2 - 6 } Wet Days & Multiyear SD & 52 & 46 & 48 & 77 \\
\hline Mean Length & Annual Mean & 3.27 & 2.96 & 3.04 & 2.97 \\
\cline { 2 - 6 } of Wet Spell & Annual SD & 0.47 & 0.44 & 0.4 & 0.54 \\
\hline
\end{tabular}

\subsection{Model Comparison for Distribution of Rainfall Depths}

This section compares the CDMC, HMC and DHMC for the mean and SD of rainfall depths (as per the assessment method discussed in section 3.4).

\subsubsection{Mean of Rainfall Depths at Daily, Monthly and Multiyear Resolutions}

As for the previous four sites, the three models perform similarly and satisfactorily for mean of rainfall depths at daily, monthly and multiyear resolutions, with a tendency to underestimate the mean of rainfall depths at all resolutions (see Figure G.11-G.13 at Appendix G.2).

\subsubsection{SD of Daily and Monthly Rainfall Depths}

As for the previous four sites, the three models show comparable satisfactory performances to reproduce the SD of rainfall depths at daily and monthly resolutions, with a tendency to overestimate the SD of rainfall depths at monthly resolution (see Figure G.14-G.15 at Appendix G.2). 


\subsubsection{SD of Multiyear Rainfall Depths}

As for the other four sites, all three models satisfactorily reproduce the SD of multiyear rainfall depths with a tendency to underestimate for the RCMs and a tendency to overestimate for the AWAP (Figure 10.8). The HMC performs better than the CDMC as the CDMC significantly underestimated the statistic for shorter multiyear resolutions (e.g. 2 to 5 overlapping years). The DHMC also tends to underestimate the SD of multiyear rainfall depths at shorter multiyear resolutions for RCM2, but performs better than the CDMC.
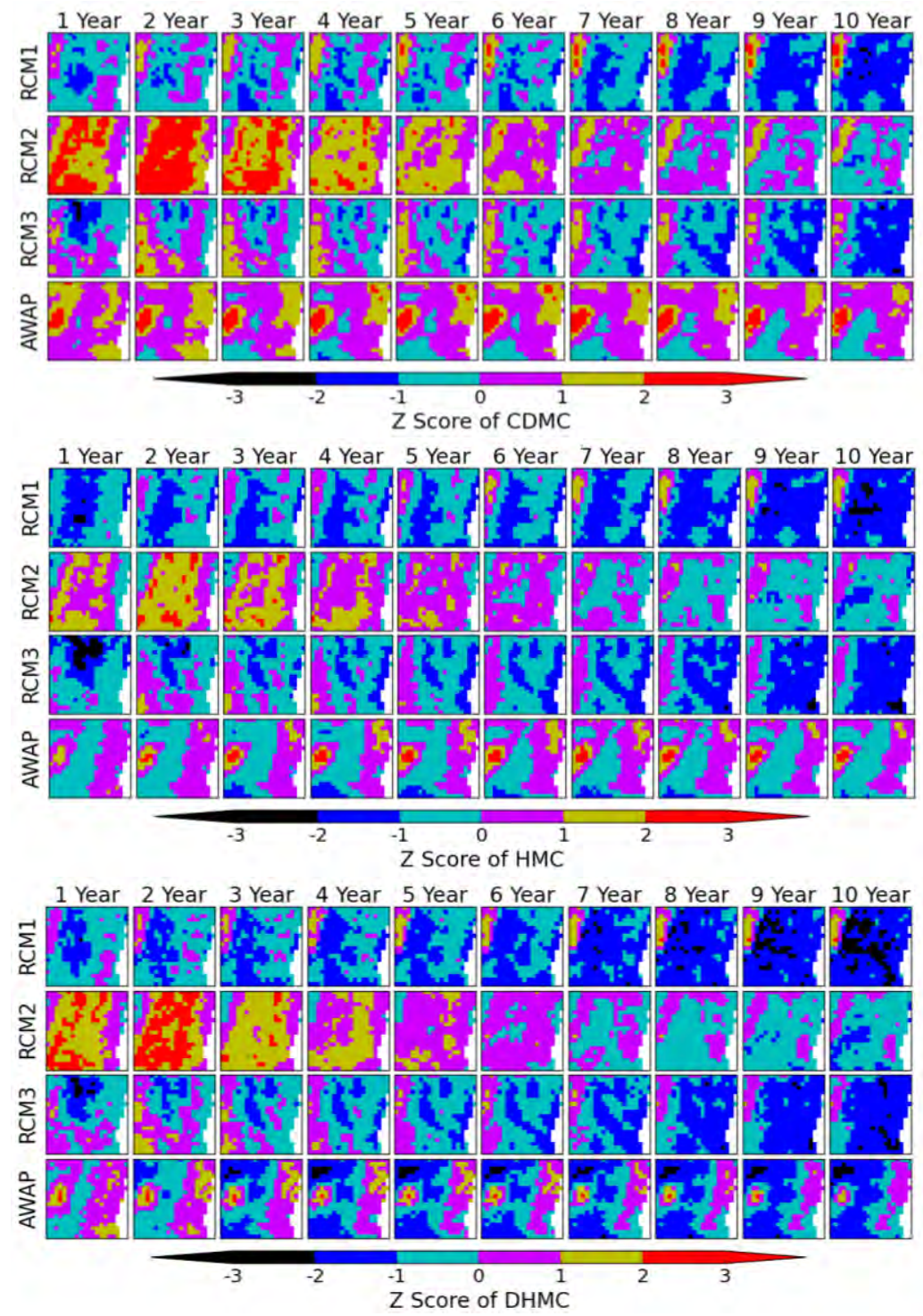

Figure 10.8: Z Scores of CDMC, HMC and DHMC for the SD of multiyear rainfall depths. 


\subsection{Model Comparison for Distribution of Wet Periods}

This section compares the CDMC, HMC and DHMC for the mean and SD of number of wet days and mean length of wet spells (as per the assessment procedures described in section 3.4).

\subsubsection{Mean of Wet Period Statistics at Monthly and Annual Resolutions}

As for the previous four sites, the CDMC, HMC, and DHMC show similar satisfactory performance to reproduce the mean of wet period statistics (number of wet days and mean length of wet spell) at monthly and annual resolutions. However, the DHMC and CDMC perform better than the HMC, as the HMC tends to overestimate the statistics (see Figure G.16G.19 at Appendix G.3).

\subsubsection{SD of Monthly Number of Wet Days and Monthly Mean Length of Wet Spells}

As for the previous four sites, the DHMC performs better than the CDMC and HMC to reproduce the SD of monthly wet period statistics (number of wet days and mean length of wet spell) of all four datasets (see Figure 10.9 and Figure G.20 at Appendix G.3). Compared to DHMC, the CDMC tends to more consistently and significantly underestimate the SD of monthly wet period statistics, while the HMC tends to significantly overestimate the statistic. 

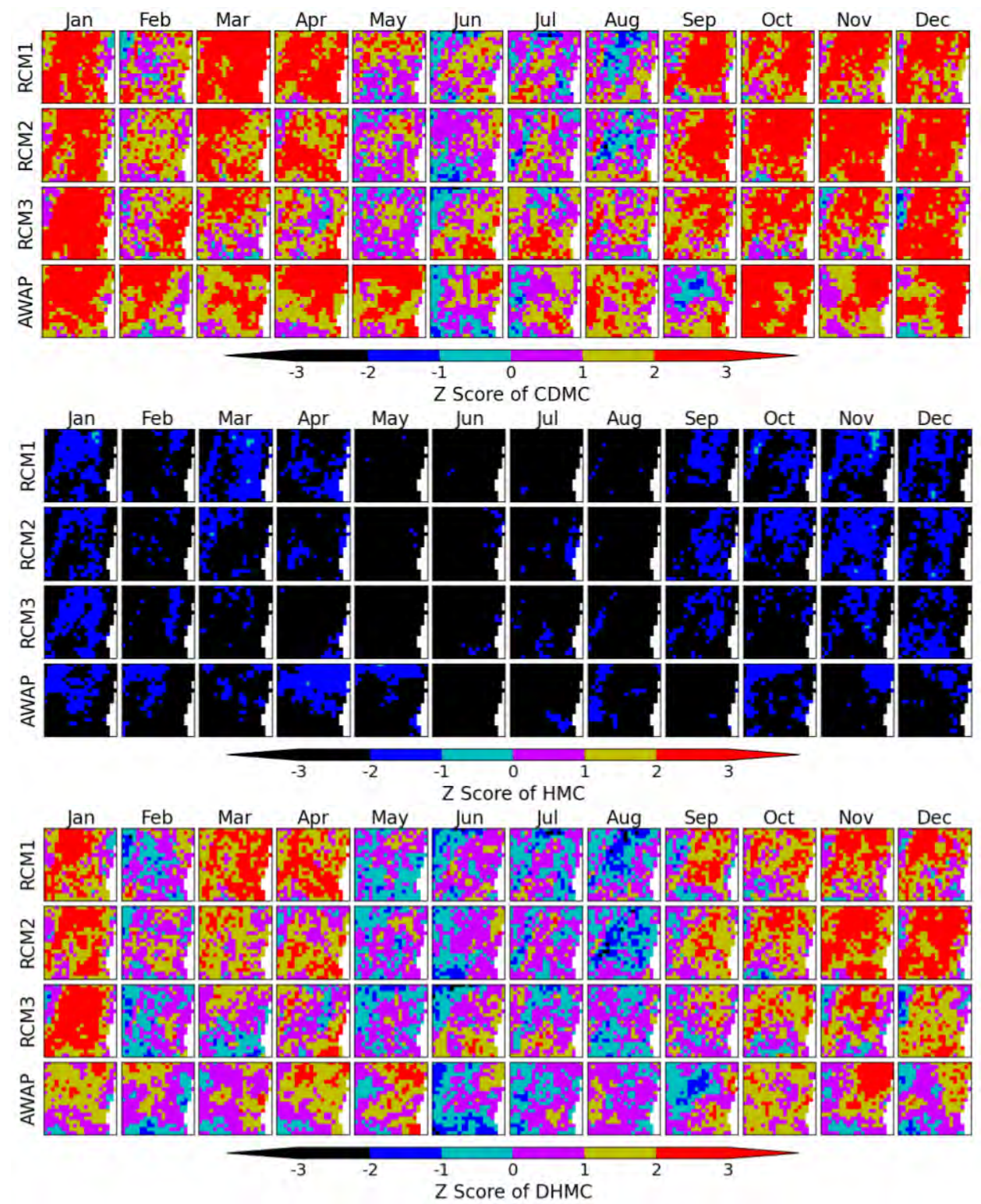

Figure 10.9: Z Scores of CDMC, HMC and DHMC for the SD of monthly number of wet days. 


\subsubsection{SD of Multiyear Number of Wet Days and Annual Mean Wet Spell Length}

As for other four sites, the DHMC performs better than the CDMC and HMC to reproduce the $\mathrm{SD}$ of multiyear number of wet days and SD of annual mean length of wet spells (see Figure 10.10 and Figure 10.11). The DHMC mostly preserves the SD of multiyear number of wet days for all datasets, with a tendency to underestimate the statistic at shorter multiyear resolution. The CDMC tends to significantly underestimate the statistic for most of the multiple years of each dataset. However, the HMC performs satisfactorily for the RCMs, but significantly underestimates the statistic for AWAP (the AWAP has higher variability of multiyear number of wet days in compare to RCMs).

For SD of annual mean length of wet spells, the DHMC mostly satisfactorily preserves the statistic, except for RCM2 (Figure 10.11). The CDMC tends to significantly underestimate the statistic for all four datasets, while the HMC tends to significantly overestimate the statistic for all RCMs. 

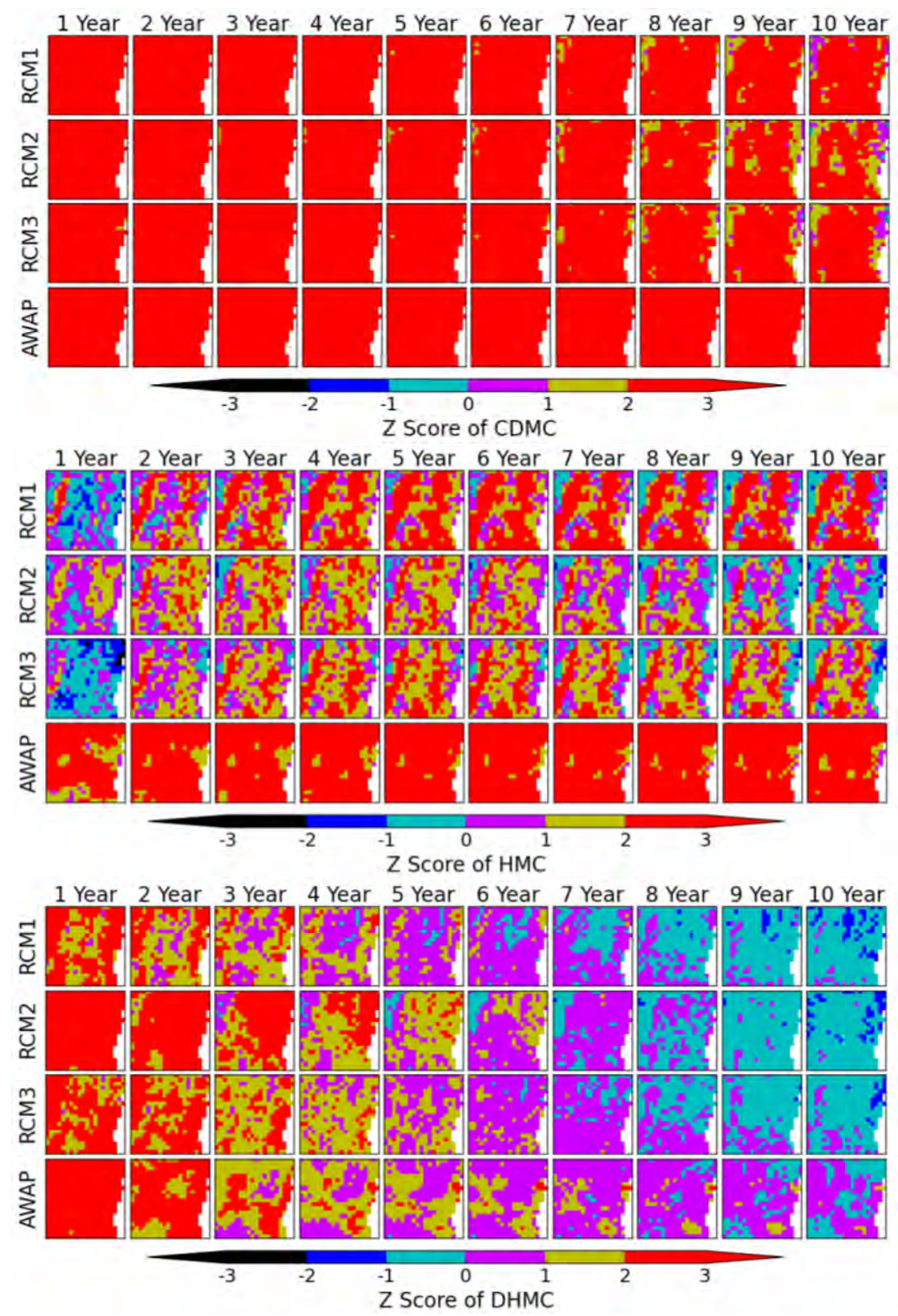

Figure 10.10: Z Scores of CDMC, HMC and DHMC for the SD of multiyear number of wet days.

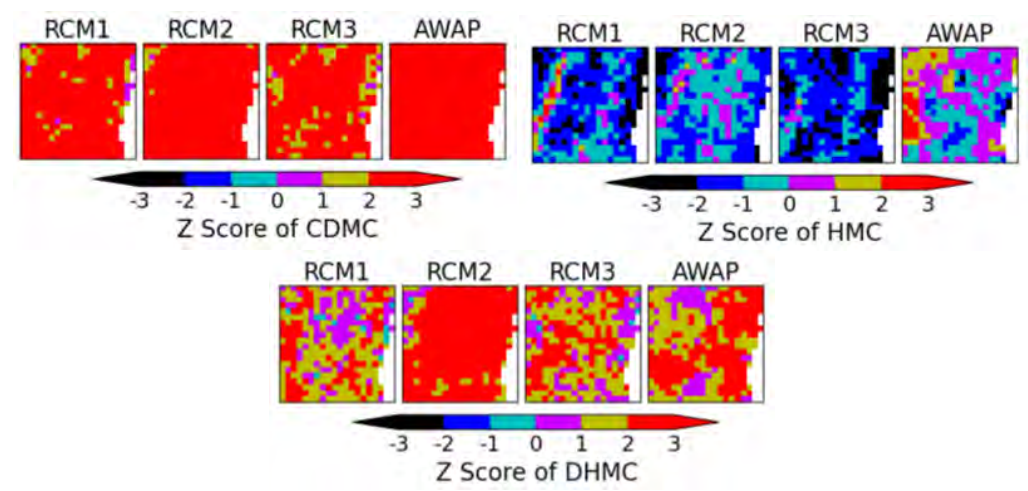

Figure 10.11: Z Scores of CDMC, HMC and DHMC for the SD of annual mean length of wet spells. 


\subsection{Summary of Model Comparison for Distribution Statistics}

Table 10.4 and Table 10.5 show the spatially and temporally averaged $\mathrm{Z}$ scores for mean and SD of rainfall depths and wet period statistics respectively. The performances of all three models are similar for rainfall depths (Table 10.4), while DHMC performs better than the CDMC and HMC for wet periods (Table 10.5), which is consistent with the findings of previous four chapters.

Table 10.4: Spatially and temporally average of the absolute value of $\mathrm{Z}$ scores for mean and SD of rainfall depths.

\begin{tabular}{|c|c|c|c|c|c|}
\hline \multirow{2}{*}{ Statistics } & \multirow{2}{*}{ Models } & \multicolumn{4}{|c|}{ Spatially and Temporally Averaged Z Scores } \\
\hline & & RCM1 & $\mathrm{RCM} 2$ & $\mathrm{RCM} 3$ & AWAP \\
\hline \multirow{3}{*}{ Daily Mean } & CDMC & 0.29 & 0.49 & 0.29 & 0.3 \\
\hline & HMC & 0.28 & 0.48 & 0.28 & 0.29 \\
\hline & DHMC & 0.3 & 0.49 & 0.29 & 0.32 \\
\hline \multirow{3}{*}{ Daily SD } & CDMC & 0.25 & 0.31 & 0.27 & 0.27 \\
\hline & HMC & 0.26 & 0.35 & 0.26 & 0.25 \\
\hline & DHMC & 0.92 & 0.87 & 0.96 & 0.95 \\
\hline \multirow{3}{*}{ Monthly Mean } & CDMC & 0.27 & 0.46 & 0.27 & 0.29 \\
\hline & HMC & 0.29 & 0.43 & 0.28 & 0.25 \\
\hline & DHMC & 0.28 & 0.46 & 0.27 & 0.3 \\
\hline \multirow{3}{*}{ Monthly SD } & CDMC & 0.56 & 0.6 & 0.65 & 0.56 \\
\hline & HMC & 0.84 & 0.68 & 0.94 & 0.86 \\
\hline & DHMC & 0.6 & 0.6 & 0.68 & 0.62 \\
\hline \multirow{3}{*}{$\begin{array}{c}\text { Multiyear } \\
\text { Mean }\end{array}$} & CDMC & 0.84 & 1.57 & 0.79 & 0.78 \\
\hline & HMC & 0.89 & 1.47 & 0.79 & 0.61 \\
\hline & DHMC & 0.83 & 1.57 & 0.78 & 0.79 \\
\hline \multirow{3}{*}{ Multiyear SD } & CDMC & 0.78 & 1.03 & 0.73 & 0.8 \\
\hline & HMC & 0.97 & 0.66 & 0.82 & 0.55 \\
\hline & DHMC & 1.09 & 0.8 & 0.83 & 0.86 \\
\hline \multirow{3}{*}{ Sum } & CDMC & 2.99 & 4.46 & 3 & 3 \\
\hline & НМС & 3.53 & 4.07 & 3.37 & 2.81 \\
\hline & DHМC & 4.02 & 4.79 & 3.81 & 3.84 \\
\hline
\end{tabular}


Table 10.5: Spatially and temporally average of the absolute value of $Z$ scores for mean and SD of wet period statistics.

\begin{tabular}{|c|c|c|c|c|c|c|}
\hline & \multirow{2}{*}{ Statistics } & \multirow{2}{*}{ Models } & \multicolumn{4}{|c|}{ Spatially and Temporally Averaged Z Scores } \\
\hline & & & RCM1 & $\mathrm{RCM} 2$ & RCM3 & AWAP \\
\hline \multirow{12}{*}{ 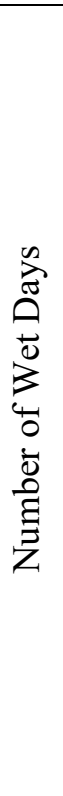 } & \multirow{3}{*}{$\begin{array}{c}\text { Monthly } \\
\text { Mean }\end{array}$} & CDMC & 0.07 & 0.08 & 0.07 & 0.07 \\
\hline & & $\mathrm{HMC}$ & 0.19 & 0.16 & 0.14 & 0.15 \\
\hline & & DHMC & 0.1 & 0.08 & 0.07 & 0.11 \\
\hline & \multirow{3}{*}{$\begin{array}{c}\text { Monthly } \\
\text { SD }\end{array}$} & CDMC & 1.75 & 1.74 & 1.55 & 1.73 \\
\hline & & HMC & 2.47 & 2.47 & 2.6 & 2.53 \\
\hline & & DHMC & 1.21 & 1.2 & 0.98 & 0.92 \\
\hline & \multirow{3}{*}{$\begin{array}{c}\text { Multiyear } \\
\text { Mean }\end{array}$} & $\mathrm{CDMC}$ & 0.03 & 0.03 & 0.03 & 0.03 \\
\hline & & $\mathrm{HMC}$ & 0.37 & 0.27 & 0.25 & 0.27 \\
\hline & & DHMC & 0.08 & 0.03 & 0.03 & 0.08 \\
\hline & \multirow{3}{*}{$\begin{array}{c}\text { Multiyear } \\
\text { SD }\end{array}$} & $\mathrm{CDMC}$ & 6.04 & 4.72 & 4.89 & 9.06 \\
\hline & & HMC & 1.98 & 1.11 & 1.36 & 3.94 \\
\hline & & DHMC & 0.96 & 1.55 & 1.1 & 1.37 \\
\hline \multirow{15}{*}{ 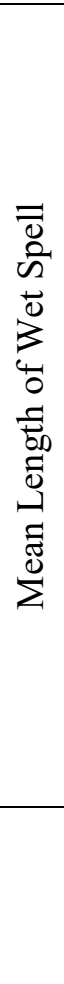 } & \multirow{3}{*}{$\begin{array}{c}\text { Monthly } \\
\text { Mean }\end{array}$} & $\mathrm{CDMC}$ & 0.63 & 0.47 & 0.45 & 0.37 \\
\hline & & HMC & 0.84 & 0.85 & 0.94 & 0.87 \\
\hline & & DHMC & 0.52 & 0.42 & 0.41 & 0.39 \\
\hline & \multirow{3}{*}{$\begin{array}{c}\text { Monthly } \\
\text { SD }\end{array}$} & CDMC & 1.24 & 1.06 & 0.97 & 0.81 \\
\hline & & $\mathrm{HMC}$ & 1.38 & 1.37 & 1.44 & 1.47 \\
\hline & & DHMC & 0.92 & 0.78 & 0.77 & 0.66 \\
\hline & \multirow{3}{*}{$\begin{array}{l}\text { Annual } \\
\text { Mean }\end{array}$} & CDMC & 0.28 & 0.23 & 0.16 & 0.23 \\
\hline & & HMC & 0.6 & 0.5 & 0.39 & 0.65 \\
\hline & & DHMC & 0.17 & 0.2 & 0.13 & 0.18 \\
\hline & \multirow{3}{*}{$\begin{array}{c}\text { Annual } \\
\text { SD }\end{array}$} & $\mathrm{CDMC}$ & 3.7 & 4.54 & 3.07 & 6.71 \\
\hline & & HMC & 1.33 & 0.93 & 1.57 & 0.84 \\
\hline & & DHMC & 1.54 & 3.32 & 1.6 & 2.03 \\
\hline & \multirow{3}{*}{ Sum } & CDMC & 9.76 & 8.1 & 7.96 & 12.07 \\
\hline & & HМC & 7.23 & 6.23 & 6.73 & 9.23 \\
\hline & & DHMC & 3.79 & 4.06 & 3.36 & 3.53 \\
\hline
\end{tabular}




\subsection{Model Comparison for Autocorrelation}

The autocorrelations of monthly rainfall and wet days in Bega site are similar to the respective results for previous three sites (Figure 10.12 and Figure 10.13). While all three models satisfactorily reproduce the seasonal and spatial patterns of the autocorrelations of monthly rainfall depths and monthly number of wet days, the HMC tends to underestimate the autocorrelations of monthly number of wet days (see Appendix G.4 and Table 10.6).

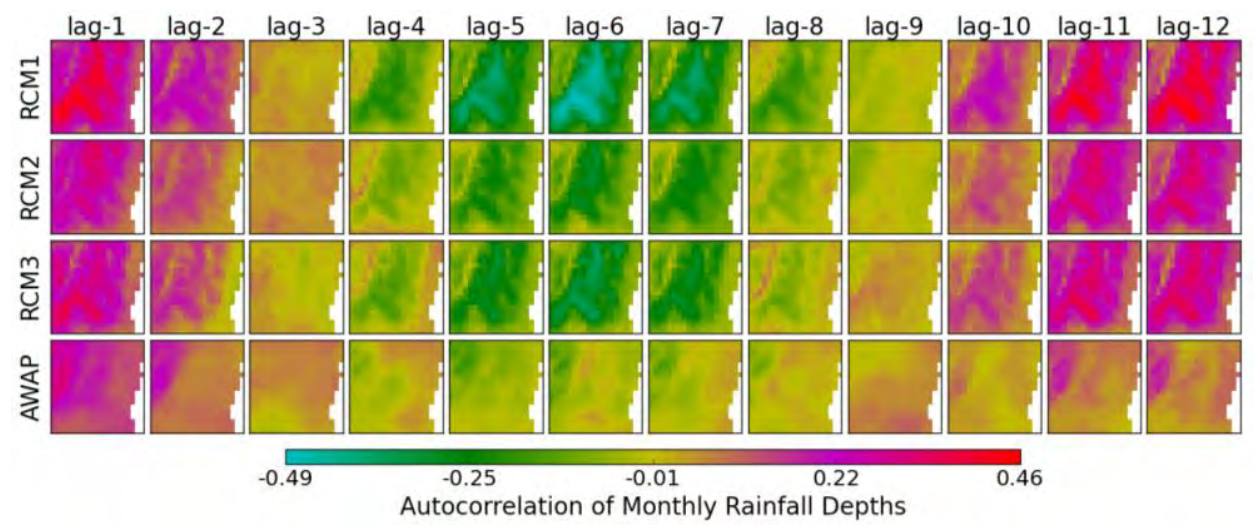

Figure 10.12: Month-to-month autocorrelations of monthly rainfall depths.

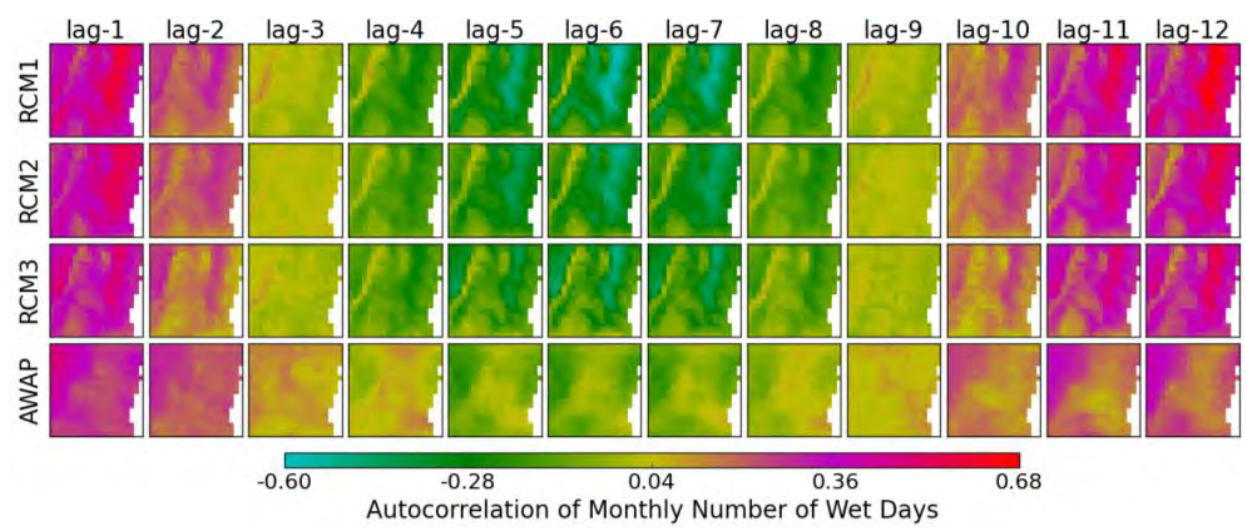

Figure 10.13: Month-to-month autocorrelations of monthly number of wet days. 
Table 10.6: Spatial (all pixels) and temporal (all lags 1-12) average of autocorrelations of monthly rainfall depths and number of wet days.

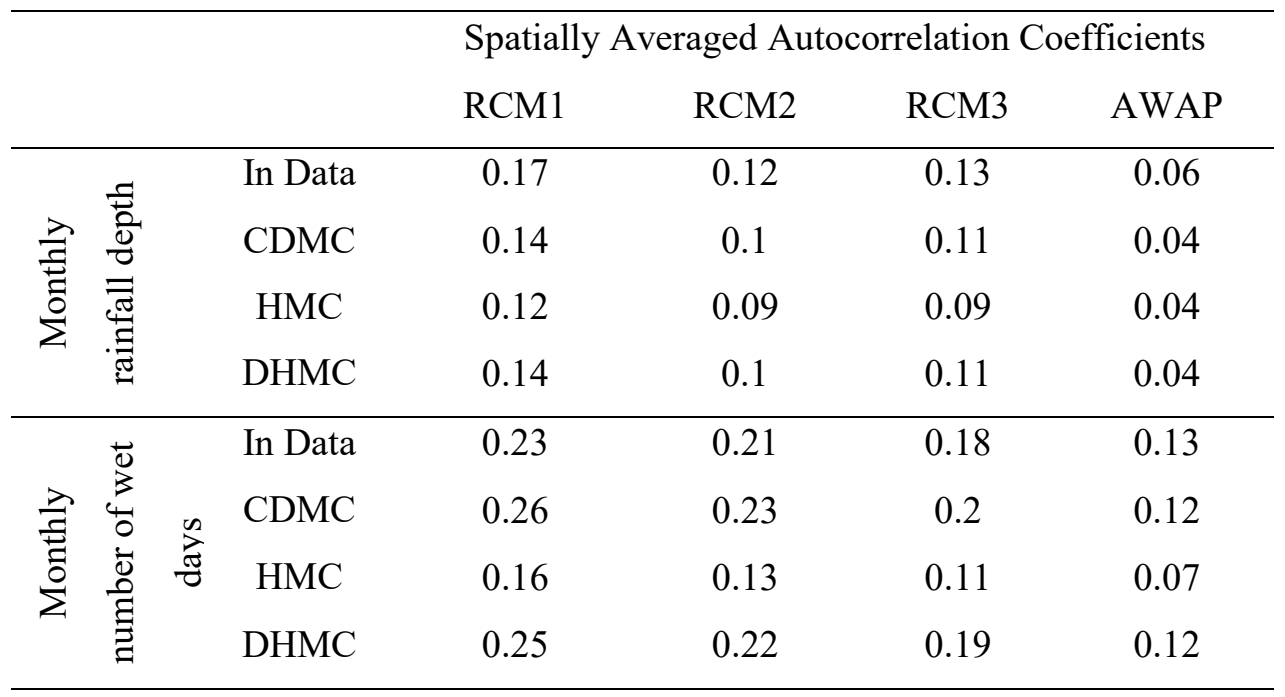

\subsection{Discussion}

This study has calibrated the CDMC, HMC and DHMC in the Bega River site using three NARCliM RCMs and AWAP gridded dataset, for the 1950-2009 period. While seasonal variability is typical of the east-Australian climate, with wet summers and dry winters, the spatial variability shows that the regions east of the escarpment along the coastline (lower elevation) has a lower number of wet days but higher mean of wet day rainfall depths (similar to the Sydney site).

This study has assessed the performance of the three models to reproduce the distribution and autocorrelations of rainfall depths and wet periods at daily to multiyear resolutions. The comparative performances of the models are similar to their performances for the previous four site. The overall performance of the DHMC is clearly better than the CDMC and HMC, with the DHMC mostly preserving the distribution and autocorrelations of rainfall depths and wet periods at daily to multiyear resolutions, although it has a tendency to underestimate the SD of wet days at shorter multiyear resolutions. While other performances are mostly comparable to the DHMC, the CDMC significantly underestimated the SD of wet period statistics at multiyear resolution, while the HMC significantly overestimated the SD of monthly wet period statistics. 
10. Comparison of MC Models in Bega River Site

\subsection{Conclusion}

The comparative assessments presented in this chapter suggest that the DHMC is a better model compared to the CDMC and HMC for stochastic simulation of rainfall in the Bega River site. These results show the ability of the DHMC to reproduce the key characteristics of rainfall in a site with least ECL influence. The DHMC performs better than the other two MC models, irrespective of the intra-annual and spatial variability of rainfall in the site. This conclusion is consistent with the conclusion for the other four sites, discussed in the previous four chapters. 


\section{Geographical Comparison of Rainfall and Model Performances}

\subsection{Introduction}

This study has assessed the comparative performance of the CDMC, HMC and DHMC (three stochastic rainfall models developed in this thesis) by calibrating them to NARCliM RCMs (reanalysis data) and AWAP gridded datasets for five case study sites along the east coast of Australia (Chapters 6 to 10). The major objective of this study is to assess the ability of the stochastic models to reproduce the distribution and autocorrelation of rainfall along the east coast of Australia. This chapter discusses the geographical comparison of the rainfall characteristics and the performance of three MC models in those five case study sites along the east coast.

\subsection{Five Case Study Sites along East Coast of Australia}

Chapter 6 showed the assessment for the Goulburn River site, which has a major hydrology field site and has been the subject of numerous hydrology research studies [Martinez et al., 2010; Chen et al., 2015]. Chapters 7 and 8 showed the assessment for the Williams River and Sydney sites respectively. The Williams River and Sydney sites are the sources of urban water supply for the Hunter and Sydney regions respectively, and they have prominent ECL impacts. Chapters 9 and 10 showed the assessment for the Richmond River site on the northern NSW coast and Bega River site on the southern NSW coast respectively. The Richmond and Bega River sites are subject to the greatest and least impact of ECL respectively. Therefore, the analysis of these five case study sites provides an understanding of the rainfall behaviour in regions of different ECL impacts, as well as the ability of the stochastic models to reproduce the key rainfall characteristics (which are particularly important for urban drought security assessment) in those regions of different ECL impacts. 


\subsection{Rainfall Variability along the East Coast of Australia}

\subsubsection{Intra-annual and Spatial Variability}

This study has examined the intra-annual and spatial variability of rainfall in each of the case study sites. Among the three RCMs, the rainfall characteristics of RCM2 has generally shown more similarities to the AWAP for each site. However, the discussion of intra-annual and spatial variability of rainfall in this section is based on the results for AWAP as AWAP gridded data is generated from ground-based observations of raingauge stations [Raupach et al., 2009].

At each of the sites, the intra-annual variability shows a wet summer (December-February) and a dry winter (June-August), with two transitional seasons of autumn (March-May) and spring (September-November). The autumn and winter rainfall in the east coast of Australia is generally influenced by the ECLs [Kiem et al., 2016], while ECLs usually contribute around $23 \%$ of annual rainfall and $40 \%$ of heavy rainfall events [Pepler et al., 2014]. The seasonal variability of both wet days and rainfall depth is distinctive in the central and northern part of the coastal NSW (i.e. Richmond River, Goulburn River, Williams River, and Sydney sites) with the possible influence of ENSO as discussed by Risbey et al. [2009]. However, the rainfall depth in the southern part of coastal NSW (i.e. Bega River site) is more uniform throughout the year with possible influence of the climate characteristics of south and south-east Australia, while the rainfall variability in south and south-east Australia is mainly linked with Indian Ocean Dipole [Risbey et al., 2009].

The MC model parameters in each study site show link with the local orography (Table 11.1). In the Goulburn and Williams River sites, the spatial variability suggests more wet days and higher intensity of rainfall at higher elevations, and more dry days and lower intensity rainfall at lower elevations. In the Sydney site, this study found that the steep escarpment adjacent to the east coastline is wetter (more wet days and higher rainfall depths) than the higher elevation regions in the west. In the Richmond River site, the summer is wetter at higher elevation, while the winter is drier at higher elevation. In the Bega River site, the spatial variability shows that the regions east of the escarpment along the coastline (lower elevation) has a lower number of wet days but higher mean of wet day rainfall depths (similar to the Sydney site). The spatial variability in the study sites suggests the importance of using high-resolution gridded data for the water security assessment in the east coast of Australia. It also suggests that multi-site version of stochastic model is required to incorporate the spatial variability particularly for large catchments (e.g. Warragamba Dam in Sydney) along the east coast. 
Table 11.1: Average of the elevation-parameters correlations (absolute values).

\begin{tabular}{ccccccc}
\hline Correlations & Data & Richmond & Williams & Goulburn & Sydney & Bega \\
\hline \multirow{6}{*}{ E vs $P_{11}$} & RCM1 & 0.25 & 0.16 & 0.3 & 0.23 & 0.49 \\
& RCM2 & 0.33 & 0.34 & 0.38 & 0.21 & 0.61 \\
& RCM3 & 0.23 & 0.13 & 0.15 & 0.23 & 0.37 \\
& AWAP & 0.3 & 0.35 & 0.28 & 0.31 & 0.31 \\
\hline \multirow{6}{*}{ E vs $\mu$} & RCM1 & 0.37 & 0.29 & 0.41 & 0.27 & 0.18 \\
& RCM2 & 0.4 & 0.36 & 0.6 & 0.3 & 0.26 \\
& RCM3 & 0.33 & 0.26 & 0.54 & 0.31 & 0.21 \\
& AWAP & 0.55 & 0.28 & 0.46 & 0.48 & 0.24 \\
\hline
\end{tabular}

\subsubsection{Multiyear Variability}

Table 11.2 and Table 11.3 show the spatially and temporally averaged values of multiyear statistics of rainfall depths and wet periods respectively. It shows that the Richmond River site is the wettest among the five study sites with higher mean rainfall depths and number of wet days. There are two possible reasons: (1) the Richmond site is in the region of highest impact of ECL where ECL is one of the major drivers of extreme rainfall, and (2) the pixels of Richmond site are mostly along the coastline, while the coastline regions are generally wetter than the inland regions. The Goulburn River site in the upper Hunter inland region is the driest among the five sites, while the Williams River in the lower Hunter coastal region is wetter than the Goulburn. The Sydney site is wetter than Goulburn but drier than Williams, probably because the Sydney site comprises both coastal and inland regions. The Bega River site is in the south of NSW coastline and drier than the Williams, probably because the Bega site is in the region of least impact of ECLs. It also indicates that there is gradual decrease of rainfall depths and number of wet days from the north to the south along the coast with an order of wetness as Richmond $>$ Williams $>$ Bega. 
Table 11.2: Spatially and temporally averaged values of the mean and SD of rainfall depth at annual and multiyear resolutions.

\begin{tabular}{ccccccc}
\hline Statistics & Data & Richmond & Williams & Goulburn & Sydney & Bega \\
\hline Annual & RCM1 & 1774 & 1471 & 1087 & 1345 & 1413 \\
Mean & RCM2 & 1525 & 1189 & 884 & 1078 & 1108 \\
& RCM3 & 1932 & 1659 & 1086 & 1495 & 1582 \\
& AWAP & 1138 & 950 & 711 & 876 & 891 \\
\hline Multiyear & RCM1 & 821 & 683 & 543 & 615 & 481 \\
SD & RCM2 & 693 & 544 & 421 & 531 & 502 \\
& RCM3 & 883 & 784 & 535 & 710 & 592 \\
& AWAP & 557 & 437 & 300 & 546 & 513 \\
\hline
\end{tabular}

Table 11.3: Spatially and temporally averaged values of the mean and SD of wet period statistics at annual and multiyear resolutions.

\begin{tabular}{|c|c|c|c|c|c|c|c|}
\hline & Statistics & Data & Richmond & Williams & Goulburn & Sydney & Bega \\
\hline \multirow{8}{*}{ 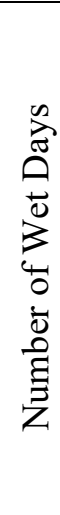 } & & RCM1 & 205 & 193 & 165 & 185 & 202 \\
\hline & Annual & RCM2 & 202 & 180 & 156 & 170 & 181 \\
\hline & Mean & RCM3 & 189 & 182 & 150 & 174 & 192 \\
\hline & & AWAP & 168 & 158 & 130 & 153 & 165 \\
\hline & & RCM1 & 44 & 40 & 40 & 41 & 52 \\
\hline & Multiyear & RCM2 & 36 & 38 & 35 & 38 & 46 \\
\hline & $\mathrm{SD}$ & $\mathrm{RCM} 3$ & 46 & 35 & 34 & 38 & 48 \\
\hline & & AWAP & 70 & 73 & 75 & 74 & 77 \\
\hline \multirow{8}{*}{ 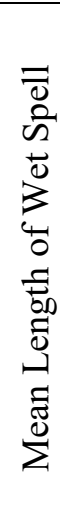 } & & RCM1 & 3.76 & 3.35 & 2.71 & 3.03 & 3.27 \\
\hline & Annual & RCM2 & 3.72 & 3.11 & 2.67 & 2.87 & 2.96 \\
\hline & Mean & RCM3 & 3.37 & 3.11 & 2.53 & 2.88 & 3.04 \\
\hline & & AWAP & 3.48 & 3.11 & 2.72 & 2.96 & 2.97 \\
\hline & & RCM1 & 0.54 & 0.47 & 0.32 & 0.39 & 0.47 \\
\hline & Annual & RCM2 & 0.54 & 0.41 & 0.32 & 0.38 & 0.44 \\
\hline & $\mathrm{SD}$ & $\mathrm{RCM} 3$ & 0.47 & 0.4 & 0.29 & 0.34 & 0.4 \\
\hline & & AWAP & 0.65 & 0.56 & 0.49 & 0.55 & 0.54 \\
\hline
\end{tabular}




\subsection{Comparison of CDMC, HMC and DHMC}

In each of these five sites, this study has compared the CDMC, HMC, and DHMC for the distribution and autocorrelations of rainfall depths and wet periods. While the detailed discussions for each site are available in Chapter 6 to 10, Table 11.4 and Table 11.5 show the sum of averaged $\mathrm{Z}$ scores for rainfall depths and wet period statistics respectably. It shows that the performance of all three models are similar for rainfall depths (as use same parameters of Gamma distribution), while DHMC performs better than CDMC and HMC for wet period statistics. For all sites, the models perform better for the RCMs than the AWAP. For both rainfall depths and wet period statistics, the performance of DHMC is best for the Richmond River site (wettest site) followed by Williams River site. This indicates that the DHMC performs better for the wetter sites, while performance of other three sites is also satisfactory. As a pragmatic conclusion the difference in the performance of all the models between the RCMs and AWAP suggest something fundamentally different between the datasets in their statistical behaviour. In a parallel project using traditional geostatistical techniques Parana Manage [2016] has suggested that the autocorrelation is markedly different between the datasets that this is consistent with a different relationship between short-term and long-term averages of rainfall for the RCMs versus AWAP.

Table 11.4: Comparison of MC models for mean and SD of rainfall depths.

\begin{tabular}{ccccccc}
\hline Statistics & Data & Richmond & Williams & Goulburn & Sydney & Bega \\
\hline \multirow{2}{*}{ RCM1 } & CDMC & 3.01 & 3.57 & 3.35 & 3.26 & 2.99 \\
& HMC & 3.09 & 3.38 & 2.99 & 3.22 & 3.53 \\
& DHMC & 2.79 & 3.35 & 3.08 & 3.22 & 4.02 \\
\hline \multirow{2}{*}{ RCM2 } & CDMC & 3.19 & 3.58 & 3.78 & 4.15 & 4.46 \\
& HMC & 3.2 & 3.32 & 3.4 & 3.61 & 4.07 \\
& DHMC & 3.04 & 3.46 & 3.67 & 4.09 & 4.79 \\
\hline \multirow{2}{*}{ RCM3 } & CDMC & 2.66 & 3.3 & 2.92 & 3.07 & 3 \\
& HMC & 2.69 & 3.13 & 2.9 & 3.15 & 3.37 \\
& DHMC & 2.6 & 3.15 & 2.87 & 3.12 & 3.81 \\
\hline \multirow{2}{*}{ AWAP } & CDMC & 3.35 & 3.13 & 3.75 & 3.47 & 3 \\
& HMC & 3.32 & 3.38 & 3.87 & 3.15 & 2.81 \\
& DHMC & 3.77 & 3.65 & 4.47 & 3.46 & 3.84 \\
\hline \multirow{2}{*}{ Sum } & CDMC & $\mathbf{1 2 . 2}$ & $\mathbf{1 3 . 6}$ & $\mathbf{1 3 . 8}$ & $\mathbf{1 4 . 0}$ & $\mathbf{1 3 . 5}$ \\
& HMC & $\mathbf{1 2 . 3}$ & $\mathbf{1 3 . 2}$ & $\mathbf{1 3 . 2}$ & $\mathbf{1 3 . 1}$ & $\mathbf{1 3 . 8}$ \\
& DHMC & $\mathbf{1 2 . 2}$ & $\mathbf{1 3 . 6}$ & $\mathbf{1 4 . 1}$ & $\mathbf{1 3 . 9}$ & $\mathbf{1 6 . 5}$ \\
\hline
\end{tabular}


Table 11.5: Comparison of MC models for mean and SD of wet period statistics.

\begin{tabular}{ccccccc}
\hline Statistics & Data & Richmond & Williams & Goulburn & Sydney & Bega \\
\hline \multirow{2}{*}{ RCM1 } & CDMC & 6.53 & 5.81 & 7.56 & 7.03 & 9.76 \\
& HMC & 6.65 & 6.33 & 8.73 & 6.01 & 7.23 \\
& DHMC & 3.26 & 3.84 & 5.08 & 4.29 & 3.79 \\
\hline \multirow{2}{*}{ RCM2 } & CDMC & 4.74 & 4.98 & 6.26 & 5.76 & 8.1 \\
& HMC & 6.63 & 6.18 & 8.71 & 6.06 & 6.23 \\
& DHMC & 3.04 & 3.22 & 4.69 & 3.91 & 4.06 \\
\hline \multirow{2}{*}{ RCM3 } & CDMC & 6.2 & 4.33 & 5.96 & 5.03 & 7.96 \\
& HMC & 6.45 & 6.46 & 8.88 & 6.21 & 6.73 \\
& DHMC & 2.86 & 3.06 & 4.19 & 3.18 & 3.36 \\
\hline \multirow{4}{*}{ AWAP } & CDMC & 10.61 & 11.1 & 16.95 & 11.61 & 12.07 \\
& HMC & 8.22 & 8.46 & 10.5 & 8.56 & 9.23 \\
& DHMC & 3.68 & 3.78 & 4.14 & 4.05 & 3.53 \\
\hline \multirow{2}{*}{ Sum } & CDMC & $\mathbf{2 8 . 1}$ & $\mathbf{2 6 . 2}$ & $\mathbf{3 6 . 7}$ & $\mathbf{2 9 . 4}$ & $\mathbf{3 7 . 9}$ \\
& HMC & $\mathbf{2 8 . 0}$ & $\mathbf{2 7 . 4}$ & $\mathbf{3 6 . 8}$ & $\mathbf{2 6 . 8}$ & $\mathbf{2 9 . 4}$ \\
& DHMC & $\mathbf{1 2 . 8}$ & $\mathbf{1 3 . 9}$ & $\mathbf{1 8 . 1}$ & $\mathbf{1 5 . 4}$ & $\mathbf{1 4 . 7}$ \\
\hline
\end{tabular}

\subsection{Conclusion}

The analysis of rainfall using NARCliM reanalysis of three RCMs and AWAP gridded datasets in the five case study sites along the east coast of Australia suggests that the rainfall in the east coast has similar intra-annual variability, but the spatial variability of rainfall in each site is influenced by local factors (e.g. orography). The comparative assessments of the CDMC, HMC and DHMC show that the models perform consistently in each site, irrespective of the intraannual and spatial variability. However, the DHMC is better than the other models for stochastic simulation of rainfall, and has the ability to reproduce the key characteristics of rainfall in each of the five case study sites with different degrees of ECL influence, which is important for urban water security. The MC models fit the RCMs better than AWAP which suggests (1) fundamental limitations in the overarching modelling framework for natural data, (2) and something fundamentally different in the statistical properties between the RCMs and AWAP. 


\section{Comparison of $M C$ and MMKD for Raingauge Data around Australia}

\subsection{Introduction}

There are a number of stochastic models available that address the underestimation of rainfall variability at monthly to multiyear resolutions. A comprehensive review of these models was presented in Chapter 2 at section 2.5. Mehrotra and Sharma [2007] proposed a model using a modified first-order Markov Chain and Kernel Density estimation focusing on the underestimation of monthly to multiyear variability. This study has referred to their model as the Modified Markov-Kernel Density (MMKD) model. The methodology of the MMKD was discussed in Chapter 2 at section 2.6. This chapter will present a comparison of the MMKD and the three MC models of this study (CDMC, HMC and DHMC).

Mehrotra et al. [2015] developed an R software package for daily rainfall simulation, based on the MMKD. This study has used this R package to assess its performance to reproduce the key statistics of rainfall depths and wet periods, and compare the relative performances with the CDMC, HMC and DHMC. The R package of MMKD is mainly a multisite version of the model, which uses a spatial dependence matrix to incorporate the spatial correlations. However, since this study compares the MMKD with the single-site MC models, the MMKD has also been run as a single-site model without using the spatial dependence matrix.

This chapter will discuss the methodological comparison between the MMKD and the three MC models. Then, the comparative performance of the models to reproduce the statistics of observed data (daily rainfall data for 30-year period of 1979-2008) at daily to multiyear resolutions for 12 stations around Australia (see Figure 3.8 and section 3.3.6) will be presented. These 12 stations were selected in such a way that they represent a wide-range of climate characteristics, and include coastal, monsoonal and arid climates, which also represent major world climatic zones. Therefore, this comparison of the MMKD and MC models is useful in a global perspective, testing their suitability for different world climates. In this chapter, the model performances for rainfall depths and wet period statistics are assessed using the method described in section 3.4. 
Chapter 13 will also present the comparison of performance between the MMKD and three MC models using raingauge data from 30 stations around Sydney (see section 3.3.7). A further comparison of the four models will be presented in Chapter 14 for validation periods (i.e. comparison for the statistics of rainfall samples with periods and/or record length different to the calibration period) using raingauge data from the Sydney station shown in Figure 3.8 (i.e. Sydney Observatory Hill Station). However, in the previous chapters, the NARCliM and AWAP data were used to compare the performance of three MC models only (and only for the calibration period). Therefore, in Chapter 15, this study will compare the performance of the MMKD and three MC models using the NARCliM and AWAP data for rainfall and streamflow generation for both calibration and validation periods.

\subsection{Methodological Comparison}

There are similarities and differences among the MMKD and three MC models (CDMC, HMC and DHMC). This section presents the methodological comparison of these three models.

\subsubsection{Common Features}

The key common features of the MMKD, CDMC, HMC and DHMC are that they all:

- Simulate rainfall at daily resolution

- Use a two-part model structure: one part to simulate the rainfall occurrences (wet or dry) and other part to simulate rainfall depths in wet days

- Use first-order MC parameters (i.e. transition probabilities of dry-to-dry and wet-to-wet days) to simulate the rainfall occurrence.

\subsubsection{Differences}

The methodological differences among the CDMC, HMC, DHMC and MMKD are shown in Table 12.1. 
12. Comparison of MC and MMKD for Raingauge Data around Australia

Table 12.1: Methodological differences among CDMC, HMC, DHMC and MMKD.

\begin{tabular}{|c|c|c|c|}
\hline & $\begin{array}{l}\text { Wet and dry day } \\
\text { simulation }\end{array}$ & $\begin{array}{c}\text { Wet day rainfall depth } \\
\text { simulation }\end{array}$ & Seasonal variability \\
\hline MMKD & $\begin{array}{l}\text { Uses modified MC } \\
\text { parameters analytically } \\
\text { derived by using additional } \\
\text { factors (e.g. wetness over } \\
\text { past periods). }\end{array}$ & $\begin{array}{l}\text { Uses nonparametric kernel- } \\
\text { density estimator, resample } \\
\text { rainfall depths from observed } \\
\text { records. The rainfall depth of } \\
\text { current day is conditioned to } \\
\text { the rainfall depth of previous } \\
\text { day. }\end{array}$ & $\begin{array}{l}\text { Uses a moving window } \\
\text { (e.g. } 30 \text { days) to } \\
\text { reproduce the seasonal } \\
\text { variability. }\end{array}$ \\
\hline CDMC & $\begin{array}{l}\text { Uses deterministic MC } \\
\text { parameters, same set of } \\
\text { parameters for each } \\
\text { simulation year. }\end{array}$ & $\begin{array}{l}\text { Uses stochastic parameters } \\
\text { (sampled from fitted } \\
\text { bivariate-lognormal } \\
\text { distribution) of Gamma } \\
\text { distribution, parameters vary } \\
\text { for each year simulation. }\end{array}$ & $\begin{array}{l}\text { Uses separate set of } \\
\text { parameters for each } \\
\text { month (e.g. } 12 \text { sets of } \mathrm{MC} \\
\text { and Gamma parameters) } \\
\text { to reproduce seasonal } \\
\text { variability. }\end{array}$ \\
\hline HМС & $\begin{array}{l}\text { Uses stochastic MC } \\
\text { parameters (sampled from } \\
\text { fitted truncated normal } \\
\text { distribution), parameters vary } \\
\text { for each year simulation. }\end{array}$ & Same as CDMC & Same as CDMC \\
\hline DHМC & $\begin{array}{l}\text { Uses decadally varied } \\
\text { deterministic MC } \\
\text { parameters. }\end{array}$ & Same as CDMC & Same as CDMC \\
\hline
\end{tabular}

\subsection{Distribution Statistics of Observed Rainfall}

From the 30-year raingauge data at each of the coastal, inland and monsoonal stations (station categories are described in section 3.3.6), this study has calculated the mean and SD of rainfall depths and wet period statistics (i.e. number of wet days and mean length of wet spells) at daily to multiyear resolutions. This section presents these distribution statistics of rainfall depths and wet periods. Section 12.4 will present comparative performance of the MMKD, CDMC, HMC and DHMC to reproduce these statistics. 


\subsubsection{Mean and SD of Daily Rainfall Depths}

For each station, the intra-annual variability of mean and SD of wet day rainfall depths (i.e. $\mu$ and $\sigma$ ) are mostly consistent with the intra-annual variability of mean and SD of monthly rainfall depths. Therefore, the intra-annual variability of monthly statistics of rainfall depths are discussed in section 12.3.2.

\subsubsection{Mean and SD of Rainfall Depth and Number of Wet Days at Monthly Resolution}

Figure 12.1 shows the mean and SD of monthly rainfall depth and number of wet days in each station.

The top six panels of Figure 12.1 show the intra-annual variability of monthly rainfall depth and number of wet days for six coastal stations. In the east coastal stations of Sydney and Brisbane, the monthly mean rainfall depth and wet days are higher in summer than winter. In the south and south-eastern stations of Adelaide and Melbourne, there are more wet days in winter than in summer. In Melbourne, the rainfall depth is higher in summer (indicates few but very heavy rainfall days) than winter. The Hobart rainfall does not necessarily follow this summer-winter trend. In Hobart, the mean rainfall depth and wet days in July to January is higher than in February to June. However, the overall wetness among months is similar to those of south-eastern stations. In Perth, the winter is significantly wetter than a very dry summer. 

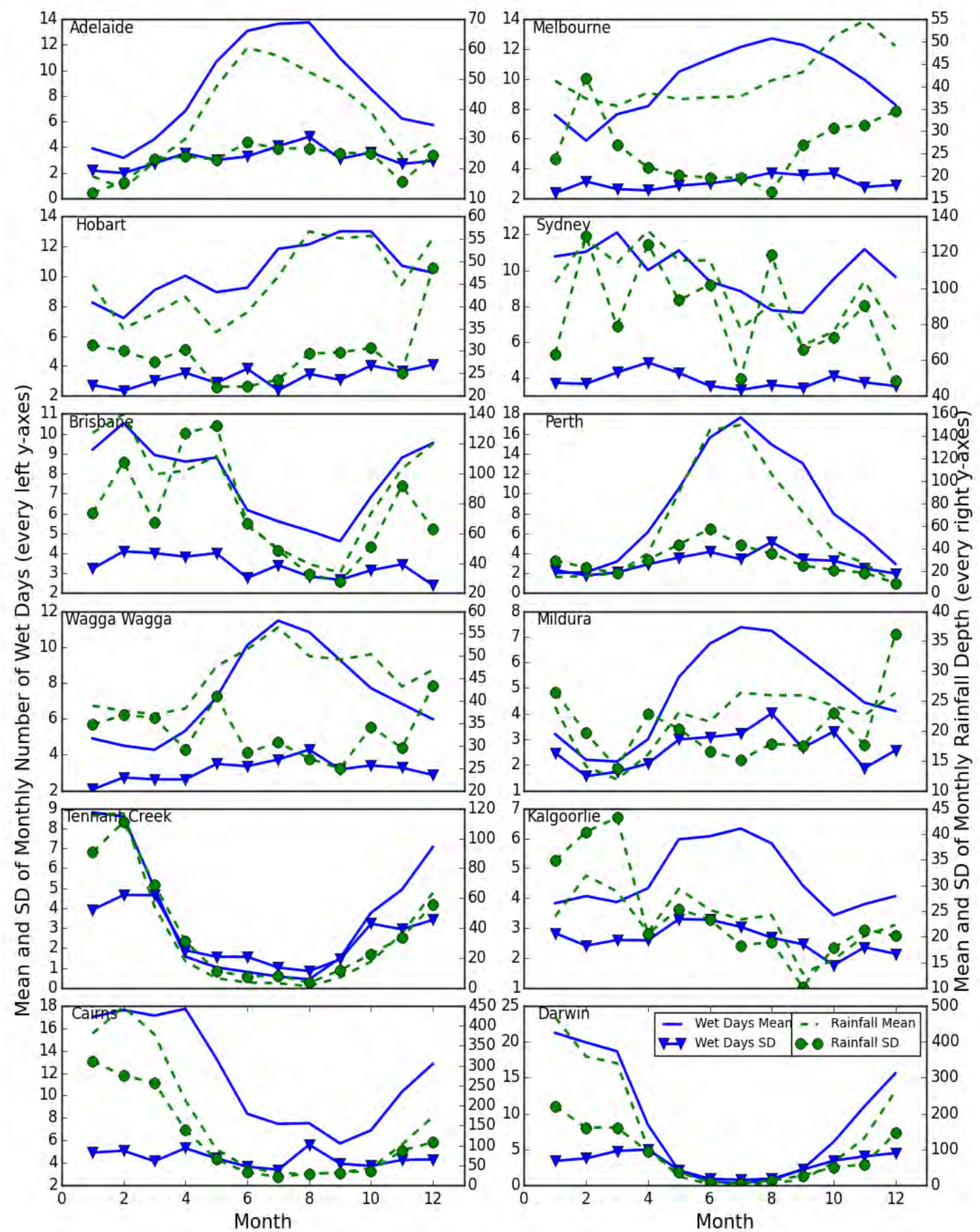

Figure 12.1: Mean and SD of monthly number of wet days and rainfall depth for the coastal (top 6 panels), inland (middle 4 panels), and monsoonal (bottom 2 panels) stations around Australia. Each left $y$-axis denotes mean and SD of number of wet days per month, and each right $y$-axis denotes mean and SD of monthly rainfall depths. Note the change in vertical scales. 
The inland stations (middle four panels in Figure 12.1) are generally drier (lower mean rainfall depth and less wet days) than the coastal stations. The intra-annual variability of inland stations is mostly influenced by the vicinity of the coastal or monsoonal climate zones. For example, the intra-annual variability of Wagga Wagga and Mildura are mostly influenced by the southeastern coastal climate (e.g. Adelaide), with winter being wetter than summer. Kalgoorlie has more wet days in winter than summer with a possible influence of Western Australia coastal climate (e.g. Perth). In Tennant Creek, the mean rainfall depth and number of wet days are higher in November to March (monsoon) with the influence of monsoonal climate, while the other months (e.g. May-September) are extremely dry with almost no rainfall (i.e. there were several years when no rainfall occurred in these months).

The monsoonal stations (bottom two panels in Figure 12.1) in northern Australia generally have higher mean monthly rainfall depth and number of wet days from November/December to March/April and significantly lower rainfall and less wet days (i.e. extremely dry with no rainfall) in May to October.

The SD of monthly rainfall depth and wet days does not vary significantly among months (seasons) for most of the stations (Figure 12.1). Although the seasonal trend is not very strong, the intra-annual variability of SD mostly follows the trend of mean.

The wetness of a station is generally represented by the mean of rainfall depth and number of wet days. The SD of the rainfall depth and number of wet days of a month represent how they vary over the years of the sampling period (e.g. over the 30-year period). Generally, the intraannual variability among months is stronger in mean of the rainfall depth and wet days than the $\mathrm{SD}$ of the same. The intra-annual variability of mean rainfall depth follows the trend of mean number of wet days in most the stations, irrespective of climate zone. 


\subsubsection{Mean and SD of Multiyear Rainfall Depth and Number of Wet Days}

Table 12.2 shows the mean of annual number of wet days and rainfall depths. For coastal stations, the annual mean number of wet days is generally about 100 days, with annual mean rainfall depths around $500 \mathrm{~mm}$ (except Sydney, Perth and Brisbane). Compared to the coastal stations, the monsoonal stations are generally wetter (higher mean annual wet days and rainfall depths), while the inland stations are generally drier. The ratios of rainfall depth and wet days are generally around five in coastal stations indicating consistent mild rainfall in most of the wet days, while the ratio is around 15 in monsoonal stations indicating very heavy rainfall in most of the wet days. Although Sydney and Brisbane are coastal stations, both have higher depth/days ratio as the east coastal stations generally experience heavy rainfall in a considerable number of wet days per year. The depth/days ratio for inland stations are also around 5, except Tennant Creek with monsoonal influence has a higher ratio of 10:1.

Figure 12.2 shows the SD of rainfall depth and number of wet days in 1 to 10 overlapping years. In general, the value of each SD is highly correlated to the corresponding mean value (i.e. the SD values are higher in the stations with higher respective mean). In most of the stations, the trend is similar in both SD of rainfall depth and number of wet days.

Table 12.2: Mean of annual number of wet days and rainfall depth.

\begin{tabular}{llccc}
\hline Climate Zone & Location & \multicolumn{2}{c}{ Annual Mean } & \multicolumn{2}{c}{$\begin{array}{c}\text { Mean Depth/ } \\
\text { Mean Wet Days }\end{array}$} \\
\cline { 1 - 3 } & Adelaide & Wet Days & Rainfall Depth & M.3 \\
& Brisbane & 101 & 439 & 11.4 \\
Coastal & Hobart & 124 & 547 & 4.4 \\
& Melbourne & 118 & 504 & 4.3 \\
& Perth & 102 & 720 & 7.1 \\
& Sydney & 119 & 1201 & 10.1 \\
\hline \multirow{3}{*}{ Inland } & Wagga Wagga & 88 & 547 & 6.2 \\
& Mildura & 57 & 262 & 4.6 \\
& Tennant Creek & 44 & 443 & 10.1 \\
& Kalgoorlie & 56 & 278 & 5.0 \\
\hline \multirow{2}{*}{ Monsoonal } & Cairns & 142 & 1962 & 13.8 \\
& Darwin & 107 & 1778 & 16.6 \\
\hline
\end{tabular}



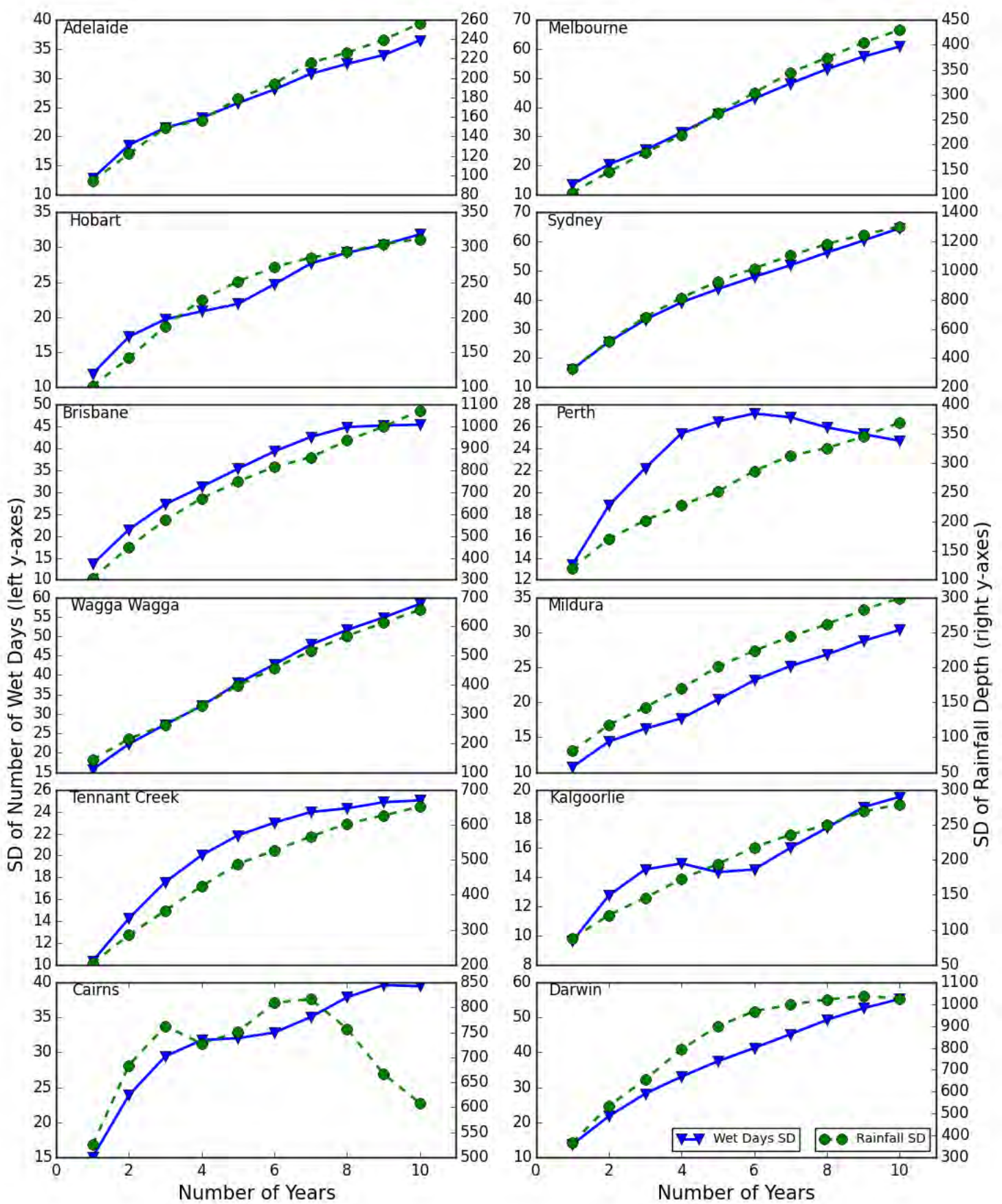

Figure 12.2: SD of multiyear number of wet days (each left $y$-axis) and multiyear rainfall depth (each right $\mathbf{y}$-axis) for 1 to 10 overlapping years. Note the change in vertical scale. 


\subsubsection{Mean and SD of Mean Length of Wet Spells at Monthly and Annual Resolution}

The seasonal trend of the mean and SD of monthly mean length of wet spells $\mu_{w e t}$ (calculated as per section 3.4.2.1) mostly follows the trend of the mean and SD of monthly number of wet days respectively for each station, and hence are shown in Appendix H.1. The mean of $\mu_{w e t}$ usually varies between 1.5 and 2.5 days for coastal station, and below 1.5 days for inland stations. For the monsoonal stations, the monsoonal (e.g. November to March) wet spells are the longest with mean of monthly $\mu_{\text {wet }}$ between 2.0 to 5.5 days. The intra-annual variability of $\mathrm{SD} \mu_{w e t}$ mostly follows the trend of mean $\mu_{w e t}$ for each station.

Figure 12.3 shows the mean and SD of annual mean length of wet spells $\left(\mu_{w e t}\right)$ in each station (calculated as per section 3.4.2.2). For coastal stations, the mean of annual $\mu_{w e t}$ are around 2.0 days, with Perth and Sydney having slightly longer wet spells in comparison with other coastal stations. For inland stations, the mean of annual $\mu_{w e t}$ is around 1.5 days in Mildura and Kalgoorlie, while just below 2.0 days in Wagga Wagga and Tennant Creek. The longest wet spells are for monsoonal stations (mean $\mu_{w e t}$ are around 3 days). The SDs of annual $\mu_{w e t}$ are numerically very small (less than 0.5 ) for all stations.

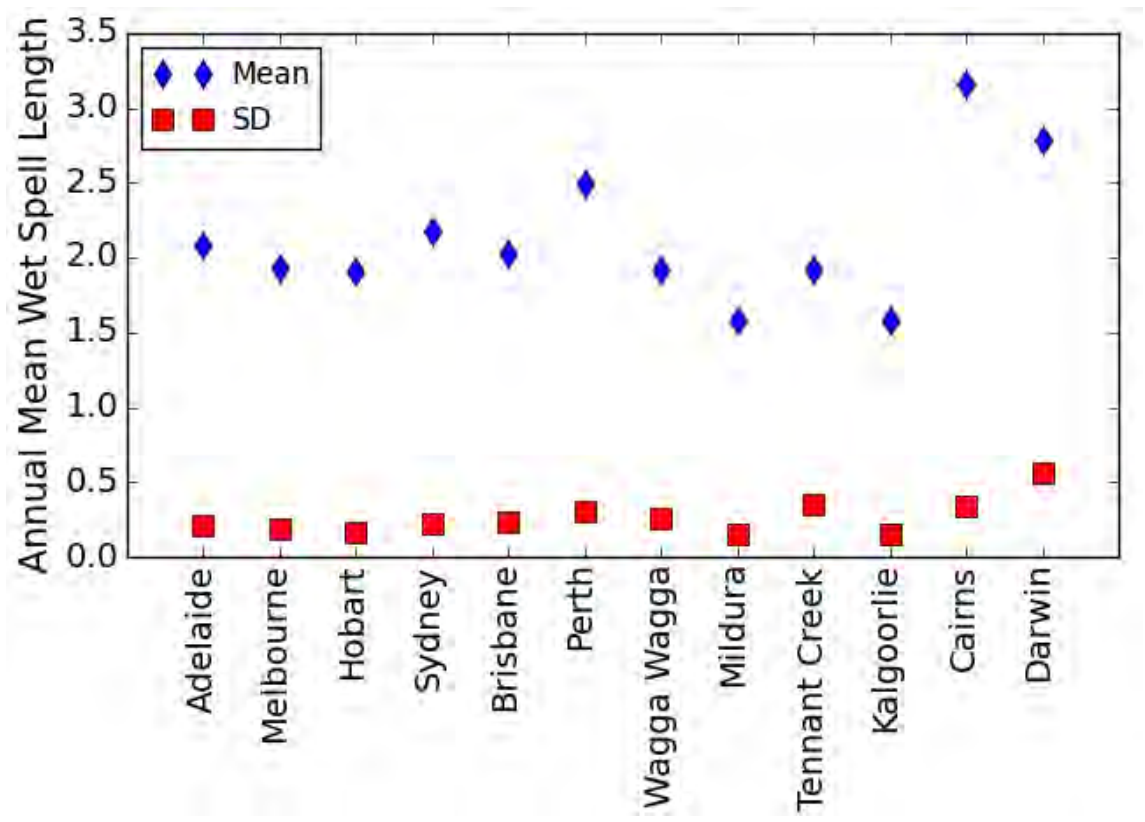

Figure 12.3: Mean and SD of annual mean length of wet spells. 


\subsection{Model Comparison for Distribution Statistics}

This section compares the performance of MMKD and three MC models (CDMC, HMC and DHMC) for reproducing the mean and SD of rainfall depths and wet period statistics. The $\mathrm{Z}$ scores of each model are calculated using the method described in section 3.4.3.

\subsubsection{Mean and SD of Wet Day Rainfall Depths}

Figure 12.4 shows the comparison for reproduction of the mean of wet day rainfall depths, while Figure 12.5 shows the same comparison for SD. For mean of wet day rainfall depths, the CDMC, HMC and DHMC (which have similar performances) tend to underestimate and MMKD tends to overestimate the mean. The three MC models have significantly underestimated the mean for some months of a few stations (see Perth, Tennant Creek, Mildura and Darwin in Figure 12.4). Such months of respective stations are generally extremely dry with almost no rainfall occurring in those months for several years (discussed in section 12.3.2), and hence, fitting a bivariate-lognormal distribution to the Gamma parameters is inadequate for those months. Therefore, the CDMC, HMC and DHMC which use stochastically sampled Gamma parameters from fitted bivariate-lognormal distribution to generate wet day rainfall depths cannot adequately generate those very small rainfalls in extremely dry conditions. Use of deterministic Gamma parameters (same as APMC) instead of stochastic Gamma parameters for these extremely dry months might resolve this issue. The MMKD can generate these very small rainfalls as the model resamples rainfall depths from observed records. However, this failure of the CDMC, HMC and DHMC is negligible, as those small rainfalls in extreme dry conditions generally have no contribution to the streamflow and reservoir water. For SD of wet day rainfall depths (Figure 12.5), the performance of each model is mostly consistent with their respective performance for mean of wet day rainfall depths. 
12. Comparison of MC and MMKD for Raingauge Data around Australia
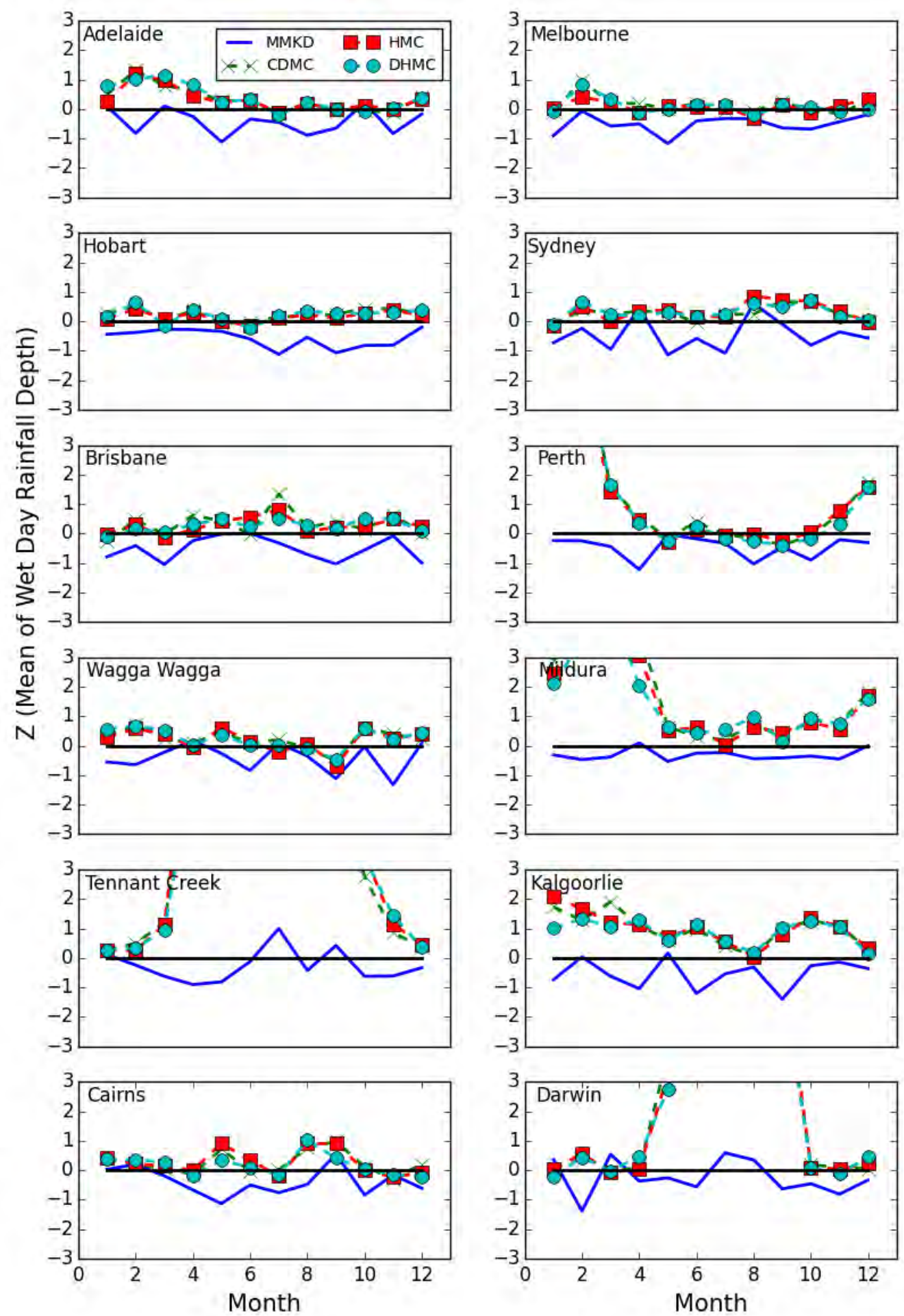

Figure 12.4: Comparison of model performances for mean of daily rainfall depths. 

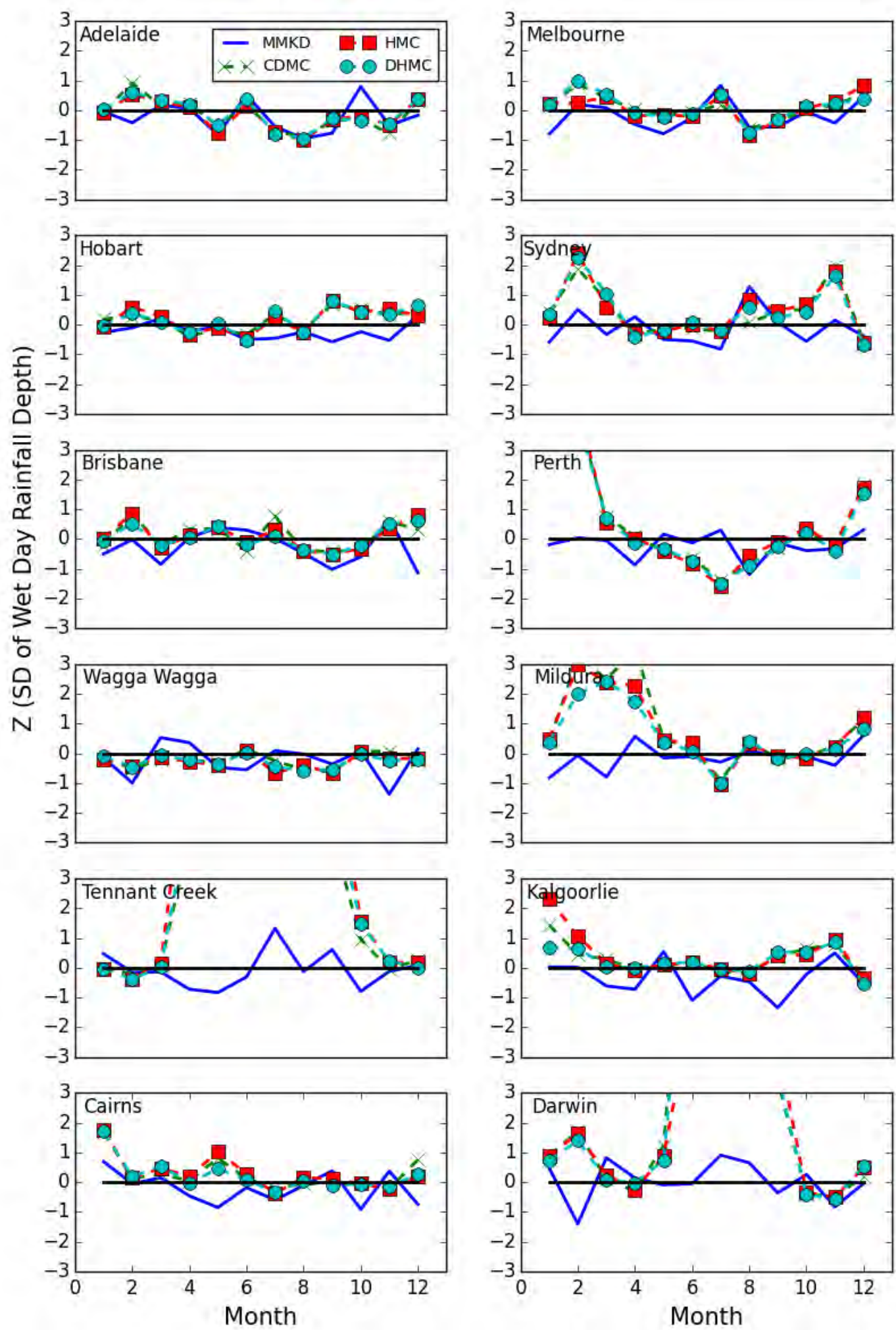

Figure 12.5: Comparison of model performances for SD of daily rainfall depths. 


\subsubsection{Mean and SD of Monthly Rainfall Depths}

For mean and SD of monthly rainfall depths, the performance of each model is mostly consistent with their respective performances for mean and SD of wet day rainfall depths (see Figure H.2-H.3 at Appendix H.2, and compare with Figure 12.4 and Figure 12.5 for mean and $\mathrm{SD}$ respectively).

\subsubsection{Mean and SD of Multiyear Rainfall Depths}

Figure 12.6 compares the models for the mean of multiyear rainfall depths, while Figure 12.7 compares for the SD.

For the mean (Figure 12.6), while all four models are mostly satisfactory, the CDMC, HMC and DHMC (which have a similar performance) tend to underestimate and the MMKD tends to overestimate the statistic. For coastal and monsoonal stations, the MC models perform better than MMKD with $\mathrm{Z}$ scores closer to zero, while the MMKD performs better than the MC models for the inland stations.

For the SD (Figure 12.7), all four models are mostly satisfactory. However, for all stations except Cairns (monsoonal station), all four models show a slight tendency to overestimate the $\mathrm{SD}$ of rainfall depths for shorter multiyear resolutions (e.g. 1 to 3 overlapping years) and underestimate the SD for longer multiyear resolutions (e.g. 7 to 10 overlapping years). 

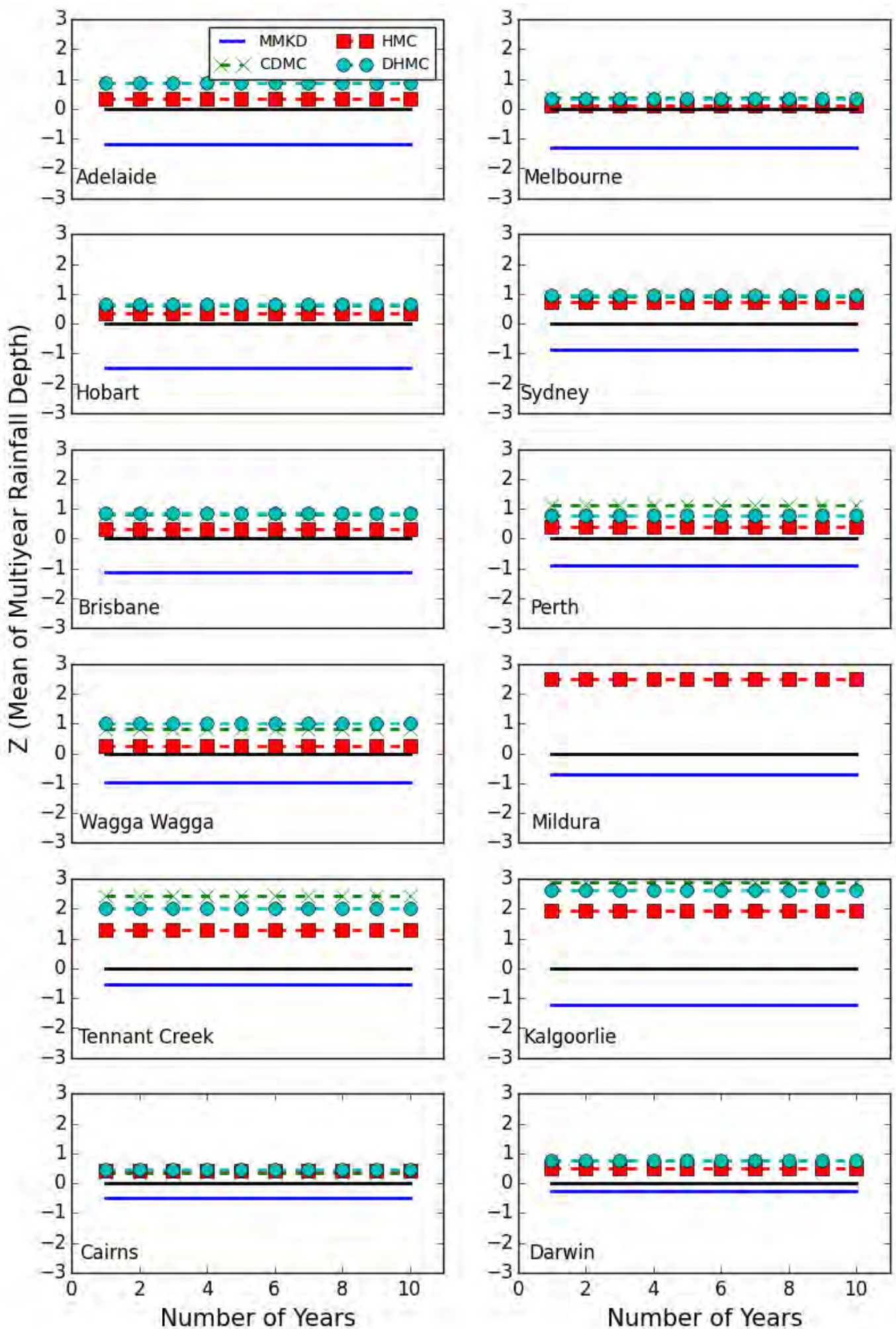

Figure 12.6: Comparison of model performances for mean of multiyear rainfall depths. 
12. Comparison of MC and MMKD for Raingauge Data around Australia
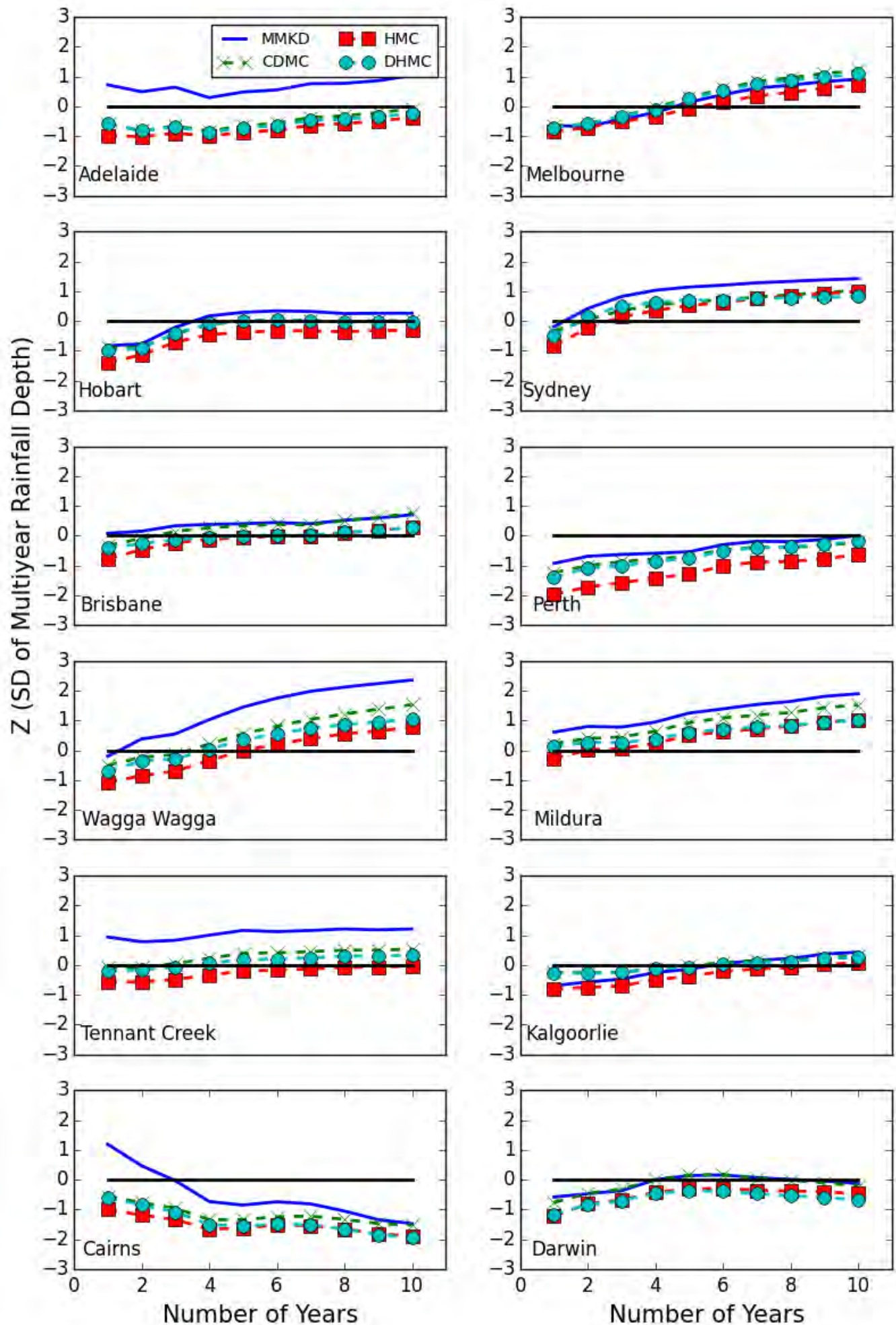

Figure 12.7: Comparison of model performances for SD of multiyear rainfall depths. 


\subsubsection{Mean and SD of Monthly and Multiyear Number of Wet Days}

Figure 12.8 compares the CDMC, HMC, DHMC and MMKD for the mean of monthly number of wet days, while Figure 12.9 compares their performances for the SD. For mean and SD of monthly number of wet days, CDMC and DHMC perform similarly to MMKD with no underor over-estimation bias. However, HMC tends to overestimate the mean, while it significantly overestimates ( $Z$ scores mostly less than -2$)$ the SD.

For the mean of multiyear number of wet days, the comparative performances of the models are consistent with their respective performances for mean of monthly number of wet days (see Figure H.4 at Appendix H.2). For SD of multiyear number of wet days (Figure 12.10), the CDMC fails to preserve the statistic, with a tendency of underestimation. The HMC, DHMC and MMKD preserve the SD of multiyear number of wet days, while the trend of $Z$ scores (increasing with years) is similar in the HMC and MMKD. The HMC and MMKD tend to overestimate the statistic at shorter multiyear resolutions (e.g. 1 to 3 overlapping years) and underestimate at longer multiyear resolutions (e.g. 7 to 10 overlapping years). In contrast, the DHMC tends to underestimate the statistic at shorter multiyear resolutions and overestimate at longer multiyear resolutions. 
12. Comparison of MC and MMKD for Raingauge Data around Australia
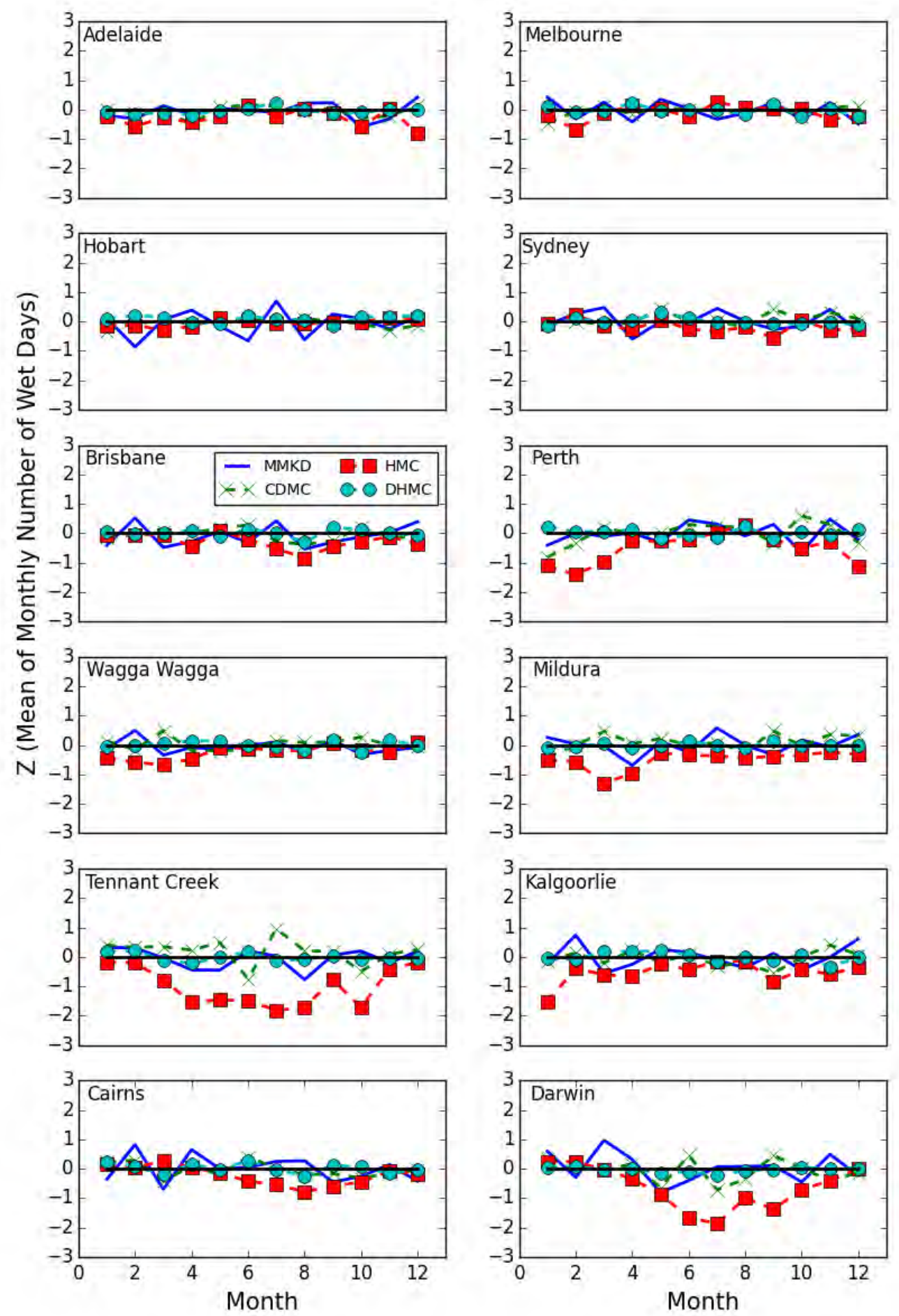

Figure 12.8: Comparison of model performances for mean of monthly number of wet days. 

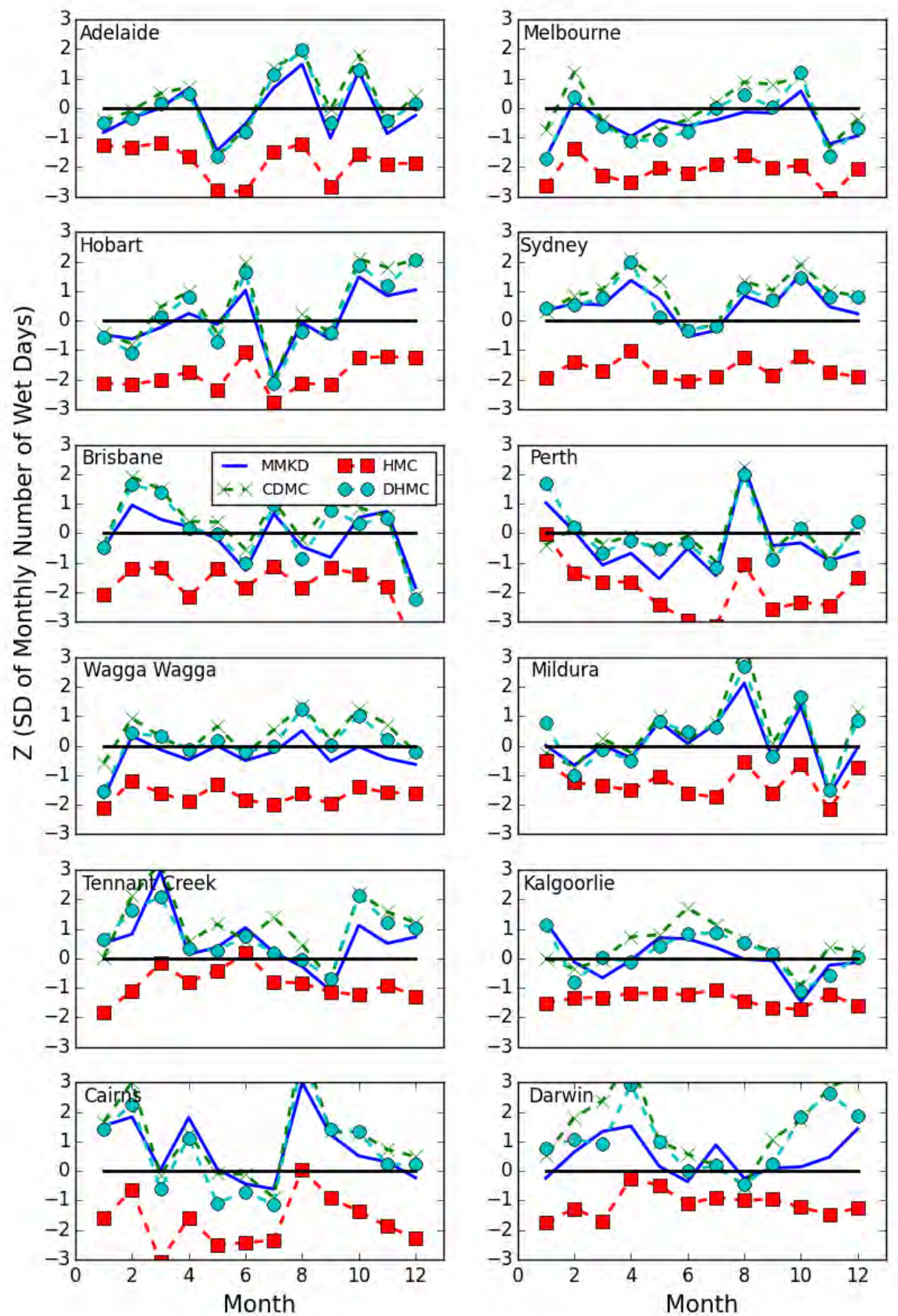

Figure 12.9: Comparison of model performances for SD of monthly number of wet days. 

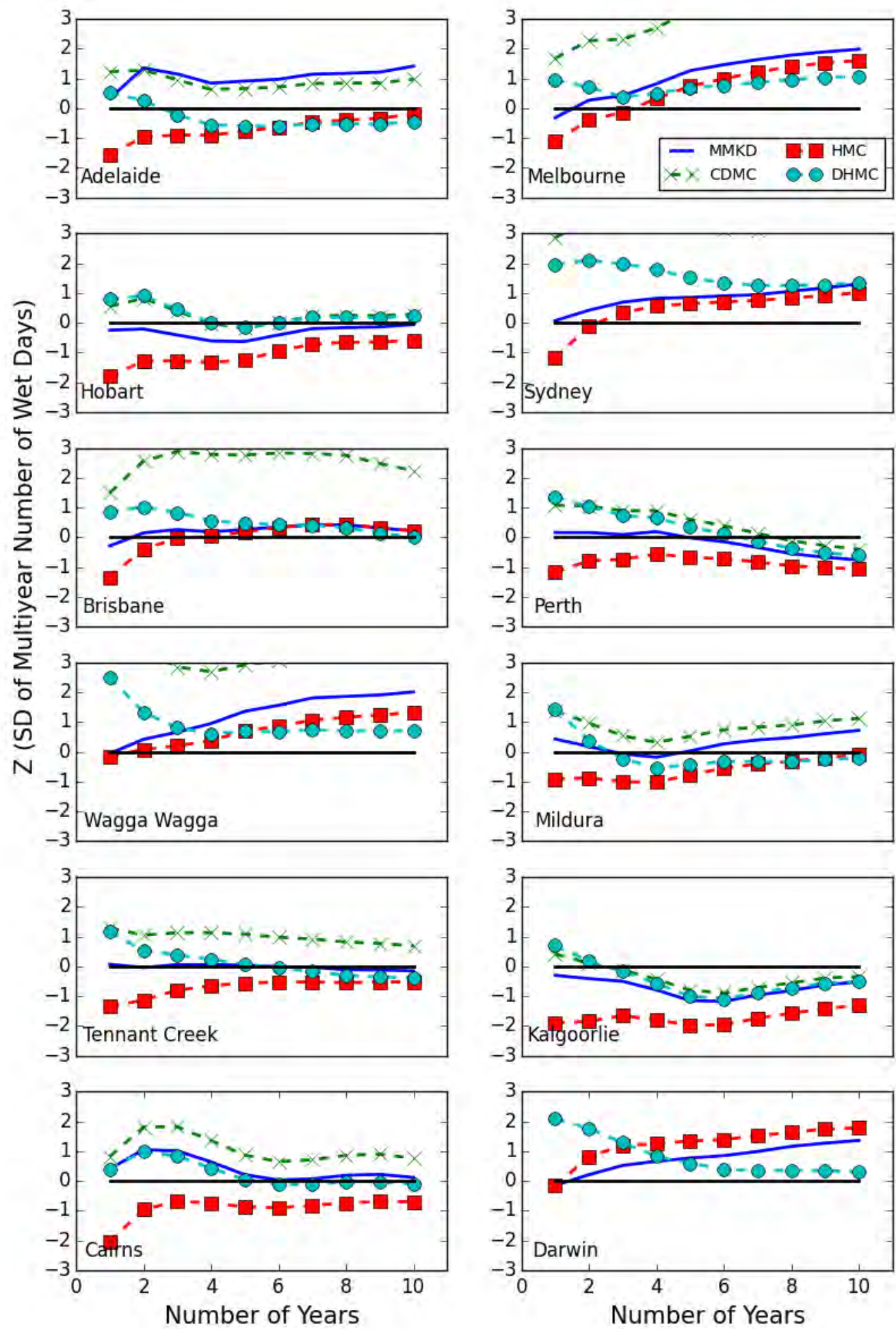

Figure 12.10: Comparison of model performances for SD of multiyear number of wet days. 


\subsubsection{Mean and SD of Monthly Mean Length of Wet Spells}

For the mean and SD of monthly mean length of wet spells, the comparative performances of the models are consistent with their respective performances for mean and SD of monthly number of wet days (see Figure H.5-H.6 at Appendix H.2). While the performances of the CDMC, DHMC and MMKD are similar and satisfactory, the HMC tends to overestimate the mean and SD of monthly mean length of wet spells.

Figure 12.11 compares the models for the mean of annual mean length of wet spells, while Figure 12.12 compares for the SD. For the mean (Figure 12.11), the performance of all four models is mostly comparable and satisfactory. For the SD (Figure 12.12), the performance of the CDMC, DHMC and MMKD is similar and satisfactory, while the HMC tends to consistently overestimate the SD.

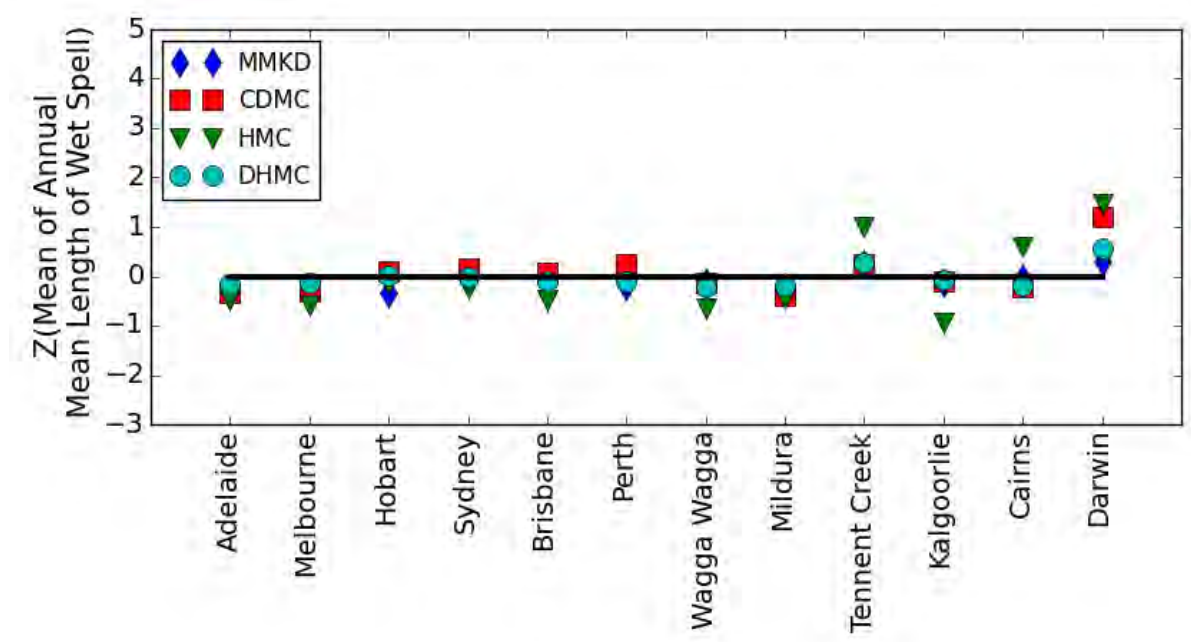

Figure 12.11: Comparison of models for mean of annual mean length of wet spells. 


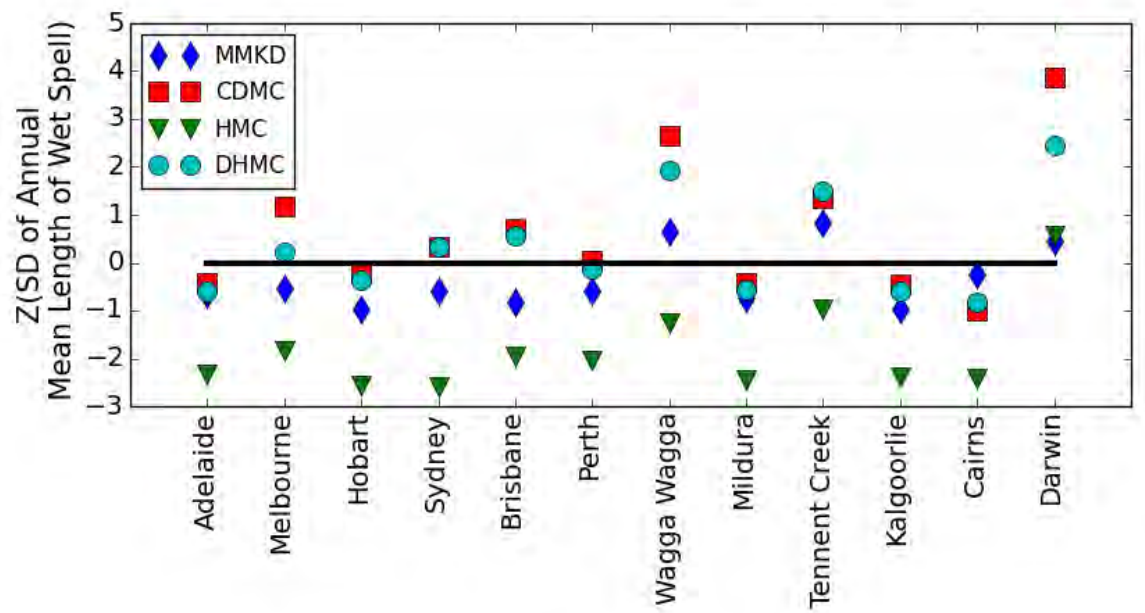

Figure 12.12: Comparison of models for SD of annual mean length of wet spells.

\subsection{Autocorrelations of Monthly Wet Days and Rainfall Depth}

Figure 12.13 shows the month-to-month autocorrelations of the monthly number of wet days and rainfall depths for coastal stations, while Figure 12.14 shows the data for the inland and monsoonal stations.

In general, the monthly autocorrelation function for wet days is stronger than for rainfall depths. Irrespective of the strength of the autocorrelation coefficients, there are clear seasonal trends for almost all stations. The autocorrelations of both the monthly number of wet days and monthly rainfall depths are strong for Adelaide, Perth, Tennant Creek, Cairns and Darwin. However, for Melbourne, Hobart, Brisbane, Wagga Wagga and Mildura, the autocorrelations for monthly number of wet days are strong, but the autocorrelations for monthly rainfall depths are weak. For Sydney and Kalgoorlie, the autocorrelations for both wet days and rainfall depths are weak. 
12. Comparison of MC and MMKD for Raingauge Data around Australia
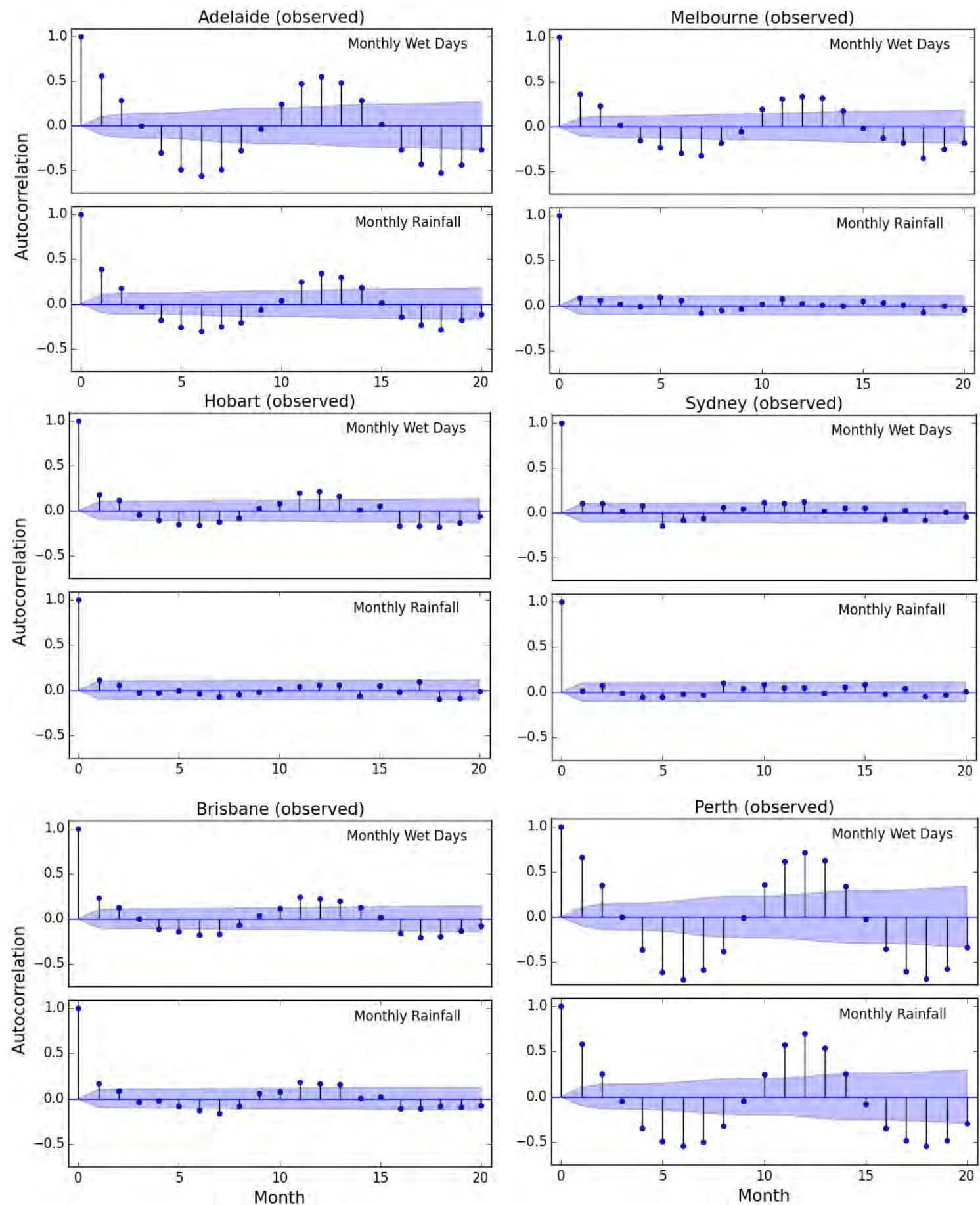

Figure 12.13: Month-to-month autocorrelations of monthly number of wet days and monthly rainfall depths for coastal stations. 
12. Comparison of MC and MMKD for Raingauge Data around Australia
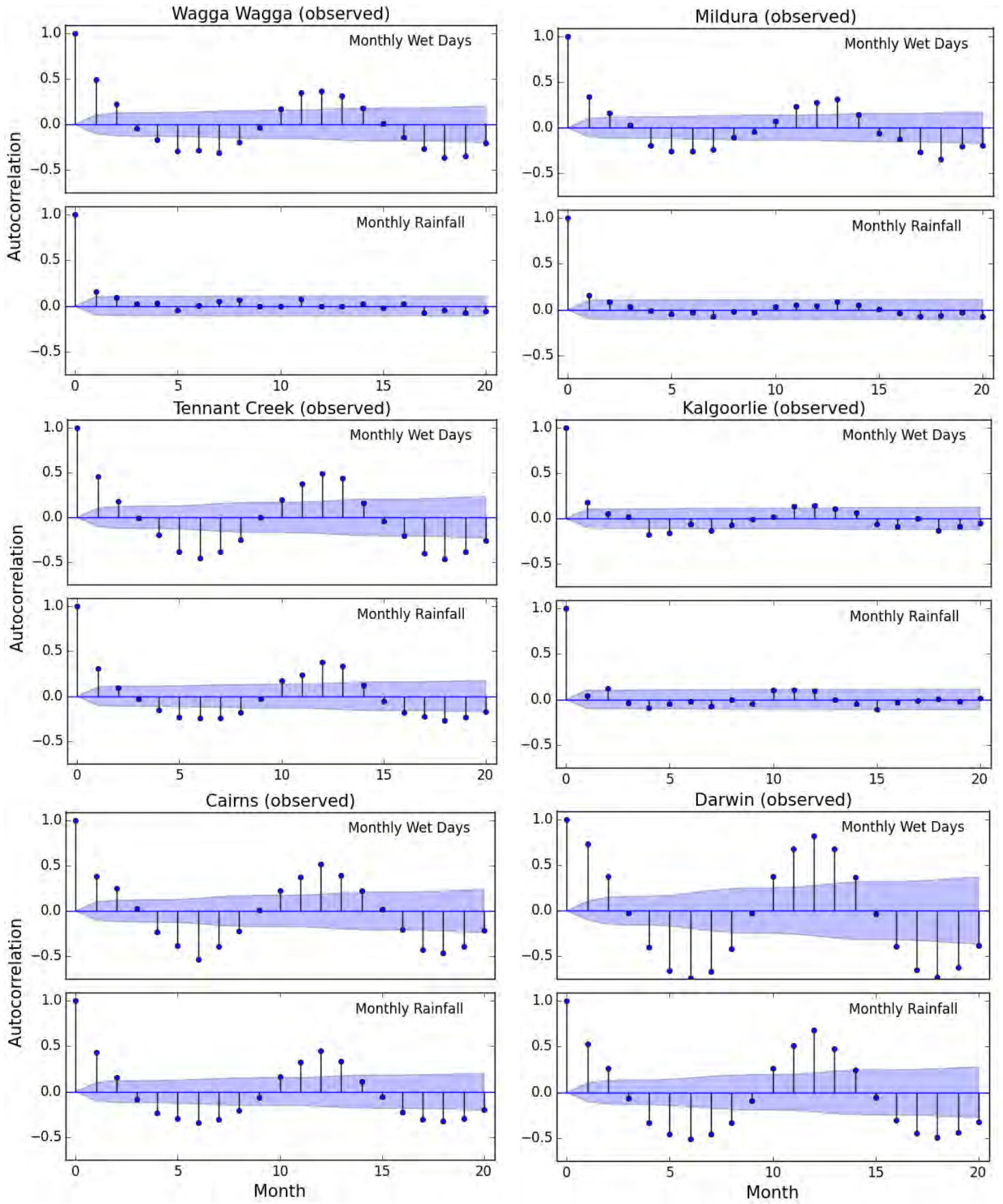

Figure 12.14: Month-to-month autocorrelations of monthly number of wet days and monthly rainfall depths for the inland and monsoonal stations. 


\subsection{Model Comparison for Autocorrelations}

Since the observed autocorrelations of monthly number of wet days and monthly rainfall depths (shown in Figure 13 and 14) are generally strong at lag-1 and lag-12, Figure 12.15 compares the four models for the autocorrelations at lag-1 and lag-12. Compared to the observed autocorrelations, the CDMC, DHMC and MMKD show comparable satisfactory performances to reproduce the autocorrelations of monthly number of wet days and monthly rainfall depths with a tendency to underestimate. However, the HMC considerably underestimates the autocorrelations in most of the stations. The performance of each model is consistent to the above performance for all lags between lag-1 and lag-20 (see Appendix H.3).
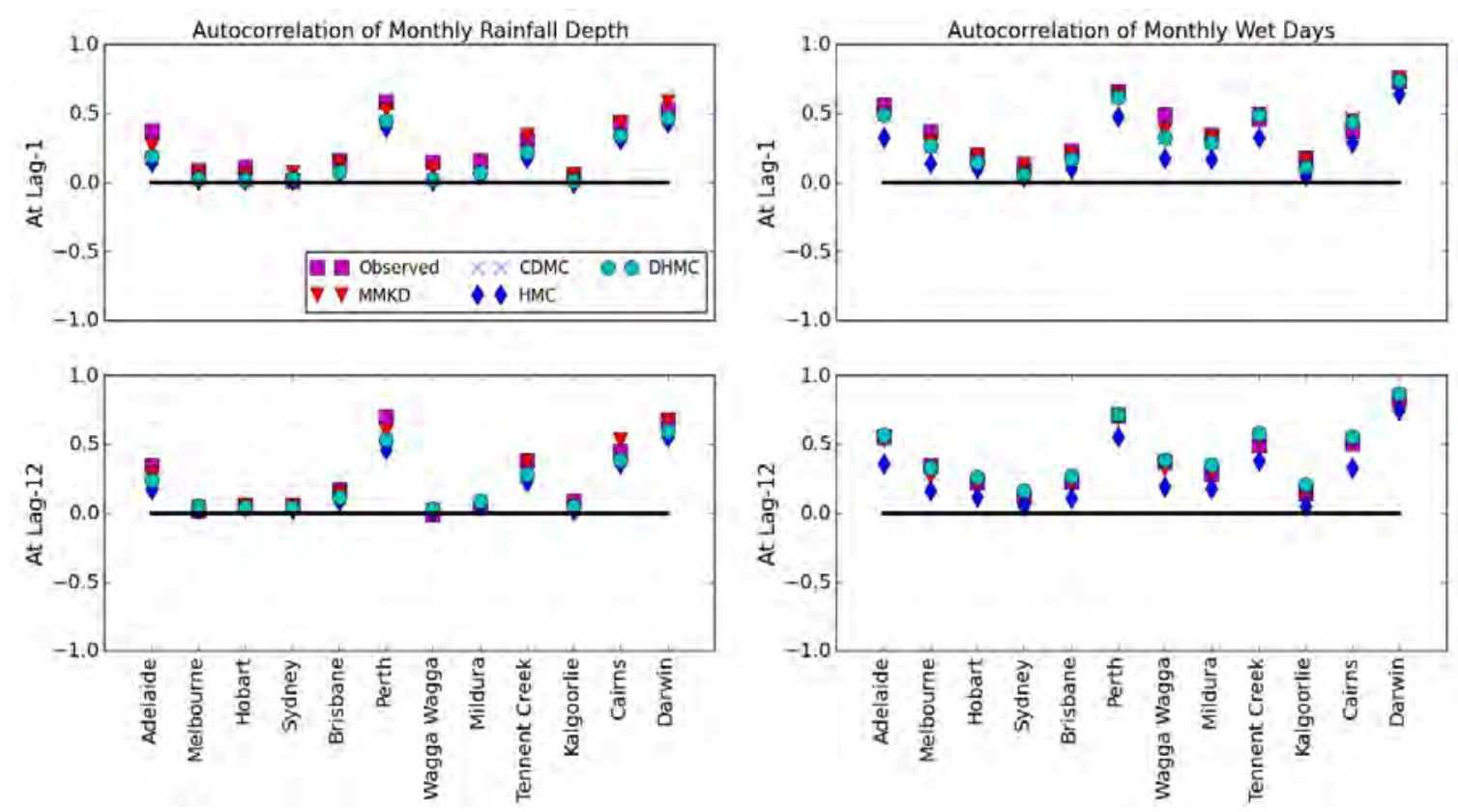

Figure 12.15: Comparison of model performances for autocorrelations of monthly number of wet days and monthly rainfall depths. 


\subsection{Insights for Simulation of Mean of Multiyear Rainfall Depths}

The DHMC and MMKD have shown comparable satisfactory performances to reproduce the distribution and autocorrelations of rainfall depths and wet periods at daily to multiyear resolutions. The key difference of performances between these two models is that the DHMC (like CDMC and HMC) tends to underestimate the mean of multiyear rainfall depths, while the MMKD tends to overestimate this mean (Figure 12.16). However, as shown in Chapter 5, the stochastic sampling of Gamma distribution parameters causes the underestimation of mean of multiyear rainfall depths in DHMC (as APMC and DPMC with deterministic Gamma parameters do not consistently underestimate the mean). To test the reason for the overestimation of mean in the MMKD, this study ran a Combined Model by using the rainfall occurrence simulation of MMKD and rainfall depths simulation of DHMC for the Sydney station. While the performance of the Combined Model for other statistics are comparable to the DHMC and MMKD (see Appendix H.4), Figure 12.16 shows that the Combined Model tends to underestimate the mean of multiyear rainfall depths. Since only the rainfall depth simulation of MMKD by the resampling process (kernel density technique) has been changed in the Combined Model, it suggests that the resampling process (not the modified Markov Chain process) causes the overestimation of mean rainfall depths in MMKD.
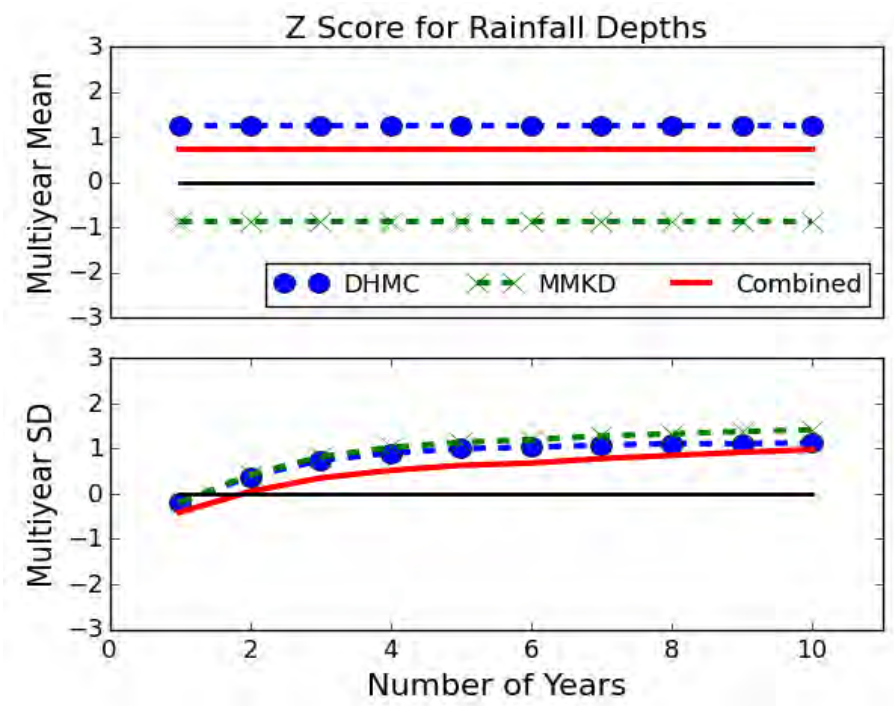

Figure 12.16: Comparison of DHMC, MMKD and Combined Model (occurrence from MMKD and depth from DHMC) to reproduce the mean of multiyear rainfall depths. 


\subsection{Discussion}

This chapter includes a discussion of the comparison of the performances of the CDMC, HMC, DHMC and MMKD to reproduce the mean and SD of rainfall depths, mean length of wet spells, and number of wet days at daily to multiyear resolutions. The comparative performances of the four models were assessed for six coastal, four inland, and two monsoonal stations (a total 12 stations around Australia). The distribution statistics of rainfall depths and wet periods show that the coastal stations are generally wetter than the inland stations. The monsoonal stations show extreme wet conditions in monsoon and extreme dry condition in other seasons.

Methodologically, there are similarities and differences among the MC models (CDMC, HMC and DHMC) and MMKD. All four models use the traditional two-part model structure and simulate rainfall occurrence and depths using separate methods. For rainfall occurrence simulation, all four models use first-order MC parameters (e.g. the transition probabilities of wet and dry days). However, for rainfall depths simulation, the MC models use Gamma distribution parameters, while MMKD resamples the rainfall depths from observed records using a nonparametric kernel-density estimator. To incorporate the multiyear rainfall variability, the MC models use stochastic parameters that vary from year-to-year, while MMKD uses memory of past periods using analytically derived equations. In the CDMC and DHMC, the stochastic parameters are used for only Gamma distribution (and deterministic parameters for MC), while the HMC uses the stochastic parameters of both Gamma distribution and MC. In the CDMC, the deterministic MC parameters are the same for all simulation years, while in the DHMC the deterministic MC parameters vary for each decade. The MC models generate rainfall depth independently for each wet day, while in the MMKD, the rainfall depth of each wet day is conditional to the rainfall depth of the previous day. For seasonal variability, the MC models use separate parameters for every month, while MMKD uses a moving window. The methodology of MMKD is complex [Mehrotra et al., 2015], while the methodology of the MC models (except HMC) are relatively simple and straightforward.

For mean and SD of rainfall depths at daily, monthly and multiyear resolutions, the performance of each model is similar for the coastal, inland and monsoonal stations. The four models show comparable satisfactory performances to reproduce the mean and SD of rainfall depths at daily, monthly and multiyear resolutions. However, the CDMC, HMC and DHMC tend to underestimate the mean rainfall depths at all resolutions (this appear to be caused by the stochastic Gamma parameters), while the MMKD tends to overestimate the same statistics 
(caused by resampling process by using the kernel-density technique). For SD of daily rainfall depths, the CDMC, HMC and DHMC tend to underestimate and MMKD tends to overestimate the statistic. All four models show satisfactory and comparable performances for SD of rainfall depths at monthly and multiyear resolutions. While overall performances are mostly comparable, for mean annual rainfall depths, the CDMC, HMC and DHMC tend to perform better for the coastal and monsoonal stations (wetter stations), and the MMKD tends to perform better for the inland stations (drier stations).

For coastal, inland and monsoonal stations, the DHMC and MMKD show comparable satisfactory performances to reproduce the mean and SD of wet period statistics (e.g. number of wet days and mean length of wet spells) at monthly to multiyear resolutions. The HMC satisfactorily reproduces the SD of wet period statistics for multiyear resolutions, but significantly overestimates the SD of wet period statistics for monthly resolution. The CDMC satisfactorily reproduces the SD of wet period statistics for monthly resolution, but significantly underestimates the statistics at multiyear resolutions. For SD of multiyear number of wet days, the MMKD and HMC tend to underestimate the statistic at longer multiyear resolutions (e.g. 7 to 10 overlapping years), while the DHMC tends to underestimate the statistic at shorter multiyear resolutions (e.g. 1 to 3 overlapping years).

The comparison for autocorrelations of monthly rainfall depths and monthly number of wet days shows that the performances of CDMC, DHMC and MMKD are similar and satisfactory, while the HMC tends to underestimate the autocorrelations. This finding is the same for all coastal, inland and monsoonal stations.

\subsection{Conclusion}

The MC models are methodologically simple and straightforward in comparison with the relative complexity of MMKD. Despite the methodological simplicity, the DHMC shows comparable satisfactory performance as MMKD to reproduce the distribution and autocorrelations of rainfall depths and wet periods at daily to multiyear resolutions. The other MC models, CDMC and HMC, also show comparable performance to reproduce the distribution of rainfall depths at all resolutions, but fail to preserve the distribution of wet periods (i.e. number of wet days and length of wet spell) at all resolutions. 
Each of the four models has advantages and limitations in terms of their usability for urban drought security assessment. Each model has preserved most of the distribution and autocorrelations of rainfall depths and wet periods at daily, monthly and multiyear resolutions. The ability of these models to reproduce most of the monthly and multiyear variability of rainfall depths and wet periods is important for urban drought security assessment as the urban water systems usually vary at monthly and multiyear resolutions. However, each model has limitations, which are critical in determining their value for drought assessment. The MMKD has a consistent tendency to overestimate the mean of rainfall depths at daily to multiyear resolutions, which indicates that the droughts simulated by the MMKD are likely to be less severe. The HMC has a consistent tendency to overestimate the variability of wet periods for monthly resolutions, which indicates that the HMC is less likely to simulate long periods of drought and flood. In contrast, the CDMC has shown a consistent tendency to underestimate the variability of wet periods at multiyear resolutions, which indicates that the CDMC is likely to simulate a stable climate over the years and thus underestimate the potential drought risks. The DHMC has shown similar, or better performances, than the MMKD for most of the statistics, although it has a tendency to underestimate the mean of rainfall depths (similar to CDMC and HMC), particularly at multiyear resolutions. 


\section{Comparison of $M C$ and MMKD for Raingauge Data around Sydney}

\subsection{Introduction}

This chapter presents the comparison of performances of the MMKD and the three MC models using raingauge data from 30 stations around Sydney (see section 3.3.7). In this chapter, the model performances for distribution statistics of rainfall depths and wet period statistics (i.e. number of wet days and mean length of wet spells) are assessed using the method described in section 3.4 .

\subsection{Mean and SD of Daily and Monthly Rainfall Depths}

Figure 13.1 and Figure 13.2 compare the mean and SD of daily rainfall depths, while Figure 13.3 and Figure 13.4 compare for the mean and SD of monthly rainfall depths.

For the mean and SD of daily rainfall depths (Figure 13.1 and Figure 13.2), all four models show satisfactory performances and the performances of the three MC models are similar. For some months, the MMKD tends to overestimate the mean and SD, while the MC models tend to underestimate it.

For the mean of monthly rainfall depths (Figure 13.3), the MMKD tends to overestimate and the MC models tend to underestimate the statistic. For the SD of monthly rainfall depths, the performances are similar in all four models with a slight tendency to overestimate the SD.

These findings for the 30 Sydney stations are consistent with the respective findings in the previous chapter for 12 stations around Australia. 
Z (Mean of Wet Day Rainfall Depths)

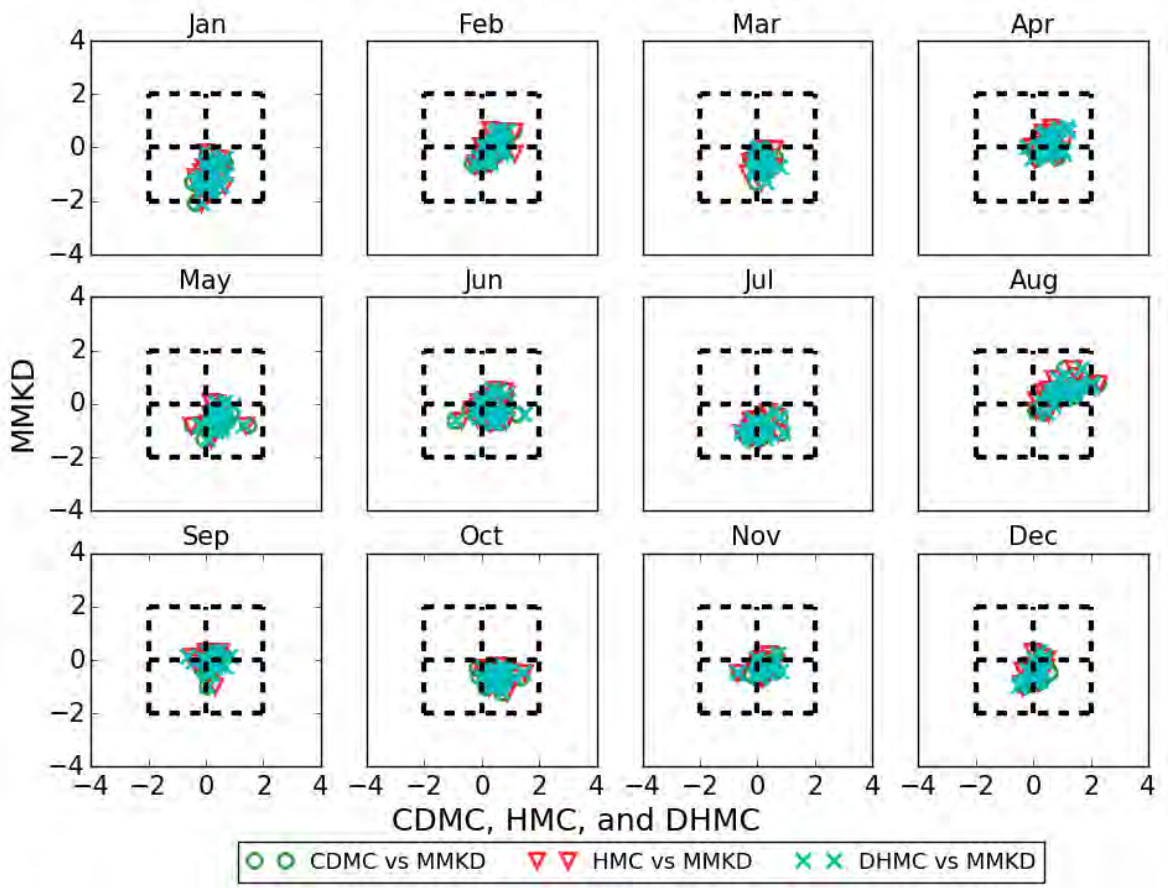

Figure 13.1: Comparison of model performances for mean of wet day rainfall depths.

Z (SD of Wet Day Rainfall Depths)

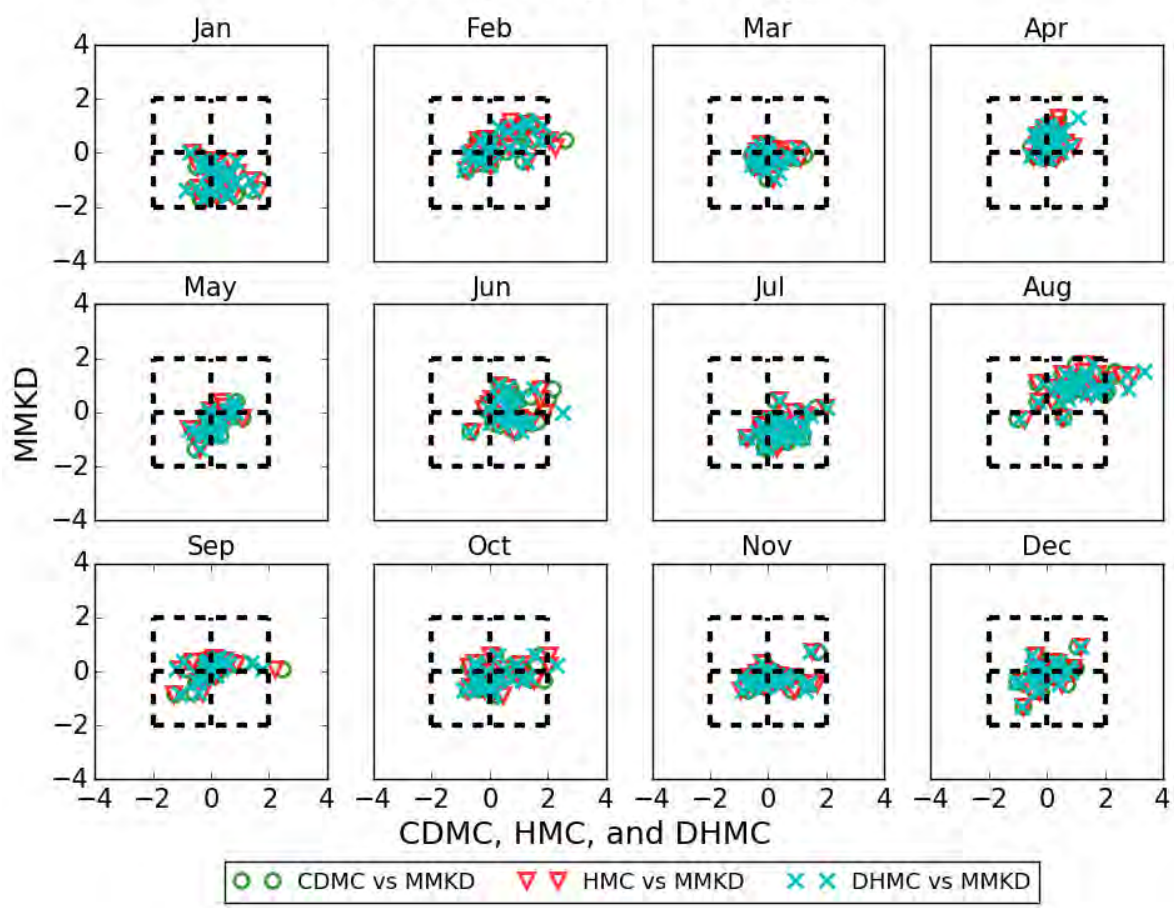

Figure 13.2: Comparison of model performances for SD of wet day rainfall depths. 


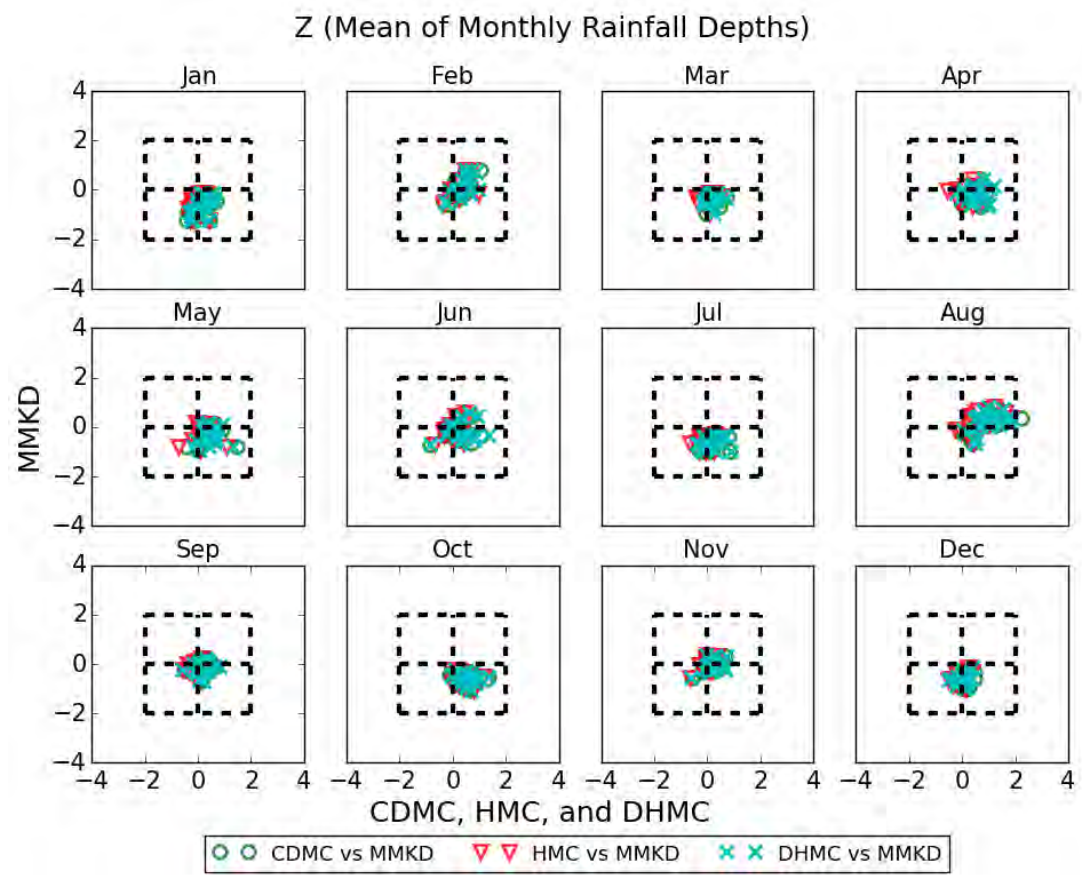

Figure 13.3: Comparison of model performances for mean of monthly rainfall depths.

Z (SD of Monthly Rainfall Depths)
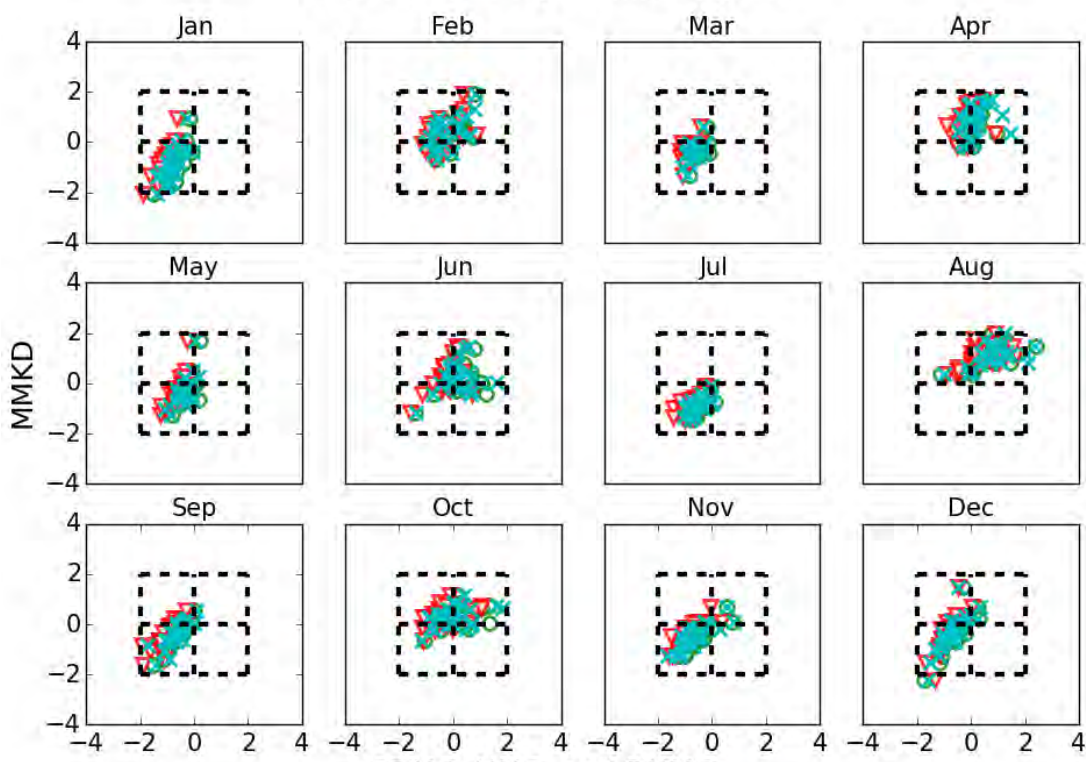

CDMC, HMC, and DHMC

\begin{tabular}{lll}
\hline$\circ$ CDMC vs MMKD & $\nabla \nabla$ HMC vs MMKD & $\times \times$ DHMC vs MMKD
\end{tabular}

Figure 13.4: Comparison of model performances for SD of monthly rainfall depths. 


\subsection{Mean and SD of Multiyear Rainfall Depths}

Figure 13.5 compares the performances of the four models for the mean of annual rainfall depths ( $\mathrm{Z}$ scores are also the same for 2 to 10 overlapping years), while Figure 13.6 compares for the SD of multiyear (e.g. 1 to 10 overlapping years) rainfall depths. Similar to daily and monthly mean, the MMKD tends to overestimate and the MC models tend to underestimate the annual mean (Figure 13.5). At shorter multiyear resolutions (1 to 3 years), the CDMC and DHMC reproduce the SD better than the MMKD and HMC, which tend to overestimate (Figure 13.6). However, at longer multiyear resolutions (6 to 10 years), the CDMC underestimates the SD more than the other models. Therefore, the overall performance of the DHMC is slightly better than the MMKD, CDMC and HMC.

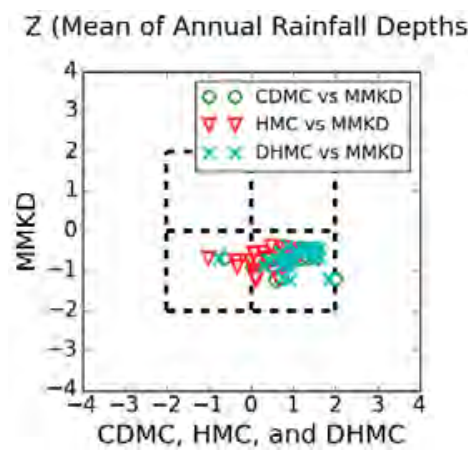

Figure 13.5: Comparison of model performances for mean of annual rainfall depths.

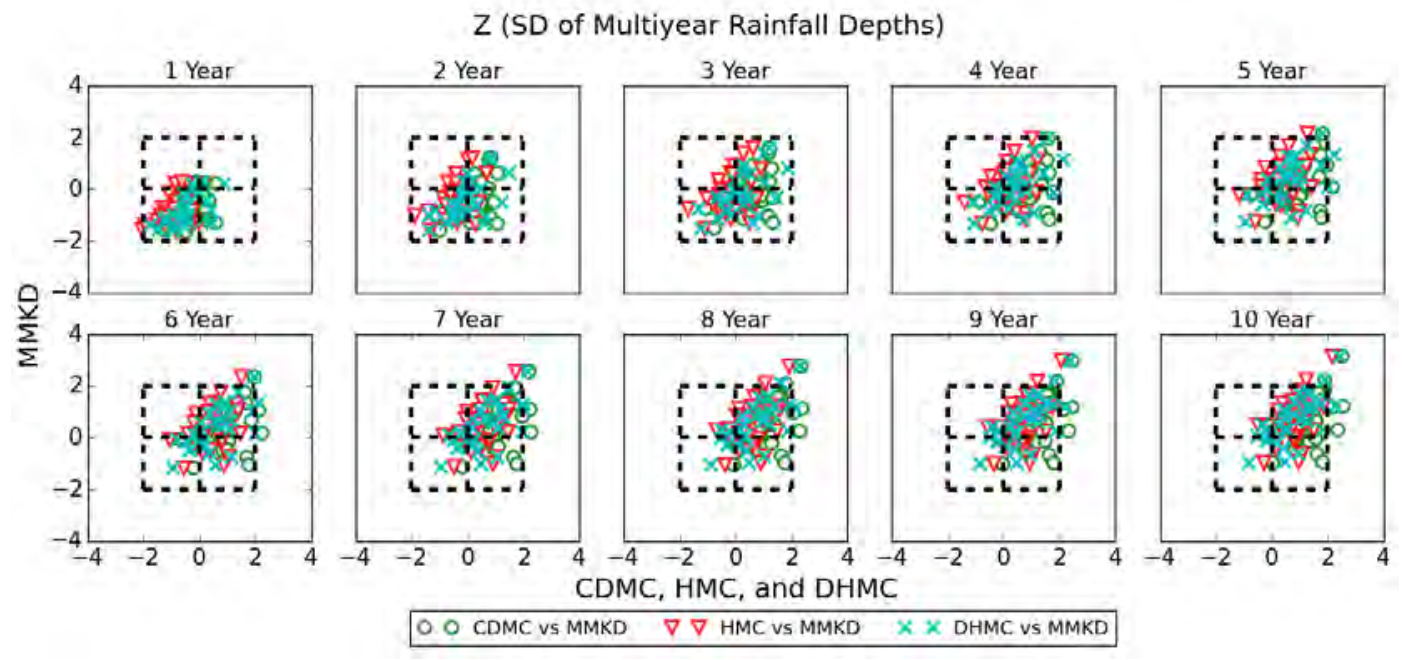

Figure 13.6: Comparison of model performances for SD of multiyear rainfall depths. 


\subsection{Mean and SD of Monthly and Multiyear Number of Wet Days}

Figure 13.7 and Figure 13.8 compare for the mean and SD of monthly number of wet days respectively. Figure 13.9 and Figure 13.10 compares the mean of annual wet days ( $\mathrm{Z}$ scores for multiple years are the same as $Z$ scores for annual resolution) and SD of multiyear wet days respectively. For the mean of wet days at monthly and annual resolutions (Figure 13.7 and Figure 13.9), the performances of the CDMC, DHMC and MMKD are similar and satisfactory, while the HMC tends to overestimate it.

For the SD of monthly number of wet days (Figure 13.8), the DHMC and MMKD have a similar performance and performed better than the other models. While the DHMC and MMKD slightly underestimate the SD of monthly number of wet days, the CDMC underestimates the statistic more consistently and significantly, while the HMC tends to overestimate the SD. For the SD of multiyear number of wet days (Figure 13.10), the MMKD and DHMC perform satisfactorily, while the DHMC tends to underestimate the SD at shorter multiyear resolutions (i.e. 1 to 3 overlapping years). The HMC also preserves the SD with a consistent tendency to underestimate. However, the CDMC tends to consistently and significantly underestimate the SD of multiyear wet days.

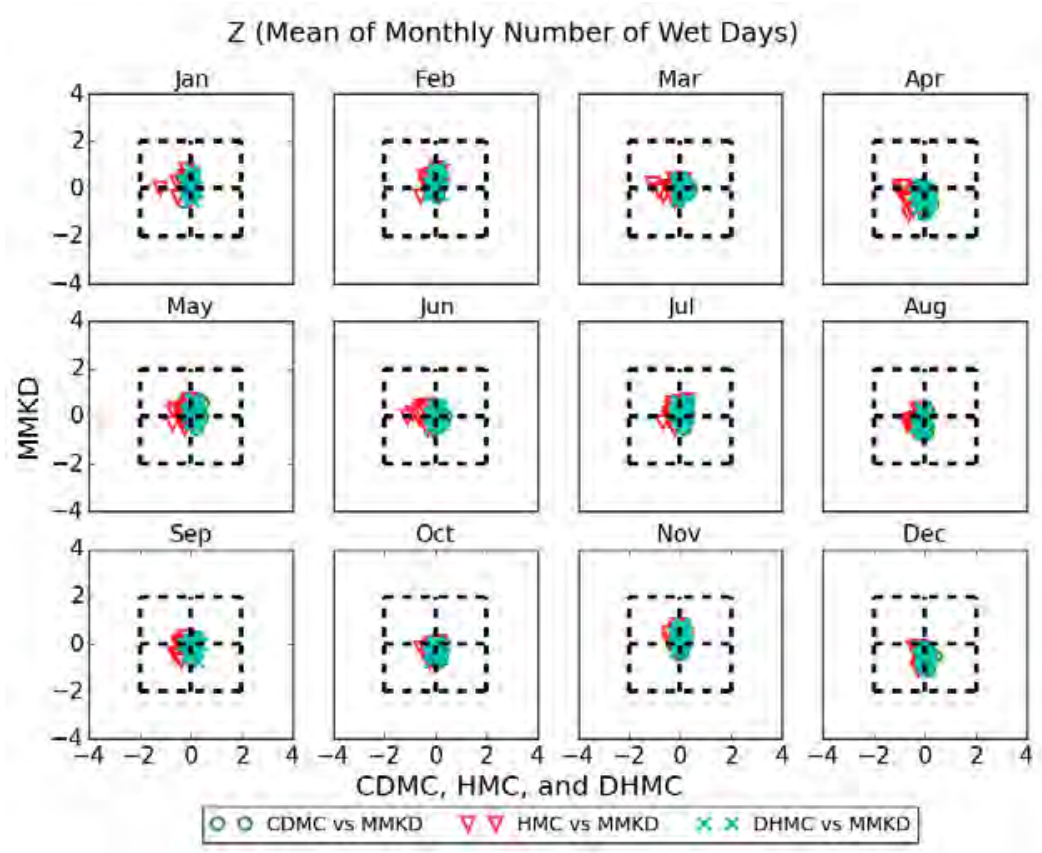

Figure 13.7: Comparison of model performances for mean monthly number of wet days. 
13. Comparison of MC and MMKD for Raingauge Data around Sydney

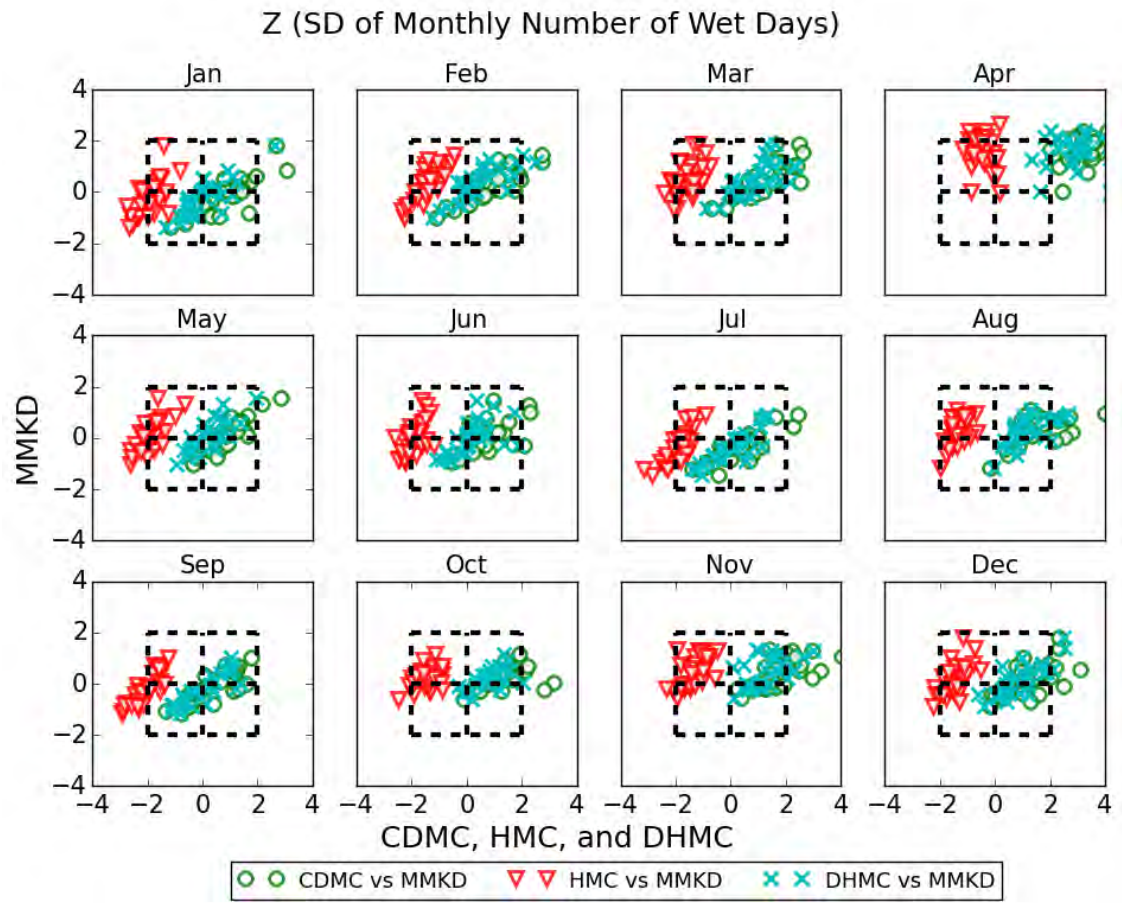

Figure 13.8: Comparison of model performances for SD of monthly number of wet days.

Z (Mean of Annual Number of Wet Days)

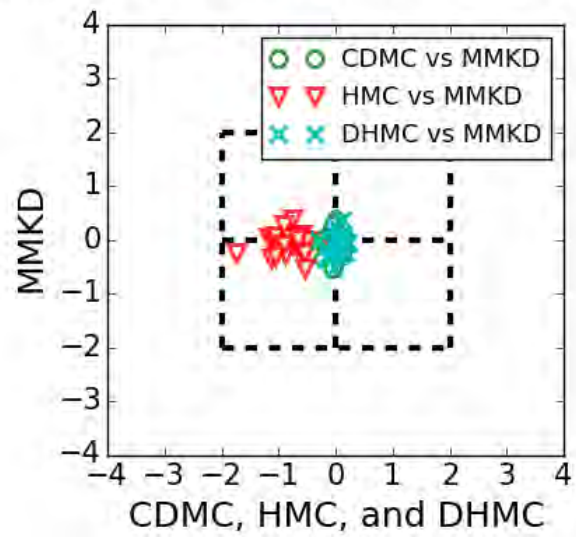

Figure 13.9: Comparison of model performances for mean of annual number of wet days. 


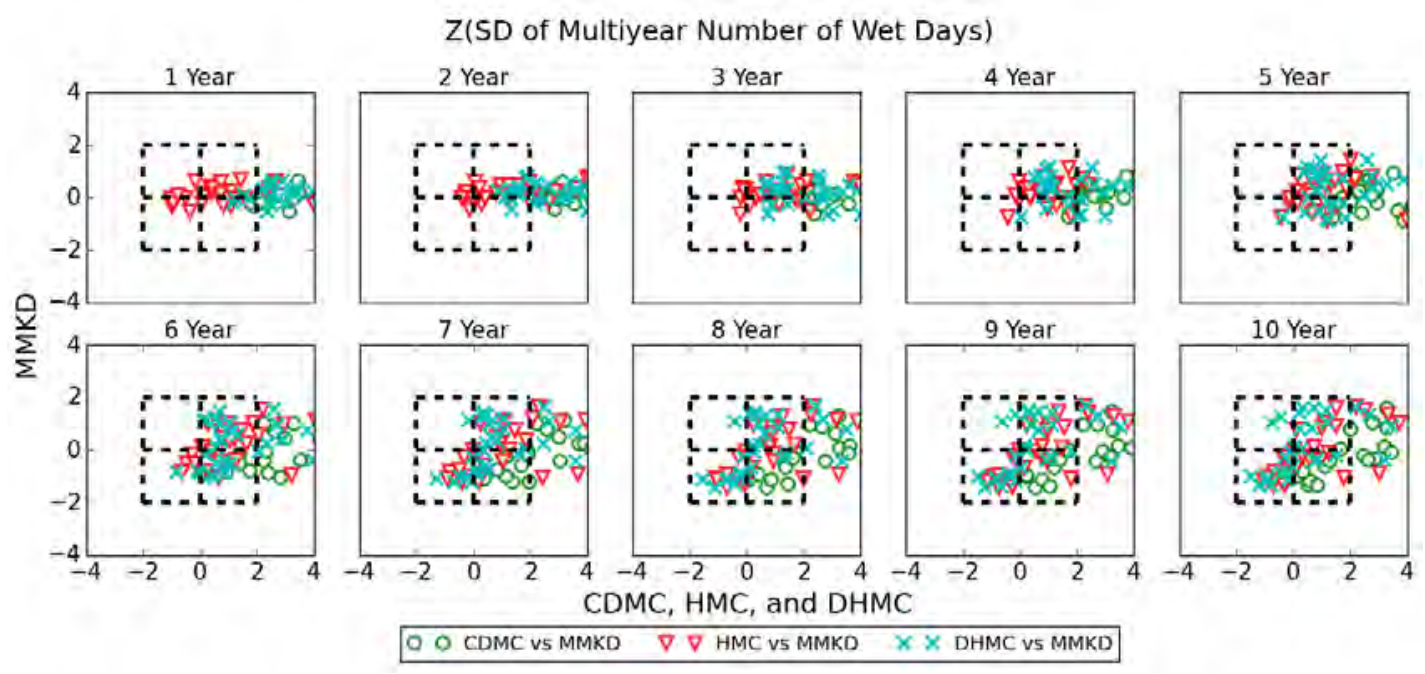

Figure 13.10: Comparison of model performances for SD of multiyear number of wet days.

\subsection{Mean and SD of Monthly and Annual Mean Length of Wet Spells}

Figure 13.11 and Figure 13.12 compares the mean and SD (respectively) of monthly mean length of wet spells $\mu_{w e t}$, while Figure 13.13 compares the same for annual $\mu_{w e t}$.

All four models have shown comparable satisfactory performances to reproduce the mean of monthly mean length of wet spells (Figure 13.11). The performances of CDMC, DHMC and MMKD are similar and satisfactory for the SD of monthly mean length of wet spells, while the HMC tends to overestimate the SD (Figure 13.12).

For the mean of annual mean length of wet spells (Figure 13.13), the performance of all four models is similar and satisfactory. For the SD of annual mean length of wet spells (Figure 13.13), the DHMC and MMKD have a similar performance and perform better than the other models, with a slight tendency to underestimate. The HMC tends to overestimate and CDMC tends to underestimate the SD of annual mean length of wet spells. 


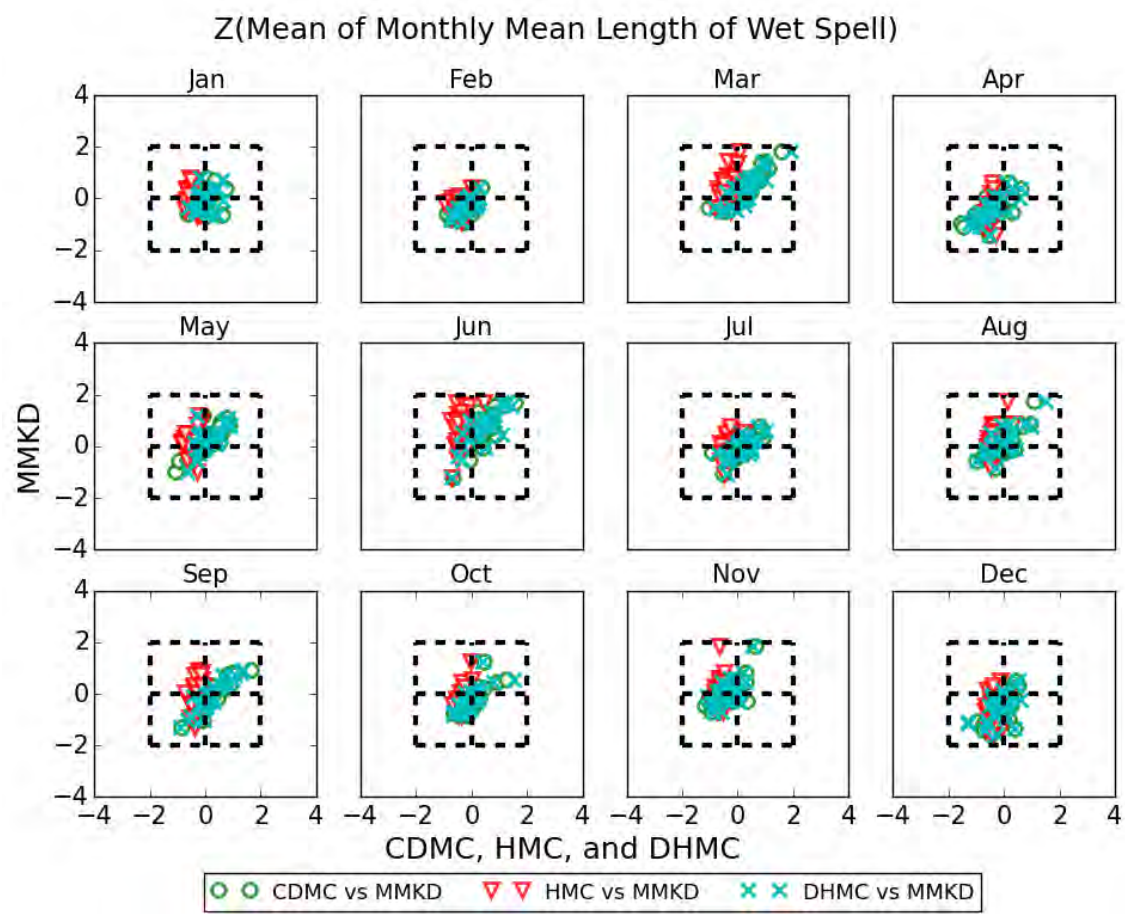

Figure 13.11: Comparison of model performances for mean of monthly mean length of wet spells.

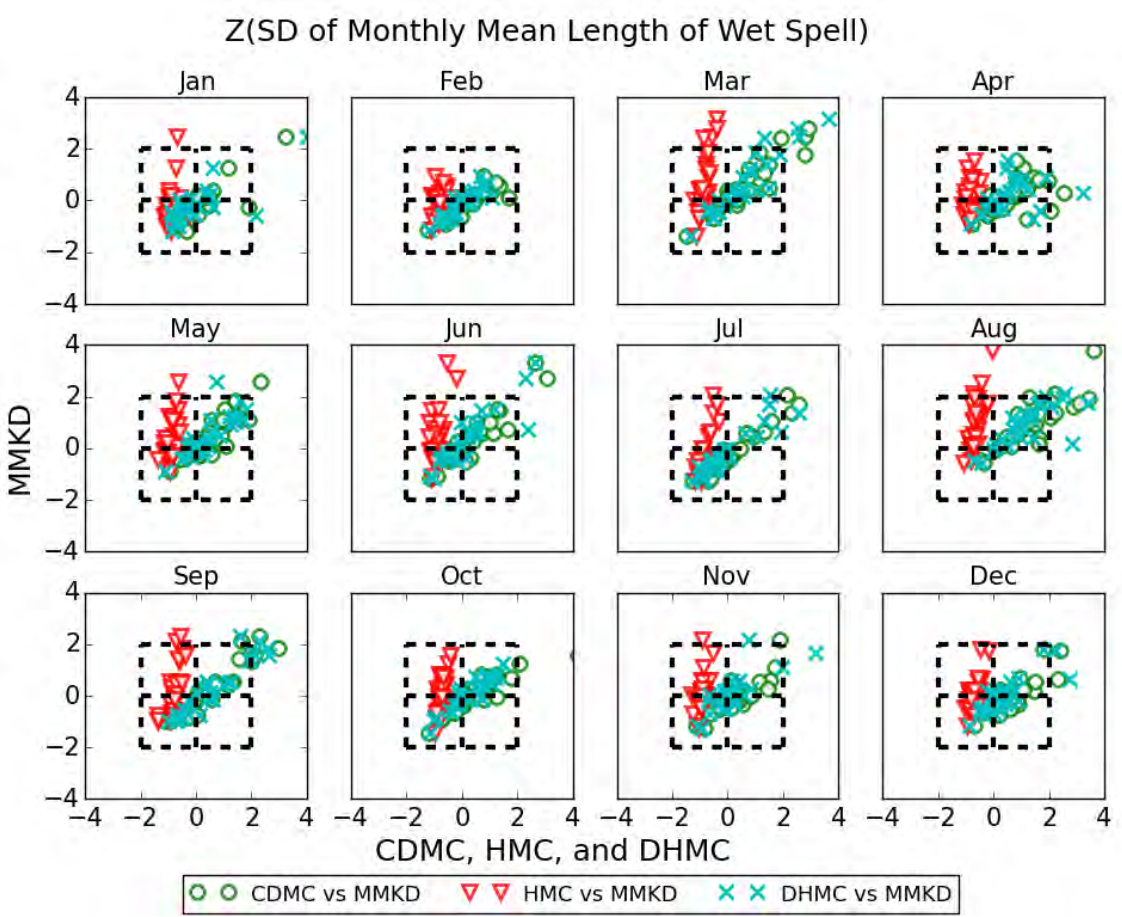

Figure 13.12: Comparison of model performances for SD of monthly mean length of wet spells. 
$Z$ (Mean of Annual Mean Length of Wet Spell) Z (SD of Annual Mean Length of Wet Spell)
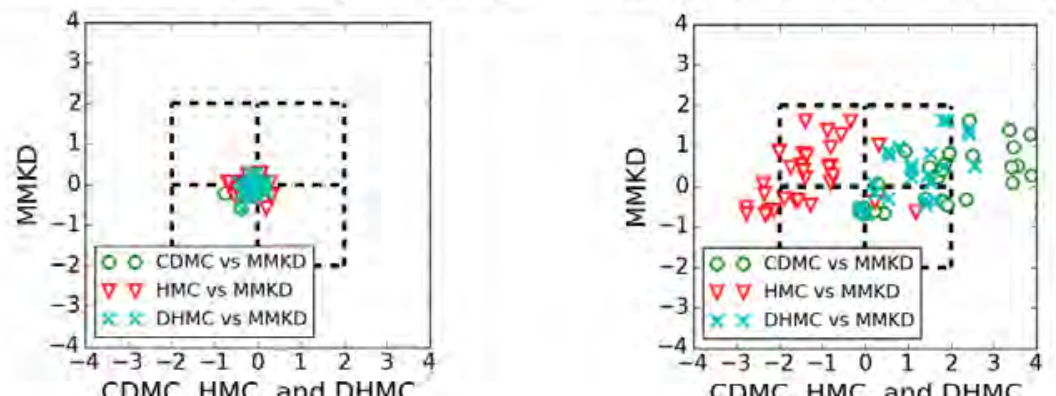

Figure 13.13: Comparison of model performances for mean (left) and SD (right) of the annual mean length of wet spells.

\subsection{Discussion and Conclusion}

This chapter has provided a comparison of how well the CDMC, HMC, DHMC and MMKD reproduce the mean and SD of rainfall depths, mean length of wet spells, and number of wet days at daily to multiyear resolutions for the 30 raingauge stations around Sydney. The overall performances of each model in Sydney stations is mostly consistent with the performances for 12 stations around Australia (shown in previous chapter).

The key findings are:

- All four models preserve the mean and SD of rainfall depths at daily to multiyear resolutions. The MC models tend to underestimate the mean of rainfall depths and the MMKD tends to overestimate the mean of rainfall depths at all resolutions.

- The DHMC and MMKD show comparable performances to reproduce the mean and SD of wet period statistics at both monthly and multiyear resolutions. The CDMC fails to preserve the SD of wet period statistics at multiyear resolution, while the HMC fails to preserve the SD of wet period statistics at monthly resolution.

- The HMC tends to overestimate the SD of all statistics except the SD of daily rainfall depths.

- Overall, the performance of the DHMC and MMKD is comparable, satisfactory, and better than the performance of the CDMC and HMC. 


\section{Comparison of MC and MMKD for Validation Periods}

\subsection{Introduction}

This chapter will discuss the comparison among CDMC, HMC, DHMC, and MMKD to reproduce the rainfall statistics for validation periods, i.e. the statistics of samples with different period and/or record length than the calibration period. This comparison is done using a 150year daily rainfall data of 1860-2009 period for the raingauge station in Sydney Observatory Hill shown in Figure 3.8 (one of the 12 raingauge stations around Australia which was used in Chapter 12). Each of the models is calibrated to a 30-year sample of 1979-2008. Then, the statistics of each model are compared with statistics of the calibration period and four validation periods:

- A 30-year validation period of 1940-1969,

- A 60-year validation period of 1910-1969,

- A 100-year validation period of 1910-2009,

- A 150-year validation period of 1860-2009.

This chapter provides a discussion of the observed statistics of rainfall depths and wet periods at daily, monthly and multiyear resolutions for both the calibration and validation periods. Then, the comparison of the four models against the observed statistics will be presented. The performance of each model for the validation periods is assessed by calculating $\mathrm{Z}$ scores as outlined in section 3.9.

\subsection{Observed Statistics}

This section presents the mean and SD of rainfall depths, mean length of wet spells, and number of wet days (at daily to multiyear resolutions), calculated from the data samples for calibration and validation periods. 


\subsubsection{Mean and SD of Rainfall Depths}

Figure 14.1 shows the mean and SD of rainfall depths at daily and monthly resolutions, while Figure 14.2 shows the same statistics for multiyear resolution.

At daily resolution, the mean of wet day rainfall depths in the calibration period (1979-2008) is higher than the mean of wet day rainfall depths in all validation periods for most of the months (i.e. seasons), while the statistic is mostly similar in all validation periods. Similarly, the SD of wet day rainfall depths in validation periods is comparable to each other, but the SD of wet day rainfall depths in the calibration period is different for some months.

At monthly resolution, the mean of monthly rainfall depths in both calibration and all validation periods is mostly comparable. Compared to the mean of wet day rainfall depths, this indicates that the monthly rainfall totals are similar in each sample, but the higher mean of wet day rainfall depths in the calibration period might be because of less frequent (less wet days), more intense rainfall in that period. However, while the SD of monthly rainfall depths in all validation periods is similar, the SD of monthly rainfall depths in the calibration period is different for some months.

At multiyear resolution, the mean of multiyear rainfall depths is similar in both the calibration and validation periods. However, the SD of multiyear rainfall depths is higher in the calibration period of 1979-2008 and the 30-year validation period of 1940-1969, while the statistic is similar in other three validation periods. This indicates that the multiyear rainfall variability observed in shorter recent past records is higher than the multiyear rainfall variability observed in longer past records. 

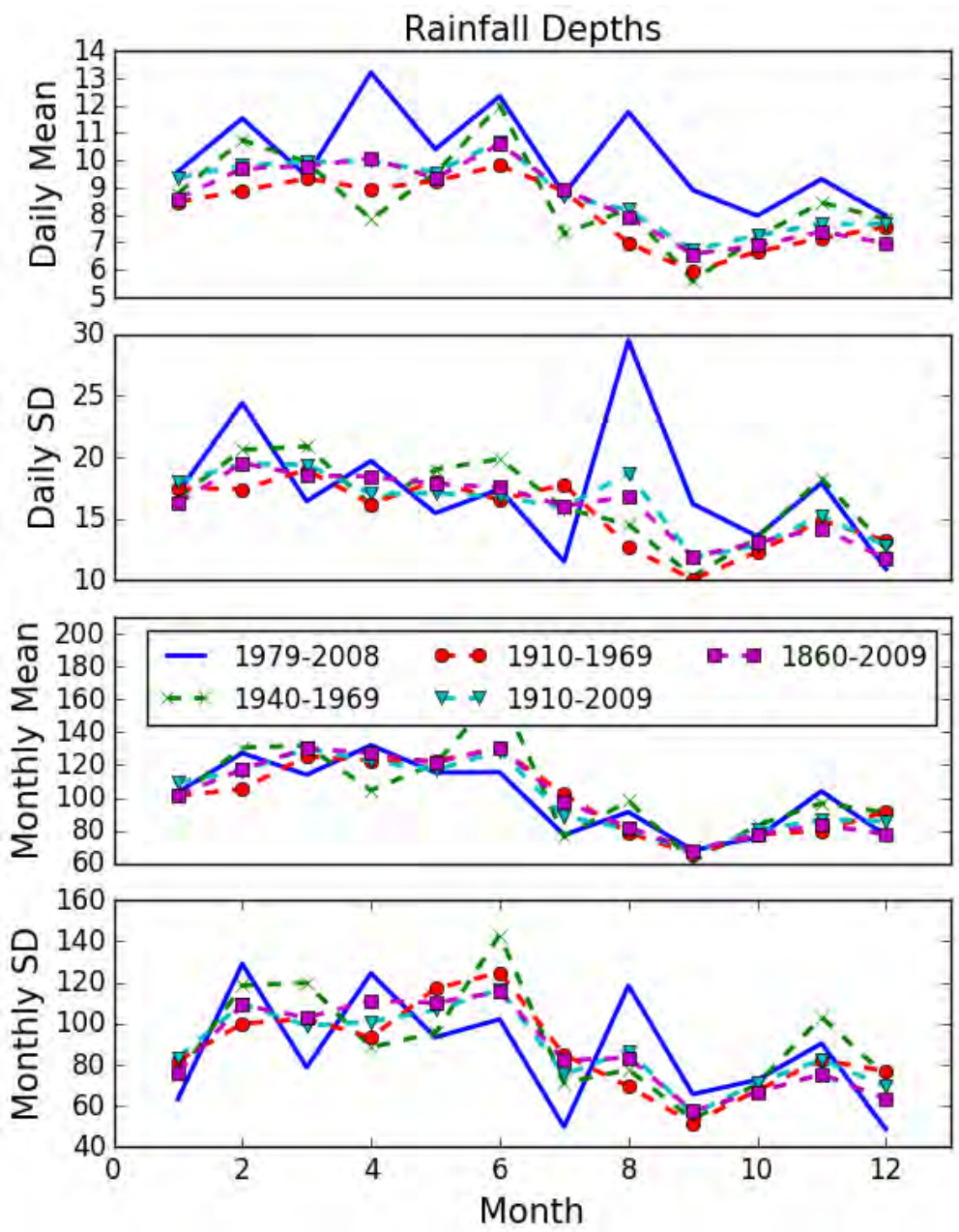

Figure 14.1: Mean and SD of the daily and monthly rainfall depths. The daily statistics are the mean and SD for the days on which rain occurs and does not include dry days.
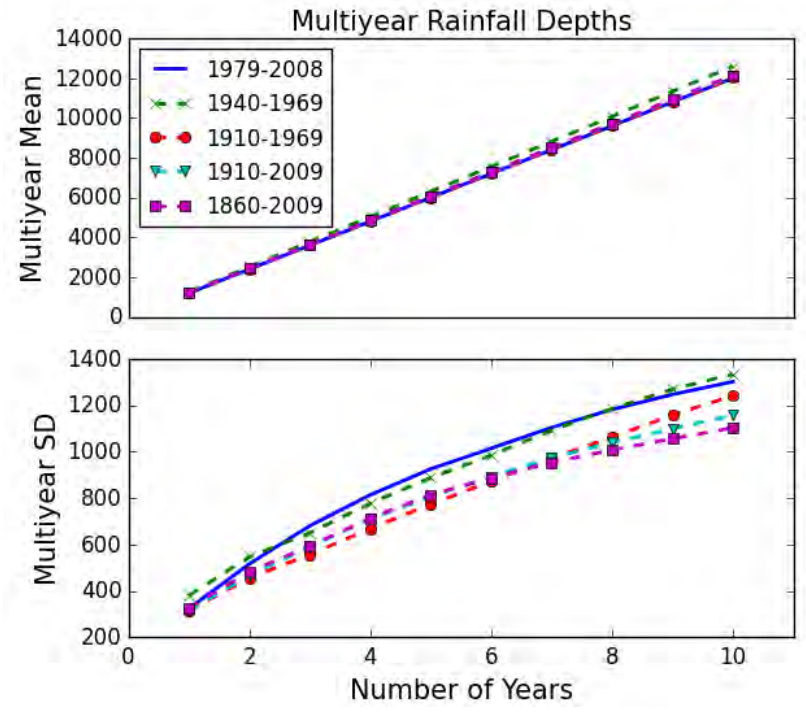

Figure 14.2: Mean and SD of the multiyear rainfall depths. 


\subsubsection{Mean and SD of Number of Wet Days}

Figure 14.3 shows the mean and SD of monthly and multiyear number of wet days in the calibration and validation periods.

Similar to the wet spell lengths, the mean and SD of the monthly number of wet days in the calibration period is less than the validation periods (Figure 14.3). It also consistent with the calibration period having less frequent more intense rainfall, so that the mean of wet day rainfall depths in the calibration period is higher than in validation periods, although the mean of monthly rainfall depths is similar in all samples. While the seasonal signals of the mean of monthly number of wet days in all samples are mostly similar, the seasonal signal of the SD of monthly number of wet days in the calibration period is different from the validation periods. Moreover, the seasonal signals of the statistic in two shorter validation periods (i.e. 30-year and 60 -year samples) are slightly different from the seasonal signals in the two longer validation periods (i.e. 100-year and 150-year samples).

At multiyear resolution, the mean of multiyear number of wet days is also smaller in the calibration period (Figure 14.3). However, the SD in the calibration period and two shorter validation periods are similar and lower than the two longer validation periods.
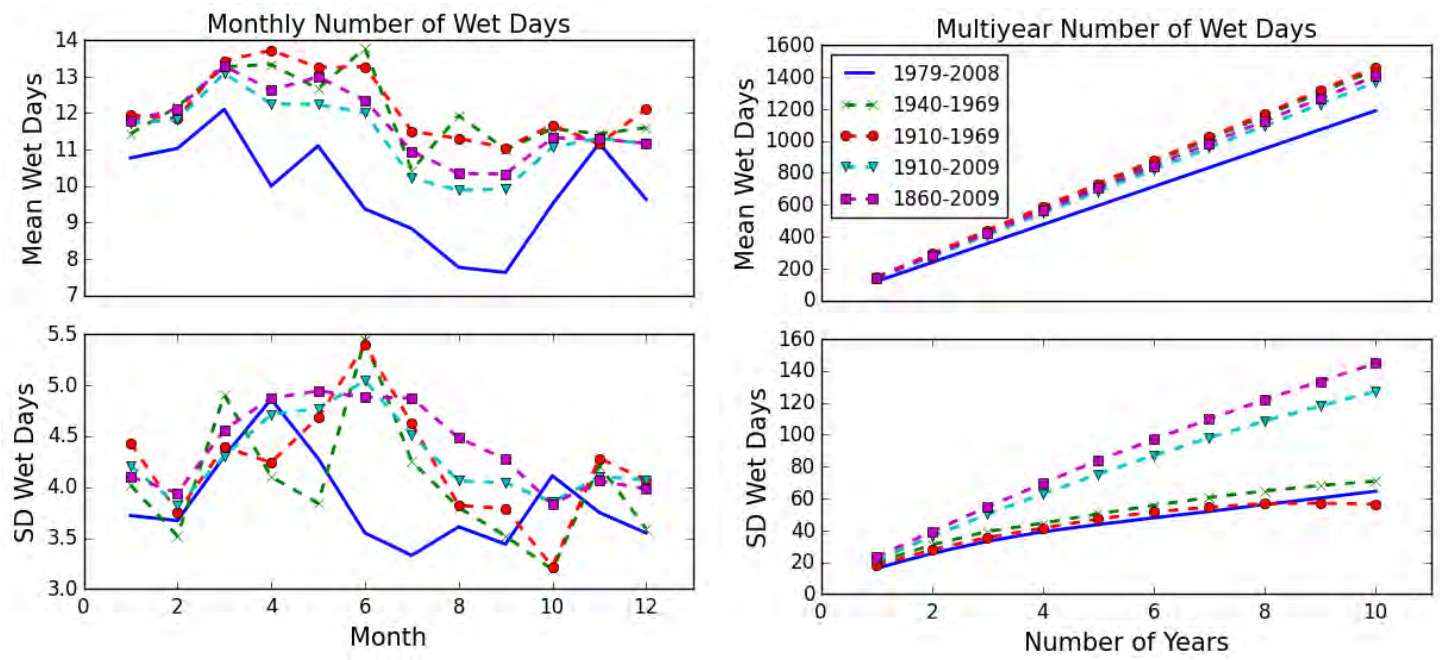

Figure 14.3: Mean and SD of the monthly (left) and multiyear (right) number of wet days. 


\subsubsection{Mean and SD of Mean Length of Wet Spells $\left(\mu_{w e t}\right)$}

Figure 14.4 shows the mean and SD of monthly $\mu_{w e t}$ for the calibration and four validation periods. The mean and SD of monthly $\mu_{w e t}$ in the calibration period is lower (i.e. wet spell lengths are shorter) than the four validation periods for all months. This is consistent with the findings in the previous section that the mean of wet day rainfall depths is higher in the calibration period but the mean of monthly rainfall depths is similar for both calibration and validation periods, because the wet spell lengths are shorter in calibration period.

Similar to the monthly resolution, the mean and SD of annual $\mu_{\text {wet }}$ (Table 14.1) are lower in the calibration period than in validation periods. The mean and SD of annual $\mu_{w e t}$ are similar in all validation periods, while the SD of annual $\mu_{\text {wet }}$ in the longest validation period 1860-2009 is higher than the other three validation periods.
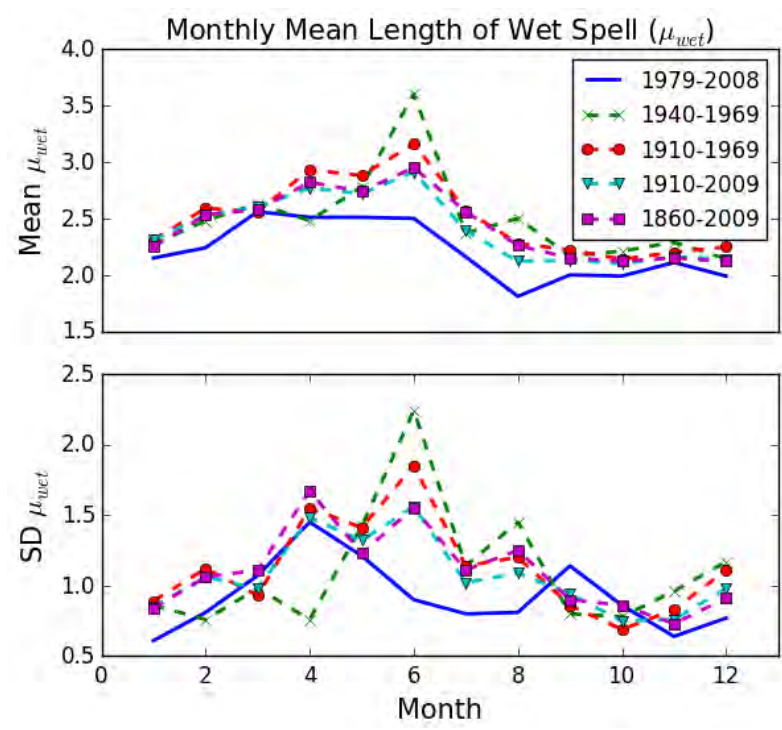

Figure 14.4: Mean and SD of the monthly mean length of wet spells.

Table 14.1: Mean and SD of the annual mean length of wet spells.

\begin{tabular}{cccccc}
\hline Annual $\boldsymbol{\mu}_{\text {wet }}$ & $\mathbf{1 9 7 9 - 2 0 0 8}$ & $\mathbf{1 9 4 0 - 1 9 6 9}$ & $\mathbf{1 9 1 0 - 1 9 6 9}$ & $\mathbf{1 9 1 0 - 2 0 0 9}$ & $\mathbf{1 8 6 0 - 2 0 0 9}$ \\
\hline Mean & 2.18 & 2.42 & 2.46 & 2.37 & 2.40 \\
SD & 0.22 & 0.29 & 0.27 & 0.29 & 0.35 \\
\hline
\end{tabular}




\subsection{Model Comparison for Calibration Period}

The comparison of model performance for the calibration period (1979-2008) was discussed in Chapter 12 (as one of the 12 stations around Australia). The key findings of the comparison for the calibration period include the following:

- For mean and SD of rainfall depths at daily to multiyear resolutions, the performances of all four models are similar and satisfactory, while the MMKD tends to overestimate and the MC models tend to underestimate the mean.

- For the mean number of wet days at monthly and multiyear resolutions, performances of all four models are similar and satisfactory. However, for the SD of number of wet days, the DHMC and MMKD have a similar performance and preserve the SD for both monthly and multiyear resolutions. The CDMC preserves the SD at monthly resolution but underestimates at multiyear resolution, and the $\mathrm{HMC}$ overestimates the $\mathrm{SD}$ at monthly resolution but preserves at multiyear resolution.

- For mean length of wet spells at monthly and annual resolutions, the CDMC, DHMC and MMKD show comparable satisfactory performances to reproduce the mean and $\mathrm{SD}$, while the HMC can reproduce the mean but significantly overestimates the SD.

- Overall, the performances of the DHMC and MMKD are comparable and better than the CDMC and HMC.

\subsection{Model Comparison for Validation Period}

This section compares the models for the statistics of the four independent validation periods (i.e. 1940-1969, 1910-1969, 1910-2009 and 1860-1909), when models are calibrated to 19792008.

\subsubsection{Mean and SD of Daily and Monthly Rainfall Depths}

Figure 14.5 shows the comparison of performances to reproduce the mean and SD of daily and monthly rainfall depths for the four validation periods. For the daily mean and SD for the validation periods, the MC models perform better than the MMKD as the MMKD tends to overestimate. This might be because the calibration period has higher wet day rainfall, while MC models generally underestimate and MMKD overestimates the daily statistics in calibration. However, for the monthly mean and SD, performances of all four models are 
similar. At the daily resolution, MMKD appears to be very similar to the calibration data because of its resampling of the calibration data, which contrasts with the sampling from the Gamma distribution of the MC models.
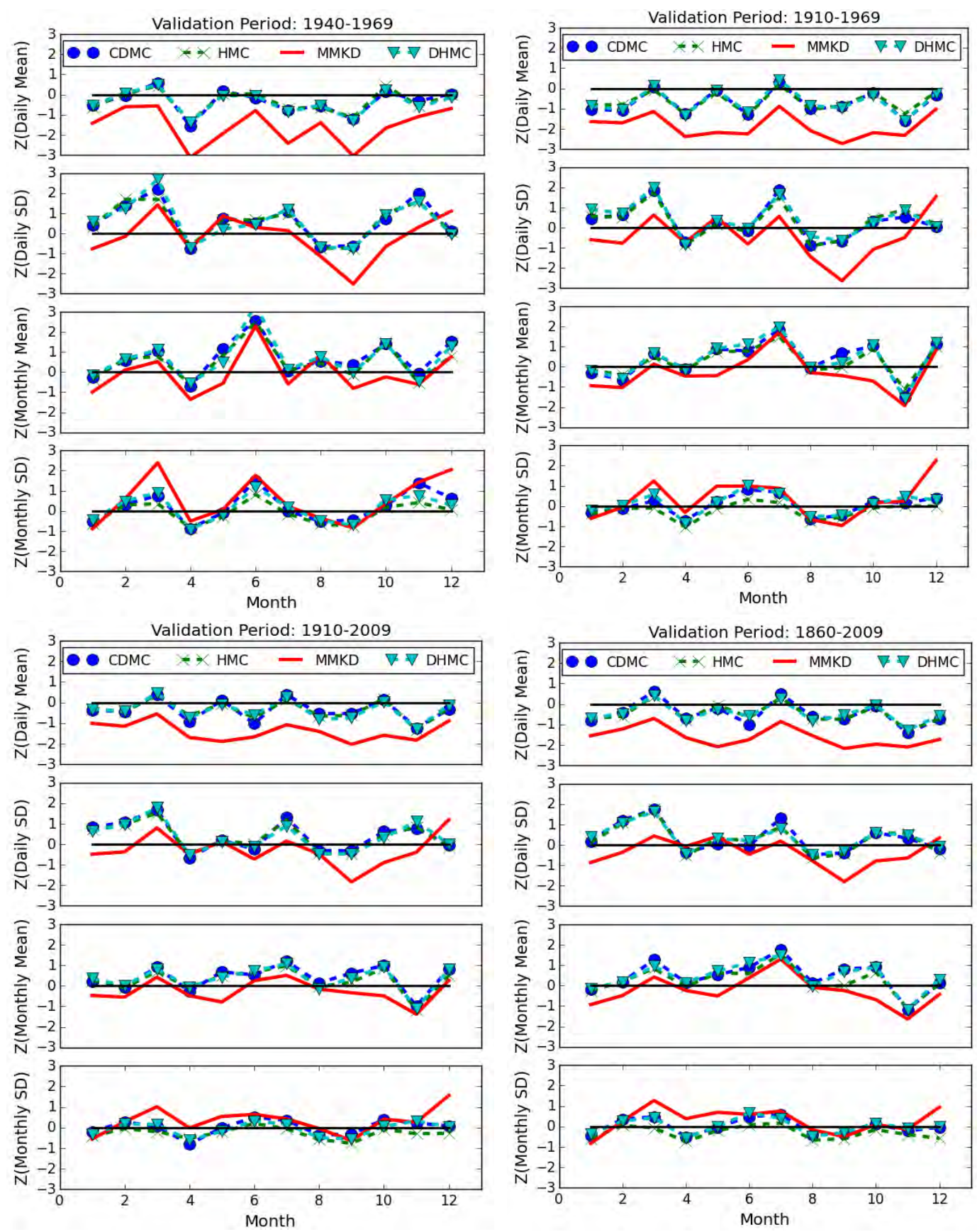

Figure 14.5: Comparison of performances to reproduce the mean and SD of daily and monthly rainfall depths for four validation periods. 


\subsubsection{Mean and SD of Multiyear Rainfall Depths}

Figure 14.6 compares the mean and SD of multiyear rainfall depths for the four validation periods. The MC models tend to underestimate the mean of multiyear rainfall depths, while the MMKD tends to overestimate. Overall, the MMKD performs slightly better than the MC models for the mean, while the MC models perform slightly better than the MMKD for the SD. However, these performances of the models might be linked with differences of the rainfall statistics between the calibration and validation periods (discussed in section 14.2.1).
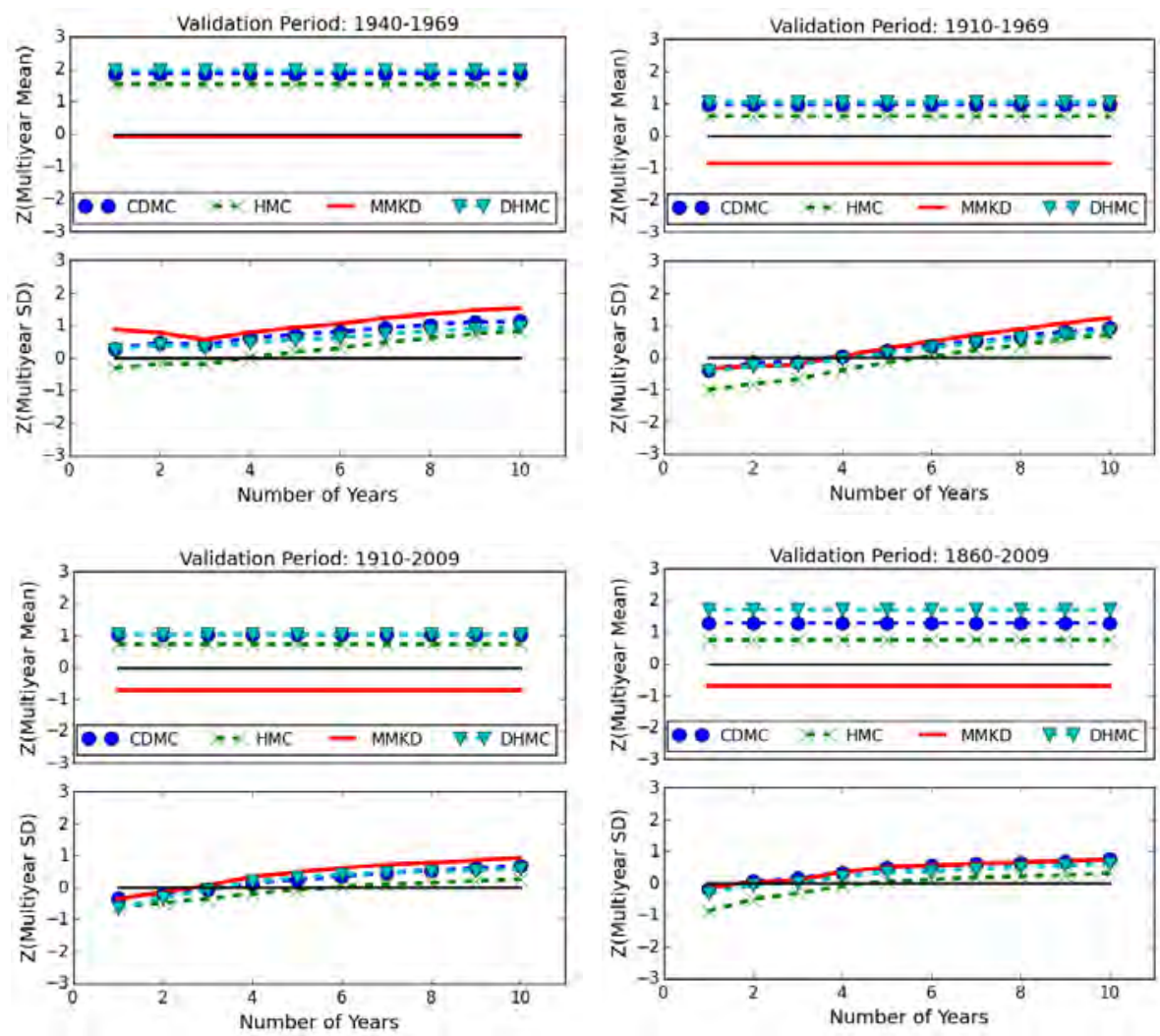

Figure 14.6: Comparison of performances to reproduce the mean and SD of multiyear rainfall depths for four validation periods.

\subsubsection{Mean and SD of Monthly Number of Wet Days}

Figure 14.7 compares the models for the mean and SD of monthly number of wet days. For the mean, all four models tend to underestimate the statistic, which might be because the models are calibrated to a period with less wet days (see section 14.2.2). For the SD, the models have mostly preserved the statistic for the two shorter validation periods of 1940-1969 and 19101969. However, the MMKD, CDMC and DHMC tend to underestimate the SD of monthly number of wet days, while the HMC tends to overestimate it. Overall, the HMC preserved the 
SD of monthly number of wet days with a tendency of overestimation, while the other models tended to consistently and significantly underestimate the SD. This better performance of HMC is probably because the HMC significantly overestimated the SD for the calibration period with less number of wet days, which enables the HMC to match the SD of validation periods.
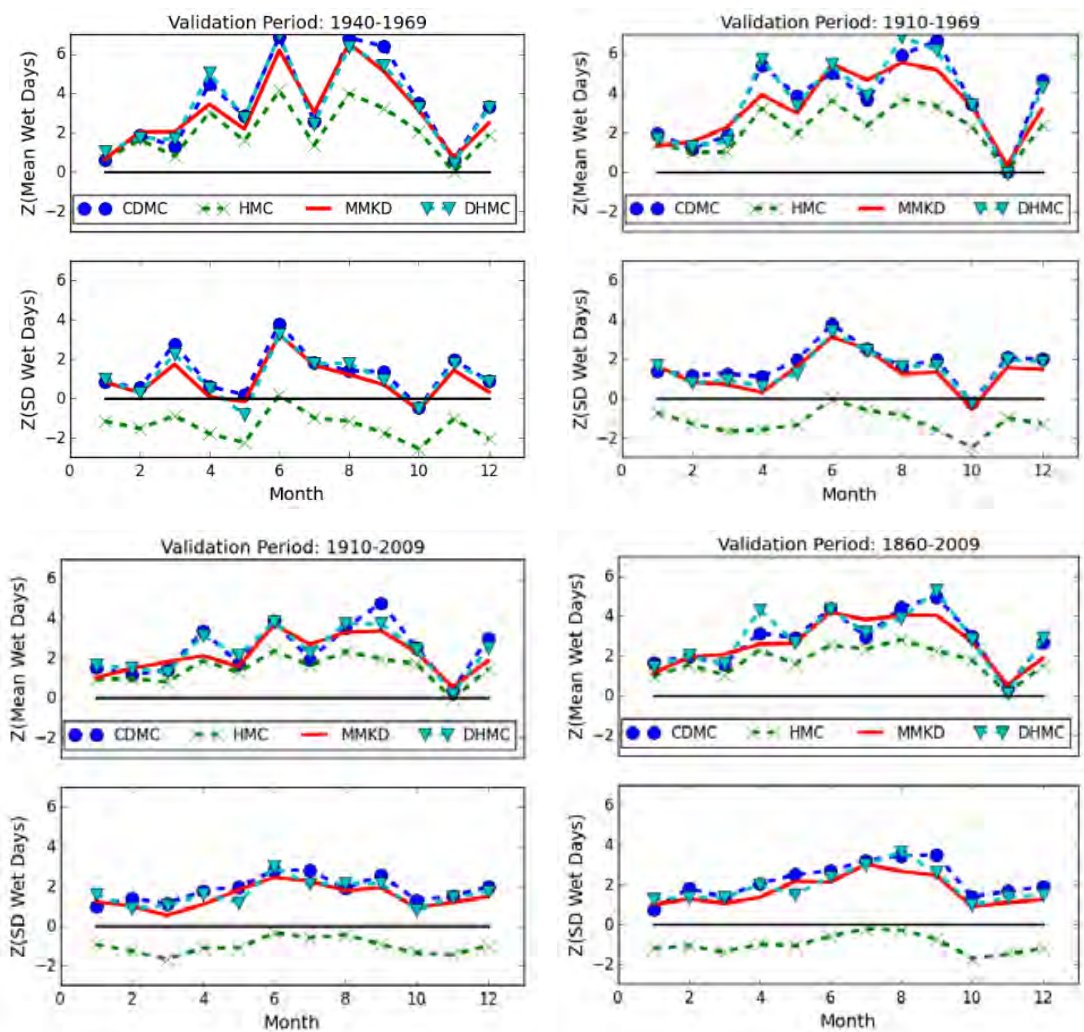

Figure 14.7: Comparison of performances to reproduce the mean and SD of monthly number of wet days in four validation periods.

\subsubsection{Mean and SD of Multiyear Number of Wet Days}

Figure 14.8 compares the models for the mean and SD of multiyear number of wet days. For the mean, all four models fail to preserve the mean with a tendency to underestimate, probably as the models are calibrated to a period having less wet days compared to the validation periods. The MMKD, HMC and DHMC have preserved the SD of multiyear number of wet days with a tendency underestimate for the shorter validation periods of 1940-1969 and 19101969. However, the statistic has been significantly underestimated in MMKD, HMC and DHMC for the longer validation periods of 1910-2009 and 1860-2009. The CDMC has significantly underestimated the statistic for all four validation periods. These results indicate that the performances of the stochastic models are likely to be dependent on the rainfall characteristics of the calibration period, and a 30-year calibration period might not be sufficient to incorporate the long-term variability. 

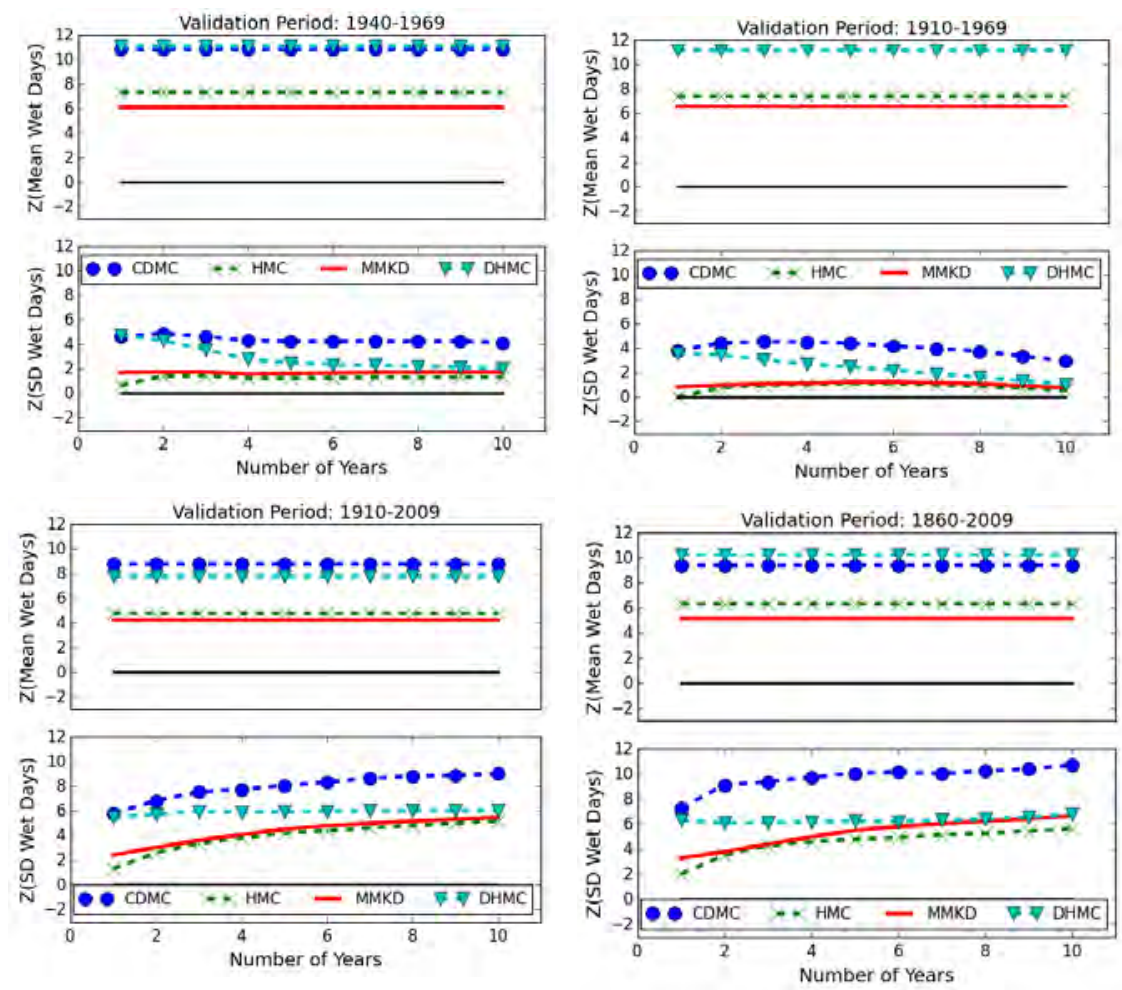

Figure 14.8: Comparison of performances to reproduce the mean and SD of multiyear number of wet days in four validation periods.

\subsubsection{Mean and SD of Monthly Mean Length of Wet Spells}

Figure 14.9 compares the models for the mean and SD of monthly $\mu_{w e t}$. For the mean of monthly $\mu_{w e t}$, the HMC satisfactorily preserves the statistic and performs better than the other models, which have similar performances and tend to consistently and significantly underestimate the mean. The HMC also preserves the SD of monthly $\mu_{\text {wet }}$ in all four validation periods with a tendency to overestimate. The HMC's performance is also better than the other three models as they significantly underestimate the SD. These performances are also consistent with the respective performances of the models for monthly number of wet days. The HMC probably performs better because it overestimates the mean and SD of wet spell lengths, when the models are calibrated to a period with shorter wet spells. 

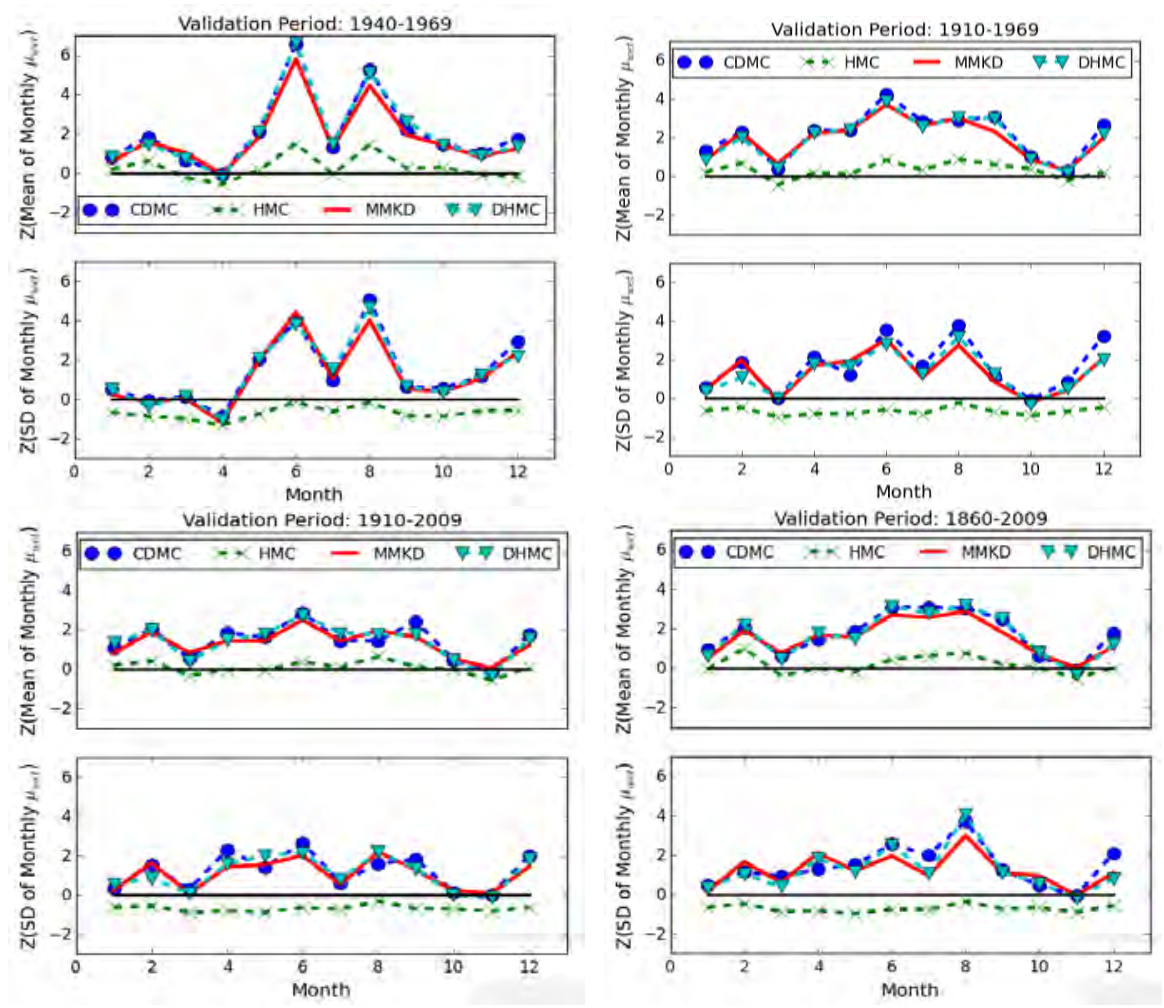

Figure 14.9: Comparison of performances to reproduce the mean and SD of monthly mean length of wet spells in four validation periods.

\subsubsection{Mean and SD of Annual Mean Length of Wet Spells}

Figure 14.10 compares the models for the mean and SD of annual $\mu_{w e t}$. For both mean and SD, the performances of the models are consistent with their respective performances for the mean and SD of multiyear number of wet days (discussed in section 14.4.4).
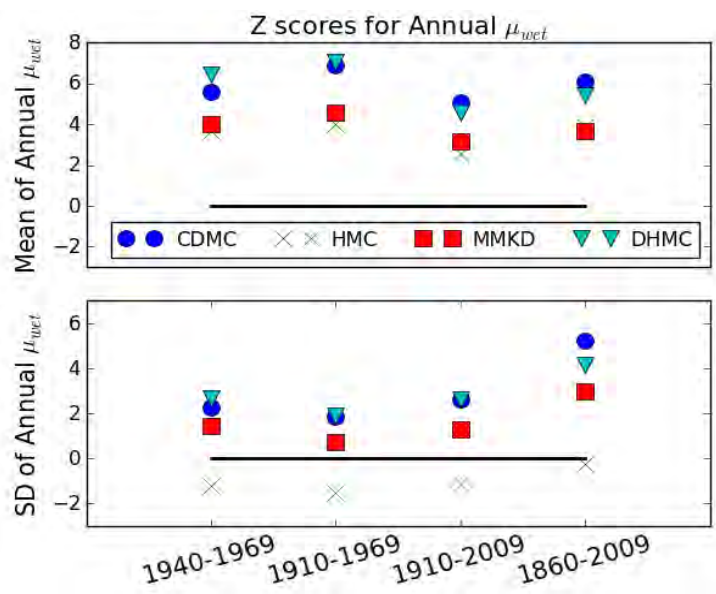

Figure 14.10: Comparison of performances to reproduce the mean and SD of annual mean length of wet spells for four validation periods. 


\subsection{Discussion and Conclusion}

This chapter has compared the performances of the four models to reproduce the mean and SD of rainfall depths, mean length of wet spells, and number of wet days at daily to multiyear resolutions for four validation periods of rainfall data, in addition to the calibration period to which parameters of each model are calibrated.

The observed statistics show that there are considerable differences in the characteristics of rainfall depths and wet periods among the data samples. Particularly, the characteristics of samples with shorter record lengths (e.g. 30-year and 60-year samples) are different than the samples with longer record lengths (e.g. 100-year and 150-year samples) and the characteristics of the calibration period are different from the characteristics of the validation periods.

At daily and monthly resolutions, the seasonality of the mean and SD of rainfall depths are mostly similar in the four validation periods, while slightly different in the calibration period. The mean of wet day rainfall in the calibration period of 1979-2008 is higher than the four validation periods, while the mean of monthly rainfall depths in the calibration and validation periods is mostly similar. This is because the calibration period has fewer wet days and the wet days are wetter (i.e. higher rainfall depths) than the wet days of the validation periods. This also indicates possible climate change impacts on the calibration period of recent past relative to the validation periods of longer past, while Wasko and Sharma [2015] found steeper temporal distribution of rainfall depths (higher rainfall in less wet days) at higher temperatures within Australian storm. While the multiyear mean of rainfall depths is similar in all samples, the SD of multiyear rainfall depths is higher in the two samples of 1979-2008 and 1940-1969. This indicates that the multiyear rainfall variability observed in shorter recent past records is higher than the multiyear rainfall variability observed in longer past records. This also indicates that the parameters of each model are calibrated to the sample, which has higher multiyear variability of rainfall depths than the other samples.

The monthly wet spell lengths and number of wet days indicate that the calibration period had shorter wet spells and less number of wet days in almost all seasons compare to the four validation periods. Also, the monthly wet period variability in calibration period is considerably less than the validation periods. Similar results are found for the mean and SD of annual mean length of wet spells, and the mean of multiyear number of wet days. However, the SD of multiyear number of wet days in the calibration period of 1979-2008 and two shorter validation 
periods of 1940-1969 and 1910-1969 are similar and considerably lower than the same in two longer validation periods of 1910-2009 and 1860-2009.

For the calibration period, while all four models perform similarly and satisfactorily for the mean and SD of rainfall depths, the MC models tend to underestimate and the MMKD tends to overestimate the mean of multiyear rainfall depths. For mean of wet period statistics in calibration, the performances of all four models are similar and satisfactory. For SD of wet period statistics, the DHMC and MMKD have a similar performance and perform satisfactorily and better than the other models. The CDMC significantly underestimates the SD of multiyear number of wet days, while the HMC significantly overestimates the SD of monthly number of wet days and mean length of wet spells. Therefore, the overall performance of DHMC and MMKD is comparable, satisfactory and better than the CDMC and HMC. These conclusions are consistent with the findings presented in Chapters 12 and 13.

For the four validation periods, the models' performances are influenced by the difference in rainfall characteristics in the calibration and validation periods. The distributions of rainfall depths in the calibration and validation periods are mostly similar and so the performance of each model for rainfall depths statistics of validation periods is consistent with their respective performance for the calibration period. But, for wet period statistics, the calibration period has considerably smaller mean and SD of wet days and wet spell lengths. Therefore, the DHMC and MMKD tend to significantly underestimate the mean and SD of wet period statistics of the wetter validation periods, although these two models perform satisfactorily and better than other models for calibration period. This indicates the sensitivity of the models to the wet period characteristics of the calibration period. This also indicates that the 30 -year record length of calibration period might not be sufficient to represent the wet period characteristics of a long historical climate. However, since the HMC overestimates the wet period statistics of the calibration period, it has shown better performances than the other models to preserve the statistics of wetter validation periods. 


\section{Comparison of MC and MMKD for Streamflow Generation}

\subsection{Introduction}

This chapter compares the CDMC, HMC, DHMC and MMKD for the generation of streamflow from simulated rainfall using the SimHyd hydrology model (the SimHyd model is described in section 2.7). This comparison is important to assess the usability of each model for urban drought security assessment. It will give an understanding of how well the models can reproduce the synthetic streamflow, which can in turn be used in reservoir simulation for drought assessment. It will also provide information about how the limitations of each model in rainfall generation is reflected in the streamflow generation. However, this analysis is only a preliminary study with following key limitations which should be addressed in future works:

- The SimHyd model was not calibrated to the data used in this analysis. Instead, the SimHyd was run by using previously calibrated parameters (see Table 2.1) of Mortazavi et al. [2013], monthly mean daily catchment average potential evapotranspiration (provided by HWC and previously used by Lockart et al. [2016]), while only the daily rainfall input was used from RCM1, AWAP, and three MC models of this study.

- A single time series of area-averaged daily rainfall data was used for each subcatchment as the MC models of this study are single-site models. This might not be sufficient to incorporate the spatial variabilities of rainfall in this site as discussed in Chapter 7. A more sophisticated analysis can be performed using multi-site version of the models, but was not done here.

- This analysis has used only RCM1 and AWAP data, while RCM2 could be a better choice considering RCM2 has more similarities to AWAP. However, the analysis was started with RCM1 and the results indicated that the performances of the MC models for streamflow generation are mostly consistent with their respective performances for rainfall generation, which was further supported by the similar results for AWAP. Therefore, this study has not repeated the same analysis for RCM2 and RCM3. 


\section{Comparison of MC and MMKD for Streamflow Generation}

This comparison is conducted for three sub-catchments (Grahamstown, Tillegra and Chichester) of the Williams River site of the lower Hunter region (Figure 3.10). These subcatchments are components of the SimHyd hydrology model for the Hunter Water urban water infrastructure. For each of the three sub-catchments, area-averaged daily rainfall timeseries of RCM1 and AWAP, and monthly averaged potential evapotranspiration data have been used in the SimHyd model to generate streamflow (see section 3.2.4 and 3.3.8). The 60-year daily rainfall data for 1950-2009 has been divided into two 30-year samples of 1950-1979 and 1980-2009. The parameters of each model are calibrated to the data for 1980-2009 (i.e. the calibration period), while the data for $1950-1979$ is used for validation. The assessment procedures of the performances of each model to reproduce the statistics of rainfall depths and streamflow volumes have been described in section 3.10 .

This chapter will present the detailed results for Grahamstown and Tillegra only. The results for Chichester are similar to the results for Tillegra, and so the Chichester results are only briefly discussed in this chapter while the detailed results are provided in Appendix I. The results for monthly resolution rainfall and streamflow are also only briefly discussed with detailed results in Appendix I, as the monthly results are mostly consistent with the daily results. 


\subsection{Justification of Using Single-site Rainfall Model}

The streamflow analysis of this study has used the single-site rainfall model calibrated to areaaveraged rainfall data for the pixels of NARCliM and AWAP, as shown in sections 3.2.4 and 3.3.8. To check the effects of using a single-site model and area-averaged rainfall data, this study has combined the rainfall data of adjacent NARCliM pixels around Gummun in three square-shaped pixel windows, namely one pixel $(10 \mathrm{~km} \times 10 \mathrm{~km})$, four pixels $(20 \mathrm{~km} \times 20 \mathrm{~km})$ and nine pixels $(30 \mathrm{~km} \times 30 \mathrm{~km})$, as shown in the boxes of Figure 15.1. The $P_{11}$ and $\mu$ parameters of these three pixel windows show the typical seasonal variation and the obvious increasing of rainfall frequency and intensity with the increase in pixel-area. However, the steady change of parameters in different pixel windows suggests that the rainfall-hydrology analysis for small-sized catchments (e.g. Grahamstown, Tillegra and Chichester of around 200 $\mathrm{km}^{2}$ area) can be done using a single-site rainfall model calibrated to area-averaged rainfall data.

This study has also calculated the correlation between daily rainfall timeseries $\left(r_{t s}\right)$ in pairs of pixels separated by different distances (e.g. 10, 20, 30, 40 and $50 \mathrm{~km}$ ) to test the similarity of rainfall in adjacent pixels. The $r_{t s}$ values of all pixel-pairs for an inter-pixel distance are shown in Figure 15.2. It shows the distributions of $r_{t s}$ for pairs of pixels separated by 10, 20, 30, 40 and $50 \mathrm{~km}$ distances for RCM2 at the Williams River site. This study has found strong correlations between rainfalls of closest pixel-pairs (i.e. at inter-pixel distance of $10 \mathrm{~km}$ and 20 $\mathrm{km}$ ), which gradually decreases with distance. Similar results have been found for the other RCMs and AWAP. This supports the assumption that the rainfall-hydrology analysis for smallsized catchments of around $200 \mathrm{~km}^{2}$ area can be done using a single-site rainfall model calibrated to area-averaged rainfall data. 

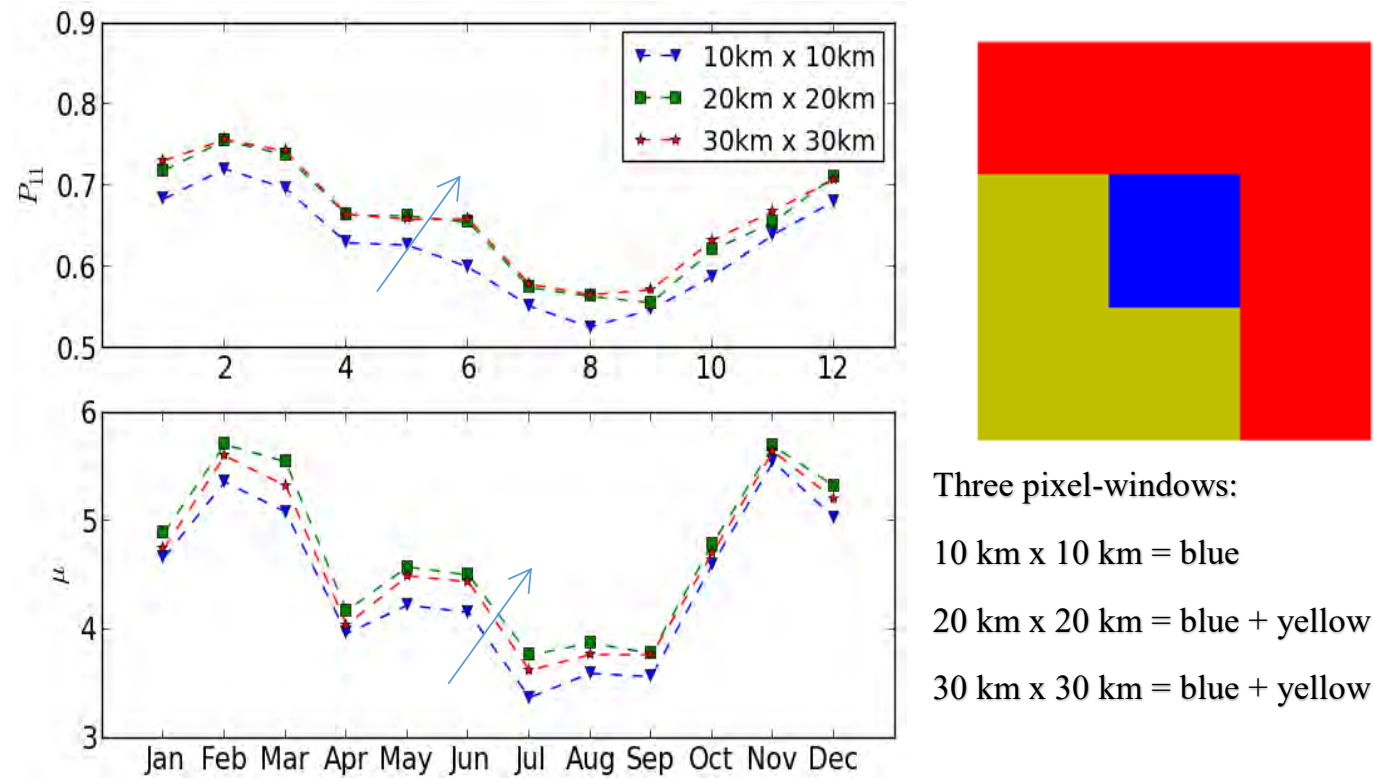

Three pixel-windows:

$10 \mathrm{~km} \times 10 \mathrm{~km}=$ blue

$20 \mathrm{~km} \times 20 \mathrm{~km}=$ blue + yellow

$30 \mathrm{~km} \times 30 \mathrm{~km}=$ blue + yellow + red

Figure 15.1: The boxes in the right figure show three pixel windows (around Gummun) of three different sizes. The figures in left panel show changes of the wet-to-wet probabilities $\left(P_{11}\right)$ and mean of wet day rainfall depths $(\mu)$ with the changes in size of pixel windows.

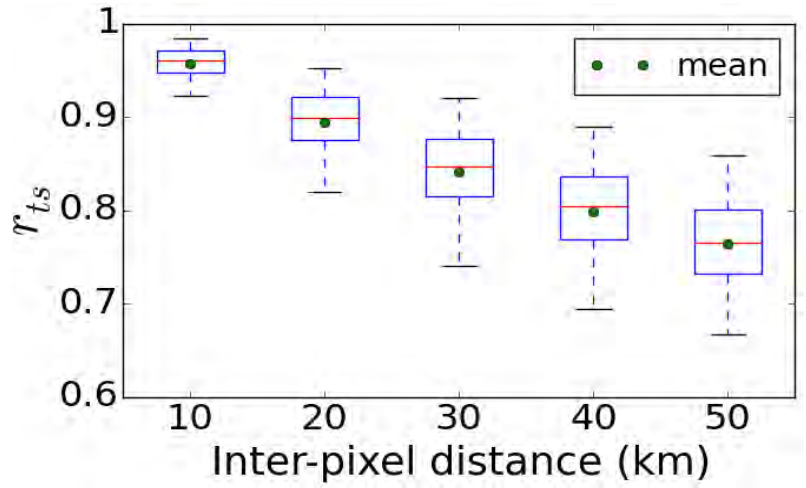

Figure 15.2: Distribution of correlation of daily rainfall timeseries $\left(r_{t s}\right)$ between pairs of pixels at different distances for RCM2 at Williams River site. 


\subsection{Distribution of Rainfall and Streamflow}

This section will present the distribution statistics (mean, SD, lowest $5^{\text {th }}$ and highest $95^{\text {th }}$ percentiles) of rainfall depths and streamflow volumes for both calibration (1980-2009) and validation (1950-1979) periods. The rainfall and streamflow at Chichester are similar to the rainfall and streamflow at Tillegra and so are shown in Appendix I.2.1.

\subsubsection{Daily Rainfall Depths and Streamflow Statistics}

Figure 15.3 shows the distribution statistics of daily rainfall depths and streamflow volumes for Grahamstown and Figure 15.4 shows the same statistics for Tillegra.

For all three catchments, the seasonal trend of streamflow volume is mostly consistent with the seasonal trend of rainfall depths, which indicates that the seasonal variability of streamflow generated by SimHyd is mostly regulated by the seasonal variability of rainfall depths. However, for a few exceptions, the seasonal trend of streamflow volume is different to the seasonal trend of rainfall depths (e.g. for Grahamstown, mean daily streamflow volume of February to April shows a different trend compare to the corresponding mean daily rainfall depths), which might be influenced by other parameters or inputs of the SimHyd model (e.g. evapotranspiration). For Grahamstown, the seasonality shows the wetter condition (higher mean, SD and percentile) in February-May and drier conditions in September-October (Figure 15.3). The seasonality for Tillegra and Chichester are similar, with wettest conditions in November-March, which gradually changes to driest conditions in July-October (Figure 15.4 and Figure I.1 at Appendix I.2.1). Overall, the Tillegra and Chichester sub-catchments are wetter (higher mean and SD) than the Grahamstown sub-catchment. The rainfall and streamflow in Tillegra and Chichester are similar and slightly different to the rainfall and streamflow in Grahamstown, which is because of the geography of the catchments, as the Tillegra and Chichester are of similar size and located in higher elevation of the Williams River site, while Grahamstown is located in lower elevation of the site (Figure 3.10 and Table 3.3).

For all three catchments, the RCM1 is wetter (higher mean and percentiles) and more variable (higher SD) than the AWAP for both calibration and validation periods (consistent with the findings presented in Chapter 7). 

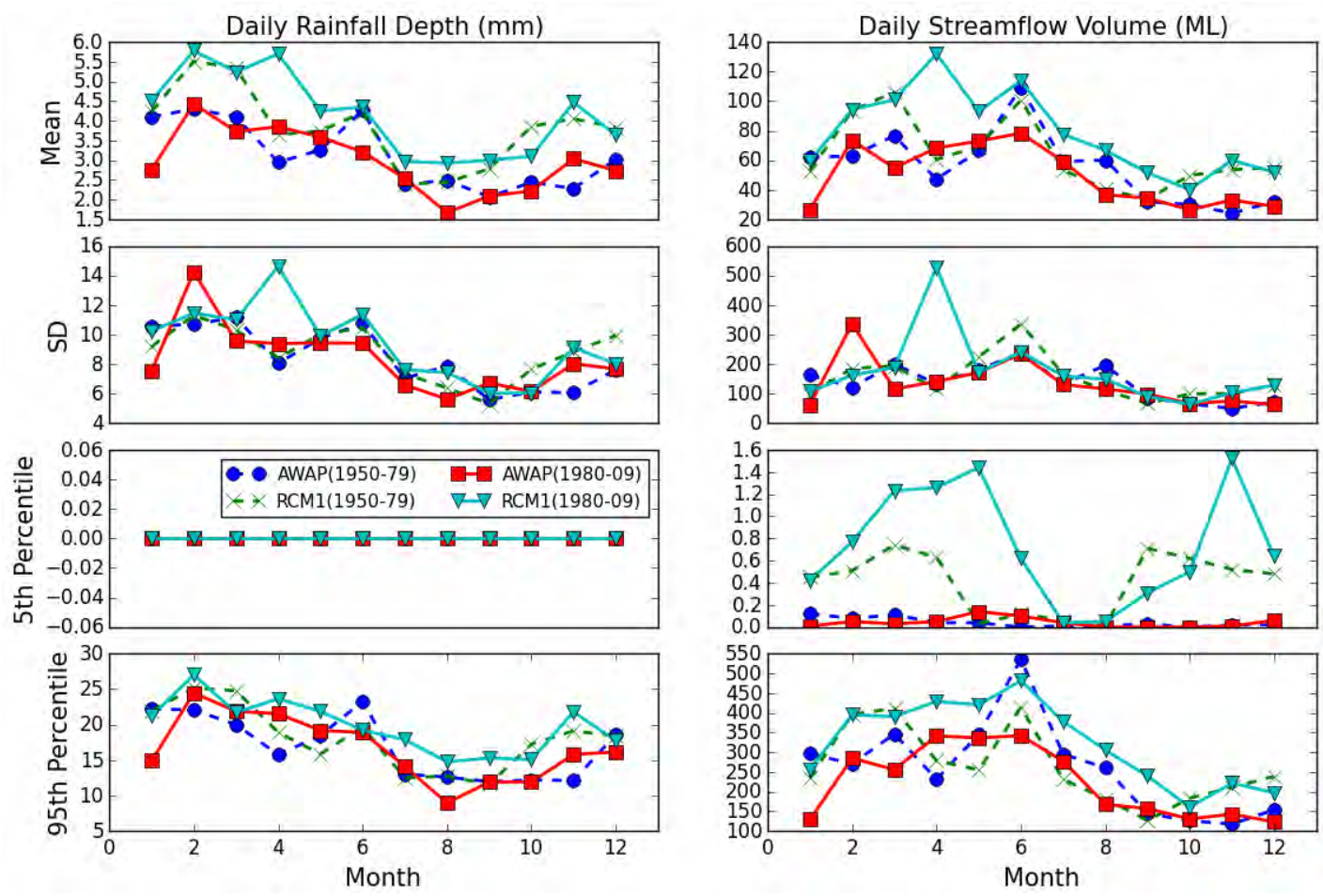

Figure 15.3: Distribution statistics of daily rainfall depths and streamflow volumes for the calibration (1980-2009) and validation (1950-1979) periods at Grahamstown catchment.
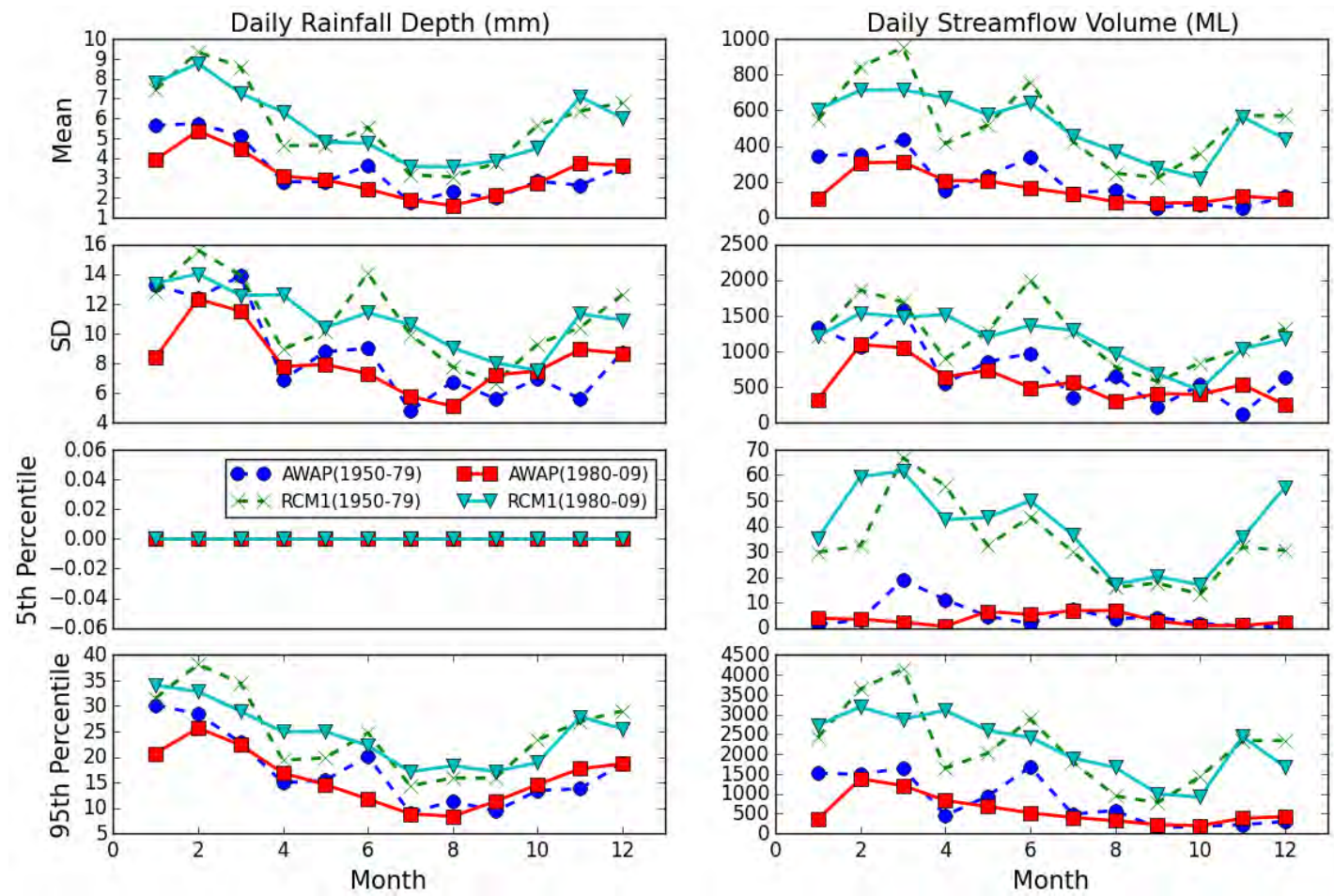

Figure 15.4: Distribution statistics of daily rainfall depths and streamflow volumes for the calibration (1980-2009) and validation (1950-1979) periods at Tillegra catchment. 


\subsubsection{Monthly Rainfall Depths and Streamflow Volumes}

For all three catchments, the seasonal trend of the mean, SD, lowest $5^{\text {th }}$ and highest $95^{\text {th }}$ percentiles of rainfall depths and streamflow volumes at monthly resolution is similar to the respective statistics at daily resolution (see Appendix I.2).

\subsubsection{Multiyear Rainfall Depths and Streamflow Volumes}

Figure 15.5 shows the distribution statistics of multiyear rainfall depths and streamflow volumes for Grahamstown and Figure 15.6 shows the same statistics for Tillegra.

The multiyear statistics of rainfall depths and streamflow volumes show the differences between the calibration and validation periods, which were not clear in daily and monthly statistics. In all three catchments, and for both RCM1 and AWAP, the calibration period (19802009 ) is drier (lower mean, SD, and percentiles) than the validation period (1950-1979), except for RCM1 for Grahamstown, where the calibration period is wetter than the validation period. The calibration period is likely to be drier than the validation period as it comprises several ElNiño conditions and the associated 'millennium drought' period (2001-2009) which affected the eastern seaboard of Australia, including the lower Hunter [Verdon-Kidd and Kiem, 2009]. However, the wetter calibration period of RCM1 for Grahamstown might be because of the anomalies in the NARCliM data discussed by Parana Manage [2016].

Similar to the daily and monthly statistics, the multiyear statistics show that the RCM1 is wetter than the AWAP and that Tillegra and Chichester are wetter than Grahamstown. 

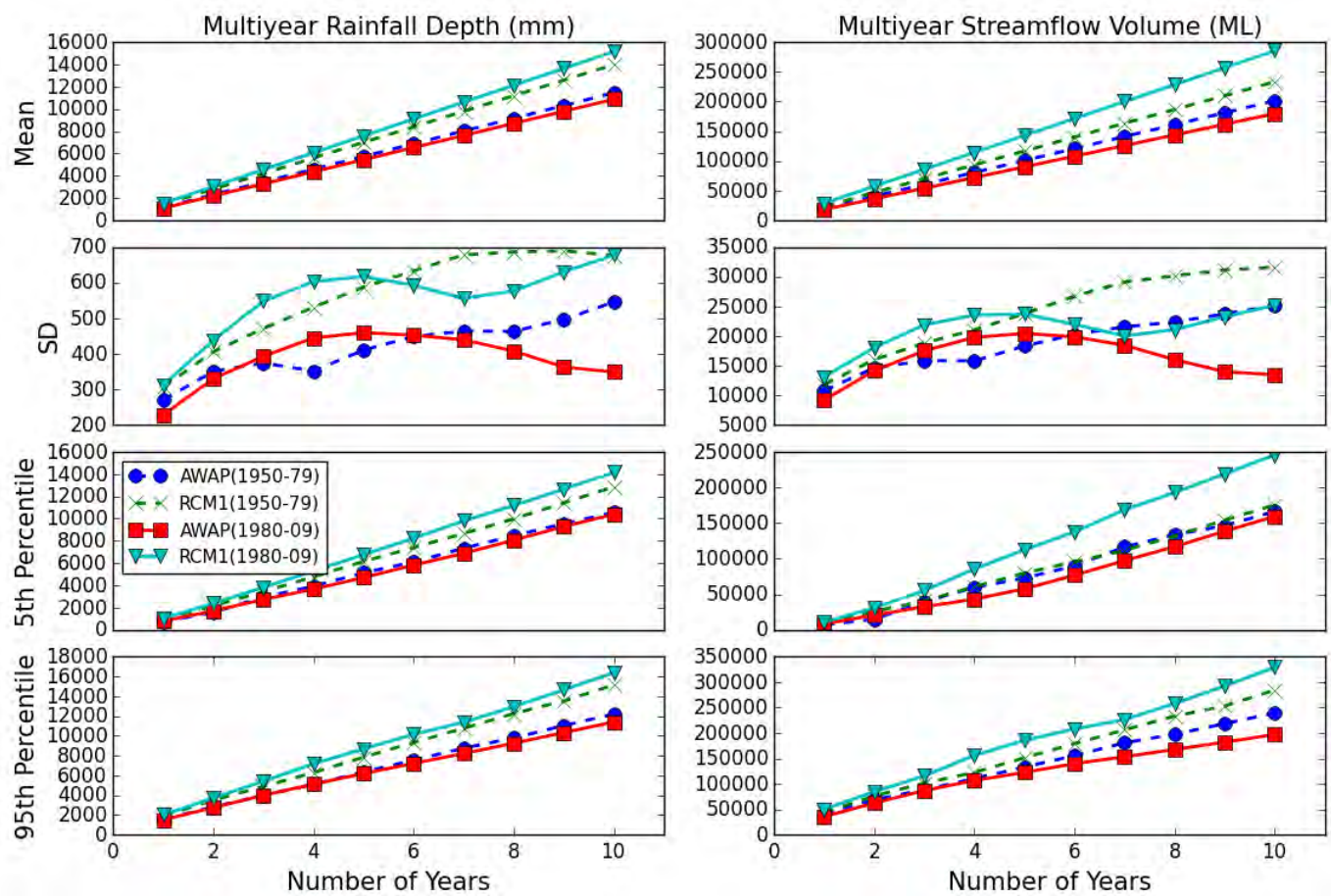

Figure 15.5: Distribution statistics of multiyear rainfall depths and streamflow volumes for the calibration (1980-2009) and validation (1950-1979) periods at Grahamstown.
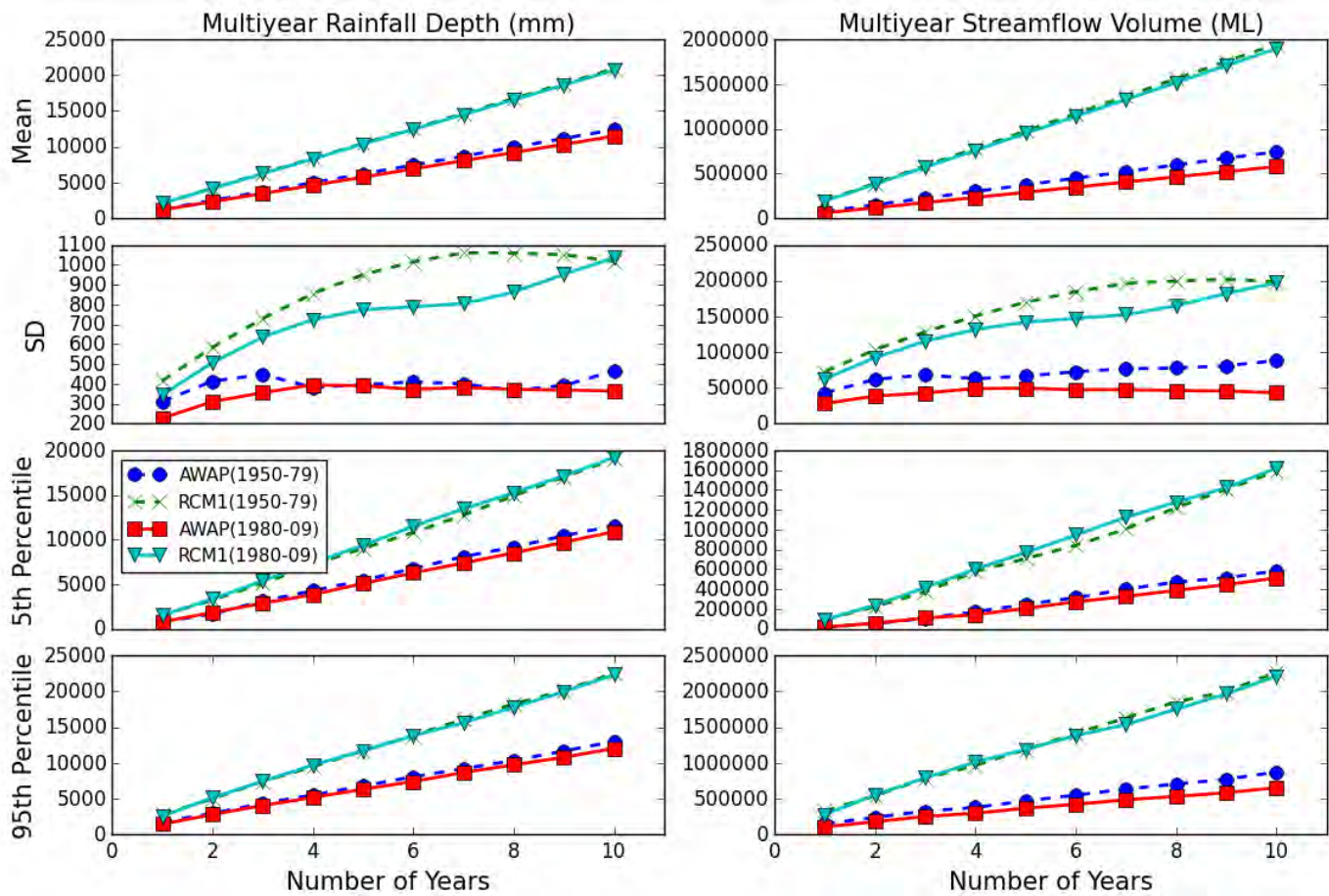

Figure 15.6: Distribution statistics of multiyear rainfall depths and streamflow volumes for the calibration (1980-2009) and validation (1950-1979) periods at Tillegra. 


\subsection{Model Comparison for Distribution Statistics}

This section compares the models for the distribution statistics (i.e. mean, SD, lowest $5^{\text {th }}$ and highest $95^{\text {th }}$ percentiles) of rainfall depths and streamflow volumes for the calibration and validation periods. While results will be discussed for all three catchments, the results for Chichester are similar to Tillegra and are shown in Appendices I.3.1 and I.3.2.

\subsubsection{Daily Rainfall Depths and Streamflow Volumes}

Figure 15.7 and 15.8 show the model comparison for the distribution of daily rainfall depths and streamflow volumes of RCM1 for the calibration and validation periods, respectively, at Grahamstown. Figure 15.9 and Figure 15.10 show the same comparisons at Tillegra.

For both Grahamstown and Tillegra, the performance of each model for streamflow volumes is consistent with their respective performance for rainfall depths, which is because the low natural storage in the catchments means that the variability of streamflow mostly follows the variability of rainfall, as discussed in section 15.3.

For both catchments, all four models show satisfactory performances to reproduce the daily rainfall depths and streamflow volumes. For the calibration period, the CDMC, HMC and DHMC perform similarly and tend to underestimate the mean, SD and $95^{\text {th }}$ percentile, while the MMKD tends to overestimate the mean, SD and $95^{\text {th }}$ percentile (consistent with the comparison results presented in Chapters 12 and 13). There is a perfect fit for the $5^{\text {th }}$ percentile rainfall because this percentile is zero rainfall, though the streamflow does not drop to zero for the $5^{\text {th }}$ percentile. For validation, the performance of all four models is similar, although the performance of each model for the validation period is, as expected, poorer than their respective performance for the calibration period. The performance of each model for Chichester is similar to Tillegra as the rainfall and streamflow of Chichester are similar to Tillegra (see section 15.4.4 and Appendix I.3.1). Similar trends of performance of each model has been found for the rainfall depths and streamflow volumes of AWAP at each catchment (see section 15.4.4 and Appendices I.3.2-I.3.4). Section 15.4.4 will summarise the model comparisons for rainfall and streamflow of RCM1 and AWAP at all three catchments by presenting temporally averaged $\mathrm{Z}$ scores (calculated as per section 3.8.1). 

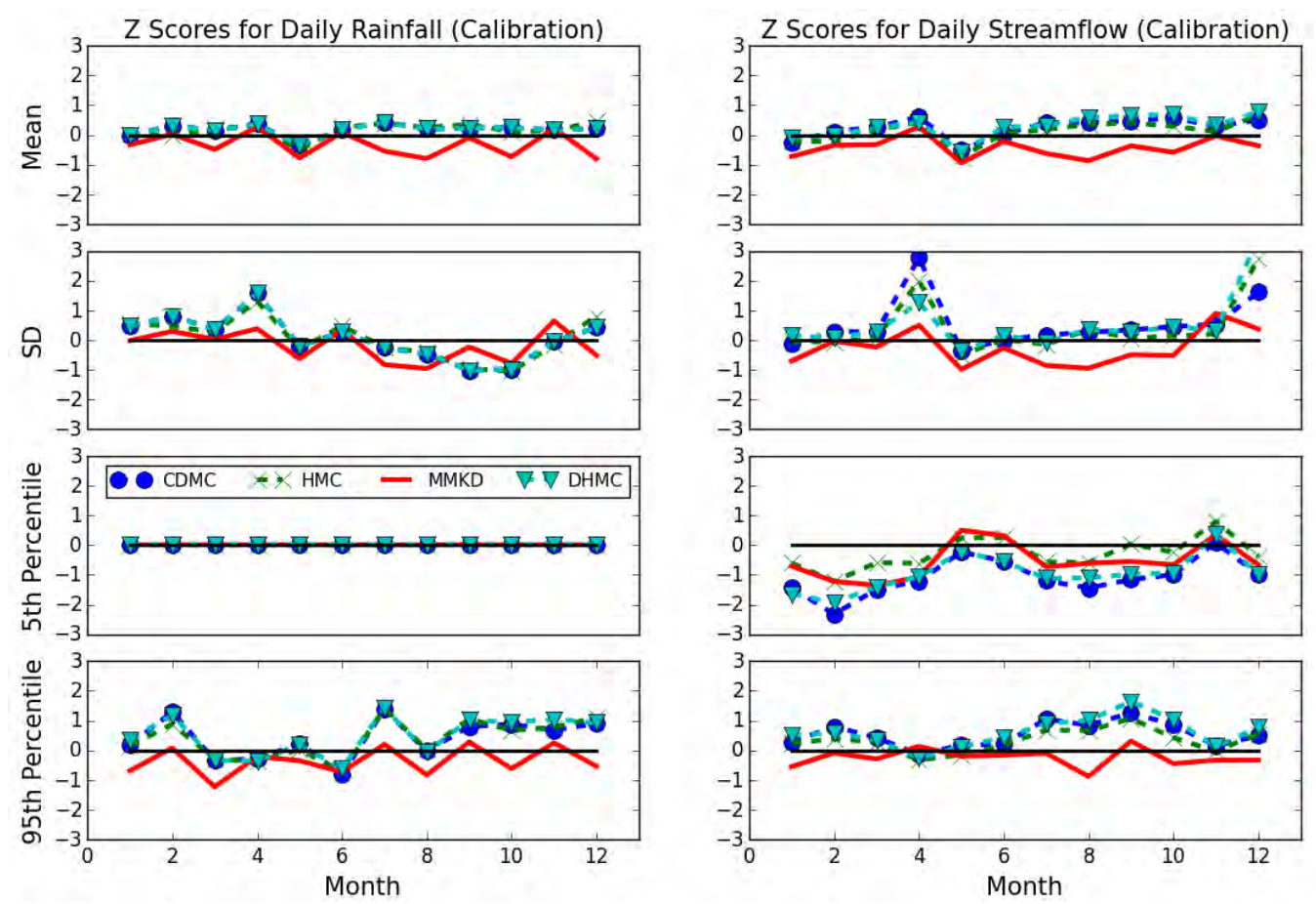

Figure 15.7: Model comparison to reproduce the distribution statistics of daily rainfall and streamflow for calibration (1980-2009) period of RCM1 at Grahamstown.
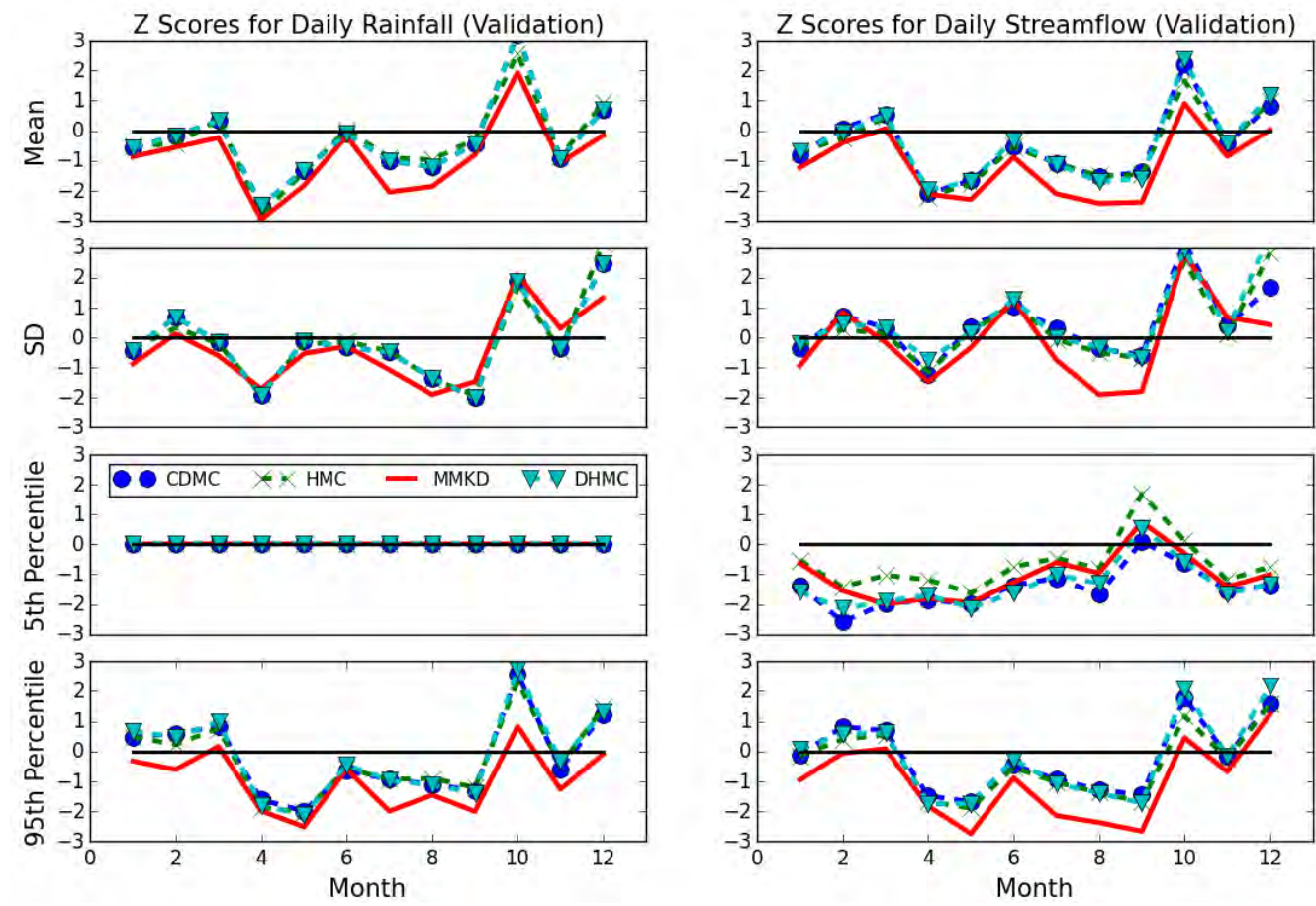

Figure 15.8: Model comparison to reproduce the distribution statistics of daily rainfall and streamflow for validation (1950-1979) period of RCM1 at Grahamstown. 

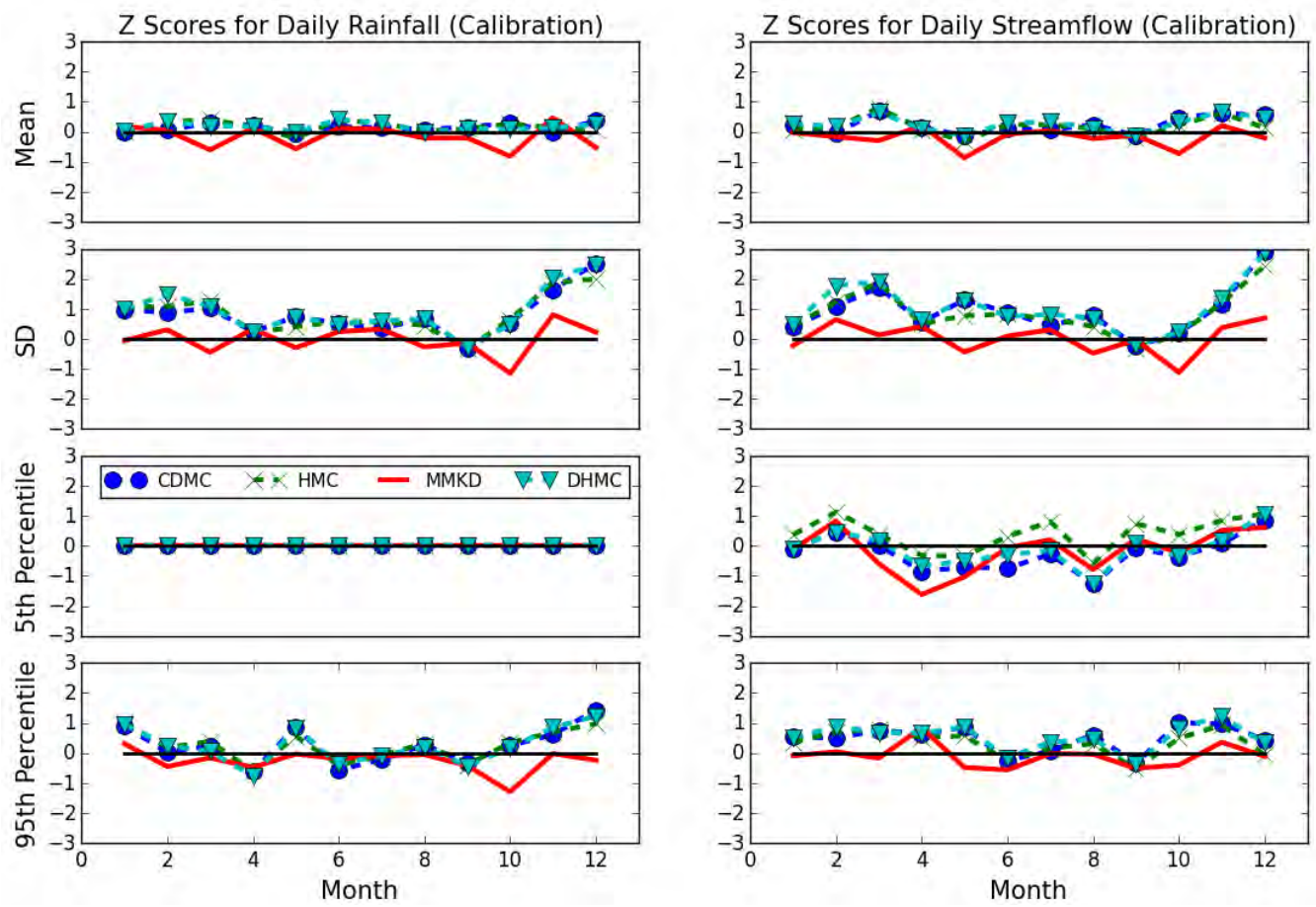

Figure 15.9: Model comparison to reproduce the distribution statistics of daily rainfall and streamflow for calibration (1980-2009) period of RCM1 at Tillegra.
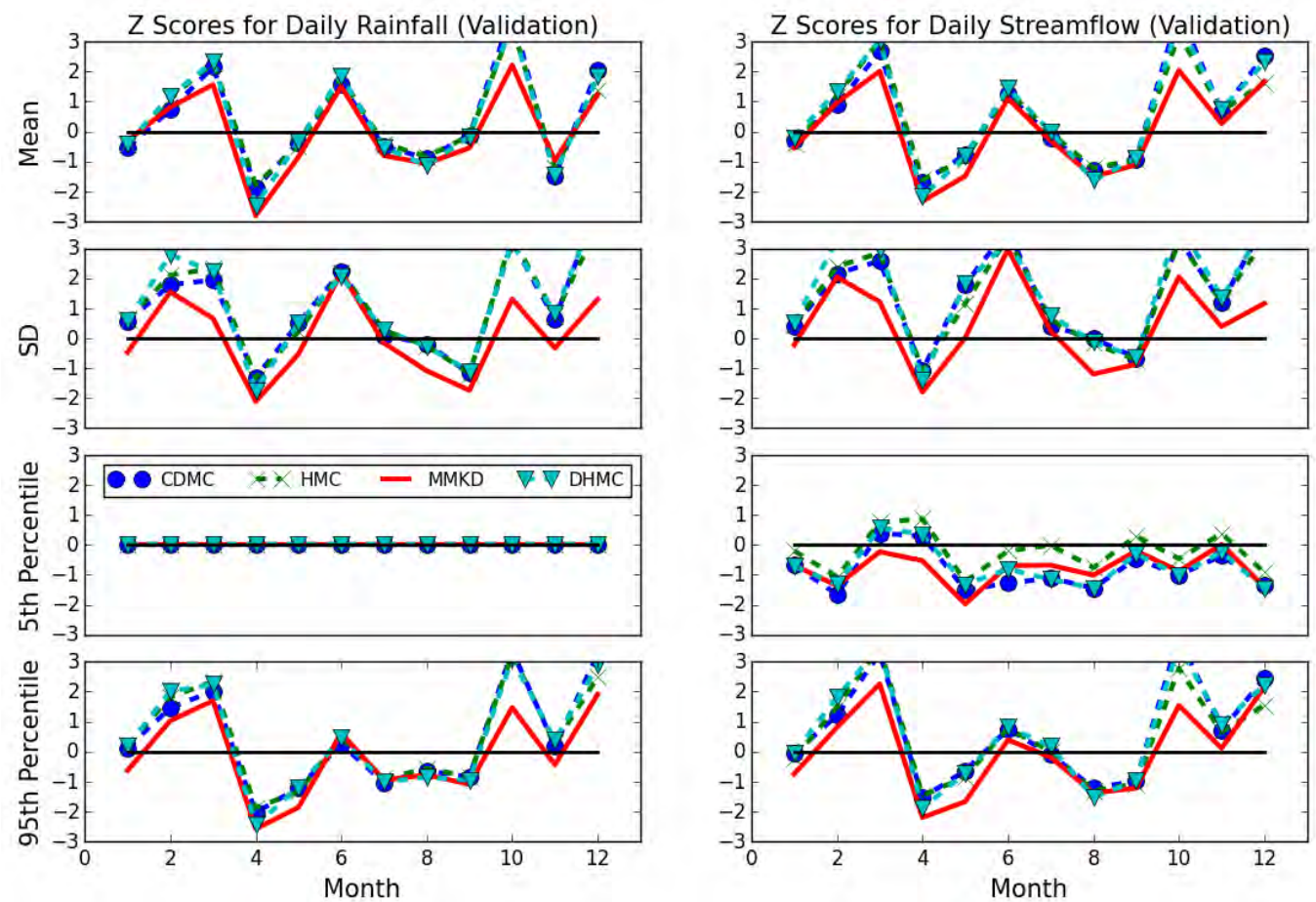

Figure 15.10: Model comparison to reproduce the distribution statistics of daily rainfall and streamflow for validation (1950-1979) period of RCM1 at Tillegra. 


\section{Comparison of MC and MMKD for Streamflow Generation}

\subsubsection{Monthly Rainfall Depths and Streamflow Volumes}

The performances of each model to reproduce the monthly rainfall depths and streamflow volume are similar to their respective performances for daily rainfall depths and streamflow volumes. Hence, the monthly results are shown in Appendix I.3.5 and briefly discussed in section 15.4 .4 by using temporally averaged $\mathrm{Z}$ scores of each model.

\subsubsection{Multiyear Rainfall Depths and Streamflow Volumes}

Figure 15.11 and Figure 15.12 show the comparison of how well the models reproduce the distribution of multiyear rainfall depths and streamflow volumes of RCM1 for calibration and validation periods, respectively, at Grahamstown. Figure 15.13 and Figure 15.14 show the same comparisons at Tillegra.

For the calibration period of RCM1, at both Grahamstown and Tillegra, the performances of all four models are comparable and satisfactory to reproduce the multiyear rainfall and streamflow statistics, including multiyear SD. The three MC models perform similarly and tend to underestimate the mean of multiyear rainfall and streamflow, and the MMKD tends to overestimate the mean (consistent with the comparisons presented in Chapters 12 and 13).

For the validation period of RCM1 at Grahamstown, all four models tend to overestimate the mean of multiyear rainfall and streamflow (Figure 15.12), probably because the parameters of each model are calibrated to a wetter sample (the calibration period of RCM1 at Grahamstown is wetter than the validation period, see Figure 15.5). The MC models perform better and have a lower overestimate of the mean of the validation than the MMKD (Figure 15.12), as the MC models usually underestimate the mean of calibration while MMKD overestimates the mean of calibration. In contrast, for the validation period of RCM1 at Tillegra, the models tend to underestimate the multiyear mean (Figure 15.14), probably as the calibration period is drier than the validation period (Figure 15.6), while the MMKD performs better than the MC models. This suggests that if the model parameters are calibrated to a wetter sample, then the MC models perform better than the MMKD for validation, while in contrast, if the model parameters are calibrated to a drier sample, the MMKD performs better than the MC models. The performances of each model for the calibration and validation periods are consistent for AWAP at Grahamstown and Tillegra, and RCM1 and AWAP at Chichester (see section 15.4.4 and Appendix I.3). 
15. Comparison of MC and MMKD for Streamflow Generation
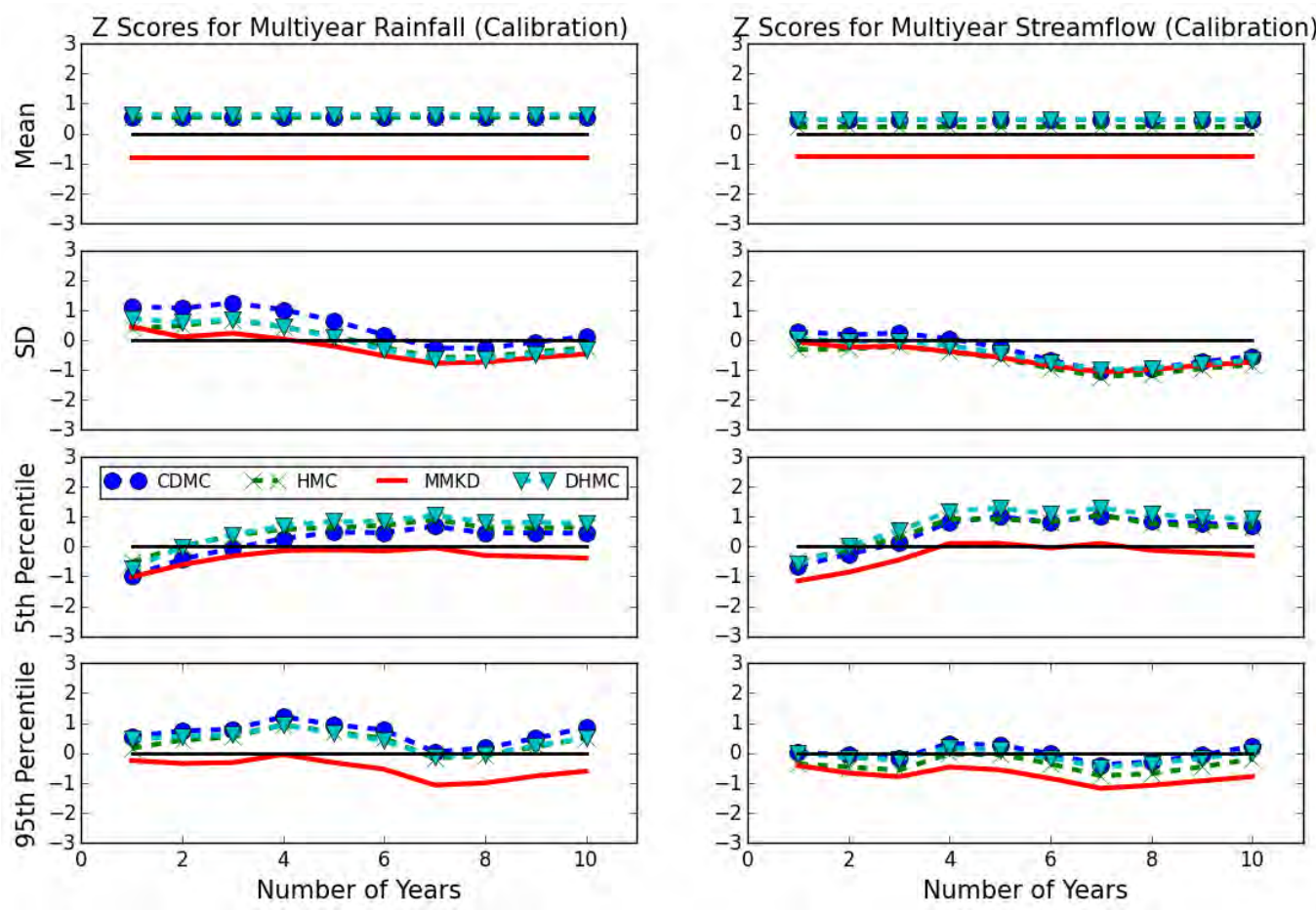

Figure 15.11: Model comparison to reproduce the distribution statistics of multiyear rainfall and streamflow for calibration (1980-2009) period of RCM1 at Grahamstown.
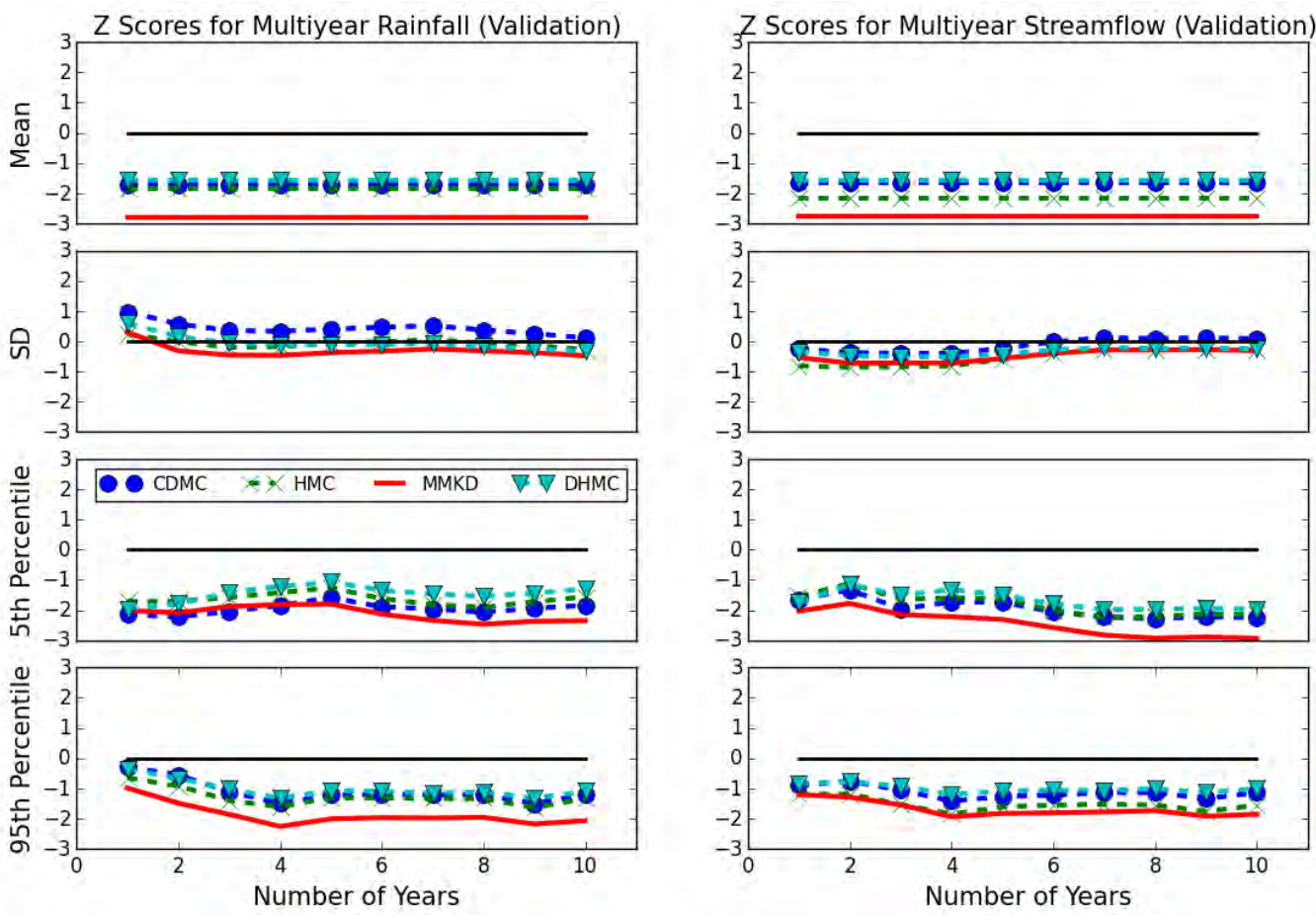

Figure 15.12: Model comparison to reproduce the distribution statistics of multiyear rainfall and streamflow for validation (1950-2079) period of RCM1 at Grahamstown. 

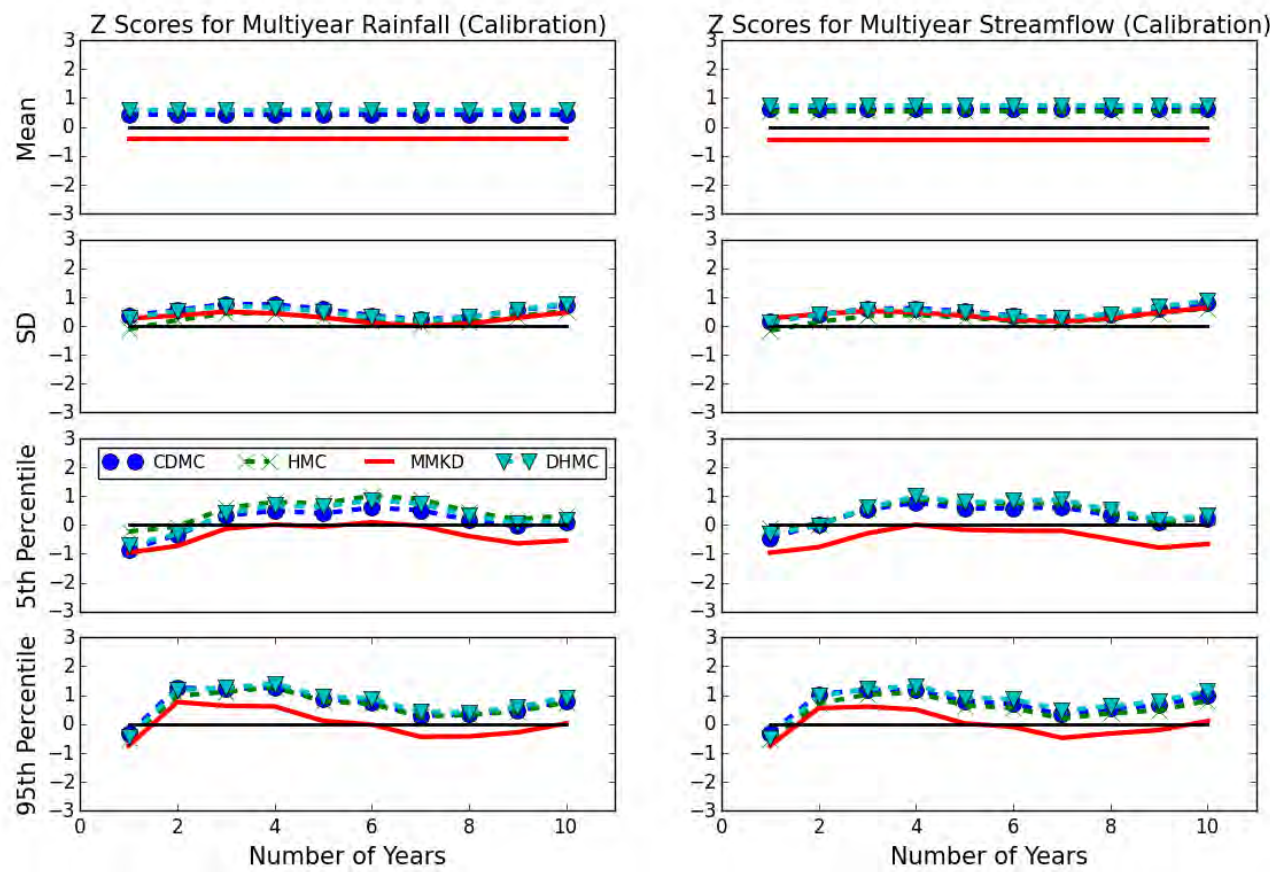

Figure 15.13: Model comparison to reproduce the distribution statistics of multiyear rainfall and streamflow for calibration (1980-2009) period of RCM1 at Tillegra.
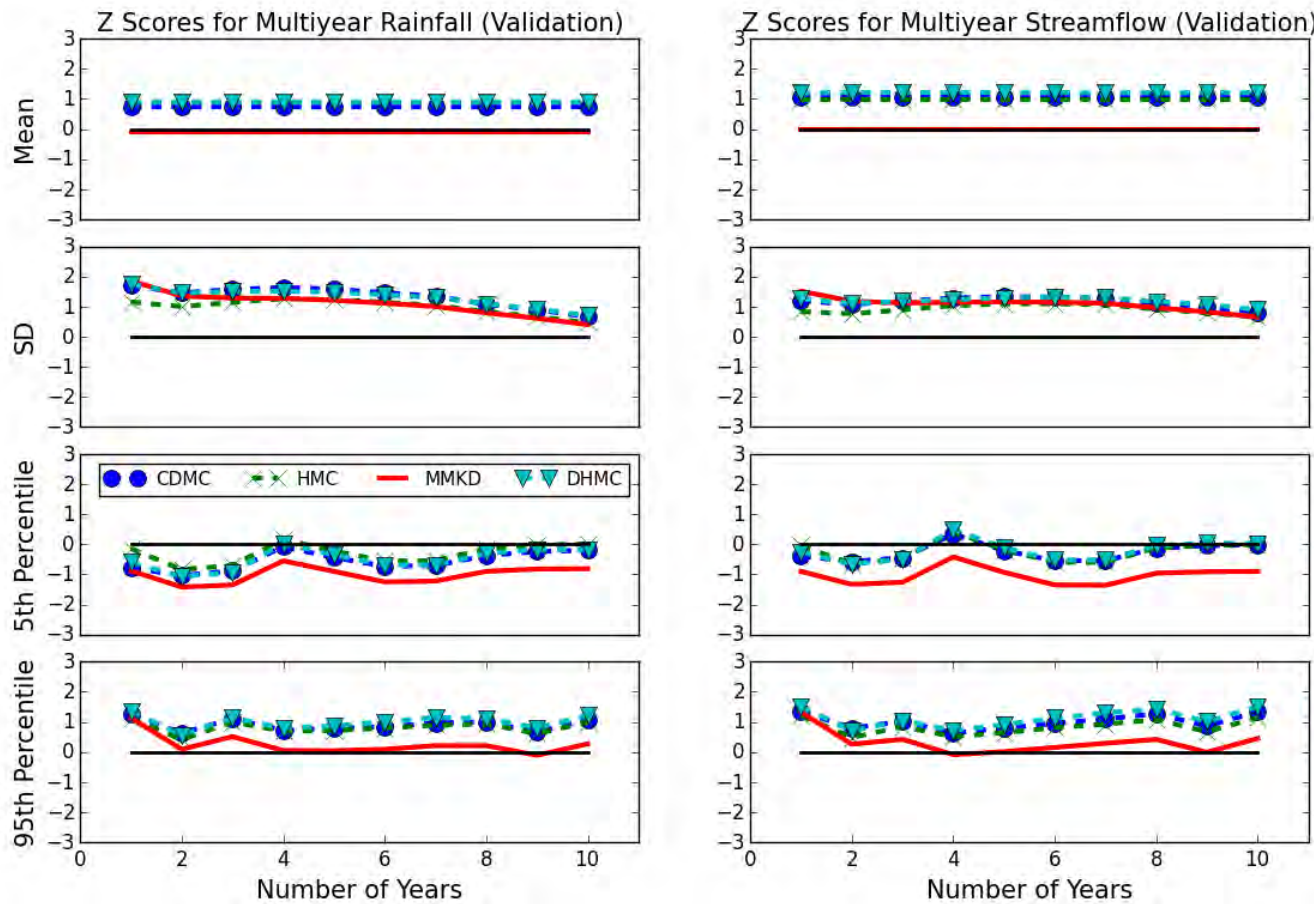

Figure 15.14: Model comparison to reproduce the distribution statistics of multiyear rainfall and streamflow for validation (1950-2079) period of RCM1 at Tillegra. 


\subsubsection{Summary}

Table 15.1 and Table 15.2 show the temporally averaged $\mathrm{Z}$ scores (calculated as per section 3.8.1) of each model for rainfall and streamflow, respectively, for the calibration period, while Table 15.3 and Table 15.4 show the temporally averaged $\mathrm{Z}$ scores for the validation period. The key findings of the model comparison for rainfall and streamflow generation are:

- The performance of each model for streamflow generation is consistent with their respective performance for rainfall generation, probably as the variability of streamflow generated by SimHyd is similar to the variability of rainfall. Moreover, performance of each model for monthly statistics is consistent with their performance for daily statistics.

- The four models perform satisfactorily for rainfall and streamflow of both RCM1 and AWAP, while the RCM1 is considerably wetter than the AWAP.

- For calibration, the MC models tend to underestimate and the MMKD tends to overestimate the mean of rainfall depths and streamflow volumes (consistent with findings presented in Chapters 12 and 13). However, the overall advantage or disadvantage of one model over another is negligible.

- For validation, the MC models perform better than MMKD if the calibration period is wetter than the validation period (e.g. RCM1 at Grahamstown). In contrast, the MMKD performs better than the MC models for validation if the calibration period is drier than the validation period (e.g. AWAP at Grahamstown and both datasets at Tillegra and Chichester).

- For both calibration and validation periods, all four models show similar satisfactory performance to preserve the SD of rainfall and streamflow at daily, monthly and multiyear resolutions.

- Model performance, for both rainfall and streamflow and across all models, was significantly worse during the validation period than the calibration period (summed $\mathrm{Z}$ scores were increased by 2 to 3 times in the validation period relative to the calibration period) and all models degraded by a similar amount, so that no model performed relatively better in the validation period. An acceptable degradation rate for the validation period was not determined. 
Table 15.1: Model comparison by the average of the absolute values of $Z$ scores for rainfall depths for calibration period (1980-2009).

\begin{tabular}{|c|c|c|c|c|c|c|c|c|c|c|c|c|c|c|}
\hline \multirow{3}{*}{\multicolumn{2}{|c|}{ 莒 }} & \multirow{3}{*}{ 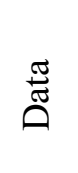 } & \multicolumn{12}{|c|}{ Averaged Z Scores } \\
\hline & & & \multicolumn{4}{|c|}{ Grahamstown } & \multicolumn{4}{|c|}{ Tillegra } & \multicolumn{4}{|c|}{ Chichester } \\
\hline & & & $\mathrm{CD}$ & $\mathrm{HN}$ & $\mathrm{DH}$ & $\mathrm{MM}$ & CD & $\mathrm{HM}$ & $\mathrm{DH}$ & $\mathrm{MM}$ & CD & HM & DH & $\mathrm{MM}$ \\
\hline \multirow{8}{*}{ 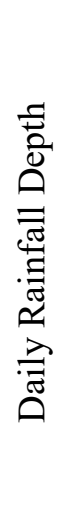 } & \multirow{2}{*}{$\sum_{\Sigma}^{\mathbb{E}}$} & R1 & 0.2 & 0.2 & 0.2 & 0.4 & 0.2 & 0.2 & 0.2 & 0.3 & 0.2 & 0.3 & 0.2 & 0.4 \\
\hline & & AW & 0.2 & 0.1 & 0.3 & 0.4 & 0.2 & 0.2 & 0.2 & 0.3 & 0.3 & 0.2 & 0.2 & 0.2 \\
\hline & \multirow{2}{*}{$\hat{\infty}$} & R1 & 0.6 & 0.6 & 0.6 & 0.5 & 0.9 & 0.9 & 1.0 & 0.4 & 1.0 & 0.9 & 0.8 & 0.5 \\
\hline & & AW & 0.6 & 0.5 & 0.6 & 0.4 & 0.4 & 0.5 & 0.5 & 0.3 & 0.6 & 0.5 & 0.6 & 0.2 \\
\hline & \multirow{2}{*}{$\stackrel{\varpi}{\hbar}$} & R1 & 0.0 & 0.0 & 0.0 & 0.0 & 0.0 & 0.0 & 0.0 & 0.0 & 0.0 & 0.0 & 0.0 & 0.0 \\
\hline & & AW & 0.0 & 0.0 & 0.0 & 0.0 & 0.0 & 0.0 & 0.0 & 0.0 & 0.0 & 0.0 & 0.0 & 0.0 \\
\hline & \multirow{2}{*}{ 营 } & R1 & 0.6 & 0.6 & 0.7 & 0.5 & 0.5 & 0.5 & 0.5 & 0.3 & 0.6 & 0.6 & 0.6 & 0.5 \\
\hline & & AW & 0.6 & 0.5 & 0.6 & 0.4 & 0.3 & 0.3 & 0.4 & 0.4 & 0.4 & 0.4 & 0.4 & 0.4 \\
\hline \multirow{8}{*}{ 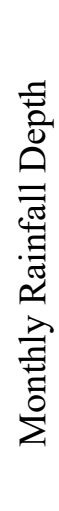 } & \multirow{2}{*}{$\stackrel{\text { है }}{\Sigma}^{\mathbb{E}}$} & R1 & 0.2 & 0.2 & 0.3 & 0.4 & 0.1 & 0.2 & 0.2 & 0.3 & 0.2 & 0.3 & 0.2 & 0.4 \\
\hline & & AW & 0.2 & 0.1 & 0.3 & 0.4 & 0.2 & 0.2 & 0.2 & 0.3 & 0.2 & 0.2 & 0.2 & 0.2 \\
\hline & \multirow{2}{*}{$\hat{\imath}$} & R1 & 0.3 & 0.6 & 0.3 & 0.6 & 0.5 & 0.6 & 0.5 & 0.7 & 0.4 & 0.6 & 0.4 & 0.7 \\
\hline & & AW & 0.4 & 0.7 & 0.4 & 0.6 & 0.4 & 0.6 & 0.4 & 0.4 & 0.4 & 0.6 & 0.4 & 0.4 \\
\hline & \multirow{2}{*}{ 站 } & $\mathrm{R} 1$ & 0.6 & 0.7 & 0.6 & 0.7 & 0.5 & 0.9 & 0.6 & 0.9 & 0.6 & 0.8 & 0.6 & 1.0 \\
\hline & & AW & 1.1 & 0.7 & 1.1 & 1.2 & 0.6 & 0.8 & 0.5 & 0.7 & 0.6 & 0.8 & 0.6 & 0.7 \\
\hline & \multirow{2}{*}{ 萦 } & $\mathrm{R} 1$ & 0.3 & 0.5 & 0.4 & 0.5 & 0.6 & 0.7 & 0.6 & 0.7 & 0.5 & 0.6 & 0.5 & 0.7 \\
\hline & & AW & 0.4 & 0.5 & 0.4 & 0.6 & 0.5 & 0.5 & 0.5 & 0.6 & 0.5 & 0.5 & 0.5 & 0.5 \\
\hline \multirow{8}{*}{ 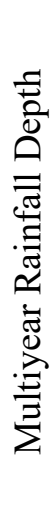 } & \multirow{2}{*}{$\sum_{\Sigma}^{\Xi}$} & R1 & 0.5 & 0.5 & 0.6 & 0.8 & 0.4 & 0.6 & 0.6 & 0.4 & 0.7 & 0.9 & 0.5 & 0.4 \\
\hline & & AW & 0.6 & 0.3 & 0.8 & 1.0 & 0.4 & 0.5 & 0.5 & 0.6 & 0.7 & 0.6 & 0.6 & 0.5 \\
\hline & \multirow{2}{*}{ के } & $\mathrm{R} 1$ & 0.6 & 0.4 & 0.5 & 0.4 & 0.5 & 0.3 & 0.5 & 0.3 & 0.5 & 0.2 & 0.3 & 0.4 \\
\hline & & AW & 0.9 & 1.2 & 0.8 & 0.9 & 1.2 & 1.3 & 1.2 & 1.3 & 1.2 & 1.3 & 1.3 & 1.4 \\
\hline & \multirow{2}{*}{$\stackrel{F}{n}$} & R1 & 0.5 & 0.6 & 0.7 & 0.3 & 0.4 & 0.5 & 0.5 & 0.4 & 0.5 & 0.7 & 0.5 & 0.4 \\
\hline & & AW & 1.3 & 1.2 & 1.4 & 0.3 & 0.8 & 1.1 & 1.2 & 0.2 & 1.2 & 1.3 & 1.2 & 0.5 \\
\hline & \multirow{2}{*}{ 萦 } & $\mathrm{R} 1$ & 0.7 & 0.4 & 0.5 & 0.5 & 0.8 & 0.7 & 0.8 & 0.4 & 0.9 & 0.7 & 0.6 & 0.5 \\
\hline & & $\mathrm{AW}$ & 0.2 & 0.5 & 0.2 & 1.1 & 0.5 & 0.6 & 0.4 & 1.3 & 0.4 & 0.4 & 0.4 & 1.2 \\
\hline \multirow{2}{*}{\multicolumn{2}{|c|}{ Sum }} & $\mathbf{R}$ & 5.1 & 5.3 & 5.4 & 5.6 & 5.4 & 6.1 & 6.0 & 5.1 & 6.1 & 6.6 & 5.2 & 5.9 \\
\hline & & $\mathbf{A W}$ & 6.5 & 6.3 & 6.9 & 7.3 & 5.5 & 6.6 & 6.0 & 6.4 & 6.5 & 6.8 & 6.4 & 6.2 \\
\hline
\end{tabular}

$\mathrm{CD}=\mathrm{CDMC}, \mathrm{HM}=\mathrm{HMC}, \mathrm{DH}=\mathrm{DHMC}, \mathrm{MM}=\mathrm{MMKD}, \mathrm{R} 1=\mathrm{RCM} 1, \mathrm{AW}=\mathrm{AWAP}$ 
Table 15.2: Model comparison by the average of the absolute values of $\mathrm{Z}$ scores for streamflow volume for calibration period (1980-2009).

\begin{tabular}{|c|c|c|c|c|c|c|c|c|c|c|c|c|c|c|}
\hline & \multirow{3}{*}{ 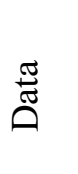 } & \multicolumn{12}{|c|}{ Averaged Z Scores } \\
\hline & & & \multicolumn{4}{|c|}{ Grahamstown } & \multicolumn{4}{|c|}{ Tillegra } & \multicolumn{4}{|c|}{ Chichester } \\
\hline & & & $\mathrm{CL}$ & $\mathrm{HM}$ & $\mathrm{DH}$ & MM & $\mathrm{CD}$ & $\mathrm{HM}$ & $\mathrm{DH}$ & MM & $\mathrm{CD}$ & HM & $\mathrm{DH}$ & $\mathrm{MM}$ \\
\hline \multirow{8}{*}{ 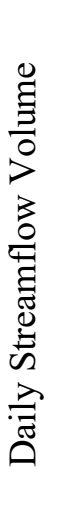 } & \multirow{2}{*}{ 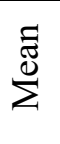 } & R1 & 0.4 & 0.3 & 0.4 & 0.5 & 0.3 & 0.3 & 0.3 & 0.3 & 0.4 & 0.4 & 0.3 & 0.3 \\
\hline & & AW & 0.2 & 0.2 & 0.2 & 0.5 & 0.3 & 0.2 & 0.2 & 0.3 & 0.4 & 0.3 & 0.4 & 0.3 \\
\hline & \multirow{2}{*}{ हे } & R1 & 0.6 & 0.5 & 0.6 & 0.6 & 1.0 & 0.9 & 1.1 & 0.4 & 1.2 & 1.1 & 1.0 & 0.5 \\
\hline & & AW & 0.4 & 0.4 & 0.3 & 0.5 & 0.5 & 0.5 & 0.5 & 0.4 & 0.6 & 0.5 & 0.6 & 0.4 \\
\hline & \multirow{2}{*}{ 点 } & R1 & 1.1 & 0.5 & 1.0 & 0.7 & 0.5 & 0.6 & 0.4 & 0.6 & 0.8 & 0.4 & 0.7 & 0.5 \\
\hline & & AW & 0.5 & 0.4 & 0.8 & 0.8 & 1.0 & 1.2 & 1.0 & 1.0 & 0.9 & 0.7 & 0.8 & 1.0 \\
\hline & \multirow{2}{*}{ 营 } & $\mathrm{R} 1$ & 0.6 & 0.4 & 0.6 & 0.3 & 0.6 & 0.5 & 0.6 & 0.3 & 0.6 & 0.6 & 0.6 & 0.4 \\
\hline & & AW & 0.4 & 0.2 & 0.4 & 0.4 & 0.2 & 0.3 & 0.3 & 0.4 & 0.3 & 0.2 & 0.2 & 0.3 \\
\hline \multirow{8}{*}{ 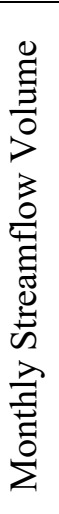 } & \multirow{2}{*}{$\sum_{\mathbb{\Xi}}^{\stackrel{\Xi}{\Sigma}}$} & R1 & 0.4 & 0.3 & 0.4 & 0.5 & 0.3 & 0.3 & 0.3 & 0.3 & 0.4 & 0.4 & 0.3 & 0.3 \\
\hline & & AW & 0.2 & 0.2 & 0.2 & 0.5 & 0.3 & 0.2 & 0.2 & 0.3 & 0.4 & 0.3 & 0.4 & 0.3 \\
\hline & \multirow{2}{*}{ हิ } & R1 & 0.4 & 0.5 & 0.4 & 0.5 & 0.5 & 0.5 & 0.5 & 0.6 & 0.4 & 0.4 & 0.4 & 0.6 \\
\hline & & AW & 0.4 & 0.5 & 0.4 & 0.5 & 0.4 & 0.4 & 0.3 & 0.4 & 0.4 & 0.4 & 0.4 & 0.4 \\
\hline & \multirow{2}{*}{$\stackrel{F}{n}$} & R1 & 0.5 & 0.4 & 0.4 & 0.7 & 0.7 & 0.9 & 0.6 & 0.5 & 0.6 & 0.6 & 0.6 & 0.7 \\
\hline & & AW & 0.7 & 0.8 & 0.6 & 0.9 & 0.9 & 1.1 & 0.9 & 1.0 & 0.9 & 0.7 & 0.8 & 1.1 \\
\hline & \multirow{2}{*}{$\stackrel{\tilde{n}}{\sigma}$} & R1 & 0.3 & 0.3 & 0.4 & 0.4 & 0.4 & 0.5 & 0.4 & 0.6 & 0.3 & 0.4 & 0.4 & 0.6 \\
\hline & & AW & 0.4 & 0.5 & 0.4 & 0.6 & 0.4 & 0.4 & 0.3 & 0.5 & 0.5 & 0.4 & 0.4 & 0.3 \\
\hline \multirow{8}{*}{ 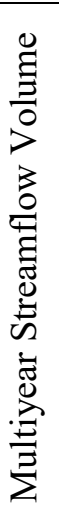 } & \multirow{2}{*}{ 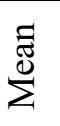 } & R1 & 0.5 & 0.2 & 0.5 & 0.8 & 0.6 & 0.5 & 0.8 & 0.5 & 0.9 & 0.9 & 0.7 & 0.4 \\
\hline & & AW & 0.1 & 0.3 & 0.2 & 1.0 & 0.3 & 0.0 & 0.4 & 0.6 & 0.6 & 0.3 & 0.5 & 0.6 \\
\hline & \multirow{2}{*}{ 命 } & R1 & 0.5 & 0.7 & 0.5 & 0.6 & 0.5 & 0.3 & 0.5 & 0.4 & 0.4 & 0.2 & 0.3 & 0.3 \\
\hline & & AW & 0.8 & 1.0 & 0.7 & 0.7 & 1.3 & 1.4 & 1.3 & 1.3 & 1.2 & 1.2 & 1.2 & 1.3 \\
\hline & \multirow{2}{*}{ 站 } & R1 & 0.7 & 0.7 & 0.9 & 0.3 & 0.4 & 0.5 & 0.5 & 0.5 & 0.7 & 0.9 & 0.7 & 0.4 \\
\hline & & AW & 1.0 & 0.8 & 1.1 & 0.5 & 1.3 & 1.2 & 1.6 & 0.3 & 1.3 & 1.2 & 1.2 & 0.3 \\
\hline & \multirow{2}{*}{ 芌 } & R1 & 0.2 & 0.4 & 0.2 & 0.8 & 0.8 & 0.6 & 0.9 & 0.4 & 0.9 & 0.7 & 0.5 & 0.4 \\
\hline & & AW & 0.4 & 0.8 & 0.3 & 0.9 & 0.5 & 0.9 & 0.5 & 1.1 & 0.4 & 0.5 & 0.4 & 1.1 \\
\hline \multirow{2}{*}{\multicolumn{2}{|c|}{ Sum }} & D1 & 6.2 & 5.2 & 6.3 & 6.7 & 6. & 6. & 6.9 & 5.4 & 7.6 & 7.0 & 6.5 & 5.4 \\
\hline & & $\mathbf{A W}$ & 5.9 & 6.1 & 5.6 & 7.8 & 7.4 & 7.8 & 7.5 & 7.6 & 7.9 & 6.7 & 7.3 & 7.4 \\
\hline
\end{tabular}

$\mathrm{CD}=\mathrm{CDMC}, \mathrm{HM}=\mathrm{HMC}, \mathrm{DH}=\mathrm{DHMC}, \mathrm{MM}=\mathrm{MMKD}, \mathrm{R} 1=\mathrm{RCM} 1, \mathrm{AW}=\mathrm{AWAP}$ 
Table 15.3: Model comparison by the average of the absolute values of $Z$ scores for rainfall depth for validation period (1950-1979).

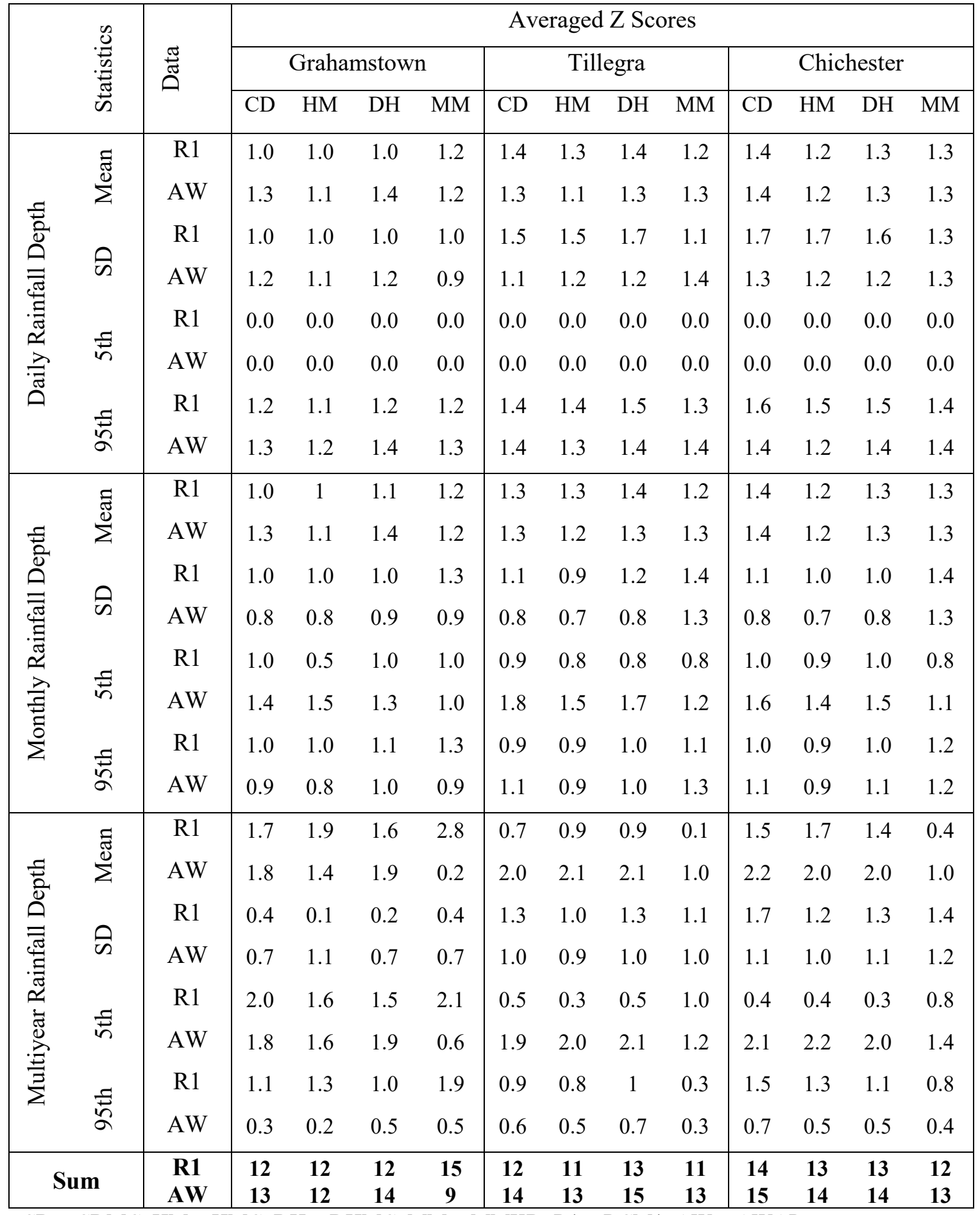

$\mathrm{CD}=\mathrm{CDMC}, \mathrm{HM}=\mathrm{HMC}, \mathrm{DH}=\mathrm{DHMC}, \mathrm{MM}=\mathrm{MMKD}, \mathrm{R} 1=\mathrm{RCM} 1, \mathrm{AW}=\mathrm{AWAP}$ 
Table 15.4: Model comparison by the average of the absolute values of $Z$ scores for streamflow volume for validation period (1950-1979).

\begin{tabular}{|c|c|c|c|c|c|c|c|c|c|c|c|c|c|c|}
\hline & & & & & & & & & $\overline{Z S}$ & & & & & \\
\hline &. & $\frac{\pi}{\pi}$ & & Srah & nsto & & & & egra & & & Chic & hester & \\
\hline & & & $\mathrm{CD}$ & $\mathrm{HM}$ & $\mathrm{DH}$ & $\mathrm{MM}$ & $\mathrm{CD}$ & $\mathrm{HM}$ & $\mathrm{DH}$ & $\mathrm{MM}$ & $\mathrm{CD}$ & HM & $\mathrm{DH}$ & $\mathrm{MM}$ \\
\hline & च & $\mathrm{R} 1$ & 1.1 & 1.1 & 1.1 & 1.3 & 1.4 & 1.4 & 1.5 & 1.3 & 1.5 & 1.4 & 1.4 & 1.3 \\
\hline$\stackrel{\Xi}{\Xi}$ & $\Sigma$ & AW & 1.0 & 0.9 & 1.1 & 1.0 & 1.4 & 1.2 & 1.3 & 1.4 & 1.5 & 1.3 & 1.3 & 1.2 \\
\hline$\overline{0}$ & & $\mathrm{R} 1$ & 0.9 & 0.8 & 0.9 & 1.1 & 1.8 & 1.7 & 2.0 & 1.2 & 2.2 & 2.1 & 2.1 & 1.3 \\
\hline 朵 & 会 & $\mathrm{AW}$ & 0.7 & 0.6 & 0.6 & 0.8 & 1.2 & 1.2 & 1.3 & 1.4 & 1.2 & 1.1 & 1.2 & 1.1 \\
\hline छ & $\sigma$ & $\mathrm{R} 1$ & 1.5 & 1.0 & 1.5 & 1.2 & 1.0 & 0.6 & 0.9 & 0.8 & 1.4 & 0.8 & 1.3 & 1.0 \\
\hline$\stackrel{ \pm}{\infty}$ & $\bar{n}$ & $\mathrm{AW}$ & 1.4 & 1.2 & 1.1 & 1.0 & 1.5 & 1.7 & 1.6 & 1.2 & 1.5 & 1.3 & 1.6 & 1.4 \\
\hline $\overrightarrow{\bar{\Xi}}$ & $=$ & $\mathrm{R} 1$ & 1.0 & 1.0 & 1.1 & 1.3 & 1.4 & 1.3 & 1.5 & 1.2 & 1.7 & 1.6 & 1.7 & 1.4 \\
\hline & $n$ & $\mathrm{AW}$ & 1.2 & 1.0 & 1.5 & 1.1 & 1.4 & 1.2 & 1.4 & 1.5 & 1.6 & 1.3 & 1.5 & 1.5 \\
\hline & $\approx$ & $\mathrm{R} 1$ & 1.1 & 1.1 & 1.1 & 1.3 & 1.4 & 1.4 & 1.5 & 1.3 & 1.5 & 1.4 & 1.4 & 1.3 \\
\hline$\Xi$ & $\Sigma^{e}$ & AW & 1.0 & 0.9 & 1.1 & 1.0 & 1.4 & 1.2 & 1.3 & 1.4 & 1.5 & 1.3 & 1.3 & 1.2 \\
\hline$>$ & 0 & $\mathrm{R} 1$ & 0.9 & 0.9 & 0.9 & 1.3 & 1.2 & 1.0 & 1.2 & 1.4 & 1.3 & 1.1 & 1.2 & 1.5 \\
\hline$\stackrel{0}{\square}$ & $\bar{n}$ & AW & 0.7 & 0.7 & 0.8 & 0.9 & 0.9 & 0.8 & 0.8 & 1.4 & 0.8 & 0.7 & 0.8 & 1.2 \\
\hline Ðే & $=$ & $\mathrm{R} 1$ & 0.9 & 0.6 & 0.8 & 1.1 & 0.8 & 0.8 & 0.8 & 0.6 & 1.0 & 0.8 & 1.0 & 0.7 \\
\hline $\begin{array}{l}\infty \\
\geq\end{array}$ & $\dot{n}$ & AW & 1.8 & 2.0 & 1.7 & 1.3 & 1.7 & 2.0 & 1.8 & 1.3 & 1.9 & 1.9 & 1.9 & 1.6 \\
\hline 营 & $\mp$ & $\mathrm{R} 1$ & 1.1 & 1.0 & 1.2 & 1.3 & 1.1 & 1.0 & 1.2 & 1.2 & 1.1 & 1.1 & 1.2 & 1.3 \\
\hline & $n$ & AW & 0.9 & 0.8 & 1.0 & 1.0 & 1.0 & 0.9 & 1.0 & 1.3 & 1.2 & 1.1 & 1.1 & 1.3 \\
\hline & $\approx$ & $\mathrm{R} 1$ & 1.6 & 2.1 & 1.6 & 2.8 & 1.1 & 1.0 & 1.2 & 0.0 & 1.8 & 1.9 & 1.7 & 0.5 \\
\hline$\Xi$ & $\sum^{0}$ & AW & 1.0 & 0.5 & 1.2 & 0.0 & 2.3 & 2.1 & 2.4 & 1.8 & 2.5 & 2.0 & 2.2 & 1.3 \\
\hline $\begin{array}{l}3 \\
3\end{array}$ & 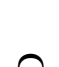 & $\mathrm{R} 1$ & 0.2 & 0.5 & 0.4 & 0.5 & 1.2 & 0.9 & 1.2 & 1.1 & 1.6 & 1.1 & 1.2 & 1.4 \\
\hline 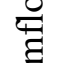 & $\bar{n}$ & AW & 0.6 & 0.8 & 0.5 & 0.5 & 0.4 & 0.5 & 0.4 & 0.5 & 0.8 & 0.7 & 0.8 & 0.9 \\
\hline 总 & $\cong$ & R1 & 1.9 & 1.8 & 1.7 & 2.5 & 0.3 & 0.3 & 0.3 & 1.0 & 0.3 & 0.4 & 0.3 & 0.8 \\
\hline 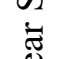 & \pm & AW & 1.8 & 1.4 & 1.9 & 0.6 & 2.1 & 2.0 & 2.3 & 1.0 & 2.3 & 2.2 & 2.2 & 1.4 \\
\hline 害 & $\mp$ & R1 & 1.1 & 1.5 & 1.0 & 1.7 & 1.0 & 0.8 & 1.1 & 0.3 & 1.6 & 1.3 & 1.2 & 0.8 \\
\hline & $n$ & AW & 0.2 & 0.2 & 0.4 & 0.2 & 0.9 & 0.6 & 1.0 & 0.8 & 0.9 & 0.6 & 0.7 & 0.3 \\
\hline & & $\mathbf{R}$ & 13 & 13 & 13 & 17 & 14 & 12 & 14 & 11 & 17 & 15 & 16 & 13 \\
\hline & & $\mathbf{A W}$ & 12 & 11 & 13 & 9 & 16 & 15 & 17 & 15 & 18 & 16 & 17 & 14 \\
\hline
\end{tabular}

$\mathrm{CD}=\mathrm{CDMC}, \mathrm{HM}=\mathrm{HMC}, \mathrm{DH}=\mathrm{DHMC}, \mathrm{MM}=\mathrm{MMKD}, \mathrm{R} 1=\mathrm{RCM} 1, \mathrm{AW}=\mathrm{AWAP}$ 


\subsection{Model Comparison for Autocorrelation}

Figure 15.15 compares the models for autocorrelations of monthly rainfall and streamflow of RCM1 at Grahamstown, while Figure 15.16 shows comparisons at Tillegra. The autocorrelations at Chichester are similar to Tillegra and are shown in Appendix I.4.

For Grahamstown, the autocorrelations of monthly rainfall depths are weaker than the autocorrelations of monthly streamflow volumes, while for Tillegra and Chichester, the autocorrelations are stronger in rainfall depths. All four models show satisfactory performances to reproduce the autocorrelations.
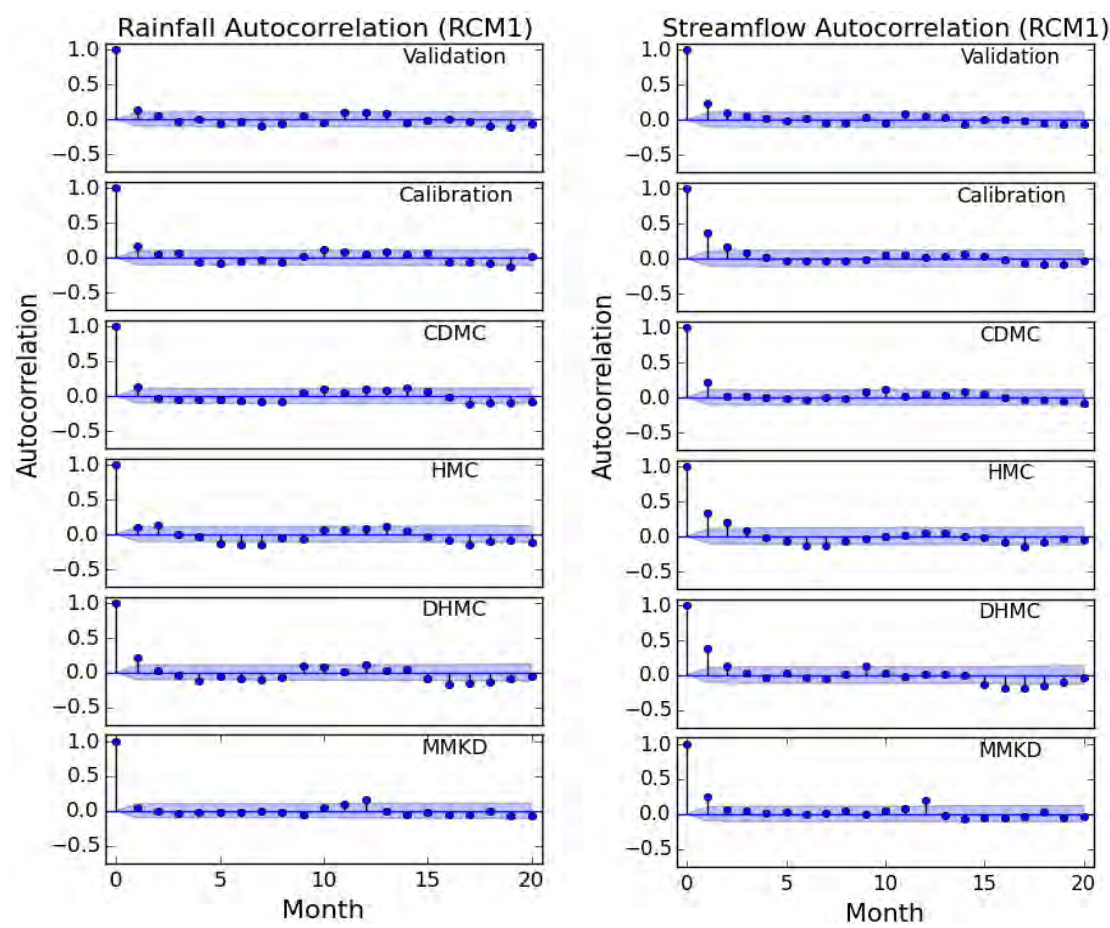

Figure 15.15: Model Comparison for the autocorrelations of monthly rainfall (left panel) and streamflow (right panel) for RCM1 at Grahamstown. 

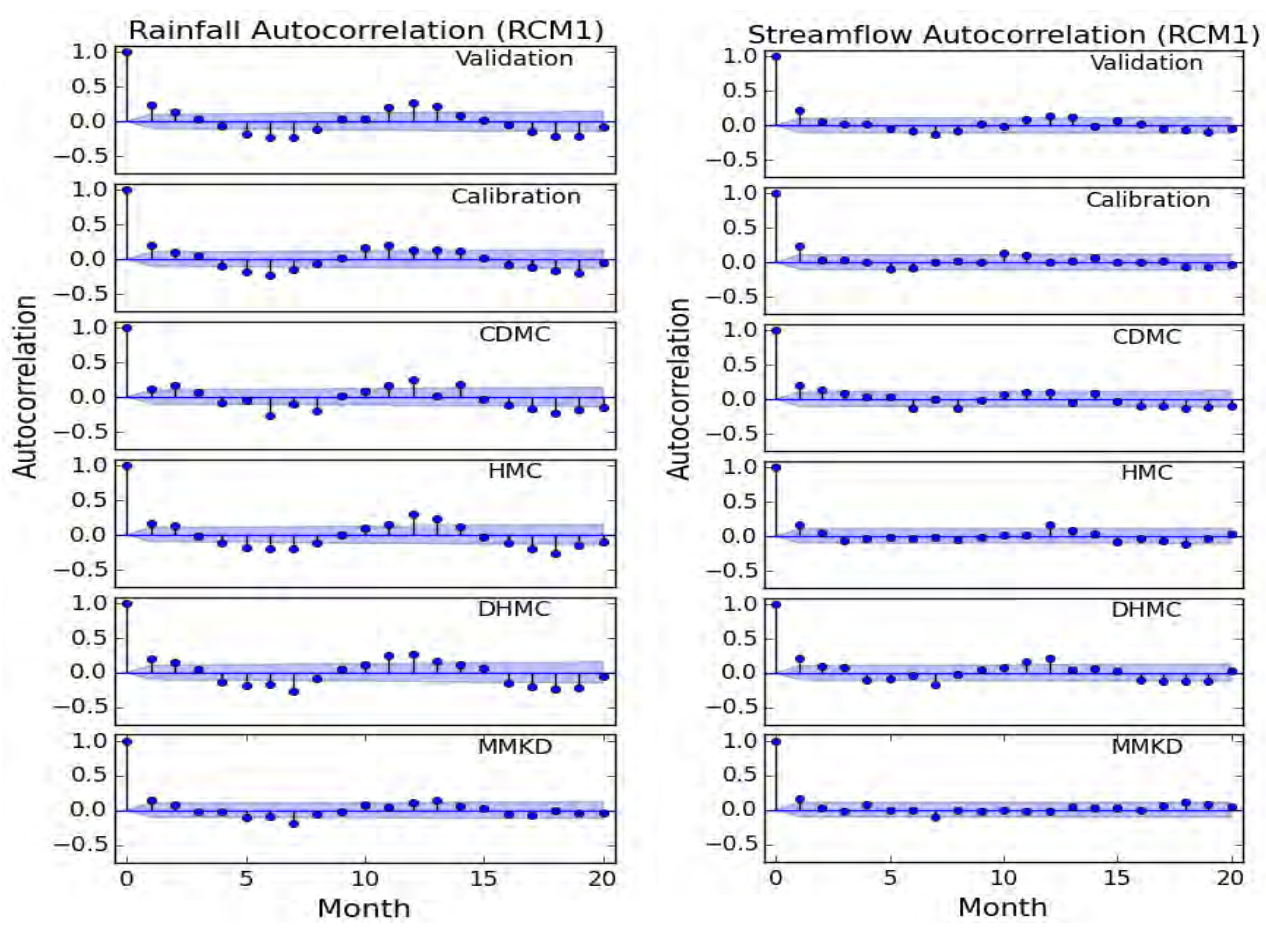

Figure 15.16: Model Comparison for the autocorrelations of monthly rainfall (left panel) and streamflow (right panel) for RCM1 at Tillegra.

\subsection{Discussion and Conclusion}

This chapter has compared the CDMC, HMC, DHMC and MMKD for rainfall and streamflow generation at Grahamstown, Tillegra, and Chichester sub-catchments of the Williams River site. The models are calibrated to RCM1 and AWAP for the 1980-2009 period and validated against the same datasets as for the 1950-1979 period.

Among the three sub-catchments, Grahamstown is located in the lower elevation of the Williams River site, while Tillegra and Chichester are two adjacent and geographically similar sub-catchments located in the higher elevation. This might explain why the rainfall and streamflow characteristics (e.g. distribution and autocorrelations, seasonal variability) of Tillegra and Chichester are similar, but different from the Grahamstown. Overall, the Tillegra and Chichester sub-catchments are wetter (higher mean and SD) than the Grahamstown subcatchment. At daily resolution, the streamflow and rainfall variabilities are similar because the Grahamstown, Tillegra and Chichester with areas of 120, 196 and $199 \mathrm{~km}^{2}$ (see Table 3.3) have time of concentrations significantly less than a day of 4.7, 5.6 and 5.7 hours (time of concentrations of east Australian catchments are given by 0.76 Area $^{0.38}$ ) respectively. Therefore, the storage effects at daily resolutions are small for these catchments, and in turn, the streamflow and rainfall variabilities become similar. 
In each of the three sub-catchments, and for both RCM1 and AWAP, the calibration period (1980-2009) is drier (lower mean, SD, and percentiles) than the validation period (1950-1979), except for the RCM1 for Grahamstown where the calibration period is wetter than the validation period. The calibration period is likely to be drier than the validation period as it comprises several El-Niño conditions and the associated 'millennium drought' period (20012009) which affected the eastern seaboard of Australia, including the lower Hunter [VerdonKidd and Kiem, 2009]. However, the wetter calibration period of RCM1 for Grahamstown might be because of the anomaly of NARCliM data discussed by Parana Manage [2016]. At each sub-catchment, the streamflow characteristics of calibration and validation periods are consistent with the respective rainfall characteristics. The seasonal variability of rainfall and streamflow at monthly resolution are consistent with the seasonal variability at daily resolution. Moreover, at each sub-catchment, the RCM1 is wetter than the AWAP.

At each of the three sub-catchments, the performance of the four models are satisfactory for the distribution and autocorrelations of rainfall and streamflow (consist with the findings presented in Chapters 12-14 for rainfall generation). The performances of each model for streamflow volumes are mostly consistent with their respective performances for rainfall depths. This is probably because the variability of daily streamflow generated by SimHyd is mostly consistent with the variability of input daily rainfall.

One of the most important findings is that all four models have shown similar satisfactory performances to reproduce the SD of multiyear rainfall depths and streamflow volumes. While the performances of the models are similar, and the overall advantages or disadvantages of one model over other models are mostly negligible, the key differences among the model performances are:

- For calibration, the MC models tend to underestimate and the MMKD tends to overestimate the mean of rainfall depths and streamflow volumes (consistent with findings presented in Chapters 12 and 13).

- For validation, the MC models perform better than the MMKD if the calibration period is wetter than the validation period (e.g. RCM1 at Grahamstown). In contrast, the MMKD performs better than the MC models for validation if the calibration period is drier than the validation period (e.g. AWAP at Grahamstown).

The three MC models use different MC parameters and perform differently for the variability of the wet period statistics (see Chapter 5). However, the performances of the MC models for 
15. Comparison of MC and MMKD for Streamflow Generation

the variability of rainfall depths are very similar (as they use the same Gamma parameters), accordingly, their performances for variability of streamflow volumes are also similar. This indicates that the streamflow generation using SimHyd hydrology model might be more sensitive to the rainfall depth simulation than the wet period simulation of the rainfall model/s. 


\section{Conclusion}

\subsection{Overview}

As part of the ESCCI-ECL project, the aim of this thesis was to develop and evaluate stochastic rainfall models that can be calibrated to available climate data for the eastern seaboard of Australia (e.g. NARCliM, AWAP and raingauge) and, in turn, the stochastic models can be used in stochastic streamflow generation to evaluate the influence of ECL on urban drought security. This chapter provides a conclusion as to how the thesis has achieved its aim, and identifies the major findings of the thesis.

\subsection{Selection of Modelling Structure and Relevant Issues}

In Chapter 2, the review of existing literature showed that the stochastic rainfall model should capture the rainfall distribution and autocorrelations at both short (daily) and long (monthly to multiyear) resolutions to incorporate the influence of ECLs in the assessment of urban drought security of reservoirs, because the ECLs often occur for a few days or a week and substantially contribute to reservoir water levels, while reservoir water levels generally only vary significantly at monthly to multiyear resolutions. Therefore, this study has developed and evaluated stochastic models for simulation of daily rainfall data, and assessed the ability of the models to reproduce the rainfall distribution and autocorrelations at daily, monthly and multiyear resolutions. Among the different model structures, the two-part MC models are most widely used and suitable for satisfactory reproduction of the variability and autocorrelations of rainfall and wet-dry periods.

One of the major issues of daily stochastic rainfall models is that the daily models often tend to underestimate the rainfall variability at monthly to multiyear resolutions. The available models that attempted to resolve the underestimation of long-term variability have advantages as well as limitations. Therefore, this study has developed MC models for daily rainfall simulation focusing on their ability to preserve the rainfall characteristics at daily to multiyear resolutions. 


\subsection{Development of Markov Chain Daily Rainfall Models}

Chapter 4 showed the methodology and justification of relevant assumptions of five MC models (APMC, DPMC, CDMC, HMC and DHMC) that have been developed and examined by this study. These five models use the first-order MC process with two parameters of dry-todry $\left(P_{00}\right)$ and wet-to-wet $\left(P_{11}\right)$ day transition probabilities for simulation of wet and dry days, and Gamma distribution with two parameters of mean $(\mu)$ and SD $(\sigma)$ of wet day rainfall depths for simulation of wet day rainfall depths. To incorporate intra-annual seasonal variability, each model uses a separate set of parameters for each month. However, each model uses different parameterisation of $\mathrm{MC}$ and Gamma distribution to incorporate the distribution and autocorrelation of rainfall depths and wet periods at daily to multiyear resolutions.

\subsection{Comparison of Five MC Models for Rainfall Generation}

Chapter 5 showed the preliminary assessment of the five MC models to reproduce the distribution and autocorrelation of rainfall depths and wet periods of NARCliM RCM2 and AWAP for a single location at Gummun. The statistics of each model have been compared with statistics of RCM2 and AWAP for the same period to which the parameters of each model were calibrated. The key findings are as follows:

- The first model, APMC, with deterministic parameters of MC and Gamma distribution preserves the mean of rainfall depths and wet periods statistics (e.g. number of wet days and mean length of wet spells) at daily, monthly and multiyear resolutions. The model can also preserve the variability of daily rainfall depths (as the parameters of the model are calibrated to daily data) and autocorrelations of monthly rainfall depths and wet days. However, the APMC significantly underestimates the monthly and multiyear variability of rainfall depths and multiyear variability of wet periods. This suggests that the deterministic parameters of MC and Gamma distribution can incorporate the shortterm variability, but cannot incorporate the long-term variability of rainfall depths and wet periods. This conclusion is consistent with the finding of several previous studies, including Wang and Nathan [2007].

- The second model, DPMC, with decadally varied MC and Gamma parameters performs similarly to the APMC in reproducing the rainfall depths, and hence, preserves the mean but underestimates the variability of rainfall depths at monthly to multiyear resolutions. However, the DPMC performs better than the APMC in reproducing the variability of wet periods at monthly to multiyear resolutions. This 
suggests that the decadally varied deterministic Gamma parameters are not useful for long-term variability of rainfall depths, but the decadally varied MC parameters are useful for long-term variability of wet periods. Other models similar to DPMC that were examined (e.g. models with parameters varying for each 5-year period and models with parameters varying for phases of IPO or ENSO), also showed similar performances as DPMC and systematically underestimate the variability of rainfall depths at monthly to multiyear resolutions. The conclusion was that the use of deterministic Gamma parameters in DPMC-like models is not adequate to satisfactorily reproduce the long-term variability of rainfall depths.

- The third model, CDMC, with deterministic parameters of MC (i.e. the same as APMC) and stochastic parameters of Gamma distribution preserves the variability of rainfall depths at daily to multiyear resolutions. However, the CDMC significantly underestimates the multiyear variability of number of wet days, which is consistent with APMC as CDMC uses the same MC parameters as APMC.

- The fourth model, HMC, with stochastic parameters of both MC and Gamma distribution performs in a similar way to the CDMC in reproducing the rainfall depths, and hence, preserves the rainfall depths variability at daily to multiyear resolutions. The HMC also introduces more variability in the wet period simulation and preserves the multiyear variability of number of wet days, but significantly overestimates the wet period variability at monthly resolution and tends to underestimate the month-to-month autocorrelations of monthly rainfall depths and monthly wet days. The consistent tendency to overestimate the variability of wet periods and underestimating autocorrelations indicates that the variability introduced by the stochastic MC parameters in the HMC is excessive. This study further examined two other variants of the HMC by using a monthly lag-1 autocorrelation equation in the stochastic parameterisation of the MC process and using a multivariate sampling of MC-Gamma parameters respectively. However, these alternative HMC also performed similarly to the standard HMC and systematically overestimated the variability of wet periods. The conclusion was that the stochastic parameters of the MC process are not useful for satisfactory reproduction of wet period variability at monthly to multiyear resolutions, although the stochastic parameters of Gamma distribution are useful to incorporate the variability of rainfall depths at daily to multiyear resolutions.

- The fifth and final model, DHMC, uses decadally varied MC parameters (i.e. the same as DPMC) with stochastic parameters of Gamma distribution (i.e. the same as CDMC). The DHMC satisfactorily preserves almost all of the key statistics of rainfall depths and 
wet periods, including the rainfall depths variability at daily to multiyear resolutions (similar to CDMC and $\mathrm{HMC}$ ), wet periods variability at monthly to multiyear resolutions (better than CDMC and HMC), and month-to-month autocorrelations of monthly rainfall depths and monthly wet days (similar to CDMC and better than HMC). However, the DHMC tends to underestimate the variability of wet days at shorter multiyear resolutions (i.e. for 1 to 3 multiple years), although it satisfactorily reproduces the variability of wet days at longer multiyear resolutions as well as the variability of annual mean length of wet spells.

Since the preliminary assessment presented in Chapter 5 using data for the Gummun climate station shows that the APMC and DPMC cannot incorporate the long-term variability of rainfall depths, this study rejected these two models. Further assessments of the CDMC, HMC and DHMC were undertaken (using gridded data of NARCliM RCMs and AWAP) in five case study catchments (Goulburn River, Williams River, Sydney, Richmond River and Bega River sites) along the east coast of NSW, and the results were presented in Chapters 6-10.

These five case study sites were selected based on their ECL influence and importance for urban water security. The analysis of rainfall using NARCliM reanalysis with three RCMs and AWAP gridded datasets in five case study sites suggests that the rainfall in each site has similar intra-annual variability, but the spatial variability of rainfall in each site is influenced by the local factors (e.g. orography). The comparative assessments of the CDMC, HMC and DHMC show that each model performs consistently between sites irrespective of the intra-annual and spatial variability. As for Gummun, the overall performance of the DHMC is satisfactory and better than the other two models (CDMC, HMC) in reproducing the distribution and autocorrelations of rainfall depths and wet periods of NARCliM RCMs and AWAP (to which the model parameters were calibrated) for each of the five case study sites. This suggests consistent satisfactory performance of the DHMC for catchments with different degrees of ECL influence and importance for urban water security.

\subsection{Comparison of MC and MMKD for Rainfall Generation}

In Chapter 12 and 13, the CDMC, HMC and DHMC models developed in this study were compared with the MMKD of Mehrotra and Sharma [2007], the latter which was developed to address the underestimation of long-term variability. Raingauge data for 12 stations around 
Australia (covering coastal, inland and monsoonal climate zones) and 30 stations around Sydney (those used by Mehrotra et al. [2015]) were used for this comparison.

The MC models are methodologically simple and straightforward in comparison to the relative complexity of the MMKD. Despite the methodological simplicity, the DHMC shows comparable satisfactory performance as the MMKD to reproduce the distribution and autocorrelations of rainfall depths and wet periods at daily to multiyear resolutions. The other two MC models, CDMC and HMC, also show comparable performance to reproduce the distribution of rainfall depths at all resolutions, but fail to preserve the distribution of wet periods at all resolutions. However, the MC models tend to underestimate the mean of rainfall depths particularly at multiyear resolutions, while the MMKD tends to overestimate the mean of rainfall depths at all resolutions. Further investigation shows that the stochastic Gamma parameters in MC models and kernel-density technique for resampling rainfall depths in MMKD are responsible for the under- and over-estimation (respectively) of mean rainfall depths.

This study compared the performance of the CDMC, HMC, DHMC and MMKD in reproducing the rainfall depths and wet period statistics for validation periods independent of model calibration. While the performance of each model for validation periods was only slightly worse than for the calibration period, the MC models showed slightly better relative performance than the MMKD for validation periods. These results were presented in Chapter 14.

\subsection{Comparison of MC and MMKD for Streamflow Generation}

This study compared the performances of the CDMC, HMC, DHMC and MMKD in reproducing the distribution and autocorrelation of rainfall depths and streamflow volumes at daily, monthly and multiyear resolutions for calibration and validation periods of RCM1 and AWAP at the Grahamstown, Tillegra and Chichester sub-catchments of the Williams River site. The SimHyd hydrology model was used for streamflow generation. These results were presented in Chapter 15.

The performances of each model for distribution of streamflow volumes are mostly consistent with their respective performances for distribution of rainfall depths. While the performances of 
the models are similar, and the overall advantages or disadvantages of one model over other models are mostly negligible, the key differences among the model performances are:

- For calibration, the MC models tend to underestimate and the MMKD tends to overestimate the mean of rainfall depths and streamflow volumes (consistent with previous findings in Chapters 12 and 13 for rainfall generation).

- For validation, the MC models perform better than the MMKD for the mean and SD of rainfall and streamflow if the calibration period is wetter than the validation period. In contrast, the MMKD performs better than the MC models for validation if the calibration period is drier than the validation period.

The performances of the CDMC, HMC and DHMC in reproducing the variability of rainfall depths are similar and, accordingly, their performances for variability of streamflow volumes are also similar, although models performed differently when reproducing the variability of the wet period statistics. This indicates that the streamflow generation using SimHyd hydrology model might be more sensitive to the rainfall depth simulation than the wet period simulation of the rainfall models.

\subsection{Future Works}

Among the five MC models of this study, the overall performance of the DHMC is better than the other four MC models and comparable to the existing MMKD. While the DHMC has great potential to be used in hydrological and agricultural impact studies (e.g. urban drought security assessment), there are two important places where the performance of the DHMC can be improved:

- The DHMC tends to underestimate the mean of multiyear rainfall depths, which is probably linked to the use of stochastic Gamma parameters. Therefore, the stochastic sampling of Gamma parameters might be improved to overcome this limitation.

- The performance of the DHMC suggests that the use of decadally varied MC parameters are effective to incorporate the long-term variability of wet periods, although the use of decadally varied Gamma parameters are not effective in incorporating the long-term variability of rainfall depths. However, the DHMC has shown a tendency to underestimate the wet period variability at shorter multiyear resolutions (e.g. 1 to 3 years). This limitation might be overcome by using other subsamples (e.g. according to the ENSO phases) instead of decadal samples for parameter 
calibration. This study tested the sub-samples according to the phases of IPO and ENSO climate modes with a focus on incorporating the long-term variability of rainfall depths but did not use in DHMC because DHMC had not been developed at the time this analysis was performed. A more comprehensive assessment of such ideas might improve the wet period simulation of the DHMC.

The future application of the DHMC for hydrology analysis requires a multisite version of the model. Although this study has used the single-site DHMC for small-sized catchments of around $200 \mathrm{~km}^{2}$ area, the single-site model is unlikely to incorporate the spatial variability of rainfall for larger catchments. A multisite DHMC might be derived by using spatially correlated parameters according to the structure of the multisite model by Wilks [1999b]. A similar model structure was also adopted by other studies, including Srikanthan and Pegram [2009] and Mehrotra et al. [2015]. The multisite DHMC will also enable the assessment of hydrology and urban drought security of larger and important water systems, such as Sydney Catchment.

In line with an objective of this study, the DHMC can be used for the analysis of ECL and nonECL rainfall in the east coast of Australia to evaluate the influence of ECL in the urban water security. This will depend upon having a rainfall record to calibrate to that distinguishes between ECL rainfall and non-ECL rainfall. Such a record is in the process of development in parallel projects in ESCCI-ECL but was not completed in time to be used in this thesis. The structure of the MC models suits the parallel simulations of ECL and non-ECL rainfall and subsequently constructing a composite rainfall record by simply adding those two records together.

\subsection{Concluding Remarks}

This study has developed five stochastic daily rainfall models focusing on their ability to preserve the distribution and autocorrelation of rainfall depths and wet periods at daily to multiyear resolutions. Among the five models, the DHMC showed consistent satisfactory performance to reproduce the distribution and autocorrelation statistics of rainfall depths, wet periods and streamflow volumes. Compared to an existing model, MMKD, the DHMC has shown comparable satisfactory performance, although the methodology of DHMC is simpler and more straightforward than the MMKD. Therefore, the DHMC developed in this study has the potential to be used by future studies for evaluation of urban drought security. 


\section{References}

Ailliot, P., D. Allard, V. Monbet, and P. Naveau (2015), Stochastic Weather Generators: An Overview of Weather Type Models, Journal of the French Statistical Society, 156(1), 101-113.

Alfieri, L., F. Pappenberger, F. Wetterhall, T. Haiden, D. Richardson, and P. Salamon (2014), Evaluation of Ensemble Streamflow Predictions in Europe, Journal of Hydrology, 517, 913-922, doi:10.1016/j.jhydrol.2014.06.035.

Allan, R. J., J. Lindesay, and D. E. Parker (1996), El Niño, Southern Oscillation, and Climatic Variability, CSIRO Publishing, Collingwood, Victoria, Australia.

Andréassian, V., C. Perrin, and C. Michel (2004), Impact of Imperfect Potential Evapotranspiration Knowledge on the Efficiency and Parameters of Watershed Models, Journal of Hydrology, 286 (1-4), 19-35, doi:10.1016/j.jhydrol.2003.09.030.

Baigorria, G. A., and J. W. Jones (2010), Gist: A Stochastic Model for Generating Spatially and Temporally Correlated Daily Rainfall Data, Journal of Climate, 23(22), 5990-6008, doi:10.1175/2010JCLI3537.1.

Bardossy, A., and E. J. Plate (1992), Space-Time Model for Daily Rainfall Using Atmospheric Circulation Patterns, Water Resources Research, 28(5), 1247-1259, doi:10.1029/91wr02589.

Beecham, S., M. Rashid, and R. K. Chowdhury (2014), Statistical Downscaling of Multi-Site Daily Rainfall in a South Australian Catchment Using a Generalized Linear Model, International Journal of Climatology, 34(14), 3654-3670, doi:10.1002/joc.3933.

Bellone, E., J. P. Hughes, and P. Guttorp (2000), A Hidden Markov Model for Downscaling Synoptic Atmospheric Patterns to Precipitation Amounts, Climate Research, 15(1), 1-12, doi:10.3354/cr015001.

BoM (2013), Daily Rainfall Data, http://www.bom.gov.au/Climate/Data, Bureau of Meteorology, Australia.

Boughton, W. C. (1999), A Daily Rainfall Generating Model for Water Yield and Flood Studies, 21 pp, CRC for Catchment Hydrology, Monash University, Melbourne.

Box, G. E. P., and G. M. Jenkins (1970), Time Series Analysis, Forecasting and Control 1st ed., 537 pp., Holden-Day, San Francisco, CA.

Buchtele, J., M. Buchtelová, M. Fortová, and M. Dubrovský (1999), Runoff Changes in Czech River Basins - the Outputs of Rainfall-Runoff Simulations Using Different Climate Change Scenarios, J. Hydrol. Hydromech, 47, 180-194.

Burton, A., C. G. Kilsby, H. J. Fowler, P. S. P. Cowpertwait, and P. E. O'Connell (2008), Rainsim: A Spatial-Temporal Stochastic Rainfall Modelling System, Environmental Modelling \& Software, 23(12), 1356-1369, doi:10.1016/j.envsoft.2008.04.003.

Carpenter, G. (2007), Australian East Coast Storm 2007: Impact of East Coast Lows, 40 pp.

Chang, T. J., M. L. Kavvas, and J. W. Delleur (1984), Modelling of Sequences of Wet and Dry Days by Binary Discrete Autoregressive Moving Average Processes, Journal of Climate and Applied Meteorology, 23(9), 1367-1378, doi:10.1175/1520-0450(1984)023<1367.

Chapman, T. (1998), Stochastic Modelling of Daily Rainfall: The Impact of Adjoining Wet Days on the Distribution of Rainfall Amounts, Environmental Modelling and Software, 13(3-4), 317-324, doi:10.1016/s1364-8152(98)00036-X.

Chen, J., and F. P. Brissette (2014), Comparison of Five Stochastic Weather Generators in Simulating Daily Precipitation and Temperature for the Loess Plateau of China, International Journal of Climatology, 34(10), 3089-3105, doi:10.1002/joc.3896.

Chen, J., F. P. Brissette, and R. Leconte (2010), A Daily Stochastic Weather Generator for Preserving Low-Frequency of Climate Variability, Journal of Hydrology, 388(3-4), 480-490, doi:10.1016/j.jhydrol.2010.05.032.

Chen, M., G. Willgoose, and P. M. Saco (2015), Evaluation of the Hydrology of the Ibis Land Surface Model in a Semi-Arid Catchment, Hydrological Processes, 29(5), 653-670, doi:10.1002/hyp.10156.

Chiew, F., and M. J. Leahy (2003), Inter-Decadal Pacific Oscillation Modulation of the Impact of El Niño/Southern Oscillation on Australian Rainfall and Streamflow, paper presented at MODSIM03, Modelling and Simulation Society of Australia and New Zealand, Volume (01).

Chiew, F., M.C. Peel, and A. Western (2002), Application and Testing of the Simple Rainfall-Runoff Model Simhyd, Water Resources Publications, LLC. 
Chiew, F., and L. Siriwardena (2005), Estimation of Simhyd Parameter Values for Application in Ungauged Catchments, in MODSIM 2005 International Congress on Modelling and Simulation, edited, pp. 2883-2889, Modelling and Simulation Society of Australia and New Zealand, Melbourne.

Chun, K. P., H. S. Wheater, and C. Onof (2013), Comparison of Drought Projections Using Two Uk Weather Generators, Hydrological Sciences Journal, 58(2), 295-309, doi:10.1080/02626667.2012.754544.

Cowpertwait, P. S. P., P. E. O'Connell, A. V. Metcalfe, and J. A. Mawdsley (1996), Stochastic Point Process Modelling of Rainfall. I. Single-Site Fitting and Validation, Journal of Hydrology, 175(1), 17-46, doi:10.1016/S0022-1694(96)80004-7.

Cox, D. R., and H. D. Miller (1977), The Theory of Stochastic Processes, Taylor \& Francis.

Cui, L., and G. Kuczera (2005), Optimizing Water Supply Headworks Operating Rules under Stochastic Inputs: Assessment of Genetic Algorithm Performance, Water Resources Research, 41(5), W05016, doi:10.1029/2004wr003517.

Dastorani, M., M. Mirzavand, M. T. Dastorani, and S. J. Sadatinejad (2016), Comparative Study among Different Time Series Models Applied to Monthly Rainfall Forecasting in Semi-Arid Climate Condition, Natural Hazards, 81(3), 1811-1827, doi:10.1007/s11069-016-2163-x.

DECCW (2010), Nsw Climate Impact Profile, 168 pp, Department of Environment, Climate Change and Water, NSW, Australia.

Diment, G. A. (1991), Wide Use of a Generalised Headworks and Resources Model: Realm, in Proceedings of International Hydrology and Water Resources Symposium, edited, pp. 579-583, Perth, Australia, Institution of Engineers, Australia, National Conference Publication 91(22).

Dubrovský, M., J. Buchtele, and Z. Žalud (2004), High-Frequency and Low-Frequency Variability in Stochastic Daily Weather Generator and Its Effect on Agricultural and Hydrologic Modelling, Climatic Change, 63(1), 145-179, doi:10.1023/b:clim.0000018504.99914.60.

Duc, H. N., K. Rivett, K. MacSween, and L. Le-Anh (2015), Association of Climate Drivers with Rainfall in New South Wales, Australia, Using Bayesian Model Averaging, Theoretical and Applied Climatology, 1-17, doi:10.1007/s00704-015-1622-8.

Dunn, P. K. (2004), Occurrence and Quantity of Precipitation can be Modelled Simultaneously, International Journal of Climatology, 24(10), 1231-1239, doi:10.1002/joc.1063.

Evans, J. P., L. Fita, D. Argüeso, and Y. Liu (2013), Initial NARCliM Evaluation, in 20th International Congress on Modelling and Simulation edited, Adelaide, Australia.

Evans, J. P., F. Ji, C. Lee, P. Smith, D. Argüeso, and L. Fita (2014), Design of a Regional Climate Modelling Projection Ensemble Experiment - NARCliM, Geoscientific Model Development, 7(2), 621-629, doi:10.5194/gmd-7-621-2014.

Evans, J. P., and M. F. McCabe (2010), Regional Climate Simulation over Australia's Murray-Darling Basin: A Multitemporal Assessment, Journal of Geophysical Research: Atmospheres, 115(D14), D14114, doi:10.1029/2010jd013816.

Fatichi, S., V. Y. Ivanov, and E. Caporali (2011), Simulation of Future Climate Scenarios with a Weather Generator, Advances in Water Resources, 34(4), 448-467, doi:10.1016/j.advwatres.2010.12.013.

Fisher, R. A., and L. H. C. Tippett (1928), Limiting Forms of the Frequency Distribution of the Largest or Smallest Member of a Sample, Mathematical Proceedings of the Cambridge Philosophical Society, 24(02), 180-190, doi:10.1017/S0305004100015681.

Folland, C. (2008), Interdecadal Pacific Oscillation Time Series, Met Office Hadley Centre for Climate Change, Exeter, UK.

Fowler, H. J., S. Blenkinsop, and C. Tebaldi (2007), Linking Climate Change Modelling to Impacts Studies: Recent Advances in Downscaling Techniques for Hydrological Modelling, International Journal of Climatology, 27(12), 1547-1578, doi:10.1002/joc.1556.

Frost, A. J., R. Srikanthan, M. A. Thyer, and G. Kuczera (2007), A General Bayesian Framework for Calibrating and Evaluating Stochastic Models of Annual Multi-Site Hydrological Data, Journal of Hydrology, 340(3-4), 129-148, doi:10.1016/j.jhydrol.2007.03.023.

Furrer, E. M., and R. W. Katz (2007), Generalized Linear Modeling Approach to Stochastic Weather Generators, Climate Research, 34(2), 129.

Furrer, E. M., and R. W. Katz (2008), Improving the Simulation of Extreme Precipitation Events by Stochastic Weather Generators, Water Resources Research, 44(12), W12439, doi:10.1029/2008wr007316.

Gabriel, K. R., and J. Neumann (1962), A Markov Chain Model for Daily Rainfall Occurrence at Tel Aviv, Quarterly Journal of the Royal Meteorological Society, 88(375), 90-95, doi:10.1002/qj.49708837511. 
Gergis, J., and L. Ashcroft (2013), Rainfall Variations in South-Eastern Australia Part 2: A Comparison of Documentary, Early Instrumental and Palaeoclimate Records, 1788-2008, International Journal of Climatology, 33(14), 2973-2987, doi:10.1002/joc.3639.

Gil-Alana, L. A. (2009), Modelling Australian Annual Mean Rainfall Data: A New Approach Based on Fractional Integration, Australian Meteorological and Oceanographic Journal, 58(2), 119-128.

Glenis, V., V. Pinamonti, J. W. Hall, and C. G. Kilsby (2015), A Transient Stochastic Weather Generator Incorporating Climate Model Uncertainty, Advances in Water Resources, 85, 14-26, doi:10.1016/j.advwatres.2015.08.002.

Green, J. R. (1964), Two Probability Models for Sequences of Wet or Dry Days, Monthly Weather Review, 93(3), 155-156, doi:10.1175/1520-0493(1965)093<0155.

Hanaish, I. S., K. Ibrahim, and A. A. Jemain (2013), On the Potential of Bartlett Lewis Rectangular Pulse Models for Simulating Rainfall in Peninsular Malaysia, Hydrological Sciences Journal, 58(8), 1690-1703, doi:10.1080/02626667.2013.838322.

Hansen, J. W., and T. Mavromatis (2001), Correcting Low-Frequency Variability Bias in Stochastic Weather Generators, Agricultural and Forest Meteorology, 109(4), 297-310, doi:10.1016/S0168-1923(01)00271-4.

Harper, B., and K. Granger (2000), Natural Hazards and the Risks they Pose to South-East Queensland Chapter 5: East Coast Low Risks, Australian Geological Survey Organisation.

Harrold, T. I., A. Sharma, and S. J. Sheather (2003), A Nonparametric Model for Stochastic Generation of Daily Rainfall Amounts, Water Resources Research, 39(12), 1343, doi:10.1029/2003wr002570.

Hendon, H. H., D. W. J. Thompson, and M. C. Wheeler (2007), Australian Rainfall and Surface Temperature Variations Associated with the Southern Hemisphere Annular Mode, Journal of Climate, 20(11), 2452-2467, doi:10.1175/JCLI4134.1.

Holland, G. J., A. H. Lynch, and L. M. Leslie (1987), Australian East-Coast Cyclones. Part I: Synoptic Overview and Case Study, Monthly Weather Review, 115(12), 3024-3036, doi:10.1175/15200493(1987)115<3024:aeccpi>2.0.co;2.

Hong, N. M., T. Y. Lee, and Y. J. Chen (2016), Daily Weather Generator with Drought Properties by Copulas and Standardized Precipitation Indices, Environmental Monitoring and Assessment, 188(6), 383, doi:10.1007/s10661-016-5395-z.

Hopkins, L. C., and G. J. Holland (1997), Australian Heavy-Rain Days and Associated East Coast Cyclones: 1958-92, Journal of climate, 10(4), 621-635, doi:10.1175/15200442(1997)010<0621:ahrdaa>2.0.co;2.

Hundecha, Y., M. Pahlow, and A. Schumann (2009), Modeling of Daily Precipitation at Multiple Locations Using a Mixture of Distributions to Characterize the Extremes, Water Resources Research, 45(12), W12412, doi:10.1029/2008wr007453.

HWC (2012), SoMo Documentation - Hunter Water Headworks Simulation Model, Version 2012.3c.

IPCC (2007), Climate Change 2007: The Physical Science Basis, edited by Solomon, S., D. Qin, M. Manning, Z. Chen, M. Marquis, K.B. Averyt, M. Tignor, and H.L. Miller, Cambridge University Press, Cambridge, United Kingdom.

Jeffrey, S.J., J.O. Carter, K.B. Moodie and A.R. Beswick (2001), Using Spatial Interpolation to Construct a Comprehensive Archive of Australian Climate Data, Environmental Modelling and Software, 16(4), 309-330, doi: 10.1016/S1364-8152(01)00008-1.

Ji, F., J. P. Evans, J. Teng, Y. Scorgie, D. Argüeso, and A. Di Luca (2016), Evaluation of Long-Term Precipitation and Temperature Weather Research and Forecasting Simulations for Southeast Australia, Climate Research, 67(2), 99-115.

Jothityangkoon, C., M. Sivapalan, and N. R. Viney (2000), Tests of a Space-Time Model of Daily Rainfall in Southwestern Australia Based on Nonhomogeneous Random Cascades, Water Resources Research, 36(1), 267-284, doi:10.1029/1999wr900253.

Kaczmarska, J., V. Isham, and C. Onof (2014), Point Process Models for Fine-Resolution Rainfall, Hydrological Sciences Journal-Journal Des Sciences Hydrologiques, 59(11), 1972-1991, doi:10.1080/02626667.2014.925558.

Katz, R. W., and M. B. Parlange (1993), Effects of an Index of Atmospheric Circulation on Stochastic Properties of Precipitation, Water Resources Research, 29(7), 2335-2344, doi:10.1029/93WR00569.

Katz, R. W., M. B. Parlange, and C. Tebaldi (2003), Stochastic Modeling of the Effects of Large-Scale Circulation on Daily Weather in the Southeastern U.S, Climatic Change, 60(1), 189-216, doi:10.1023/a:1026054330406. 
Katz, R. W., and X. Zheng (1999), Mixture Model for Overdispersion of Precipitation, Journal of Climate, 12(8), 2528-2537, doi:10.1175/1520-0442(1999)012<2528:MMFOOP>2.0.CO;2.

Kiem, A. S., and S. W. Franks (2004), Multi-Decadal Variability of Drought Risk, Eastern Australia, Hydrological Processes, 18(11), 2039-2050, doi:10.1002/hyp.1460.

Kiem, A. S., C. Twomey, N. Lockart, G. Willgoose, G. Kuczera, A.F.M.K. Chowdhury, N. Parana Manage, and L. Zhang (2016), Links between East Coast Lows and the Spatial and Temporal Variability of Rainfall Along the Eastern Seaboard of Australia, Journal of Southern Hemisphere Earth Systems Science, 66, 162-176.

Kim, D., H. Cho, C. Onof, and M. Choi (2016), Let-It-Rain: A Web Application for Stochastic Point Rainfall Generation at Ungaged Basins and Its Applicability in Runoff and Flood Modeling, Stochastic Environmental Research and Risk Assessment, 1-21, doi:10.1007/s00477-016-12346.

Kuczera, G. (1997), WATHNET-Generalised Water Supply Headworks Simulation Using Network Linear Programming, Version 3, School of Engineering, University of Newcastle, Australia.

Kuczera, G., L. Cui, R. Gilmore, A. Graddon, M. Mortazavi, and C. Jefferson (2009), Addressing the Shortcomings of Water Resource Simulation Models Based on Network Linear Programming, paper presented at 32nd Hydrology and Water Resources Symposium., Engineers Australia, Newcastle, Australia.

Lall, U., B. Rajagopalan, and D. G. Tarboton (1996), A Nonparametric Wet/Dry Spell Model for Resampling Daily Precipitation, Water Resources Research, 32(9), 2803-2823, doi:10.1029/96wr00565.

Lennartsson, J., A. Baxevani, and D. Chen (2008), Modelling Precipitation in Sweden Using Multiple Step Markov Chains and a Composite Model, Journal of Hydrology, 363(1-4), 42-59, doi:10.1016/j.jhydrol.2008.10.003.

Li, C., V. P. Singh, and A. K. Mishra (2013), A Bivariate Mixed Distribution with a Heavy-Tailed Component and Its Application to Single-Site Daily Rainfall Simulation, Water Resources Research, 49(2), 767-789, doi:10.1002/wrcr.20063.

Liu, Y., W. Zhang, Y. Shao, and K. Zhang (2011), A Comparison of Four Precipitation Distribution Models Used in Daily Stochastic Models, Advances in Atmospheric Sciences, 28(4), 809-820, doi:10.1007/s00376-010-9180-6.

Lockart, N., G. Willgoose, G. Kuczera, A.F.M.K. Chowdhury, N. Parana Manage, L. Zhang, and C. Twomey (2016), Case Study on the Use of Dynamically Downscaled Climate Model Data for Assessing Water Security in the Lower Hunter Region of the Eastern Seaboard of Australia, Journal of Southern Hemisphere Earth Systems Science(66), 177-202.

Martinez, C., G. R. Hancock, J. D. Kalma, T. Wells, and L. Boland (2010), An Assessment of Digital Elevation Models and their Ability to Capture Geomorphic and Hydrologic Properties at the Catchment Scale, Int. J. Remote Sens., 31(23), 6239-6257, doi:10.1080/01431160903403060.

McAneney, J., R. Crompton, K. Chen, and L. Hunter (2007), A Century of Damage - Property Losses Due to Natural Perils., Journal of the Australian and New Zealand Institute of Insurance and Finance, 30(3), 16-22.

McLachlan, G. J., and T. Krishnan (1997), The Em Algorithm and Extensions, Wiley.

McMahon, T. A., A. S. Kiem, M. C. Peel, P. W. Jordan, and G. G. S. Pegram (2008), A New Approach to Stochastically Generating Six-Monthly Rainfall Sequences Based on Empirical Mode Decomposition, Journal of Hydrometeorology, 9(6), 1377-1389, doi:10.1175/2008JHM991.1.

Mehrotra, R., J. Li, S. Westra, and A. Sharma (2015), A Programming Tool to Generate Multi-Site Daily Rainfall Using a Two-Stage Semi Parametric Model, Environmental Modelling \& Software, 63(0), 230-239, doi:10.1016/j.envsoft.2014.10.016.

Mehrotra, R., and A. Sharma (2007), A Semi-Parametric Model for Stochastic Generation of Multi-Site Daily Rainfall Exhibiting Low-Frequency Variability, Journal of Hydrology, 335(1-2), 180193, doi:10.1016/j.jhydrol.2006.11.011.

Mielke, P. W. (1973), Another Family of Distributions for Describing and Analyzing Precipitation Data, Journal of Applied Meteorology, 12(2), 275-280, doi:10.1175/1520-0450(1973)012<0275.

Montanari, A., R. Rosso, and M. S. Taqqu (1997), Fractionally Differenced ARIMA Models Applied to Hydrologic Time Series: Identification, Estimation, and Simulation, Water Resources Research, 33(5), 1035-1044, doi:10.1029/97wr00043.

Mortazavi, M., G. Kuczera, and L. Cui (2012), Multiobjective Optimization of Urban Water Resources: Moving toward More Practical Solutions, Water Resources Research, 48(3), W03514, doi:10.1029/2011wr010866. 
Mortazavi, M., G. Kuczera, A. S. Kiem, B. Henley, B. Berghout, and E. Turner (2013), Robust Optimisation of Urban Drought Security for an Uncertain Climate, 74 pp, National Climate Change Adaptation Research Facility, Gold Coast.

Naveau, P., R. Huser, P. Ribereau, and A. Hannart (2016), Modeling Jointly Low, Moderate, and Heavy Rainfall Intensities without a Threshold Selection, Water Resources Research, 52(4), 27532769, doi:10.1002/2015WR018552.

Ng, W. W., and U. S. Panu (2010), Comparisons of Traditional and Novel Stochastic Models for the Generation of Daily Precipitation Occurrences, Journal of Hydrology, 380(1-2), 222-236, doi:10.1016/j.jhydrol.2009.11.002.

NOAA (2014), Climate Indices: Monthly Atmospheric and Ocean Time Series: http://www.esrl.noaa.gov/psd/Data/Climateindices/List/, edited, National Centres for Environmental Prediction (NCEP) - NOAA.

Onof, C., and H. S. Wheater (1993), Modelling of British Rainfall Using a Random Parameter BartlettLewis Rectangular Pulse Model, Journal of Hydrology, 149(1), 67-95, doi:10.1016/00221694(93)90100-N.

Onof, C., and H. S. Wheater (1994), Improved Fitting of the Bartlett-Lewis Rectangular Pulse Model for Hourly Rainfall, Hydrological Sciences Journal, 39(6), 663-680, doi:10.1080/02626669409492786.

Parana Manage, N. (2016), Statistical Testing of Downscaled Rainfall Data for Water Security Assessment, The University of Newcastle, Australia.

Pepler, A. Coutts-Smith, and B. Timbal (2014), The Role of East Coast Lows on Rainfall Patterns and Inter-Annual Variability across the East Coast of Australia, International Journal of Climatology, 34(4), 1011-1021, doi:10.1002/joc.3741.

Pepler, A., and C. Rakich (2010), Extreme Inflow Events and Synoptic Forcing in Sydney Catchments, in 17th National Conference of the Australian Meteorological and Oceanographic Society, edited, p. 6, IOP Publishing, doi:10.1088/1755-1315/11/1/012010.

Piantadosi, J., J. Boland, and P. Howlett (2009), Generating Synthetic Rainfall on Various TimescalesDaily, Monthly and Yearly, Environmental Modeling \& Assessment, 14(4), 431-438, doi:10.1007/s10666-008-9157-3.

Pittock, B., D. Abbs, R. Suppiah, and R. Jones (2006), Climatic Background to Past and Future Floods in Australia, in Advances in Ecological Research, edited by P. Aldo, pp. 13-39, Academic Press, doi:10.1016/S0065-2504(06)39002-2.

Power, S., T. Casey, C. Folland, A. Colman, and V. Mehta (1999), Inter-Decadal Modulation of the Impact of ENSO on Australia, Climate Dynamics, 15(5), 319-324, doi:10.1007/s003820050284.

Racsko, P., L. Szeidl, and M. Semenov (1991), A Serial Approach to Local Stochastic Weather Models, Ecological Modelling, 57(1-2), 27-41, doi:10.1016/0304-3800(91)90053-4.

Rajagopalan, B., and U. Lall (1999), A K-Nearest-Neighbor Simulator for Daily Precipitation and Other Weather Variables, Water Resources Research, 35(10), 3089-3101, doi:10.1029/1999wr900028.

Ramesh, N. I., and C. Onof (2014), A Class of Hidden Markov Models for Regional Average Rainfall, Hydrological Sciences Journal, 59(9), 1704-1717, doi:10.1080/02626667.2014.881484.

Raupach, M. R., P. R. Briggs, V. Haverd, E. A. King, M. Paget, and C. M. Trudinger (2009), Australian Water Availability Project (AWAP), CSIRO Marine and Atmospheric Research Component: Final Report for Phase 3, 67 pp, Centre for Australian Weather and Climate Research (Bureau of Meteorology and CSIRO), Melbourne, Australia.

Richardson, C. W. (1981), Stochastic Simulation of Daily Precipitation, Temperature, and Solar Radiation, Water Resources Research, 17(1), 182-190, doi:10.1029/WR017i001p00182.

Risbey, J.S., M.J. Pook, P.C. McIntosh, M.C. Wheeler, and H.H. Hendon (2009), On the Remote Drivers of Rainfall Variability in Australia, Monthly Weather Review, 137, 3233-3253, doi: 10.1175/2009MWR2861.1.

Rodriguez-Iturbe, I., D. R. Cox, and V. Isham (1988), A Point Process Model for Rainfall: Further Developments, Proceedings of the Royal Society of London. A. Mathematical and Physical Sciences, 417(1853), 283-298, doi:10.1098/rspa.1988.0061.

Rodríguez-Iturbe, I., B. F. de Power, and J. B. Valdés (1987), Rectangular Pulses Point Process Models for Rainfall: Analysis of Empirical Data, Journal of Geophysical Research: Atmospheres, 92(D8), 9645-9656, doi:10.1029/JD092iD08p09645.

Roldan, J., and D. A. Woolhiser (1982), Stochastic Daily Precipitation Models, 1. A Comparison of Occurrence Processes., Water Resource Research., 18, 1451-59. 
Rüdiger, C., T. Wells, G. Hancock, C. Martinez, H. M. Hemakumara, B. Jacobs, J. D. Kalma, M. Thyer, J. P. Walker, and G. Willgoose (2007), Goulburn River Experimental Catchment Data Set, Water Resources Research, 43(10), doi:10.1029/2006wr005837.

Salas, J. (1993), Analysis and Modeling of Hydrologic Time Series, in Handbook of Hydrology, Edited by D. Maidment, edited, pp. 19.11-19.72, McGraw-Hill, New York, USA.

Semenov, M. A. (2008), Simulation of Extreme Weather Events by a Stochastic Weather Generator, Climate Research, 35(3), 203-212.

Sharda, V. N., and P. K. Das (2005), Modelling Weekly Rainfall Data for Crop Planning in a Sub-Humid Climate of India, Agricultural Water Management, 76(2), 120-138, doi:10.1016/j.agwat.2005.01.010.

Sharif, M., and D. H. Burn (2006), Simulating Climate Change Scenarios Using an Improved K-Nearest Neighbor Model, Journal of Hydrology, 325(1-4), 179-196, doi:10.1016/j.jhydrol.2005.10.015.

Sharma, A., and R. Mehrotra (2010), Rainfall Generation. In: Testik, Firt Y., Gebremichael, Mekonnen (Eds.), Rainfall: State of the Science, AGU Geophysical Monograph Series.

Speer, M. (2008), On the Late Twentieth Century Decrease in Australian East Coast Rainfall Extremes, Atmospheric Science Letters, 9(3), 160-170, doi:10.1002/asl.191.

Speer, M., P. Wiles, and A. Pepler (2009), Low Pressure Systems Off the New South Wales Coast and Associated Hazardous Weather: Establishment of a Database, Australian Meteorological and Oceanographic Journal, 58, 29-39.

Srikanthan, R. (2005), Stochastic Generation of Daily Rainfall Using a Nested Transition Probability Model, in 29th Hydrology and Water Resources Symposium, Canberra, Australia.

Srikanthan, R., T. I. Harrold, A. Sharma, and T. A. McMahon (2005), Comparison of Two Approaches for Generation of Daily Rainfall Data, Stochastic Environmental Research \& Risk Assessment, 19(3), 215-226, doi:10.1007/s00477-004-0226-0.

Srikanthan, R., and T. A. McMahon (1985), Stochastic Generation of Rainfall and Evaporation Data, 301 pp., Canberra : Australian Government Publishing Service.

Srikanthan, R., and T. A. McMahon (2001), Stochastic Generation of Annual, Monthly and Daily Climate Data: A Review, Hydrol. Earth Syst. Sci., 5(4), 653-670, doi:10.5194/hess-5-653-2001.

Srikanthan, R., and G. G. S. Pegram (2009), A Nested Multisite Daily Rainfall Stochastic Generation Model, Journal of Hydrology, 371(1-4), 142-153, doi:10.1016/j.jhydrol.2009.03.025.

Srikanthan, R., and B. J. Stewart (1992), Analysis of Australian Rainfall and Rainday Data with Respect to Climatic Variability and Change, Hydrology Branch, Bureau of Meteorology.

Stern, R. D., and R. Coe (1984), A Model Fitting Analysis of Daily Rainfall Data, Journal of the Royal Statistical Society. Series A (General), 147(1), 1-34, doi:10.2307/2981736.

Tarpanelli, A., M. Franchini, L. Brocca, S. Camici, F. Melone, and T. Moramarco (2012), A Simple Approach for Stochastic Generation of Spatial Rainfall Patterns, Journal of Hydrology, 472473, 63-76, doi:10.1016/j.jhydrol.2012.09.010.

Thyer, M., and G. Kuczera (2000), Modeling Long-Term Persistence in Hydroclimatic Time Series Using a Hidden State Markov Model, Water Resources Research, 36(11), 3301-3310, doi:10.1029/2000wr900157.

Vance, T. R., J. L. Roberts, C. T. Plummer, A. S. Kiem, and T. D. van Ommen (2015), Interdecadal Pacific Variability and Eastern Australian Megadroughts over the Last Millennium, Geophysical Research Letters, 42(1), 2014GL062447, doi:10.1002/2014GL062447.

Vaze, J., D. A. Post, F. H. S. Chiew, J. M. Perraud, J. Teng, and N. R. Viney (2011), Conceptual Rainfall-Runoff Model Performance with Different Spatial Rainfall Inputs, Journal of Hydrometeorology, 12(5), 1100-1112, doi:10.1175/2011JHM1340.1.

Verdon-Kidd, D. C., and A. S. Kiem (2009), Nature and Causes of Protracted Droughts in Southeast Australia: Comparison between the Federation, WWII, and Big Dry Droughts, Geophysical Research Letters, 36(22), doi:10.1029/2009g1041067.

Verdon-Kidd, D. C., A. S. Kiem, G. Willgoose, and P. Haines (2010), East Coast Lows and the Newcastle/Central Coast Pasha Bulker Storm, 61 pp, National Climate Change Adaptation Research Facility, Gold Coast.

Verdon-Kidd, D. C., A. M. Wyatt, A. S. Kiem, and S. W. Franks (2004), Multidecadal Variability of Rainfall and Streamflow: Eastern Australia, Water Resources Research, 40(10), W10201, doi:10.1029/2004wr003234.

Vrac, M., and P. Naveau (2007), Stochastic Downscaling of Precipitation: From Dry Events to Heavy Rainfalls, Water Resources Research, 43(7), W07402, doi:10.1029/2006wr005308. 
Wang, Q. J., and R. J. Nathan (2007), A Method for Coupling Daily and Monthly Time Scales in Stochastic Generation of Rainfall Series, Journal of Hydrology, 346(3-4), 122-130, doi:10.1016/j.jhydrol.2007.09.003.

Wasko C. and A. Sharma (2015), Steeper Temporal Distribution of Rain Intensity at Higher Temperatures within Australian Storms, Nature Geoscience, 8, 527-530, doi: 10.1038/NGEO2456.

WBM (2012), Derivation of Williams River Flow Datasets, Prepared for Hunter Water Corporation, Andrew Grant and Philip Pedruco, R.M8248.001.01, Melbourne, Victoria.

Wilby, R. L., T. M. L. Wigley, D. Conway, P. D. Jones, B. C. Hewitson, J. Main, and D. S. Wilks (1998), Statistical Downscaling of General Circulation Model Output: A Comparison of Methods, Water Resources Research, 34(11), 2995-3008, doi:10.1029/98wr02577.

Wiles, P., A. Pepler, and M. Speer (2009), Inter-Annual Variability in Australian East Coast Lows and Its Relation with Key Climate Indices, paper presented at 9th Annual International Conference on Southern Hemisphere Meteorology and Oceanography (ICSHMO), Melbourne, Australia.

Wilks, D. S. (1998), Multisite Generalization of a Daily Stochastic Precipitation Generation Model, Journal of Hydrology, 210(1-4), 178-191, doi:10.1016/S0022-1694(98)00186-3.

Wilks, D. S. (1999a), Interannual Variability and Extreme-Value Characteristics of Several Stochastic Daily Precipitation Models, Agricultural and Forest Meteorology, 93(3), 153-169, doi:10.1016/S0168-1923(98)00125-7.

Wilks, D. S. (1999b), Simultaneous Stochastic Simulation of Daily Precipitation, Temperature and Solar Radiation at Multiple Sites in Complex Terrain, Agricultural and Forest Meteorology, 96(1-3), 85-101, doi:10.1016/S0168-1923(99)00037-4.

Wilson, P. S., and R. Toumi (2005), A Fundamental Probability Distribution for Heavy Rainfall, Geophysical Research Letters, 32(14), L14812, doi:10.1029/2005gl022465.

Yunus, R. M., M. M. Hasan, N. A. Razak, Y. Z. Zubairi, and P. K. Dunn (2016), Modelling Daily Rainfall with Climatological Predictors: Poisson-Gamma Generalized Linear Modelling Approach, International Journal of Climatology, doi:10.1002/joc.4784. 
Appendices 


\section{Appendices}

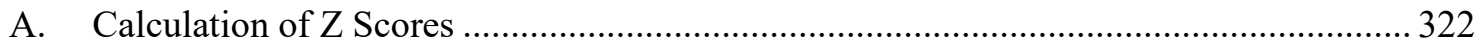

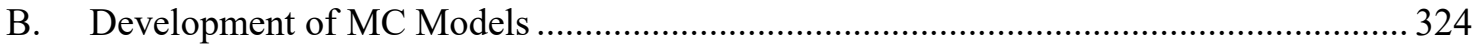

C. Comparison of MC Models in Goulburn River Site........................................................ 335

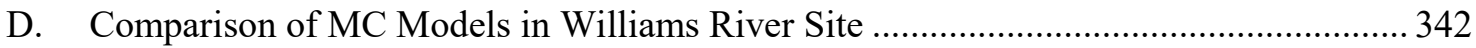

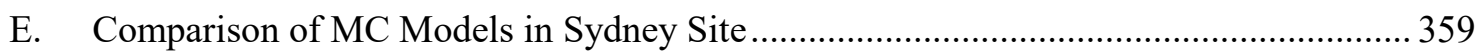

F. Comparison of MC Models in Richmond River Site ……................................................. 373

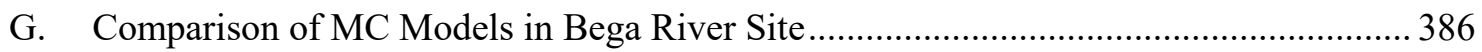

H. Comparison of MC and MMKD for Raingauge Data around Australia........................... 398

I. Comparison of MC and MMKD for Streamflow Generation.......................................... 414

\section{List of Figures in Appendices}

Figure A.1: Performance of APMC for mean and SD of rainfall depths. The black solid lines indicate the respective RCM2 values, while the blue lines with error bars indicate the APMC simulated value with $95 \%$ confidence limit ( $2 \mathrm{SD}$ ). The red dotted lines indicate the Z Scores.

323

Figure B.1: Effects of different thresholds (e.g. $0.0 \mathrm{~mm}, 0.1 \mathrm{~mm}, 0.2 \mathrm{~mm}$, and $0.3 \mathrm{~mm}$ ) for wet day rainfall depths on the APMC parameters of RCM2 (left) and AWAP (right). ......................................324

Figure B.2: Distribution of wet day rainfall depths in AWAP and APMC simulation for January, April,

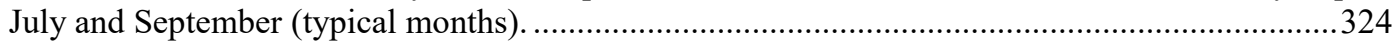

Figure B.3: Distribution of wet day rainfall depths between $0.3 \mathrm{~mm}$ and $1 \mathrm{~mm}$ in AWAP and APMC simulation for January, April, July and September (typical months). .....................................325

Figure B.4: Box-cox transformation of wet day rainfall depths in AWAP (left). Correlation of transformed rainfall depths in successive wet days (right)................................................................. 325

Figure B.5: Comparison of the decadal variability of the DPMC parameters (wet-to-wet probabilities P11 and mean wet day rainfalls $\mu$ ) with the APMC parameters....................................................326

Figure B.6: Parametric bootstrapping of DPMC parameters (P11 and $\mu$ ) for January and July as two representative months (typical of Figure 4.12) ...............................................................326

Figure B.7: Parameters (P11 and $\mu$ ) of 5-year sub-samples of RCM2 ............................................327

Figure B.8: Correlation between the $12 \times 60$ values of each parameter $(\mathrm{P} 00, \mathrm{P} 11, \mu$, and $\sigma$ ) for AWAP and climate indices (ONI, nino3.4, MEI, and PDO)............................................................. 327

Figure B.9: Correlation between the $12 \times 60$ values of each parameter $(\mathrm{P} 00, \mathrm{P} 11, \mu$, and $\sigma)$ for AWAP and

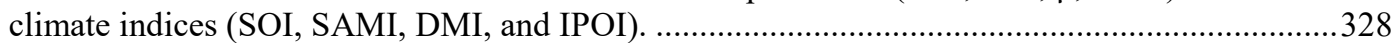

Figure B.10: Lognormal probability plots of $\mu$ for each month for AWAP. ..........................................329

Figure B.11: Lognormal probability plots of $\sigma$ for each month for AWAP. ..........................................330

Figure B.12: Correlation between $\log \mu$ and $\log \sigma$ for each month for AWAP...................................330

Figure B.13: Normal probability plots of P00 for each month for AWAP. .......................................331

Figure B.14: Normal probability plots of P11 for each month for AWAP. ........................................331

Figure B.15: Correlation between P00 and P11 for each month for AWAP......................................332

Figure B.16: Correlation between $\log \mu$ and P11 for each month for AWAP....................................332

Figure B.17: Correlation between $\log \sigma$ and P11 for each month for AWAP. ..................................333

Figure B.18: Correlation between $\log \mu$ and P00 for each month for AWAP.....................................333

Figure B.19: Correlation between $\log \sigma$ and P00 for each month for AWAP. ....................................334

Figure C.1: Intra-annual and spatial variability of the mean of monthly rainfall depths. ......................335

Figure C.2: Intra-annual and spatial variability of the SD of monthly rainfall depths. .......................335

Figure C.3: Spatial variability of the mean of annual rainfall depths............................................336

Figure C.4: Spatial variability of the SD of multiyear rainfall depths. ...............................................336

Figure C.5: Spatial variability of the mean of annual number of wet days. .......................................336

Figure C.6: Spatial variability of the SD of multiyear number of wet days. ....................................336 
Figure C.7: Intra-annual and spatial variability of the mean of monthly mean length of wet spells. ......337

Figure C.8: Intra-annual and spatial variability of the SD of monthly mean length of wet spells...........337

Figure C.9: Spatial variability of the mean of annual mean length of wet spells. .................................337

Figure C.10: Spatial variability of the SD of annual mean length of wet spells..................................337

Figure C.11: Comparison of CDMC, HMC and DHMC for the mean of monthly rainfall depths. ........338

Figure C.12: Distribution of Z scores of CDMC, HMC and DHMC for the mean and SD of rainfall depths

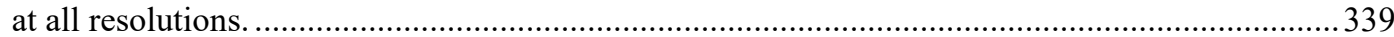

Figure C.13: Overall comparison of CDMC, HMC and DHMC for the mean and SD of number of wet days at monthly and multiyear resolutions.......................................................................... 340

Figure C.14: Overall comparison of CDMC, HMC and DHMC for the mean and SD of mean length of wet

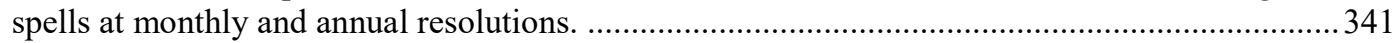

Figure D.1: Intra-annual and spatial variability of the mean of monthly rainfall depths.......................342

Figure D.2: Intra-annual and spatial variability of the SD of monthly rainfall depths. ........................342

Figure D.3: Spatial variability of the mean of annual rainfall depths................................................342

Figure D.4: Spatial variability of the SD of multiyear rainfall depths.............................................343

Figure D.5: Intra-annual and spatial variability of the mean of monthly number of wet and dry days. ...343

Figure D.6: Intra-annual and spatial variability of the SD of monthly number of wet and dry days. .....344

Figure D.7: Intra-annual and spatial variability of the mean of monthly mean length of wet and dry spells.

Figure D.8: Intra-annual and spatial variability of the SD of monthly mean length of wet and dry spells.

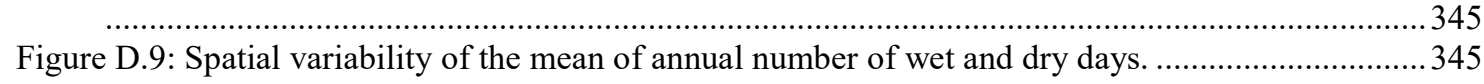

Figure D.10: Spatial variability of the mean and SD of annual mean length of wet and dry spells. .......345

Figure D.11: Spatial variability of the SD of multiyear number of wet and dry days.............................346

Figure D.12: Comparison of CDMC, HMC and DHMC for the mean of daily rainfall depths. .............347

Figure D.13: Comparison of CDMC, HMC and DHMC for the mean of monthly rainfall depths. ........348

Figure D.14: Comparison of CDMC, HMC and DHMC for the mean of annual rainfall depths...........348

Figure D.15: Comparison of CDMC, HMC and DHMC for the SD of daily rainfall depths..................349

Figure D.16: Comparison of CDMC, HMC and DHMC for the SD of monthly rainfall depths............350

Figure D.17: Comparison of CDMC, HMC and DHMC for the mean of monthly number of wet days. 351

Figure D.18: Comparison of CDMC, HMC and DHMC for the mean of annual number of wet days. ...352

Figure D.19: Comparison of CDMC, HMC and DHMC for the mean of annual mean length of wet spells.

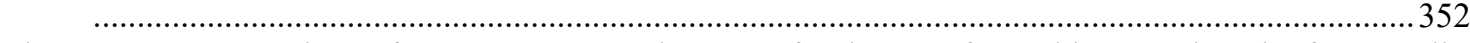

Figure D.20: Comparison of CDMC, HMC and DHMC for the SD of monthly mean length of wet spells.

353

Figure D.21: Comparison of CDMC, HMC and DHMC for the month-to-month autocorrelations of

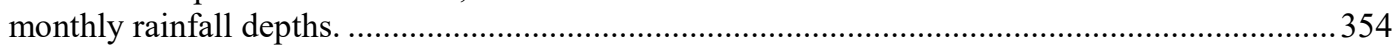

Figure D.22: Comparison of CDMC, HMC and DHMC to reproduce the month-to-month autocorrelations

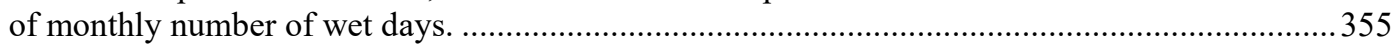

Figure D.23: Intra-annual and spatial variability of model parameters (APMC values) for reanalysis and CSIRO mk3.0 of RCM2.

356

Figure D.23: Intra-annual and spatial variability of model parameters (APMC values) for reanalysis and

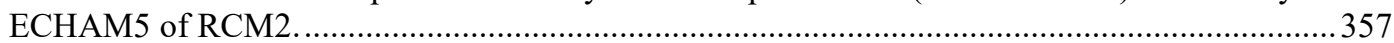

Figure D.23: Intra-annual and spatial variability of model parameters (APMC values) for reanalysis and

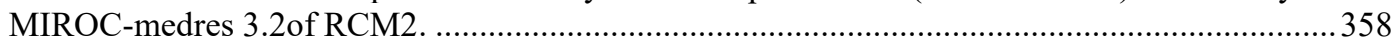

Figure E.1: Intra-annual and spatial variability of the mean of monthly rainfall depths. .......................359

Figure E.2: Intra-annual and spatial variability of the SD of monthly rainfall depths...........................359

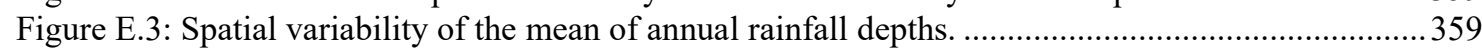

Figure E.4: Intra-annual and spatial variability of the mean of monthly number of wet days. ...............360

Figure E.5: Intra-annual and spatial variability of the SD of monthly number of wet days...................360

Figure E.6: Intra-annual and spatial variability of the mean of monthly mean length of wet spells. ......360

Figure E.7: Intra-annual and spatial variability of the SD of monthly mean length of wet spells...........361

Figure E.8: Spatial variability of the mean of annual number of wet days............................................3 361

Figure E.9: Spatial variability of the mean of annual mean length of wet spells. ...............................361

Figure E.10: Spatial variability of the SD of annual mean length of wet spells......................................361

Figure E.11: Comparison of CDMC, HMC and DHMC for the mean of daily rainfall depths...............362

Figure E.12: Comparison of CDMC, HMC and DHMC for the mean of monthly rainfall depths. .........363

Figure E.13: Comparison of CDMC, HMC and DHMC for the mean of annual rainfall depths. ...........364

Figure E.14: Comparison of CDMC, HMC and DHMC for the SD of daily rainfall depths. ..................365 
Figure E.15: Comparison of CDMC, HMC and DHMC for the SD of monthly rainfall depths. ............366

Figure E.16: Comparison of CDMC, HMC and DHMC for the mean of monthly number of wet days. 367 Figure E.17: Comparison of CDMC, HMC and DHMC for the mean of annual number of wet days....368 Figure E.18: Comparison of CDMC, HMC and DHMC for the mean of annual mean length of wet spells. .368

Figure E.19: Comparison of CDMC, HMC and DHMC for the mean of monthly mean length of wet spells. 369

Figure E.20: Comparison of CDMC, HMC and DHMC for the SD of monthly mean length of wet spells. . .370

Figure E.21: Comparison of CDMC, HMC and DHMC for the month-to-month autocorrelations of

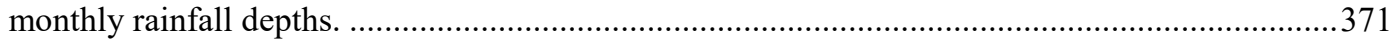

Figure E.22: Comparison of CDMC, HMC and DHMC for the month-to-month autocorrelations of

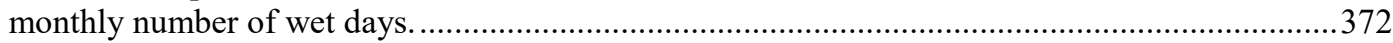

Figure F.1: Intra-annual and spatial variability of the mean of monthly rainfall depths. .....................373

Figure F.2: Intra-annual and spatial variability of the SD of monthly rainfall depths.........................373

Figure F.3: Spatial variability of the mean of annual rainfall depths. .................................................3 373

Figure F.4: Intra-annual and spatial variability of the mean of monthly number of wet days................374

Figure F.5: Intra-annual and spatial variability of the SD of monthly number of wet days. ..................374

Figure F.6: Intra-annual and spatial variability of the mean of monthly mean length of wet spells........374

Figure F.7: Intra-annual and spatial variability of the SD of monthly mean length of wet spells. ..........375

Figure F.8: Spatial variability of the mean of annual number of wet days. .......................................375

Figure F.9: Spatial variability of the mean of annual mean length of wet spells..................................375

Figure F.10: Spatial variability of the SD of annual mean length of wet spells. ................................375

Figure F.11: Comparison of CDMC, HMC and DHMC for the mean of daily rainfall depths. ..............376

Figure F.12: Comparison of CDMC, HMC and DHMC for the mean of monthly rainfall depths..........377

Figure F.13: Comparison of CDMC, HMC and DHMC for the mean of annual rainfall depths. ...........377

Figure F.14: Comparison of CDMC, HMC and DHMC for the SD of daily rainfall depths.................378

Figure F.15: Comparison of CDMC, HMC and DHMC for the SD of monthly rainfall depths. ............379

Figure F.16: Comparison of CDMC, HMC and DHMC for the mean of monthly number of wet days. 380

Figure F.17: Comparison of CDMC, HMC and DHMC for the mean of monthly mean length of wet spells.

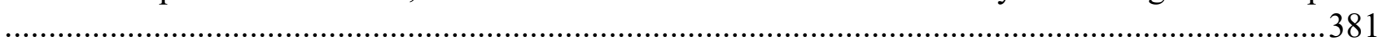

Figure F.18: Comparison of CDMC, HMC and DHMC for the mean of annual number of wet days....382 Figure F.19: Comparison of CDMC, HMC and DHMC for the mean of annual mean length of wet spells.

.382

Figure F.20: Comparison of CDMC, HMC and DHMC for the SD of monthly mean length of wet spells.

Figure F.21: Comparison of CDMC, HMC and DHMC for the month-to-month autocorrelations of monthly rainfall depths.

384

Figure F.22: Comparison of CDMC, HMC and DHMC for the month-to-month autocorrelations of

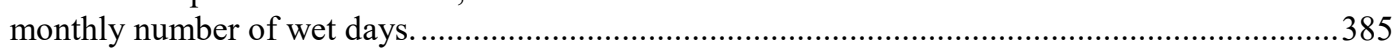

Figure G.1: Intra-annual and spatial variability of the mean of monthly rainfall depths.......................386

Figure G.2: Intra-annual and spatial variability of the SD of monthly rainfall depths. ........................386

Figure G.3: Spatial variability of the mean of annual rainfall depths..............................................386

Figure G.4: Intra-annual and spatial variability of the mean of monthly number of wet days. ................387

Figure G.5: Intra-annual and spatial variability of the SD of monthly number of wet days...................387

Figure G.6: Intra-annual and spatial variability of the mean of monthly mean length of wet spells.......387

Figure G.7: Intra-annual and spatial variability of the SD of monthly mean length of wet spells. .........388

Figure G.8: Spatial variability of the mean of annual number of wet days. ...........................................38

Figure G.9: Spatial variability of the mean of annual mean length of wet spells. .................................38

Figure G.10: Spatial variability of the SD of annual mean length of wet spells.................................388

Figure G.11: Comparison of CDMC, HMC and DHMC for the mean of daily rainfall depths. .............389

Figure G.12: Comparison of CDMC, HMC and DHMC for the mean of monthly rainfall depths. ........390

Figure G.13: Comparison of CDMC, HMC and DHMC for the mean of annual rainfall depths. ...........390

Figure G.14: Comparison of CDMC, HMC and DHMC for the SD of daily rainfall depths. ................391

Figure G.15: Comparison of CDMC, HMC and DHMC for the SD of monthly rainfall depths.............392

Figure G.16: Comparison of CDMC, HMC and DHMC for the mean of monthly number of wet days. 393

Figure G.17: Comparison of CDMC, HMC and DHMC for the mean of annual number of wet days. ..394 Figure G.18: Comparison of CDMC, HMC and DHMC for the mean of monthly mean length of wet spells. 
Figure G.19: Comparison of CDMC, HMC and DHMC for the mean of annual mean length of wet spells.

Figure G.20: Comparison of CDMC, HMC and DHMC for the SD of monthly mean length of wet spells.

Figure G.21: Comparison of CDMC, HMC and DHMC for the month-to-month autocorrelations of

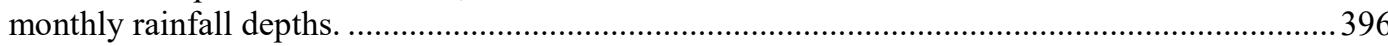

Figure G.22: Comparison of CDMC, HMC and DHMC for the month-to-month autocorrelations of

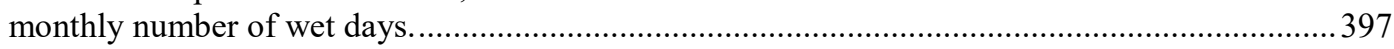

Figure H.1: Mean and SD of monthly mean length of wet spells. Note the change in vertical scale for

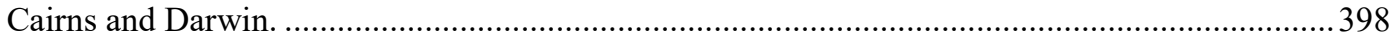

Figure H.2: Comparison of model performances for mean of monthly rainfall depths......................... 399

Figure H.3: Comparison of model performances for SD of monthly rainfall depths. ..........................400

Figure H.4: Comparison of models for mean of multiyear number of wet days. ................................ 401

Figure H.5: Comparison of model performances for mean of monthly mean length of wet spells. ........402

Figure H.6: Comparison of model performances for SD of monthly mean length of wet spells. ...........403

Figure H.7: CDMC reproduction of the autocorrelations of monthly number of wet days and monthly rainfall depths for coastal stations.

Figure H.8: CDMC reproduction of the autocorrelations of monthly number of wet days and monthly

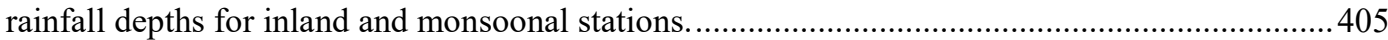

Figure H.9: HMC reproduction of the autocorrelations of monthly number of wet days and monthly rainfall depths for coastal stations.

Figure H.10: HMC reproduction of the autocorrelations of monthly number of wet days and monthly rainfall depths for inland and monsoonal stations.

Figure H.11: DHMC reproduction of the autocorrelations of monthly number of wet days and monthly rainfall depths for coastal stations.

Figure H.12: DHMC reproduction of the autocorrelations of monthly number of wet days and monthly rainfall depths for inland and monsoonal stations.

Figure H.13: MMKD reproduction of the autocorrelations of monthly number of wet days and monthly rainfall depths for coastal stations.

Figure H.14: MMKD reproduction of the autocorrelations of monthly number of wet days and monthly rainfall depths for inland and monsoonal stations.

Figure H.15: Comparison of DHMC, MMKD and Combined Model to reproduce the mean and SD of daily

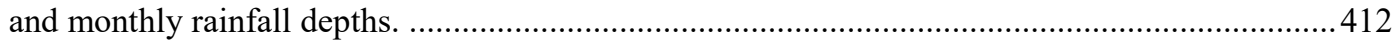

Figure H.16: Comparison of DHMC, MMKD and Combined Model to reproduce the mean and SD of

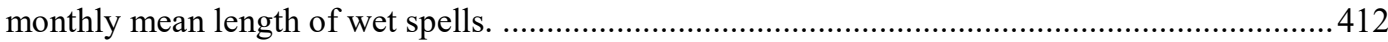

Figure H.17: Comparison of DHMC, MMKD and Combined Model to reproduce the mean and SD of

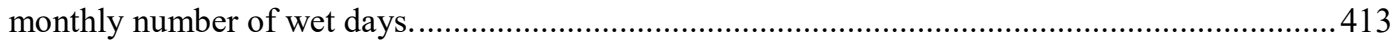

Figure H.18: Comparison of DHMC, MMKD and Combined Model to reproduce the mean and SD of

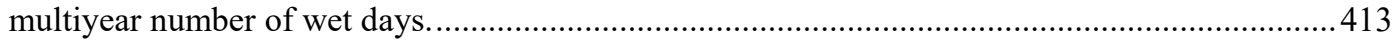

Figure I.1: Distribution statistics of daily rainfall and streamflow at Chichester. ...................................414

Figure I.2: Distribution statistics of monthly rainfall and streamflow at Chichester............................415

Figure I.3: Distribution statistics of multiyear rainfall and streamflow at Chichester........................... 415

Figure I.4: Distribution statistics of monthly rainfall and streamflow at Grahamstown. ...................... 416

Figure I.5: Distribution statistics of monthly rainfall and streamflow at Tillegra. ............................... 416

Figure I.6: Model comparison for daily rainfall and streamflow of RCM1 for calibration period at Chichester.

Figure I.7: Model comparison for daily rainfall and streamflow of RCM1 for validation period at Chichester.

Figure I.8: Model comparison for monthly rainfall and streamflow of RCM1 for calibration period at Chichester.

Figure I.9: Model comparison for monthly rainfall and streamflow of RCM1 for validation period at Chichester.

Figure I.10: Model comparison for multiyear rainfall and streamflow of RCM1 for calibration period at Chichester.

Figure I.11: Model comparison for multiyear rainfall and streamflow of RCM1 for validation period at Chichester.

Figure I.12: Model comparison for daily rainfall and streamflow of AWAP for calibration period at Chichester. 
Figure I.13: Model comparison for daily rainfall and streamflow of AWAP for validation period at Chichester.

Figure I.14: Model comparison for monthly rainfall and streamflow of AWAP for calibration period at Chichester.

Figure I.15: Model comparison for monthly rainfall and streamflow of AWAP for validation period at Chichester. .422

Figure I.16: Model comparison for multiyear rainfall and streamflow of AWAP for calibration period at Chichester. 422

Figure I.17: Model comparison for multiyear rainfall and streamflow of AWAP for validation period at Chichester. 423

Figure I.18: Model comparison for daily rainfall and streamflow of AWAP for calibration period at Grahamstown. .423

Figure I.19: Model comparison for daily rainfall and streamflow of AWAP for validation period at Grahamstown. .424

Figure I.20: Model comparison for monthly rainfall and streamflow of AWAP for calibration period at Grahamstown. 424

Figure I.21: Model comparison for monthly rainfall and streamflow of AWAP for validation period at Grahamstown. 425

Figure I.22: Model comparison for daily rainfall and streamflow of AWAP for calibration period at Tillegra. .425

Figure I.23: Model comparison for daily rainfall and streamflow of AWAP for validation period at Tillegra.

Figure I.24: Model comparison for monthly rainfall and streamflow of AWAP for calibration period at Tillegra. 426

Figure I.25: Model comparison for monthly rainfall and streamflow of AWAP for validation period at Tillegra. 427

Figure I.26: Model comparison for monthly rainfall and streamflow of RCM1 for calibration period at Grahamstown. 427

Figure I.27: Model comparison for monthly rainfall and streamflow of RCM1 for validation period at Grahamstown. .428

Figure I.28: Model comparison for monthly rainfall and streamflow of RCM1 for calibration period at Tillegra. 428

Figure I.29: Model comparison for monthly rainfall and streamflow of RCM1 for validation period at Tillegra.

Figure I.30: Model comparison for autocorrelations of monthly rainfall and streamflow of RCM1 at Chichester. 429

Figure I.31: Model comparison for autocorrelations of monthly rainfall and streamflow of AWAP at Chichester. .430

\section{List of Table in Appendices}

Table B.1 : Change of Mean and SD of simulated rainfall depths at daily and monthly resolutions with the change of variability of the parameters. 329 


\section{A. Calculation of Z Scores}

Performances of the models of this thesis to reproduce the distribution statistics (e.g. mean and SD) of rainfall depths and wet periods are assessed by calculating Z Scores. While the calculation procedure of $Z$ scores has been presented in Chapter 3 (section 3.4.3), this Appendix is presenting an example of the $\mathrm{Z}$ scores of APMC model (one of the model of this thesis) for mean and SD of rainfall depths for daily and monthly resolutions.

Figure A.1 shows the performance of APMC in reproducing the mean and SD of rainfall depth at daily and monthly resolutions. Both the bootstrapped statistics and $\mathrm{Z}$ scores are shown in this figure to explain the assessment procedure described in section 3.4.3 of Chapter 3. The black lines in each panel indicates the observed value (i.e. the statistics calculated from the data to be modelled, in this case it is RCM2 at Gummun) of the respective statistics. The blue lines and error bars indicate the expected values and $95 \%$ confidence limits of a statistic respectively in APMC simulation. These expected values and $95 \%$ confidence limits are calculated as the mean and 2 SD respectively of 1000 realisations of a statistic. The red dotted lines indicate the corresponding $\mathrm{Z}$ scores of a statistic, calculated from the comparison between the observed and simulated (mean and SD of 1000 realisations) values of the statistic using Equation 3.2 of Chapter 3. Therefore, these are two ways of analysing the comparison between the observed and simulated statistics.

For daily mean and SD, and monthly SD of rainfall depths (first three panels), the blue line of expected simulated values overlap the black line of observed values in every month, indicating a very good reproduction of the statistics in APMC simulation. This is also indicated by the red dotted line with $\mathrm{Z}$ score of almost zero in each month. However, for SD of monthly rainfall depths, the APMC significantly underestimated the statistic for most of the months as the observed values are over the upper 95\% confidence limit of the APMC simulation, and accordingly $\mathrm{Z}$ scores are greater than 2 . Similarly, if a statistic is overestimated in the model, the observed value stays below the lower $95 \%$ confidence limit, and accordingly the $\mathrm{Z}$ score becomes less than -2 . 

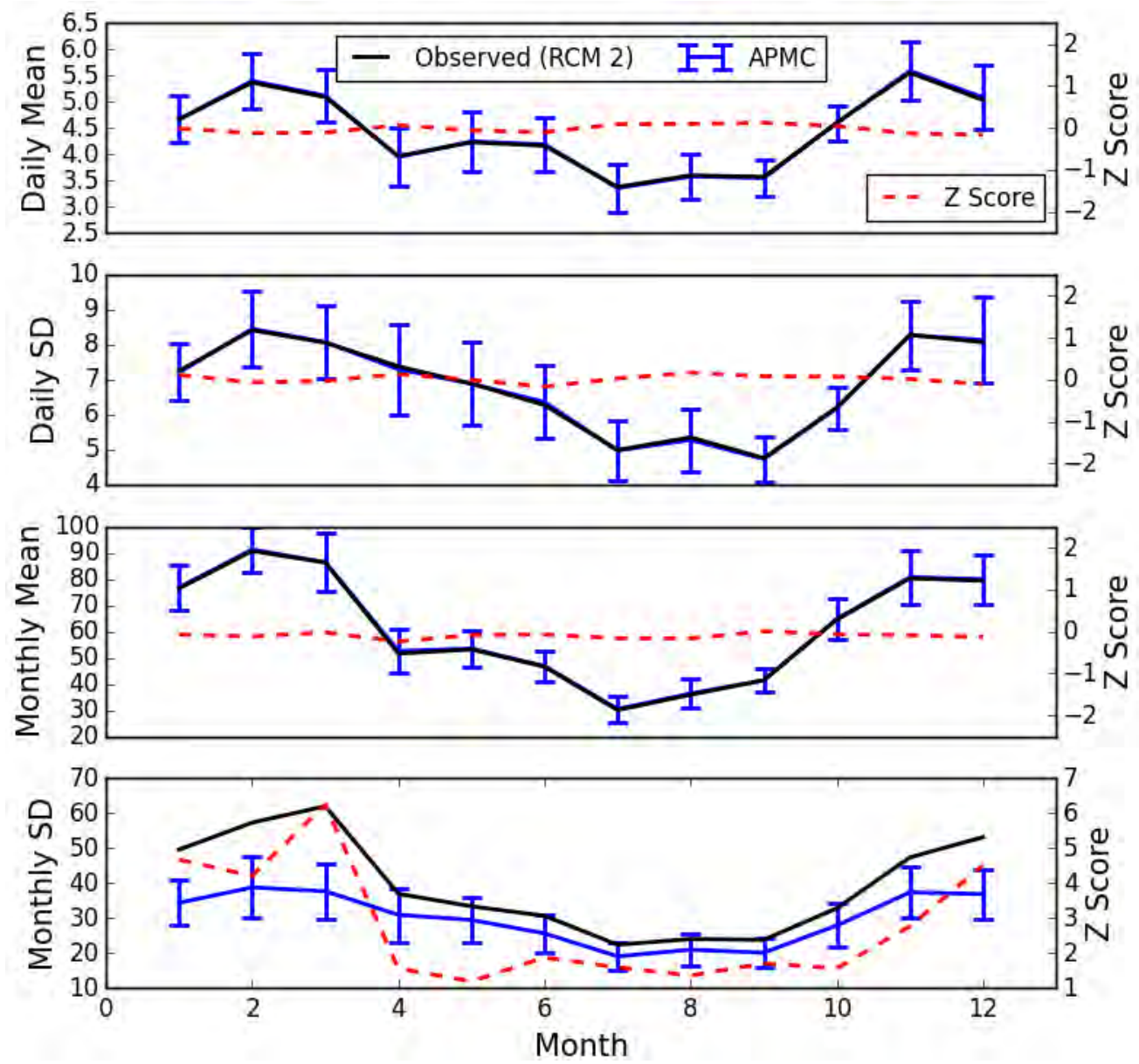

Figure A.1: Performance of APMC for mean and SD of rainfall depths. The black solid lines indicate the respective RCM2 values, while the blue lines with error bars indicate the APMC simulated value with $95 \%$ confidence limit ( 2 SD). The red dotted lines indicate the $\mathrm{Z}$ Scores. 


\section{B. Development of MC Models}

\section{B.1 Justifications of Model Assumptions}

Figures in this appendix present the additional information for justification of model assumptions discussed in Chapter 4.
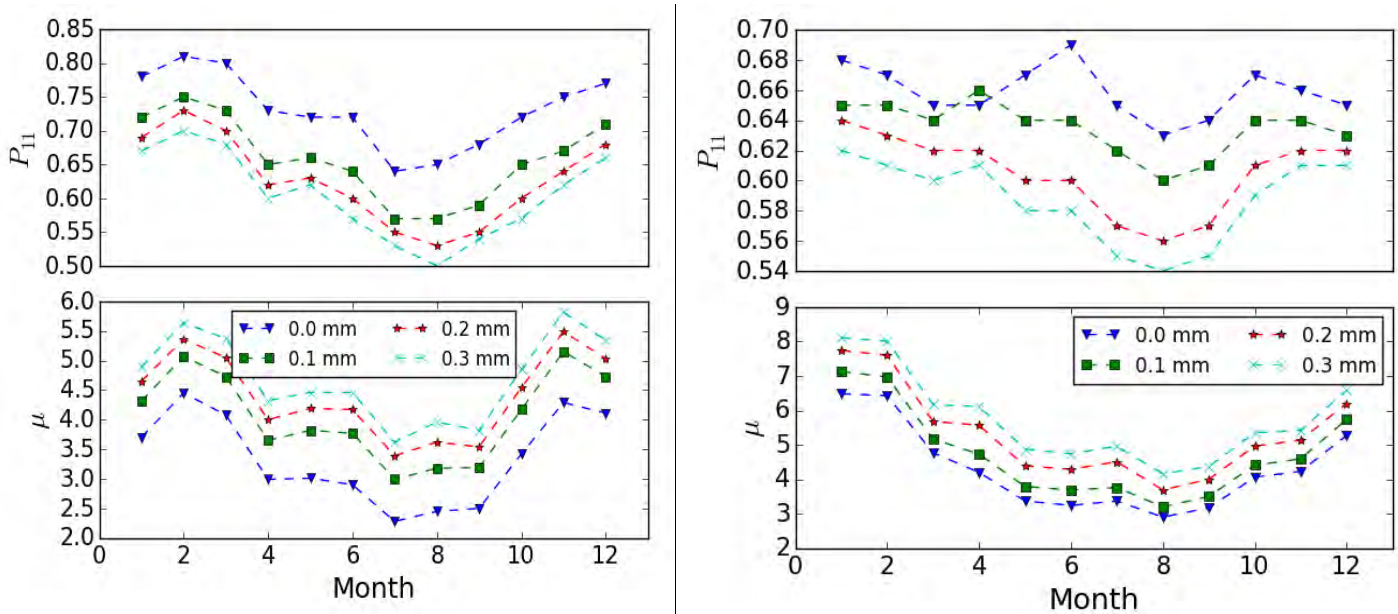

Figure B.1: Effects of different thresholds (e.g. $0.0 \mathrm{~mm}, 0.1 \mathrm{~mm}, 0.2 \mathrm{~mm}$, and $0.3 \mathrm{~mm}$ ) for wet day rainfall depths on the APMC parameters of RCM2 (left) and AWAP (right).
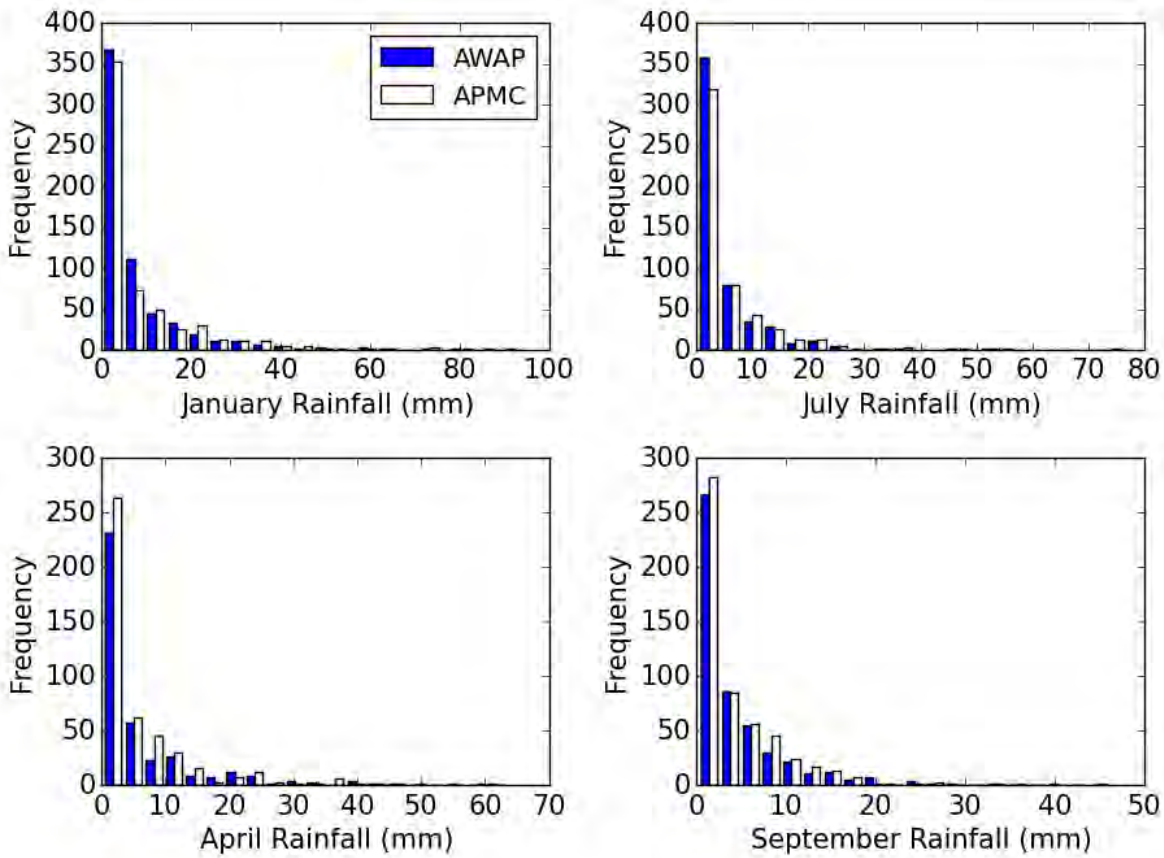

Figure B.2: Distribution of wet day rainfall depths in AWAP and APMC simulation for January, April, July and September (typical months). 

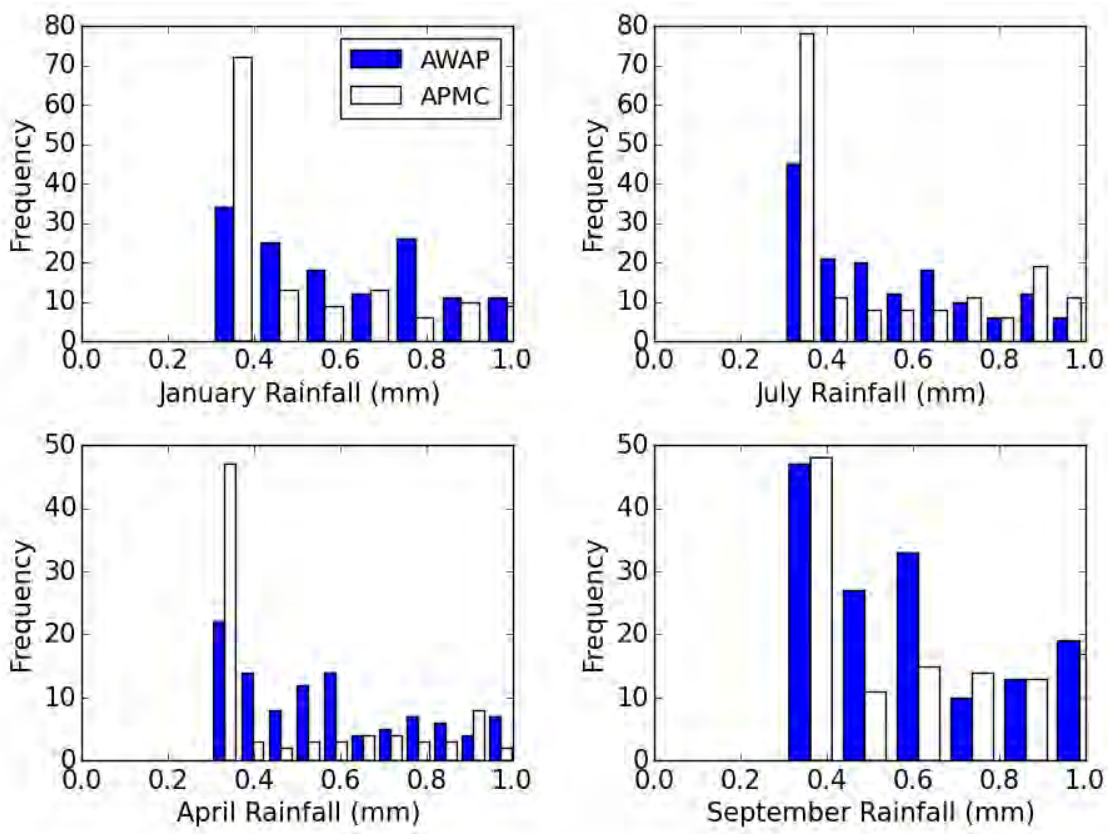

Figure B.3: Distribution of wet day rainfall depths between $0.3 \mathrm{~mm}$ and $1 \mathrm{~mm}$ in AWAP and APMC simulation for January, April, July and September (typical months).
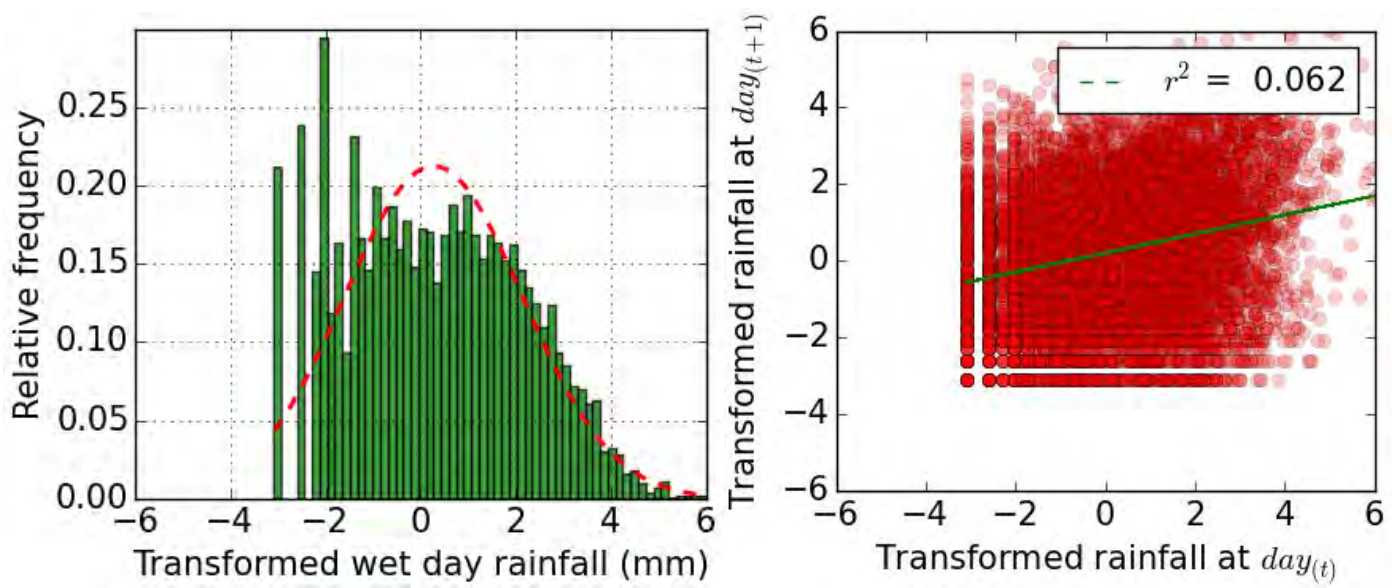

Figure B.4: Box-cox transformation of wet day rainfall depths in AWAP (left). Correlation of transformed rainfall depths in successive wet days (right). 
B. Development of MC Models

\section{B.2 DPMC Parameters for AWAP}

Figures in this appendix show the DPMC parameters calibrated to the AWAP data at Gummun.

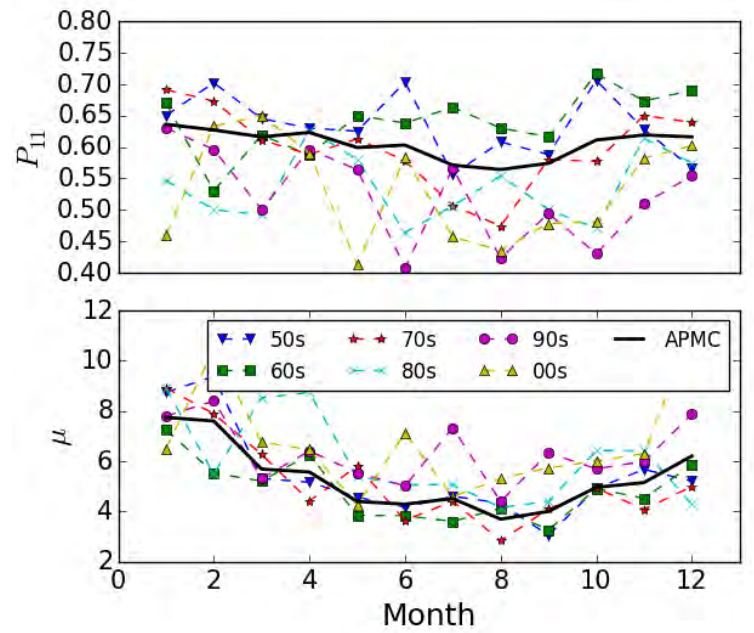

Figure B.5: Comparison of the decadal variability of the DPMC parameters (wet-to-wet probabilities $\boldsymbol{P}_{11}$ and mean wet day rainfalls $\mu$ ) with the APMC parameters.
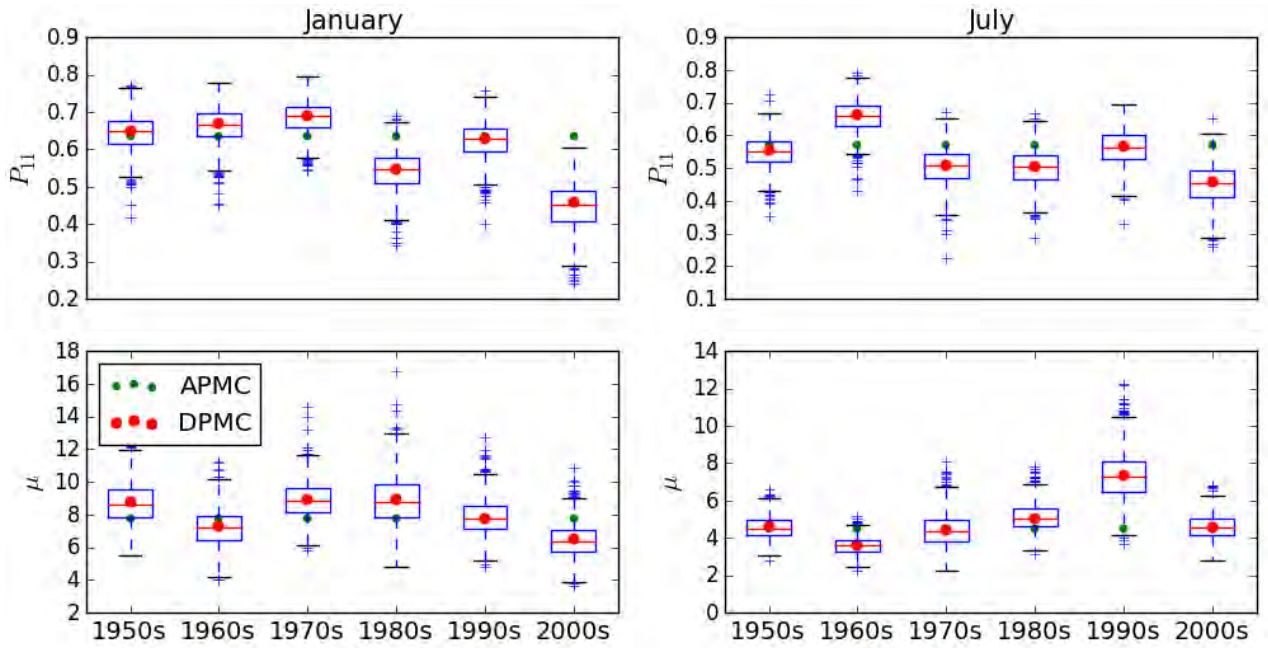

Figure B.6: Parametric bootstrapping of DPMC parameters $\left(P_{11}\right.$ and $\left.\mu\right)$ for January and July as two representative months (typical of Figure 4.12). 


\section{B.3 Potential Impact of Climate Modes}

Figures in this appendix show the parameters of DPMC-like models with parameters of 5-year sub-samples and correlations between $12 \times 60$ values of parameters and climate indices.
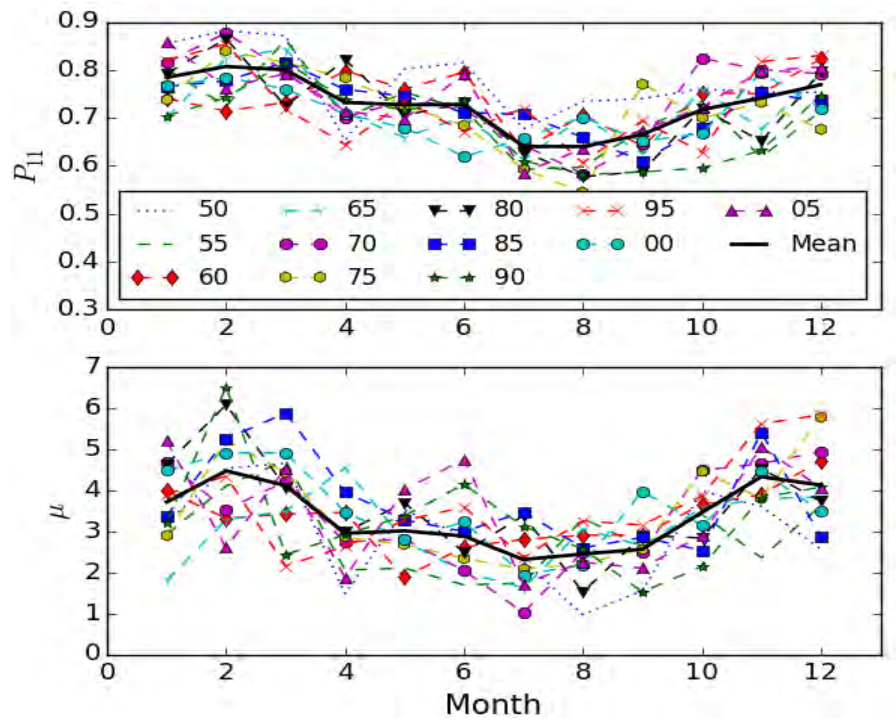

Figure B.7: Parameters $\left(P_{11}\right.$ and $\left.\mu\right)$ of 5-year sub-samples of RCM2.
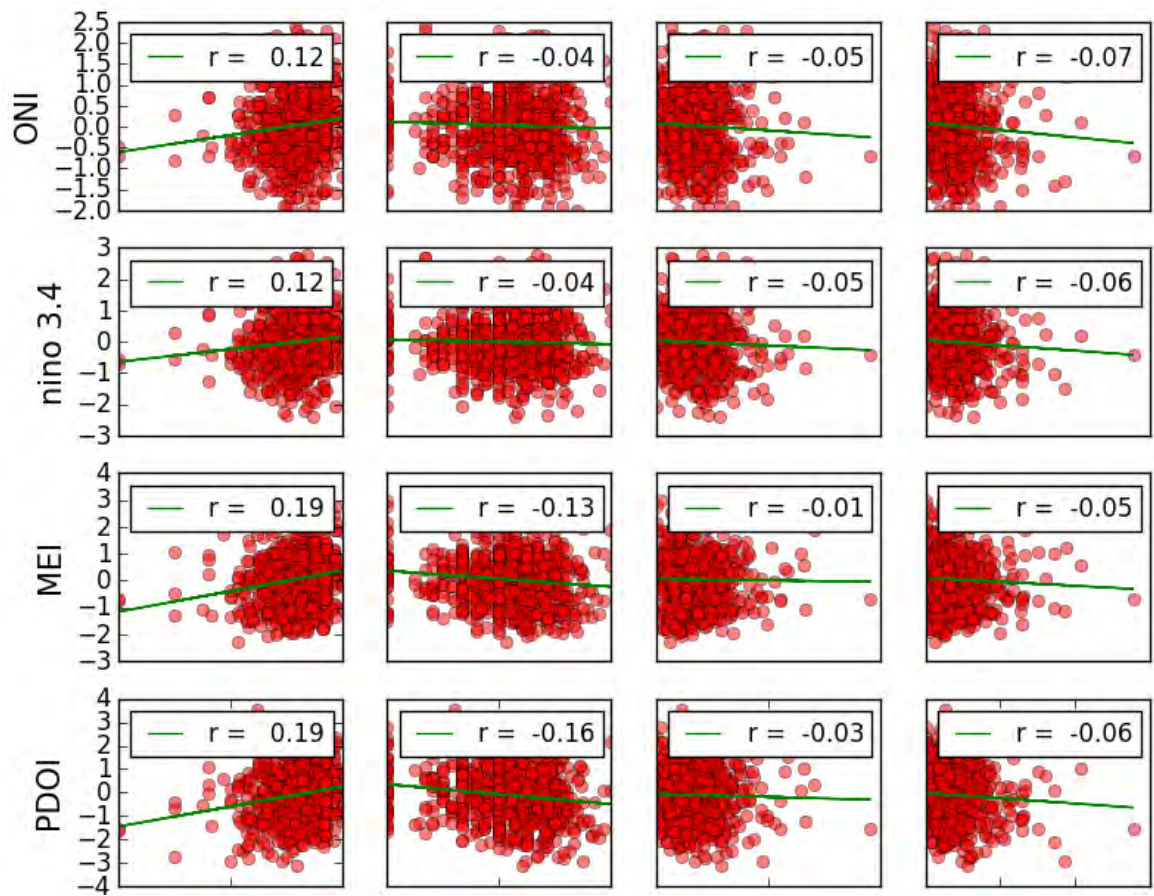

Figure B.8: Correlation between the $12 \times 60$ values of each parameter $\left(P_{00}, P_{11}, \mu\right.$, and $\left.\sigma\right)$ for AWAP and climate indices (ONI, nino3.4, MEI, and PDO). 

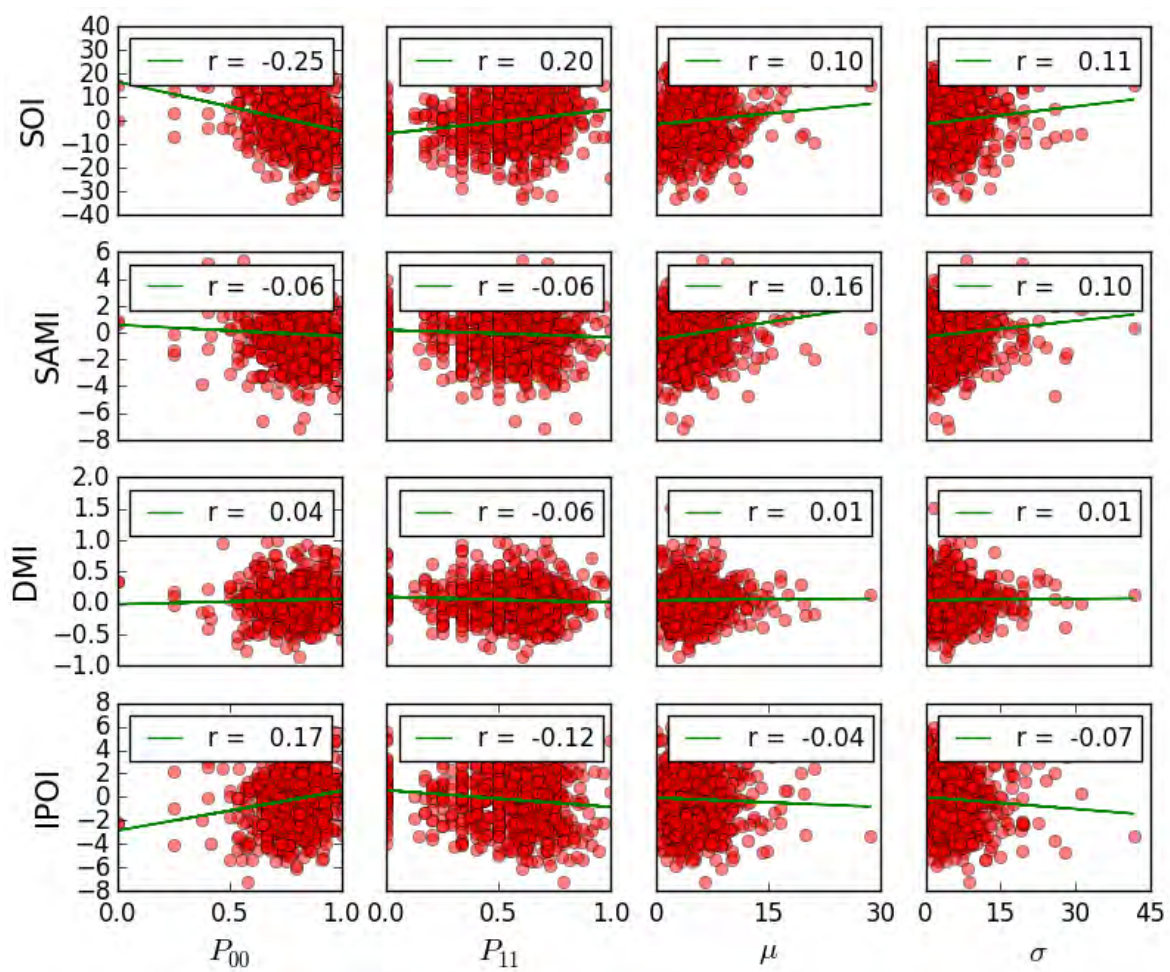

Figure B.9: Correlation between the $12 \times 60$ values of each parameter $\left(P_{00}, P_{11}, \mu\right.$, and $\left.\sigma\right)$ for AWAP and climate indices (SOI, SAMI, DMI, and IPOI).

\section{B.4 Parameters Dependence of Long-term Variability}

The APMC model with deterministic values of $P_{00, i}, P_{11, i}, \mu_{i}$, and $\sigma_{i}$ (for each month $i$ ) satisfactorily reproduces the mean of rainfall depths at all resolutions, but SD of rainfall depths at only daily resolution. The SD of rainfall depths at monthly to multiyear resolutions are underestimated by APMC. Therefore, this study has examined that the variability of which of the four parameters significantly influence the variability of rainfall depths at monthly resolution. For this examination, the parameters are calculated for each month of each year (similar to HMC). Then, the models are run using stochastic values (by sampling from uniform distributions between $5^{\text {th }}$ and $95^{\text {th }}$ percentiles of calibrated $P_{00, i}, P_{11, i}, \mu_{i}$, and $\sigma_{i}$ ) of each parameter separately as well as stochastic values of all parameters together. The simulated mean and SD of rainfall depths at daily and monthly resolutions from each model run are compared against the respective simulations of APMC (Table B.1). It shows that the stochastic variabilities of $P_{00, i}$ and $P_{11, i}$ do not significantly change the variability of simulated rainfall depths compare to the APMC simulation, while the stochastic variabilities of $\mu_{i}$, and $\sigma_{i}$ significantly increase the mean and SD of simulated rainfall depths. This suggests that the mean and SD of rainfall depths in the model is mainly regulated by the variability of Gamma parameters $\mu_{i}$, and $\sigma_{i}$, while effects of MC parameters $P_{00, i}$ and $P_{11, i}$ are marginal. This is consistent with the findings of Dubrovský et al [2004]. 
Table B.1 : Change of Mean and SD of simulated rainfall depths at daily and monthly resolutions with the change of variability of the parameters.

\begin{tabular}{lcccc}
\hline & $\begin{array}{c}\text { Daily } \\
\text { Mean }\end{array}$ & $\begin{array}{c}\text { Daily } \\
\text { SD }\end{array}$ & $\begin{array}{c}\text { Monthly } \\
\text { Mean }\end{array}$ & $\begin{array}{c}\text { Monthly } \\
\text { SD }\end{array}$ \\
\hline $\begin{array}{l}\text { With APMC values of all parameters } \\
\text { With stochastic } \boldsymbol{P}_{\mathbf{0 0}, \boldsymbol{i}} \text { and APMC of other }\end{array}$ & 2.3 & 4.5 & 41.5 & 20.5 \\
$\begin{array}{l}\text { three } \\
\text { With stochastic } \boldsymbol{P}_{\mathbf{1 1}, \boldsymbol{i}} \text { and APMC of other }\end{array}$ & 2.3 & 4.5 & 42.0 & 22.0 \\
$\begin{array}{l}\text { three } \\
\text { With stochastic } \boldsymbol{\mu}_{\boldsymbol{i}} \text { and APMC of other three }\end{array}$ & 2.8 & 5.1 & 51.0 & 21.0 \\
$\begin{array}{l}\text { With stochastic } \boldsymbol{\sigma}_{\boldsymbol{i}} \text { and APMC of other three } \\
\text { With stochastic values of all parameters }\end{array}$ & 3.2 & 7.1 & 57.0 & 30.0 \\
\hline
\end{tabular}

\section{B.5 CDMC Parameters for AWAP}

Figures in this appendix present the probability distribution and cross-correlations of CDMC parameters calibrated to AWAP data at Gummun.

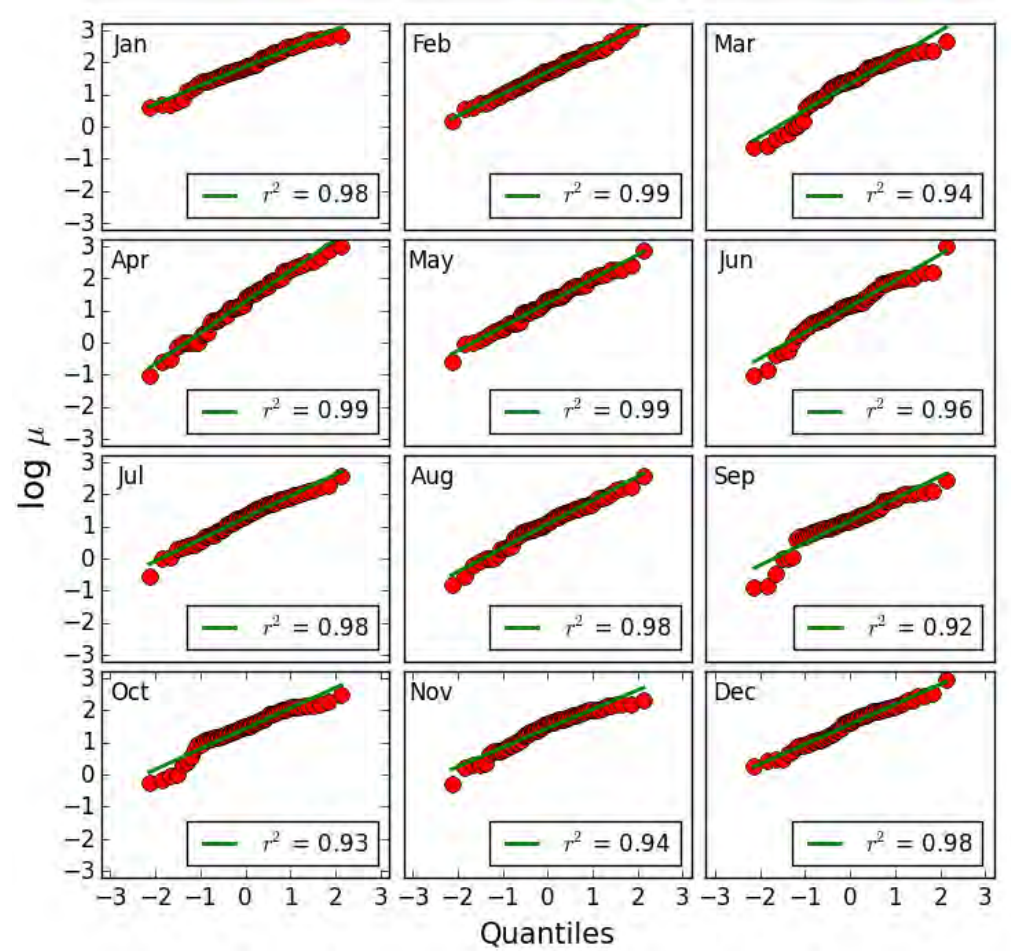

Figure B.10: Lognormal probability plots of $\boldsymbol{\mu}$ for each month for AWAP. 


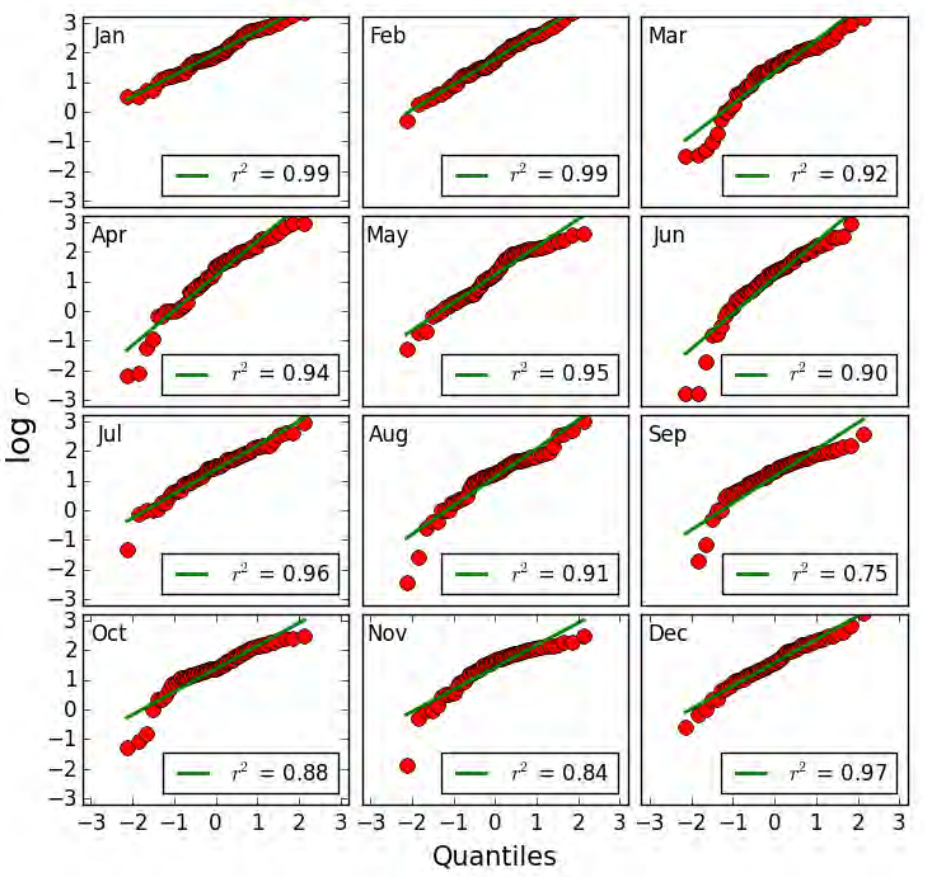

Figure B.11: Lognormal probability plots of $\sigma$ for each month for AWAP.

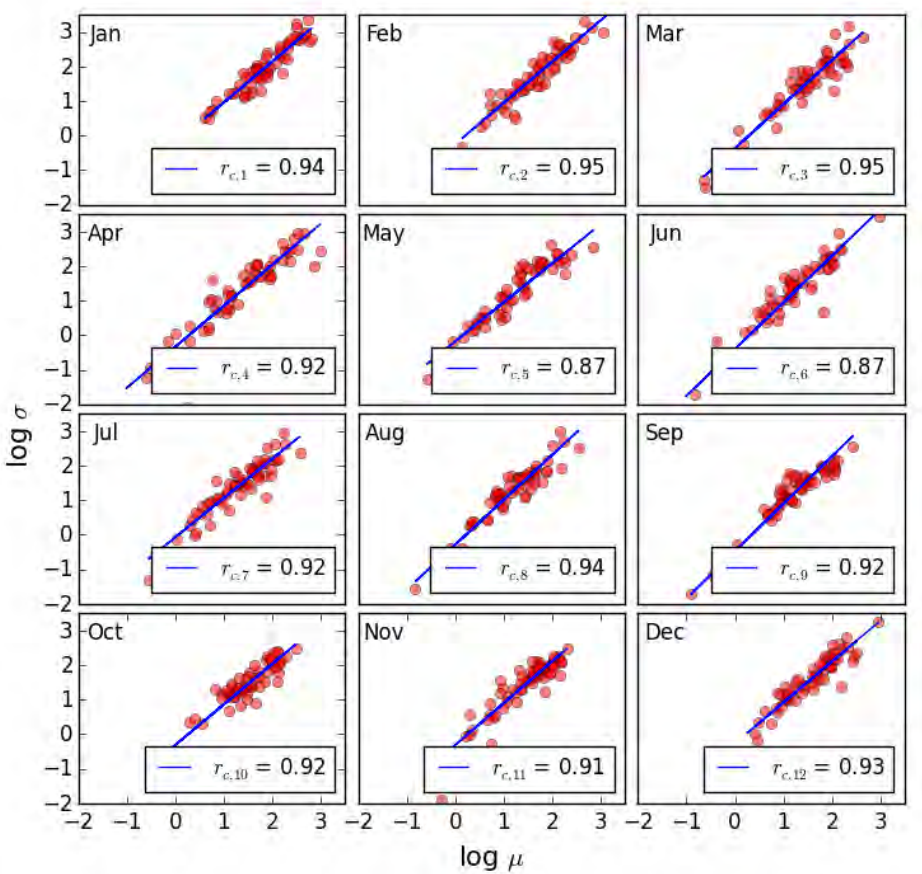

Figure B.12: Correlation between $\log \mu$ and $\log \sigma$ for each month for AWAP. 


\section{B.6 HMC Parameters for AWAP}

Figures in this appendix present the probability distribution and cross-correlations of HMC parameters calibrated to AWAP data at Gummun.

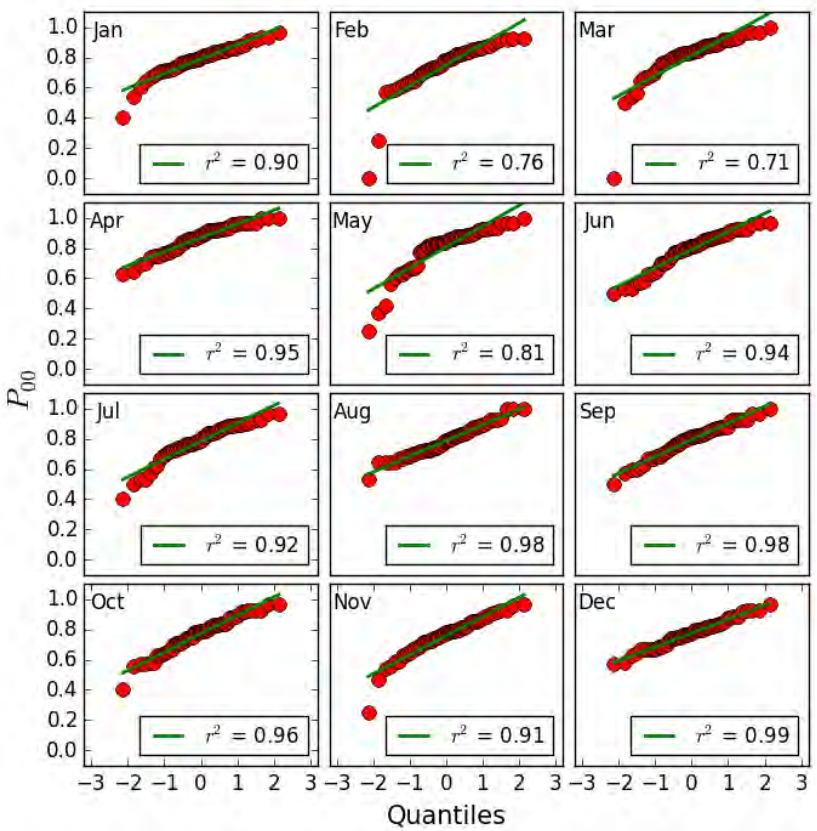

Figure B.13: Normal probability plots of $\boldsymbol{P}_{00}$ for each month for AWAP.

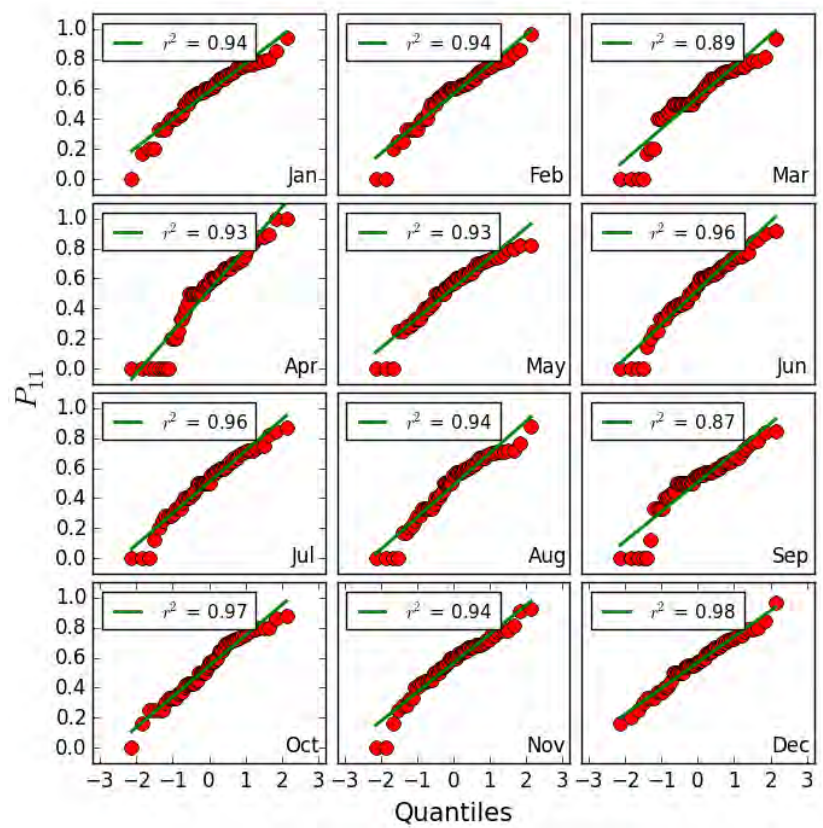

Figure B.14: Normal probability plots of $\boldsymbol{P}_{11}$ for each month for AWAP. 
B. Development of MC Models

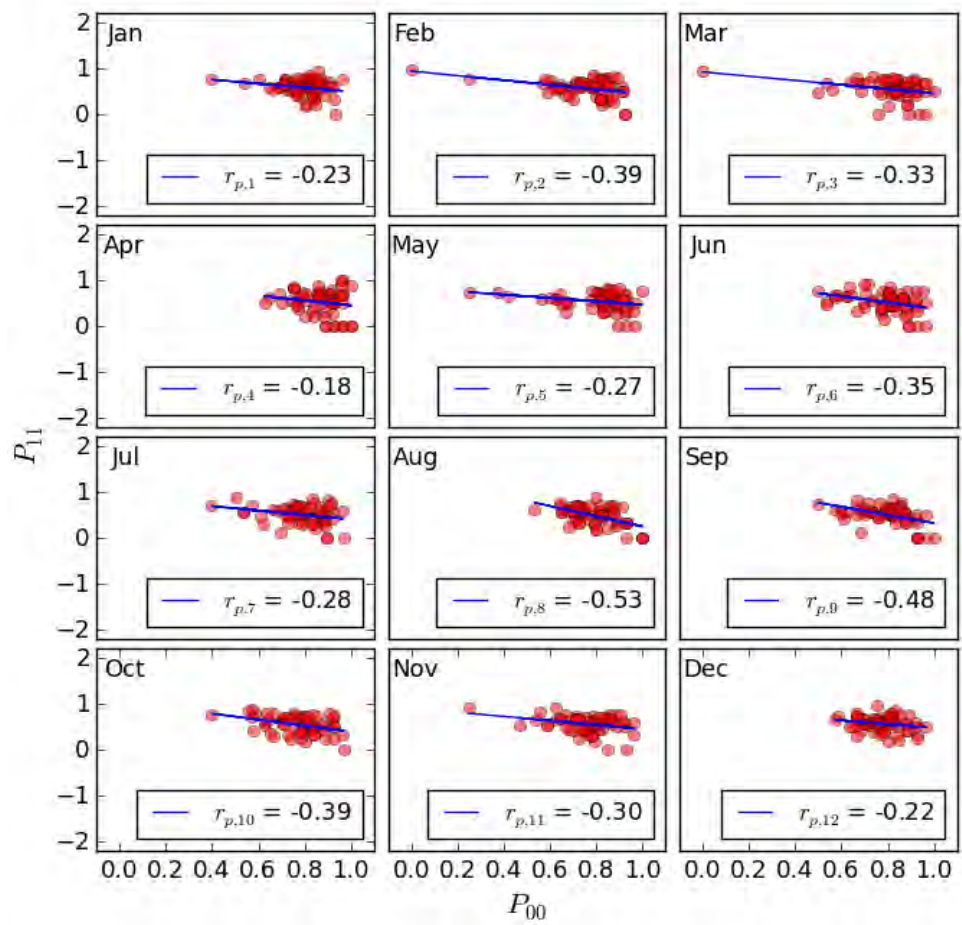

Figure B.15: Correlation between $P_{00}$ and $P_{11}$ for each month for AWAP.

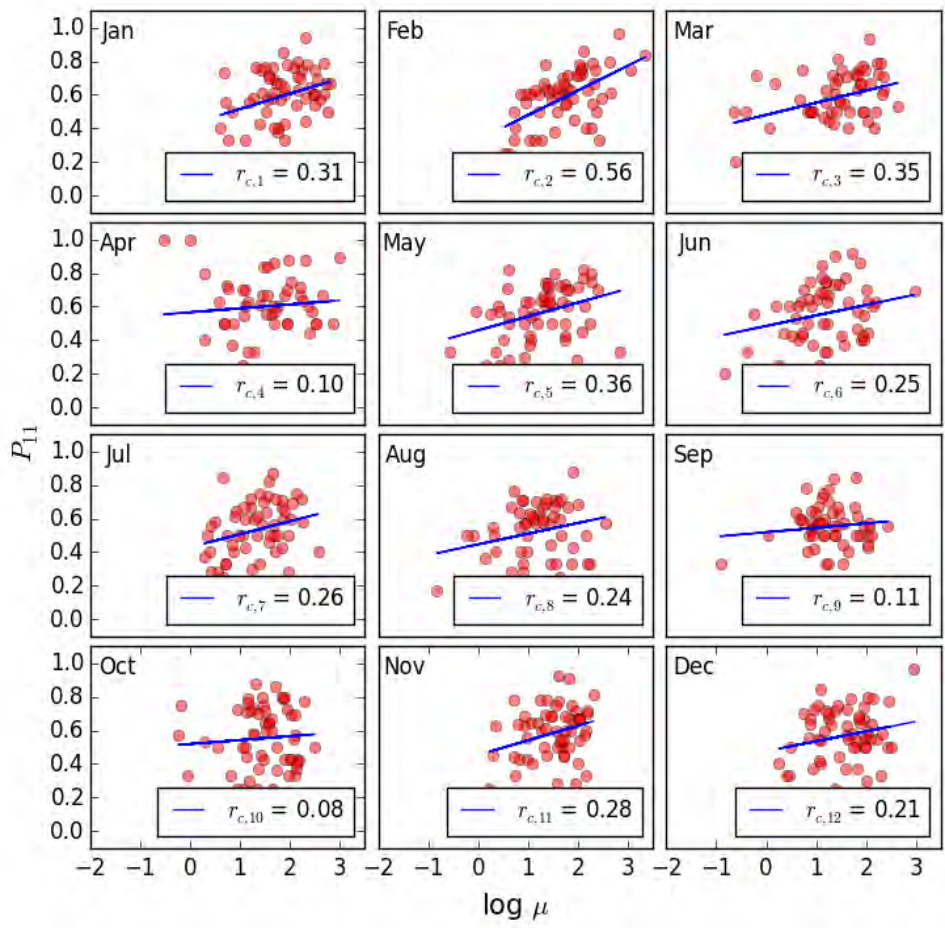

Figure B.16: Correlation between $\log \mu$ and $P_{11}$ for each month for AWAP. 


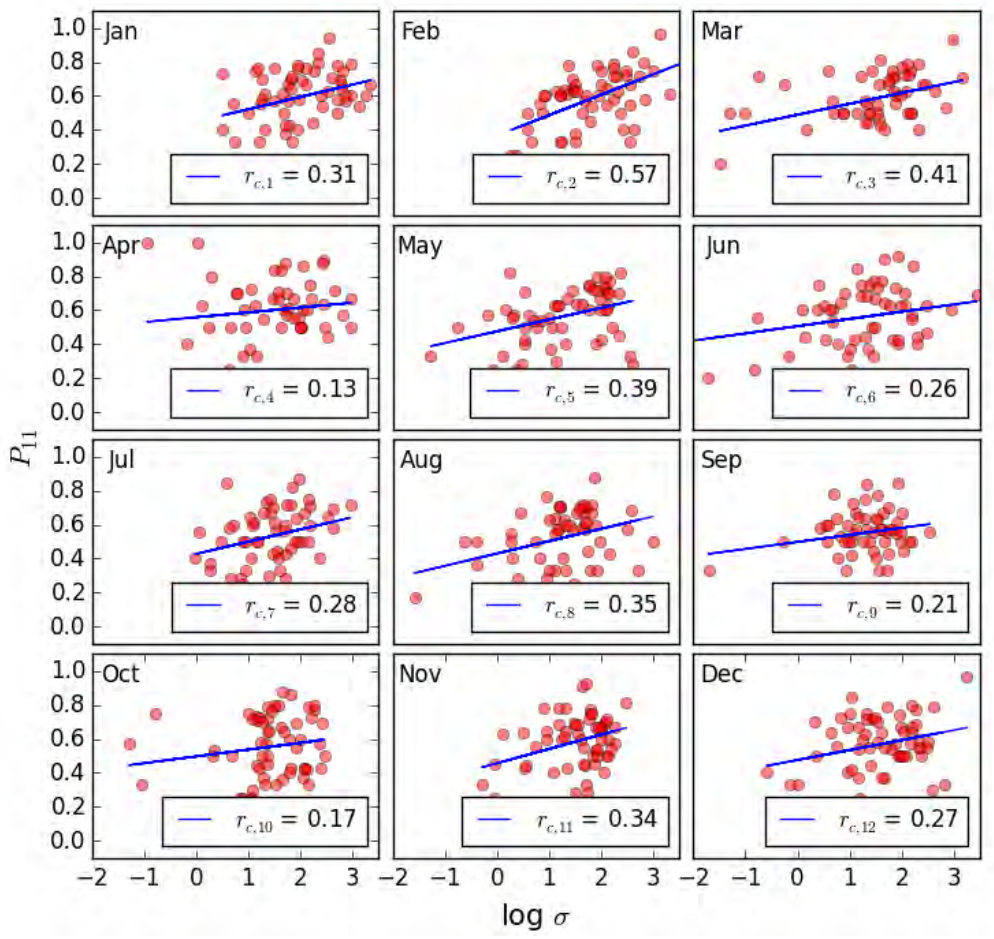

Figure B.17: Correlation between $\log \sigma$ and $P_{11}$ for each month for AWAP.

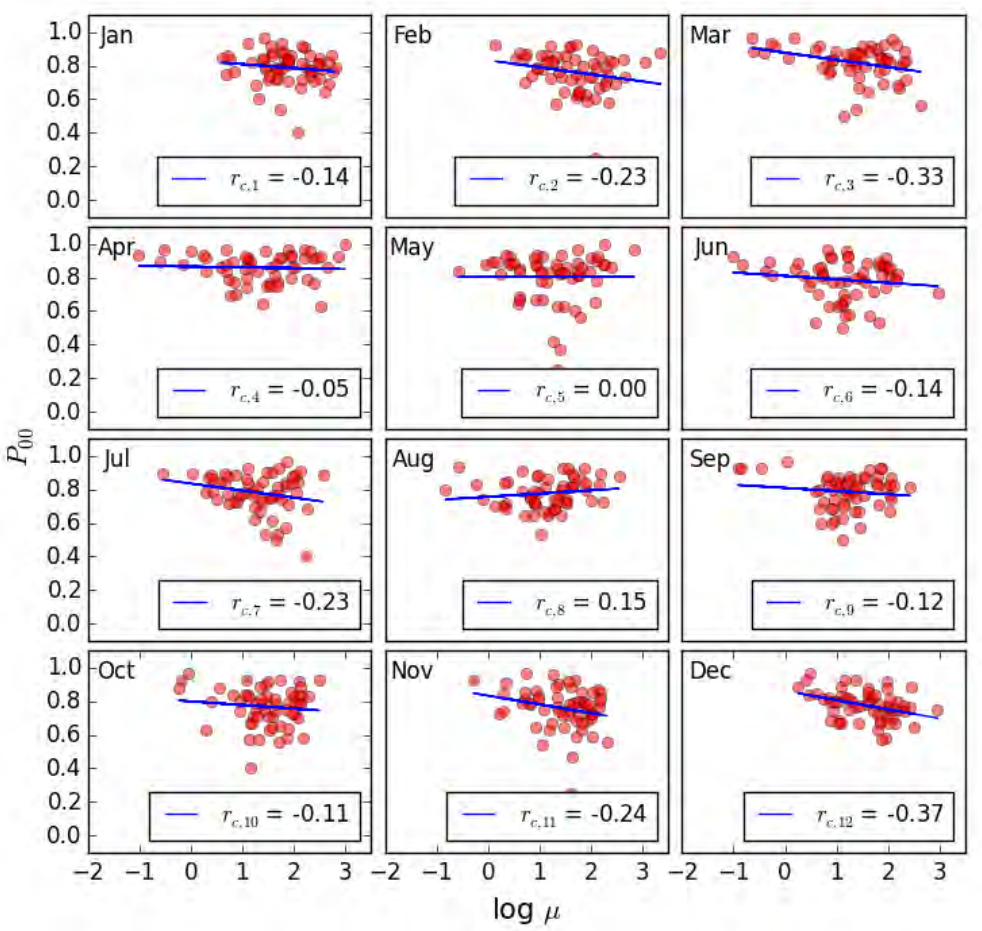

Figure B.18: Correlation between $\log \mu$ and $P_{00}$ for each month for AWAP. 


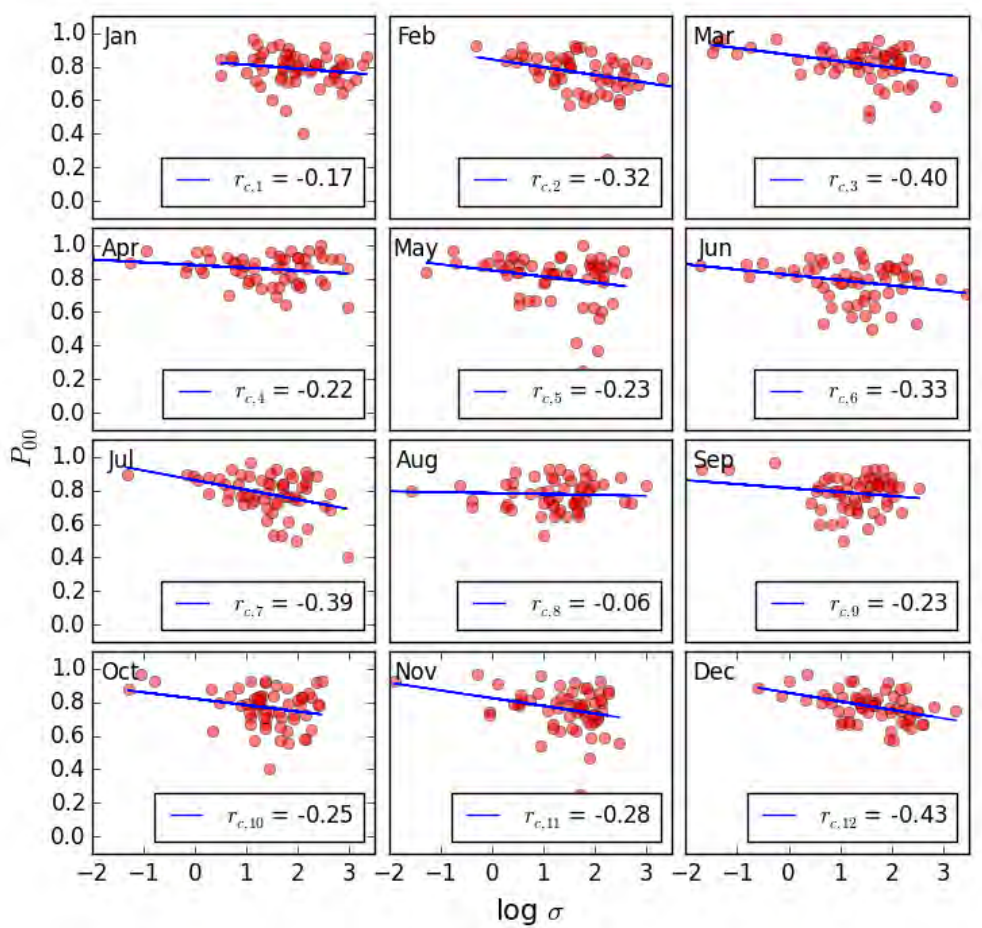

Figure B.19: Correlation between $\log \sigma$ and $\boldsymbol{P}_{00}$ for each month for AWAP. 


\section{Comparison of MC Models in Goulburn River Site}

\section{C.1 Intra-annual and Spatial Variability of Rainfall and Wet Periods}

Figures in this appendix show the intra-annual and spatial variability of the mean and SD of rainfall depths and wet periods statistics.

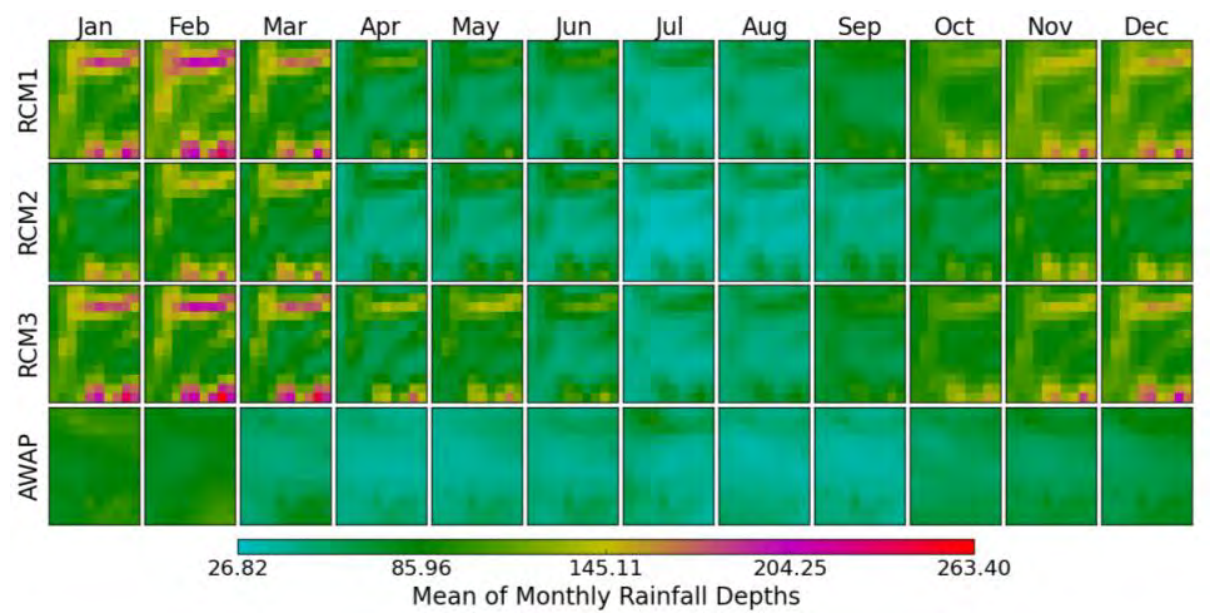

Figure C.1: Intra-annual and spatial variability of the mean of monthly rainfall depths.

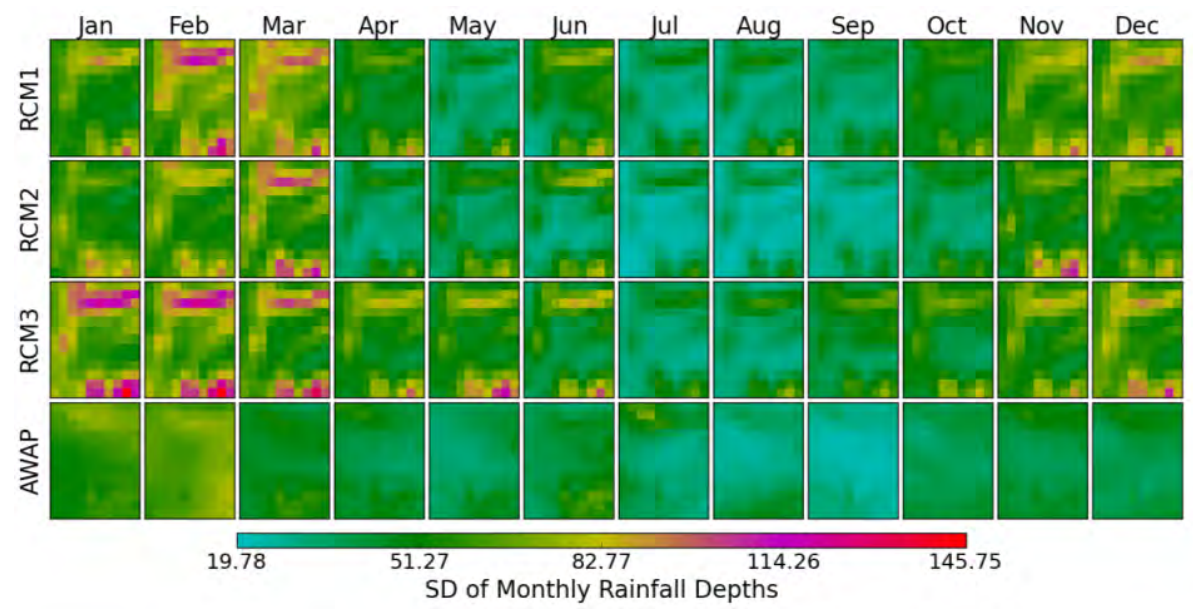

Figure C.2: Intra-annual and spatial variability of the SD of monthly rainfall depths. 


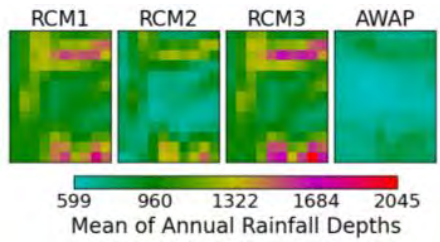

Figure C.3: Spatial variability of the mean of annual rainfall depths.

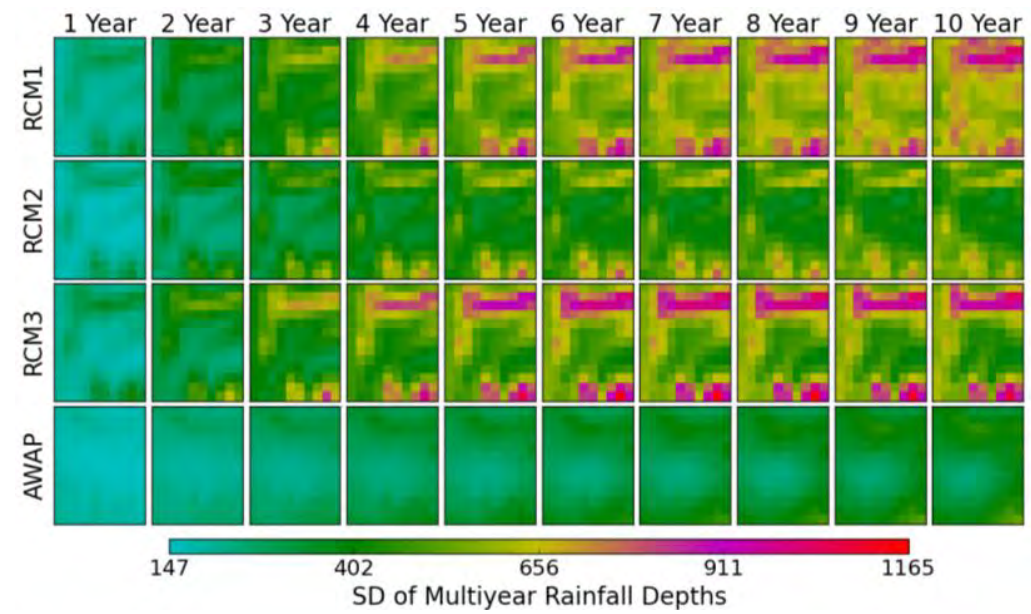

Figure C.4: Spatial variability of the SD of multiyear rainfall depths.

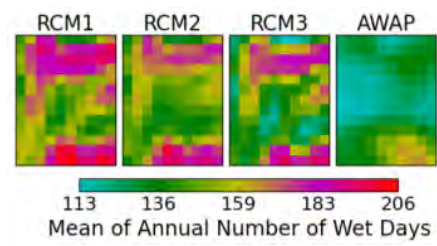

Figure C.5: Spatial variability of the mean of annual number of wet days.

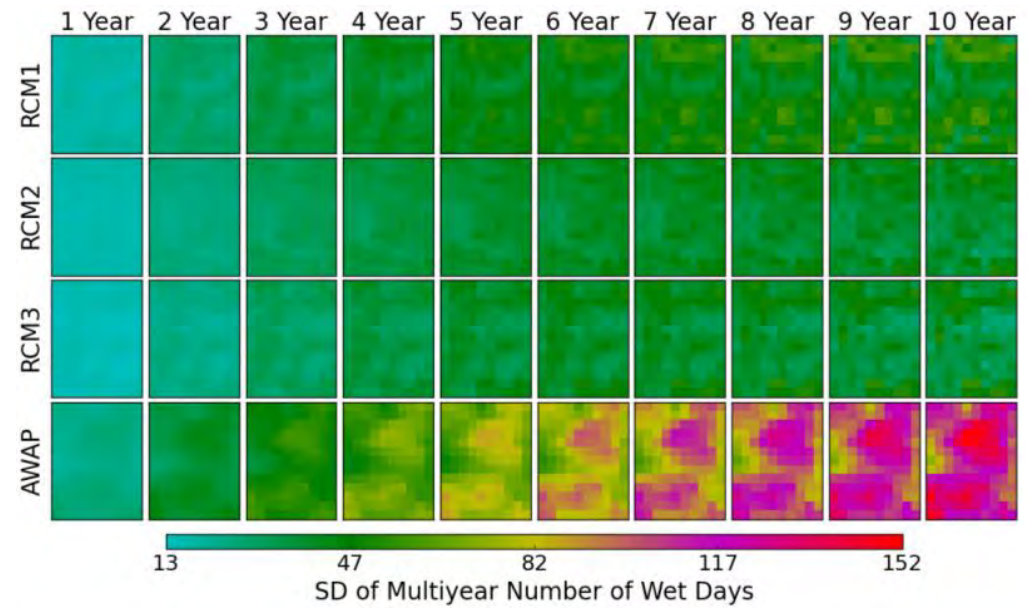

Figure C.6: Spatial variability of the SD of multiyear number of wet days. 
C. Comparison of MC Models in Goulburn River Site

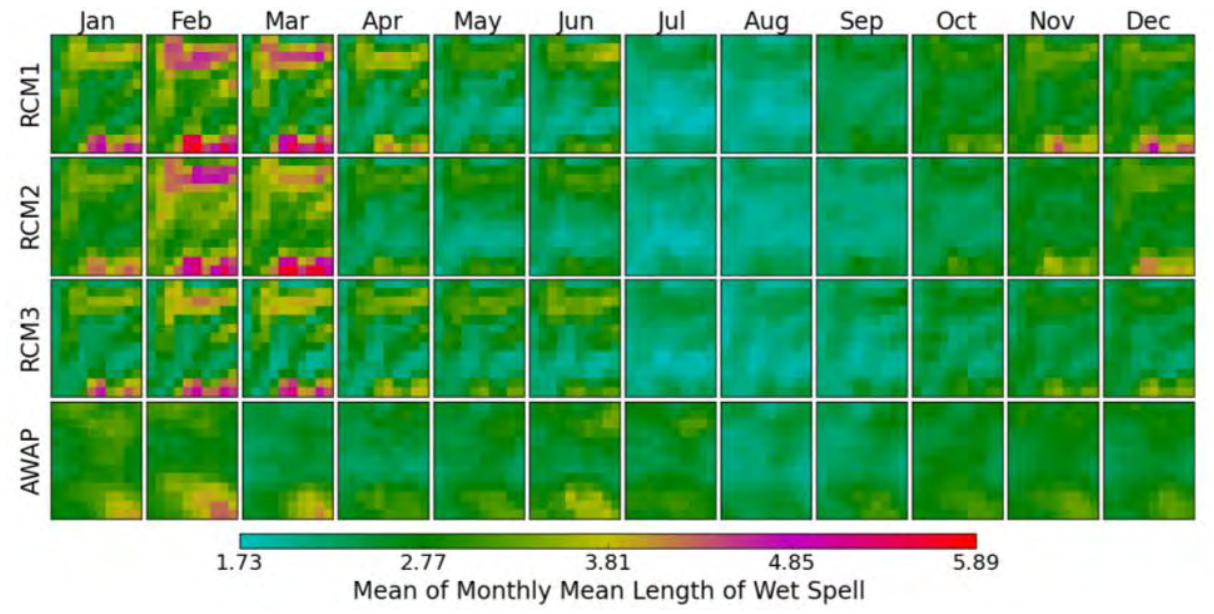

Figure C.7: Intra-annual and spatial variability of the mean of monthly mean length of wet spells.

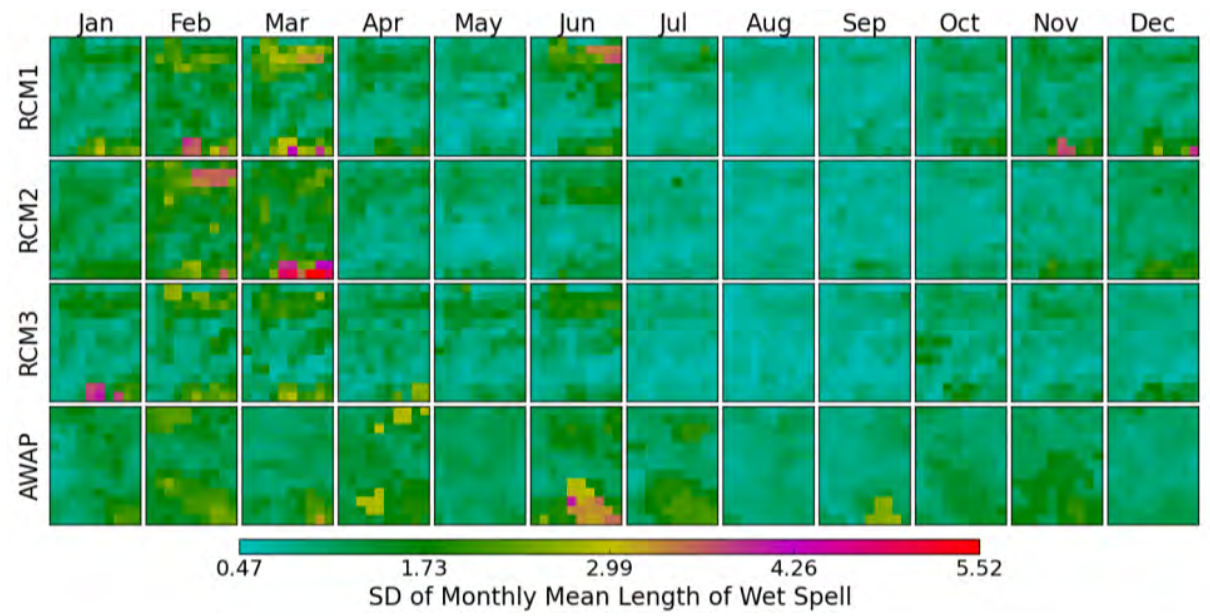

Figure C.8: Intra-annual and spatial variability of the SD of monthly mean length of wet spells.

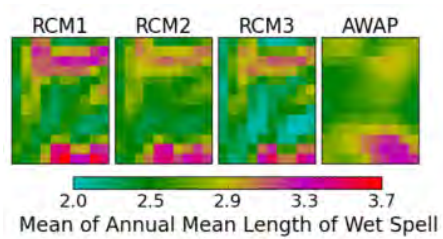

Figure C.9: Spatial variability of the mean of annual mean length of wet spells.

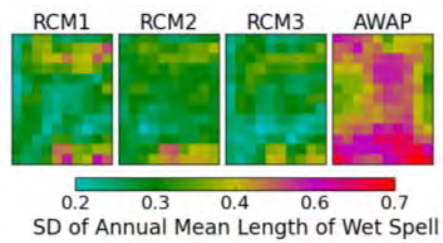

Figure C.10: Spatial variability of the SD of annual mean length of wet spells. 


\section{C.2 Comparison of MC Models for Distribution of Rainfall Depths}

Figures of this appendix compare the MC models for the mean and SD of rainfall depths.
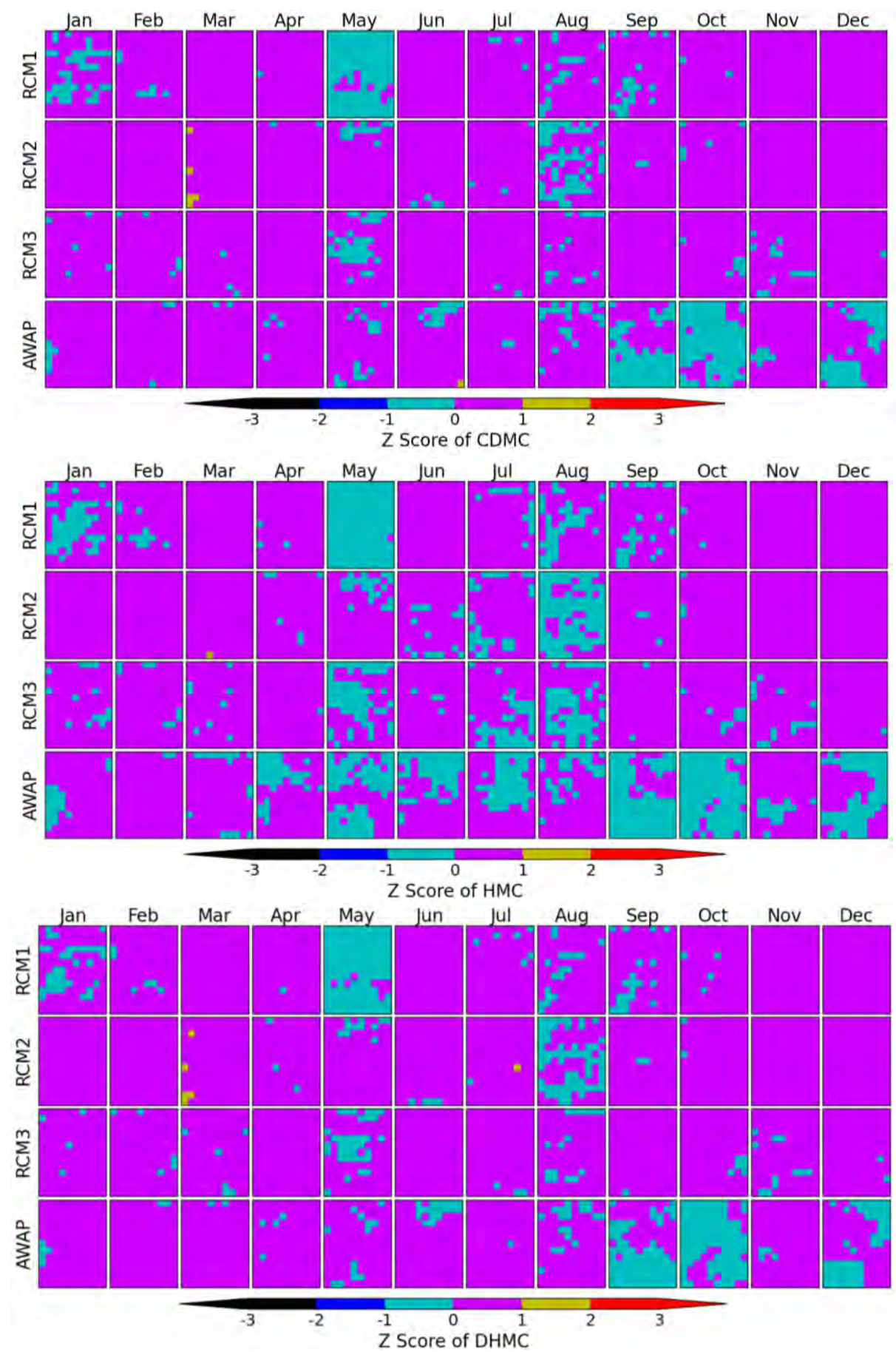

Figure C.11: Comparison of CDMC, HMC and DHMC for the mean of monthly rainfall depths. 

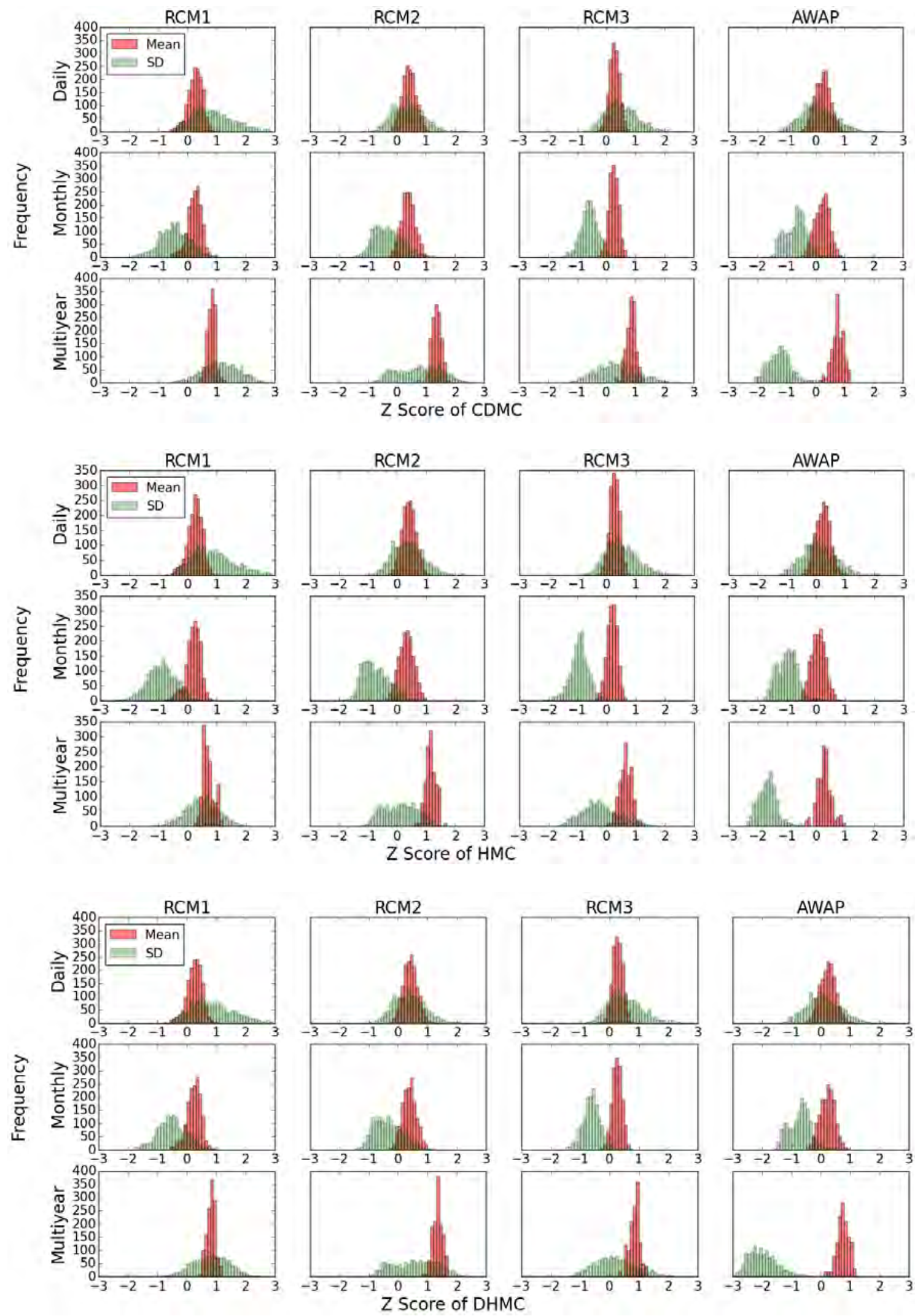

Figure C.12: Distribution of $Z$ scores of CDMC, HMC and DHMC for the mean and SD of rainfall depths at all resolutions. 


\section{C.3 Comparison of MC Models for Distribution of Wet Period Statistics}

Figures of this appendix compare the MC models for distribution of wet period statistics.
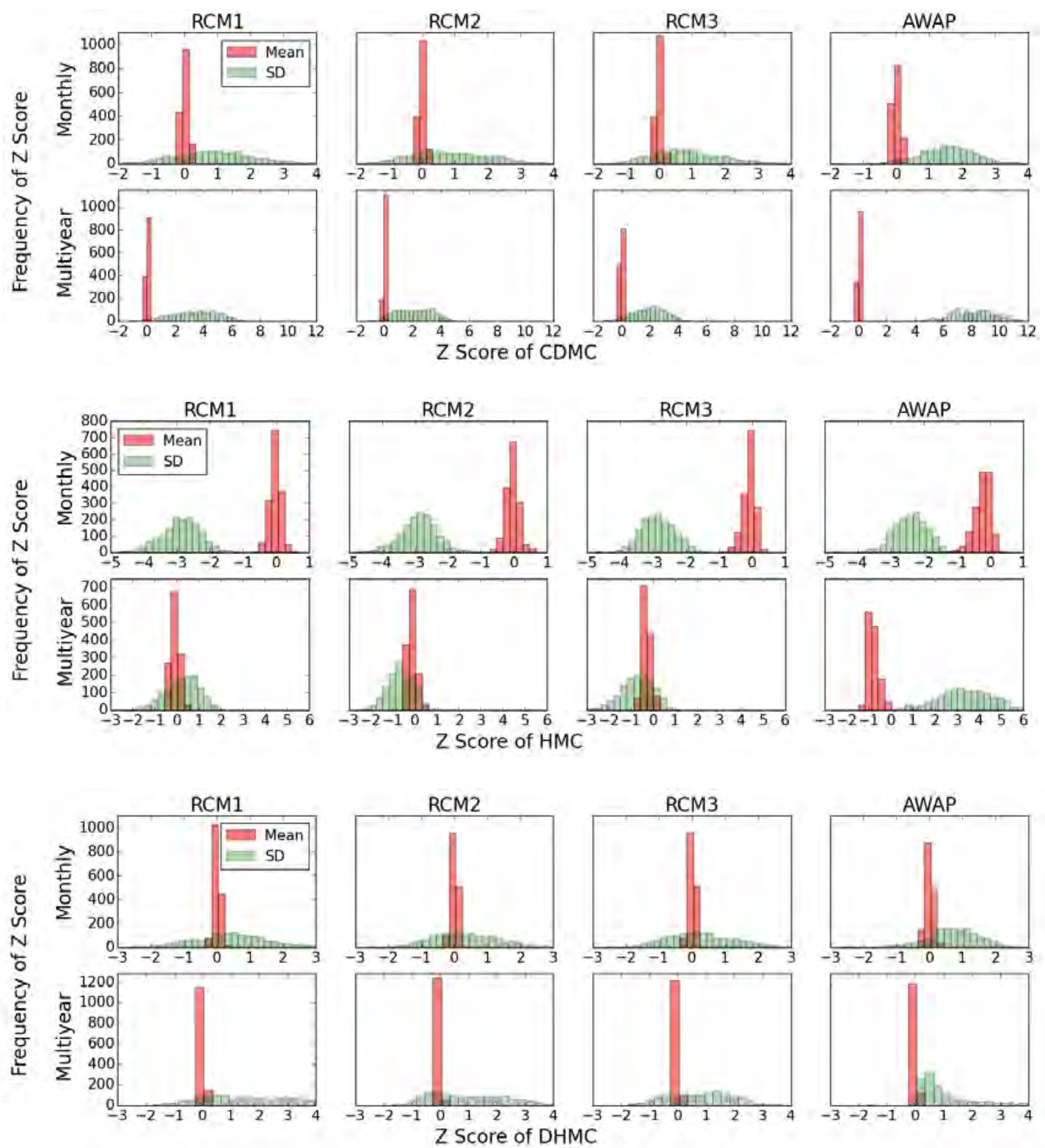

Figure C.13: Overall comparison of CDMC, HMC and DHMC for the mean and SD of number of wet days at monthly and multiyear resolutions. 
C. Comparison of MC Models in Goulburn River Site
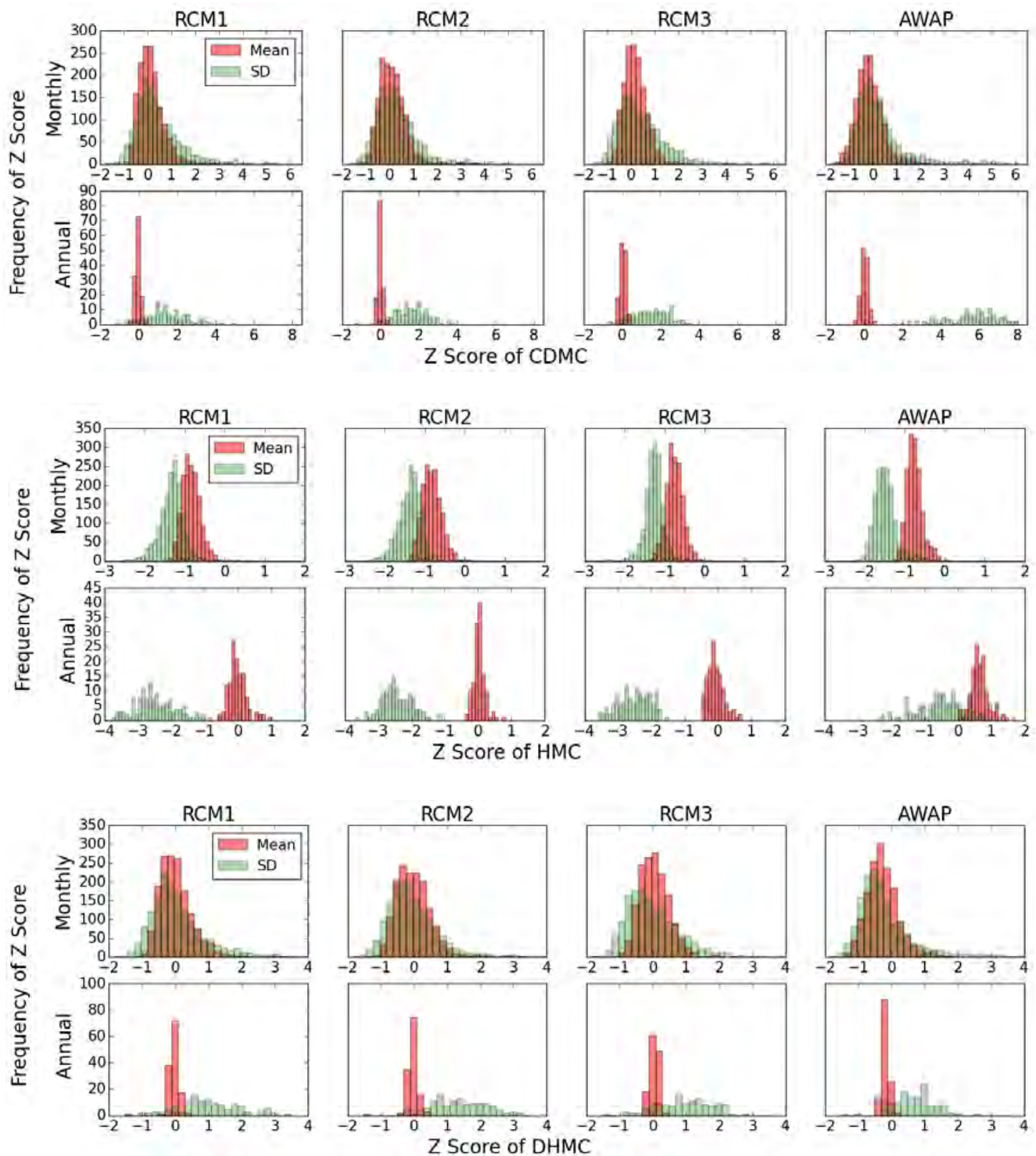

Figure C.14: Overall comparison of CDMC, HMC and DHMC for the mean and SD of mean length of wet spells at monthly and annual resolutions. 


\section{Comparison of MC Models in Williams River Site}

\section{D.1 Intra-annual and Spatial Variability of Rainfall and Wet Periods}

Figures in this appendix show the intra-annual and spatial variability of the mean and SD of rainfall depths and wet periods statistics.

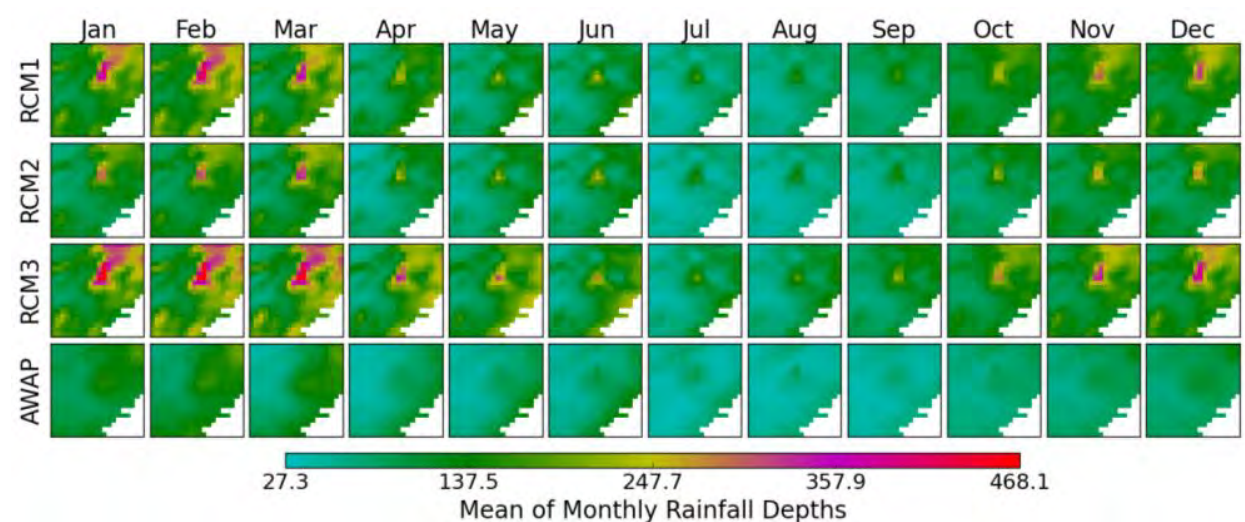

Figure D.1: Intra-annual and spatial variability of the mean of monthly rainfall depths.

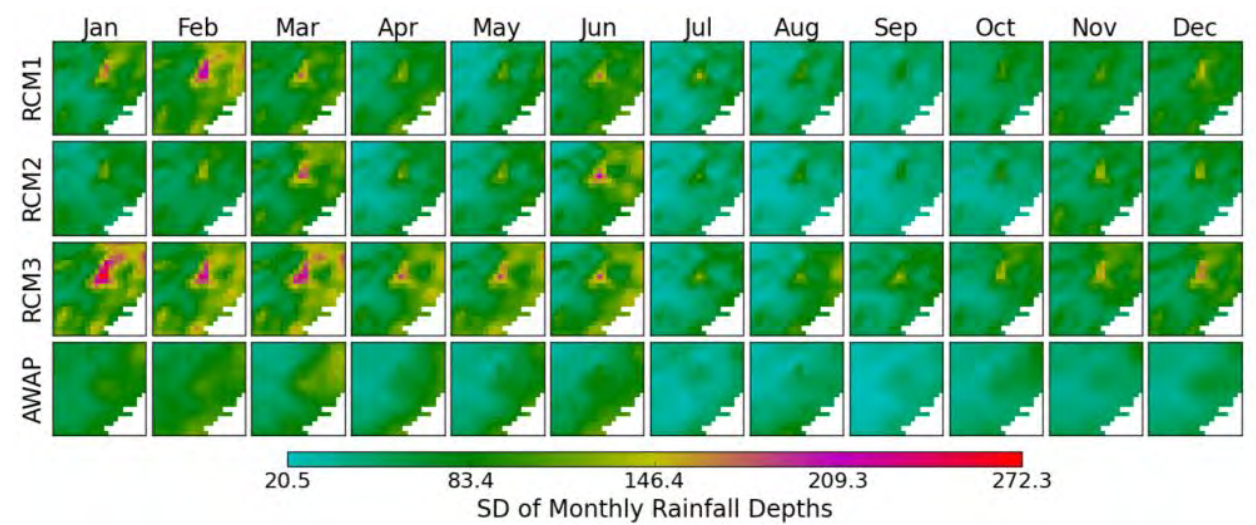

Figure D.2: Intra-annual and spatial variability of the SD of monthly rainfall depths.

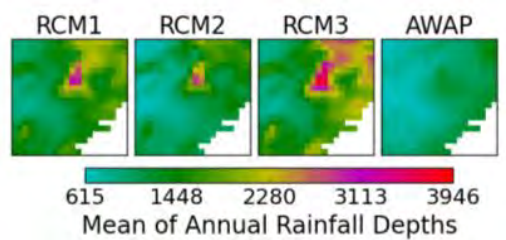

Figure D.3: Spatial variability of the mean of annual rainfall depths. 


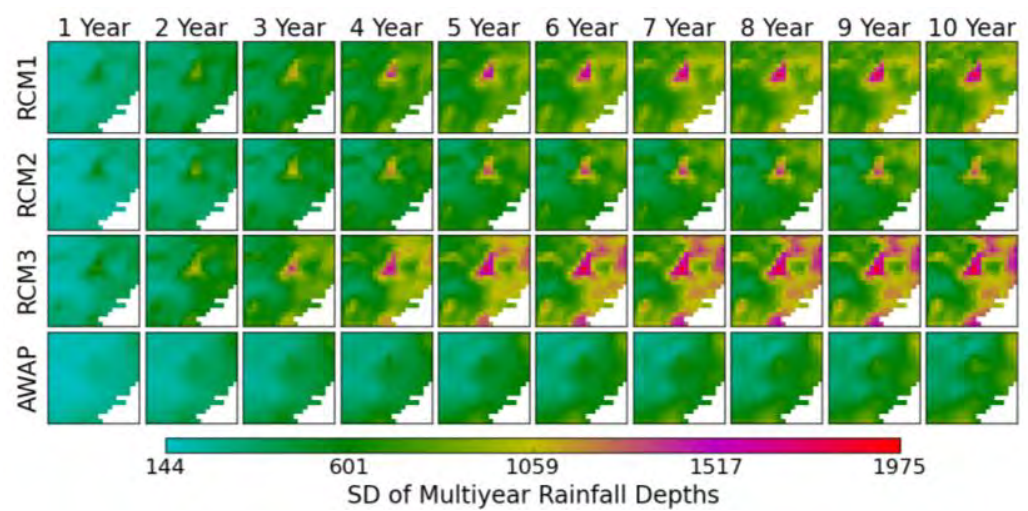

Figure D.4: Spatial variability of the SD of multiyear rainfall depths.
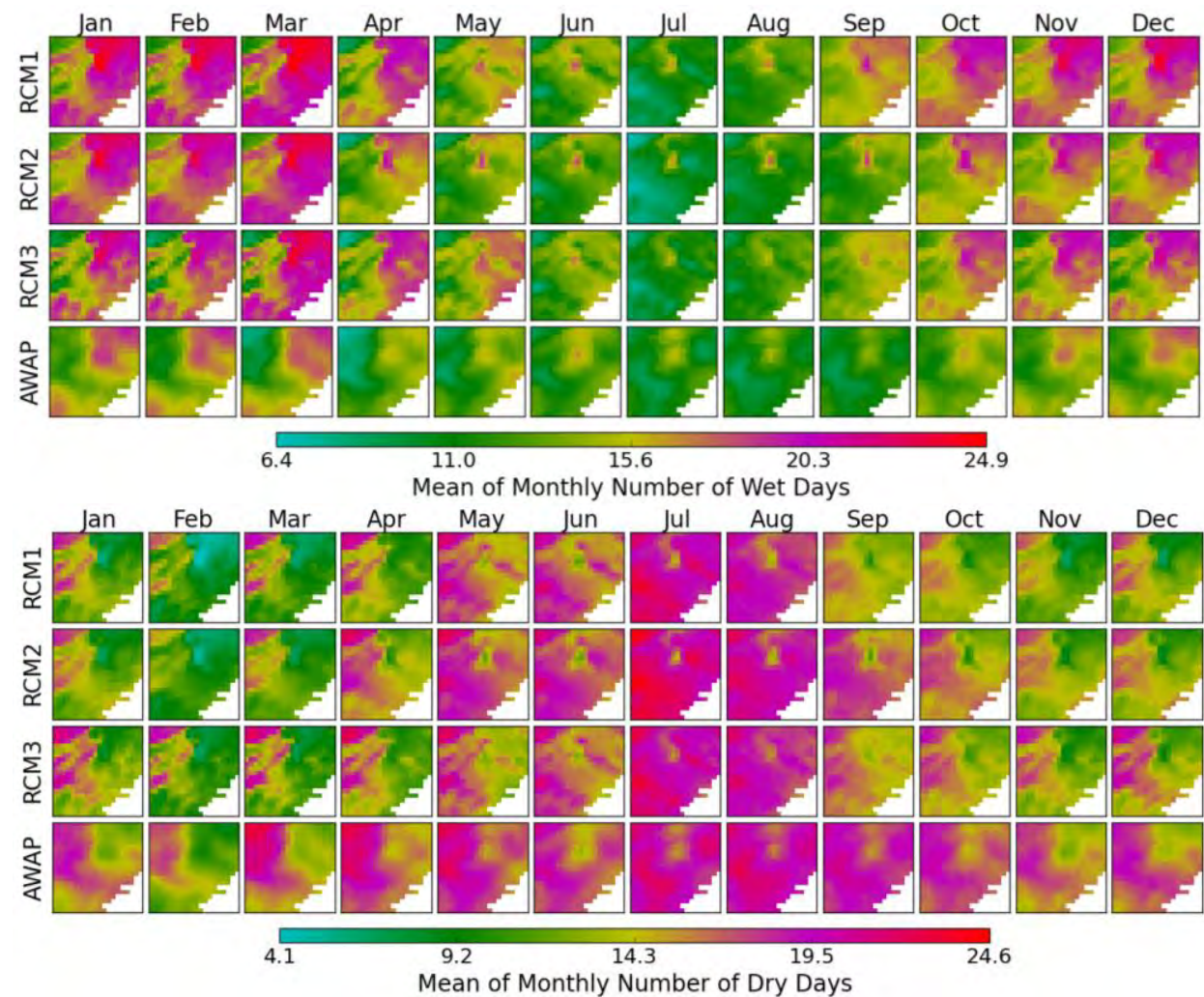

Figure D.5: Intra-annual and spatial variability of the mean of monthly number of wet and dry days. 

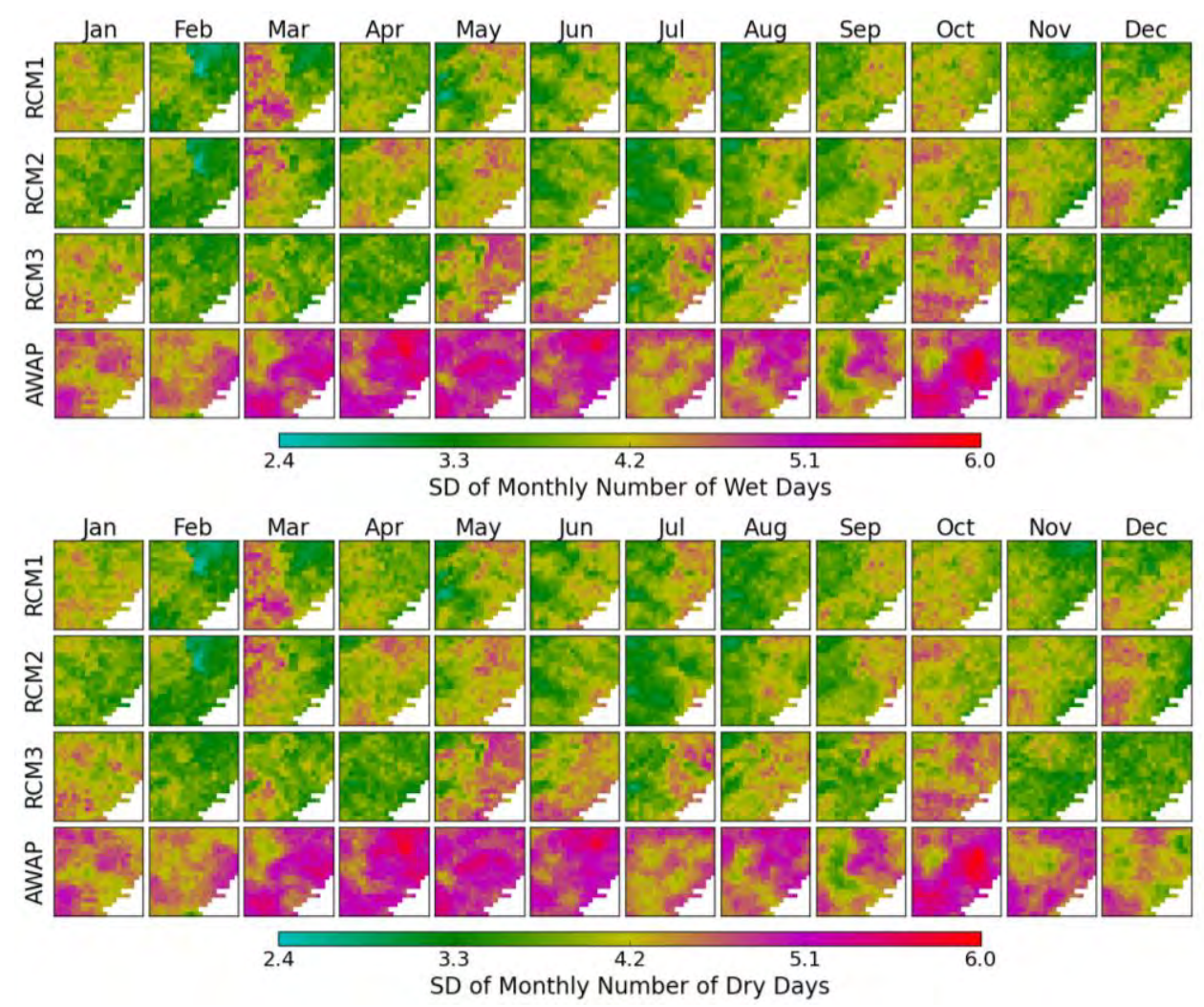

Figure D.6: Intra-annual and spatial variability of the SD of monthly number of wet and dry days.
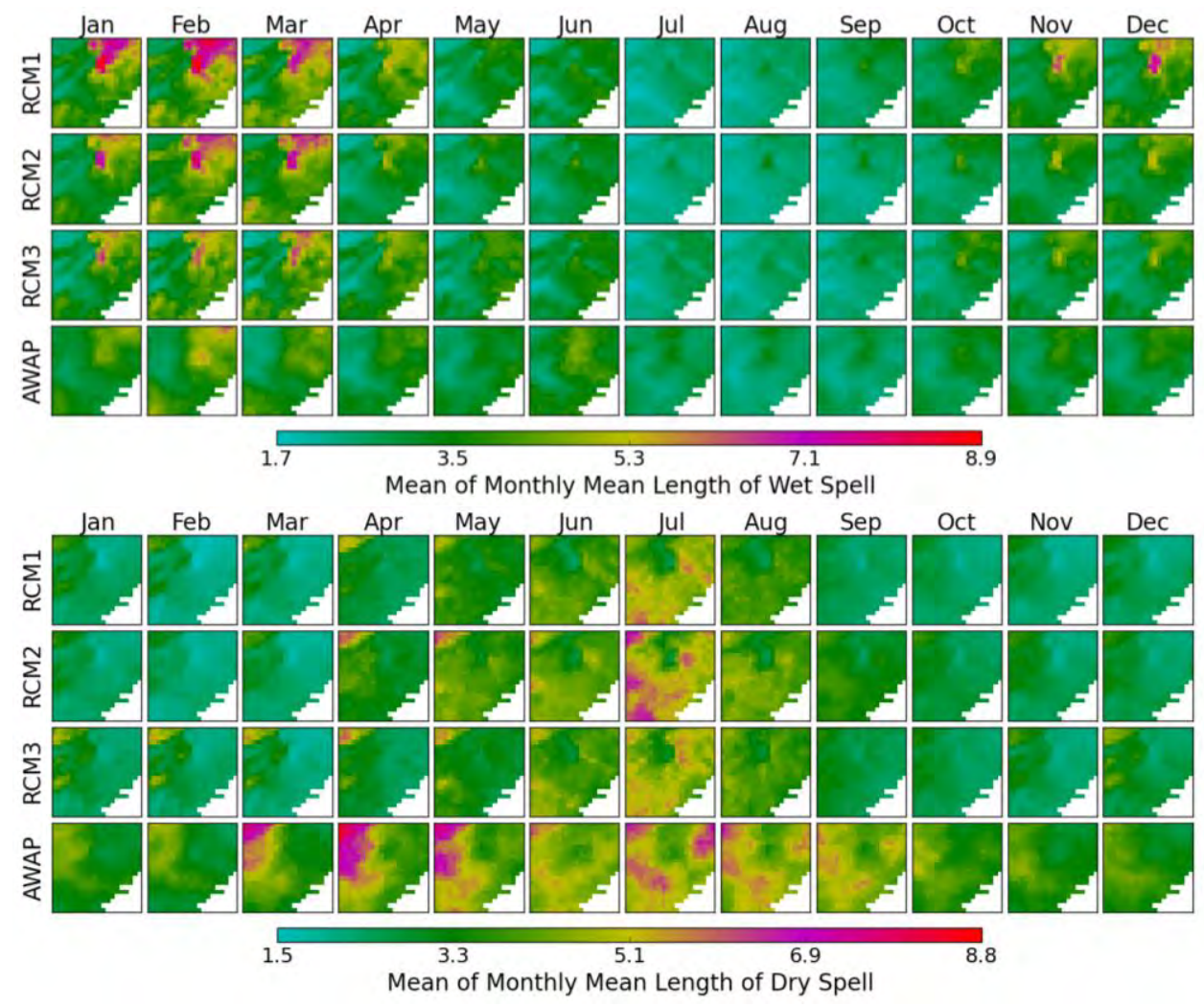

Figure D.7: Intra-annual and spatial variability of the mean of monthly mean length of wet and dry spells. 

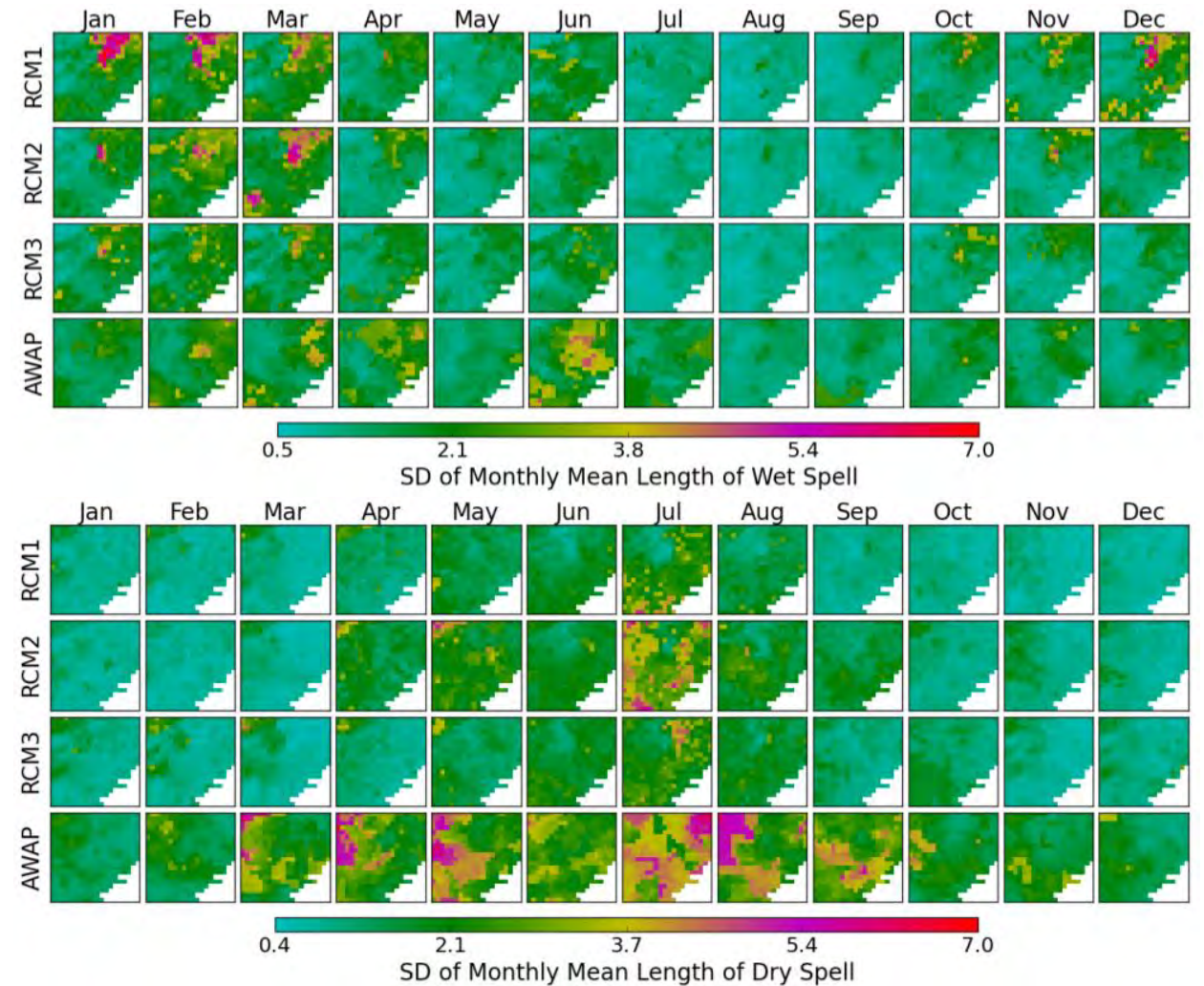

Figure D.8: Intra-annual and spatial variability of the SD of monthly mean length of wet and dry spells.
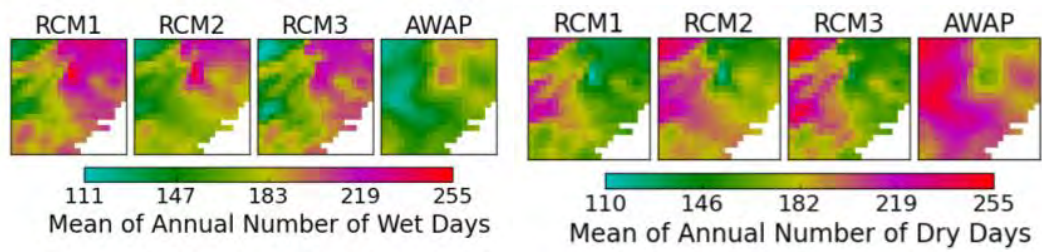

Figure D.9: Spatial variability of the mean of annual number of wet and dry days.
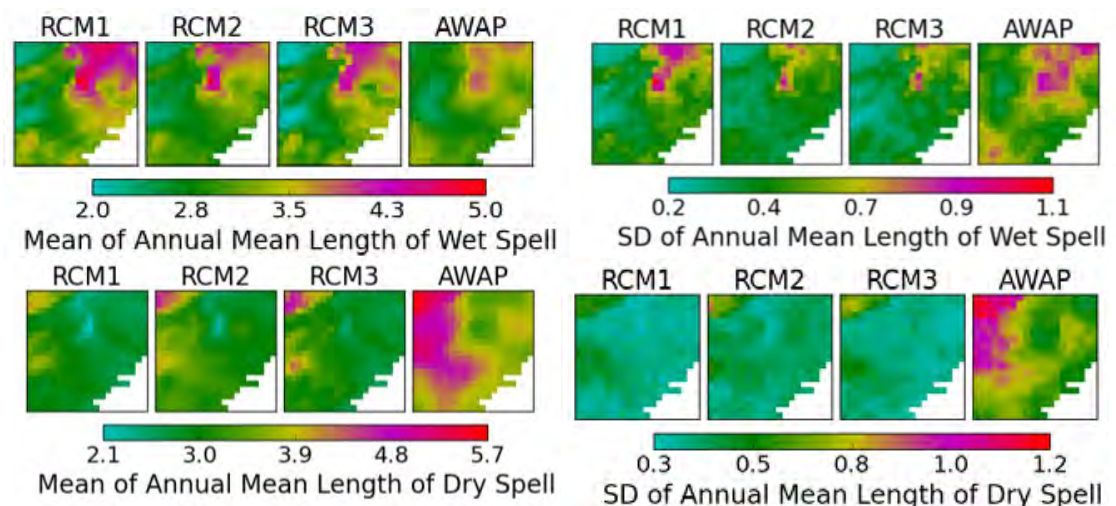

Figure D.10: Spatial variability of the mean and SD of annual mean length of wet and dry spells. 

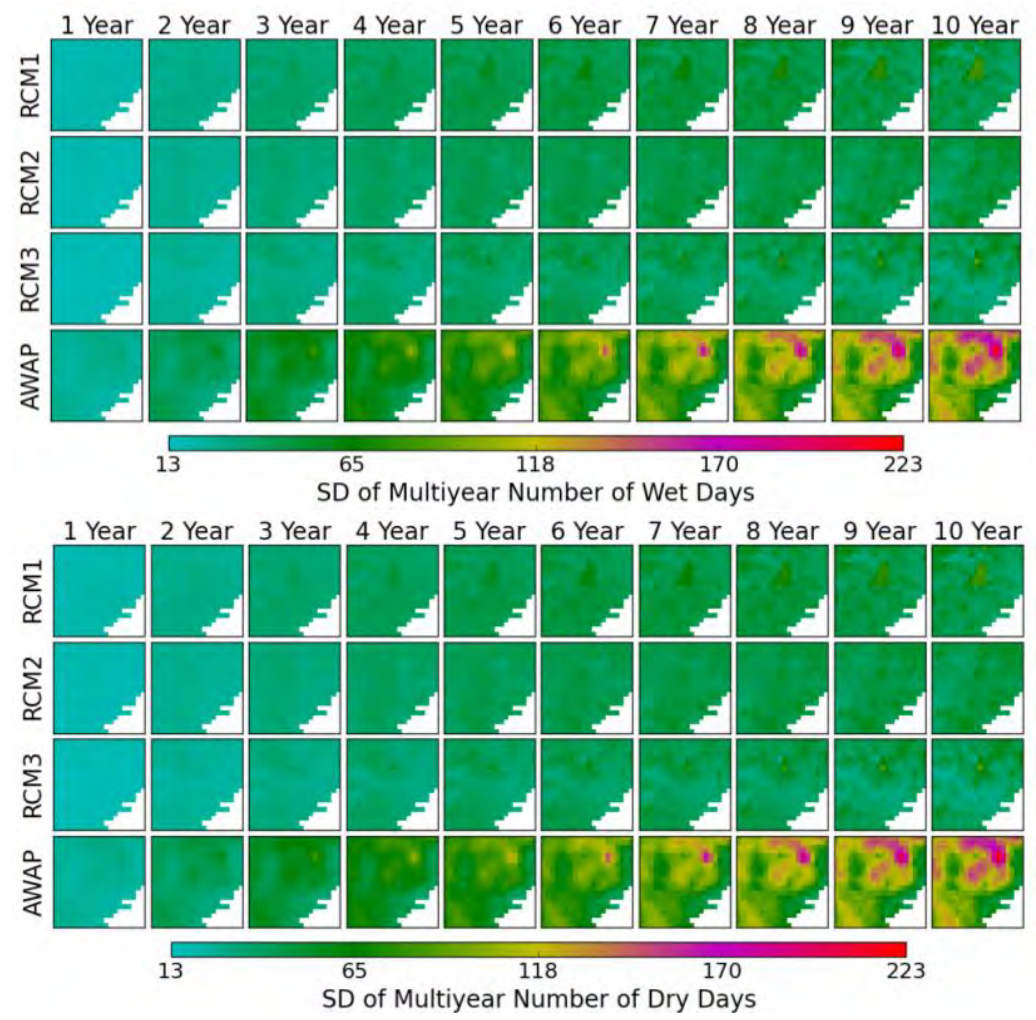

Figure D.11: Spatial variability of the SD of multiyear number of wet and dry days. 


\section{D.2 Comparison of MC Models for Distribution of Rainfall Depths}

Figures of this appendix compare the MC models for the mean and SD of rainfall depths.
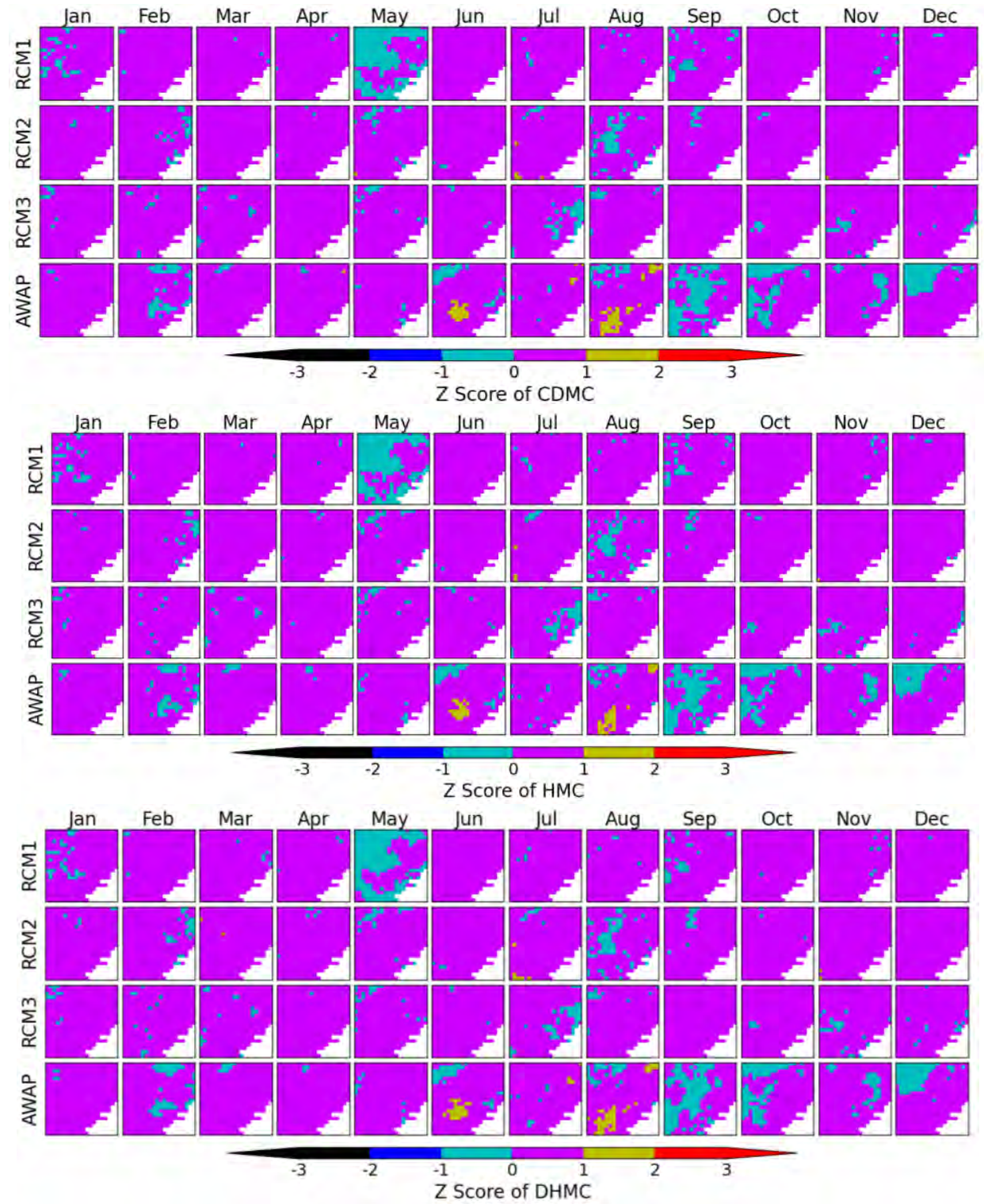

Figure D.12: Comparison of CDMC, HMC and DHMC for the mean of daily rainfall depths. 

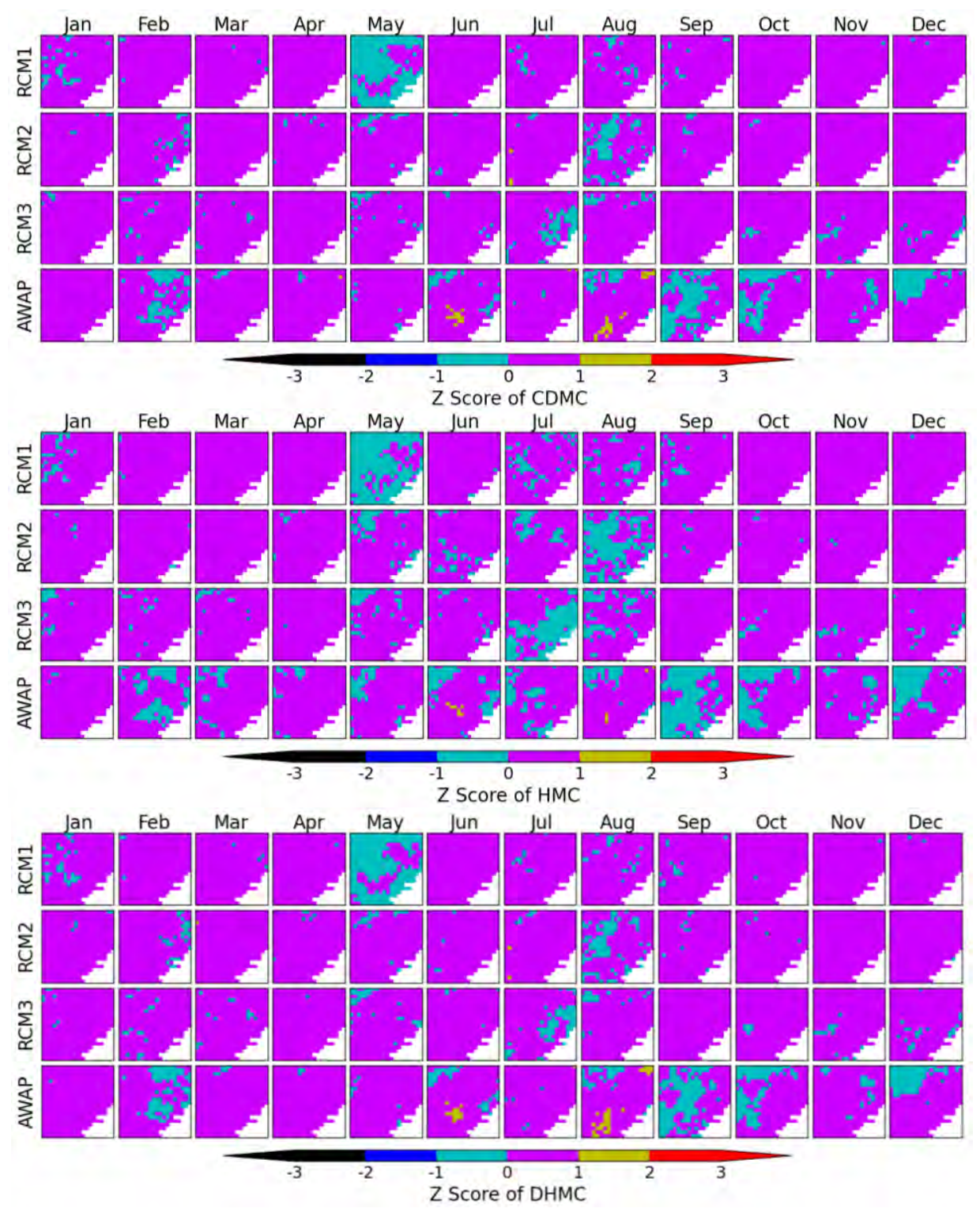

Figure D.13: Comparison of CDMC, HMC and DHMC for the mean of monthly rainfall depths.

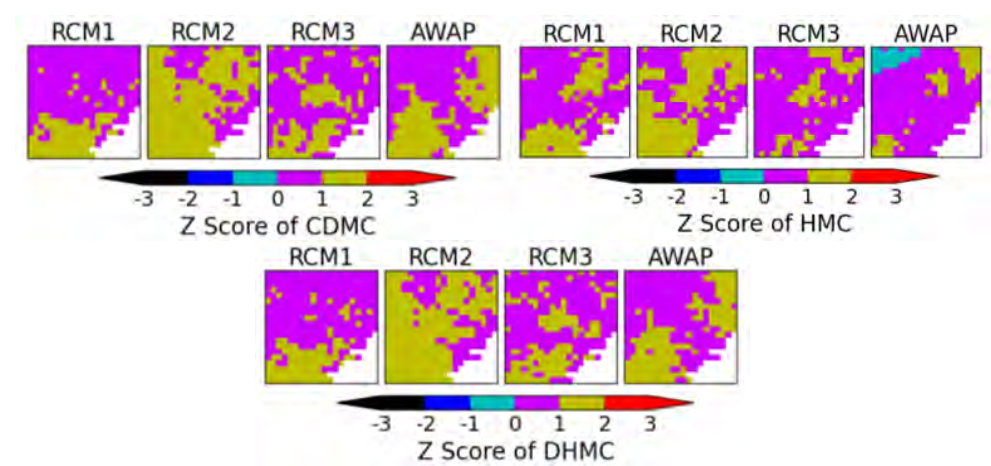

Figure D.14: Comparison of CDMC, HMC and DHMC for the mean of annual rainfall depths. Z scores for $\mathbf{2}$ to 10 multiyear resolutions are same as the $Z$ scores for annual resolution, and hence, are not shown. 

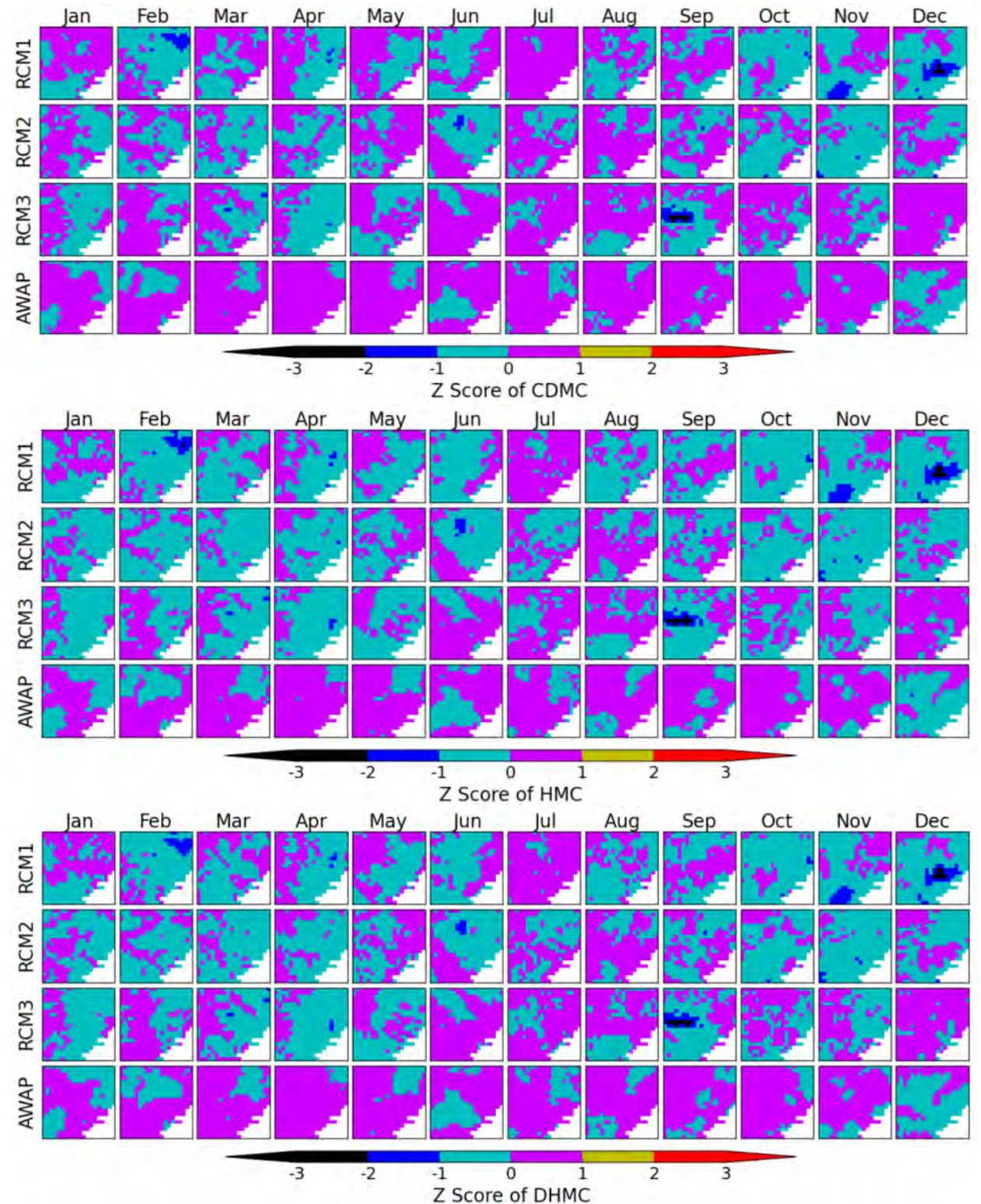

Figure D.15: Comparison of CDMC, HMC and DHMC for the SD of daily rainfall depths. 

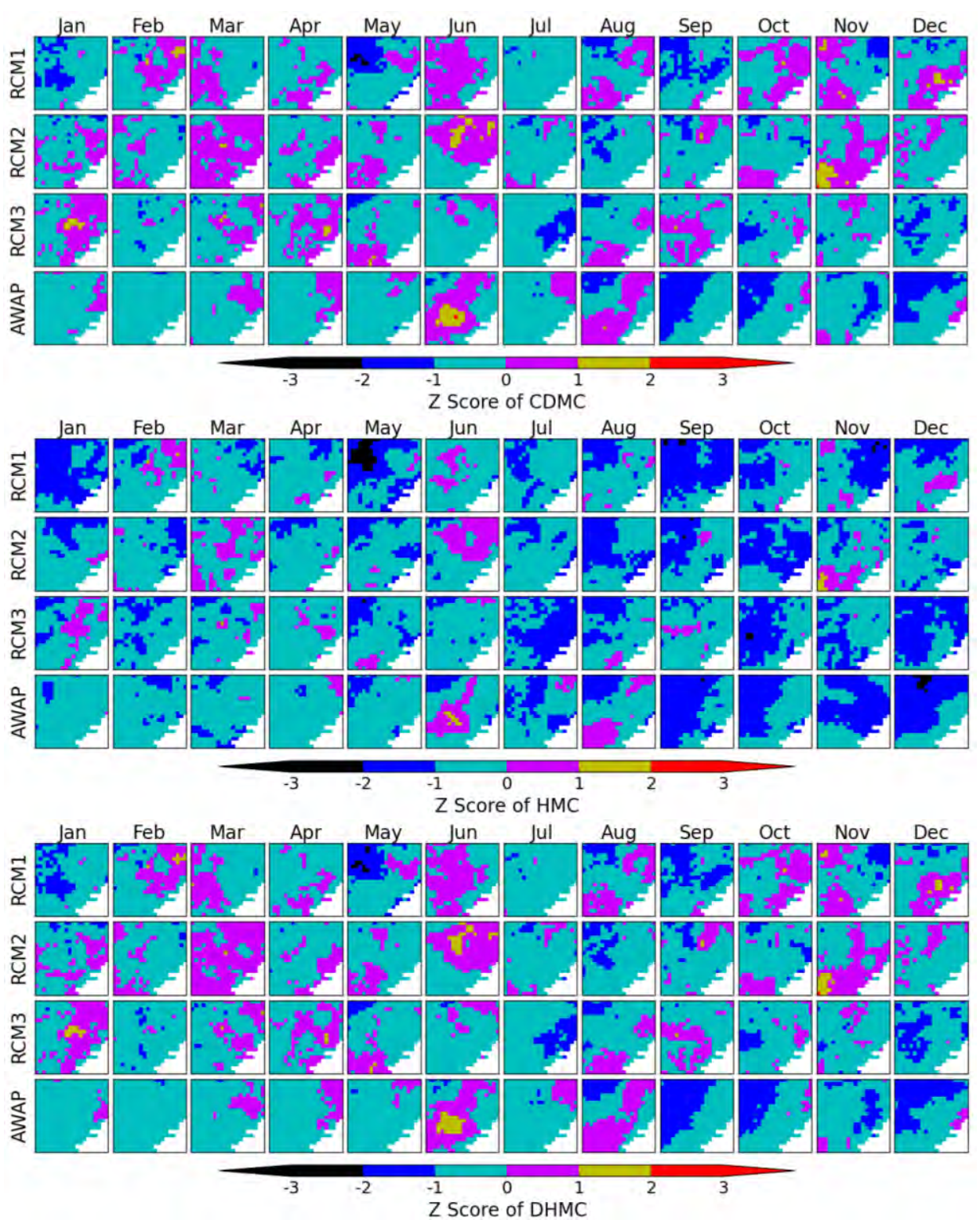

Figure D.16: Comparison of CDMC, HMC and DHMC for the SD of monthly rainfall depths. 


\section{D.3 Comparison of MC Models for Distribution of Wet Period Statistics}

Figures of this appendix compare the MC models for distribution of wet period statistics.
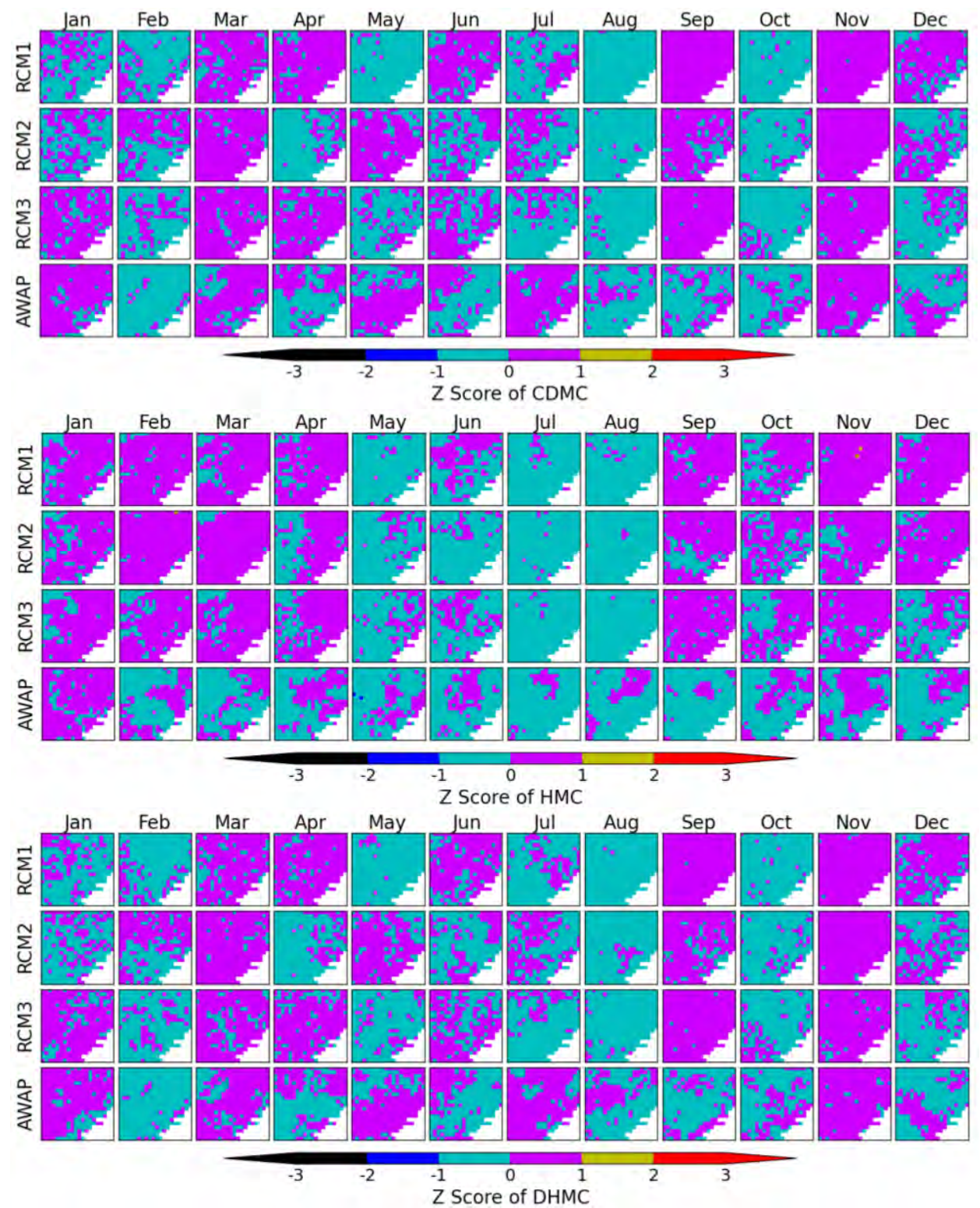

Figure D.17: Comparison of CDMC, HMC and DHMC for the mean of monthly number of wet days. 
D. Comparison of MC Models in Williams River Site
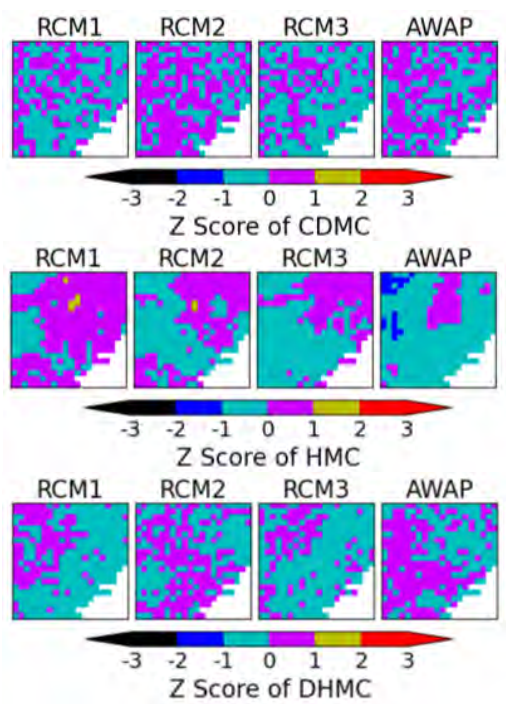

Figure D.18: Comparison of CDMC, HMC and DHMC for the mean of annual number of wet days.
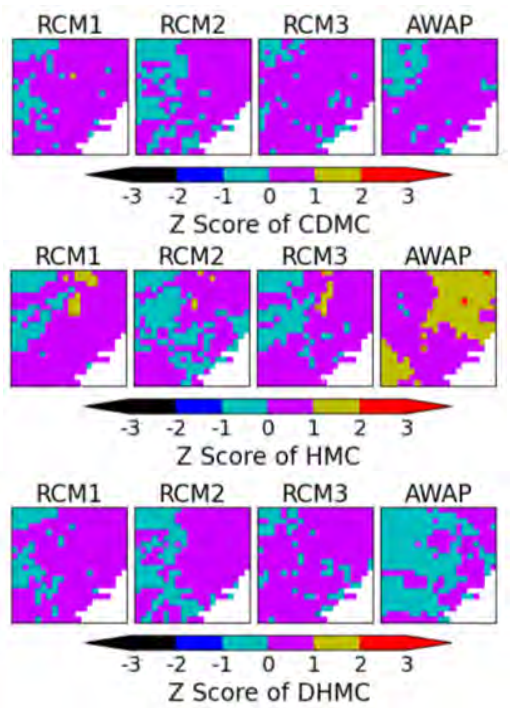

Figure D.19: Comparison of CDMC, HMC and DHMC for the mean of annual mean length of wet spells. 

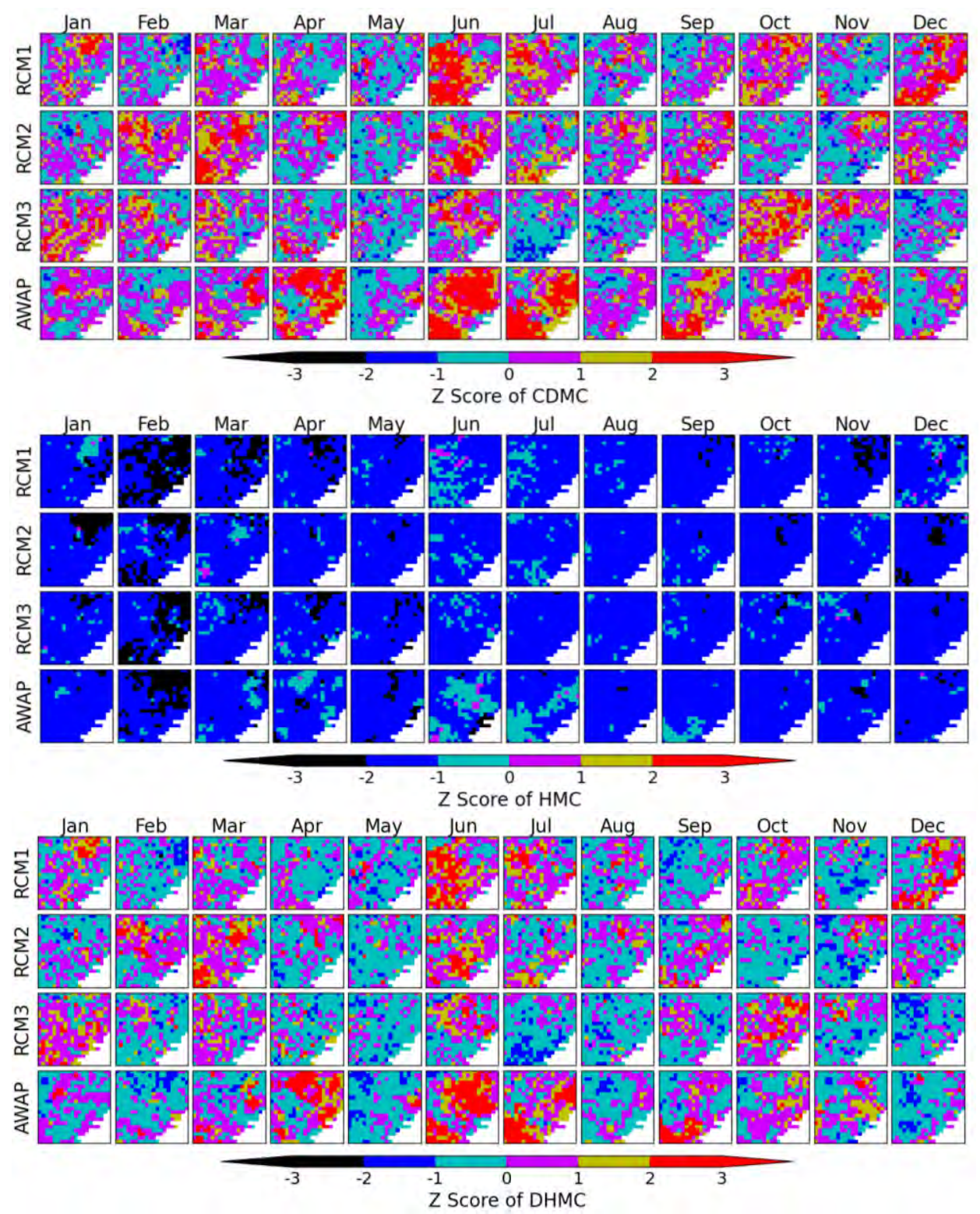

Figure D.20: Comparison of CDMC, HMC and DHMC for the SD of monthly mean length of wet spells. 


\section{D.4 Comparison of MC Models for Autocorrelations}

Figures in this appendix compare the MC models for the autocorrelations of monthly rainfall depths and number of wet days.
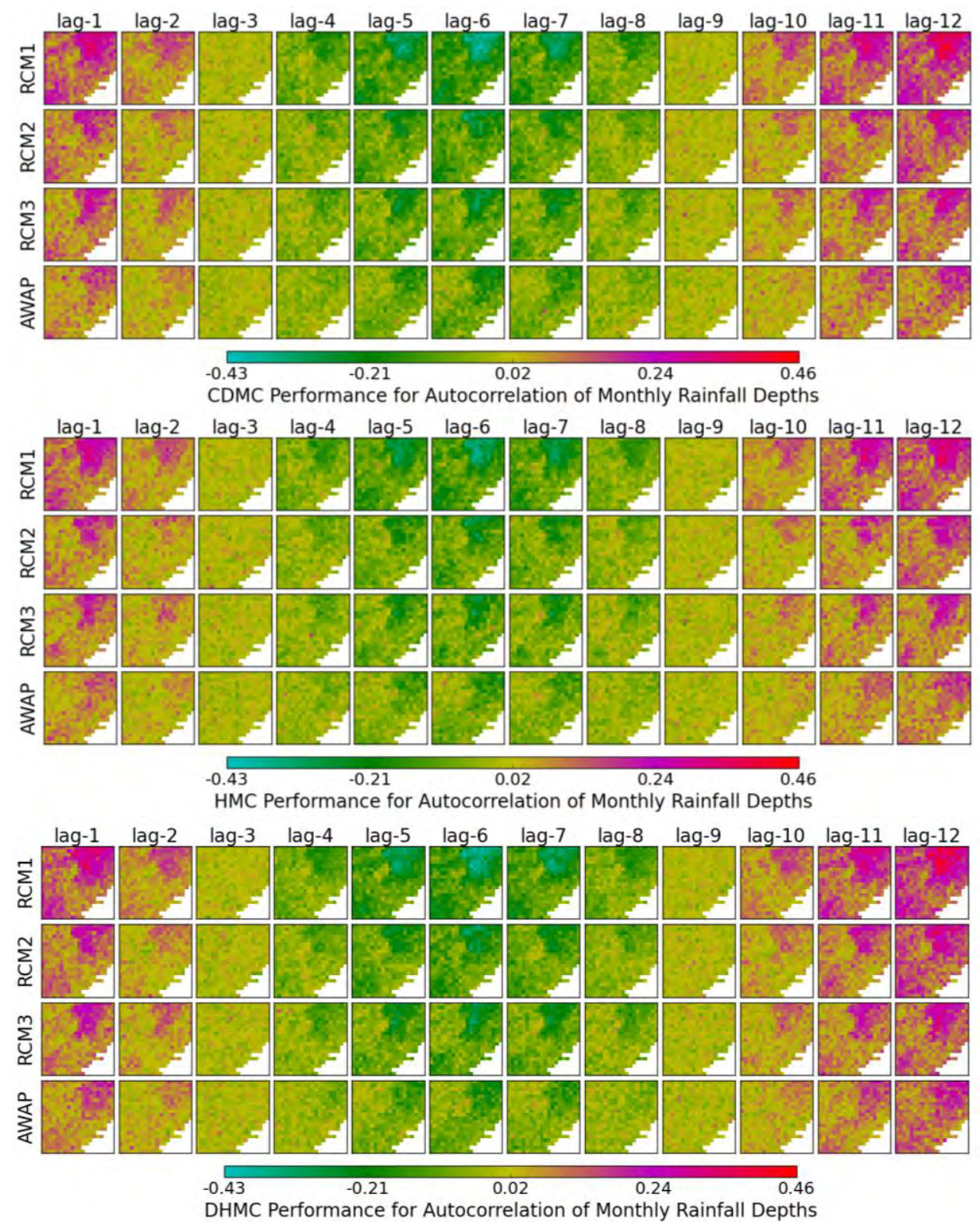

Figure D.21: Comparison of CDMC, HMC and DHMC for the month-to-month autocorrelations of monthly rainfall depths. 

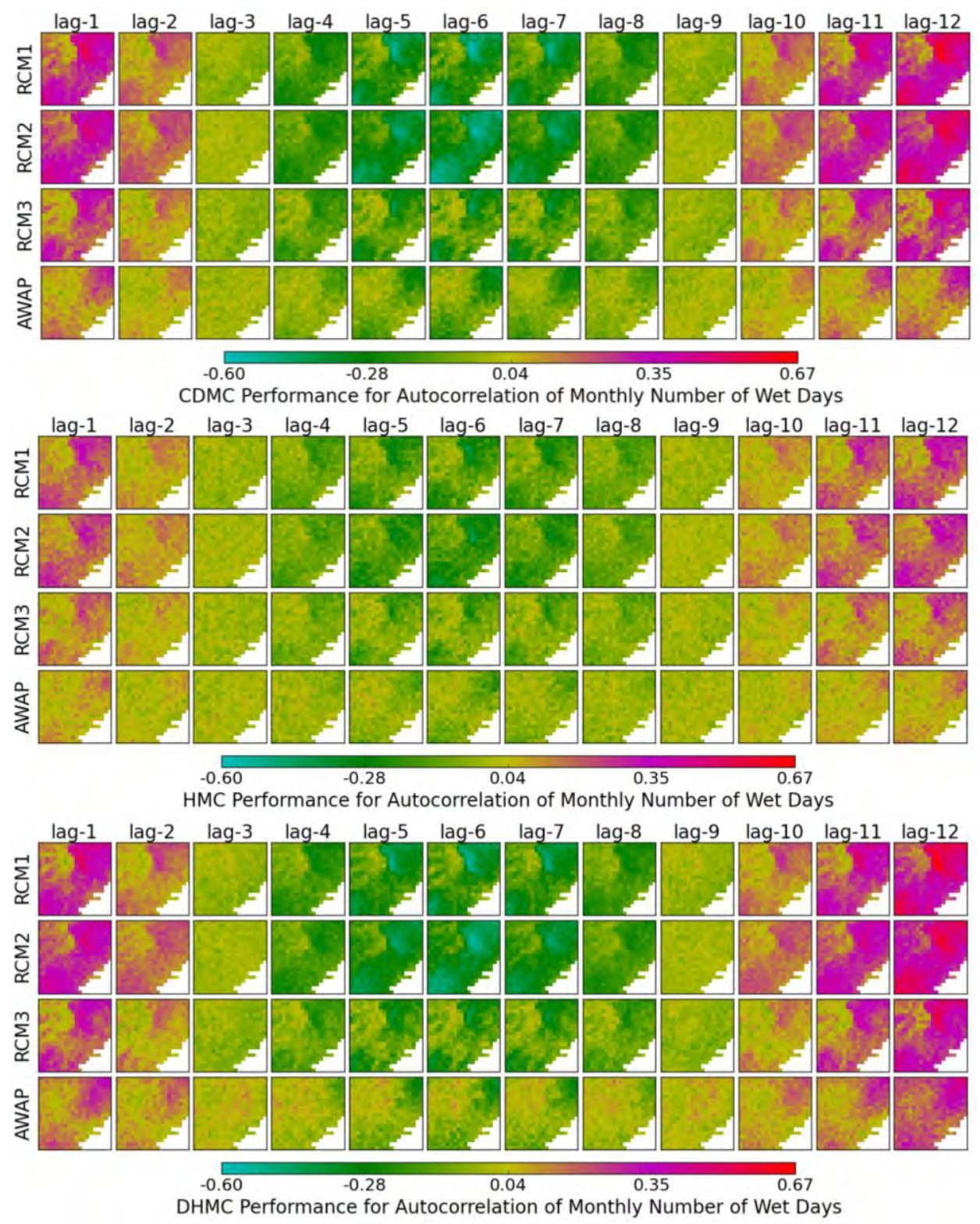

Figure D.22: Comparison of CDMC, HMC and DHMC to reproduce the month-to-month autocorrelations of monthly number of wet days.

\section{D.5 Climate Change Trend of Rainfall}

This section compares the intra-annual and spatial variability of model parameters (APMC values) between historical (1950-2009) and climate change periods (1990-2009, 2020-2039, and 2060-2079) for reanalysis and three GCMs (CSIRO mk3.0, ECHAM5, and MIROC-medres 3.2) of RCM2. 

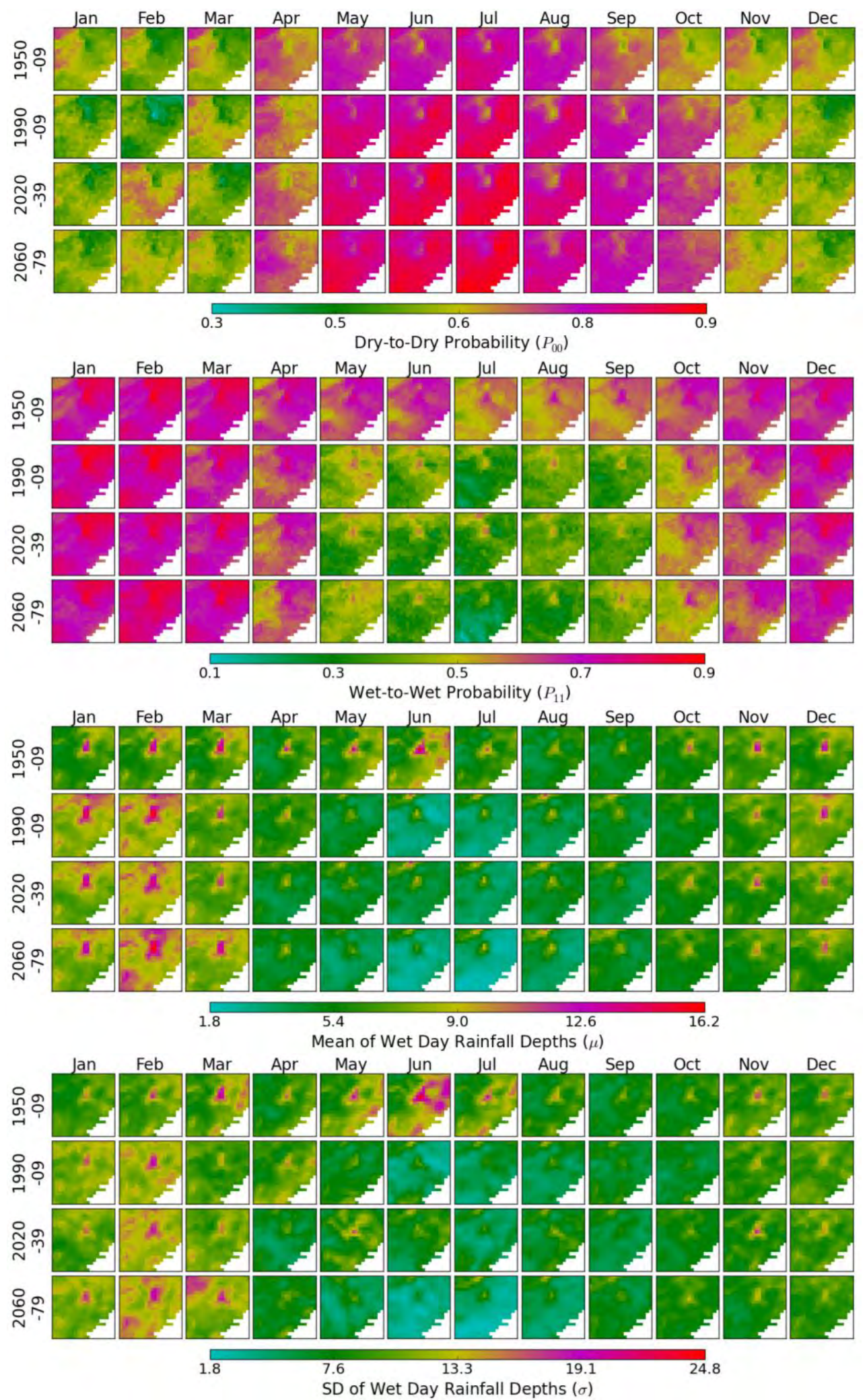

Figure D.23: Intra-annual and spatial variability of model parameters (APMC values) for reanalysis and CSIRO mk3.0 of RCM2. 

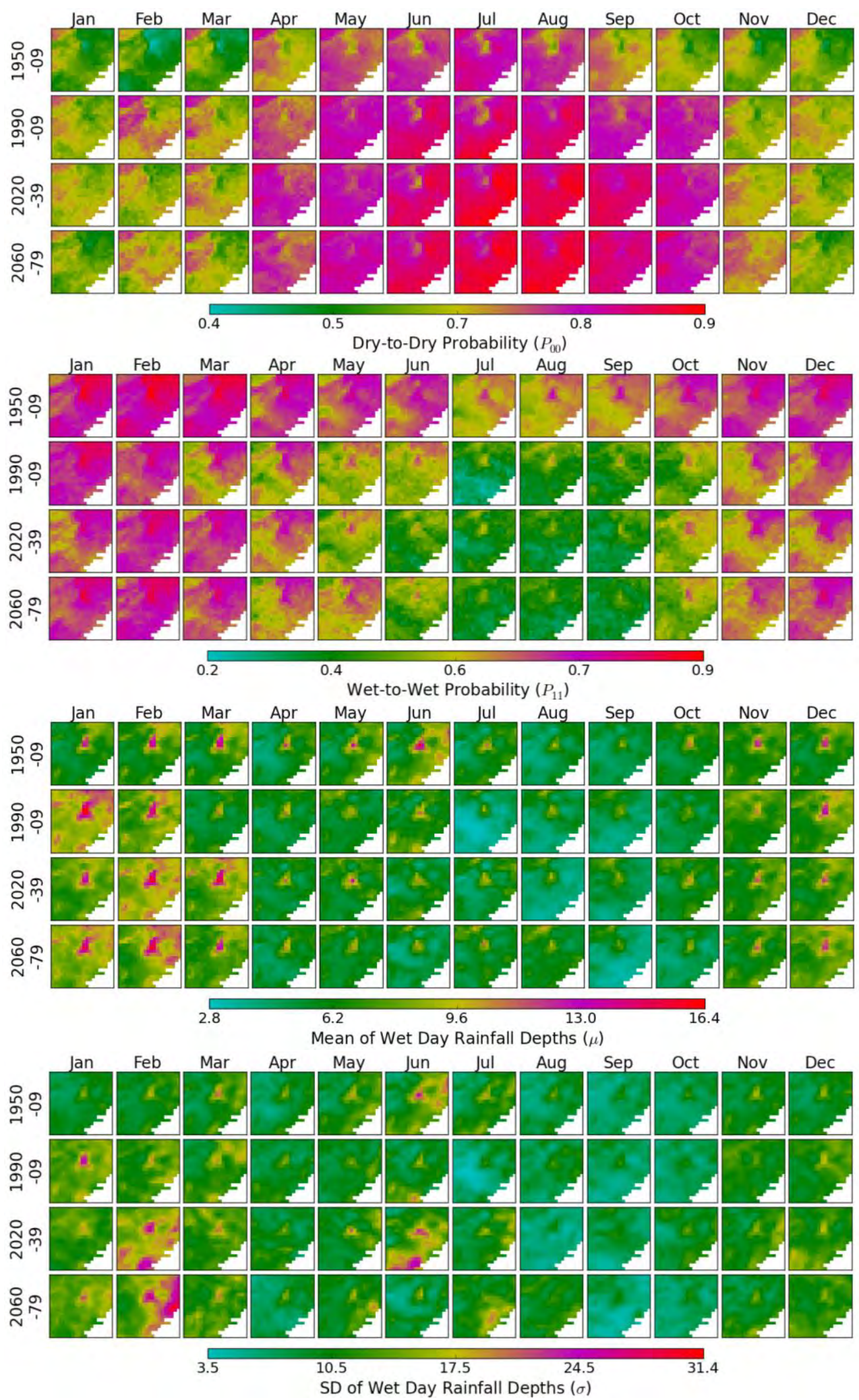

Figure D.24: Intra-annual and spatial variability of model parameters (APMC values) for reanalysis and ECHAM5 of RCM2. 

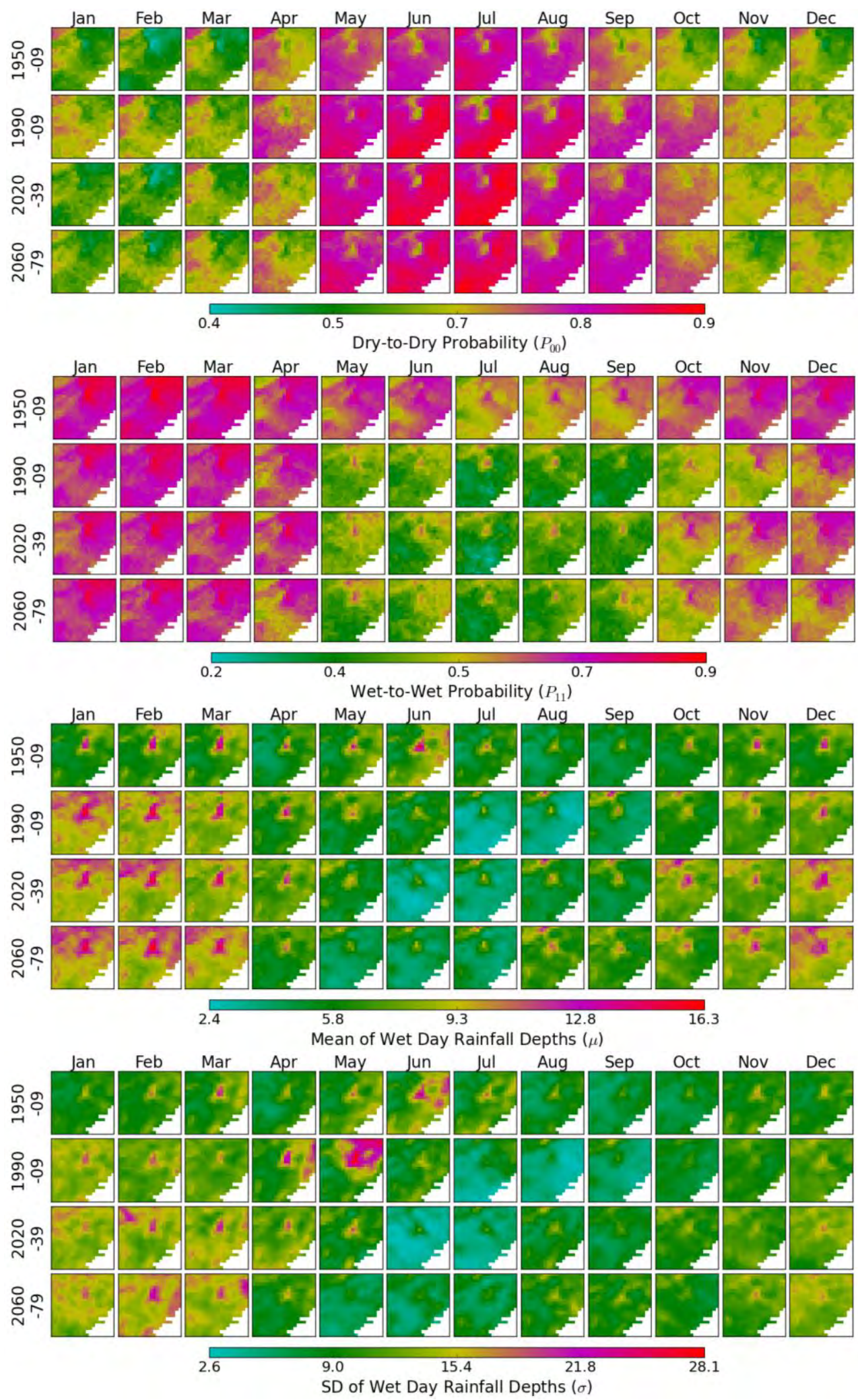

Figure D.25: Intra-annual and spatial variability of model parameters (APMC values) for reanalysis and MIROC-medres 3.2of RCM2. 


\section{E. Comparison of MC Models in Sydney Site}

\section{E.1 Intra-annual and Spatial Variability of Rainfall and Wet Periods}

Figures in this appendix show the intra-annual and spatial variability of the mean and SD of rainfall depths and wet periods statistics.

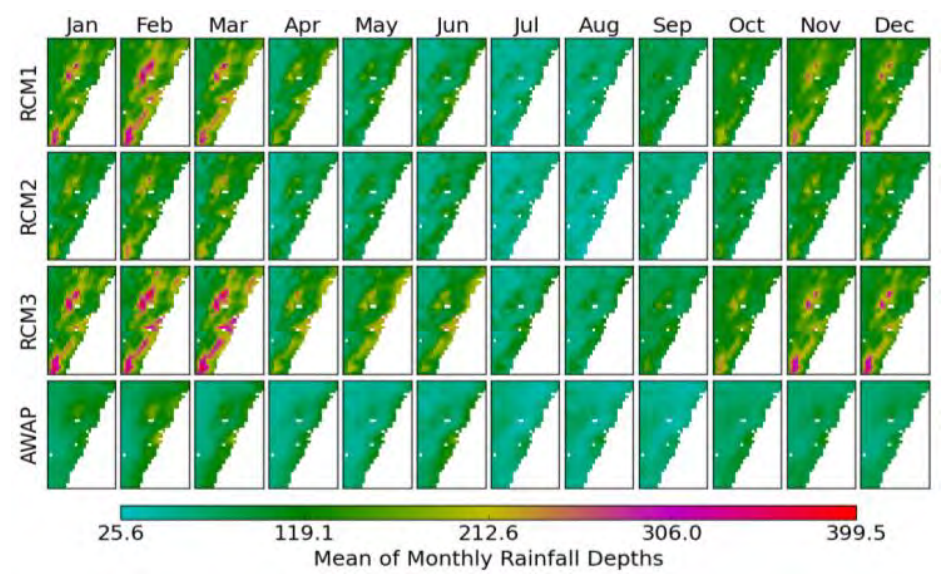

Figure E.1: Intra-annual and spatial variability of the mean of monthly rainfall depths.

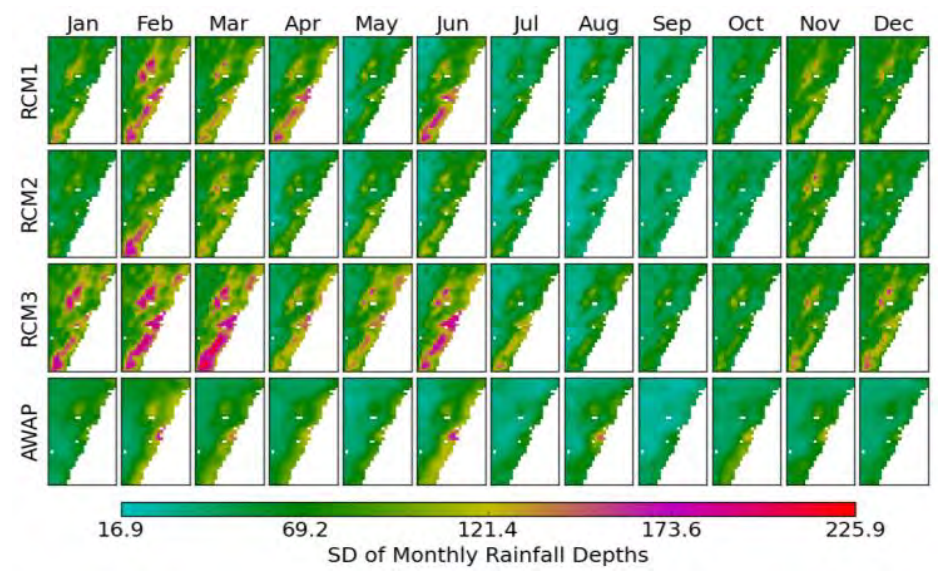

Figure E.2: Intra-annual and spatial variability of the SD of monthly rainfall depths.

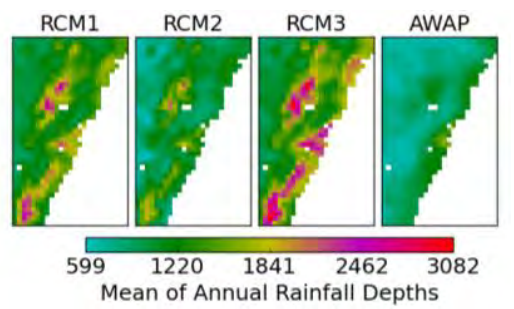

Figure E.3: Spatial variability of the mean of annual rainfall depths. 


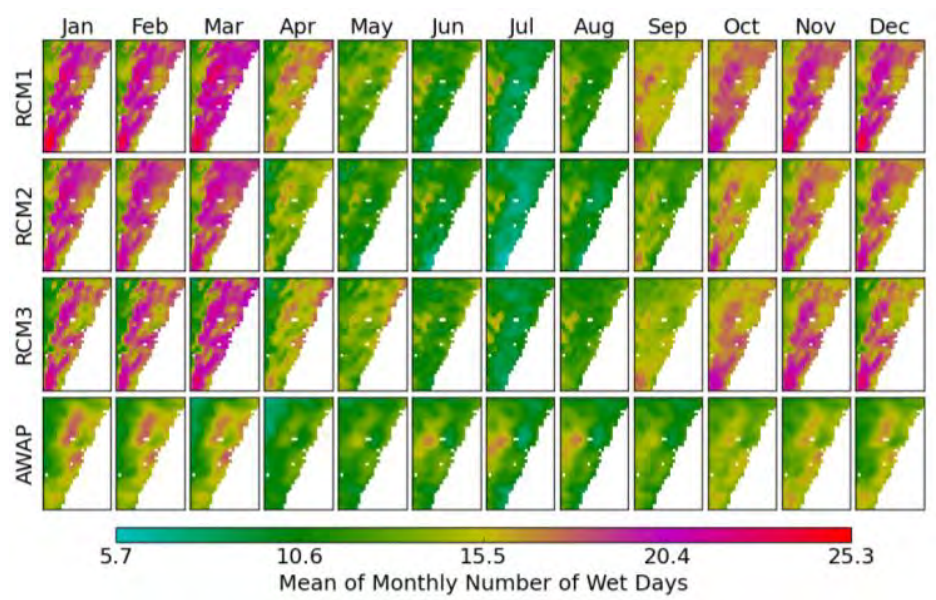

Figure E.4: Intra-annual and spatial variability of the mean of monthly number of wet days.

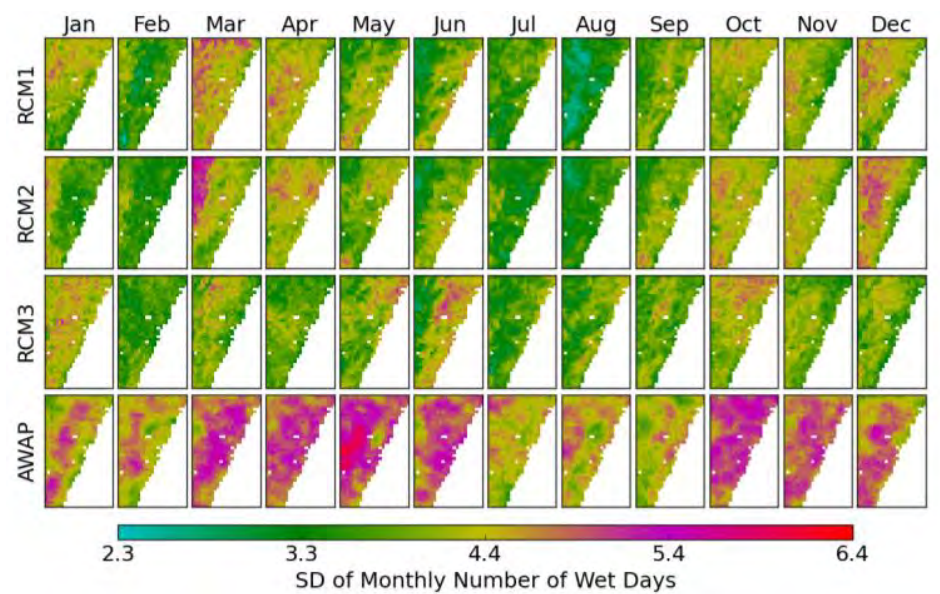

Figure E.5: Intra-annual and spatial variability of the SD of monthly number of wet days.

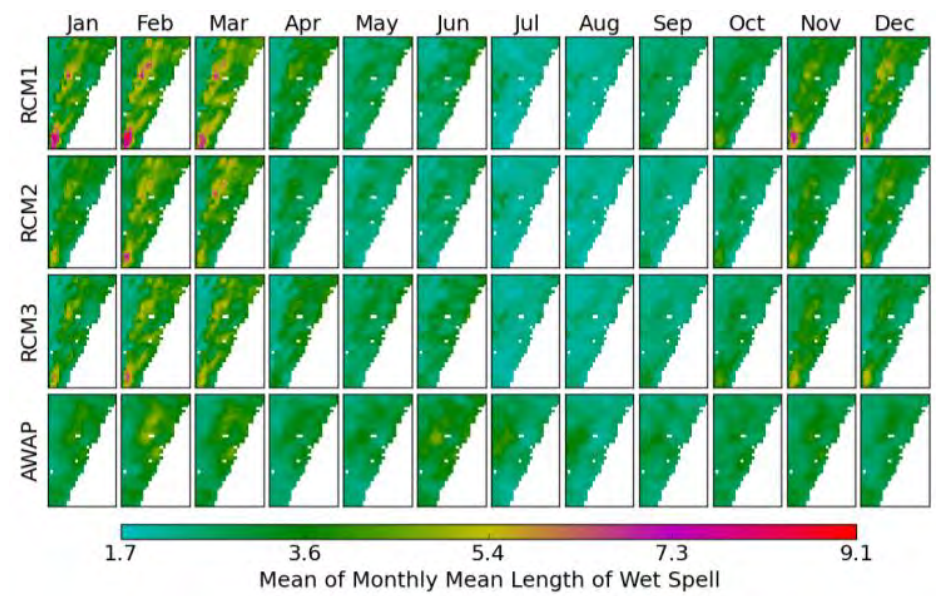

Figure E.6: Intra-annual and spatial variability of the mean of monthly mean length of wet spells. 


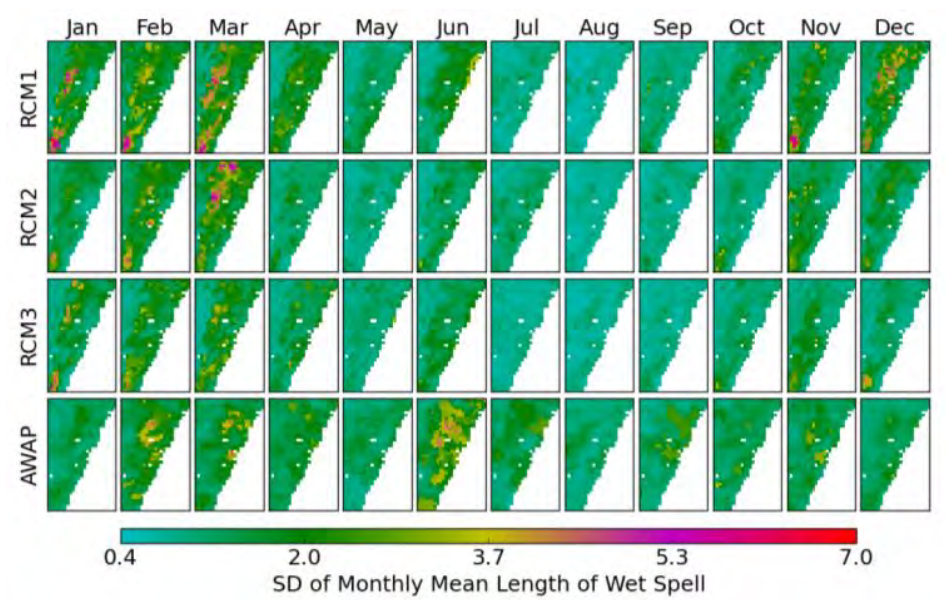

Figure E.7: Intra-annual and spatial variability of the SD of monthly mean length of wet spells.

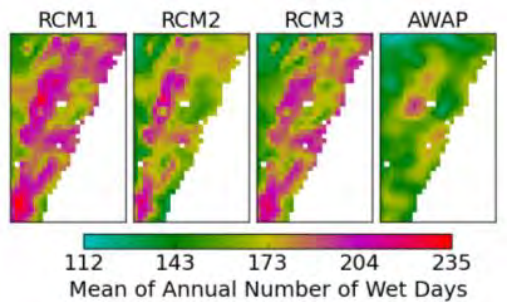

Figure E.8: Spatial variability of the mean of annual number of wet days.

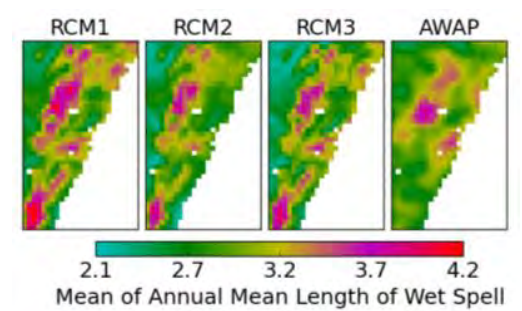

Figure E.9: Spatial variability of the mean of annual mean length of wet spells.

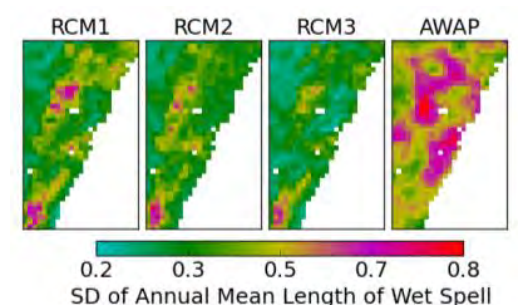

Figure E.10: Spatial variability of the SD of annual mean length of wet spells. 


\section{E.2 Comparison of MC Models for Distribution of Rainfall Depths}

Figures of this appendix compare the MC models for the mean and SD of rainfall depths.
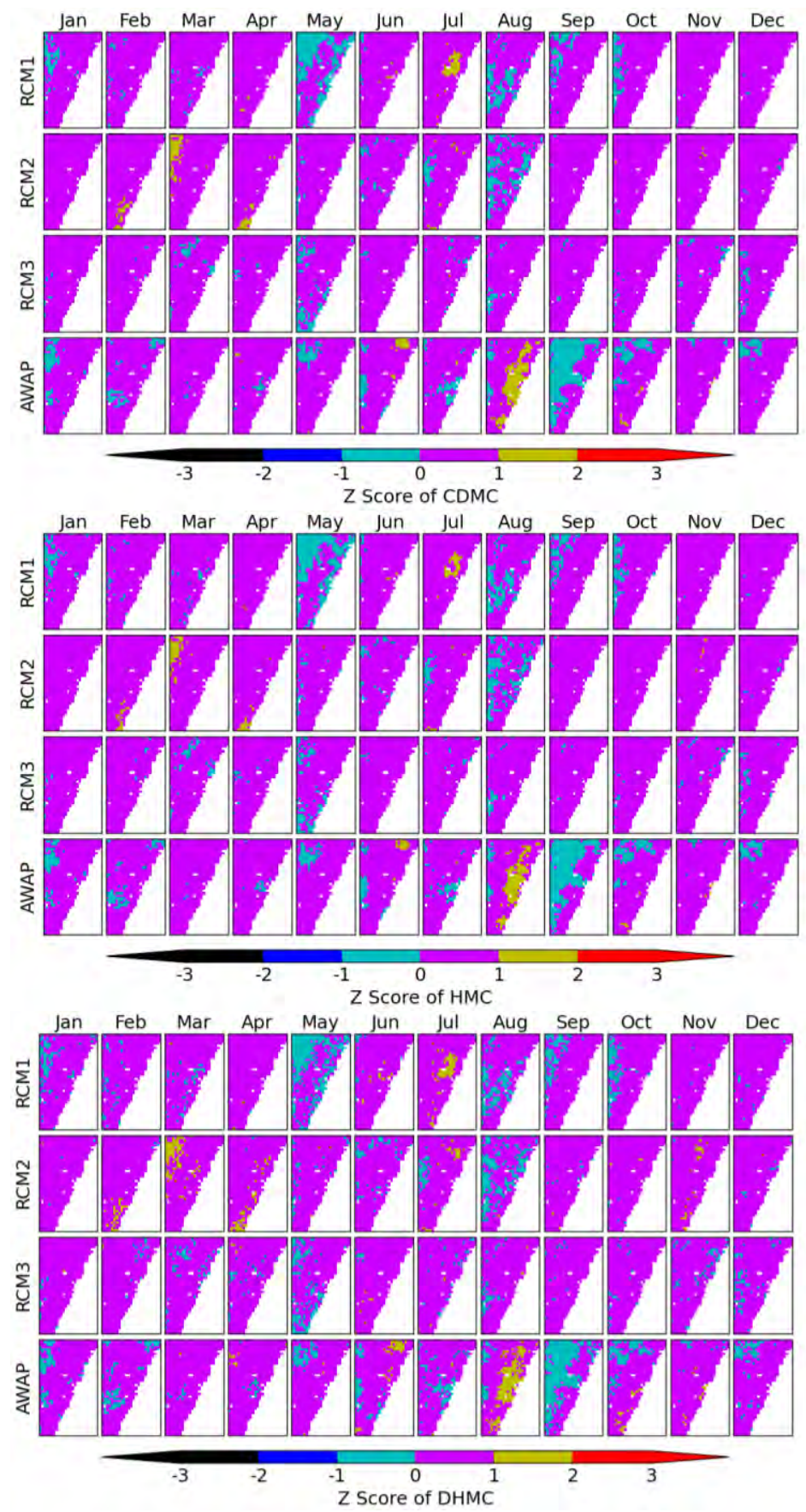

Figure E.11: Comparison of CDMC, HMC and DHMC for the mean of daily rainfall depths. 
E. Comparison of MC Models in Sydney Site
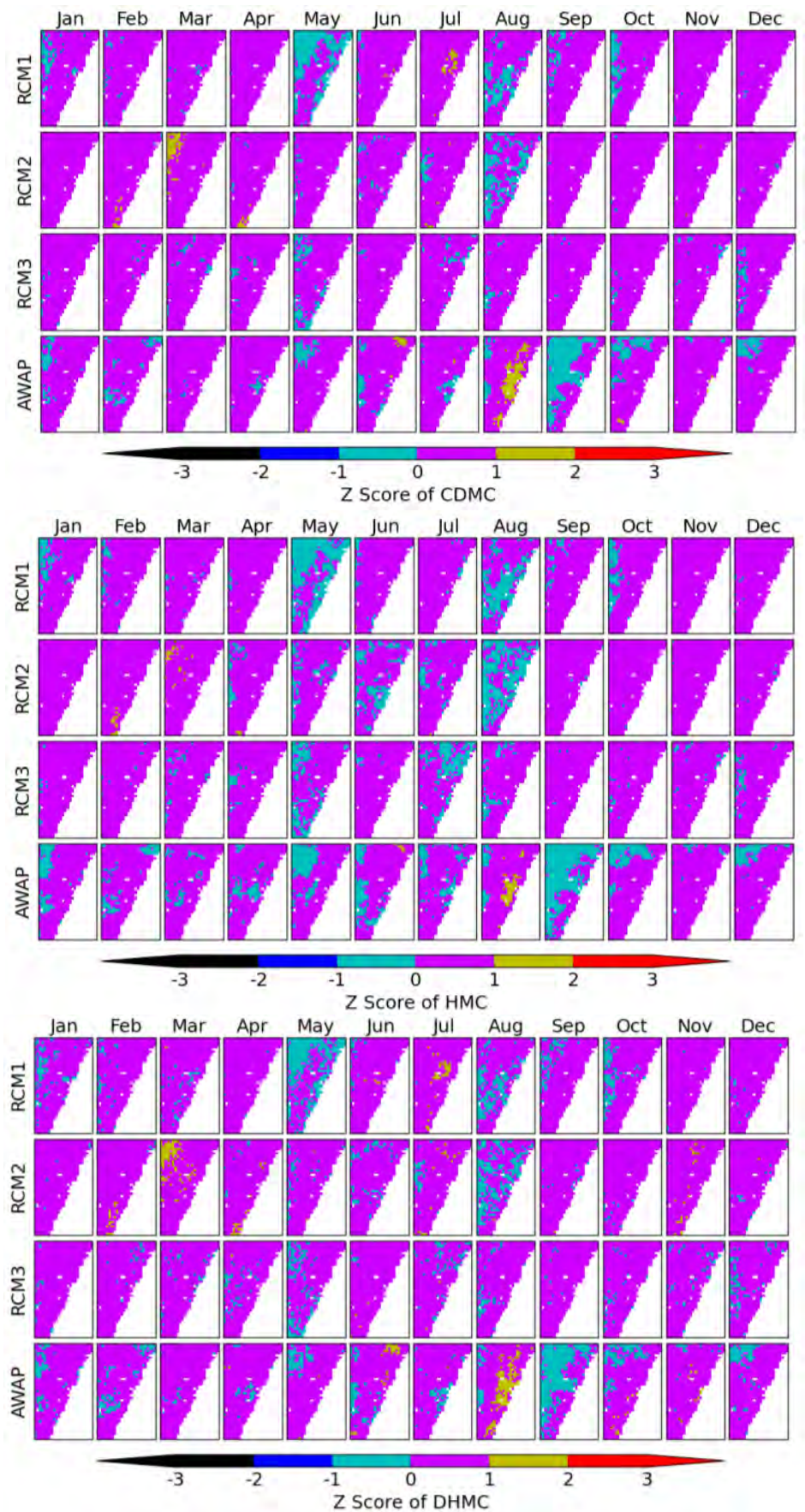

Figure E.12: Comparison of CDMC, HMC and DHMC for the mean of monthly rainfall depths. 

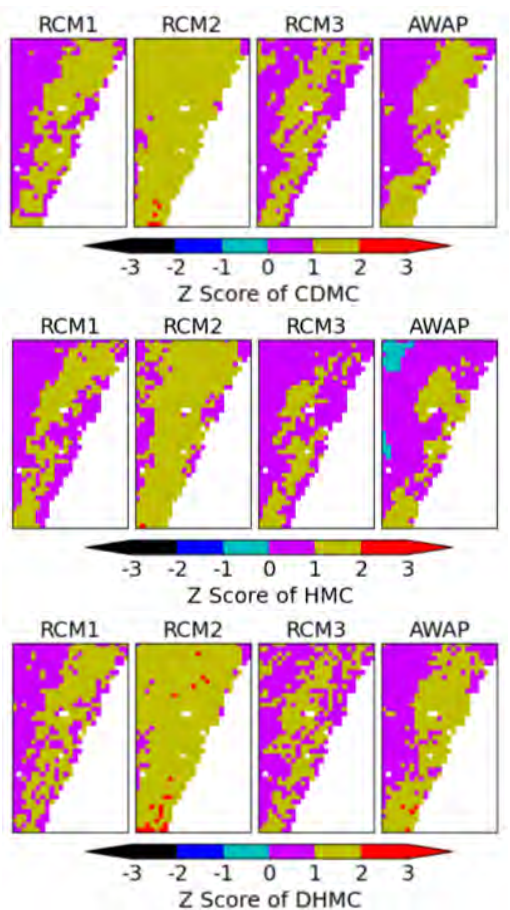

Figure E.13: Comparison of CDMC, HMC and DHMC for the mean of annual rainfall depths. 
E. Comparison of MC Models in Sydney Site
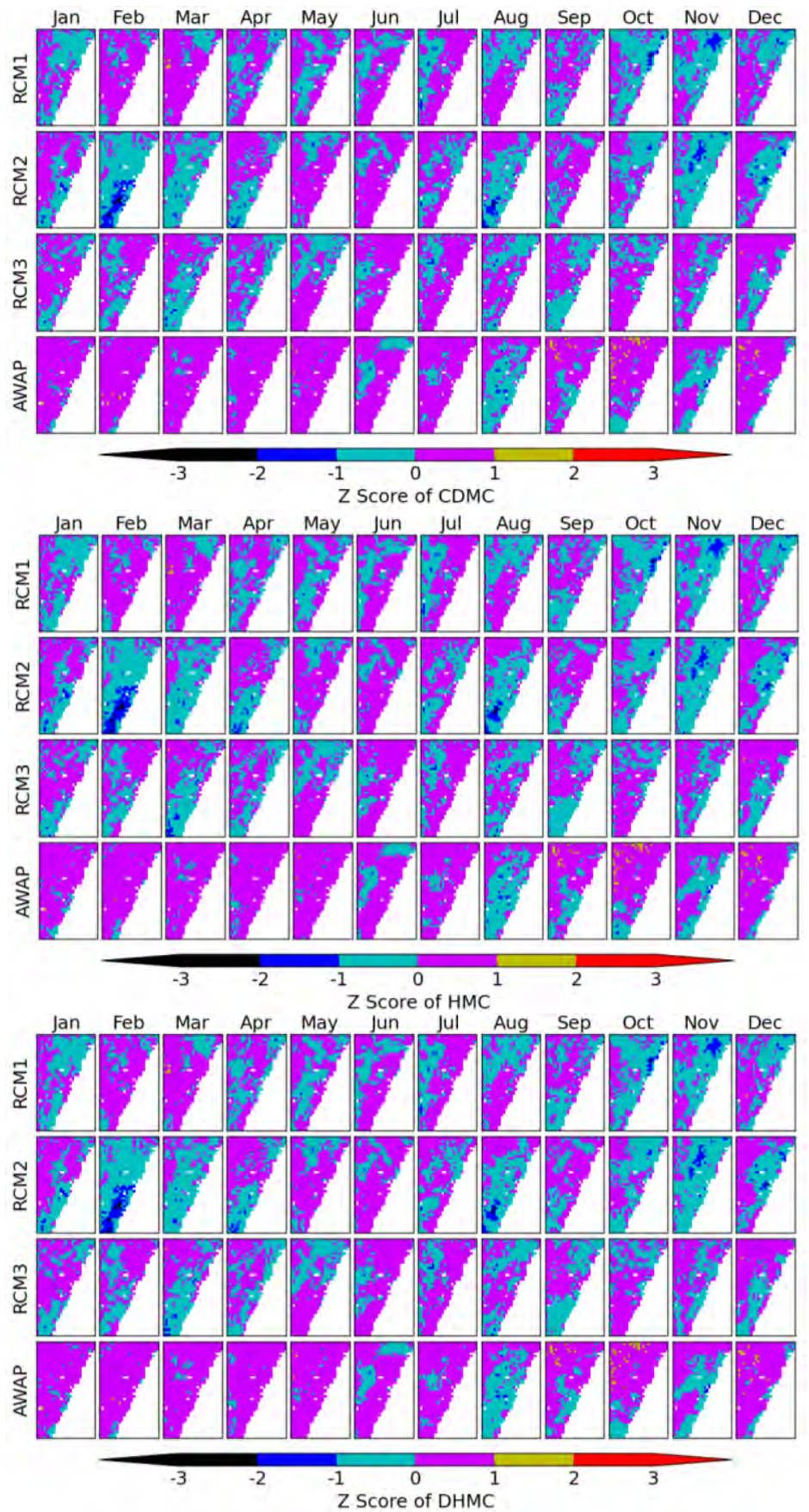

Figure E.14: Comparison of CDMC, HMC and DHMC for the SD of daily rainfall depths. 

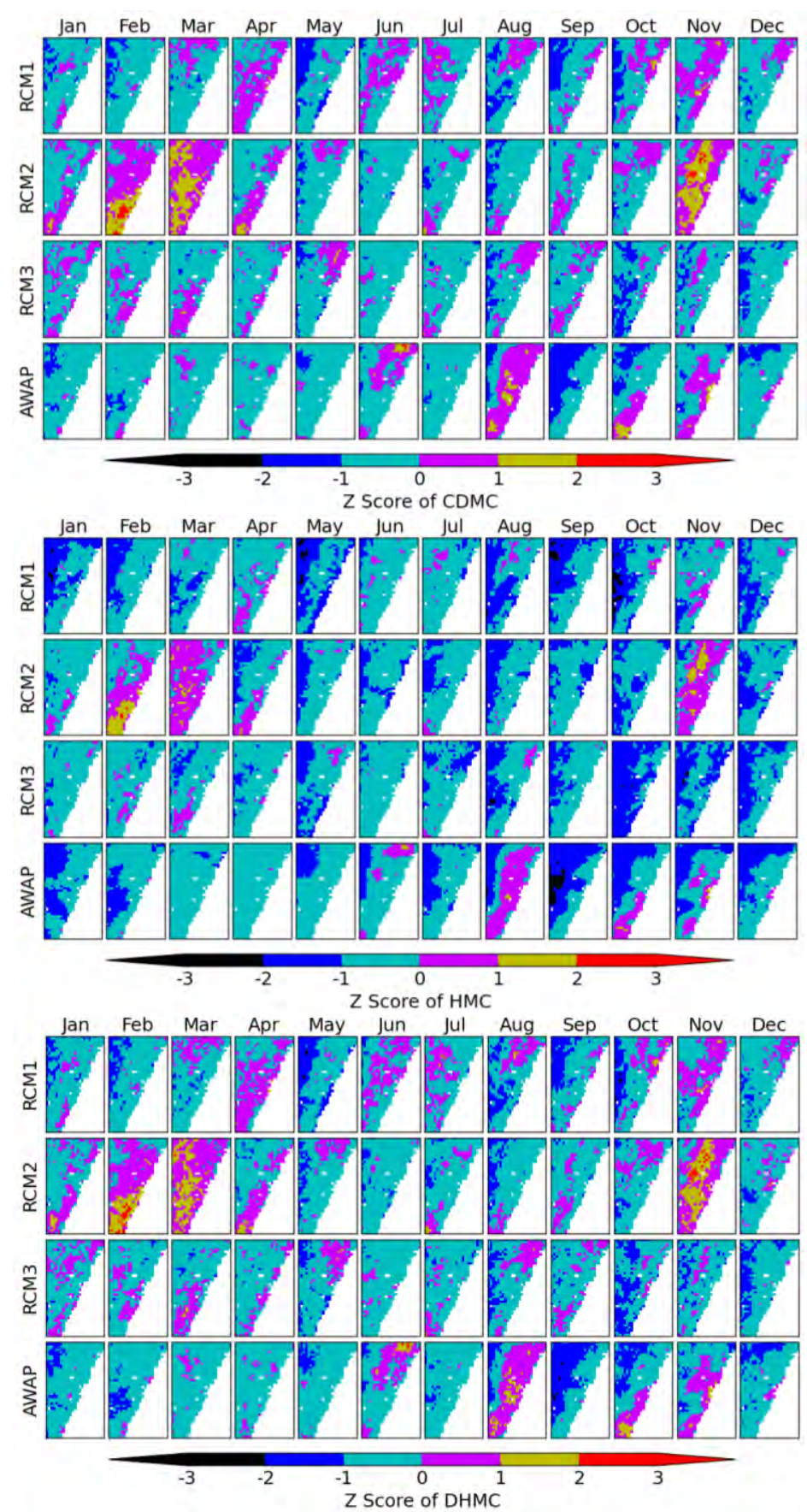

Figure E.15: Comparison of CDMC, HMC and DHMC for the SD of monthly rainfall depths. 


\section{E.3 Comparison of MC Models for Distribution of Wet Period Statistics}

Figures of this appendix compare the MC models for distribution of wet period statistics.
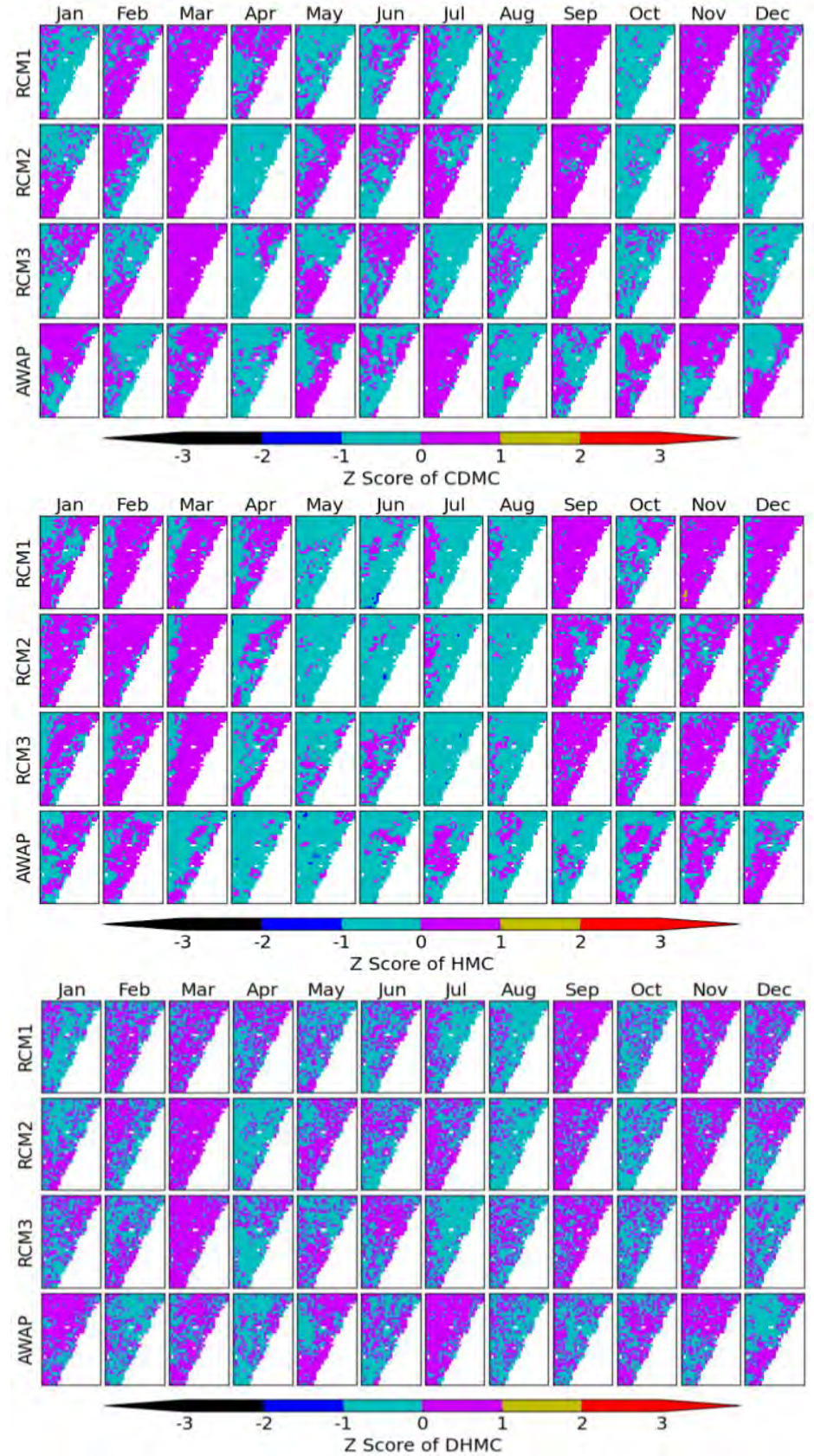

Figure E.16: Comparison of CDMC, HMC and DHMC for the mean of monthly number of wet days. 

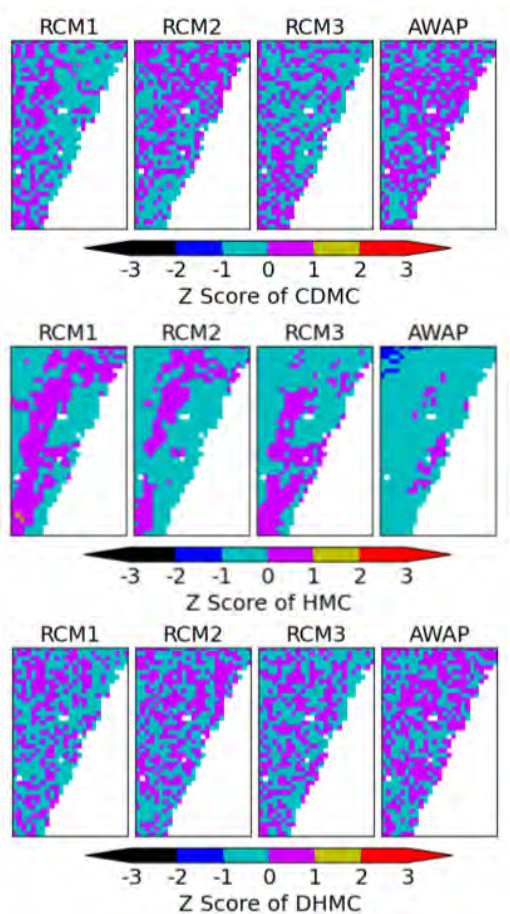

Figure E.17: Comparison of CDMC, HMC and DHMC for the mean of annual number of wet days.
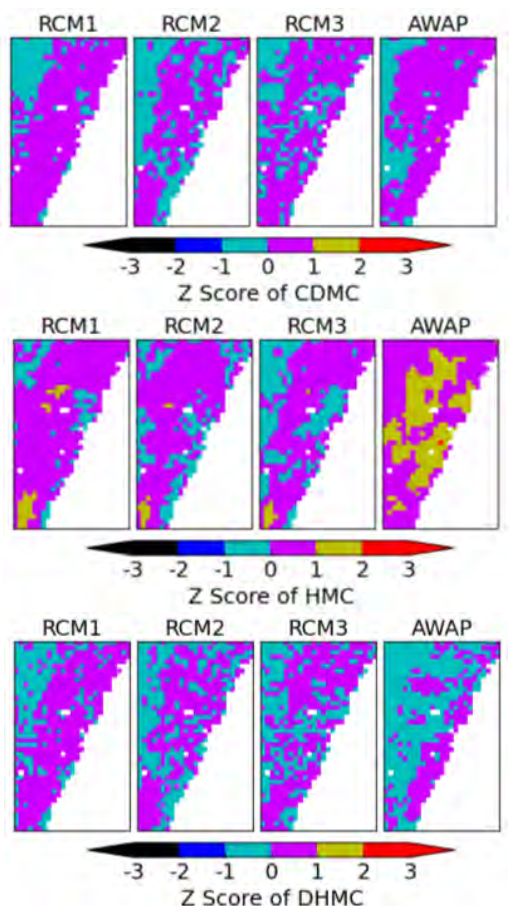

Figure E.18: Comparison of CDMC, HMC and DHMC for the mean of annual mean length of wet spells. 
E. Comparison of MC Models in Sydney Site
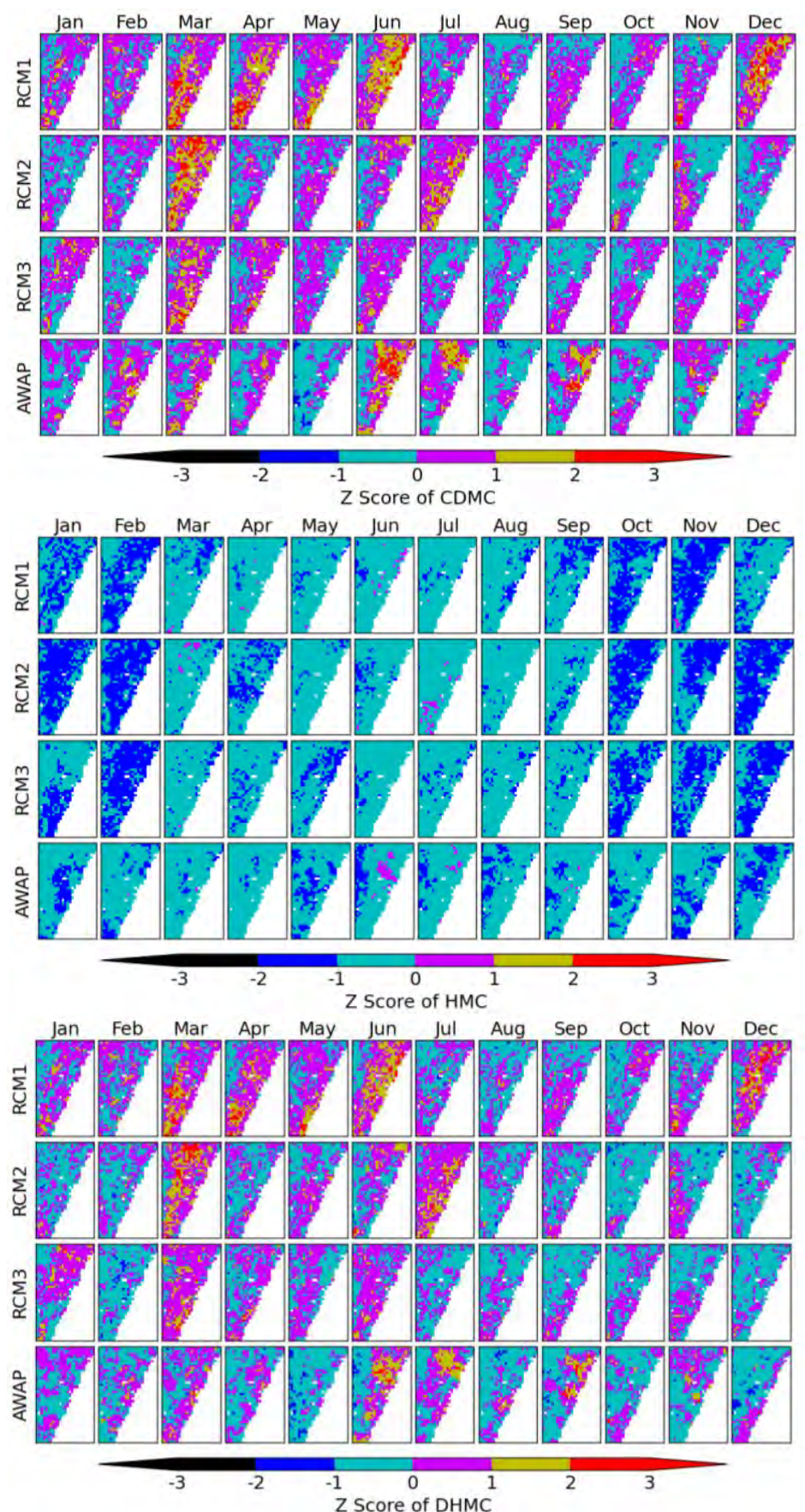

Figure E.19: Comparison of CDMC, HMC and DHMC for the mean of monthly mean length of wet spells. 

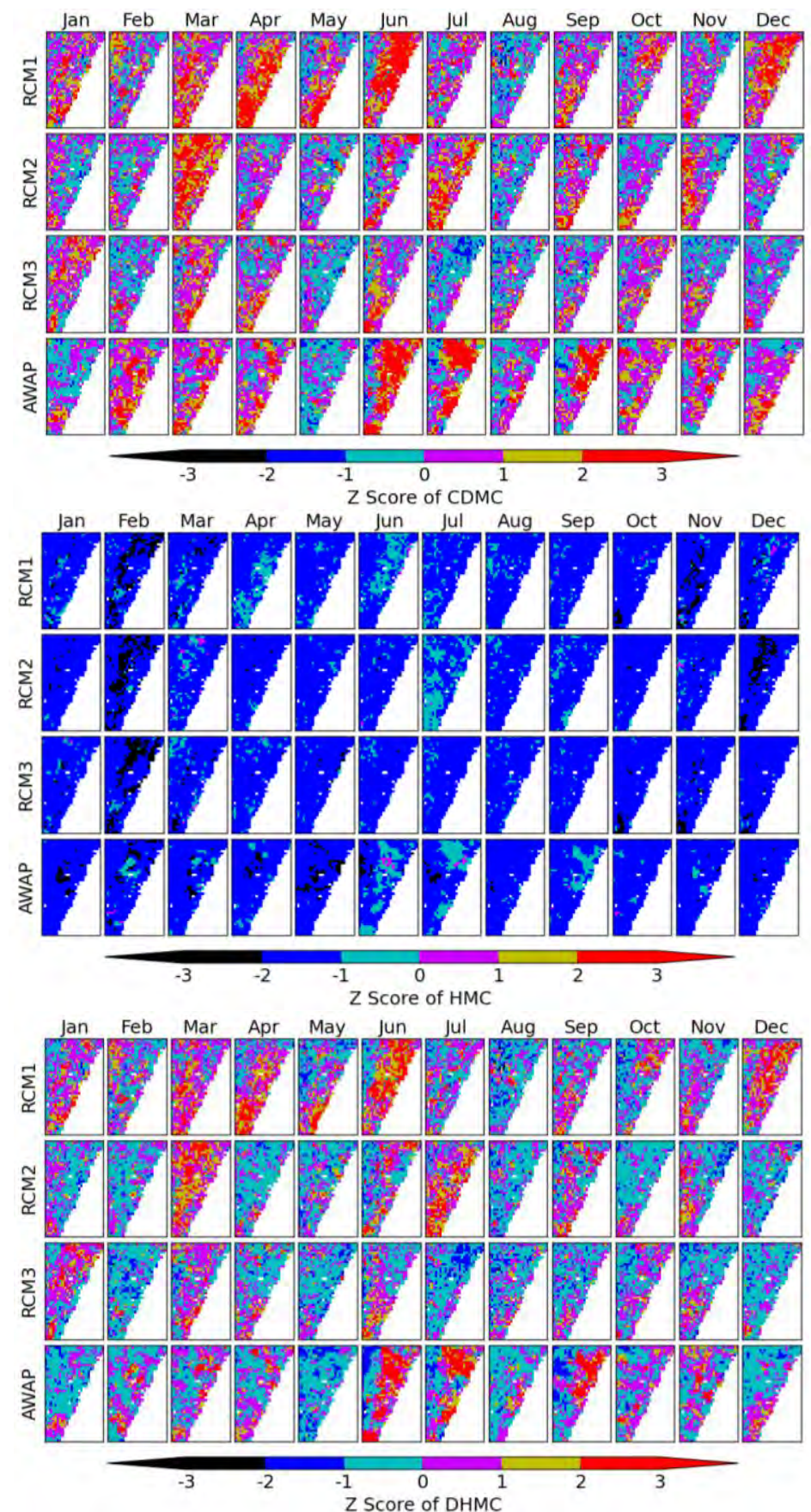

Figure E.20: Comparison of CDMC, HMC and DHMC for the SD of monthly mean length of wet spells. 


\section{E.4 Comparison of MC Models for Autocorrelations}

Figures in this appendix compare the MC models for the autocorrelations of monthly rainfall depths and number of wet days.
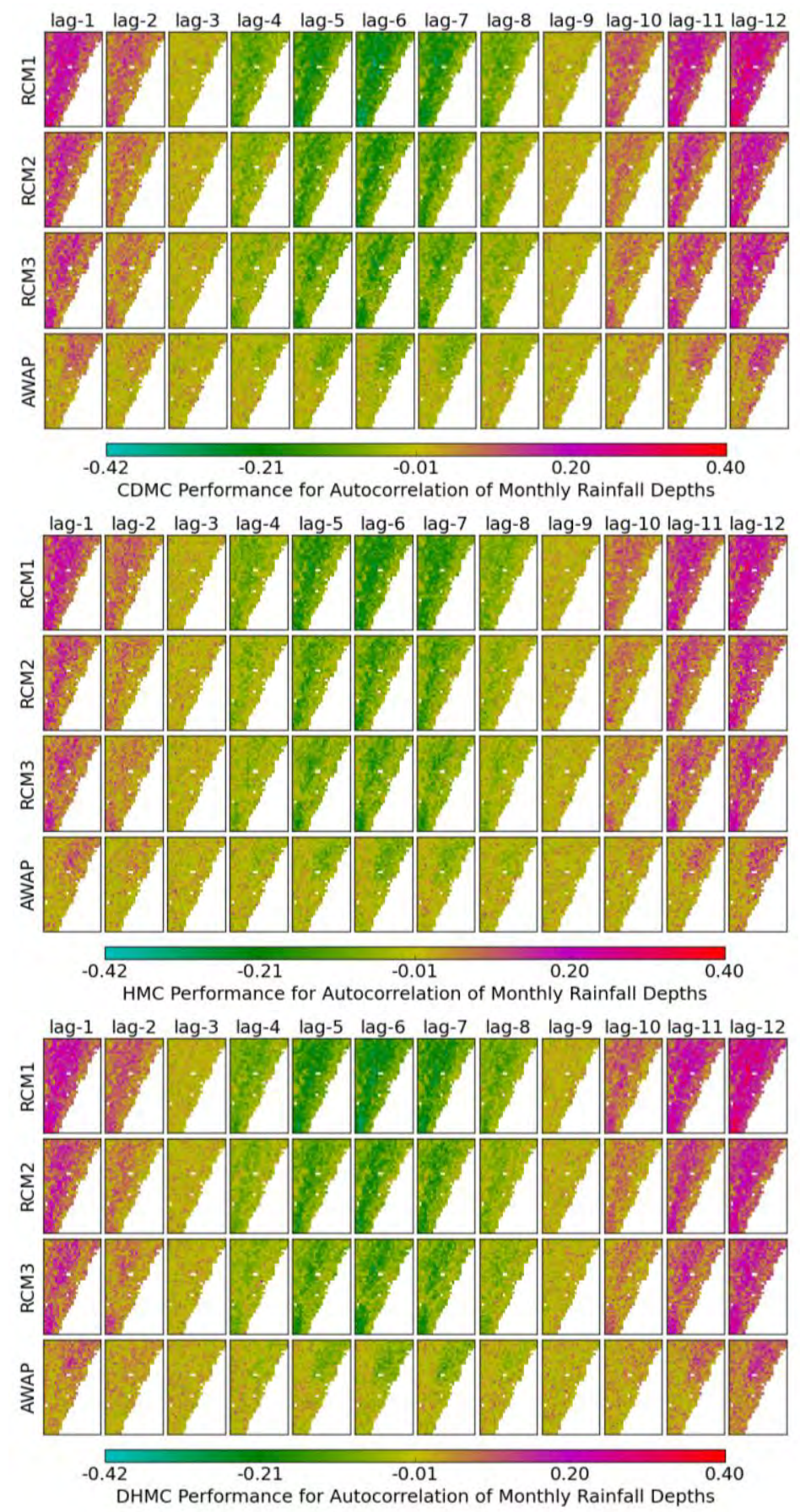

Figure E.21: Comparison of CDMC, HMC and DHMC for the month-to-month autocorrelations of monthly rainfall depths. 

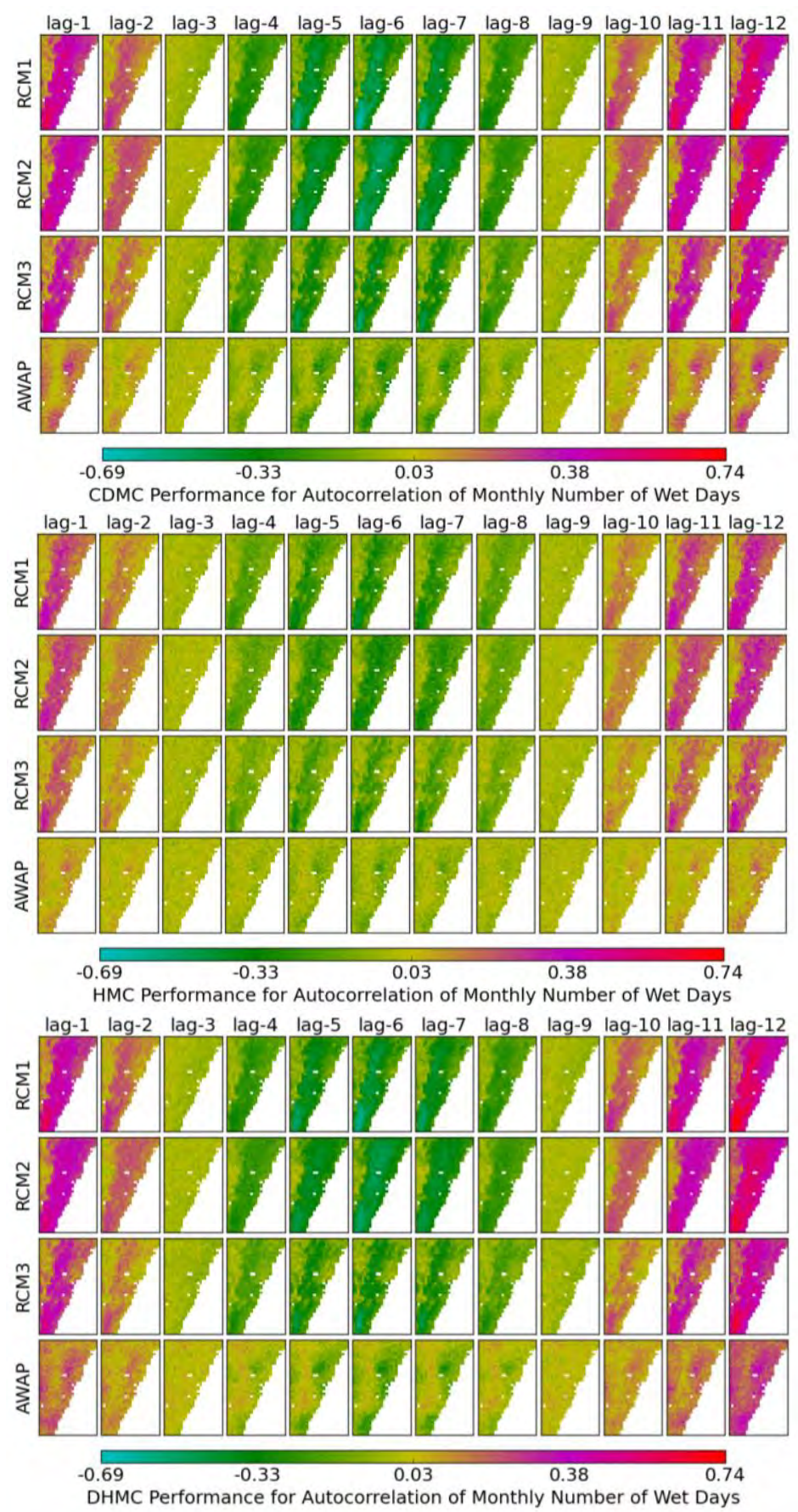

Figure E.22: Comparison of CDMC, HMC and DHMC for the month-to-month autocorrelations of monthly number of wet days. 


\section{F. Comparison of MC Models in Richmond River Site}

\section{F.1 Intra-annual and Spatial Variability of Rainfall and Wet Periods}

Figures in this appendix show the intra-annual and spatial variability of the mean and SD of rainfall depths and wet periods statistics.

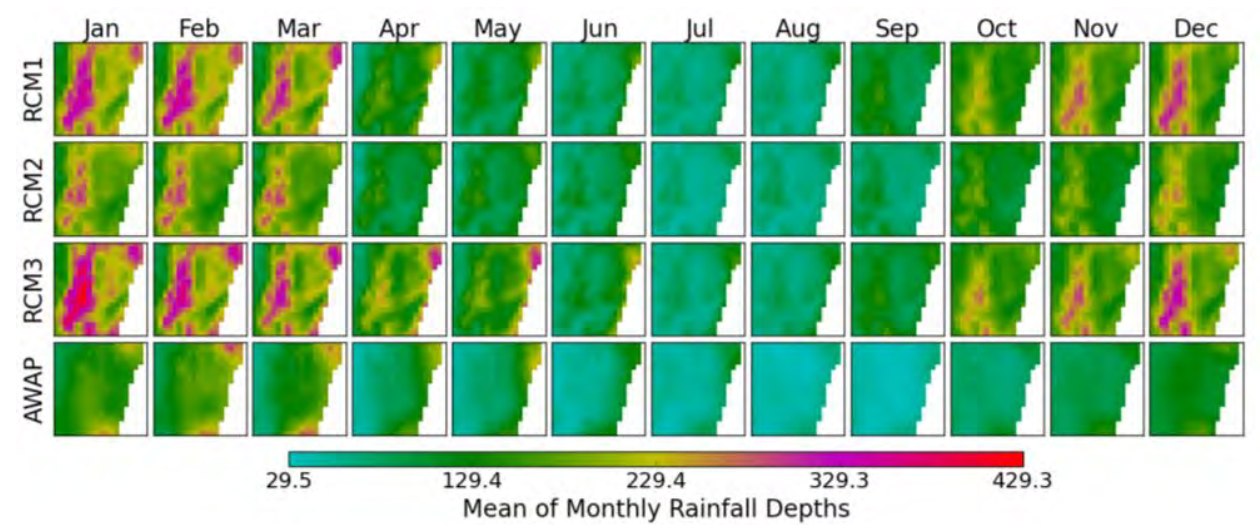

Figure F.1: Intra-annual and spatial variability of the mean of monthly rainfall depths.

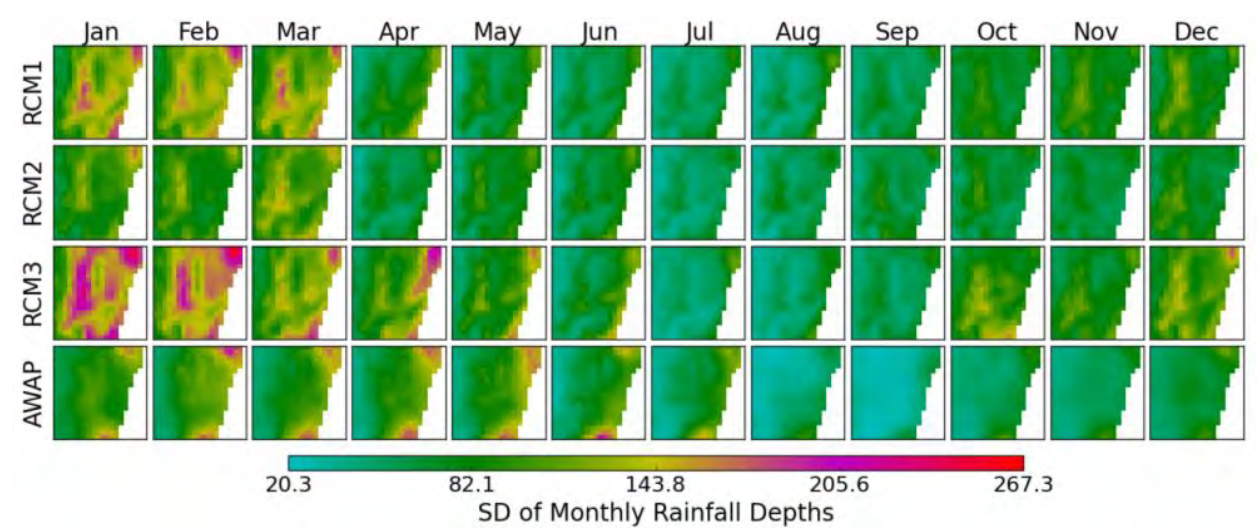

Figure F.2: Intra-annual and spatial variability of the SD of monthly rainfall depths.

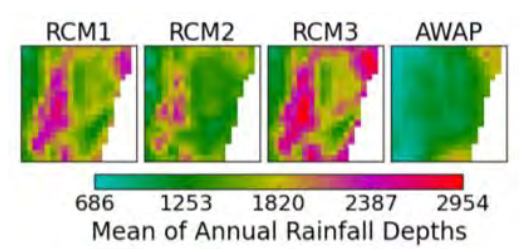

Figure F.3: Spatial variability of the mean of annual rainfall depths. 


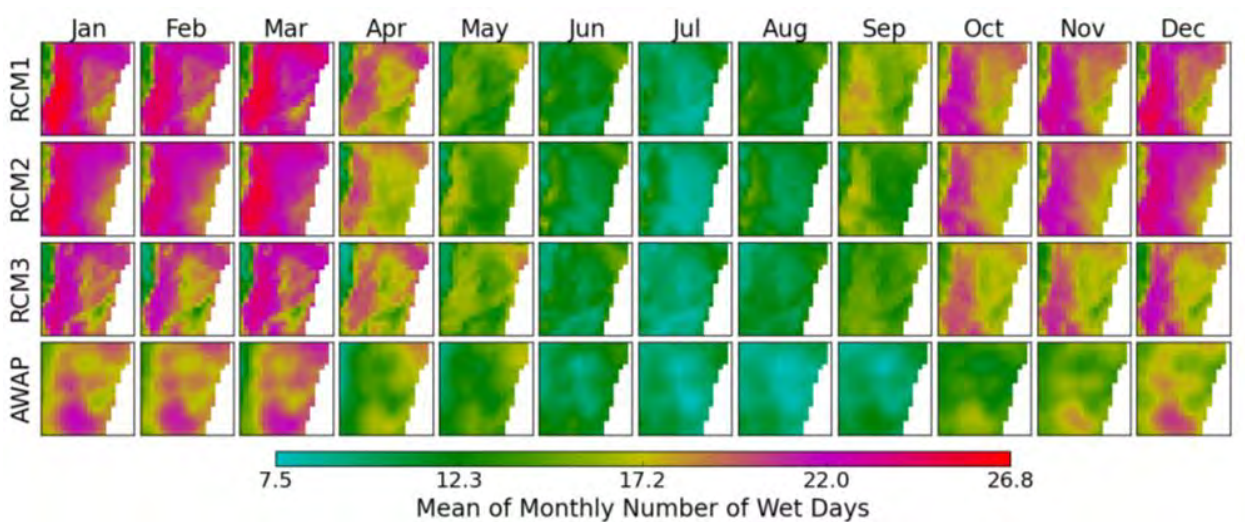

Figure F.4: Intra-annual and spatial variability of the mean of monthly number of wet days.

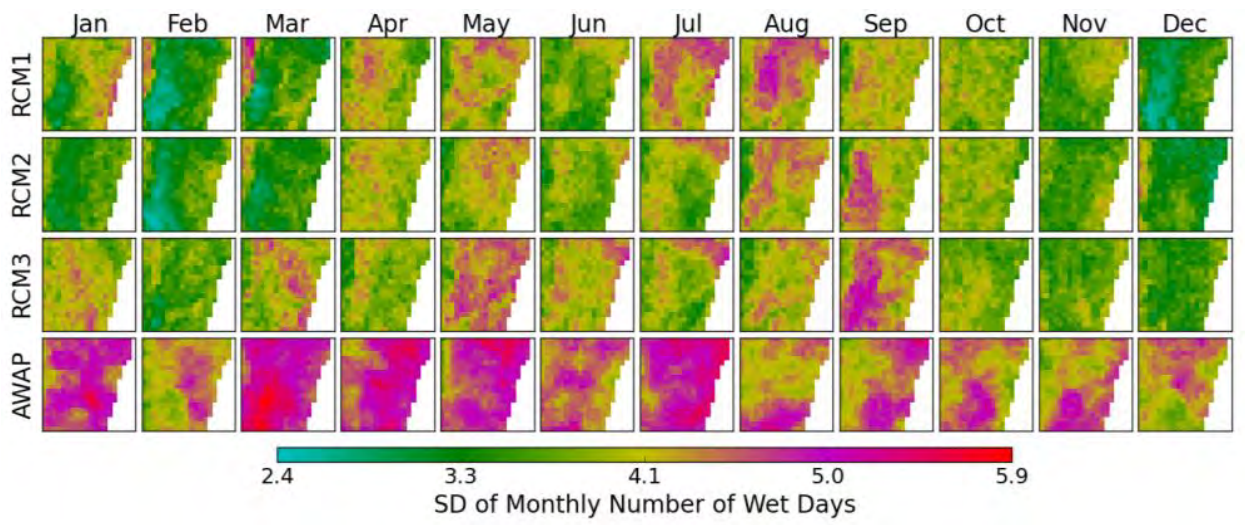

Figure F.5: Intra-annual and spatial variability of the SD of monthly number of wet days.

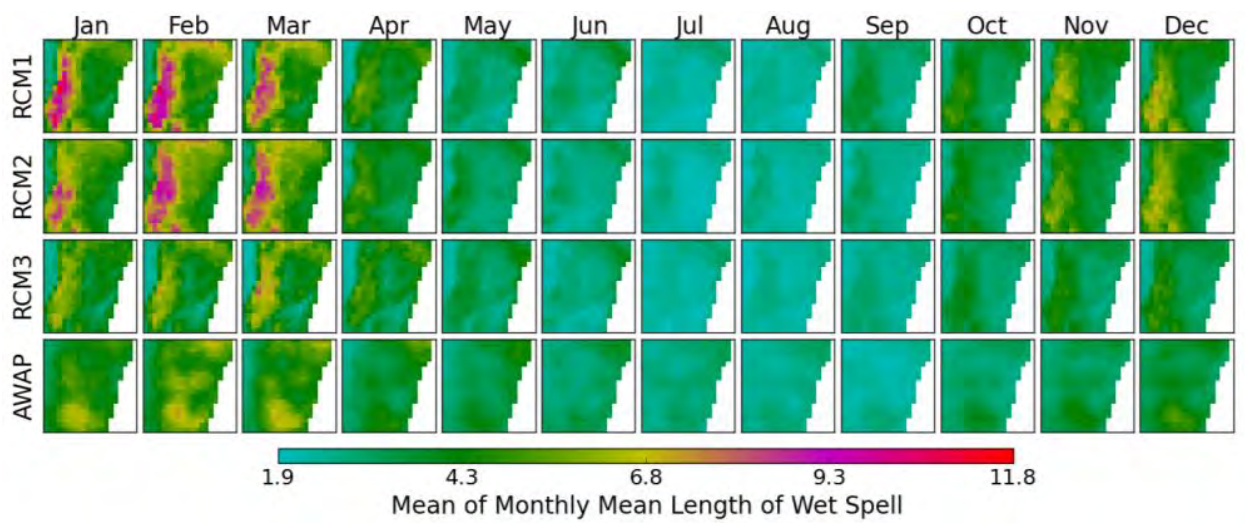

Figure F.6: Intra-annual and spatial variability of the mean of monthly mean length of wet spells. 


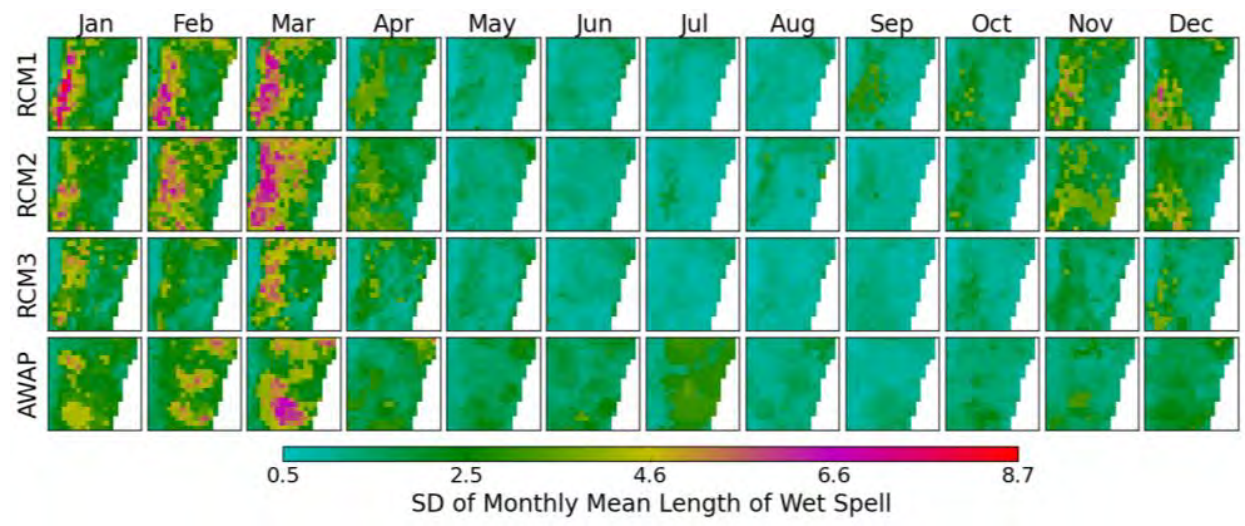

Figure F.7: Intra-annual and spatial variability of the SD of monthly mean length of wet spells.

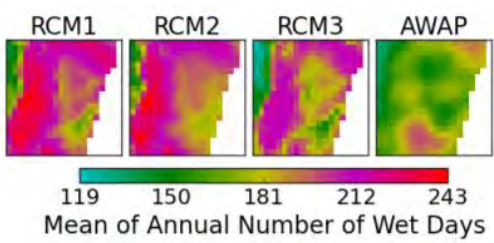

Figure F.8: Spatial variability of the mean of annual number of wet days.

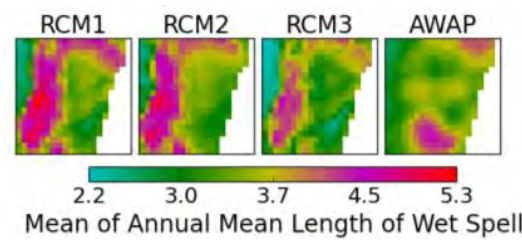

Figure F.9: Spatial variability of the mean of annual mean length of wet spells.

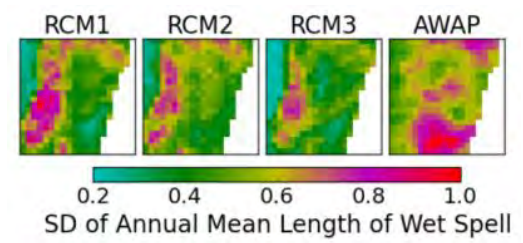

Figure F.10: Spatial variability of the SD of annual mean length of wet spells. 


\section{F.2 Comparison of MC Models for Distribution of Rainfall Depths}

Figures of this appendix compare the MC models for the mean and SD of rainfall depths.
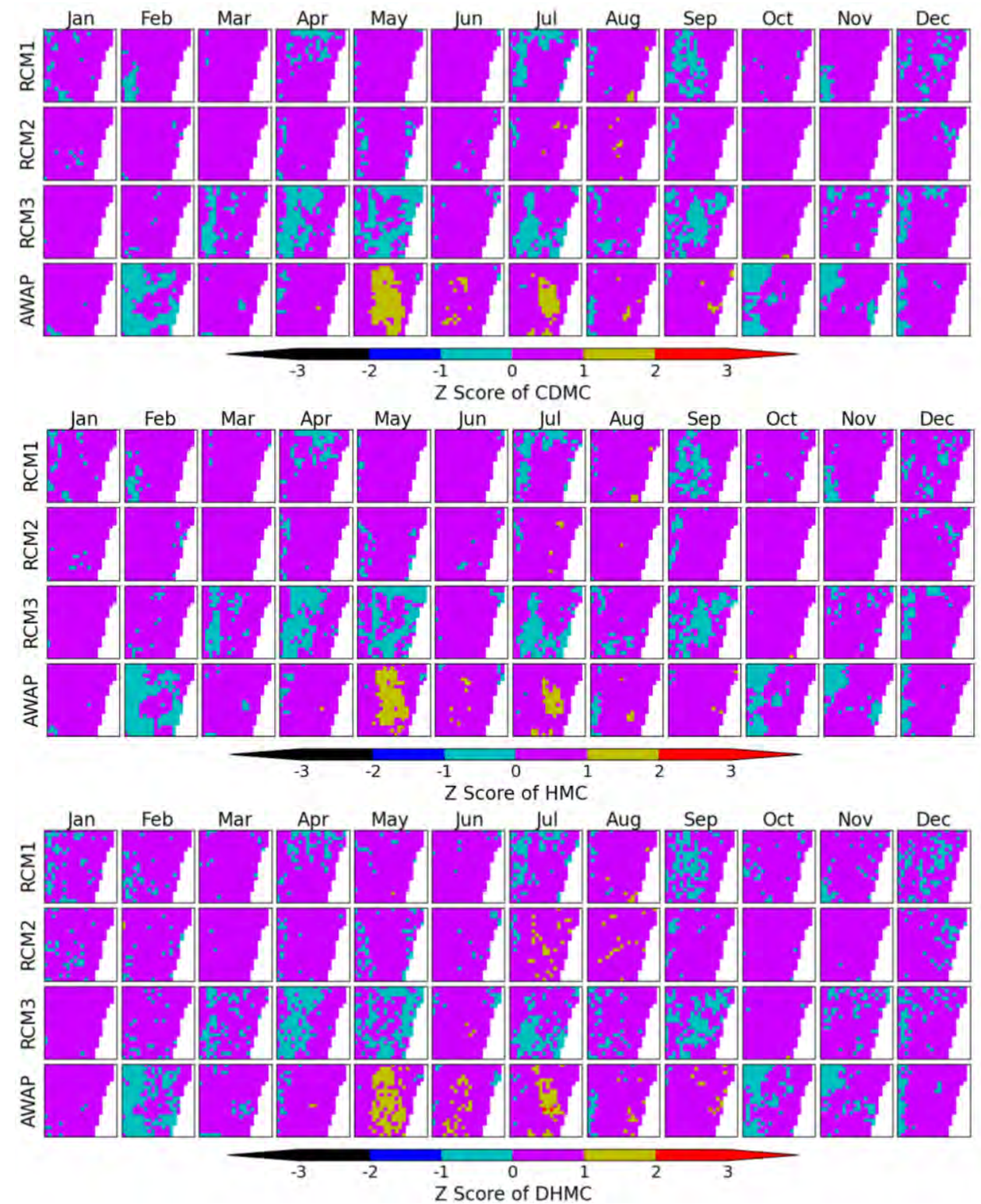

Figure F.11: Comparison of CDMC, HMC and DHMC for the mean of daily rainfall depths. 

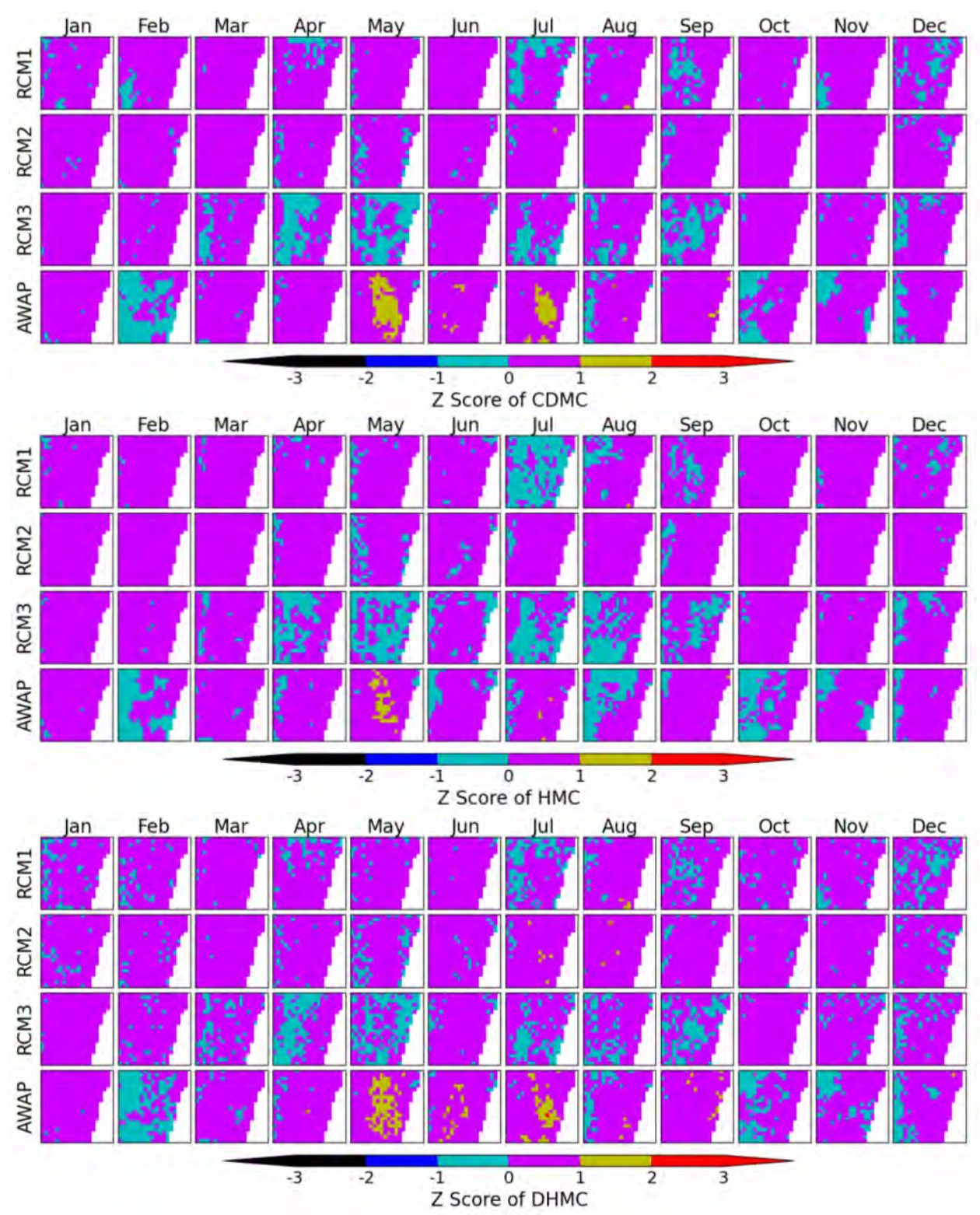

Figure F.12: Comparison of CDMC, HMC and DHMC for the mean of monthly rainfall depths.

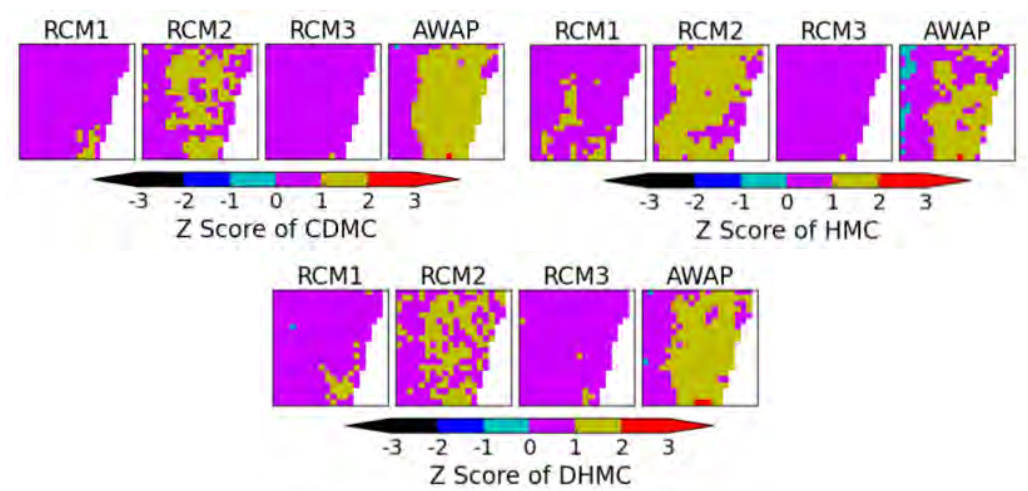

Figure F.13: Comparison of CDMC, HMC and DHMC for the mean of annual rainfall depths. 

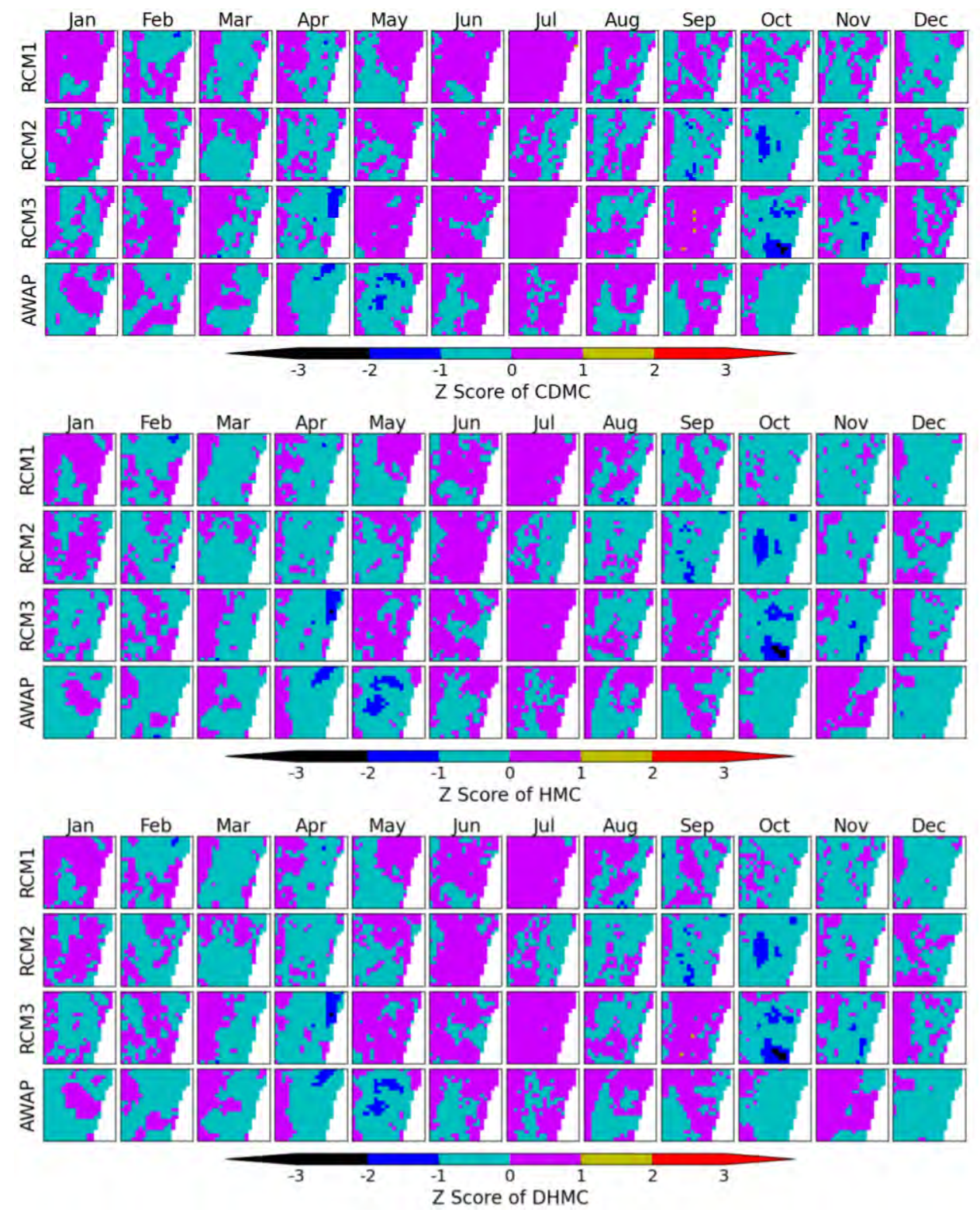

Figure F.14: Comparison of CDMC, HMC and DHMC for the SD of daily rainfall depths. 

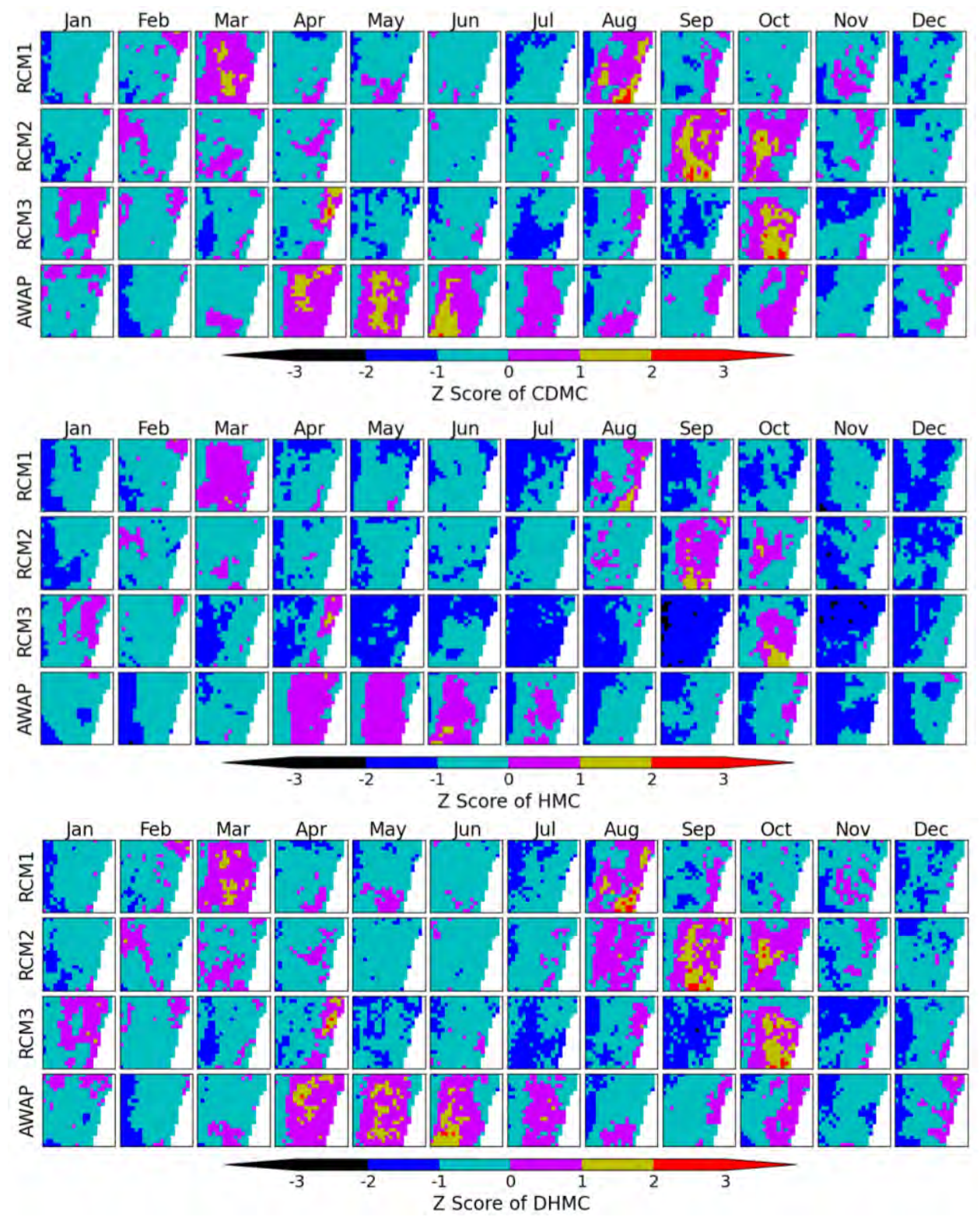

Figure F.15: Comparison of CDMC, HMC and DHMC for the SD of monthly rainfall depths. 


\section{F.3 Comparison of MC Models for Distribution of Wet Period Statistics}

Figures of this appendix compare the MC models for distribution of wet period statistics.
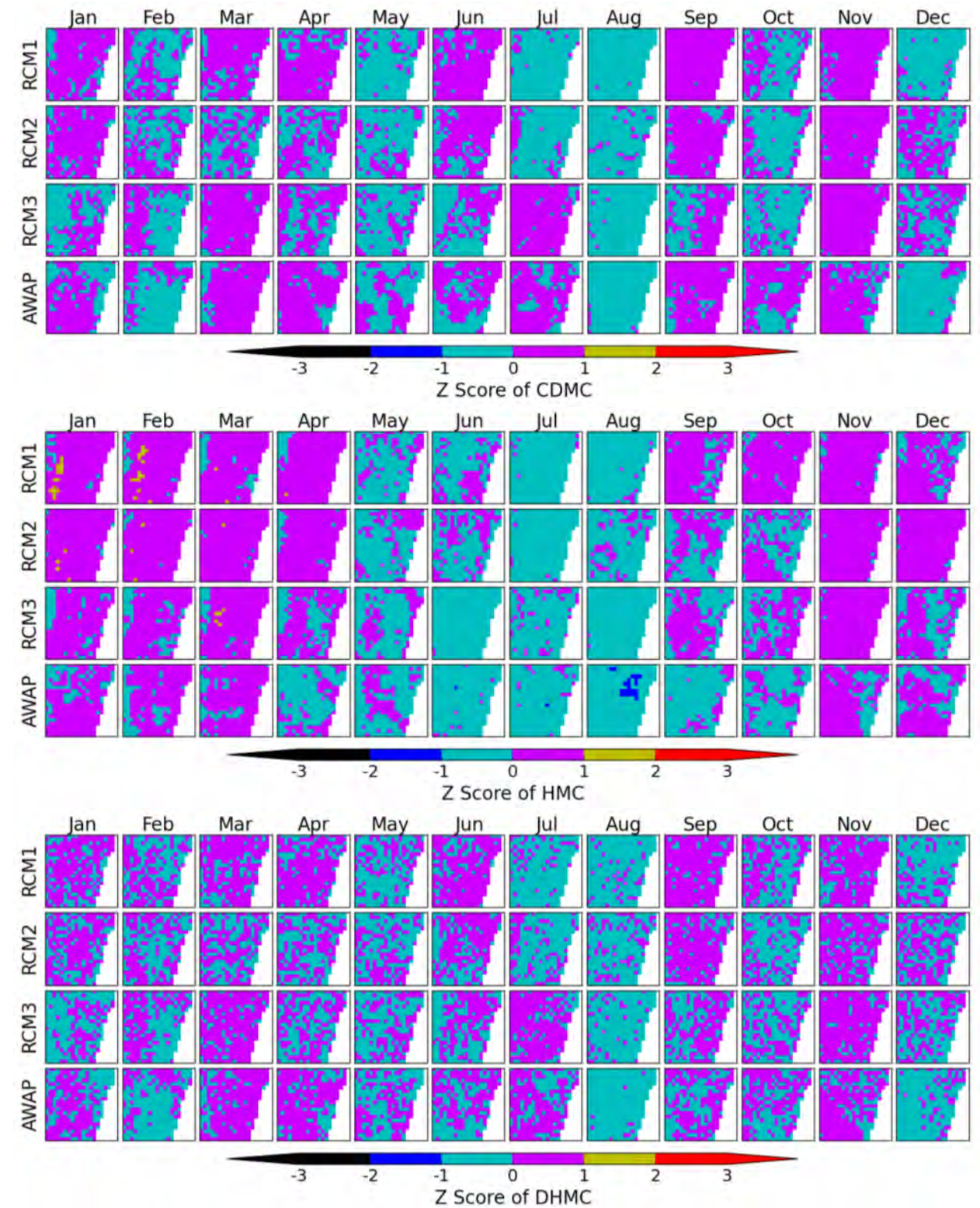

Figure F.16: Comparison of CDMC, HMC and DHMC for the mean of monthly number of wet days. 

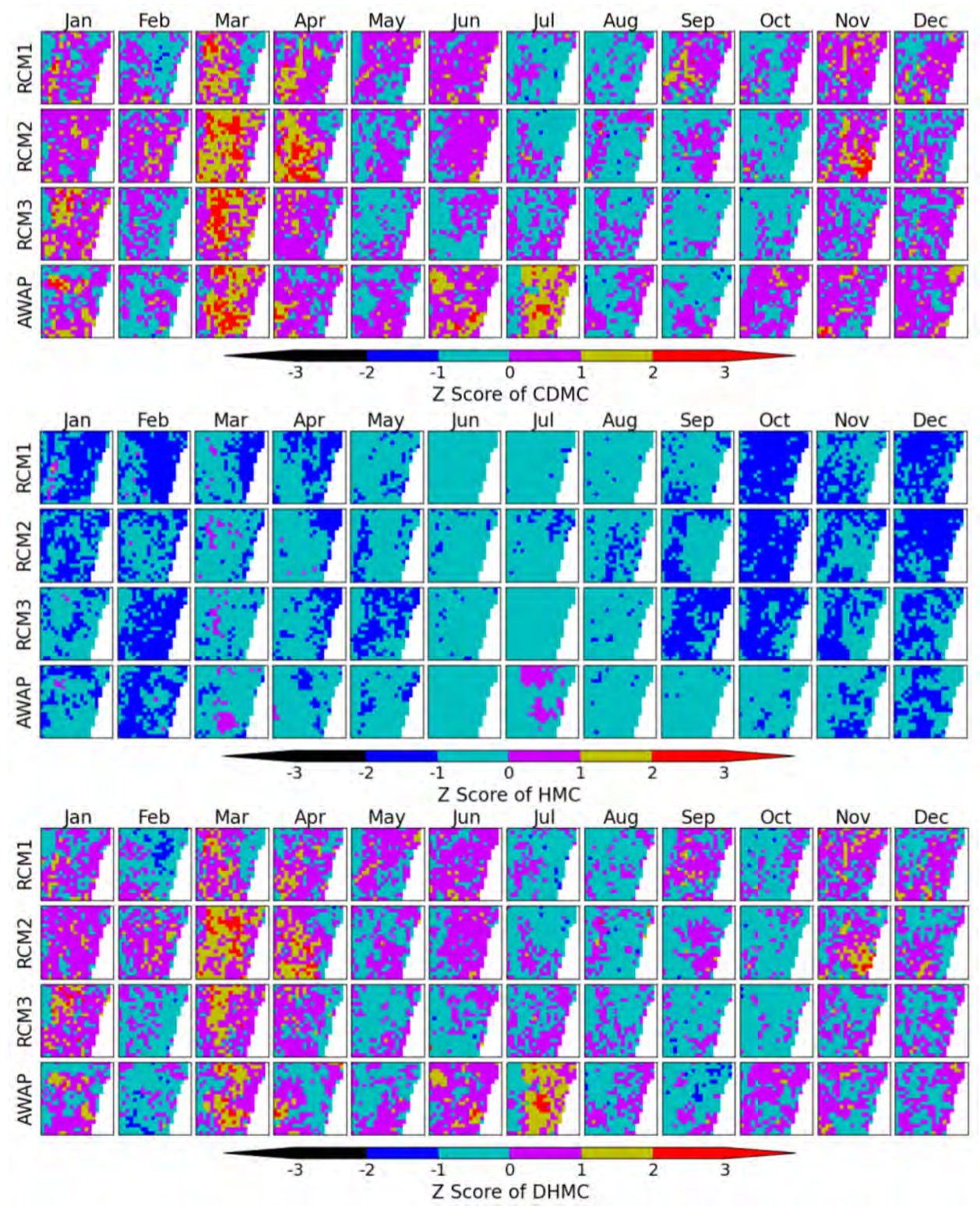

Figure F.17: Comparison of CDMC, HMC and DHMC for the mean of monthly mean length of wet spells. 
F. Comparison of MC Models in Richmond River Site
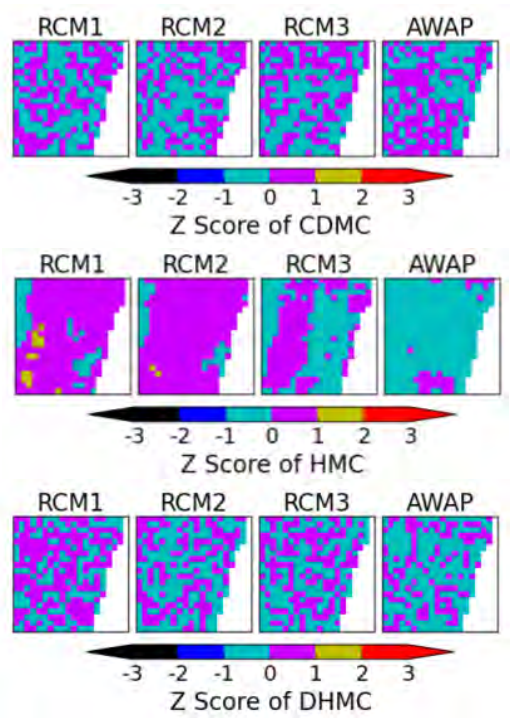

Figure F.18: Comparison of CDMC, HMC and DHMC for the mean of annual number of wet days.
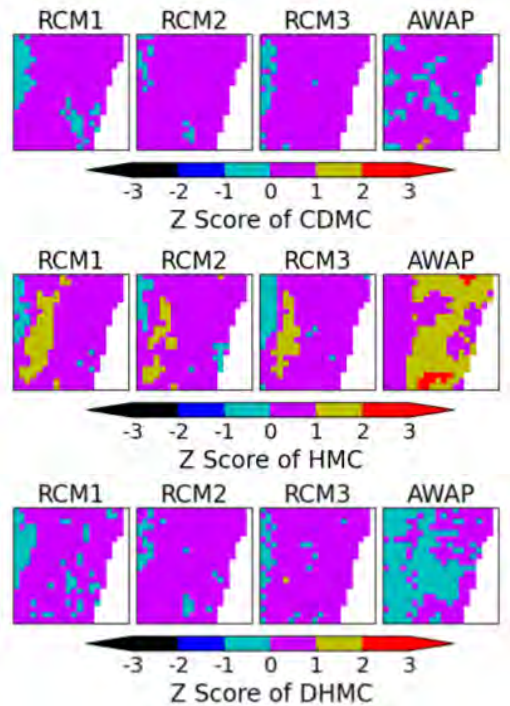

Figure F.19: Comparison of CDMC, HMC and DHMC for the mean of annual mean length of wet spells. 

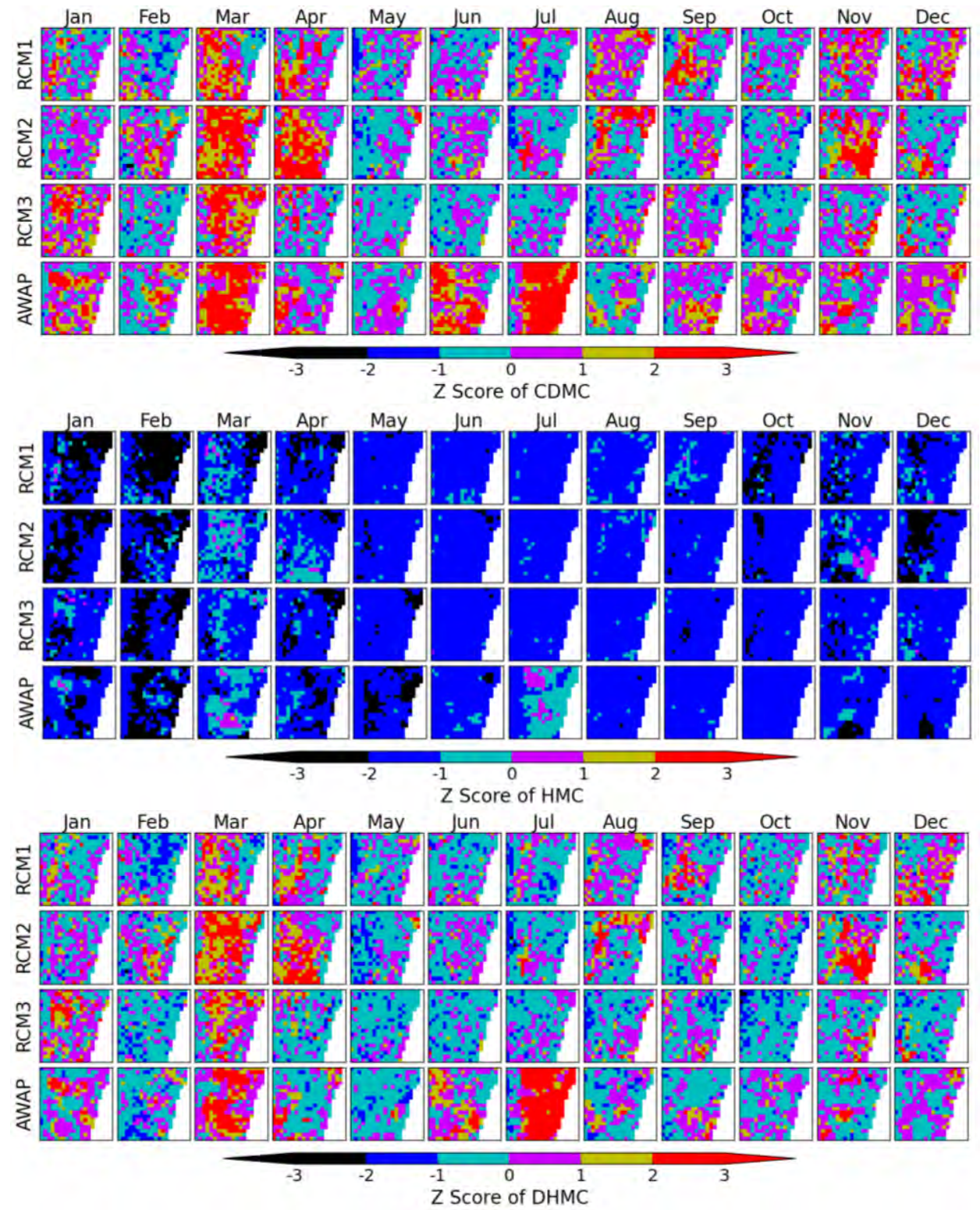

Figure F.20: Comparison of CDMC, HMC and DHMC for the SD of monthly mean length of wet spells. 


\section{F.4 Comparison of MC Models for Autocorrelations}

Figures in this appendix compare the MC models for the autocorrelations of monthly rainfall depths and number of wet days.
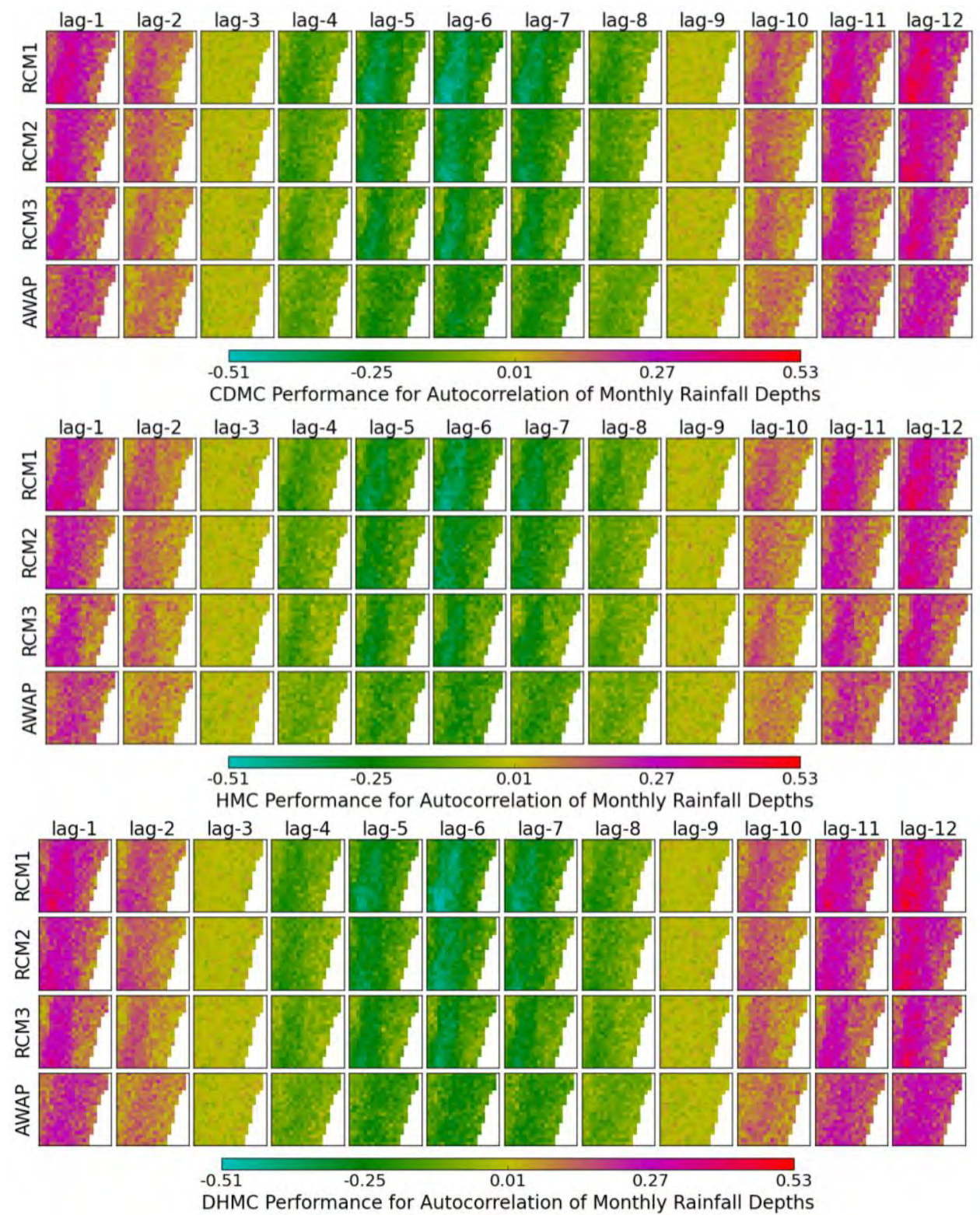

Figure F.21: Comparison of CDMC, HMC and DHMC for the month-to-month autocorrelations of monthly rainfall depths. 

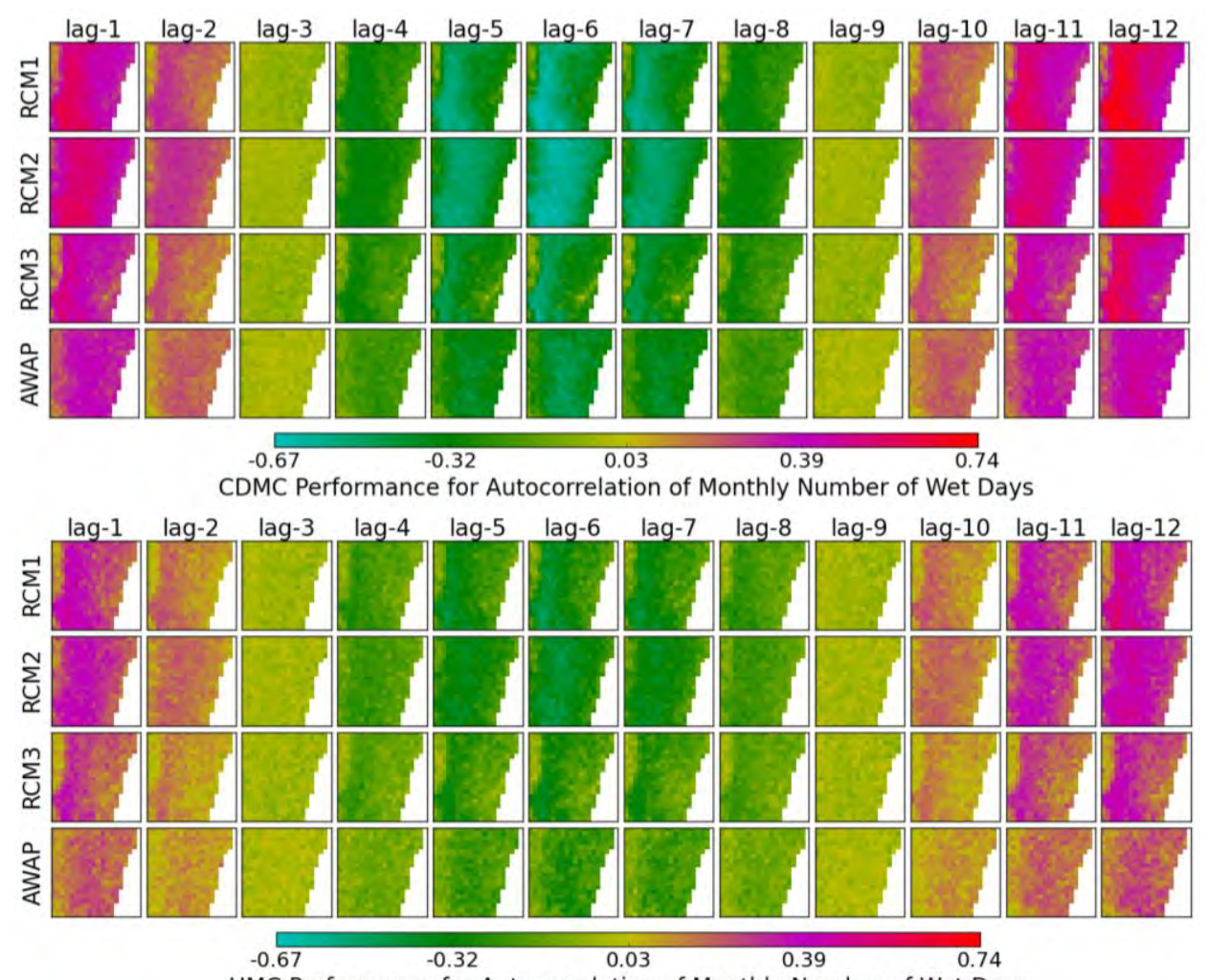

HMC Performance for Autocorrelation of Monthly Number of Wet Days

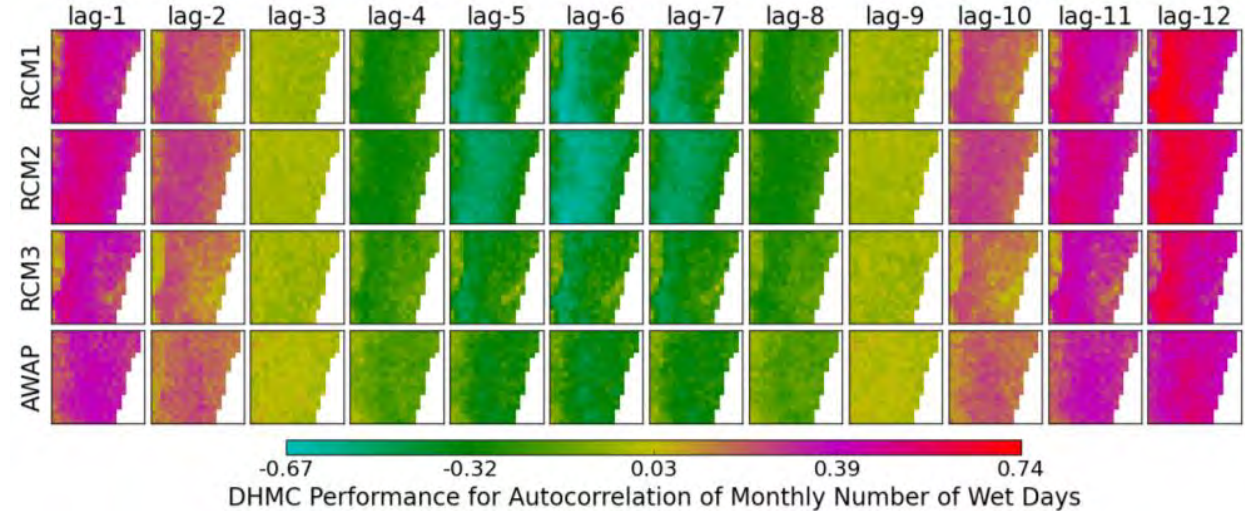

Figure F.22: Comparison of CDMC, HMC and DHMC for the month-to-month autocorrelations of monthly number of wet days. 


\section{G. Comparison of MC Models in Bega River Site}

\section{G.1 Intra-annual and Spatial Variability of Rainfall and Wet Periods}

Figures in this appendix show the intra-annual and spatial variability of the mean and SD of rainfall depths and wet periods statistics.

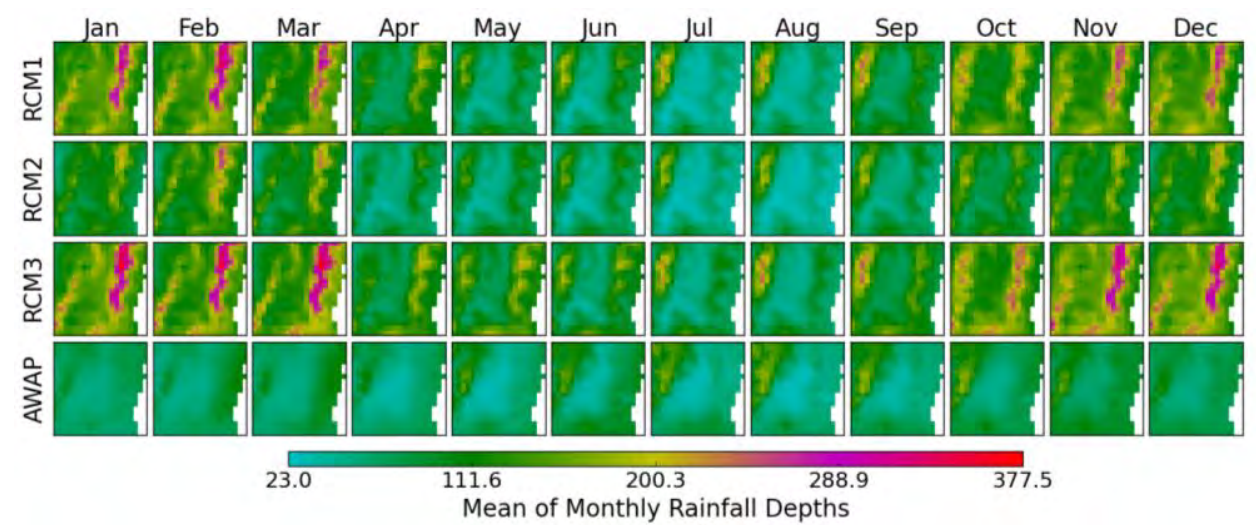

Figure G.1: Intra-annual and spatial variability of the mean of monthly rainfall depths.

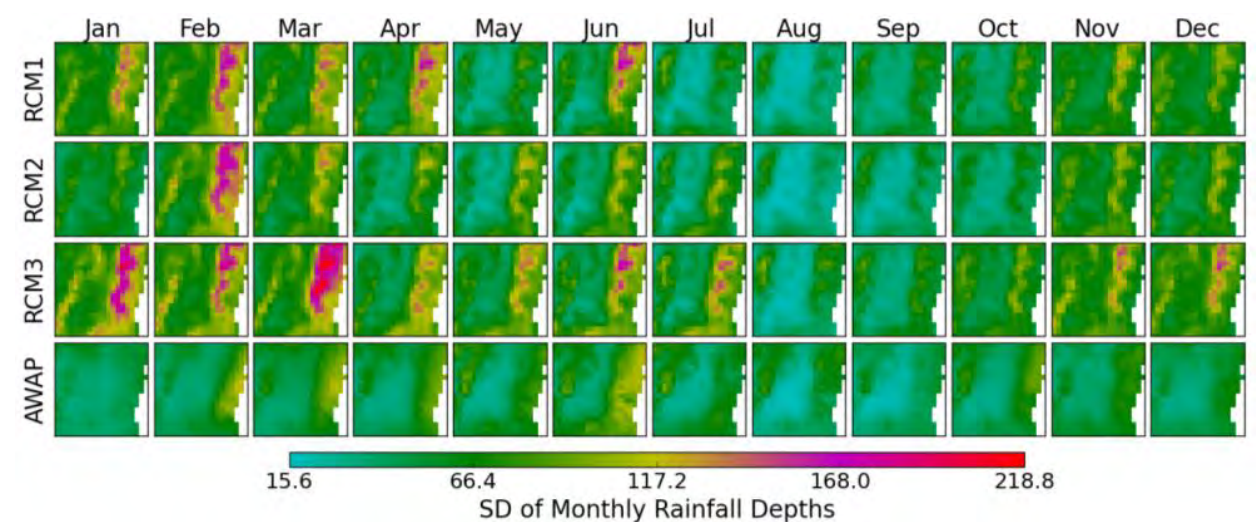

Figure G.2: Intra-annual and spatial variability of the SD of monthly rainfall depths.

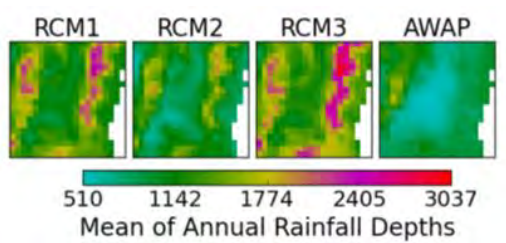

Figure G.3: Spatial variability of the mean of annual rainfall depths. 


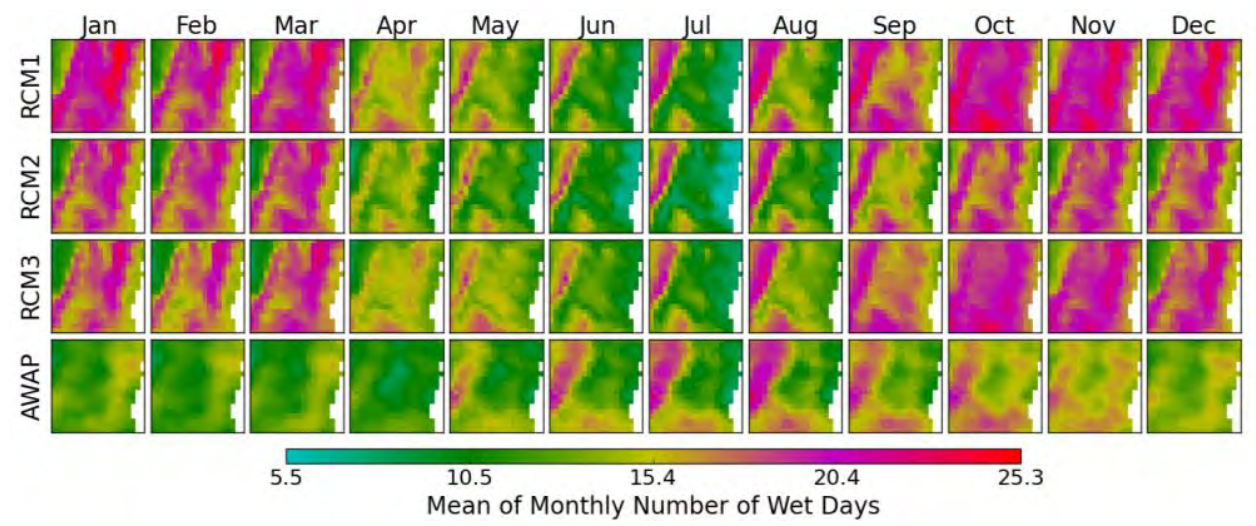

Figure G.4: Intra-annual and spatial variability of the mean of monthly number of wet days.

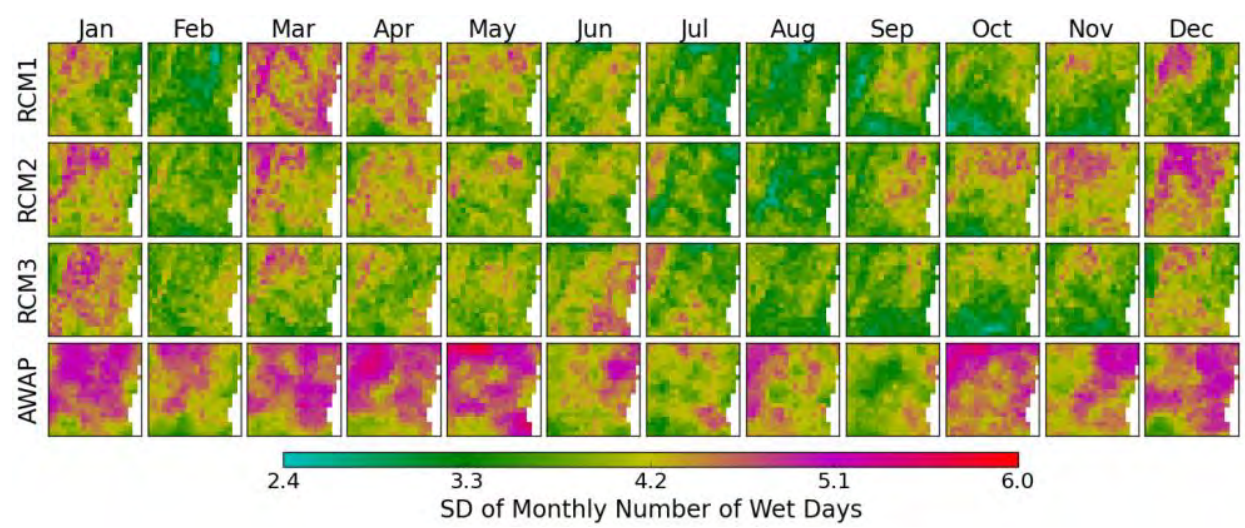

Figure G.5: Intra-annual and spatial variability of the SD of monthly number of wet days.

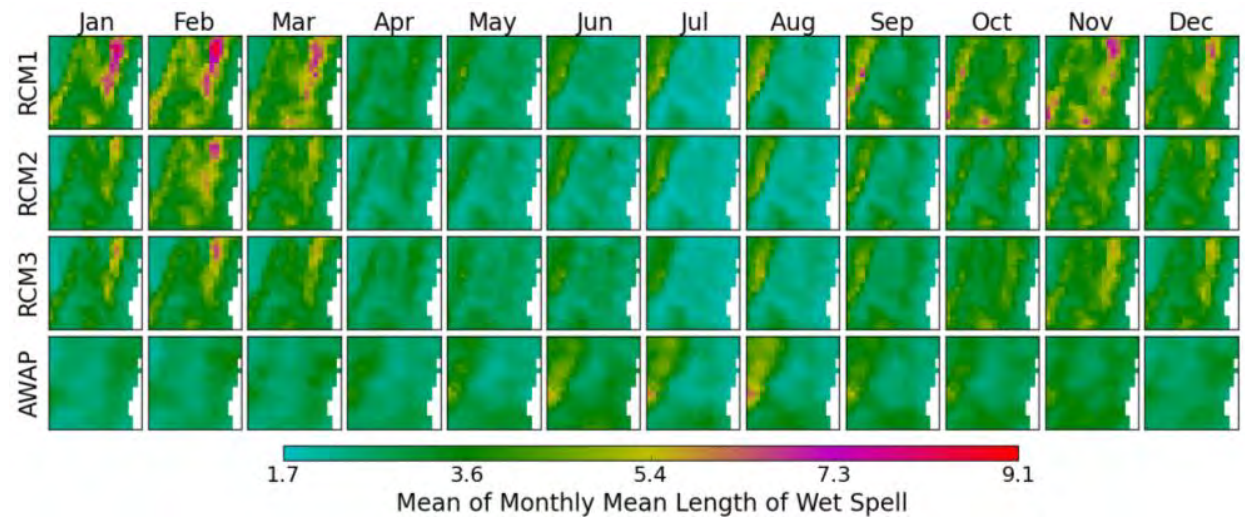

Figure G.6: Intra-annual and spatial variability of the mean of monthly mean length of wet spells. 


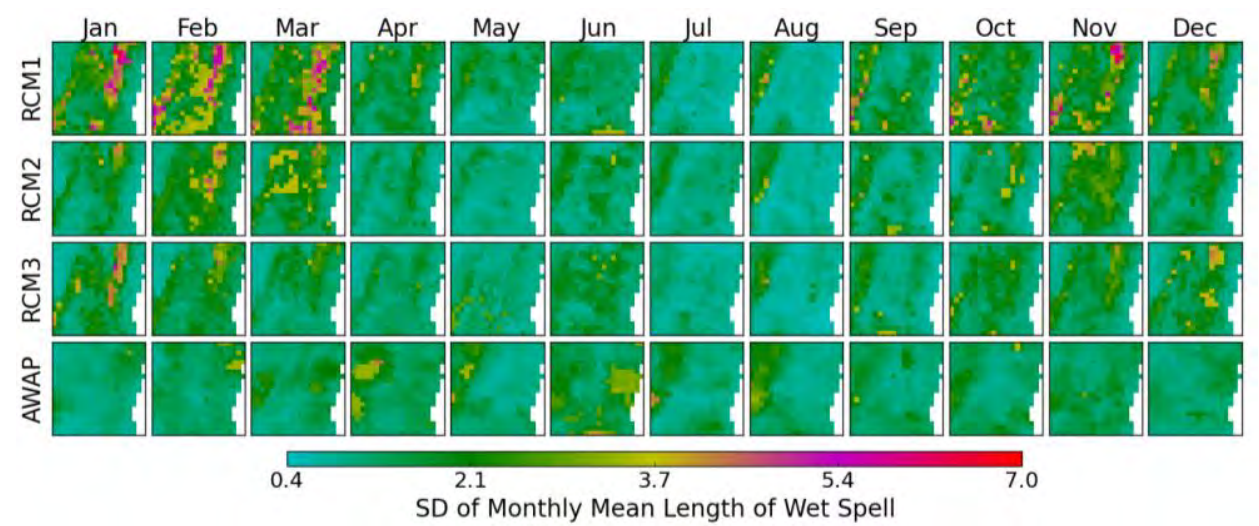

Figure G.7: Intra-annual and spatial variability of the SD of monthly mean length of wet spells.

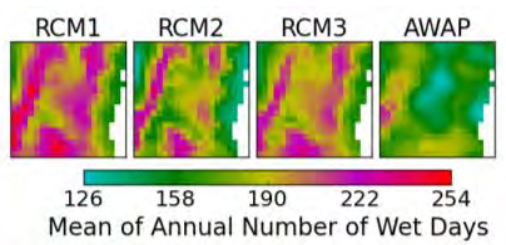

Figure G.8: Spatial variability of the mean of annual number of wet days.

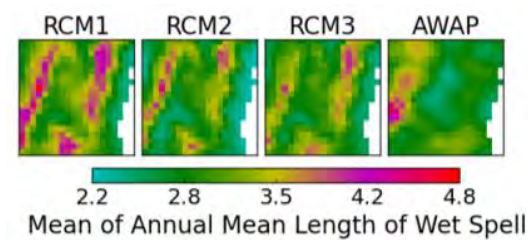

Figure G.9: Spatial variability of the mean of annual mean length of wet spells.

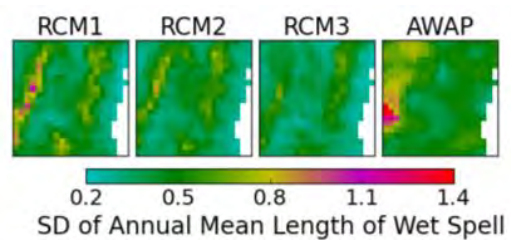

Figure G.10: Spatial variability of the SD of annual mean length of wet spells. 


\section{G.2 Comparison of MC Models for Distribution of Rainfall Depths}

Figures of this appendix compare the MC models for the mean and SD of rainfall depths.
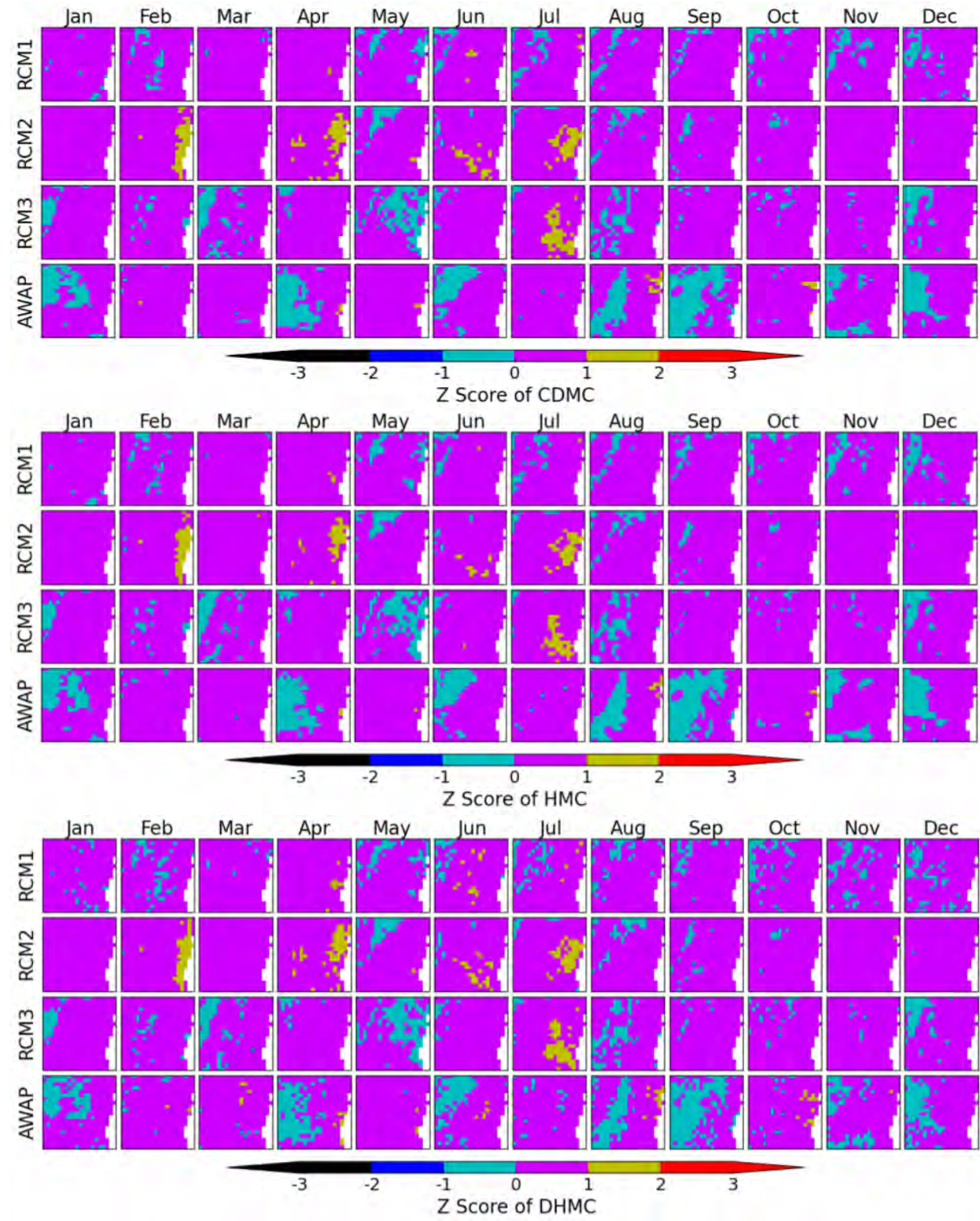

Figure G.11: Comparison of CDMC, HMC and DHMC for the mean of daily rainfall depths. 

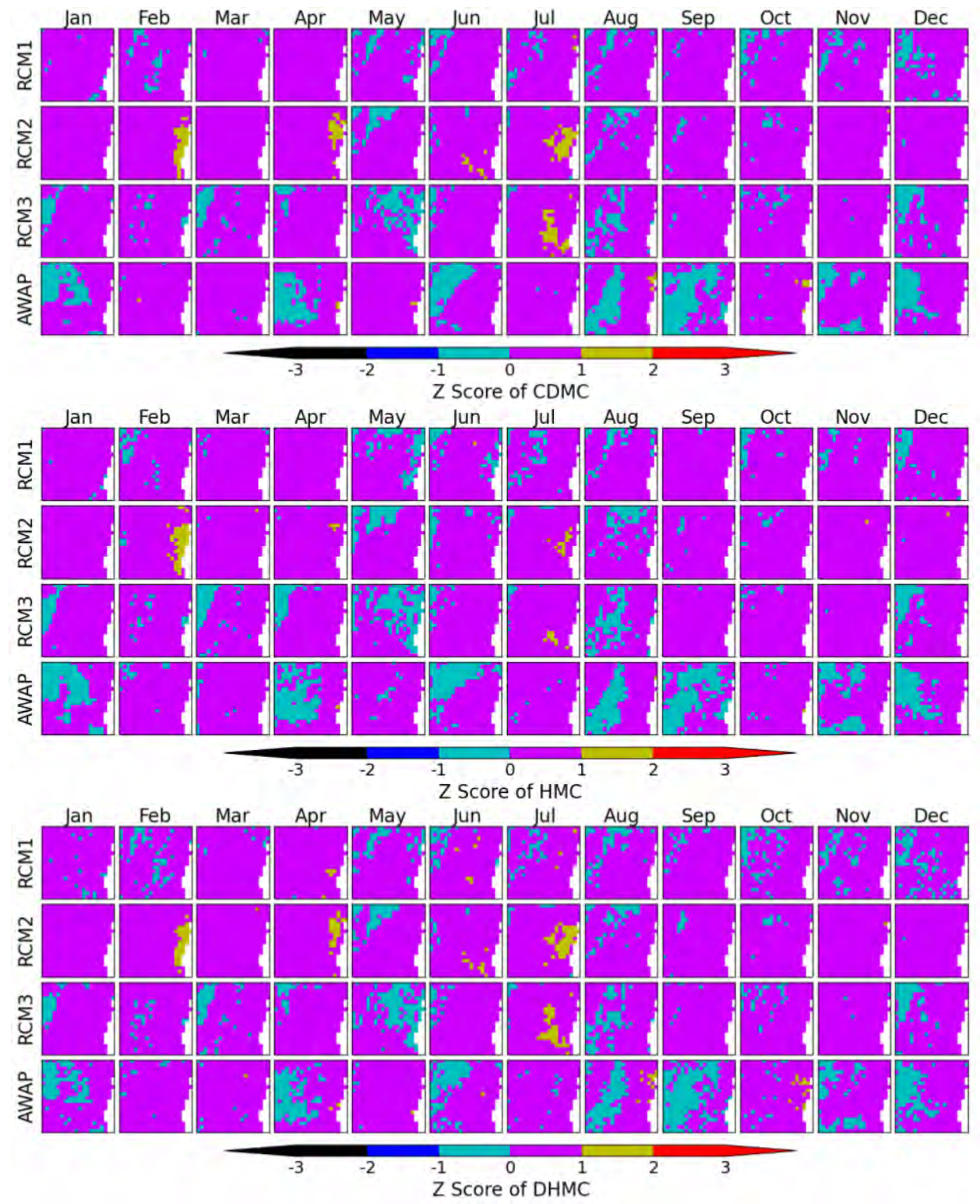

Figure G.12: Comparison of CDMC, HMC and DHMC for the mean of monthly rainfall depths.

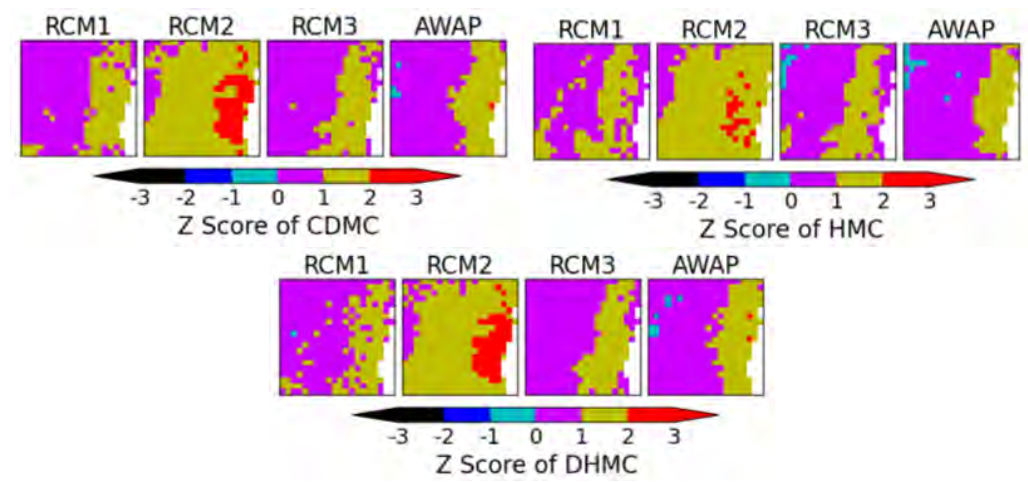

Figure G.13: Comparison of CDMC, HMC and DHMC for the mean of annual rainfall depths. 

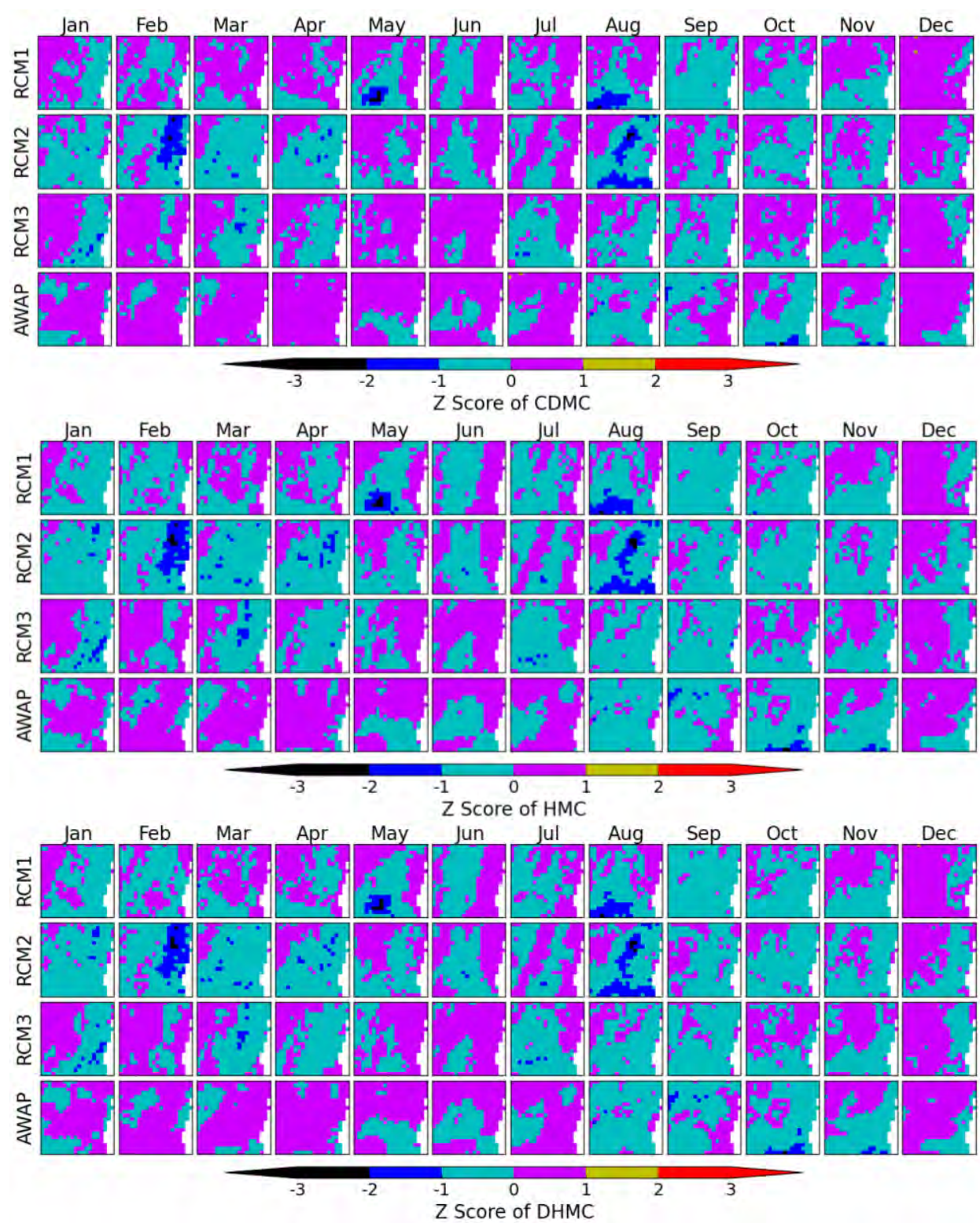

Figure G.14: Comparison of CDMC, HMC and DHMC for the SD of daily rainfall depths. 

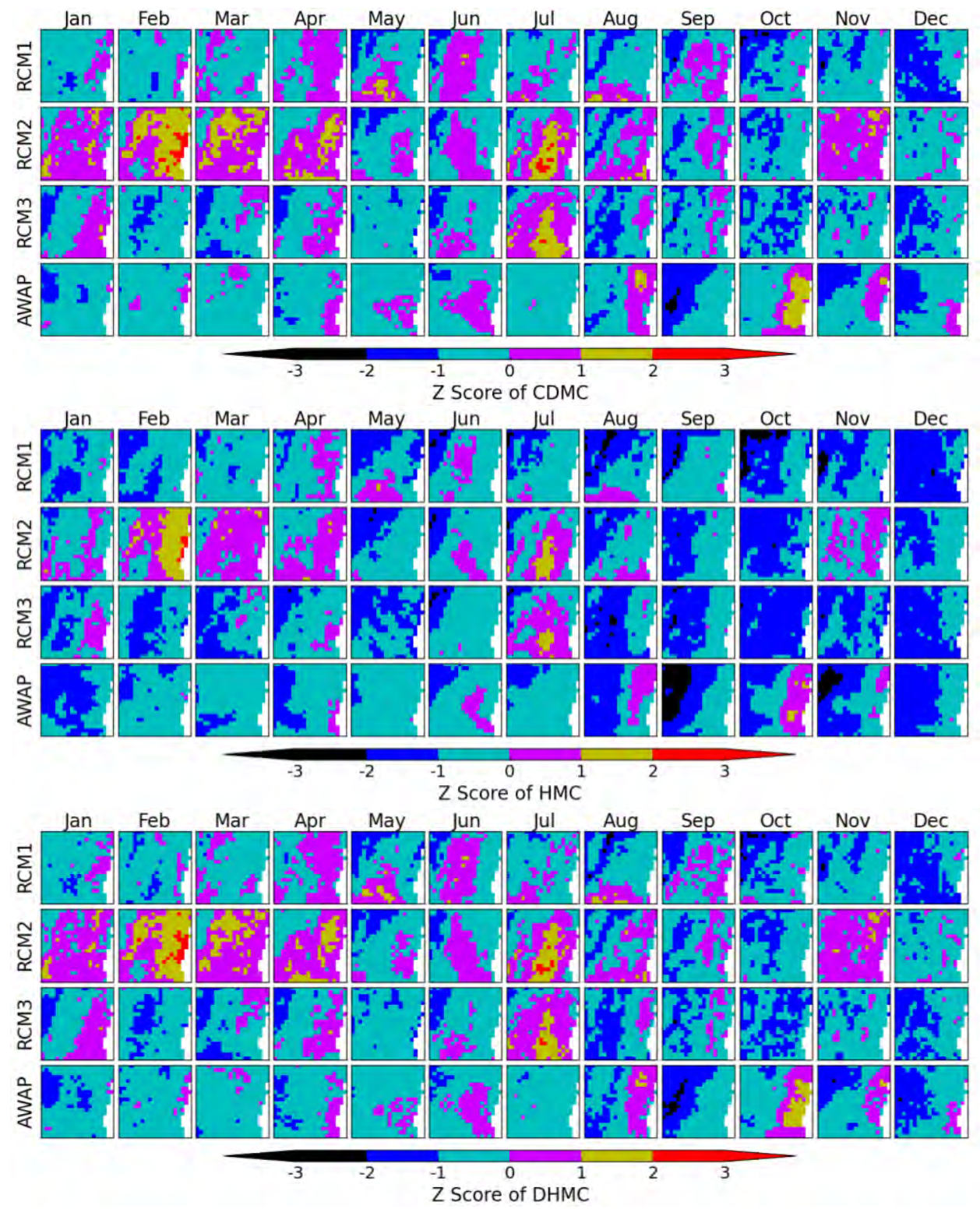

Figure G.15: Comparison of CDMC, HMC and DHMC for the SD of monthly rainfall depths. 


\section{G.3 Comparison of MC Models for Distribution of Wet Period Statistics}

Figures of this appendix compare the MC models for distribution of wet period statistics.
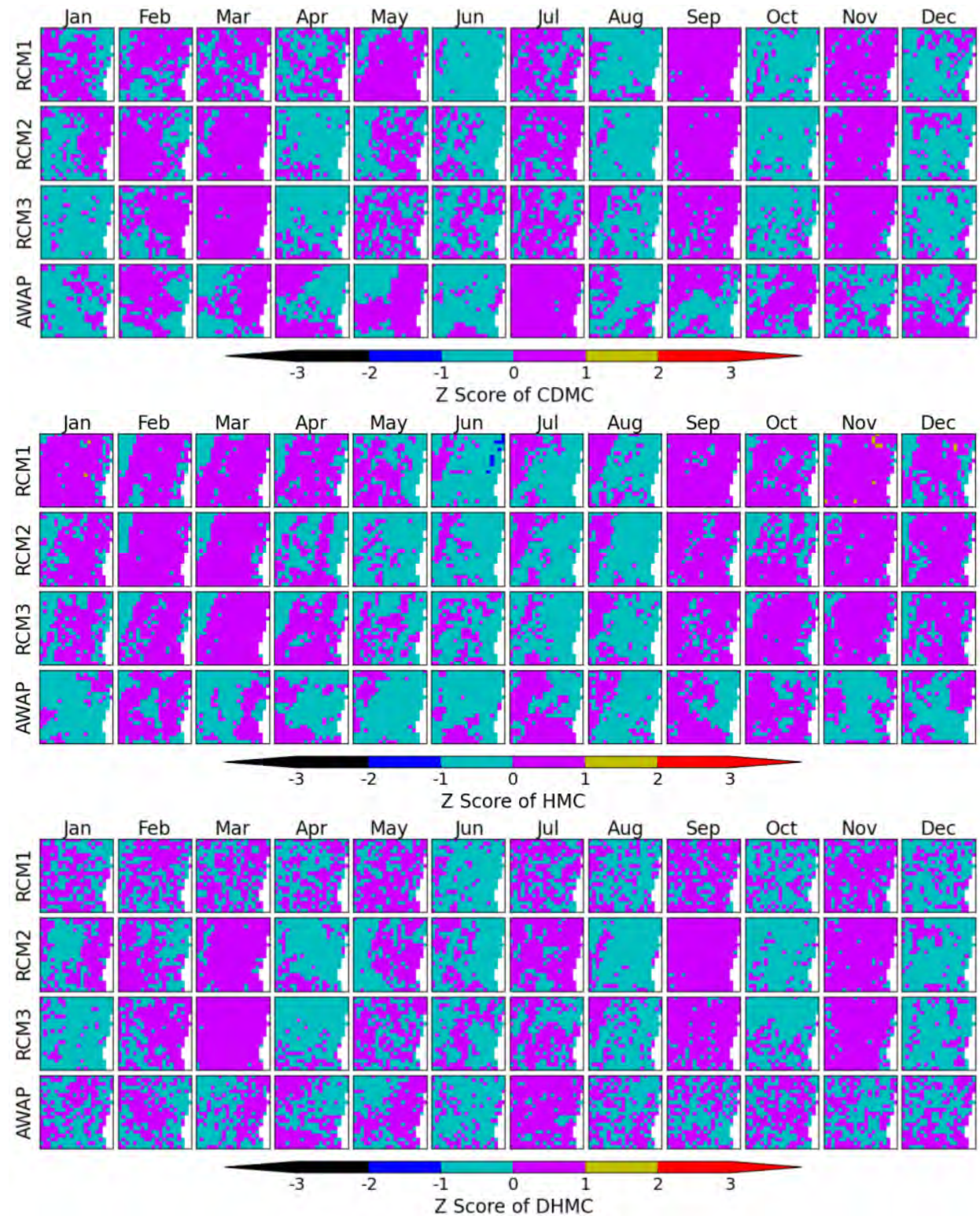

Figure G.16: Comparison of CDMC, HMC and DHMC for the mean of monthly number of wet days. 


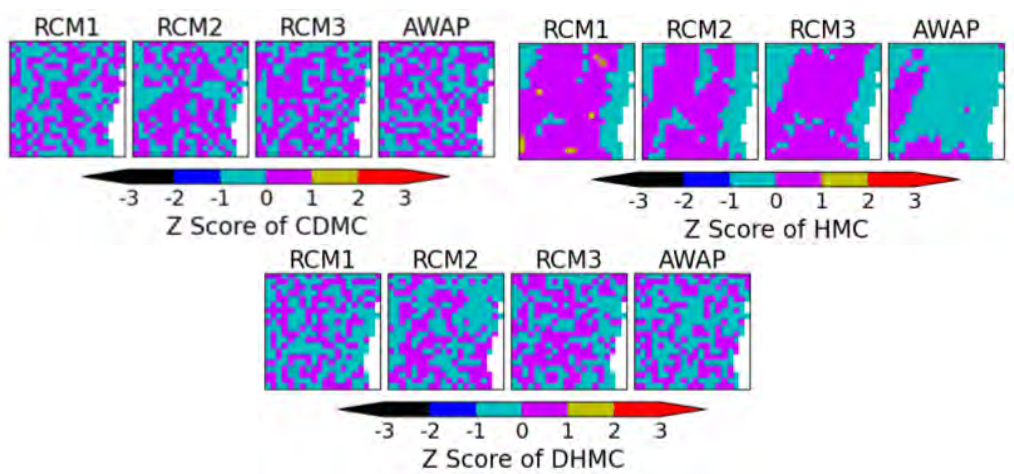

Figure G.17: Comparison of CDMC, HMC and DHMC for the mean of annual number of wet days.
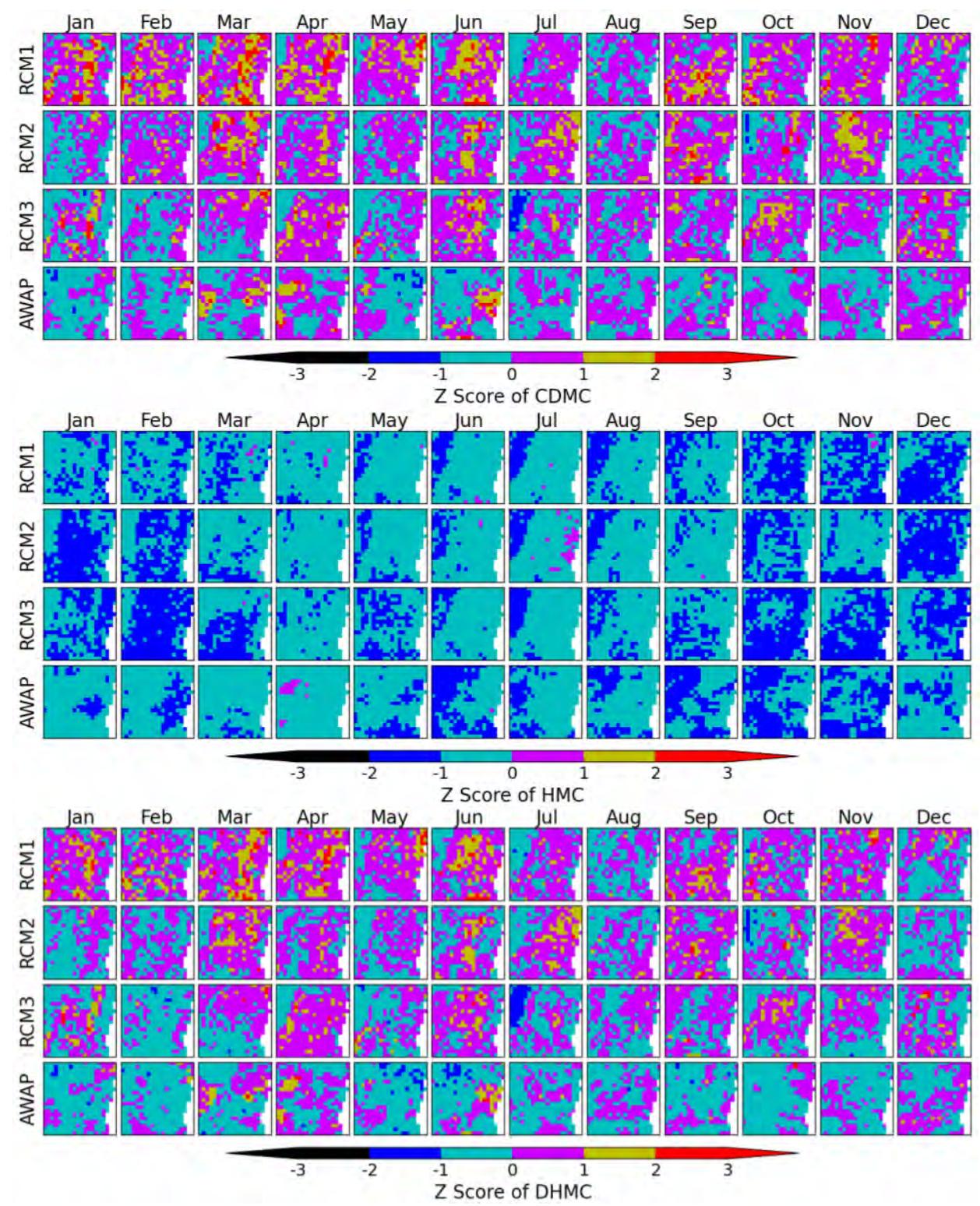

Figure G.18: Comparison of CDMC, HMC and DHMC for the mean of monthly mean length of wet spells. 


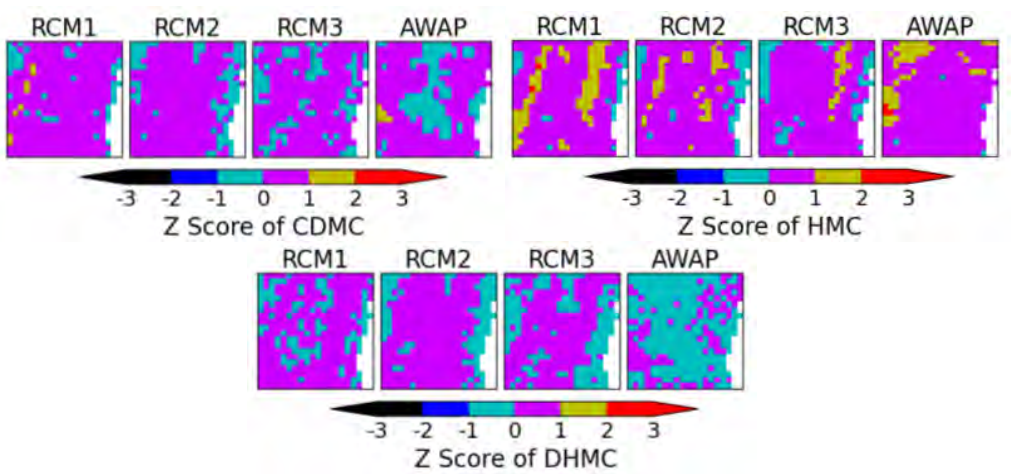

Figure G.19: Comparison of CDMC, HMC and DHMC for the mean of annual mean length of wet spells.
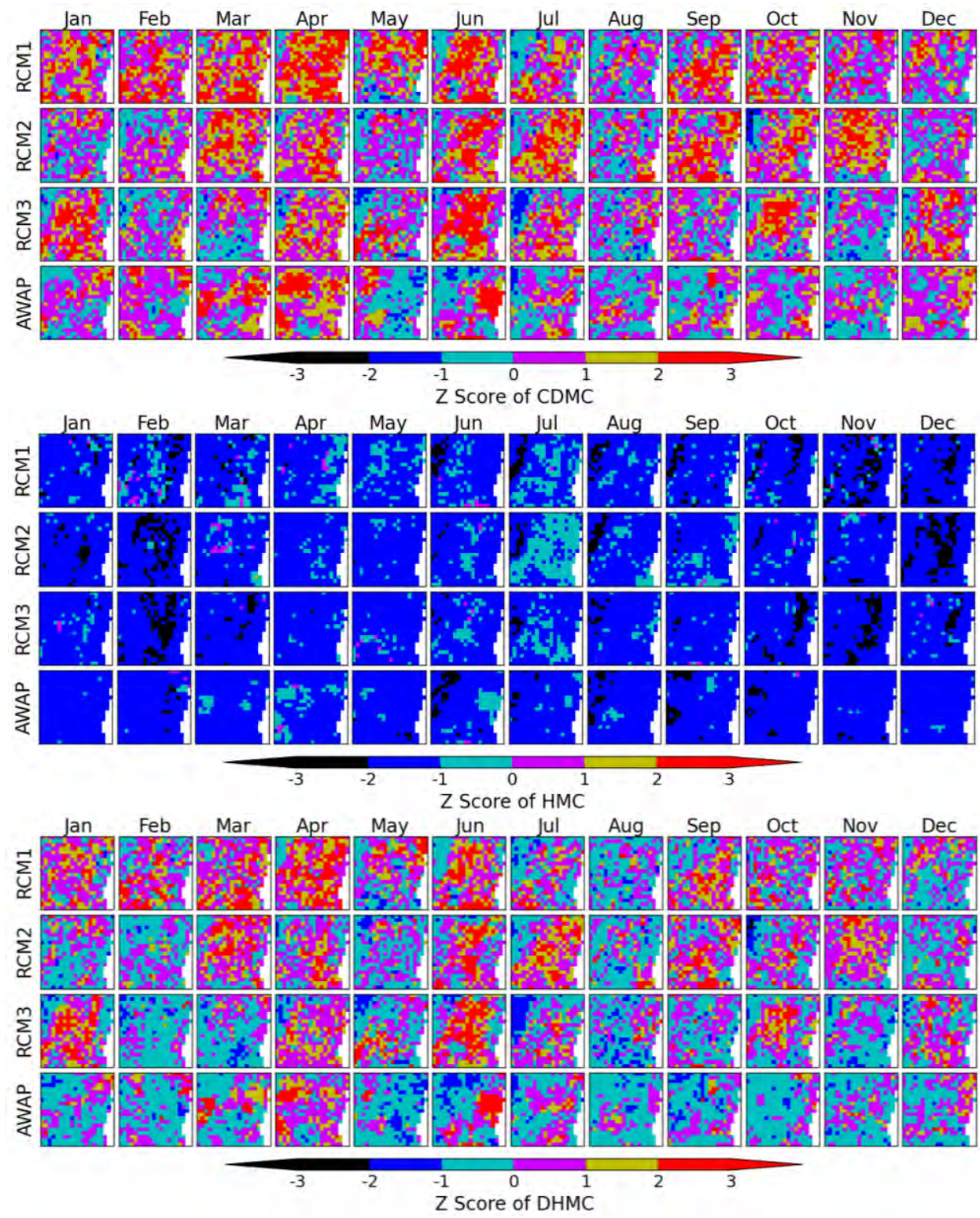

Figure G.20: Comparison of CDMC, HMC and DHMC for the SD of monthly mean length of wet spells. 


\section{G.4 Comparison of MC Models for Autocorrelations}

Figures in this appendix compare the MC models for the autocorrelations of monthly rainfall depths and number of wet days.
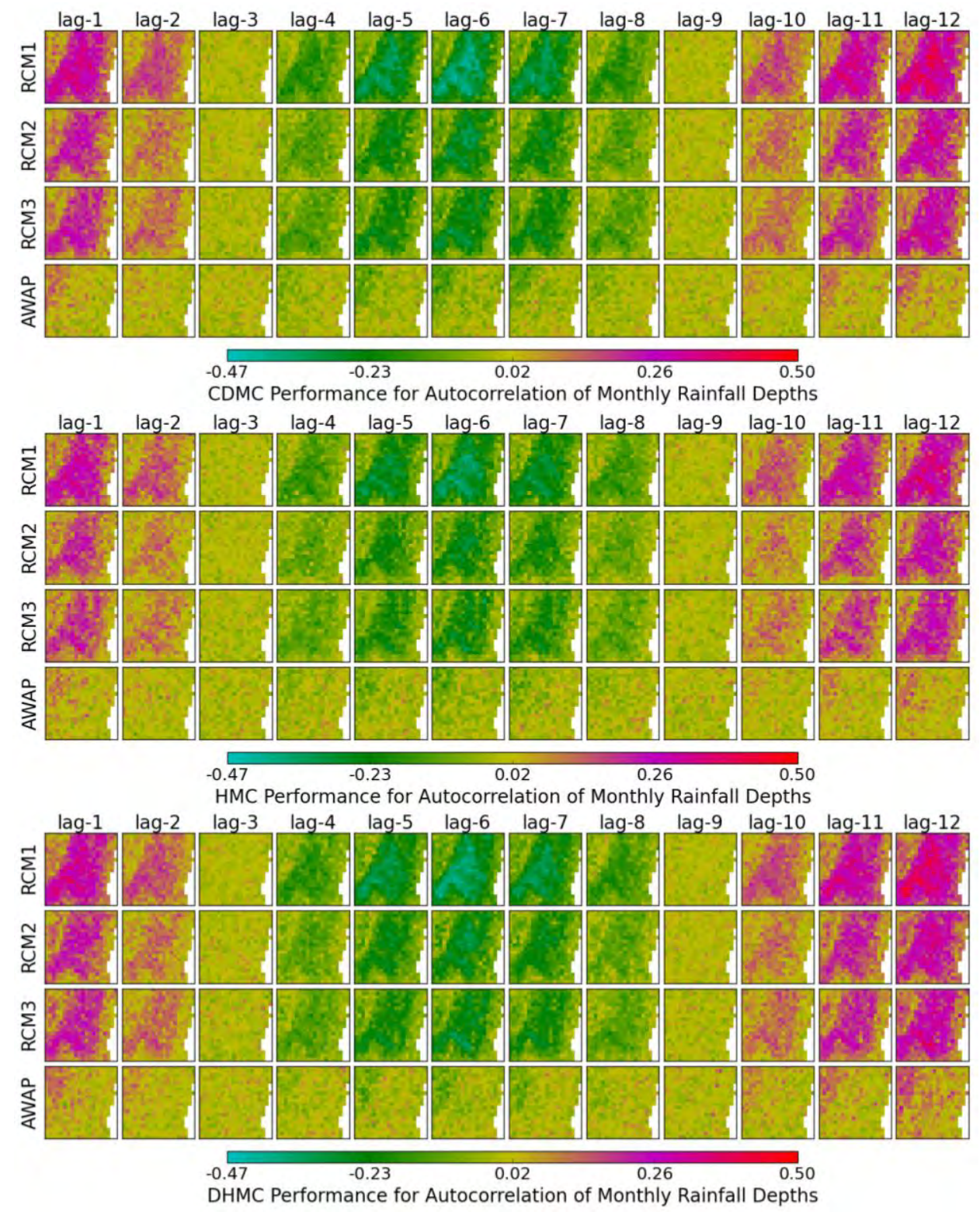

Figure G.21: Comparison of CDMC, HMC and DHMC for the month-to-month autocorrelations of monthly rainfall depths. 

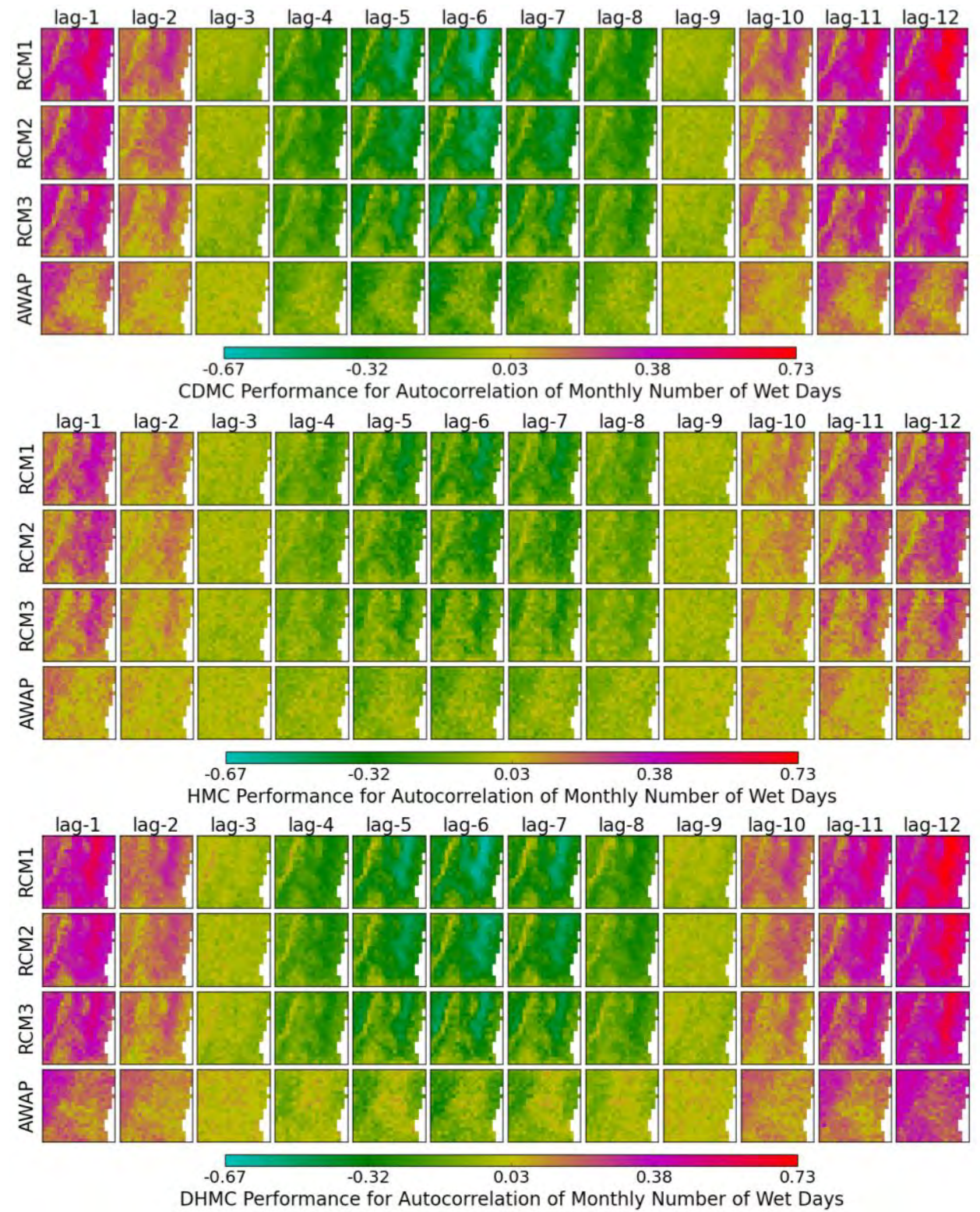

Figure G.22: Comparison of CDMC, HMC and DHMC for the month-to-month autocorrelations of monthly number of wet days. 


\section{H. Comparison of MC and MMKD for Raingauge Data around Australia}

\section{H.1 Mean and SD of Monthly Mean Length of Wet Spells}

Figure H.1 shows the mean and SD of monthly mean length of wet spells.
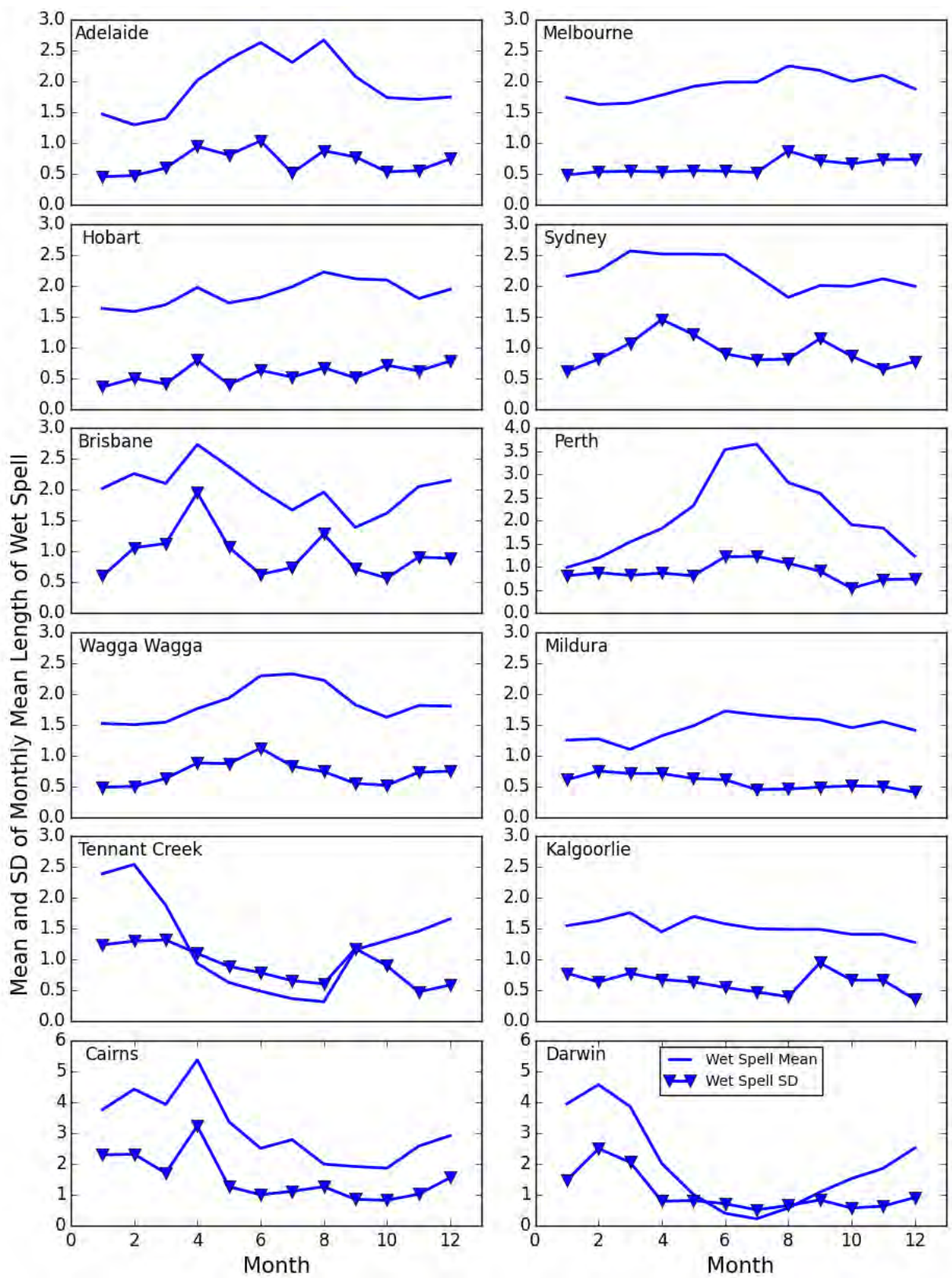

Figure H.1: Mean and SD of monthly mean length of wet spells. Note the change in vertical scale for Cairns and Darwin. 


\section{H.2 Model Comparison for Distribution Statistics}

Figures in this appendix compare the CDMC, HMC, DHMC, and MMKD for mean and SD of rainfall depths and wet period statistics.
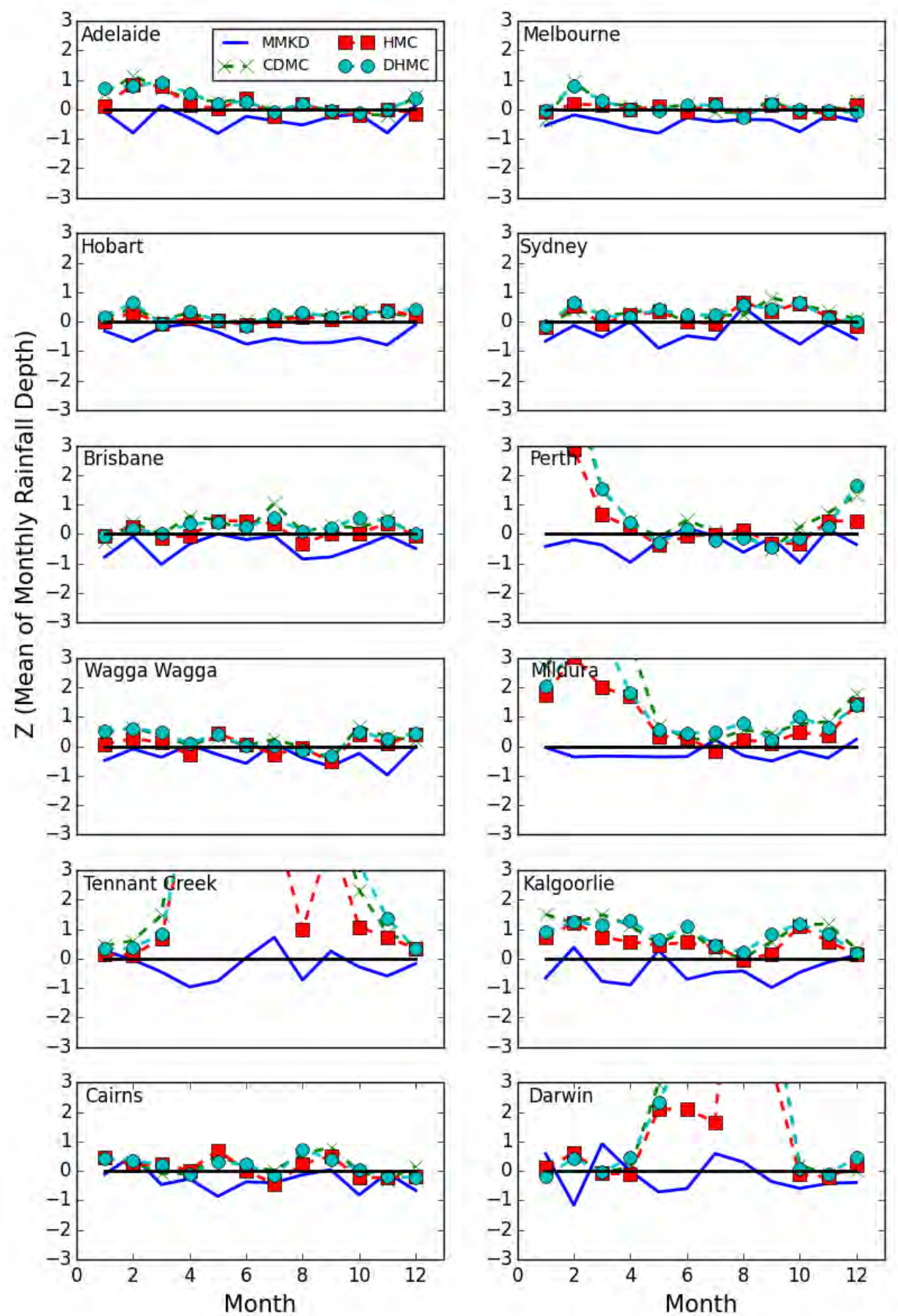

Figure H.2: Comparison of model performances for mean of monthly rainfall depths. 
H. Comparison of MC and MMKD for Raingauge Data around Australia
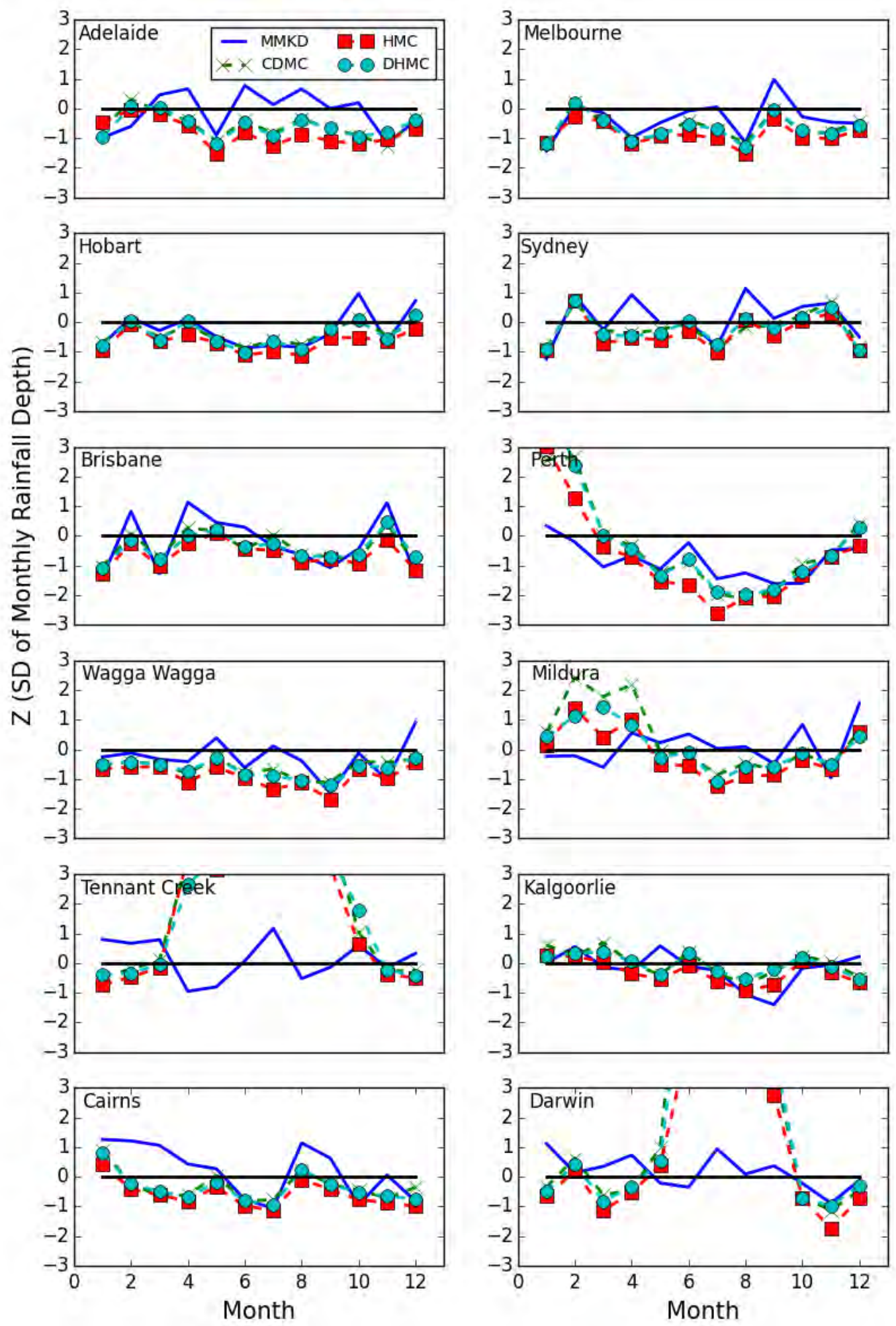

Figure H.3: Comparison of model performances for SD of monthly rainfall depths. 

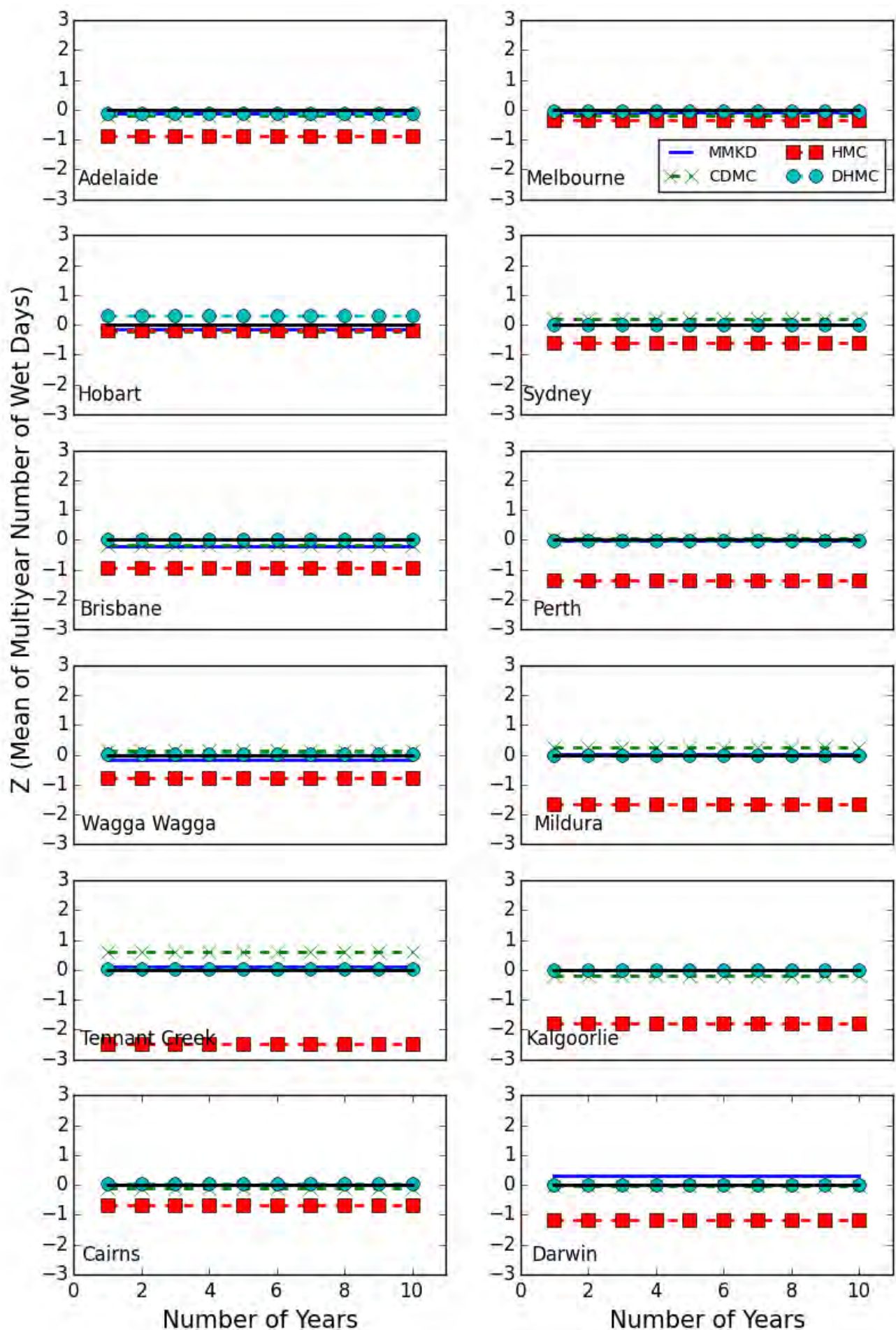

Figure H.4: Comparison of models for mean of multiyear number of wet days. 
H. Comparison of MC and MMKD for Raingauge Data around Australia
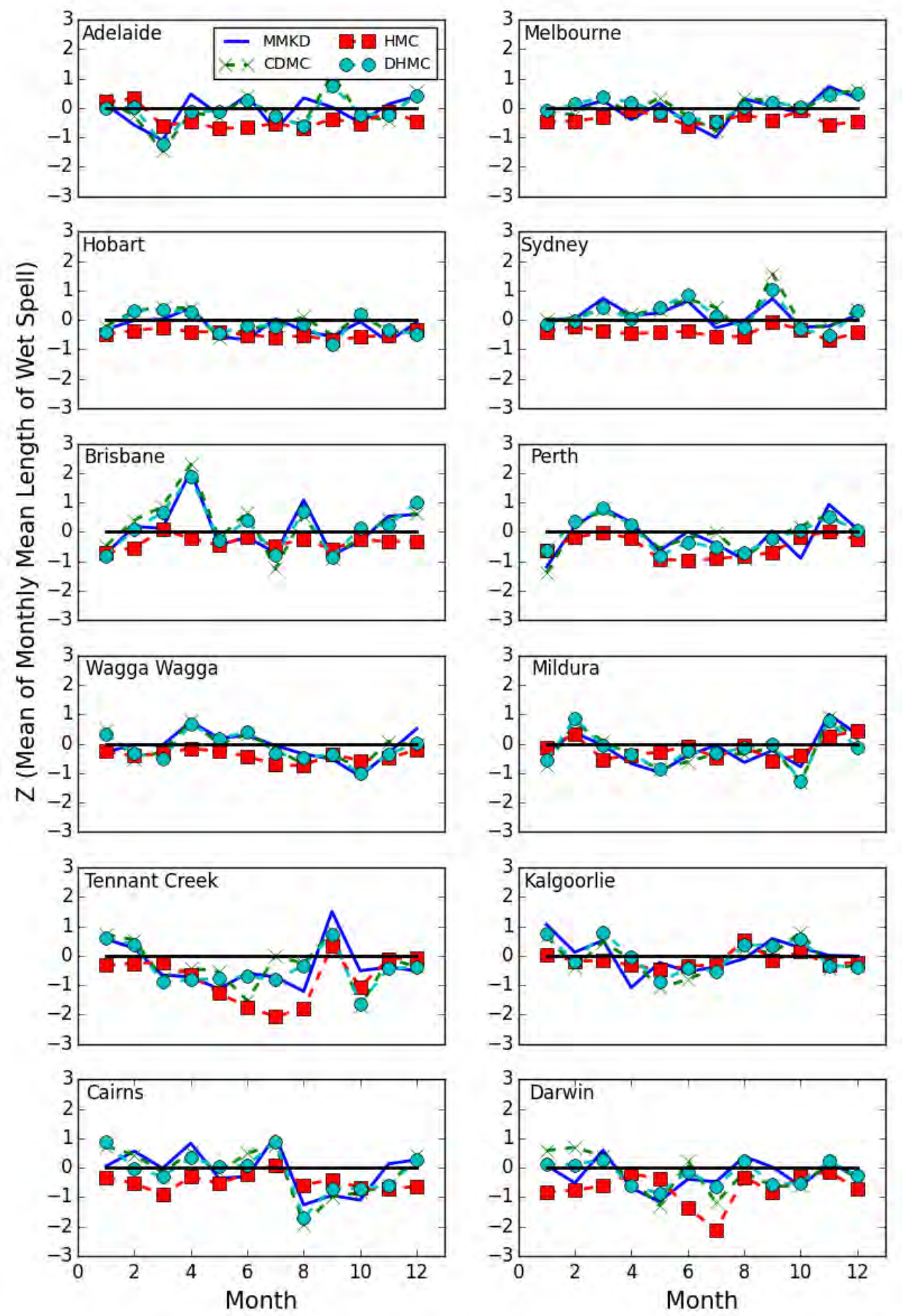

Figure H.5: Comparison of model performances for mean of monthly mean length of wet spells. 

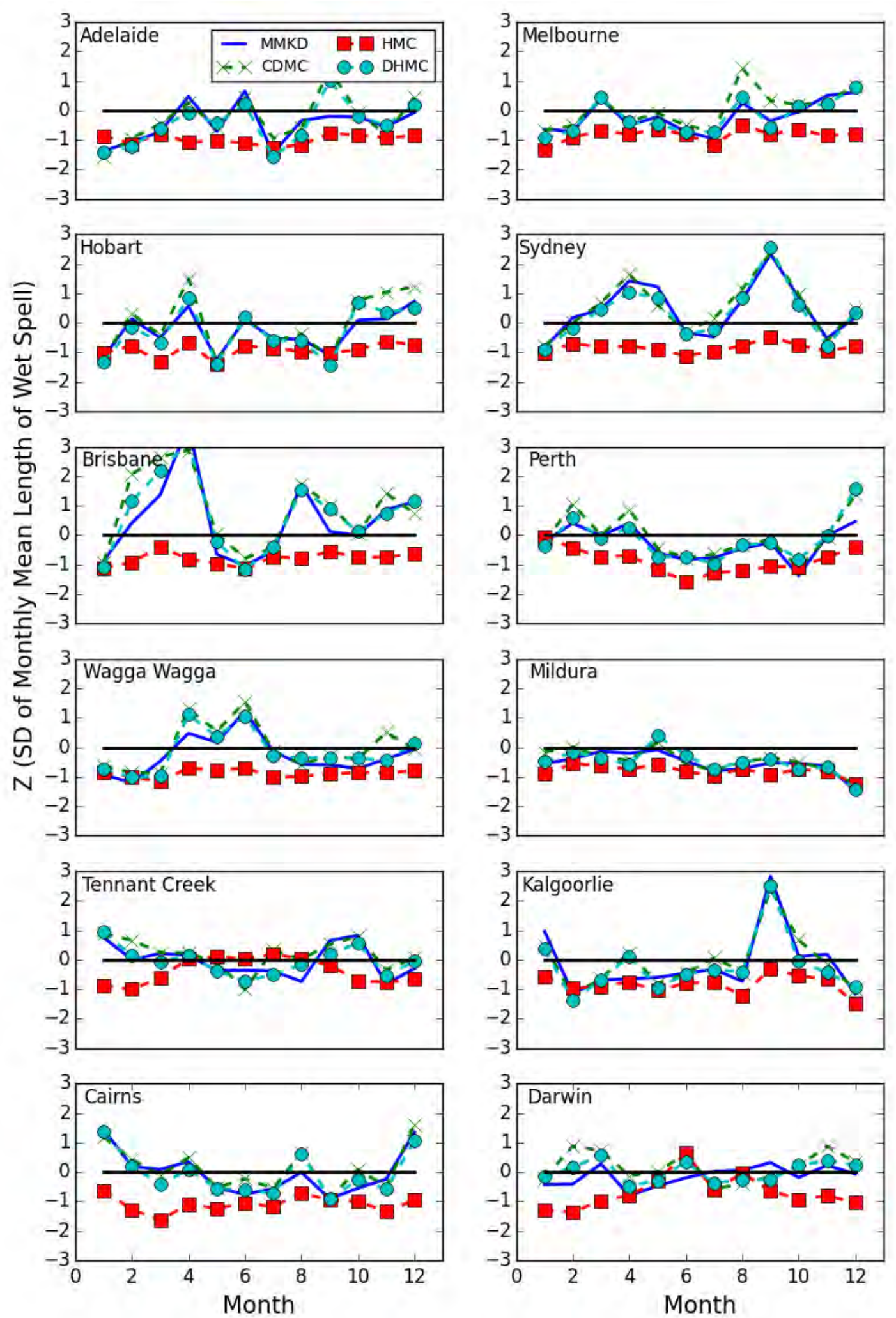

Figure H.6: Comparison of model performances for SD of monthly mean length of wet spells. 
H. Comparison of MC and MMKD for Raingauge Data around Australia

\section{H.3 Model Comparison for Autocorrelations}

The section compares the model performance for autocorrelations of monthly number of wet days and monthly rainfall depths.

\section{H.3.1 Performance of CDMC to Reproduce the Autocorrelations}
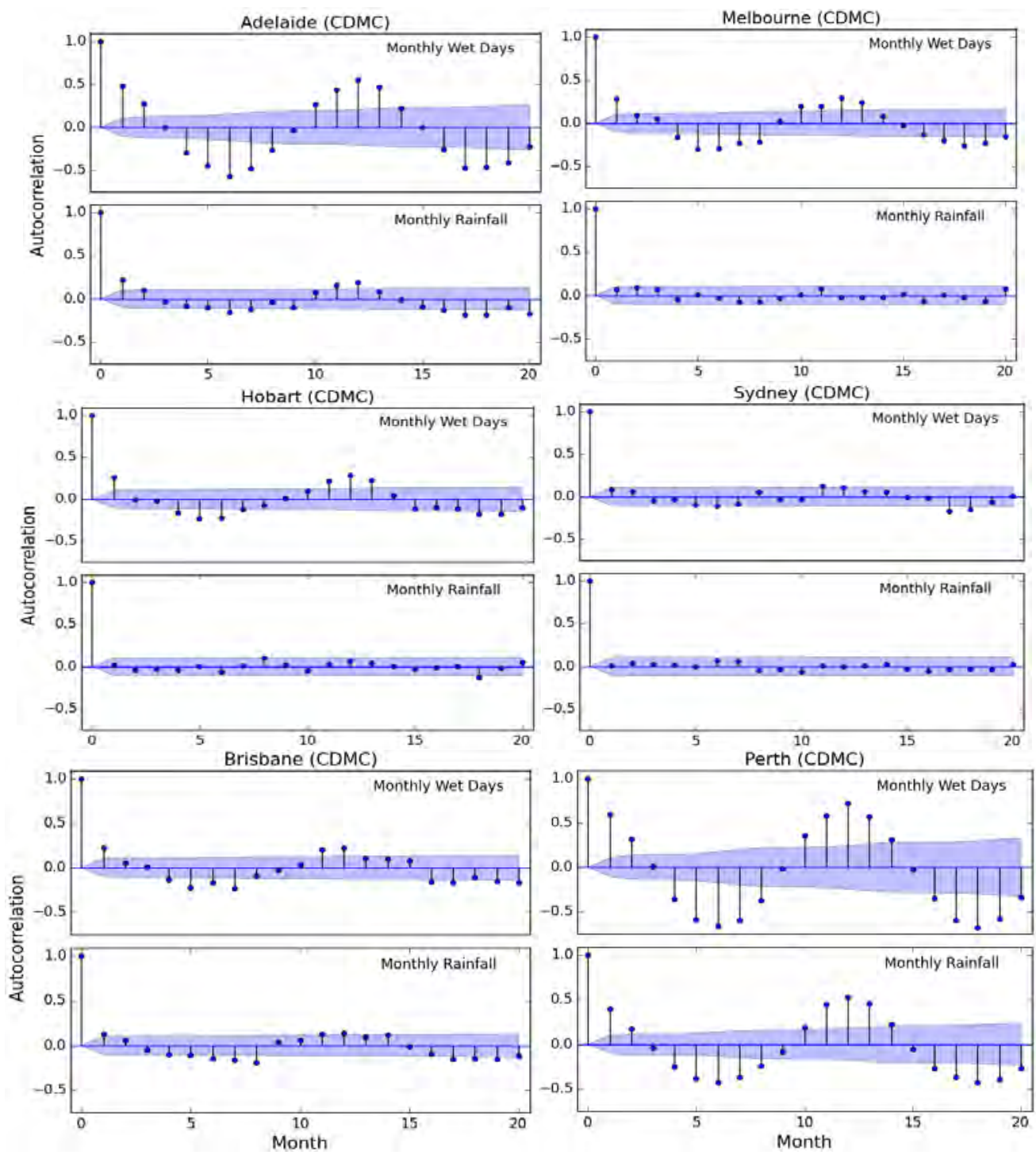

Figure H.7: CDMC reproduction of the autocorrelations of monthly number of wet days and monthly rainfall depths for coastal stations. 
H. Comparison of MC and MMKD for Raingauge Data around Australia
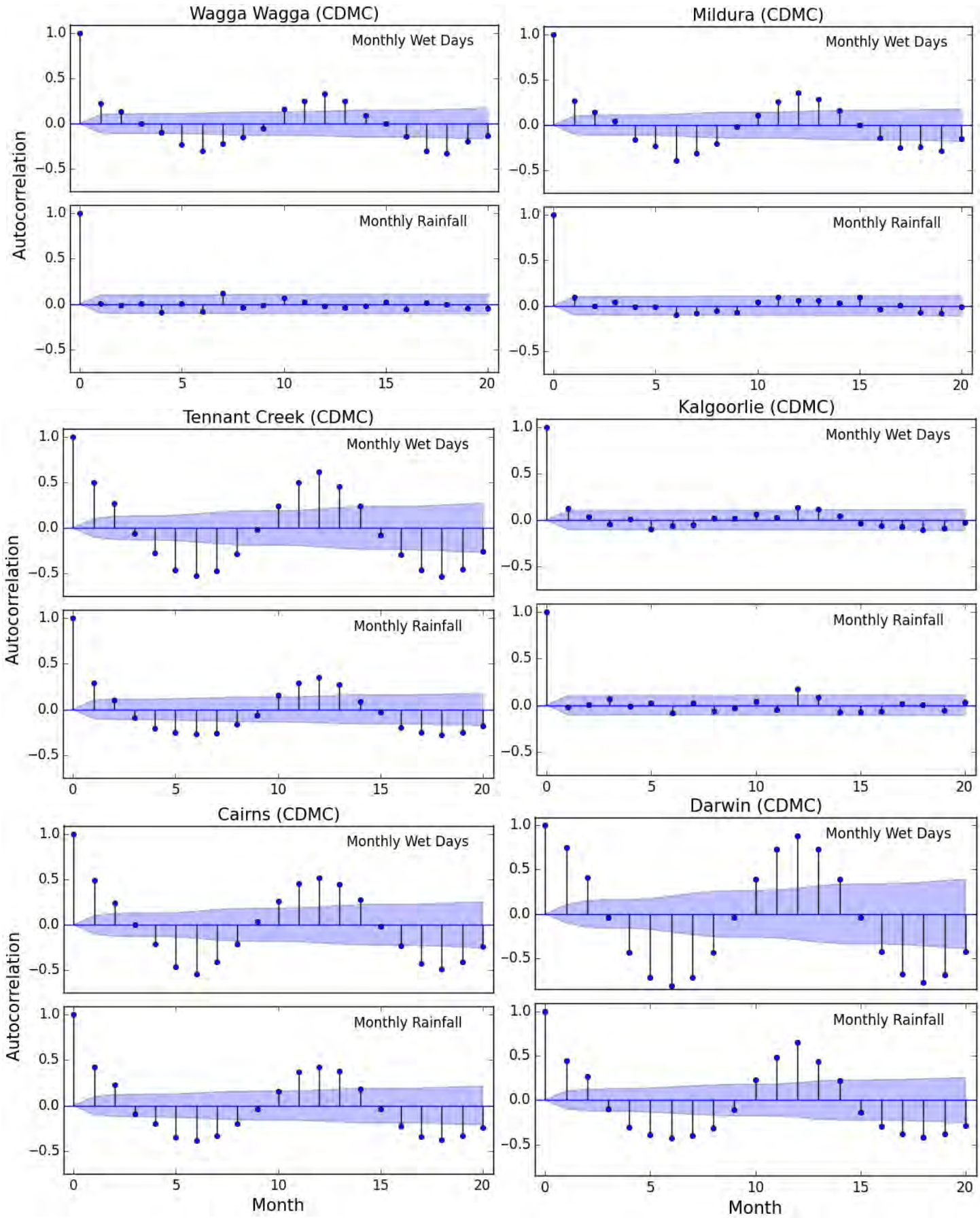

Figure H.8: CDMC reproduction of the autocorrelations of monthly number of wet days and monthly rainfall depths for inland and monsoonal stations. 
H. Comparison of MC and MMKD for Raingauge Data around Australia

\section{H.3.2 Performance of HMC to Reproduce the Autocorrelations}
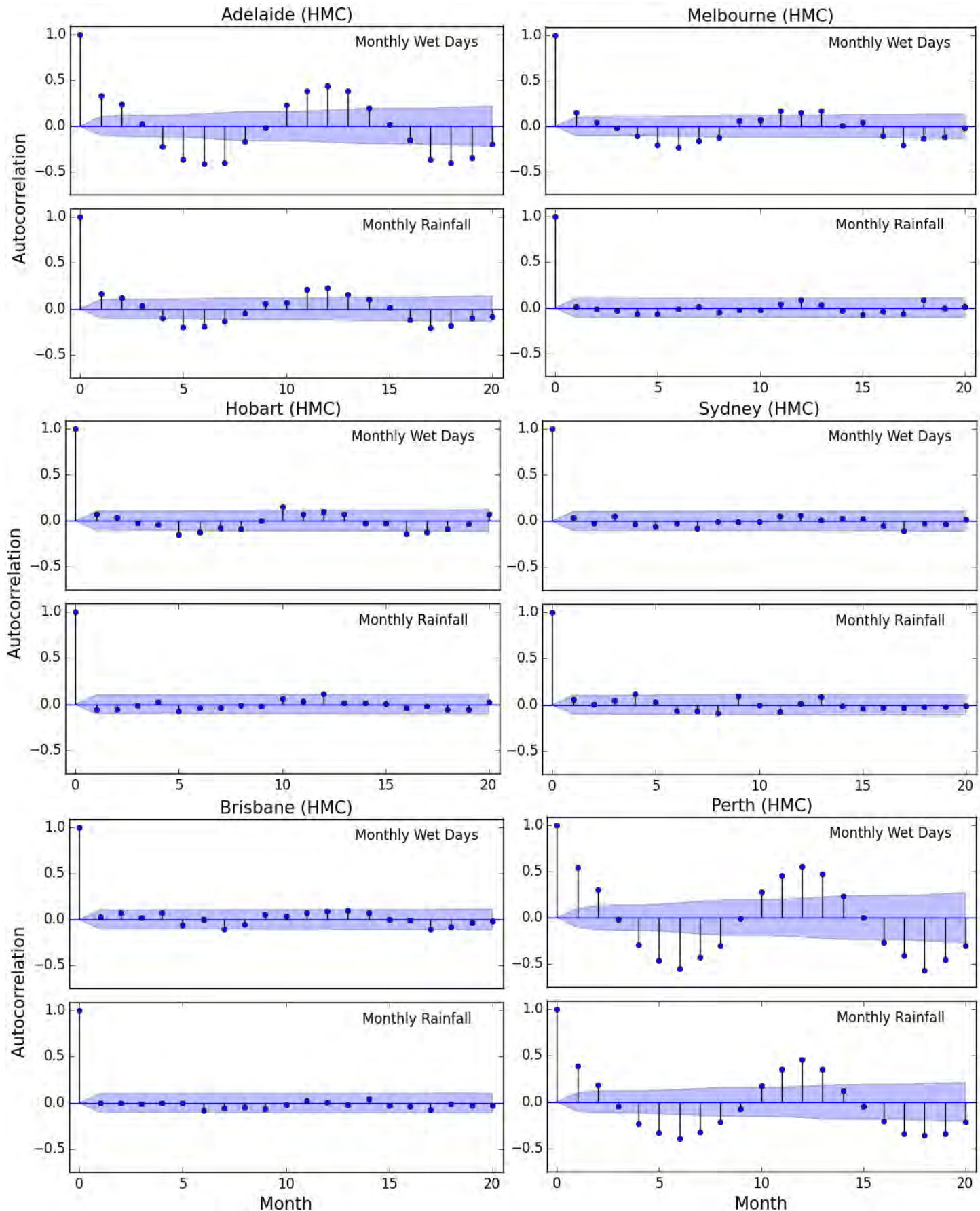

Figure H.9: HMC reproduction of the autocorrelations of monthly number of wet days and monthly rainfall depths for coastal stations. 
H. Comparison of MC and MMKD for Raingauge Data around Australia
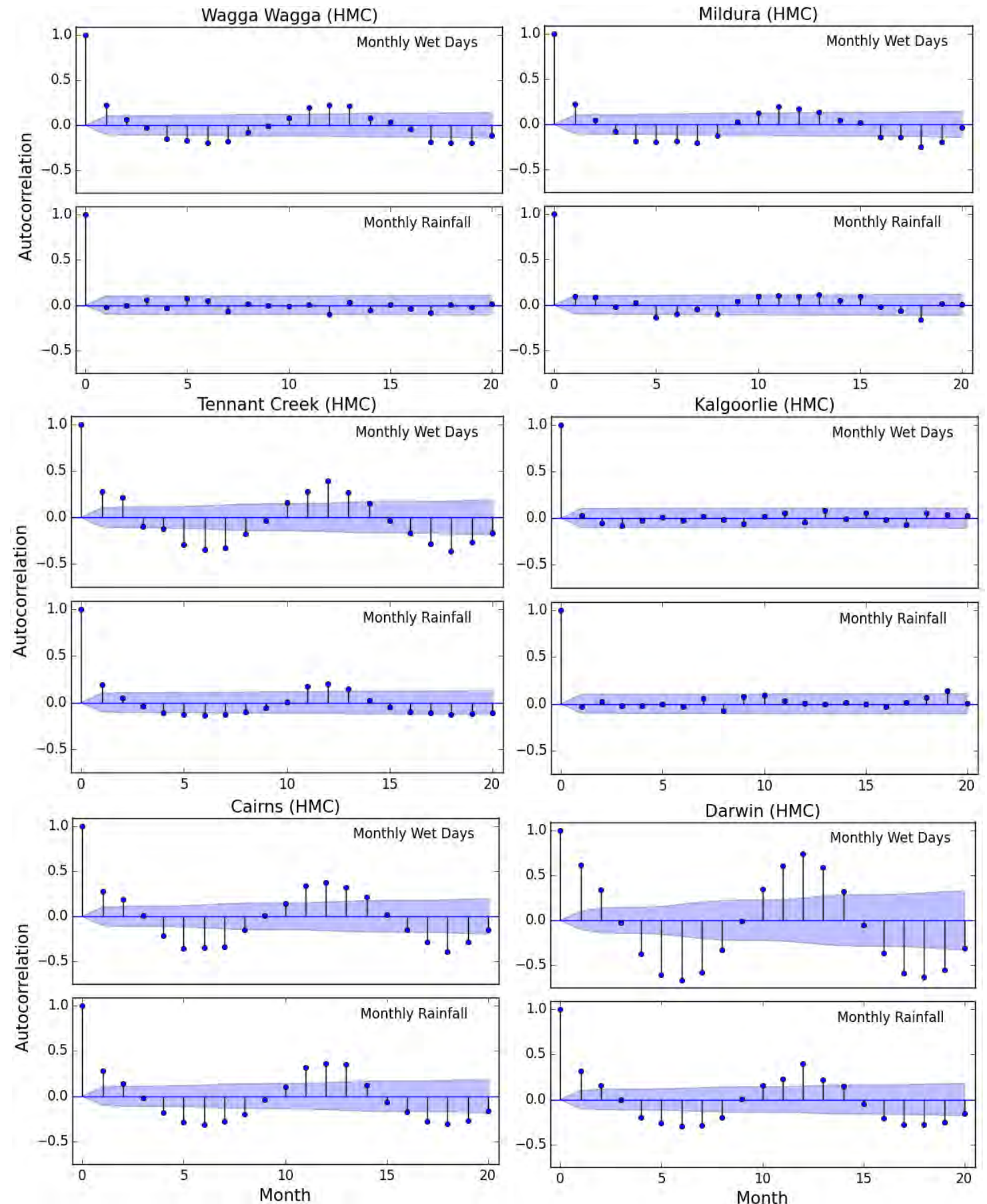

Figure H.10: HMC reproduction of the autocorrelations of monthly number of wet days and monthly rainfall depths for inland and monsoonal stations. 
H. Comparison of MC and MMKD for Raingauge Data around Australia

\section{H.3.3 Performance of DHMC to Reproduce the Autocorrelations}
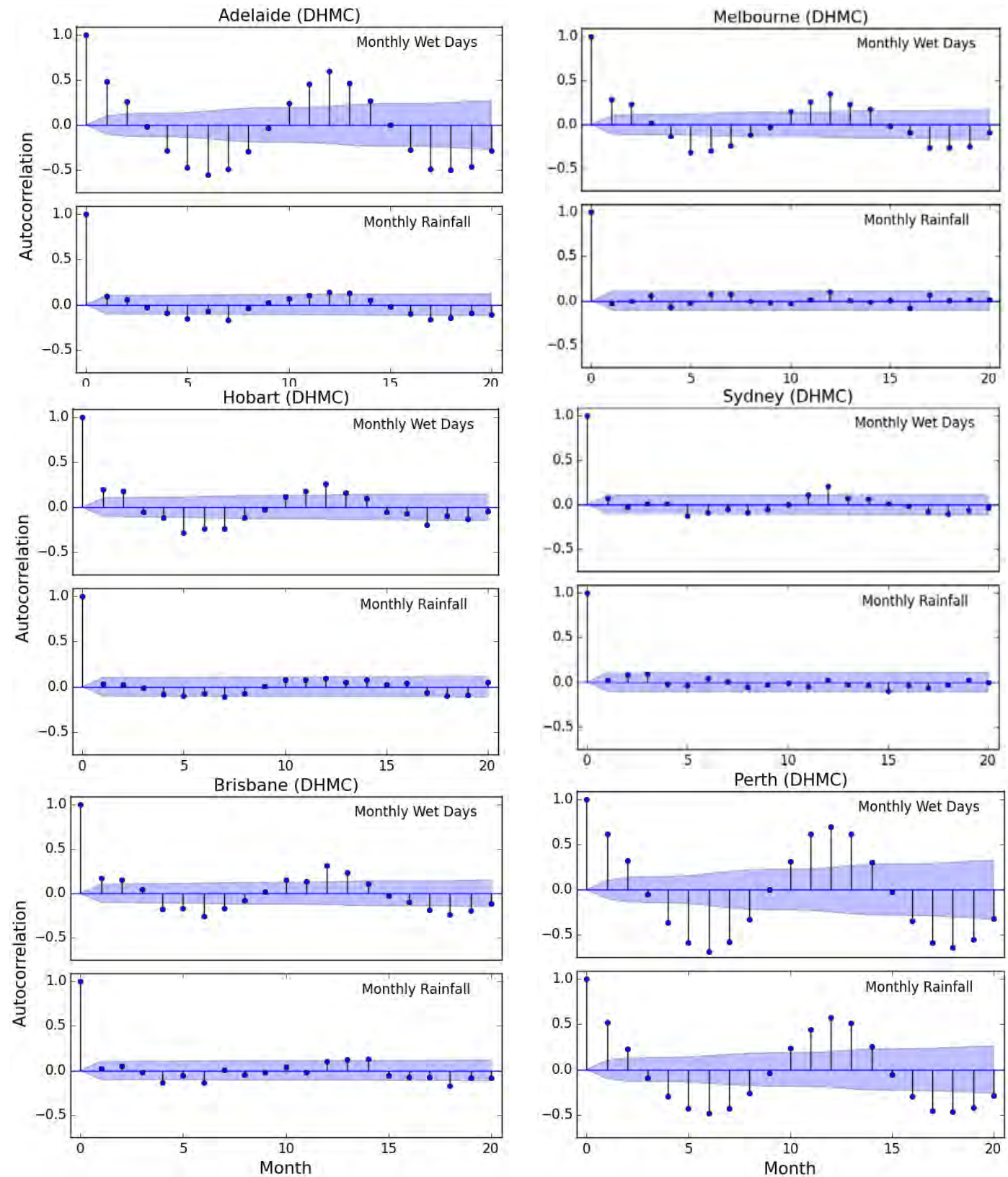

Figure H.11: DHMC reproduction of the autocorrelations of monthly number of wet days and monthly rainfall depths for coastal stations. 
H. Comparison of MC and MMKD for Raingauge Data around Australia
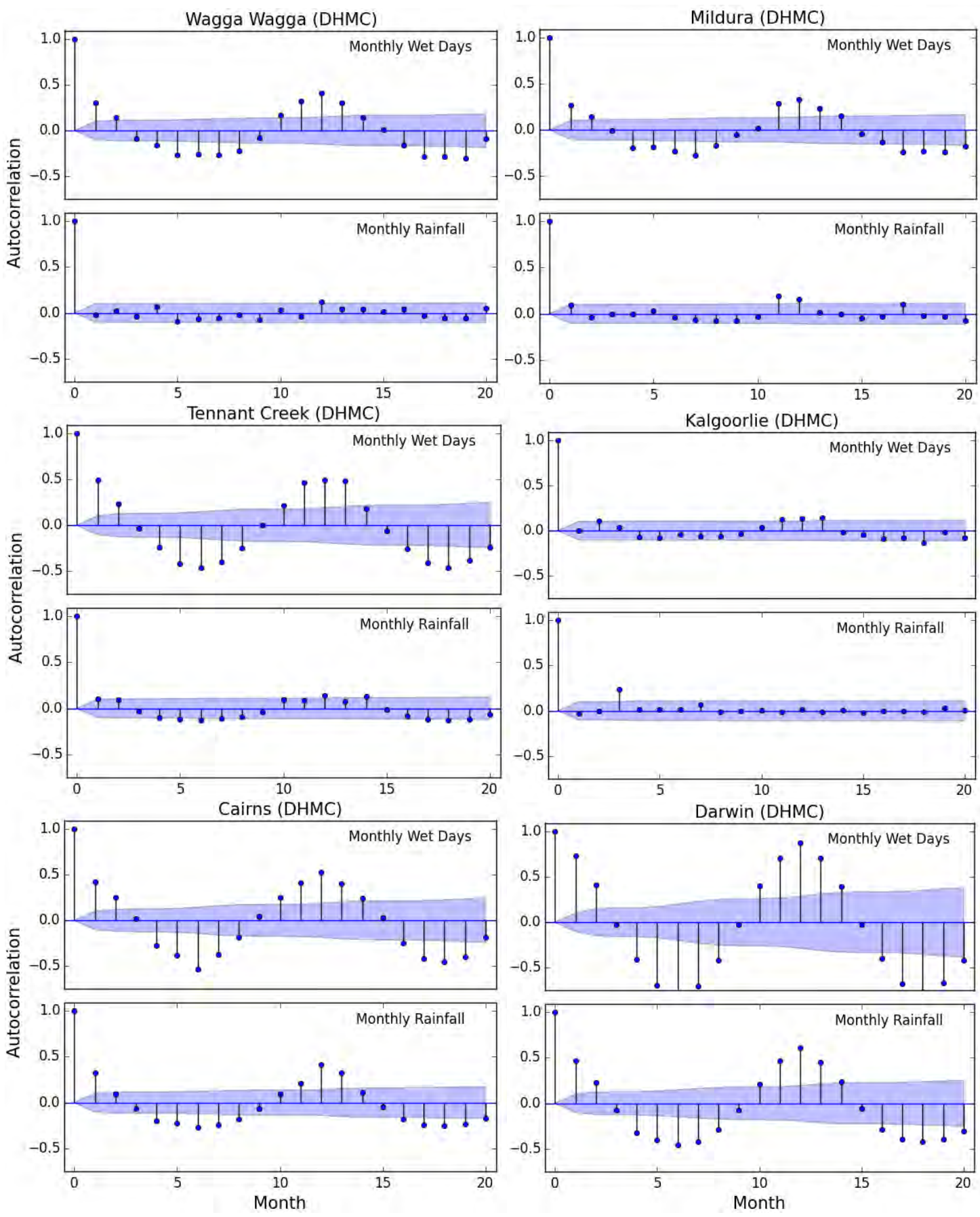

Figure H.12: DHMC reproduction of the autocorrelations of monthly number of wet days and monthly rainfall depths for inland and monsoonal stations. 
H. Comparison of MC and MMKD for Raingauge Data around Australia

\section{H.3.4 Performance of MMKD to Reproduce the Autocorrelations}
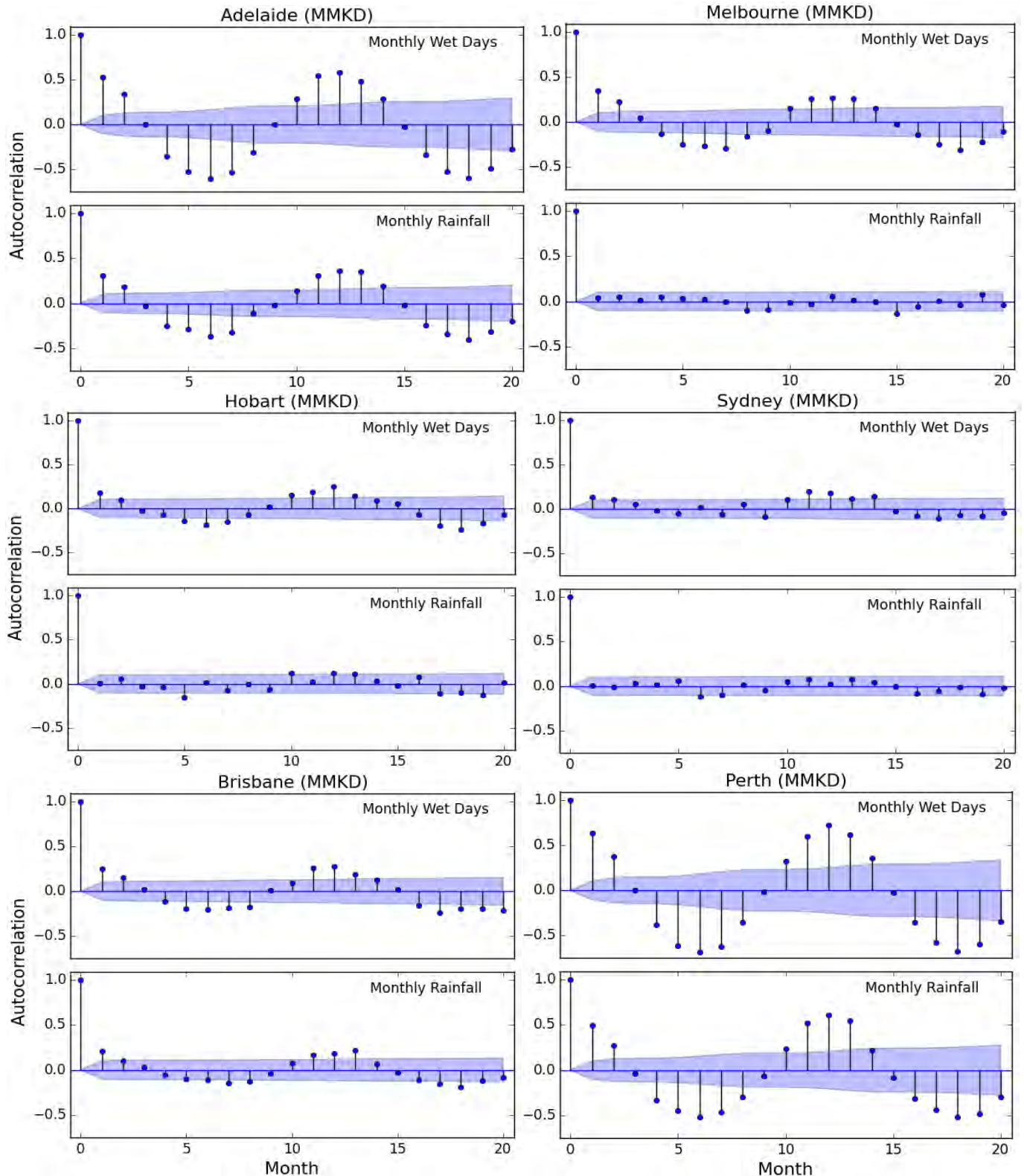

Figure H.13: MMKD reproduction of the autocorrelations of monthly number of wet days and monthly rainfall depths for coastal stations. 
H. Comparison of MC and MMKD for Raingauge Data around Australia
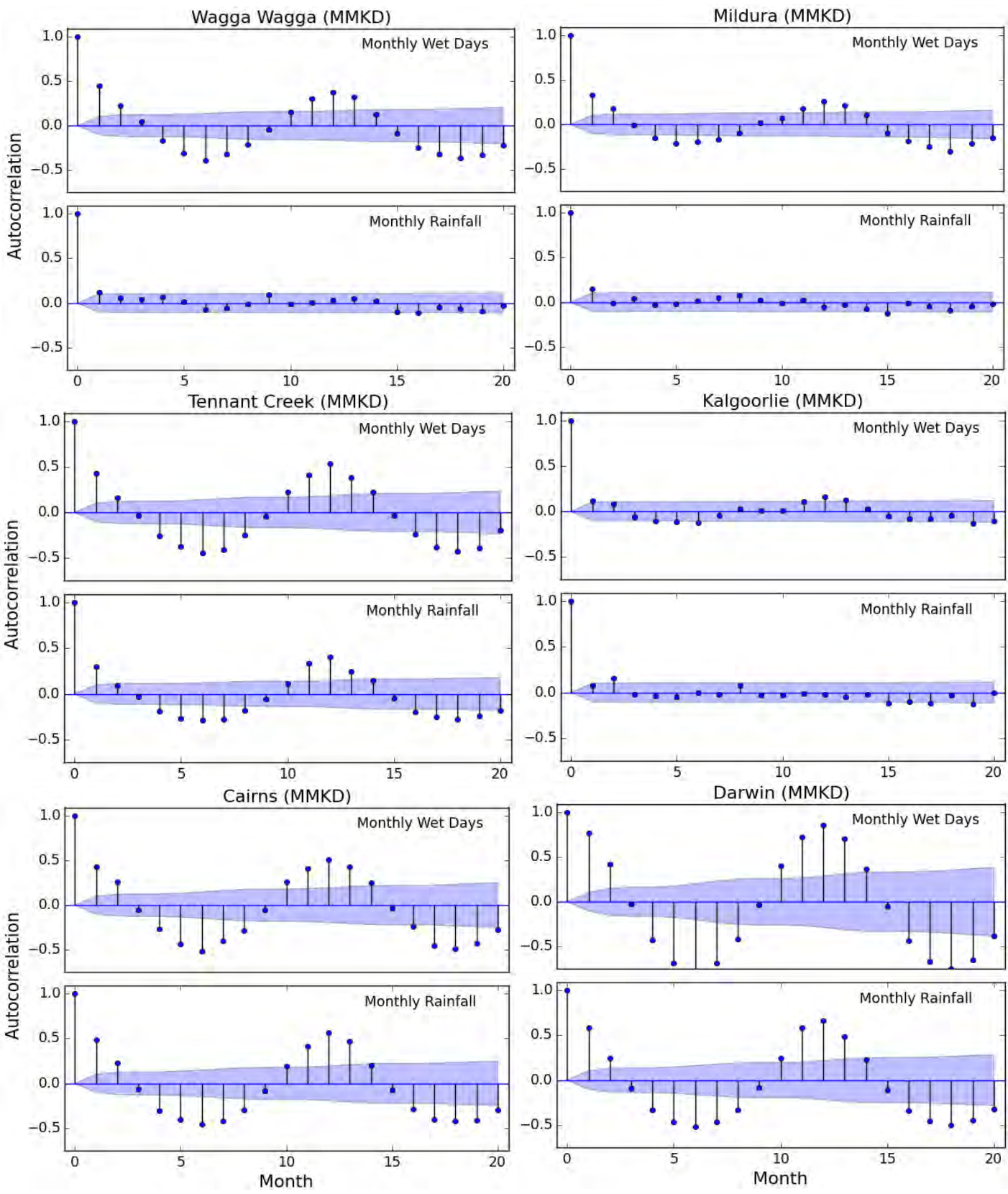

Figure H.14: MMKD reproduction of the autocorrelations of monthly number of wet days and monthly rainfall depths for inland and monsoonal stations. 


\section{H.4 Comparison of DHMC, MMKD and Combined Model}

This section compares the DHMC, MMKD and combined model of DHMC and MMKD.

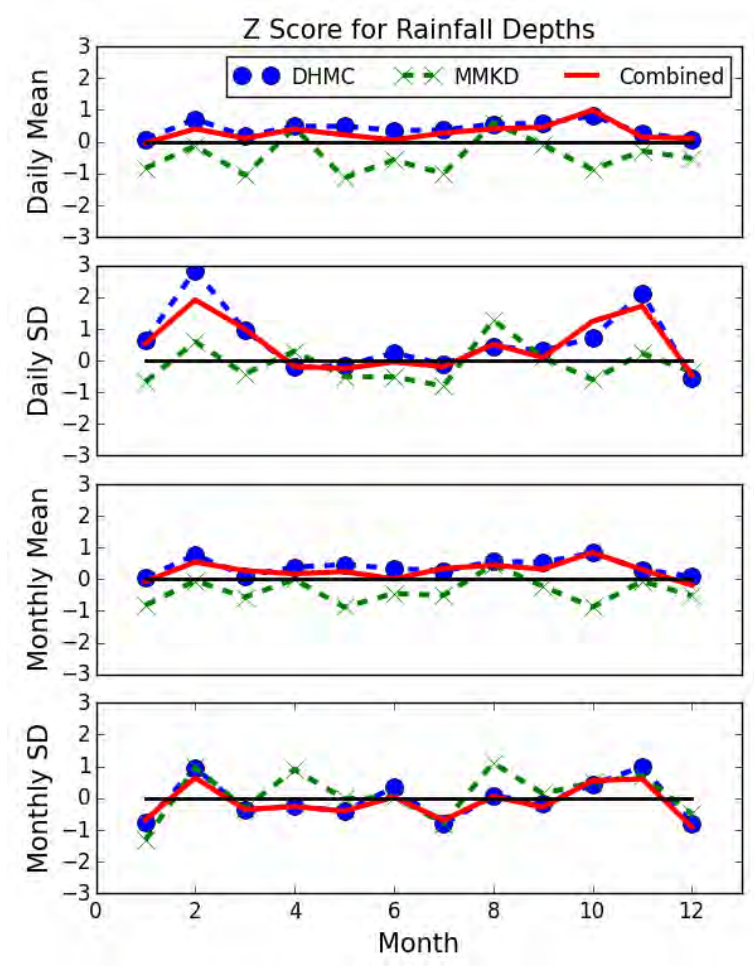

Figure H.15: Comparison of DHMC, MMKD and Combined Model to reproduce the mean and SD of daily and monthly rainfall depths.
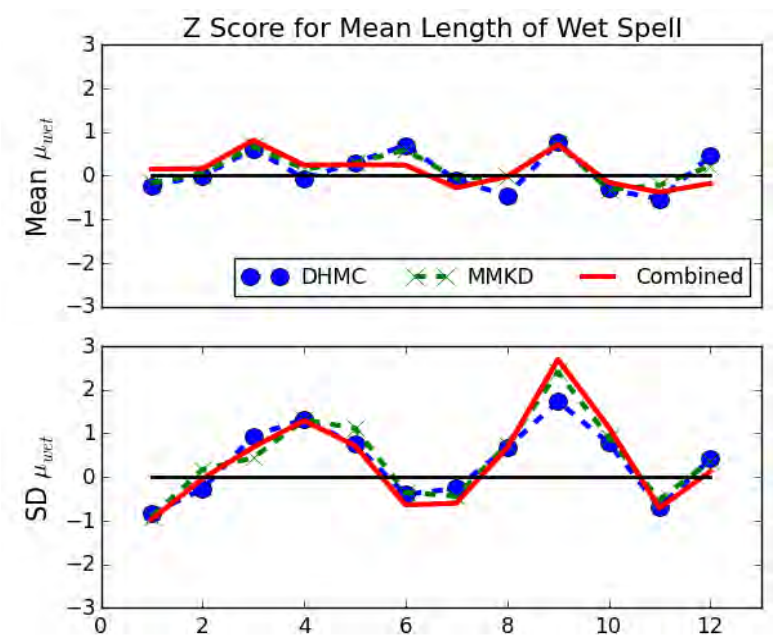

Figure H.16: Comparison of DHMC, MMKD and Combined Model to reproduce the mean and SD of monthly mean length of wet spells. 

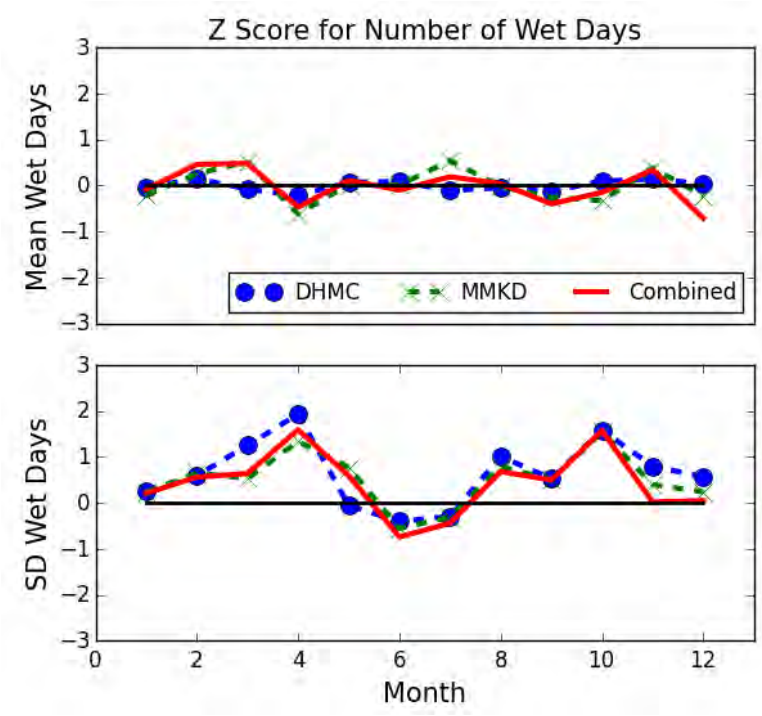

Figure H.17: Comparison of DHMC, MMKD and Combined Model to reproduce the mean and SD of monthly number of wet days.

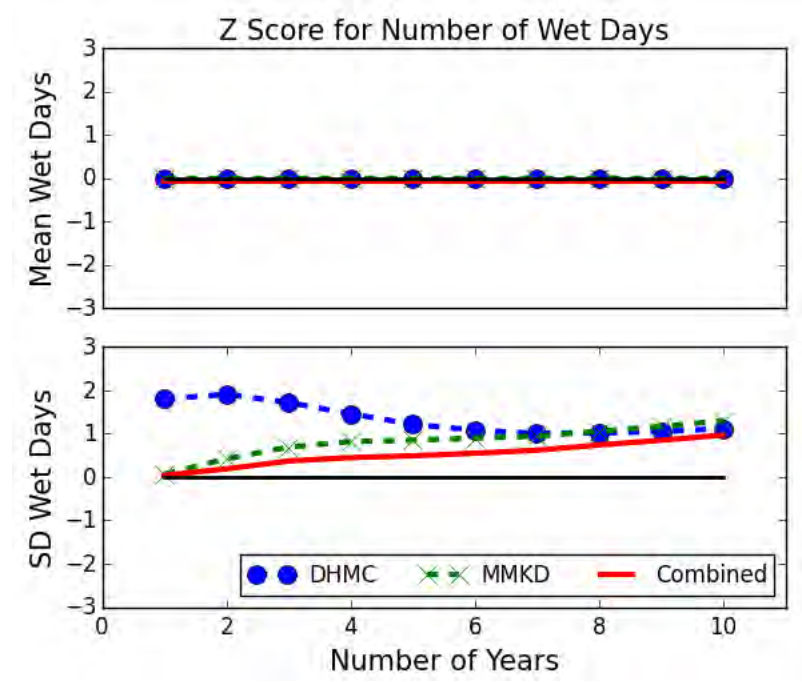

Figure H.18: Comparison of DHMC, MMKD and Combined Model to reproduce the mean and SD of multiyear number of wet days. 


\section{Comparison of MC and MMKD for Streamflow Generation}

\section{I.1 Introduction}

This appendix presents the additional information of the Chapter 15 for comparison between MC and MMKD models in streamflow generation.

\section{I.2 Distribution Statistics of Rainfall and Streamflow}

\section{I.2.1 Mean and SD of Rainfall and Streamflow at Chichester}

Figure I.1-I.3 show the distribution statistics of daily rainfall depths and streamflow volumes at Chichester for daily, monthly and multiyear resolution respectively. The characteristics of rainfall and streamflow at Chichester are similar to the rainfall and streamflow at Tillegra (discussed in section 15.3).
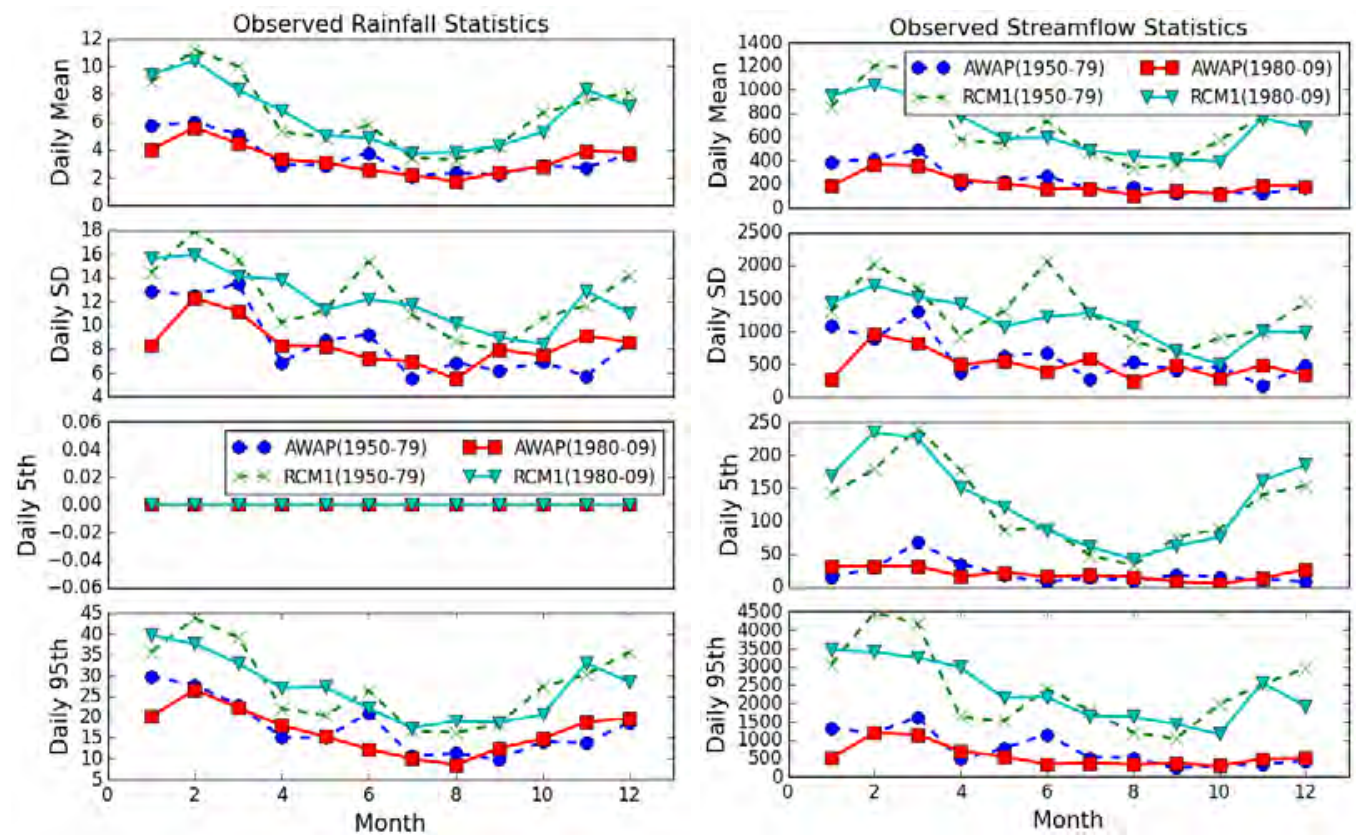

Figure I.1: Distribution statistics of daily rainfall and streamflow at Chichester. 

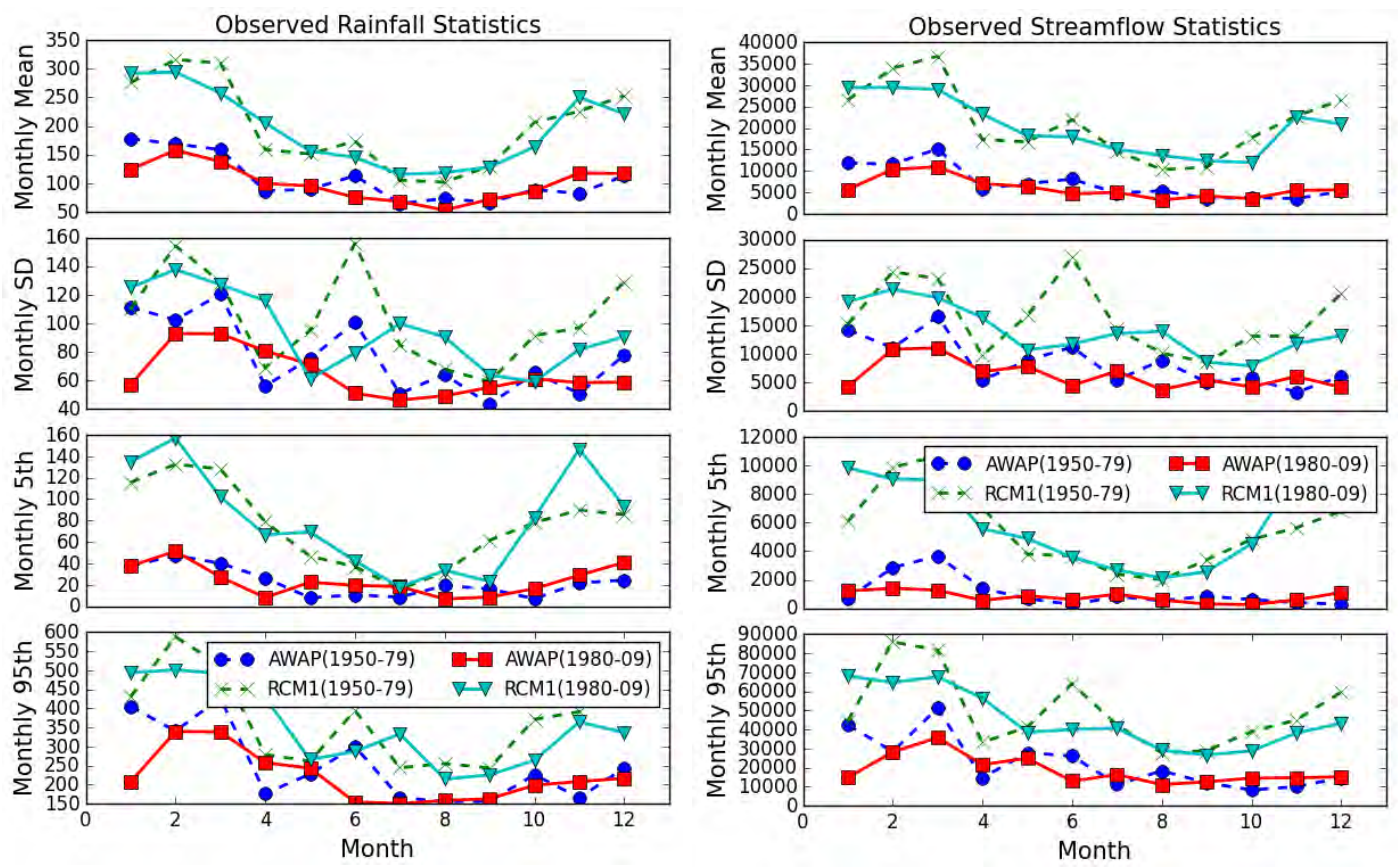

Figure I.2: Distribution statistics of monthly rainfall and streamflow at Chichester.
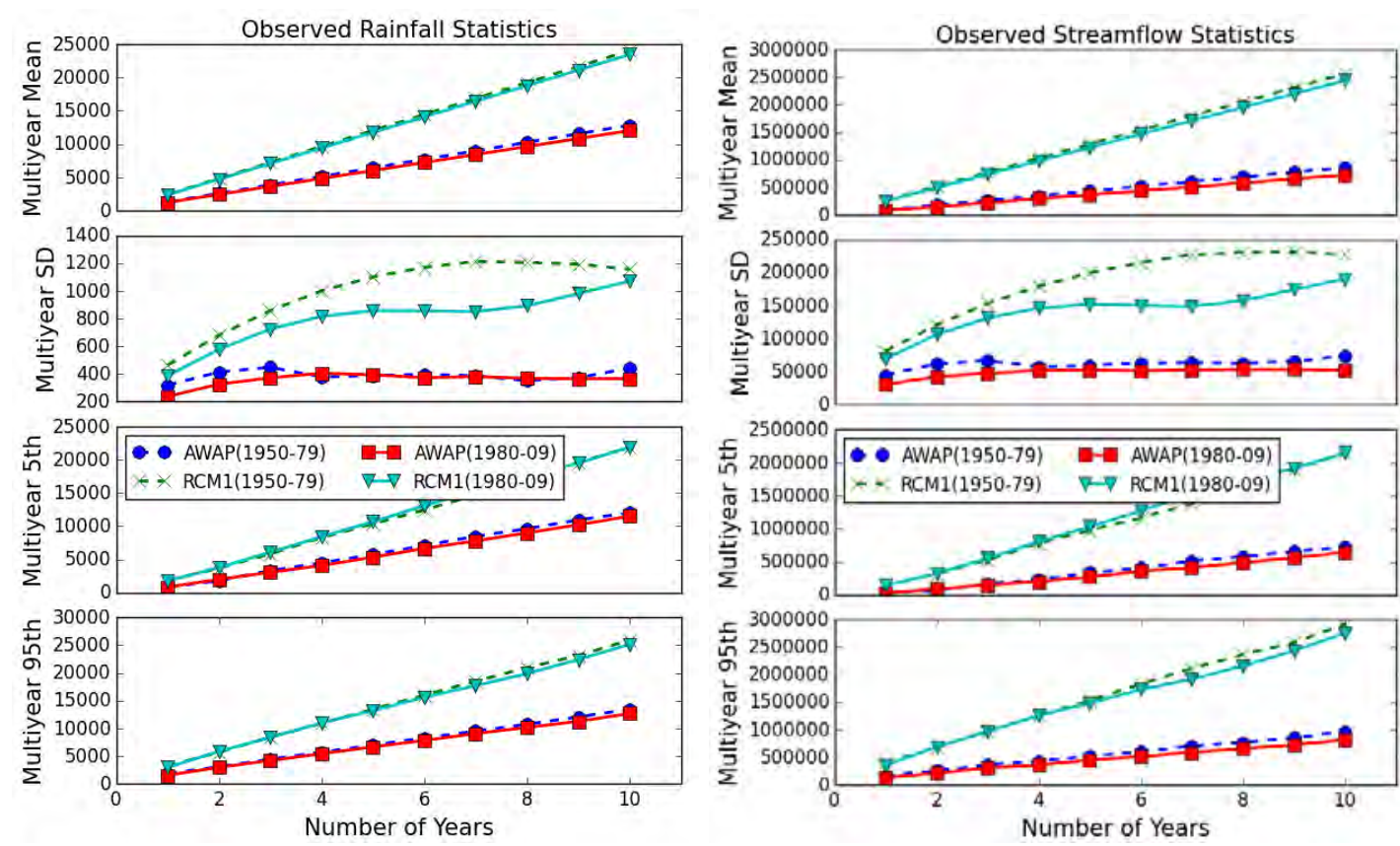

Figure I.3: Distribution statistics of multiyear rainfall and streamflow at Chichester. 


\section{I.2.2 Monthly Rainfall and Streamflow at Grahamstown and Tillegra}

Figure I.4 and Figure I.5 show the distribution of monthly rainfall depths and streamflow volumes at Grahamstown and Tillegra respectively. The monthly statistics are consistent with the respective daily statistics (discussed in section 15.3.2).
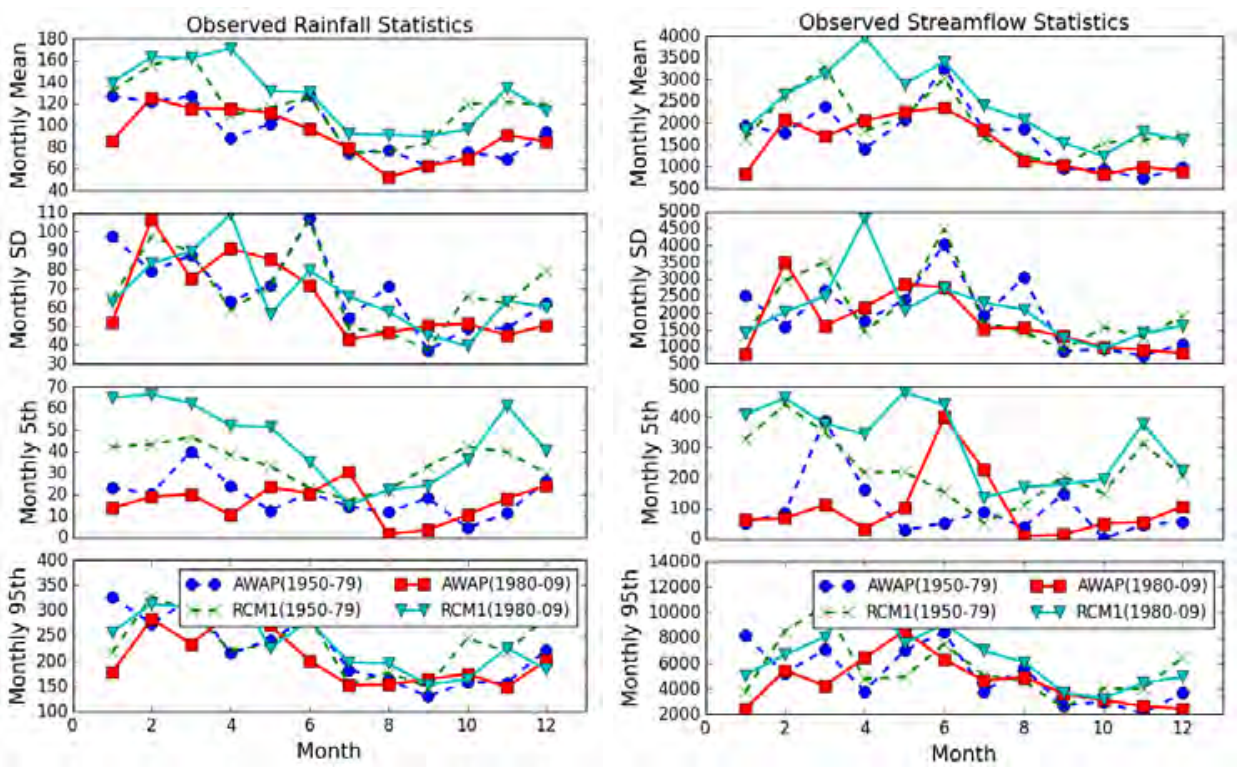

Figure I.4: Distribution statistics of monthly rainfall and streamflow at Grahamstown.
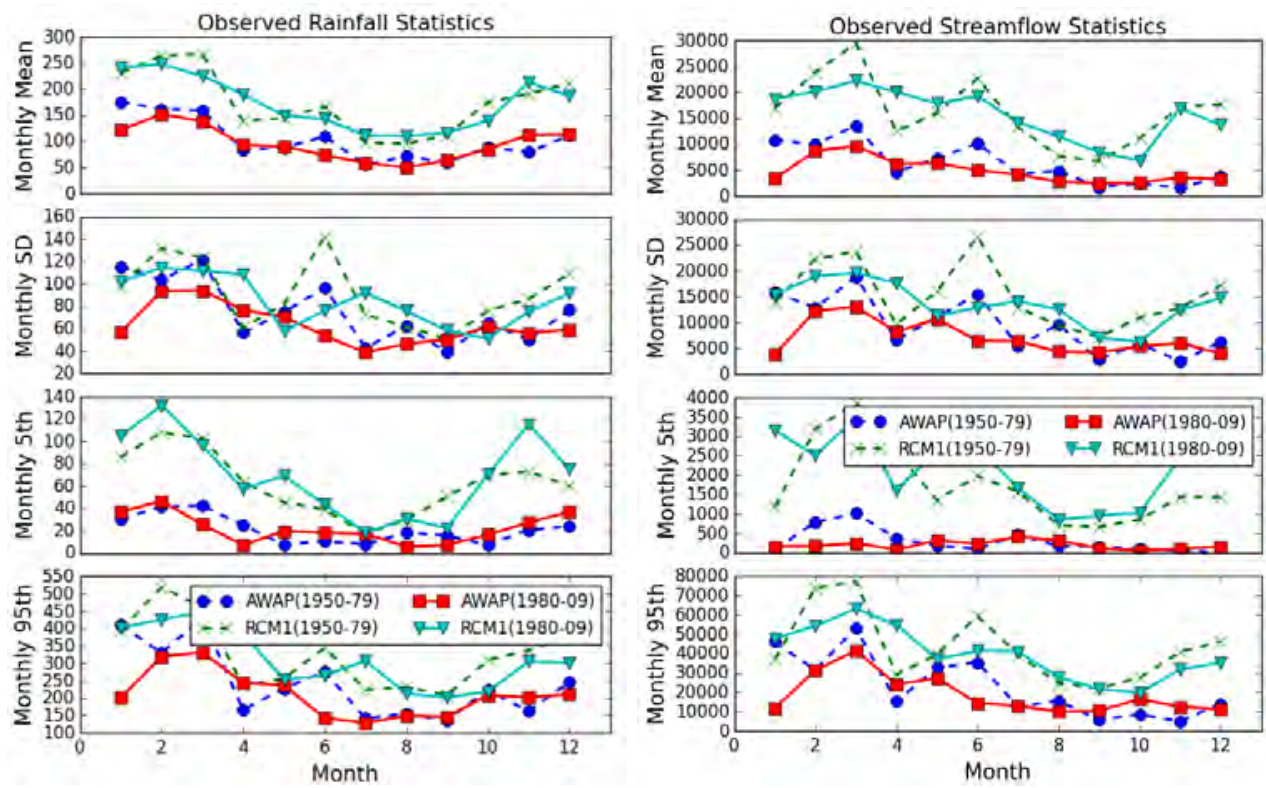

Figure I.5: Distribution statistics of monthly rainfall and streamflow at Tillegra. 


\section{I.3 Model Comparison for Distribution Statistics}

\section{I.3.1 Model Comparison for at Chichester Using RCM1}

Figure I.6-I.11 compare the models for rainfall and streamflow using RCM1 at Chichester, which are consistent with the results for Tillegra (discussed in section 15.4). Section I.3.2 will show the same comparison at Chichester using AWAP.
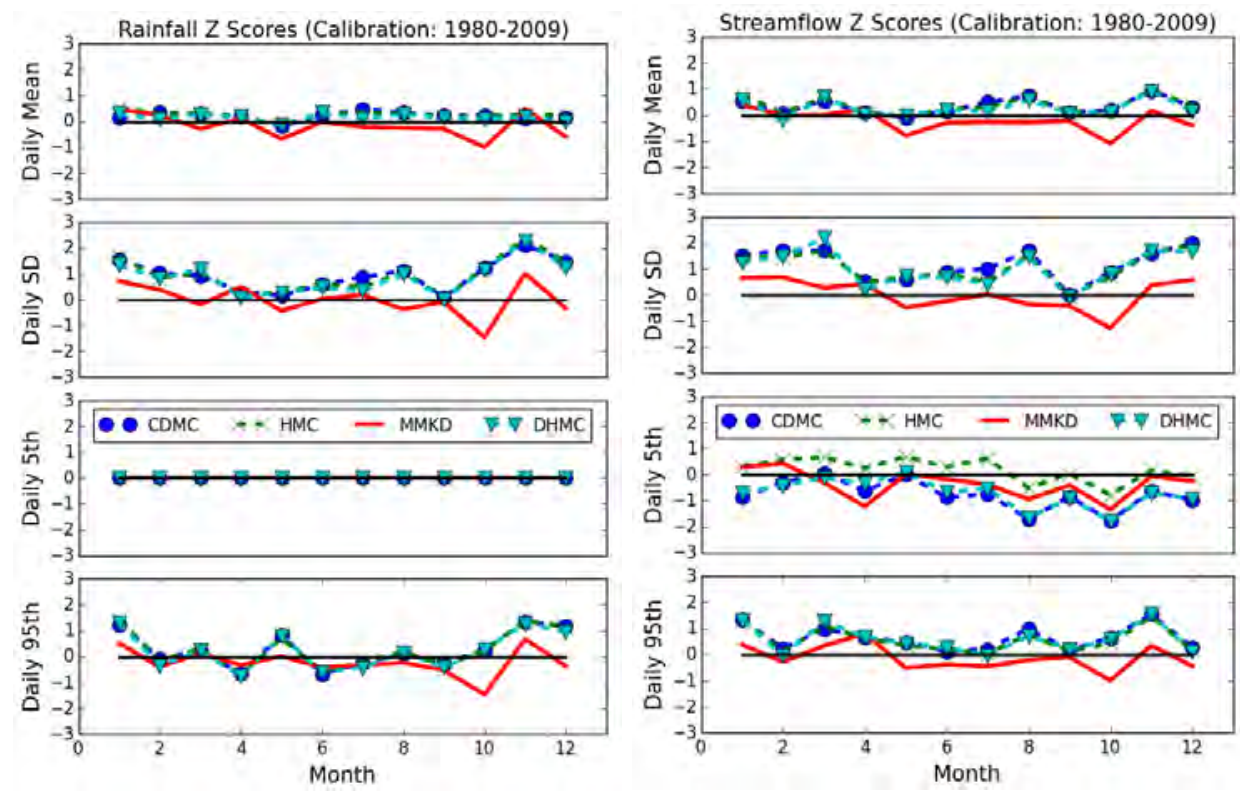

Figure I.6: Model comparison for daily rainfall and streamflow of RCM1 for calibration period at Chichester. 

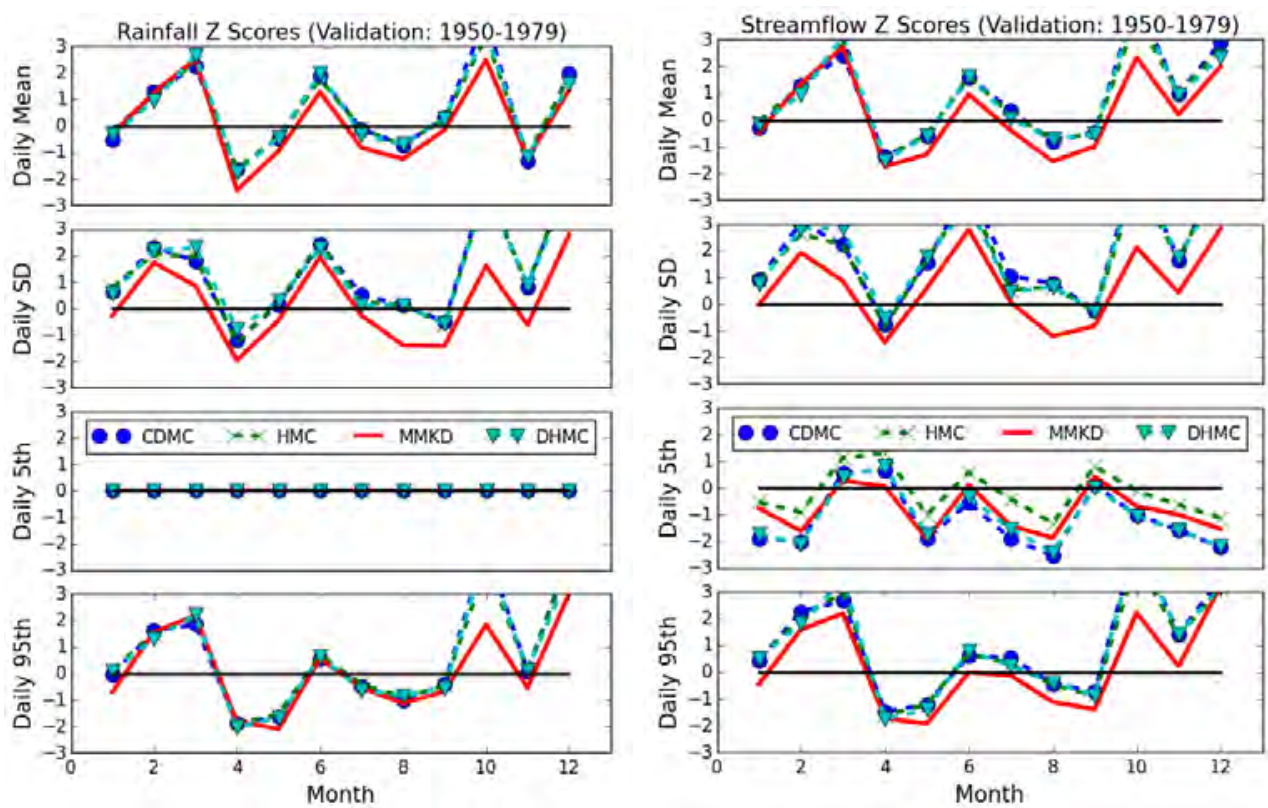

Figure I.7: Model comparison for daily rainfall and streamflow of RCM1 for validation period at Chichester.
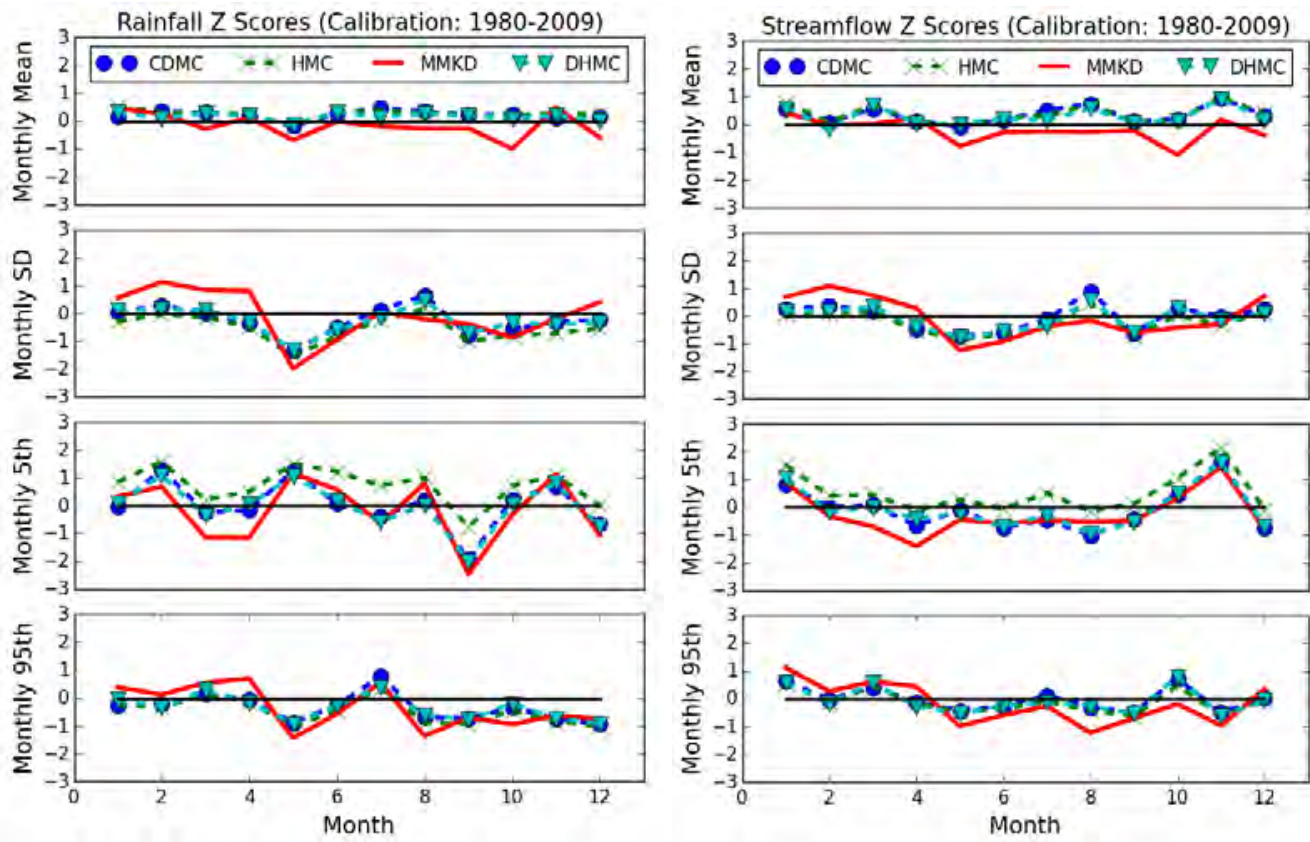

Figure I.8: Model comparison for monthly rainfall and streamflow of RCM1 for calibration period at Chichester. 

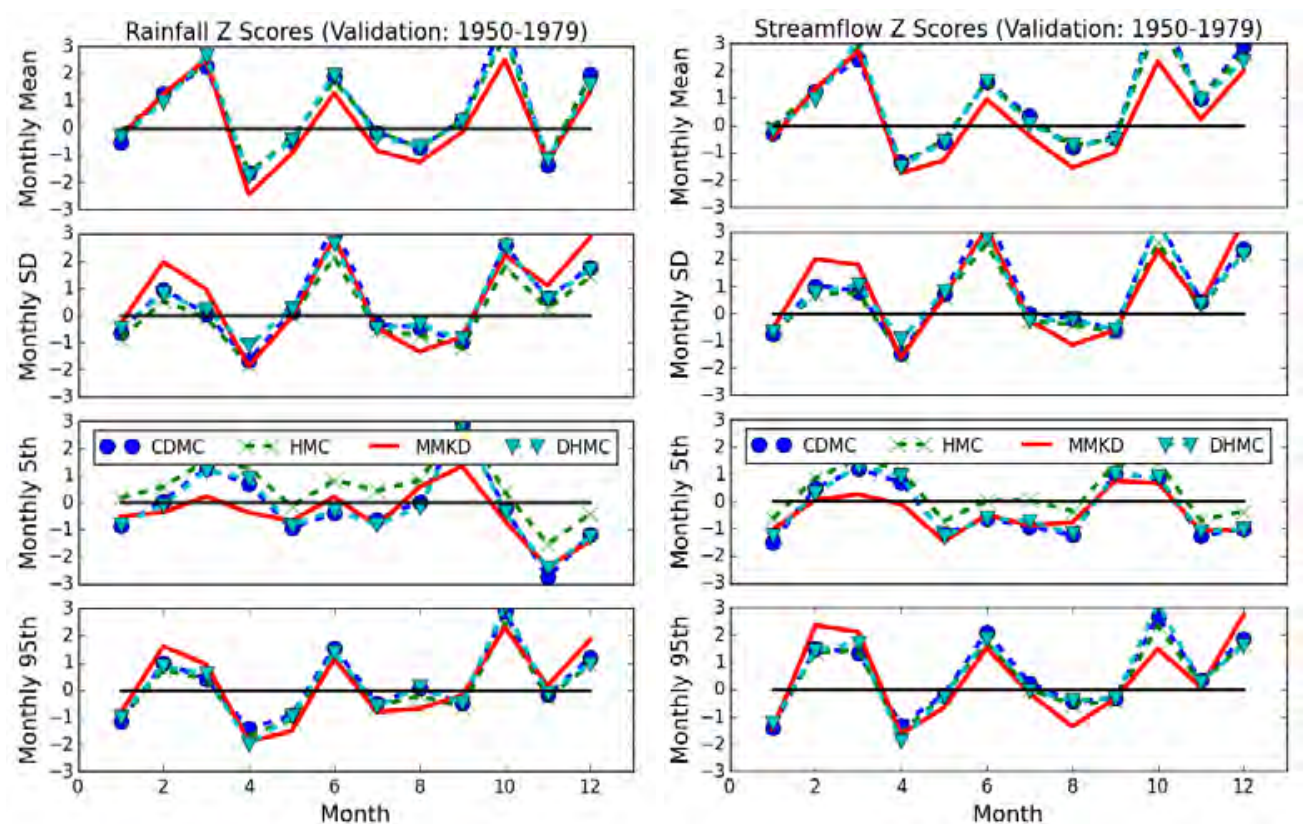

Figure I.9: Model comparison for monthly rainfall and streamflow of RCM1 for validation period at Chichester.
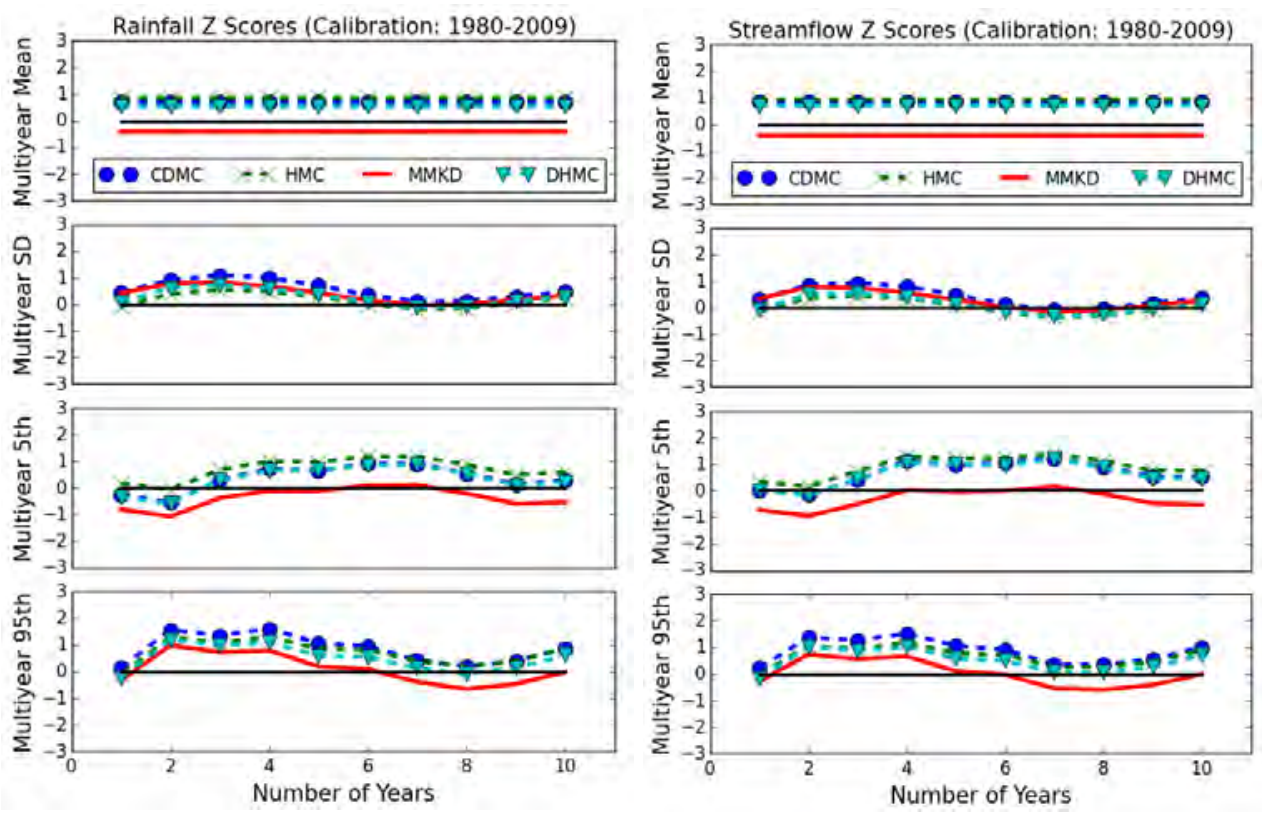

Figure I.10: Model comparison for multiyear rainfall and streamflow of RCM1 for calibration period at Chichester. 

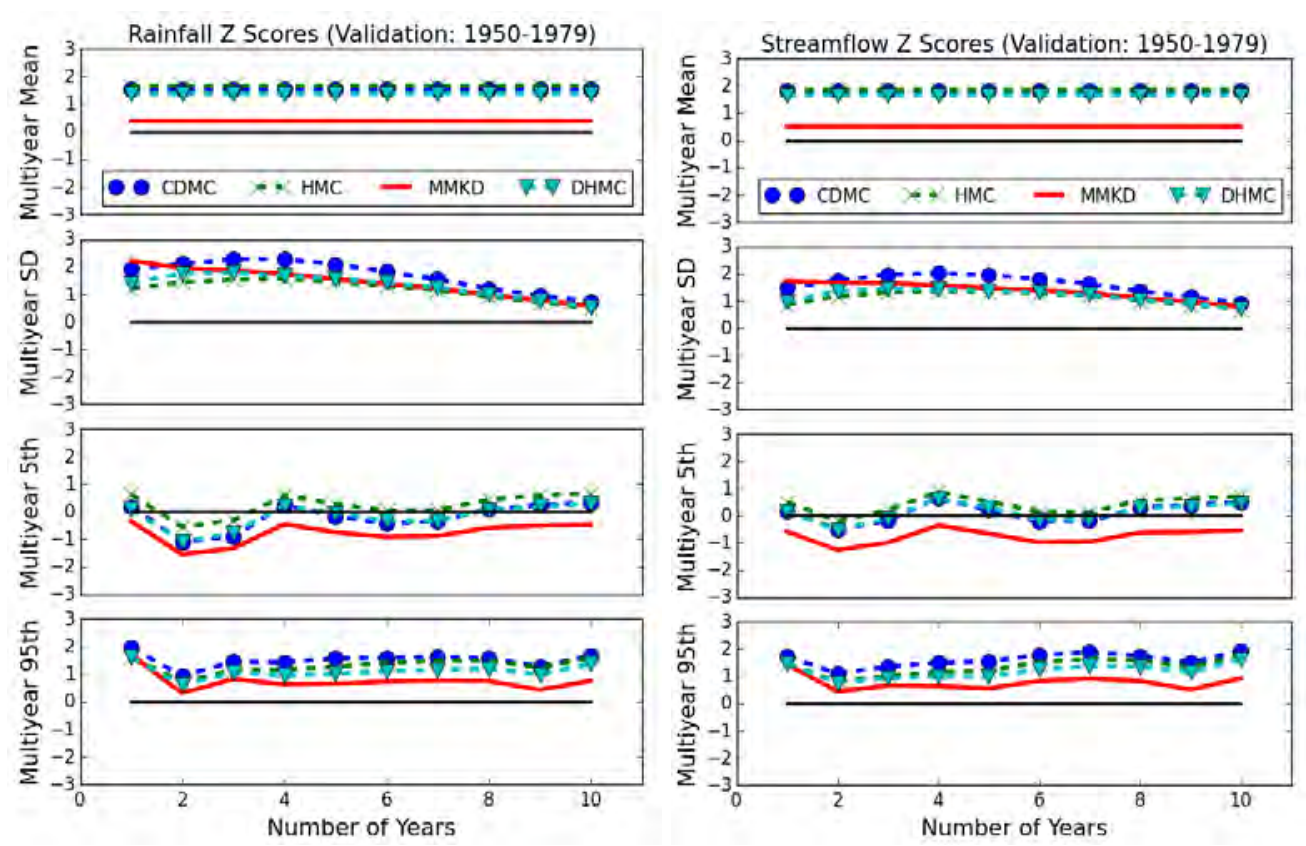

Figure I.11: Model comparison for multiyear rainfall and streamflow of RCM1 for validation period at Chichester.

\section{I.3.2 Model Comparison for at Chichester Using AWAP}

Figure I.12-I.17 compare the models for rainfall and streamflow using AWAP at Chichester, which are consistent with the results for Tillegra (discussed in section 15.4).
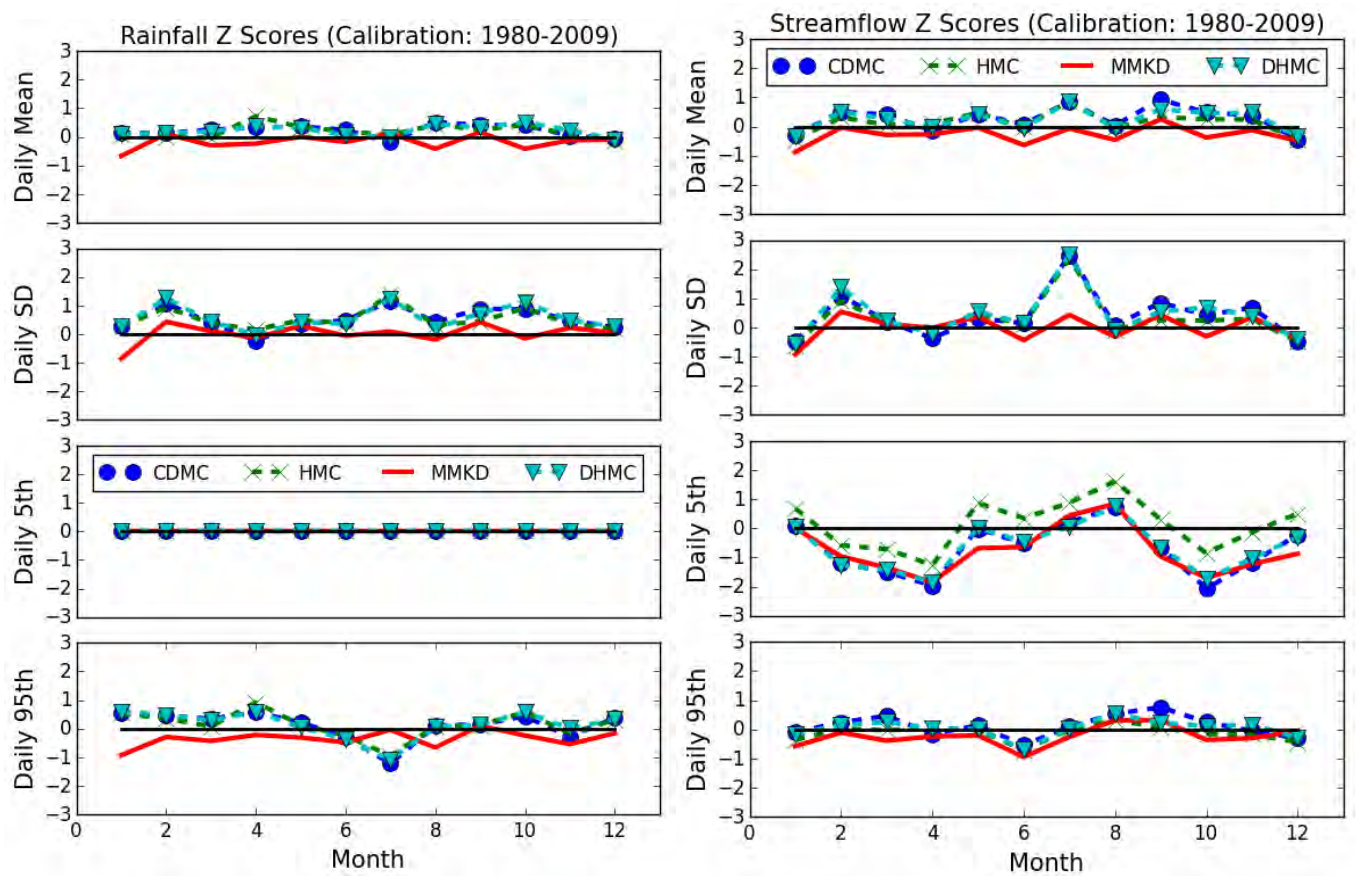

Figure I.12: Model comparison for daily rainfall and streamflow of AWAP for calibration period at Chichester. 

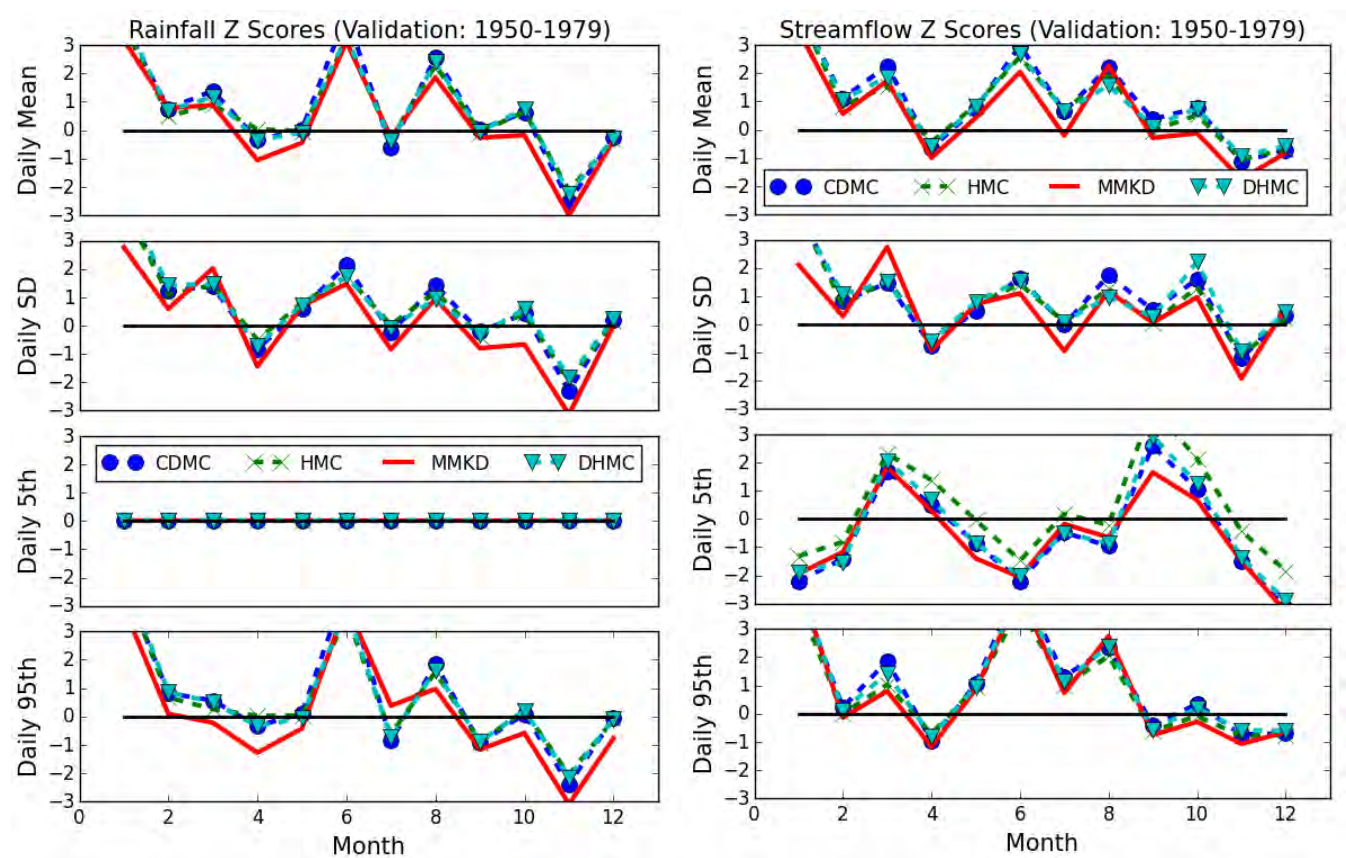

Figure I.13: Model comparison for daily rainfall and streamflow of AWAP for validation period at Chichester.
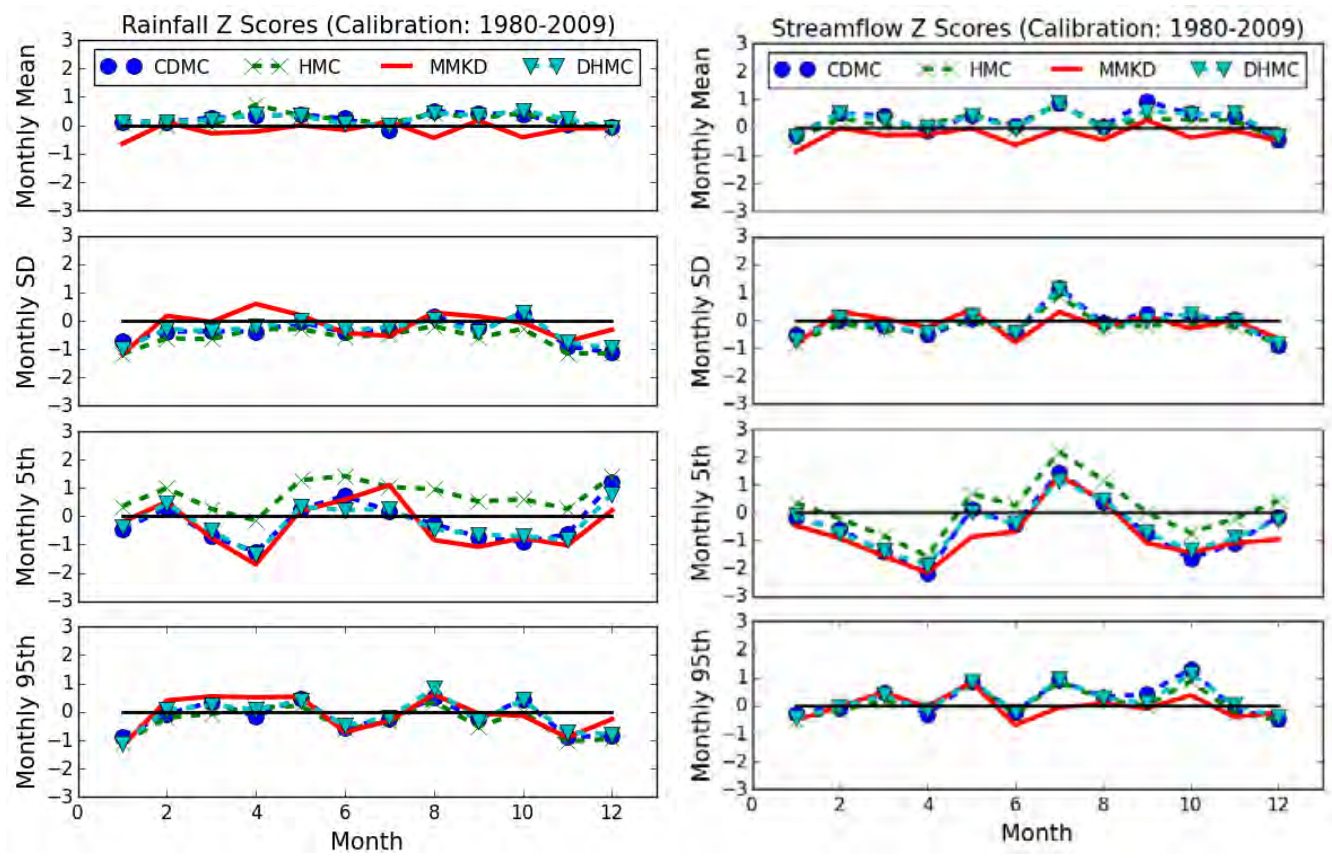

Figure I.14: Model comparison for monthly rainfall and streamflow of AWAP for calibration period at Chichester. 

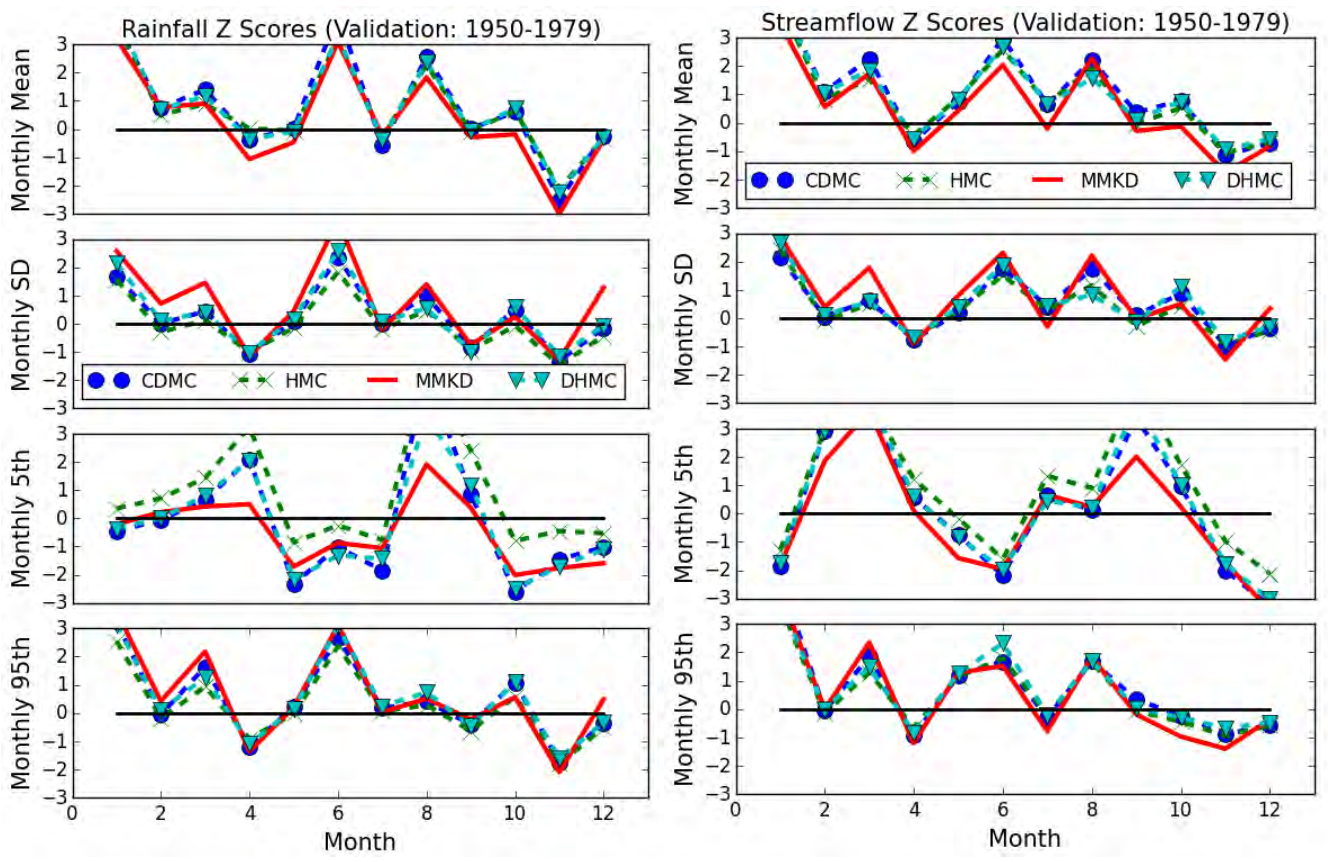

Figure I.15: Model comparison for monthly rainfall and streamflow of AWAP for validation period at Chichester.
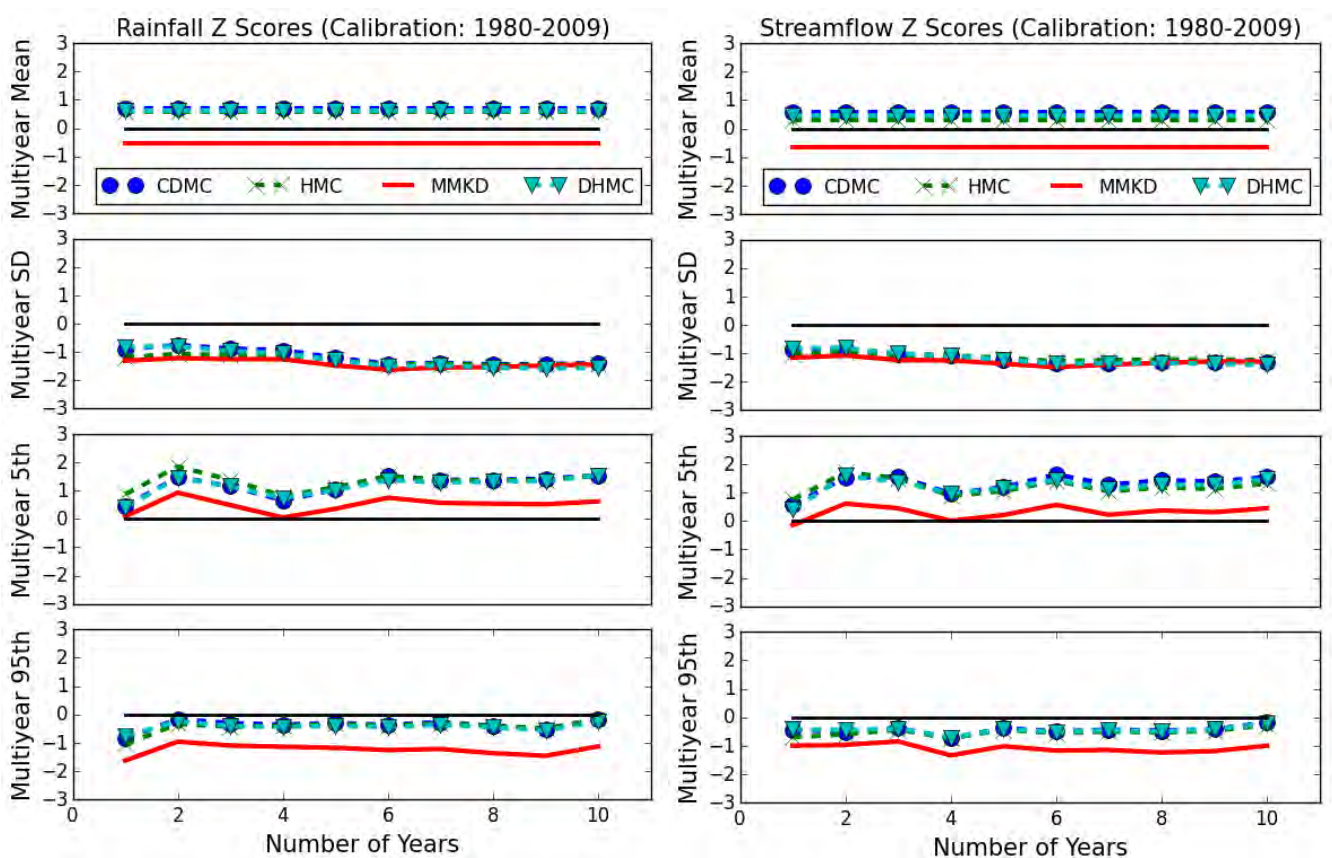

Figure I.16: Model comparison for multiyear rainfall and streamflow of AWAP for calibration period at Chichester. 

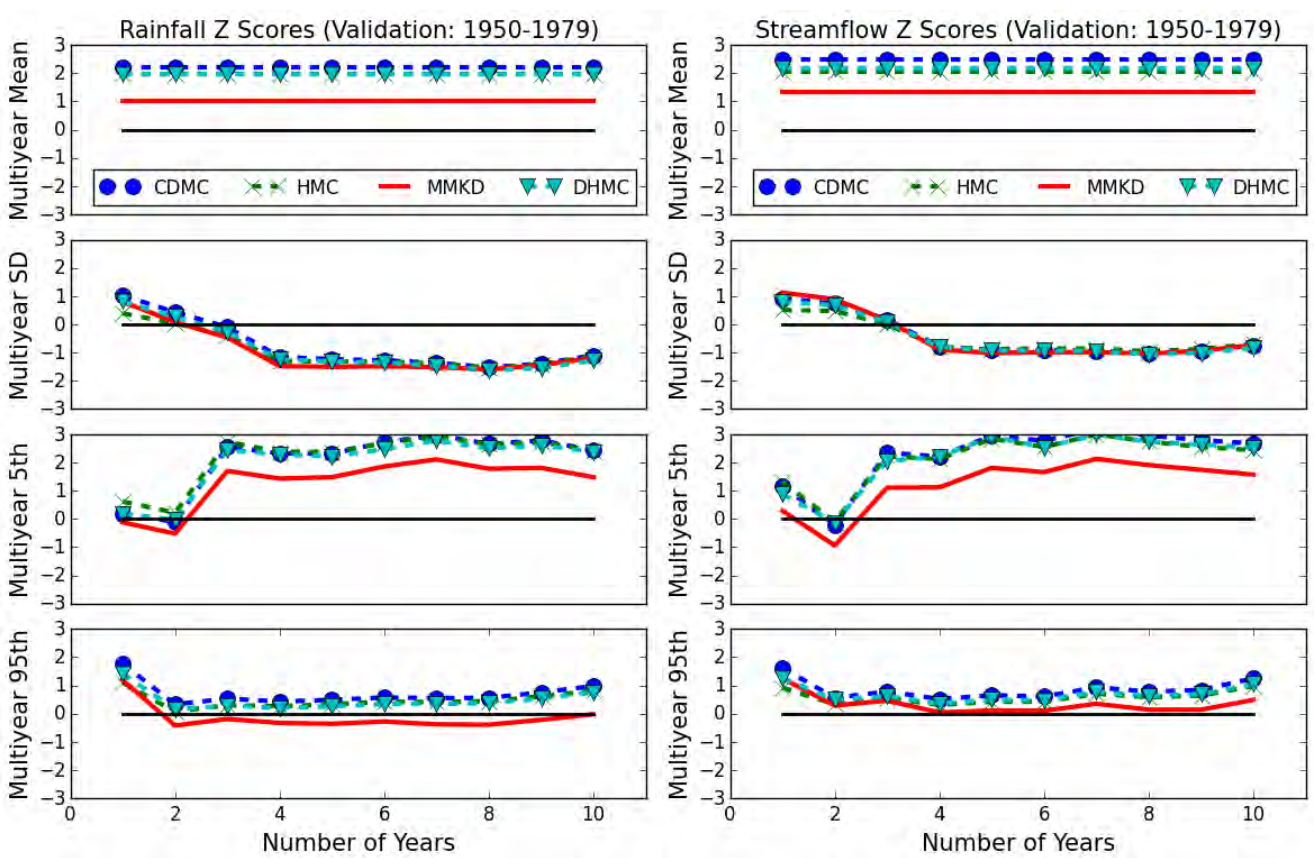

Figure I.17: Model comparison for multiyear rainfall and streamflow of AWAP for validation period at Chichester.

\section{I.3.3 Model Comparison at Grahamstown using AWAP}

Figure I.18-I.21 compare the model performances for rainfall and streamflow of AWAP at Grahamstown, which are consistent with the respective model performances for RCM1.
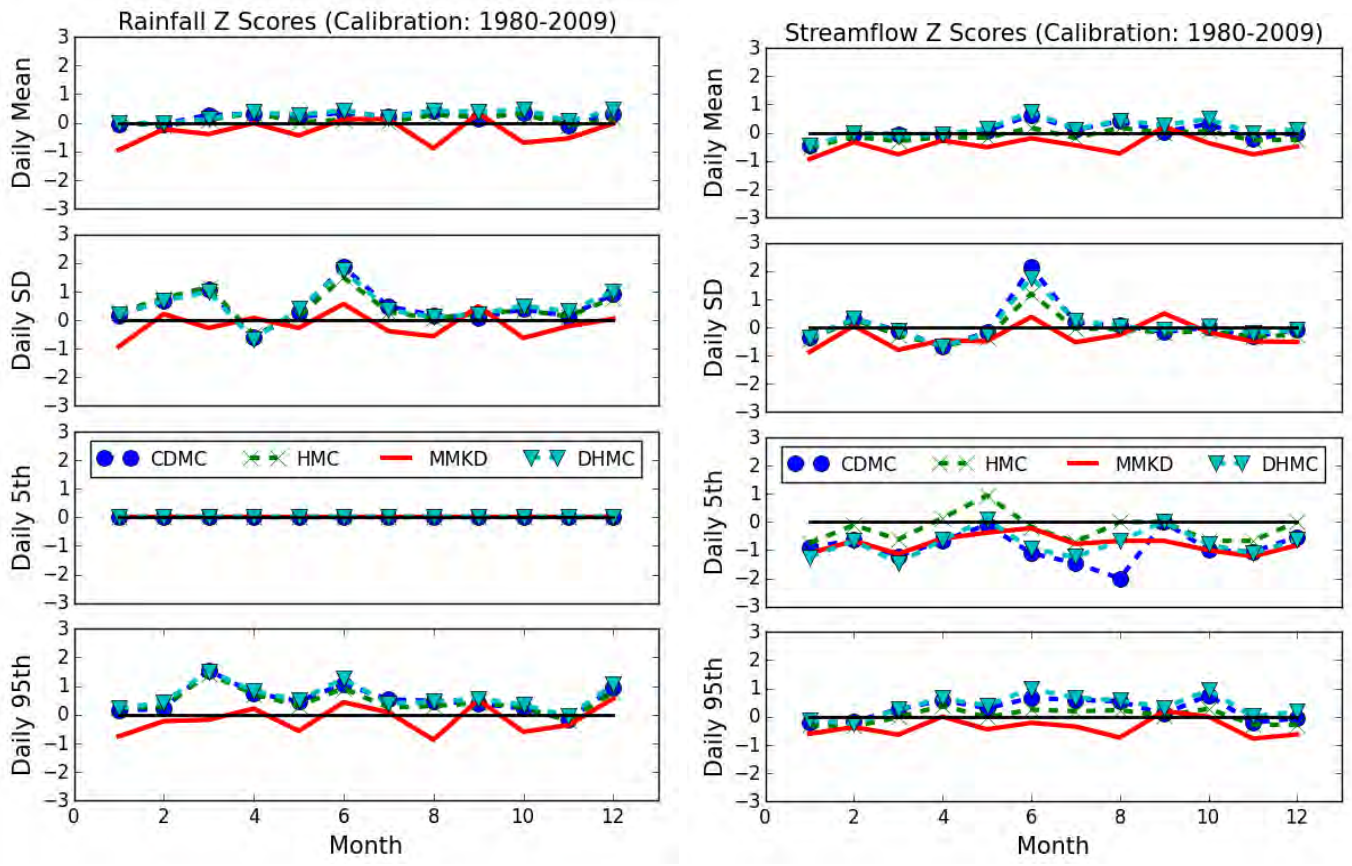

Figure I.18: Model comparison for daily rainfall and streamflow of AWAP for calibration period at Grahamstown. 

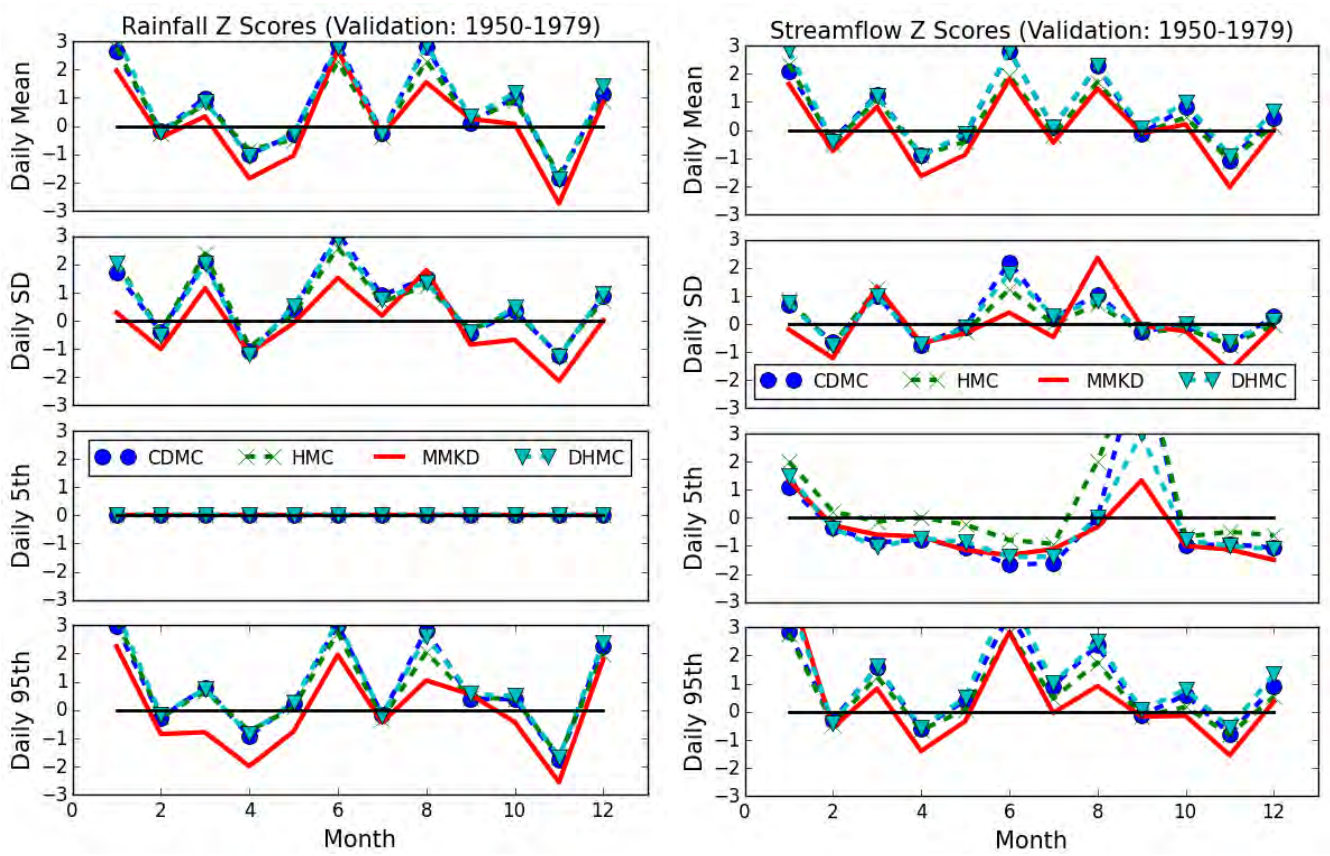

Figure I.19: Model comparison for daily rainfall and streamflow of AWAP for validation period at Grahamstown.
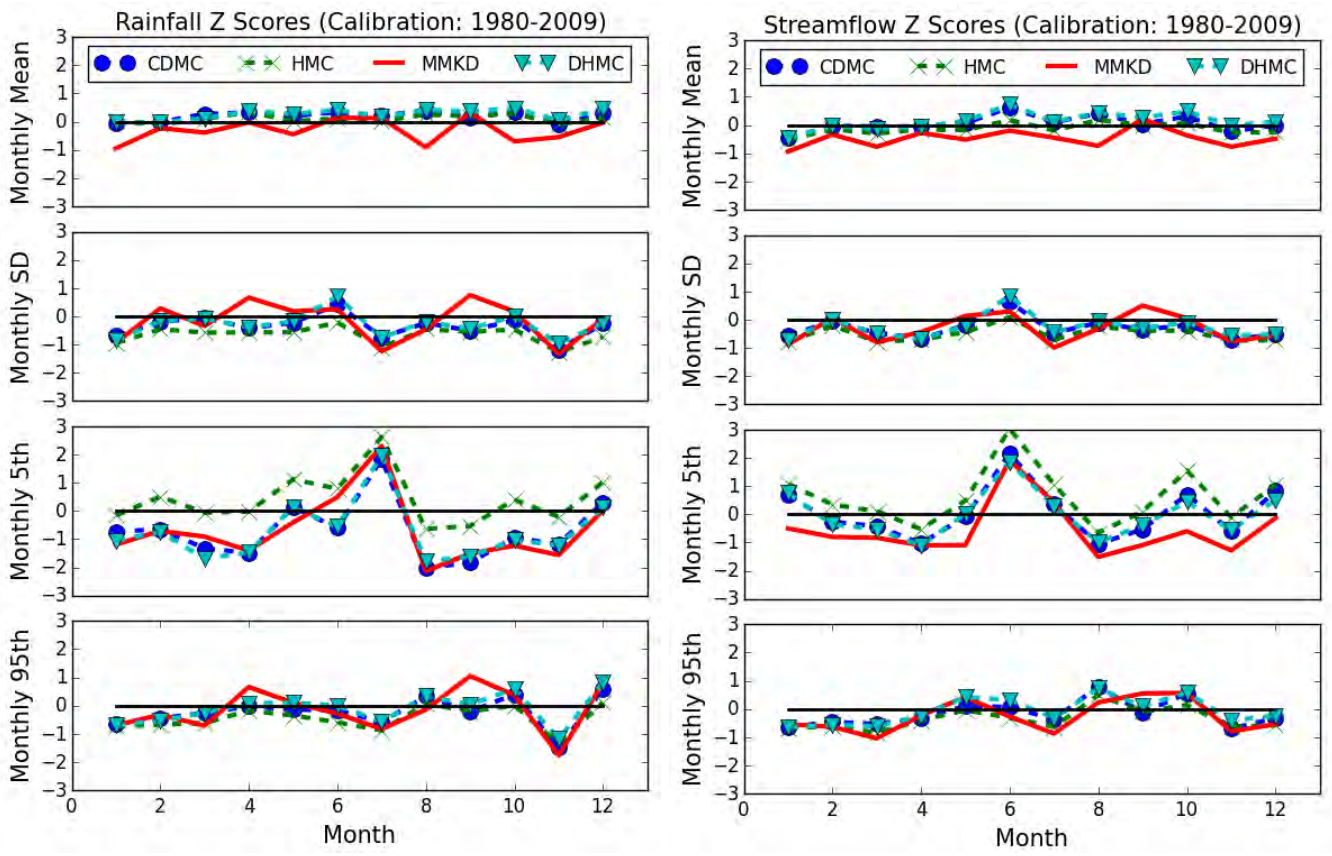

Figure I.20: Model comparison for monthly rainfall and streamflow of AWAP for calibration period at Grahamstown. 

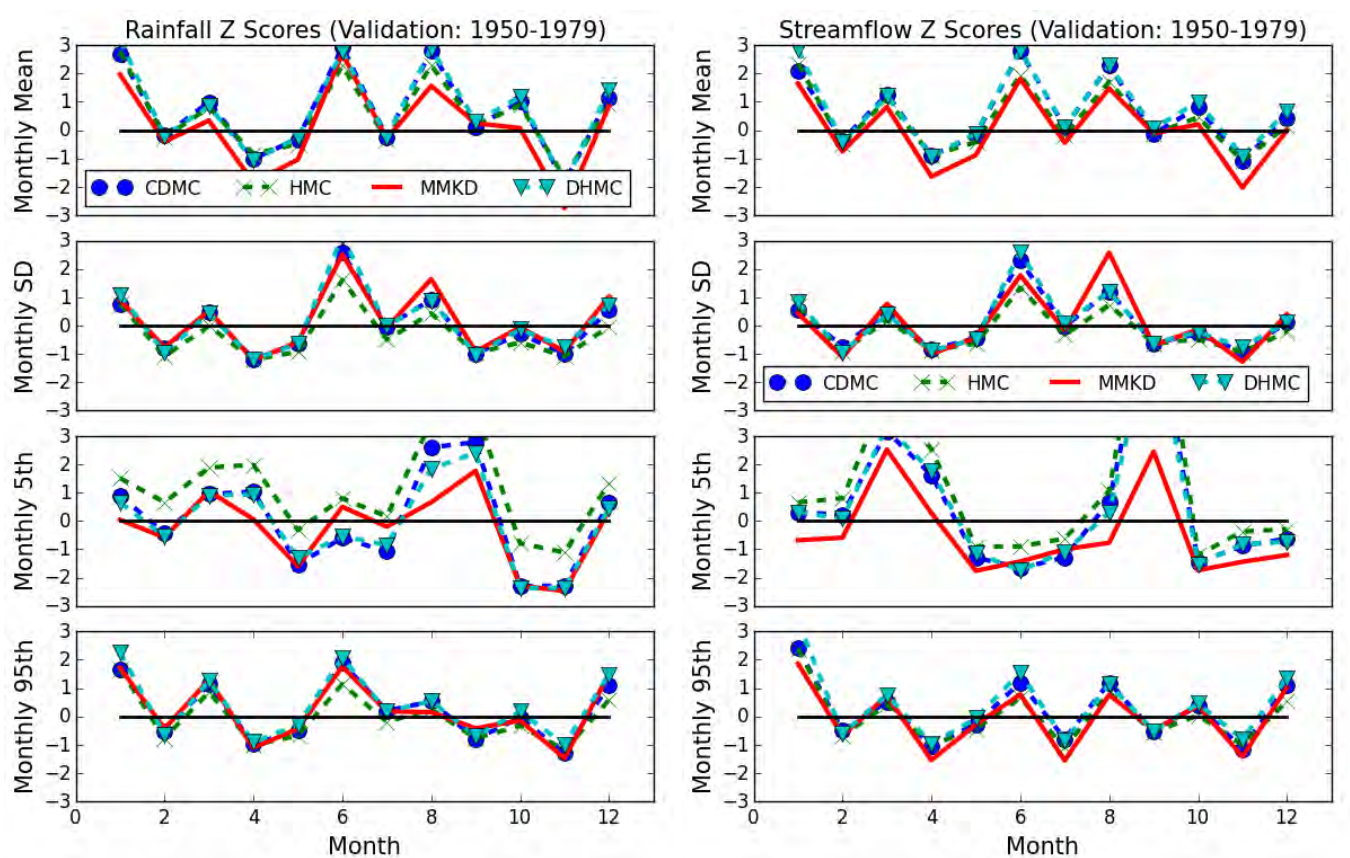

Figure I.21: Model comparison for monthly rainfall and streamflow of AWAP for validation period at Grahamstown.

\section{I.3.4 Model Comparison at Tillegra using AWAP}

Figure I.22-I.25 compare the model performances for rainfall and streamflow of AWAP at Tillegra, which are consistent with the respective model performances for RCM1.
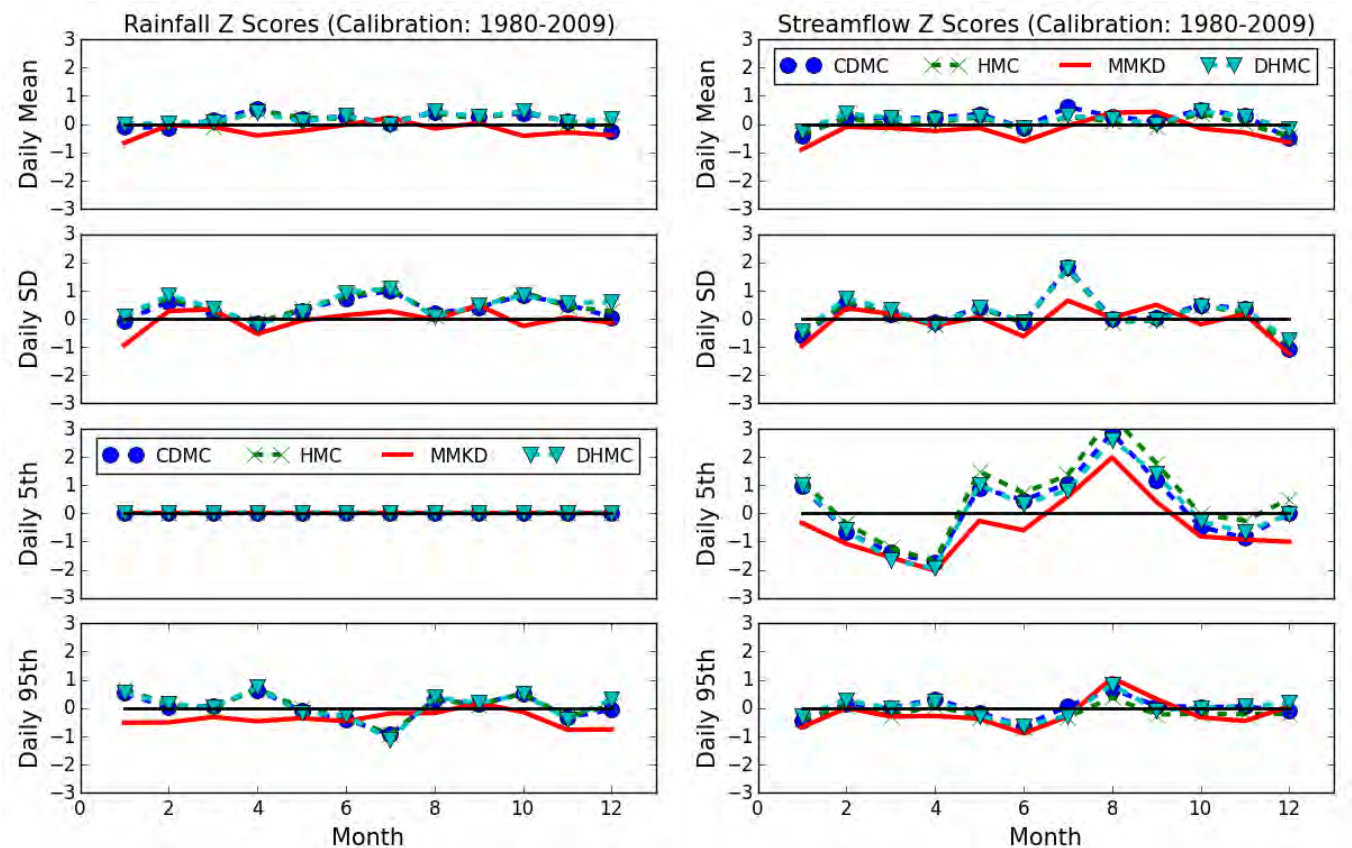

Figure I.22: Model comparison for daily rainfall and streamflow of AWAP for calibration period at Tillegra. 

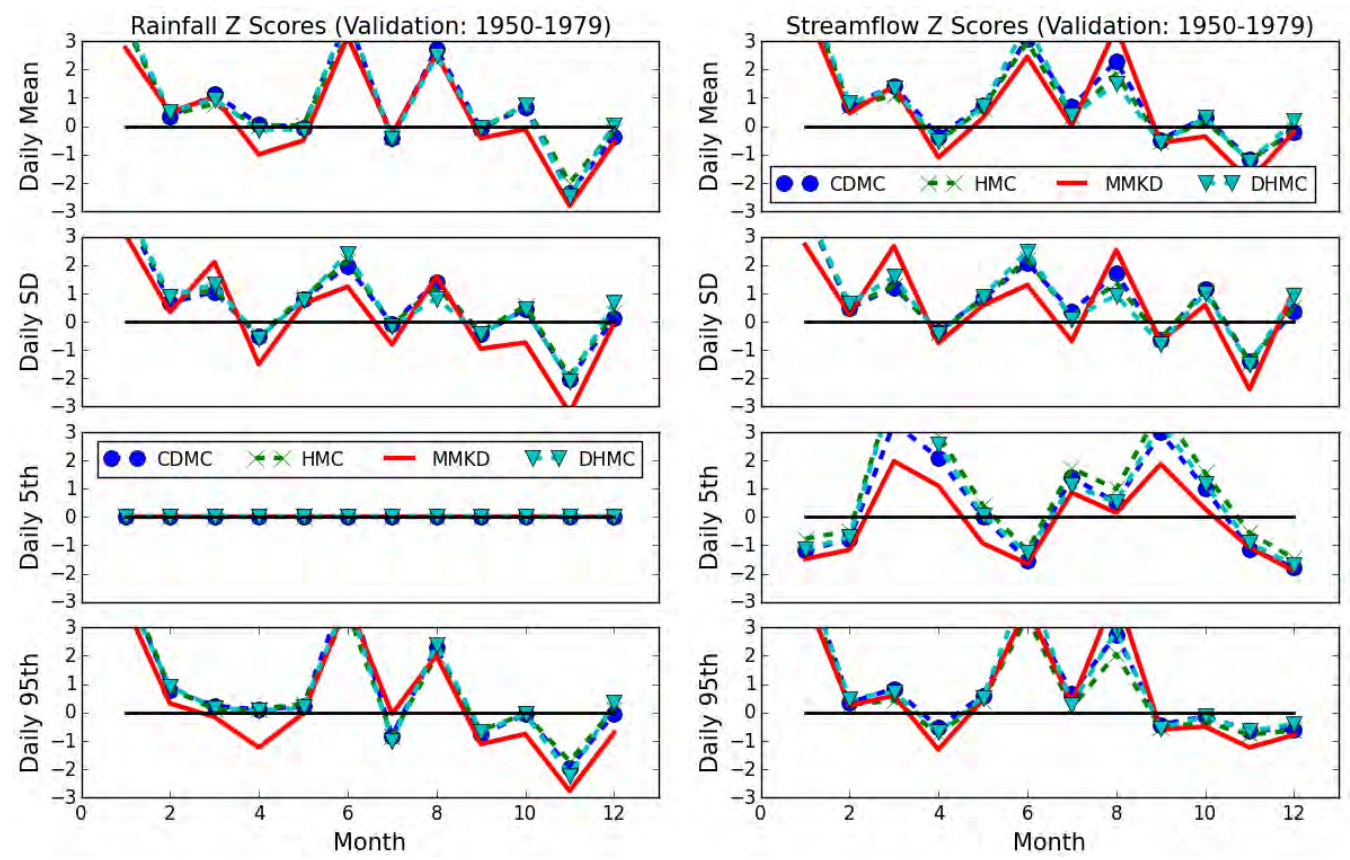

Figure I.23: Model comparison for daily rainfall and streamflow of AWAP for validation period at Tillegra.
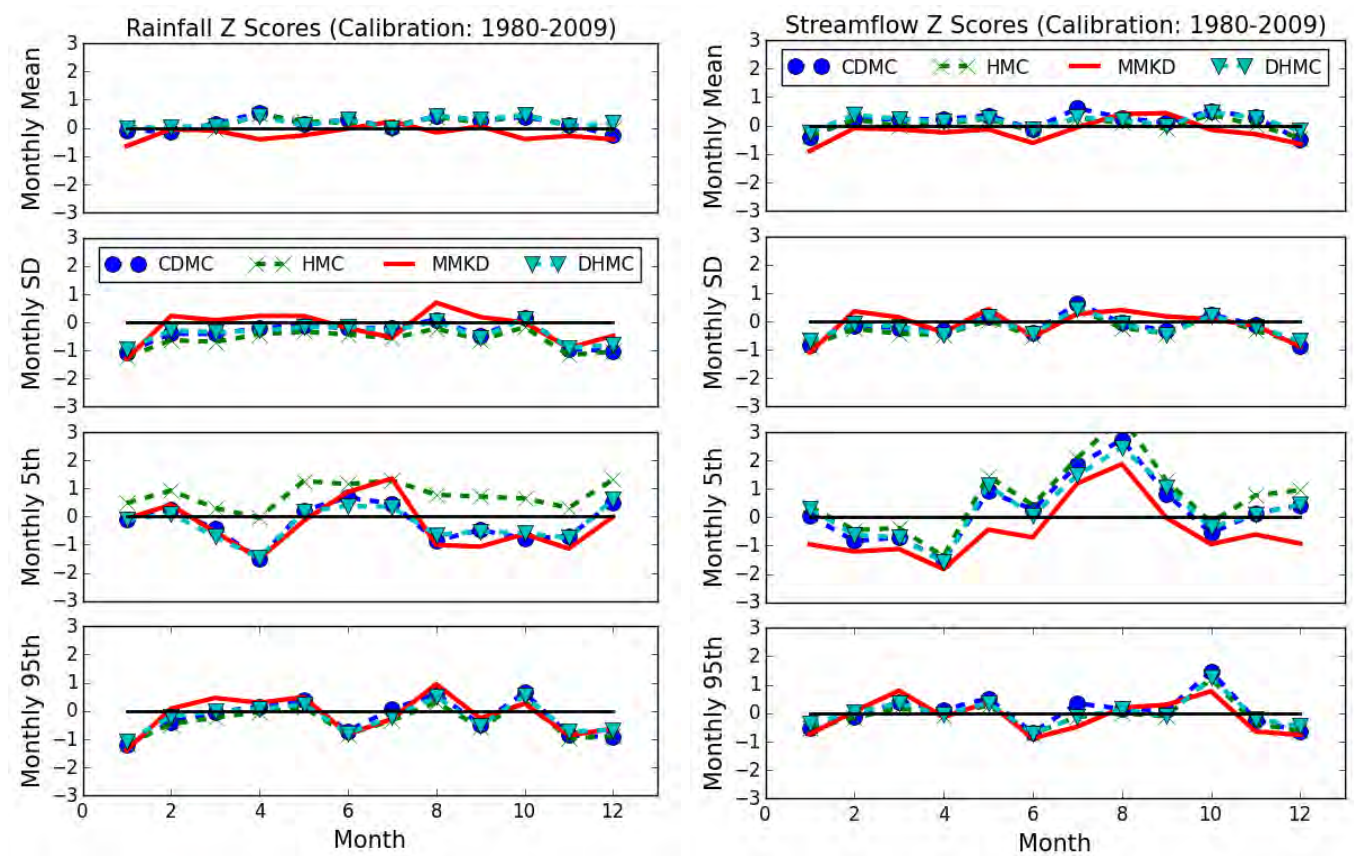

Figure I.24: Model comparison for monthly rainfall and streamflow of AWAP for calibration period at Tillegra. 

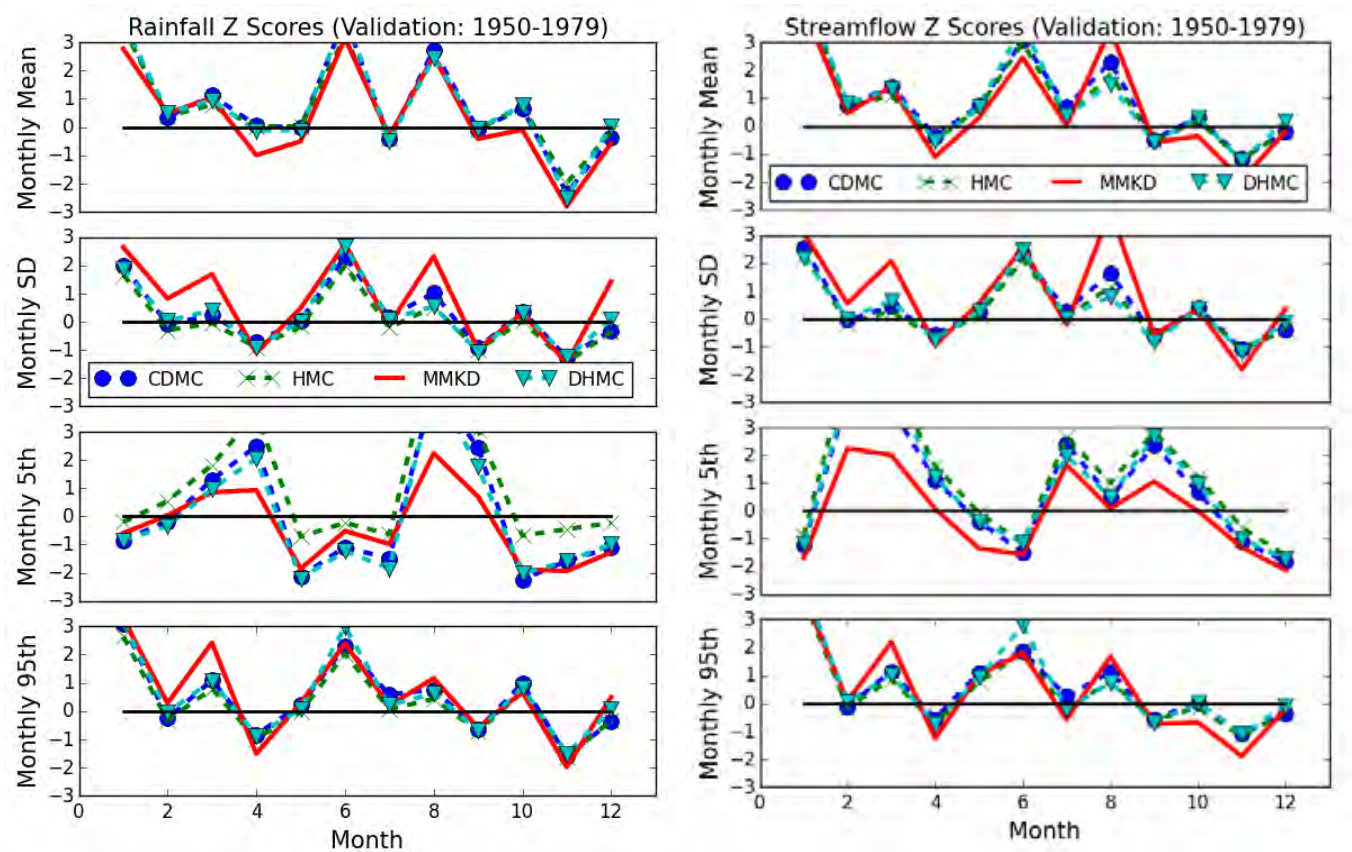

Figure I.25: Model comparison for monthly rainfall and streamflow of AWAP for validation period at Tillegra.

\section{I.3.5 Model Comparison for Monthly Statistics at Grahamstown and Tillegra}

Figures in this appendix compare the monthly rainfall and streamflow of RCM1 at Grahamstown and at Tillegra.
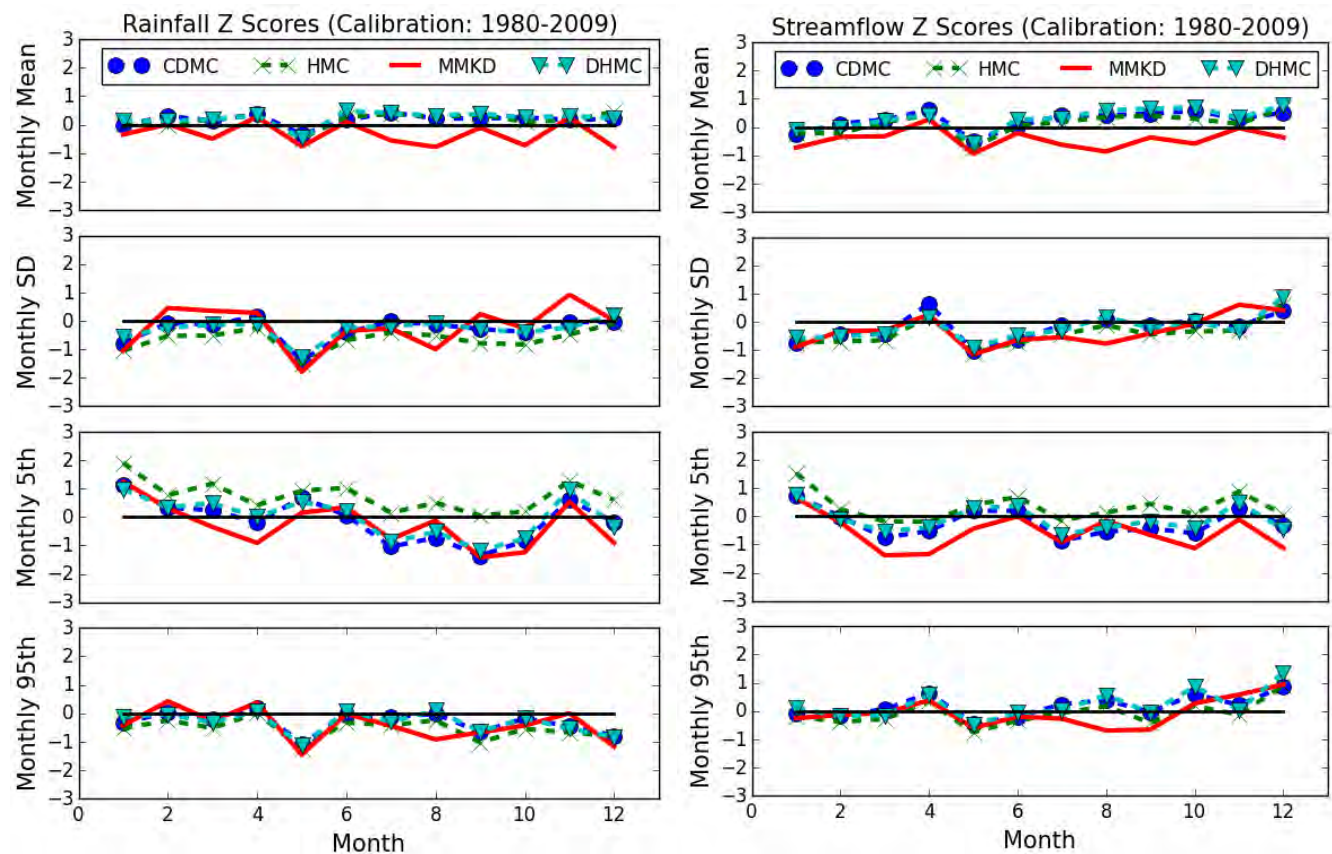

Figure I.26: Model comparison for monthly rainfall and streamflow of RCM1 for calibration period at Grahamstown. 

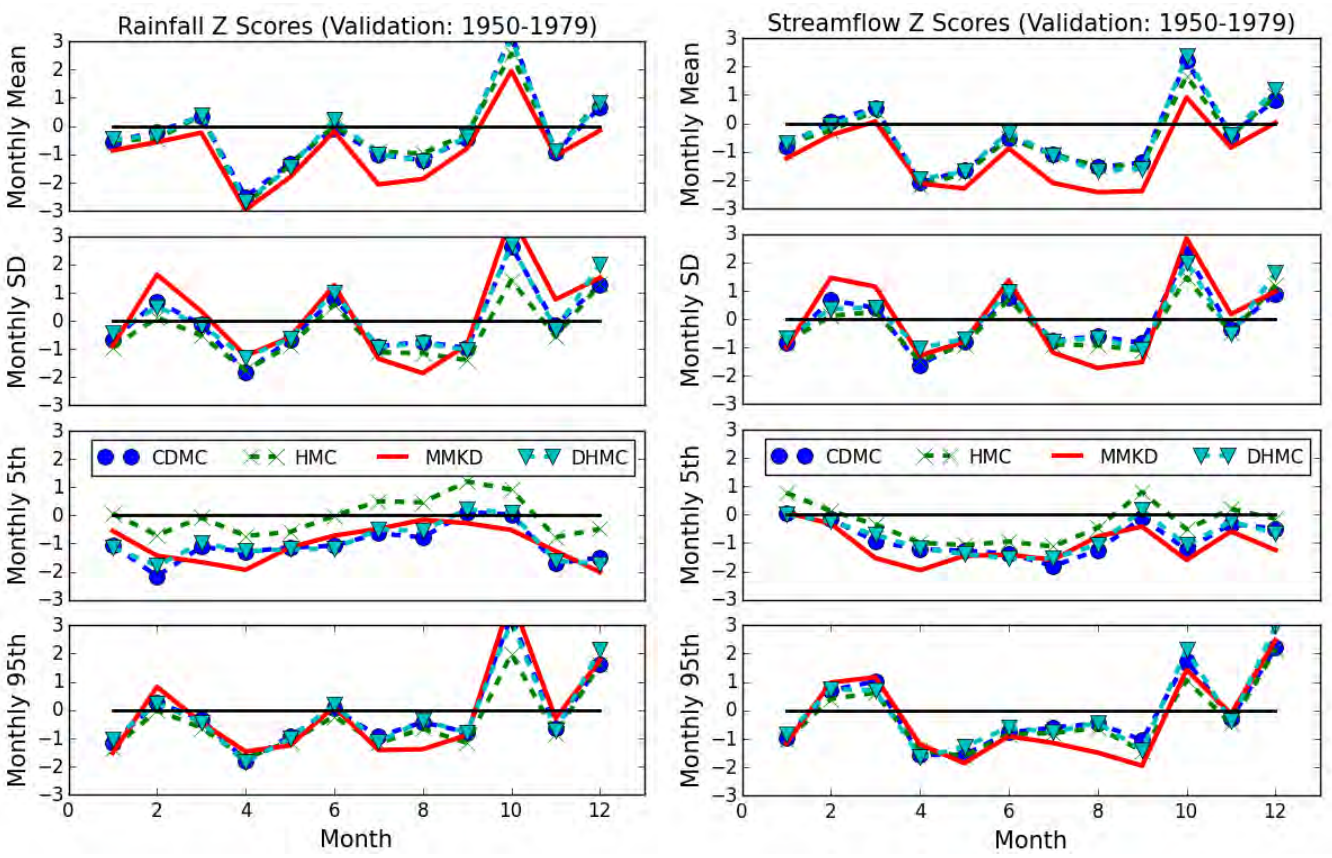

Figure I.27: Model comparison for monthly rainfall and streamflow of RCM1 for validation period at Grahamstown.
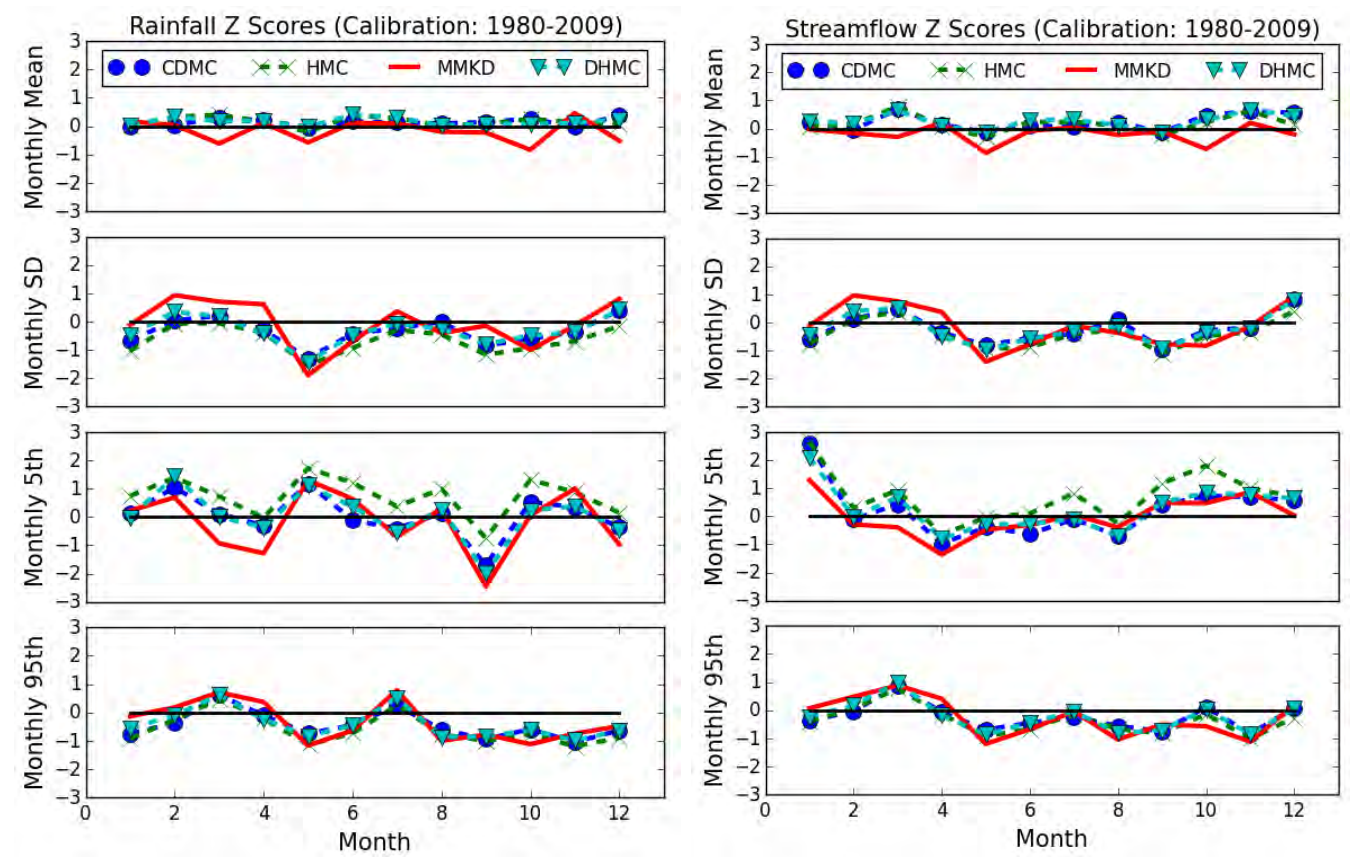

Figure I.28: Model comparison for monthly rainfall and streamflow of RCM1 for calibration period at Tillegra. 

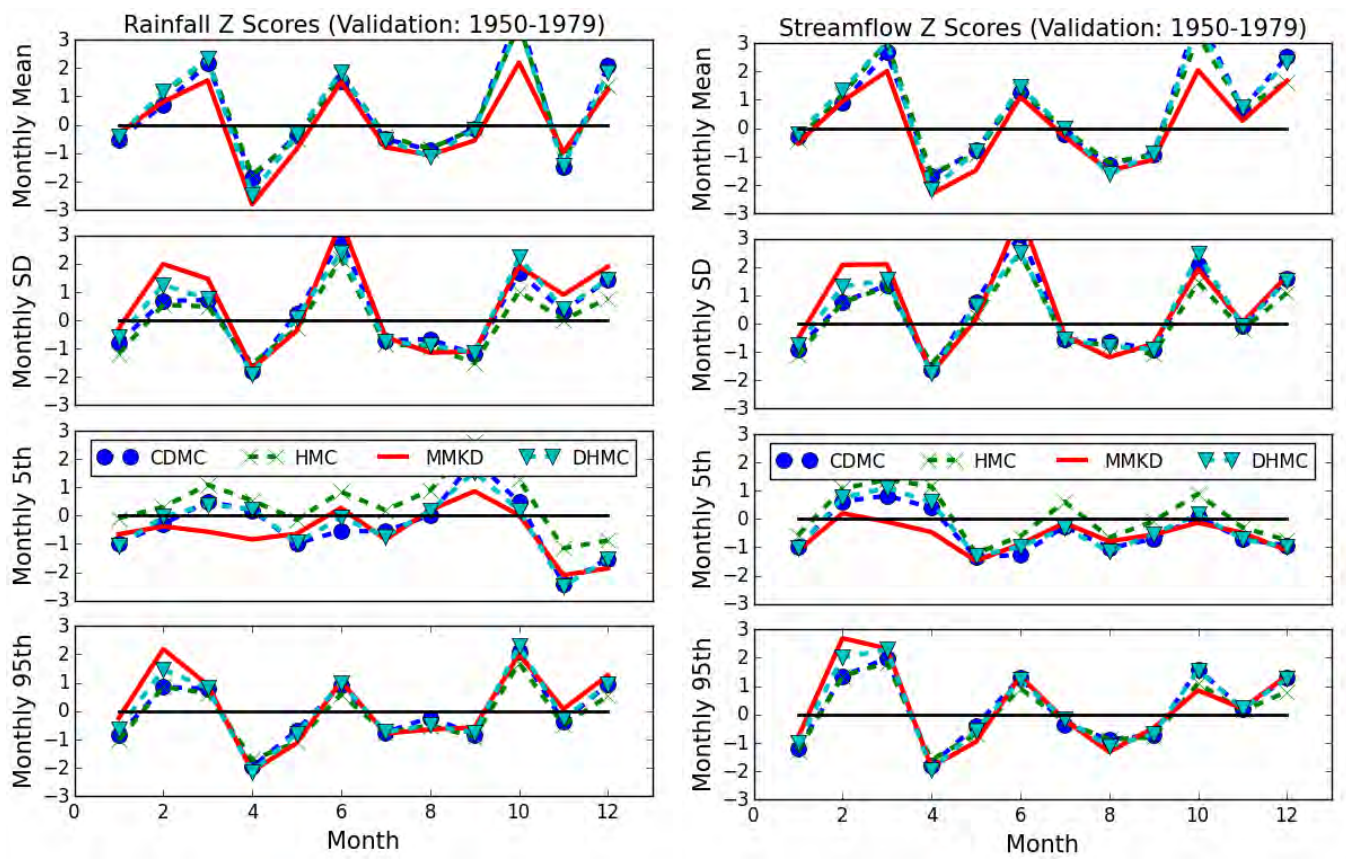

Figure I.29: Model comparison for monthly rainfall and streamflow of RCM1 for validation period at Tillegra.

\section{I.4 Model Comparison for Autocorrelation at Chichester}

Figure I.30 and Figure I31 compare the models for autocorrelations of monthly rainfall and streamflow at Chichester,
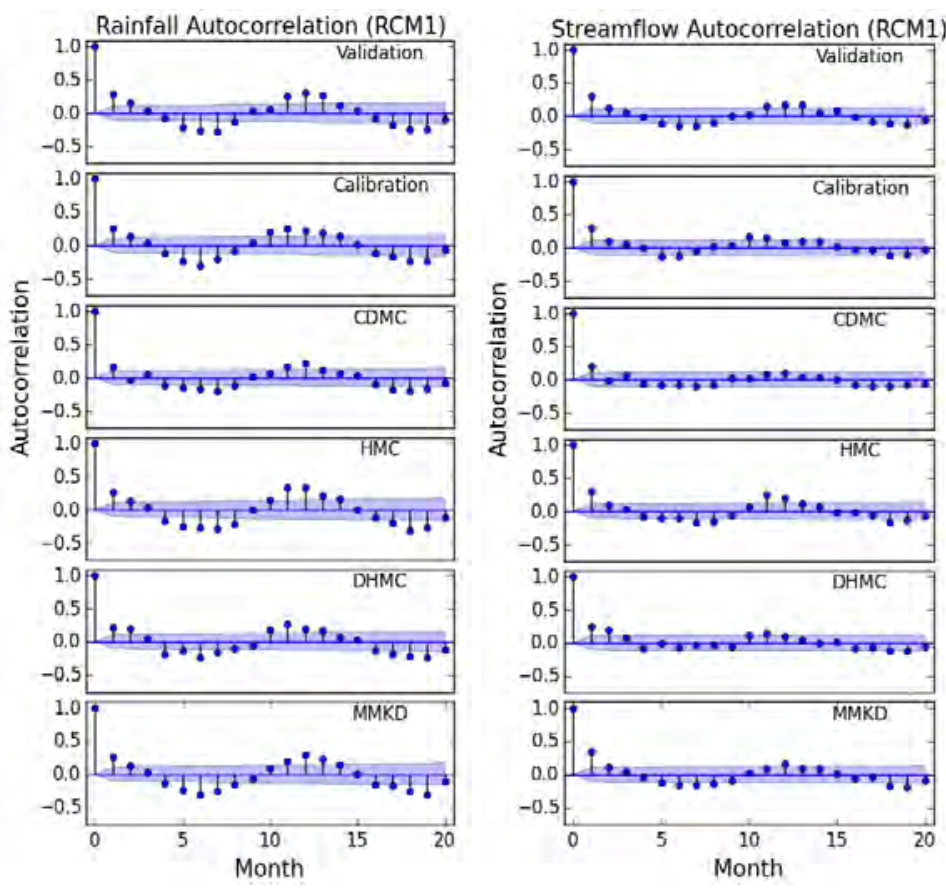

Figure I.30: Model comparison for autocorrelations of monthly rainfall and streamflow of RCM1 at Chichester. 
I. Comparison of MC and MMKD for Streamflow Generation
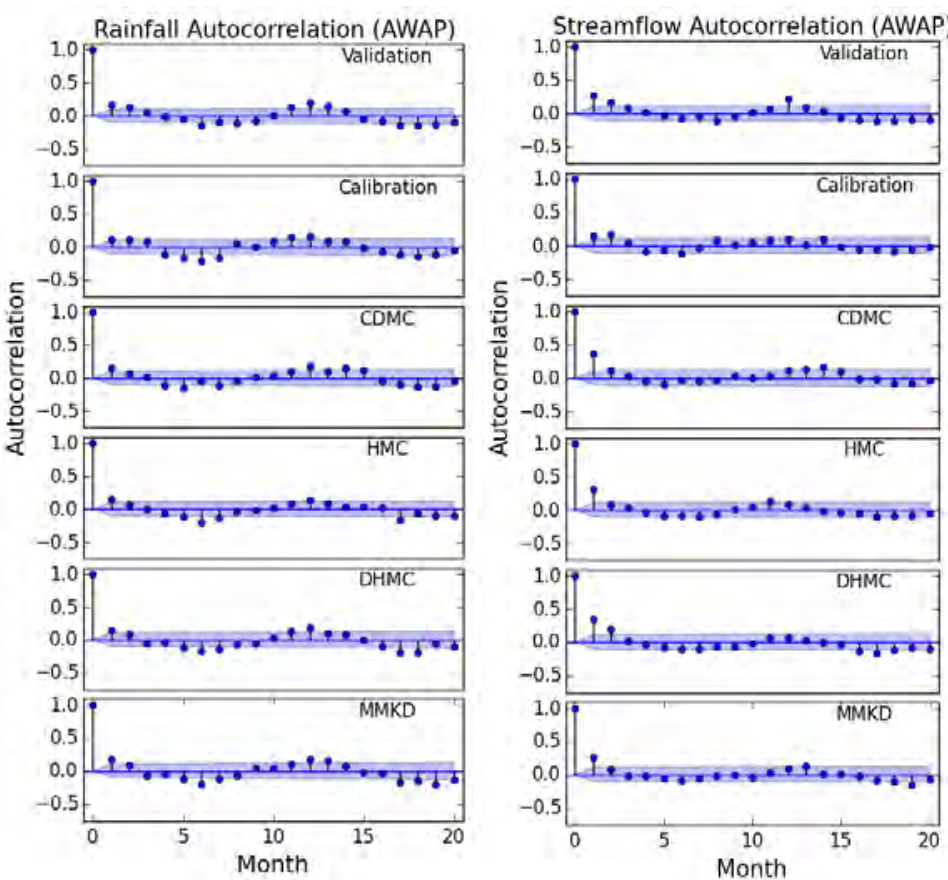

Figure I.31: Model comparison for autocorrelations of monthly rainfall and streamflow of AWAP at Chichester. 